\title{
Evolution
}

from

AGB Star

to

\section{Planetary Nebula}

\begin{abstract}
by
Emanuel Vassiliadis

A thesis submitted for the degree of Doctor of Philosophy of the Australian National University.
\end{abstract}

1st August, 1992

Mount Stromlo and Siding Spring Observatories

Institute of Advanced Studies

The Australian National University

Canberra 


\section{Errata}

page ix, line 8: Delete the.

page 9, last paragraph of section 1.3: Include ... Current deconvolution techniques of Planetary and Faint Object Camera images allow sub-arcsecond structures to be resolved.

page 11, line 10: Change ...optically thick due to dust ... to ...optically thick due to gas and dust.

page 12, line 22: Delete and $H \alpha \lambda 6563 \AA$.

page 12, line 23: Include ... Since most of the electrons arise from ionised hydrogen atoms, optical emission from non- $H$ species will likely show different structures than those observed in the radio continuum.

page 24, line 28: Delete homogeneous.

page 25, lines 17-19: Change..${ }^{14} N$ is less abundant in the $L M C$ and SMC than in the Sun by an additional factor of $\sim 2$, and the $\ldots$ to $\ldots{ }^{14} N$ is less abundant in the LMC and SMC, than in the Sun, by additional factors of $\sim 2$ and $\sim 4$, respectively, and thus...

pages 27-28: Include ... Given the present state of knowledge from observations, it is not known if equations 2.2-2.4 should show any metallicity dependence. However, if pulsation is largely responsible for determining $\dot{M}$, then such a mechanical process could plausibly be independent of metallicity. In contrast, $v_{\exp }$ probably does depend on metallicity. Recent observations of LMC OH/IR stars suggest $v_{\text {exp }}(L M C) \sim 1 / 2 \times v_{\text {exp }}($ Galaxy) (Wood et al. 1992, ApJ, 397, 552).

page 57, fig. 2.8: Change 4000 to 1000 in caption.

page 97, line 24: Include ... Some multiple shell PNe have intershell ages comparable with interpulse timescales, yet some others do not (Frank, A., Balick, B., Ė van der Veen, W. 1992, IAU Symposium 155, Planetary Nebulae, Innsbruck, Austria.

page 104, fig. 2.41: Change $Z=004$ to $Z=0.004$ in caption.

page 119, lines 15-16: Change ... in the $P N$ regime ... Pauldrach et al. to ... from the $O H / I R$ stars, through the intermediate spectral types, and to the $P N$ regime itself.

page 119, line 16: See page insert between pages 118 and 119.

Continued Next Page/ 


\section{ERrata-Cont'd.}

page 144 , table 3.4: Ignore data pertaining to $(M, Y, Z)=(2.5,0.25,0.016)$. This is a H-burning sequence, with an artificially induced phase of departure from the AGB, which is not discussed in the text.

page 165, line 9: Include ... If $\dot{M}$ is a factor of 2 larger on the $A G B$, the lower mass models might be expected to lose their envelopes in less flashes, and probably in just a single flash. Consequently, most of the ejected material would only be concentrated in a single shell. However, raising $\dot{M}$ would shorten the AGB lifetimes and affect the $A G B$ tip luminosities, which we have found to agree quite well with observations in LMC clusters (see Chapter 2).

page 175, line 13: Include $\ldots$ The negative reddening values $(H \alpha / H \beta<2.94)$ can be used to estimate the measurement errors. From 18 such cases, the mean weighted reddening constant is -0.12 with a 1-sigma error of 0.09 .

page 176, section 5.3.4: Include ... Sky rows were selected manually to avoid any regions of diffuse emission in the Magellanic Clouds, and as close as possible to the signal rows to minimize distortion effects in the spatial axis, for each CCD. page 179, section 5.4.1: Include ...Using $H \alpha / H \beta$ alone to determine the reddening constant would have been acceptable if all objects were faint. However, there were a significant number which were bright, comparable to the ones studied in Meatheringham and Dopita. To be consistent with that work, and be internally self-consistent, it was decided to persist with using all three Balmer line ratios.

pages 180-203, table 5.4: Note... Objects with $T_{e}[O I I I] \gtrsim 25000 \mathrm{~K}$ are probably akin to the highly energetic PN in the LMC reported by Dopita, Ford, \& Webster 1985, ApJ, 297, 593. The values of $T_{e}[\mathrm{OIII}]$ presented in table 5.4 are in the zero-density-limit. Consequently, proper determination of $T_{e}[O I I I]$ requires knowledge of the density.

page 205, line 2: Include ... The average c values were calculated with the inclusion of the individual negative $\mathrm{c}$ values.

page 216, line 9: Change $T_{\text {eff }}$ to $T_{e}[O I I I]$.

page 233, last line: Include ... There is a potential error due to placement of the $P N$ image off-center on the slit. From the comparison with the measurements made by $M D F W$, this error can be no more than the order of $2-3 \mathrm{~km} \mathrm{~s}^{-1}$.

page 234, fig. 6.2: Change paner to panel in caption.

page 241, table 6.4: Note ... The errors in velocity and position angle for the complete MDFW sample, and the combined MDFW and new sample, are of the order of $2 \mathrm{~km} \mathrm{~s}^{-1}$ and $3^{\circ}$, respectively. The smaller samples have errors of the order of $5 \mathrm{~km} \mathrm{~s}^{-1}$ and $10^{\circ}$, in velocity and position angle, respectively. 
To my Mother and Natalie 


\section{Disclaimer}

I hereby declare that the work in this thesis is that of the candidate alone, except where indicated below, and where indicated in the text:

Chapter 1 The majority of the text was written by the candidate. Comments were given by Dr. P. R. Wood.

Chapter 2 The majority of the models were calculated by the candidate, with close supervision by Dr. Wood. Enhancements to the Mount Stromlo Stellar Structure Code were primarily made by Dr. Wood. The candidate was responsible for changing the adopted mass loss formalism. The latter half of the asymptotic giant branch phases for the $5.0 \mathrm{M}_{\odot}$ cases were recalculated solely by Dr. Wood due to computational difficulties. The majority of the text was produced by the candidate. This work is intended for publication in the Astrophysical Journal.

Chapter 3 All models into the planetary nebula regime were calculated by the candidate, again, under close supervision by Dr. Wood. The candidate was responsible for changing the adopted mass loss formalism in the Mount Stromlo Stellar Structure Code. The majority of the text was written by the candidate, with comments given by Dr. Wood. This work is intended for publication in the Astrophysical Journal.

Chapter 4 The candidate and Dr. Wood contributed equally to the text presented. The hydrodynamic sequence presented was calculated by Dr. Wood using input calculated by the candidate in Chapter 2 . The hydrodynamic code was altered by the candidate and Dr. Wood to include the temporal evolution of mass loss and to modify the existing boundary conditions, respectively. This work is intended for publication in the Astrophysical Journal. A prelimary version of this work has been accepted for publication in the Procedings of the Astronomical Society of Australia, and will appear in 1992. 
Chapter 5 The majority of the text was written by the candidate, with comments given by Drs. M. A. Dopita and D. H. Morgan (Royal Observatory, Edinburgh). Drs. Dopita and Morgan also assisted with obtaining the observations. Dr. Morgan provided finding charts and coordinates for newly discovered planetary nebulae prior to publication. Dr. Dopita obtained supplementary $\mathrm{H} \alpha$ images, which were reduced by J. F. Bell. All spectrophotometric data was reduced by the candidate. This work has been accepted for publication in the Astrophysical Journal Supplements, and is scheduled to appear in November, 1992: The authors of the paper are E. Vassiliadis, M. A. Dopita, D. H. Morgan, and J. F. Bell.

Chapter 6 The majority of the text was written by the candidate, with comments provided by Drs. S.J. Meatheringham and M. A. Dopita. The échelle observations were obtained by the candidate, and Drs. Dopita and D. H. Morgan. The rotation solution was calculated by Dr. Meatheringham using the MINUIT program. All data was reduced by the candidate. This work has been accepted for publication in the Astrophysical Journal, and is scheduled to appear in August, 1992. The authors of the paper are E. Vassiliadis, S. J. Meatheringham, and M. A. Dopita.

Chapter 7 The majority of the text was written by Dr. M. A. Dopita, with contributions from Dr. G. H. Jacoby and the candidate. Specifically, the candidate was responsible for providing the theoretical models used in the calibration. The candidate and Dr. Dopita contributed equally to section 7.5. This work has been published in the Astrophysical Journal, volume 389, page 27, April 1992.

Signed:

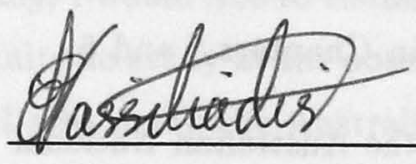

Emanuel Vassiliadis 


\section{Acknowledgements}

I wish to extend my sincere gratitude to my thesis supervisors Drs. P. R. Wood and M. A. Dopita for allowing me to undertake this research under their tutelage. I also wish to thank my thesis advisor Dr. D. J. Faulkner for his assistance during my supervisors' absence, and in his capacity as student coordinator towards the later stages of my thesis production. I thank the director of Mount Stromlo \& Siding Spring Observatories, Professor A. W. Rodgers, for giving me the opportunity to conduct this research at the Observatories.

Dr. David Morgan of the Royal Observatory, Edinburgh, was kind enough to supply an extensive sample of finding-charts for newly discovered planetary nebulae in the Large and Small Magellanic Clouds before publication. These charts were invaluable for the work presented in Chapter's 5 and 6 . I greatly appreciate the time and effort Dr. Morgan volunteered in coming to Australia, and being present for the majority of the observations conducted. I would also like to thank Jeanette Barnes at Kitt Peak National Observatory for her assistance in explaining the use of the IRAF data reduction software for the analysis of the data presented in Chapters 5 and 6 .

All observations for this thesis were carried out with the Australian National University's 2.3 metre Advanced Technology Telescope at Siding Spring Observatory. The generous allocation of observing time by the Mount Stromlo \& Siding Spring Observatories Time Allocation Committee is greatly appreciated, especially when oversubscription of observing time was higher than usual. I would also like to thank the people at Siding Spring Observatory for making my visits as pleasant as possible, and maintaining functionality of the computers and telescope during my observing runs. A very special mention should be made of Peter Young who was able to offer me assistance in rectifying major difficulties at the telescope one New Year's Day from over 600 kilometres away!

Due to the nature of the work carried out in this thesis, a substantial portion 
has heavily relied on the newly installed Unix computer systems at Mount Stromlo Observatory. Again, my gratitude is extended to past and present members of the Computer Section; in particular, Peter Young, Dr. Stephen Meatheringham and Dr. Roger Brissenden.

I am indebted to Dr. Russell Lavery and Dr. Glen Mackie for providing me with a rare view of life from the Newtonian cage of the Anglo-Australian Telescope, when I was assisting for one of their observing runs. Similary, I must thank Nicholas Achilleos for showing me the joys of observing a single white dwarf all night.

I wish to extend my most sincere well-wishes to the administrative people who really keep the observatory functioning: Gitta Benzin, Maggie Miller, Thelma Johnson and Milka Angelic.

Apart from the financial assistance granted to me from Mount Stromlo, which finally allowed me to study astronomy at a professional level, I wish to extend my sincere gratitude to my mother, Georgia Vassiliadis, for her personal sacrifice in seeing me reach this goal. My success is based on her determination to see me do well.

During my time at Stromlo, I've been extremely lucky in meeting Natalie Craig, the most beautiful woman in the world. She was the woman who had to put up with my daily emotions, and I thank her for her love and perserverance. Without the concern of Natalie, or my Mother, I don't think I would have come so far. Thank-you again to both ladies.

Finally, I would like to thank Mount Stromlo once more for initially offering me the opportunity to study at the observatory under an Australian National University scholarship. I also thank the Australian Government for funding under the Commonwealth and Australian Postgraduate Reseach Award schemes, at later stages of the thesis. 


\section{Abstract}

This thesis presents a theoretical and observational study of stellar evolution for low to intermediate mass stars $\left(\sim 1-8 \mathrm{M}_{\odot}\right)$, from the asymptotic giant branch (AGB) phase to the planetary nebula $(\mathrm{PN})$ phase.

In chapter 2 we have undertaken a series of theoretical calculations using the Mount Stromlo Stellar Structure Code to produce 22 stellar evolutionary sequences, complete from the main sequence phase through to the AGB phase. Models are calculated for initial masses $0.89,0.945,1.0,1.5,2.0,2.5,3.5$, and $5.0 \mathrm{M}_{\odot}$, and metallicities $0.016,0.008,0.004$, and $0.001 \mathrm{Z}_{\odot}$. These abundance and mass values were chosen to allow comparison with Galactic, and Magellanic Cloud stars. An important feature of these calculations is the inclusion of an empirical relationship between mass loss rate and pulsation period for AGB stars. The calculations lead naturally to a superwind phase, where the AGB is terminated by severe mass loss over the last 2-3 thermal pulse cycles. More precisely, the superwind phases occur towards the end of quiescent phases of helium shell flash cycles. The maximum AGB luminosities predicted from this work are in excellent agreement with those observed for Magellanic Cloud stars. The resulting initial-final mass relation predicts white dwarf masses $\sim 0.1 \mathrm{M}_{\odot}$ larger than the observational calibration of the relation.

In chapter 3 we continue the calculations presented in chapter 2 into the PN regime. Mass loss has also been included here, using an empirical formalism derived from observations of PN nuclei available in the literature. The evolutionary rates during the PN phase are examined, and are compared with those available in the literature. The calculations fall into two distinct groups; those where shell He burning is dominant, and those where shell $\mathrm{H}$ burning is dominant, when a star leaves the AGB phase. Low mass models are more likely to leave the AGB burning $\mathrm{He}$, as the results in Chapter 2 indicate the mass loss rate to be greatest immediately prior to a thermal pulse.

In chapter 4 we examine the hydrodynamic behaviour of the winds produced during 
the AGB phase. A preliminary investigation was conducted over one complete shell flash cycle. At a thermal pulse event, an increase in wind speed drives a shell into the slower, pre-existing, quiescent wind. Following from the thermal pulse event, there is a significant luminosity decrease which allows matter to fall back towards the stellar surface, and which results in the formation of a hollow circumstellar shell. These characteristics may have already been noted in observations previously presented in the literature.

In Chapter 5 we present optical spectroscopy in the the range $\lambda \lambda 3400-7900 \AA$ for a total of 52 planetary nebulae (PNe) in the Large Magellanic Cloud (LMC) and 18 in the Small Magellanic Cloud (SMC). This sample contains a large selection of objects of low luminosity, with measurements of line intensities down to $\sim 10 \%$ of $\mathrm{H} \beta$. Brighter objects have line intensities measured down to $\sim 2 \%$ of $\mathrm{H} \beta$. Reddening estimates from Balmer line ratios have been determined: unreddened and reddened line intensities are tabulated.

In Chapter 6 we present échelle observations of 16 LMC PNe in the [O III] $\lambda 5007 \AA$ emission line, 10 of which are previously unobserved. An important property of these objects is that they lie far from the centre of the LMC and hence, their velocities provide strong constraints on the rotation of the LMC. These data have been used in conjunction with previously published data to redetermine the position angle of the kinematic line of nodes, $\theta_{0}$, and the systemic Galactocentric velocity, $V_{0}$, of the LMC. After analysis of our data, combined with data available in the literature, we confirm the previous values derived from PNe, where $\theta_{0} \sim 170^{\circ}$ and $\mathrm{V}_{0} \sim 40 \mathrm{~km} \mathrm{~s}^{-1}$. The LMC is confirmed to consist of a single disk, and not a dual disk structure as originally proposed by Freeman, Illingworth and Oemler.

In chapter 7 , we examine the effects of stellar effective temperature, and stellar and nebular metallicities, on the emission line fluxes used in the planetary nebular cosmic distance scale determinations. An absolute calibration of this distance scale is made by determining the metallicity-corrected, cut-off in the luminosity function of PNe. The distance modulus to the LMC is derived by fitting shell He burning PN nucleus evolutionary tracks (presented in chapter 3 ) to the observations. Distance modulii, relative to the LMC, are also derived for the SMC, Galactic Bulge, M31, and the Virgo cluster, after correcting the absolute PN nuclei luminosities for both stellar age and metallicity effects, and using the observed luminosity function cut-offs for these systems. In all the cases where Cepheid distances are known, a -0.14 mag zero-point offset is found with respect to the PN distance scale. It is concluded that the principle 
source of uncertainty in the PN distance scale is the intrinsic upper luminosity cut-off of the PN nuclei which, in turn, is critically dependent upon the stellar age (and therefore, mass) distribution, and the treatment of mass loss in the stellar evolutionary models. 


\section{Contents}

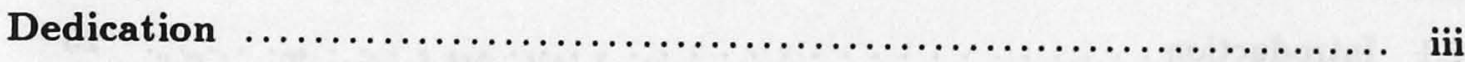

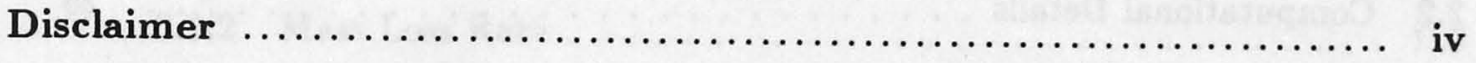

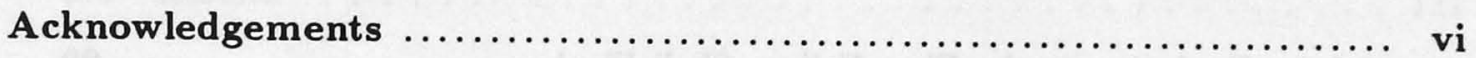

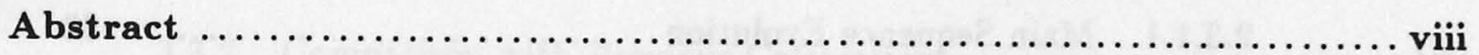

1 Introduction 1

1.1 Overview of the Evolution of Low and Intermediate Mass Stars . . . . . . . . . . . . . . . . 1

1.2 Asymptotic Giant Branch Evolution ............... 2

1.2.1 Theoretical Studies . . . . . . . . . . . . . . 2

1.2.2 Observational Identification $\ldots \ldots \ldots \ldots \ldots \ldots$

1.2.3 Mass Loss on the AGB $\ldots \ldots \ldots \ldots$

1.3 Planetary Nebulae ......................... 8

1.4 The Evolution of Planetary Nebulae . . . . . . . . . . . 9

1.4.1 AGB stars as the Progenitors of Planetary Nebulae . . . . . . . 9

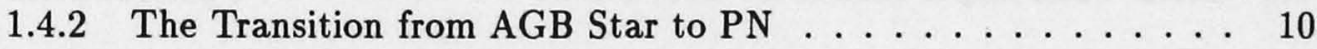

1.4.3 Evolution through the Planetary Nebula Phase . . . . . . . . 11

1.5 The Evolution of the Circumstellar Shells and

Nebulae . . . . . . . . . . . . . . . . . . . . . 12 
1.6 Planetary Nebulae in the Magellanic Clouds . . . . . . . . . . . . . 13

1.6 .1 Observational Samples . . . . . . . . . . . . . . 14

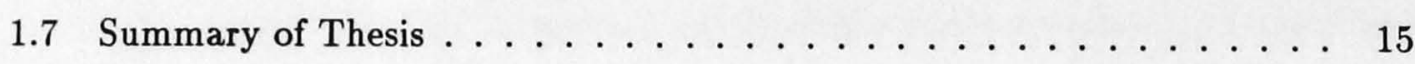

1.8 References . . . . . . . . . . . . . . . . 17

2 Evolution of Low and Intermediate Mass Stars to the End of the AGB with Mass Loss 23

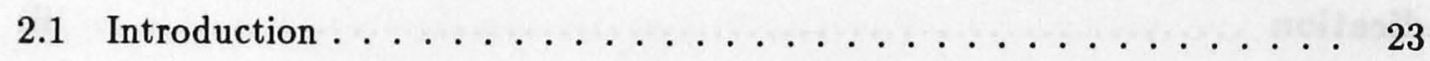

2.2 Computational Details . . . . . . . . . . . . . . 25

2.3 Results and Discussion . . . . . . . . . . . . . . . . 29

2.3.1 Evolution to the First Helium Shell Flash . . . . . . . . . . 29

2.3.1.1 Main Sequence Evolution ............ 30

2.3.1.2 First Giant Branch Evolution . . . . . . . . . 31

2.3.1.3 Core Helium Burning Evolution . . . . . . . . . 32

2.3.1.4 The Early-AGB Phase. . . . . . . . . . . 33

2.3.1.5 Surface Abundance Changes-First and Second Dredgeup .................... 34

2.3.2 AGB Evolution with Mass Loss: The Thermally Pulsing AGB Phase ..................... 59

2.3.2.1 Surface Luminosity Variations . . . . . . . . 59

2.3.2.2 Luminosity-Core Mass Relations . . . . . . . . . 60

2.3.2.3 Interpulse Timescales and TP-AGB Lifetimes . . . . 60

2.3.3 The Superwind Mass Loss Phase . . . . . . . . . . . . 97

2.3.3.1 AGB Tip Luminosities . . . . . . . . . . 98

2.3.3.2 Third Dredge-up - M, S and C stars . . . . . . 98

2.3.3.3 Hot-Bottom Burning in Massive AGB Stars . . . . . . 99 
2.3.3.4 The Initial-Final Mass Relation ... . . . . . . . 101

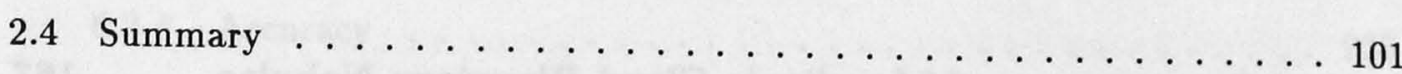

2.5 References ........................ 107

3 Post-AGB Evolution of Low to Intermediate Mass Stars. 113

3.1 Introduction . . . . . . . . . . . . . . 113

3.2 Computational Details . . . . . . . . . . . . . 116

3.2 .1 Terminal Wind Velocity . . . . . . . . . . 116

3.2 .2 Mass Loss Rate . . . . . . . . . . . . . . . . . 119

3.3 Results. . . . . . . . . . . . . . . . . . 121

3.3.1 The Calculations ................... . 121

3.3.2 Comparison with Previous Calculations $\ldots \ldots \ldots \ldots 122$

3.4 Discussion . . . . . . . . . . . . . . . . . . 148

3.4.1 The Transition Time . . . . . . . . . . . . . 148

3.4.2 PNN Tracks at High Core Mass . . . . . . . . . . . . 148

3.4.3 The Case For He Burning PNNi . . . . . . . . 151

3.4 .4 Luminosity Functions $\ldots \ldots \ldots \ldots \ldots \ldots \ldots \ldots$

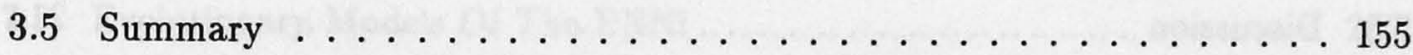

3.6 References ..................... 156

4 The Dynamical Evolution of Circumstellar Shells Surrounding Helium $\begin{array}{ll}\text { Shell Flashing AGB Stars } & 159\end{array}$

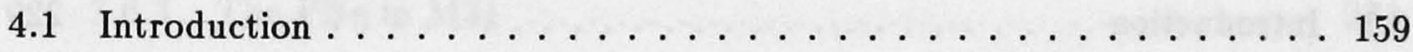

4.2 Numerical Methods . . . . . . . . . . . . . . . . . . . 160

4.3 Results and Discussion . . . . . . . . . . . . . . . . . 162

4.4 Summary . . . . . . . . . . . . . . . . 165 


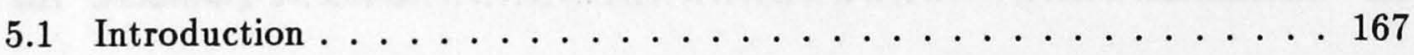

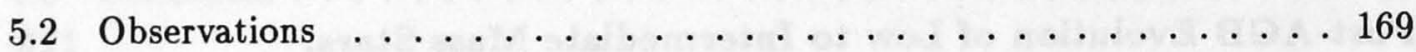

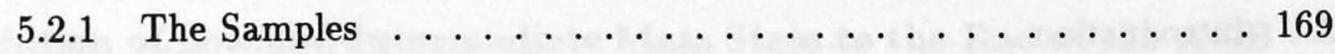

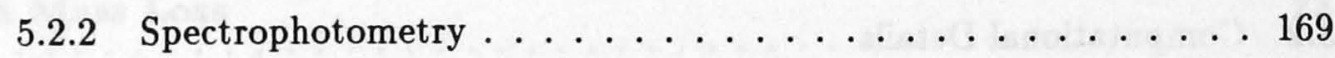

$5.2 .3 \mathrm{H} \beta$ Photometry . . . . . . . . . . . . 172

5.3 Data Reduction . . . . . . . . . . . . . 173

5.3 .1 General Procedure . . . . . . . . . . . . 173

5.3.2 Saturation of Bright Lines . . . . . . . . . . . 174

$5.3 .3 \mathrm{H} \beta$ Photometry . . . . . . . . . . . . . 175

5.3.4 Detector Efficiency and Accuracy . . . . . . . . . 176

5.4 Results. . . . . . . . . . . . . . . 178

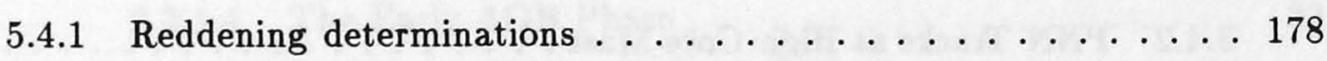

5.4 .2 Nebular Diagnostics . . . . . . . . . . 205

5.4 .3 Other Studies . . . . . . . . . . . . . 209

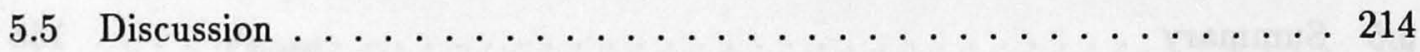

5.6 References . . . . . . . . . . . . . . . 225

6 The Kinematics of Planetary Nebulae in the Outer Fields of the Large $\begin{array}{ll}\text { Magellanic Cloud } & 229\end{array}$

6.1 Introduction . . . . . . . . . . . . . . . . 229

6.2 Observations and Data Reduction ............... 231

6.2 .1 Selection of Objects ................. 231

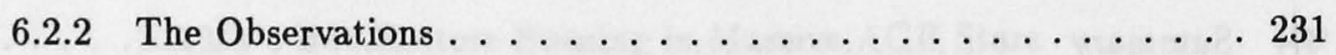


6.2 .3 Reduction Procedure ... . . . . . . . . . 231

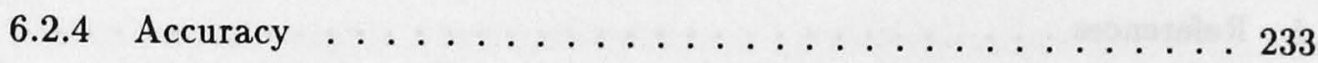

6.3 Results. . . . . . . . . . . . . . . 235

6.3 .1 Radial Velocities .................. 235

6.3.2 Expansion Velocities ................. 235

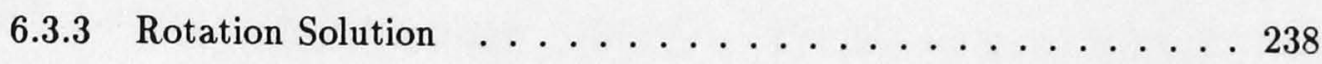

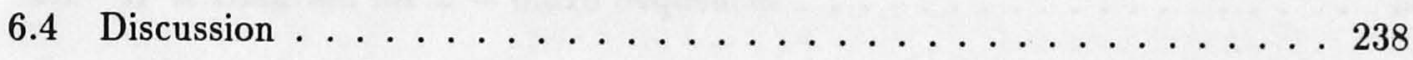

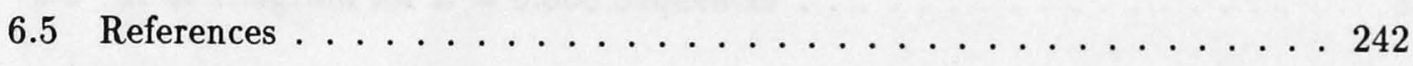

7 A Theoretical Calibration of the Planetary Nebular Cosmic Distance $\begin{array}{ll}\text { Scale } & \mathbf{2 4 5}\end{array}$

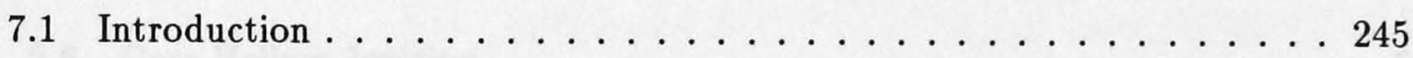

7.2 Photoionization Modelling . . . . . . . . . . . . 246

7.3 Results . . . . . . . . . . . . . . . . . . . 249

7.3 .1 The $\mathrm{H} \beta$ Flux ................... 249

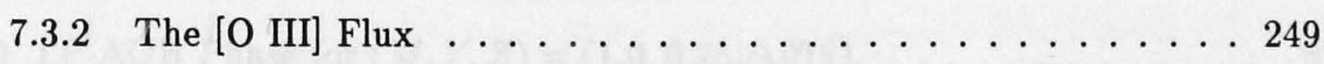

7.4 Luminosity Functions Of The PN In The Magellanic Clouds . . . . . . 253

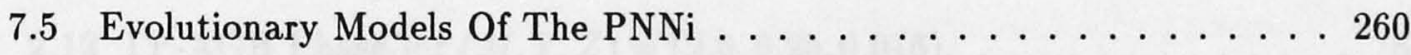

7.6 Calibration of the Planetary Nebula Distance Scale . . . . . . . . . 264

7.6.1 The PNe in the Magellanic Clouds . . . . . . . . . 265

7.6.2 The PNe in the Galactic Bulge . . . . . . . . . 269

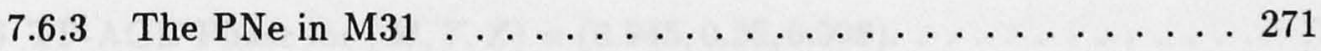

7.6.4 The PNe in the Virgo Ellipticals . . . . . . . . . 272

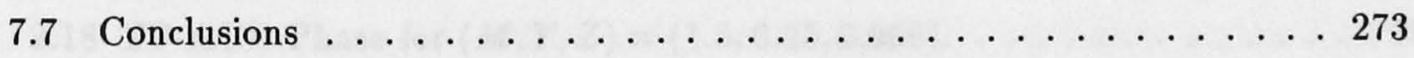

7.8 References ...................... 276 
8 Summary and Suggestions for Further Work

8.1 References . . . . . . . . . . . . . . . . 281 


\section{List of Figures}

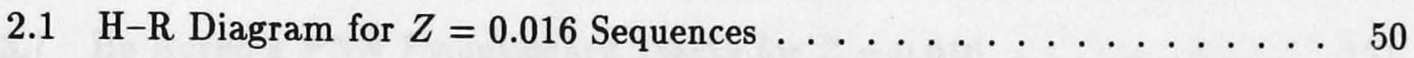

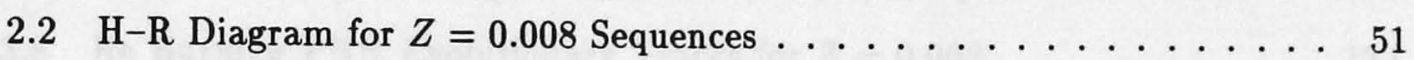

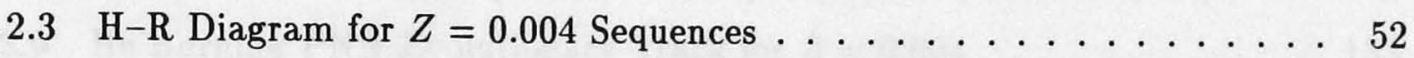

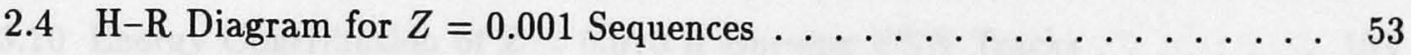

2.5 Main Sequence Turn-Off $\ldots \ldots \ldots \ldots \ldots \ldots \ldots$

2.6 Core Helium Ignition $\ldots \ldots \ldots \ldots \ldots \ldots \ldots \ldots$

2.7 Comparison with Lattanzio's Evolutionary Tracks $\ldots \ldots \ldots$. . . . . 56

2.8 Relative Abundances After First Dredge-Up . . . . . . . . . . 57

2.9 Relative Abundances After Second Dredge-Up . . . . . . . . . . 58

2.10 TP-AGB Phase for $(M, Y, Z)=(1.0,0.25,0.016) \ldots \ldots \ldots \ldots$

2.11 TP-AGB Phase for $(M, Y, Z)=(1.5,0.25,0.016) \ldots \ldots \ldots \ldots$

2.12 TP-AGB Phase for $(M, Y, Z)=(2.0,0.25,0.016) \ldots \ldots \ldots \ldots$

2.13 TP-AGB Phase for $(M, Y, Z)=(2.5,0.25,0.016) \ldots \ldots \ldots \ldots$

2.14 TP-AGB Phase for $(M, Y, Z)=(3.5,0.25,0.016) \ldots \ldots \ldots \ldots \ldots$

2.15 TP-AGB Phase for $(M, Y, Z)=(5.0,0.25,0.016) \ldots \ldots \ldots \ldots$

2.16 TP-AGB Phase for $(M, Y, Z)=(0.945,0.25,0.008) \ldots \ldots \ldots \ldots$

2.17 TP-AGB Phase for $(M, Y, Z)=(1.0,0.25,0.008) \ldots \ldots \ldots \ldots \ldots$

2.18 TP-AGB Phase for $(M, Y, Z)=(1.5,0.25,0.008) \ldots \ldots \ldots \ldots$

2.19 TP-AGB Phase for $(M, Y, Z)=(2.0,0.25,0.008) \ldots \ldots \ldots \ldots \ldots$ 
2.20 TP-AGB Phase for $(M, Y, Z)=(2.5,0.25,0.008) \ldots \ldots \ldots \ldots$

2.21 TP-AGB Phase for $(M, Y, Z)=(3.5,0.25,0.008) \ldots \ldots \ldots \ldots$

2.22 TP-AGB Phase for $(M, Y, Z)=(5.0,0.25,0.008) \ldots \ldots \ldots \ldots$

2.23 TP-AGB Phase for $(M, Y, Z)=(0.89,0.25,0.004) \ldots \ldots \ldots \ldots$

2.24 TP-AGB Phase for $(M, Y, Z)=(1.0,0.25,0.004) \ldots \ldots \ldots 1$

2.25 TP-AGB Phase for $(M, Y, Z)=(1.5,0.25,0.004) \ldots \ldots \ldots \ldots$

2.26 TP-AGB Phase for $(M, Y, Z)=(2.0,0.25,0.004) \ldots \ldots \ldots 3$

2.27 TP-AGB Phase for $(M, Y, Z)=(2.5,0.25,0.004) \ldots \ldots \ldots$

2.28 TP-AGB Phase for $(M, Y, Z)=(3.5,0.25,0.004) \ldots \ldots \ldots \ldots$

2.29 TP-AGB Phase for $(M, Y, Z)=(5.0,0.25,0.004) \ldots \ldots \ldots 6$

2.30 TP-AGB Phase for $(M, Y, Z)=(1.0,0.25,0.001) \ldots \ldots \ldots$

2.31 TP-AGB Phase for $(M, Y, Z)=(1.5,0.25,0.001) \ldots \ldots \ldots$

2.32 Thermal Pulse Morphology . . . . . . . . . . . . . . 89

2.33 Thermal Pulse Characteristics for 0.89 and $0.945 \mathrm{M}_{\odot}$ Models . . . . 90

2.34 Thermal Pulse Characteristics for $1.0 \mathrm{M}_{\odot}$ Models . . . . . . . . . . 91

2.35 Thermal Pulse Characteristics for $1.5 \mathrm{M}_{\odot}$ Models . . . . . . . . . . 92

2.36 Thermal Pulse Characteristics for $2.0 \mathrm{M}_{\odot}$ Models $\ldots \ldots \ldots \ldots \ldots$

2.37 Thermal Pulse Characteristics for $2.5 \mathrm{M}_{\odot}$ Models . . . . . . . . . . . 94

2.38 Thermal Pulse Characteristics for $3.5 \mathrm{M}_{\odot}$ Models . . . . . . . . . 95

2.39 Thermal Pulse Characteristics for $5.0 \mathrm{M}_{\odot}$ Models . . . . . . . . . 96

2.40 Mass Loss Behaviour . . . . . . . . . . . . . . . . . 103

2.41 Luminosities at the First Thermal Pulse and AGB Tip . . . . . . . . . 104

2.42 Relative Abundance Changes After Third Dredge-Up . . . . . . . . . 105

2.43 Initial-Final Mass Relations . . . . . . . . . . . . . 106

xviii 
3.1 Behaviour of Stellar Terminal Wind Velocity in the PN Regime . . . . 118

3.2 Behaviour of Adopted Mass Loss Rate in the PN Regime . . . . . . 120

3.3 H Burning PNN Evolutionary Tracks for $Z=0.016 \ldots \ldots \ldots \ldots$

3.4 H Burning PNN Evolutionary Tracks for $Z=0.008 \ldots \ldots \ldots \ldots$. . . . 128

3.5 H Burning PNN Evolutionary Tracks for $Z=0.004 \ldots \ldots$. . . . . . . 129

3.6 H Burning PNN Evolutionary Tracks for $Z=0.001 \ldots \ldots \ldots \ldots$

3.7 He Burning PNN Evolutionary Tracks for $Z=0.016 \ldots \ldots \ldots 131$

3.8 He Burning PNN Evolutionary Tracks for $Z=0.008 \ldots \ldots \ldots$. . . . 132

3.9 He Burning PNN Evolutionary Track for $Z=0.004 \ldots \ldots \ldots \ldots$

3.10 Energy Contribution of $Z=0.016$ H Burning PNN Tracks . . . . . 153

3.11 Energy Contribution of $Z=0.008$ He Burning PNN Tracks . . . . . 154

4.1 Temporal Evolution of Pulsation Period, Luminosity, Mass, and Mass Loss Rate for a $(M, Z)=(1,0.008)$ AGB Evolutionary Sequence . . . 163

4.2 Density, Temperature, and Velocity variations of Wind Material during a Thermal Pulse Event . . . . . . . . . . . . . . . . . . . . 164

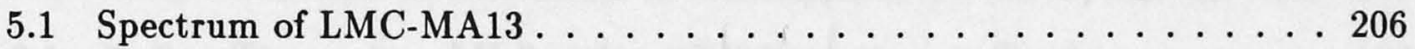

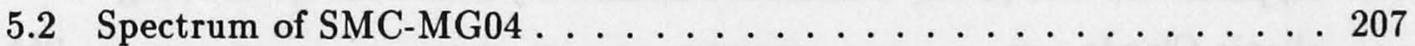

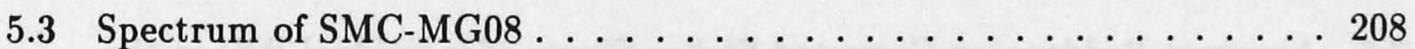

5.4 Comparison of Measured Emission Line fluxes: Aller et al. (1981) and

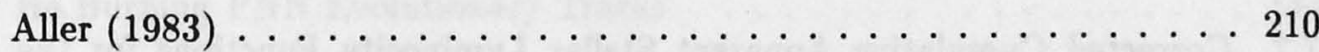

5.5 Comparison of Measured Emission Line Fluxes: Barlow (1987) _ . . . 211

5.6 Comparison of Measured Emission Line Fluxes: Monk, Barlow, and Clegg (1988). . . . . . . . . . . . . . . . . . . . . . 212

5.7 Comparison of Measured Emission Line Fluxes: Meatheringham and

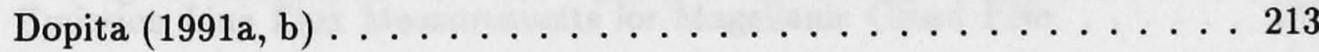

5.8 The Nebular H-R diagram for LMC PNe . . . . . . . . . . . . . . 219 
5.9 The Nebular H-R Diagram for SMC PNe . . . . . . . . . . 220

5.10 Nebular Electron Temperature Versus Nebular Excitation Class for LMC

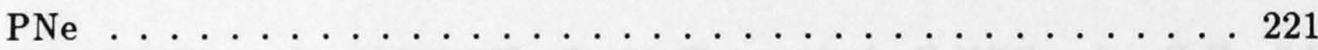

5.11 Nebular Electron Temperature Versus Nebular Excitation Class for SMC

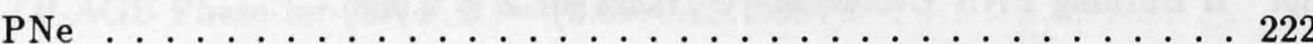

5.12 Dereddened [N III] $\lambda 6583 \AA$ Line Flux versus the Dereddened He II $\lambda 4686 \AA$ Line Flux for LMC PNe . . . . . . . . . . . . . . . . . . . . . 223

5.13 Dereddened [N III] $\lambda 6583 \AA$ Line Flux versus the Dereddened He II $\lambda 4686 \AA$ Line Flux for LMC PNe . . . . . . . . . . . . . . . . . . . . . . 224

6.1 Spatial Distribution of PNe Across the Face of the LMC. . . . . . . 232

6.2 Observed [OIII] Emission Line Profiles for a Sample of Outer-Field LMC PNe. . . . . . . . . . . . . . . . . . . . . 234

7.1 Conversion Efficiency of Stellar Flux to $\mathrm{H} \beta$ Luminosity . . . . . . . . 250

7.2 Conversion Efficiency of Stellar Flux to [OIII] $\lambda 5007 \AA$ Luminosity . . . 251

7.3 Electron Temperature as a Function of both Stellar Effective Temperature and Metallicity. . . . . . . . . . . . . . . . . 252

7.4 Raw $\mathrm{H} \beta$ and [O III] PN Luminosities as a Function of Excitation Class. 254

7.5 Apparent $\mathrm{H} \beta$ and [O III] Cumulative Luminosity Functions for the Magellanic Clouds . . . . . . . . . . . . . . . . . 255

7.6 Apparent Reddenning-Corrected Stellar Fluxes of Individual PNe in the Magellanic Clouds. . . . . . . . . . . . . . . . . . . . . 258

7.7 Corrected Cumulative Apparent Stellar Luminosity Functions for the Magellanic Clouds . . . . . . . . . . . . . . . . . 259

7.8 Theoretical and observed H-R diagram for PNNi in the LMC. . . . . . 263

7.9 The Intrinsic Cumulative Stellar Luminosity Function for LMC PNe . . 268

7.10 The Cumulative Apparent Luminosity Function for Galactic Bulge PNe 270 


\section{List of Tables}

2.1 Definitions of Equivalent Evolutionary Points. . . . . . . . . . . . 36

2.2 Equivalent Evolutionary Points-Results . . . . . . . . . . . 37

2.3 Lifetimes of Major Evolutionary Phases . . . . . . . . . . . . 45

2.4 Abundances After Major Dredge-up Episodes: $Z=0.016 \ldots \ldots$

2.5 Abundances After Major Dredge-up Episodes: $Z=0.008 \ldots \ldots . \ldots 47$

2.6 Abundances After Major Dredge-up Episodes: $Z=0.004 \ldots \ldots \ldots$. . 48

2.7 Abundances After Major Dredge-up Episodes: $Z=0.001 \ldots \ldots$. . . . 49

2.8 Central $\left({ }^{12} \mathrm{C},{ }^{16} \mathrm{O}\right)$ Abundances on the AGB . . . . . . . . 49

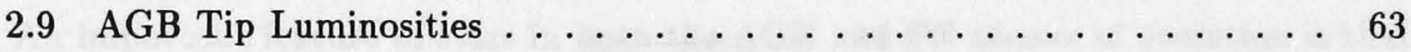

3.1 Fading Timescales of H-Burning PNN Evolutionary Tracks . . . . . 123

3.2 Fading Timescales of He Burning PNN Evolutionary Tracks . . . . . . 124

3.3 H Burning PNN Evolutionary Tracks . . . . . . . . . . . . . 134

3.4 He Burning PNN Evolutionary Tracks . . . . . . . . . . . . . 143

5.1 Cross-Reference Name List for Observed SMP Objects . . . . . . . . 170

5.2 Coordinate List for Observed SMP Objects . . . . . . . . . . 171

5.3 Estimated Errors in Measured Emission Line Fluxes . . . . . . . . . 177

5.4 Emission Line Flux Measurements for Magellanic Cloud PNe . . . . . 180

5.5 Supplementary Emission Line List . . . . . . . . . . . . . . 204 
6.1 Velocity Information for the Outer-Field PNe of the LMC. . . . . . . 236

6.2 Local Standard of Rest Velocities for the Outer-Field PNe in the LMC . 237

6.3 Expansion Velocities for the Outer-Field PNe in the LMC . . . . . . 237

6.4 Rotation Solutions for PNe in the LMC . . . . . . . . . . . 241

7.1 Ages, Core Masses, and Luminosities for PNNi . . . . . . . . . 262

7.2 The Planetary Nebular Cosmic Distance Scale . . . . . . . . . . . 275 


\section{Chapter 1}

\section{Introduction}

All single main sequence stars in the mass range $1 \lesssim M / M_{\odot} \lesssim 8$ are thought to pass through the asymptotic giant branch (AGB) and planetary nebula (PN) phases of evolution on their way to the white dwarf stellar graveyard. Very rapid mass loss occurs at the end of AGB evolution and it is this mass loss that causes stars to evolve into the PN domain. However, our current knowledge of the details of the mass loss process is still very uncertain. The first aim of this thesis is to use empirical estimates of mass loss rates during the late stages of stellar evolution to simulate AGB and PN evolution and then to see if the results of these calculations agree with the available observations.

An important feature of stars in both the AGB and PN phases of evolution is that they are very luminous. Consequently, such stars have been extensively observed in the Magellanic Clouds over the past ten years. The second part of this thesis contains new observations of planetary nebulae in the Magellanic Clouds and makes use of the stellar models produced earlier in the thesis in order to interpret these observations.

In the following sections of the Introduction, the evolution of AGB stars and planetary nebulae (PNe), along with relevant observational material, is reviewed.

\subsection{Overview of the Evolution of Low and Intermediate Mass Stars}

Low mass stars are defined as those that develop an electron-degenerate core immediately following the main sequence, core hydrogen $(\mathrm{H})$ burning phase of evolution. Following core helium (He) burning, single low mass stars pass through the AGB and 
PN phases of evolution. Intermediate mass stars are defined as those stars that do not develop electron degenerate cores following main sequence evolution, but that do develop electron-degenerate carbon-oxygen $(\mathrm{C}-\mathrm{O})$ cores following core He burning, this being the AGB phase of evolution. The term asymptotic-giant branch arises because low mass AGB stars evolve along a path in the Hertzsprung-Russell (H-R) diagram which is asymptotic to the first, or red, giant branch (FGB). The mass limits for low mass stars and intermediate mass stars are $M / M_{\odot} \lesssim 2.25$ and $2.25 \lesssim M / M_{\odot} \lesssim 8$, respectively, assuming standard stellar evolution theory. The precise limits depend on the treatment of the input physics, particularly main sequence convective core overshoot (see Chiosi 1986 for a review).

Mass loss has been found to be very important in terminating AGB evolution. If the degenerate $\mathrm{C}-\mathrm{O}$ core exceeds the Chandrasekhar limit of $1.44 \mathrm{M}_{\odot}$ (as would happen for any star more massive that $1.44 \mathrm{M}_{\odot}$ in the absence of mass loss) then the core will explode to produce a supernova. For low to intermediate mass stars with efficient mass loss, AGB evolution is believed to terminate before the Chandrasekhar core mass limit is reached. There is a possibility that the most massive AGB stars cannot lose their envelopes before the Chandrasekhar core mass limit is reached and that these stars do produce supernovae (e. g., Wood, Bessell, \& Fox 1983).

Theoretical calculations of AGB evolution suggest that when the envelope mass of an AGB star becomes sufficiently small it evolves to higher temperatures at nearly constant luminosity. As it does this, the increase in the number of ultra-violet (UV) photons emitted by the central star results in the ionisation of the previously expelled material, and hence the formation of a visible PN. Once all nuclear energy sources are extinguished, the remnant star gravitationally contracts to become a white dwarf. The characteristics of these various evolutionary phases will now be discussed in detail.

\subsection{Asymptotic Giant Branch Evolution}

\subsubsection{Theoretical Studies}

Until the mid-1960's, stellar evolution calculations had progressed as far as describing the core and shell H burning phases and core He burning phase. Many theoretical studies around this time were concerned with determining the amount of mixing associated with core He ignition at the termination of the FGB (e. g., Faulkner 1966). The tip of the FGB appeared to be well determined from theory, yet there appeared to be a 
group of giants at greater luminosities than this FGB luminosity limit.

Schwarzschild \& Härm (1965) were investigating post-core He burning evolution for low mass Population II stars and found that the evolutionary tracks of such stars approached the FGB and went beyond its limit. More importantly, they came across the phenomenon of thermal pulses, a major feature of AGB evolution. At about the same time, Weigert (1966) came across the thermal pulsing phenomenon when studying intermediate mass Population I stars.

The structure of AGB stars consists of an inert C-O core, surrounded by a He burning shell which, in turn, is surrounded by an $\mathrm{H}$ burning shell and an outer convective envelope. Thermal pulses, or He shell flashes, result from an interaction between the $\mathrm{H}$ and $\mathrm{He}$ burning shells of such stars. As $\mathrm{H}$ is burnt into He, the He layer between the $\mathrm{H}$ burning shell and the $\mathrm{C}-\mathrm{O}$ core becomes thicker and the base of this region gets hotter. Eventually, the He becomes hot enough to ignite, pushing the $\mathrm{H}$ burning shell outwards. The $\mathrm{H}$ burning shell is extinguished at this stage leaving a He burning shell to eat away at the He layer until it is too thin to maintain the temperature required for He burning. Once the He burning subsides, the $\mathrm{H}$ shell begins to contract and eventually reignites. These relaxation oscillations, an oscillation between $\mathrm{He}$ and $\mathrm{H}$ burning, continue until the AGB phase of evolution is terminated (see Iben \& Renzini 1983).

The next major theoretical advance in the study of AGB stars came when Iben (1976) was able to show that nuclear-processed material from the interior of an AGB star could be dredged-up to the surface during a thermal pulse. In particular, when the energy liberated by the intense burning of the He burning shell escaped from the core, envelope convection dug into the region which had been enriched in ${ }^{12} \mathrm{C}$ and sprocess elements. This enriched material was then transported to the stellar surface by convection. Thus, the existence of chemically-enriched objects such as carbon (C) stars and $\mathrm{S}$ stars could be explained.

It had been shown not long after Schwarzschild and Harm's (1965) original work that the solar distribution of s-process isotopes could be explained by multiple neutron exposures which result from the pulse-to-pulse overlap of the convective shells that form above the He burning zone during a thermal pulse (Saunders 1967; Ulrich 1973; Iben 1975a, b; Truran \& Iben 1977). A major problem still remaining after Iben's discovery was the source of neutrons required for the s-process. Although massive AGB stars ( $\succsim 5 \mathrm{M}_{\odot}$ ) burnt $\mathrm{He}$ at temperatures hot enough to stimulate the neutron producing 
reactions,

$$
{ }^{14} N(\alpha, \gamma){ }^{18} F\left(\beta^{+} \nu\right){ }^{18} O(\alpha, \gamma){ }^{22} N e(\alpha, n)^{25} M g
$$

the common (1.5-2 $\left.\mathrm{M}_{\odot}\right) \mathrm{C}$ stars were not able to make this reaction work. The solution to this problem was provided by Hollowell and Iben (1989a; b) who showed that semiconvection during dredge-up caused small amounts of ${ }^{1} \mathrm{H}$ (i. e., protons) to be mixed down into the $\mathrm{C}$-rich layers of the $\mathrm{H}$-exhausted core. When shell $\mathrm{H}$ burning re-ignited, the small amount of $\mathrm{H}$ present was able to convert the ${ }^{12} \mathrm{C}$ to ${ }^{13} \mathrm{C}$ via the reaction,

$$
{ }^{12} C(p, \gamma){ }^{13} N\left(\beta^{+} \nu\right){ }^{13} C
$$

there being insufficient protons around to continue further reactions of the $\mathrm{CN}$ cycle. Then, during the next He shell flash, the ${ }^{13} \mathrm{C}$ was incorporated into the convective shell where it was convected down into the hot zones where the reaction ${ }^{13} C(\alpha, n){ }^{16} O$ could occur, giving rise to the neutrons required.

Although the general features of theoretical AGB evolution now seem secure, the details are still sketchy. Firstly, dredge-up has only been obtained for rather limited ranges of abundance and stellar mass and in nearly all calculations the amount of dredge-up is less than that which appears necessary in order to form carbon stars (see Lattanzio 1989, for a review). Secondly, mass loss from the stellar surface has mostly been ignored, although it must have an important bearing on AGB evolution. In this thesis, the latter question is addressed in particular. Dredge-up is a natural part of the evolutionary calculations, although the present calculations once again do not produce dredge-up to the extent that is observed.

\subsubsection{Observational Identification}

The most definitive way of identifying AGB stars is to look for the chemical peculiarities that result from dredge-up. Clearly, the $\mathrm{C}$ stars show the results of ${ }^{12} \mathrm{C}$ dredge-up, as do the MS and S stars, which show s-process enhancements. The Magellanic Clouds have offered a large observational database for the study of M, S and C star evolution (e. g., Blanco, McCarthy, \& Blanco 1980; Westerlund et al. 1991). The chemical evolution sequence is believed to progess as $\mathrm{M} \rightarrow \mathrm{MS} \rightarrow \mathrm{S} \rightarrow \mathrm{SC} \rightarrow \mathrm{C}$, where the sub-classes are defined as: $\mathrm{C}$ stars, $C / O>1$; $\mathrm{S}$ stars, $C / O \sim 1$; and $\mathrm{M}$ stars, $C / O<1$ (see Kleinmann 1989 , for a review). Definitive evidence of this sequence is shown by the studies of giants on the AGBs of Magellanic Cloud clusters (Bessell, Wood, \& Lloyd Evans 1983; Lloyd Evans 1984). 
Two particular observations show that shell flashes have occurred recently in a star. Firstly, the s-process element Technetium (Tc) was discovered in red giants by Merrill (1952). Recent observations of ${ }^{99} \mathrm{Tc}$ in AGB stars, particularly Mira variables, have been reported by (Little et al. 1987). Since ${ }^{99} \mathrm{Tc}$, the only Tc isotope to be formed via the s-process, has a half-life of $2.1 \times 10^{5}$ years, it must have been produced recently in these stars, giving direct evidence of the recent occurrence of shell flashes (LittleMarenin 1989). The second piece of evidence for shell flashes in AGB stars is provided by the rapid period changes exhibited by the Mira variables R Hya and R Aql (Wood and Zarro 1981). In these two stars, the shell flash must be very active at the present time as the periods have changed dramatically over the last few hundred years.

A less spectacular group of AGB stars can be identified in globular clusters, where accurate photometry of giants allows a separation of AGB and FGB stars at low luminosities (before the AGB asymptotes to the FGB). No photometric separation is possible on the upper giant branch. (e. g., 47 Tuc: Hesser et al. 1987)

As noted earlier, some Mira variables show direct evidence for AGB status and it is probable that all Mira variables are AGB stars. This particular class of variable star has extremely large pulsation amplitude in the visible part of the spectrum $(>2.5$ mag by definition, typically $6 \mathrm{mag}$ ), although the bolometric pulsation amplitude is $\sim 1$ magnitude. The brightness and large pulsation amplitude of these stars allows their identification at large distances (e. g., the Magellanic Clouds and Galactic Centre, as well as throughout the Galaxy). The number of Mira variables known in the Galaxy, and catalogued in the General Catalogue of Variable Stars (Kholopov 1985), is > 15000 . In recent times, large, homogeneous samples of long period variables have been studied in the Magellanic Clouds (e. g., Hughes 1989; Hughes \& Wood 1990; Hughes, Wood, \& Reid 1991).

Mira variables are predominantly of spectral class Me. Their emission nature, dominated by $\mathrm{H} \alpha$ (Merrill 1940), varies through their pulsation cycle probably due to the passage of shocks through their outer atmospheres (e. g., Fox \& Wood 1985). The variables exhibit a period-mass-radius (P-M-R) relationship (e. g., Fox \& Wood 1983; Wood 1986) which has been particularly useful for the derivation of masses of Magellanic Cloud long period variables (Wood, Bessell and Fox 1983).

The Two Micron Sky Survey (Neugebauer \& Leighton 1969) was one of the first systematic studies to show that AGB stars had strong infrared (IR) components, due to them being surrounded by cool circumstellar envelopes. The thick circumstellar 
shells clearly meant that AGB stars were undergoing extensive mass loss. The launch of IRAS (Infrared Astronomical Satellite), and the subsequent publishing of the IRAS point source catalogue (Neugebauer et al. 1984), provided a major impetus to the study of AGB stars. Flux measurements at 12,25 , and $60 \mu \mathrm{m}$ were ideal for deriving colour temperatures of between 200 and $1000 \mathrm{~K}$ (see Habing et al. 1989, for a recent review), which closely corresponds with the optimal temperature range for detecting dust shells around AGB stars. Due to the low reddening at far infrared wavelengths, IRAS was able to see right through the Galaxy, and the AGB stars in the central bulge appeared as a prominent component of the Galactic structure (Habing 1986, 1987).

The IRAS flux measurements allow the creation of what has come to be known as the IRAS 2-colour diagram (Olnon et al. 1984). In this diagram there is a clear sequence of AGB stars from optically visible Mira variables, to pulsating dust-enshrouded AGB stars, and on to non-pulsating dusty stars. This sequence has been interpreted as a sequence of increasing mass loss during ascent of the AGB (Bedijn 1987).

At approximately the same time as the Two Micron Sky Survey became available, it was discovered that some Mira variables and IR sources were associated with strong hydroxyl (OH) $1612 \mathrm{MHz}$ maser emission (Wilson \& Barrett 1968). The emission usually consisted of twin-peaked profiles, resulting from the expansion of material in front of, and behind, the mass losing AGB star. The outflow velocities were typically of the order of $15 \mathrm{~km} \mathrm{~s}^{-1}$, and mass loss rates ranged from $10^{-7}$ to $10^{-4} \mathrm{M}_{\odot} \mathrm{yr}^{-1}$. The stars with the heaviest mass loss rates were optically obscured (being found as IR sources), leading to their designation as $\mathrm{OH} / \mathrm{IR}$ stars. A recent extensive survey of the $\mathrm{OH}$ properties of the cool sources in the IRAS Point Source Catalogue has been published by te Lintel Hekkert et al. (1991). Models of the mass outflows (Elitzur, Goldreich, \& Scoville 1976) allow an estimate of the mass loss rate.

Some optically-visible Mira variables possess $\mathrm{OH}$ maser emission, though much weaker than the $\mathrm{OH} / \mathrm{IR}$ objects (Sivagnanam et al. 1989). The dusty OH/IR stars have also been found to have variable $\mathrm{OH}$ and IR emission which is presumably the result of pulsation of the central star (Harvey et al. 1974; Engels et al. 1983; Herman \& Habing 1985; van Langevelde 1992). The periods of this variability range from $\sim 500$ to $\sim 2000$ days, which appears as a natural extension of the Mira period range of $\sim 200$ to $\sim 500$ days. As mentioned above, this merging of the Mira variables into the variable $\mathrm{OH} / \mathrm{IR}$ stars was also found in the IRAS 2-colour diagram. However, not all OH/IR stars pulsate. Herman \& Habing (1985) found a small group of objects which showed no, or irregular, variability and these stars lie at the extreme red end of the Mira to 
$\mathrm{OH} / \mathrm{IR}$ sequence in the IRAS 2-colour diagram. These stars are believed to be near the termination of the OH/IR (i. e., AGB) phase, or in the transition to the PN phase.

In addition to $\mathrm{OH}$ maser emission from the circumstellar shells of AGB stars, maser emission has been found from other molecules such as silicon oxide ( $\mathrm{SiO})$ and water $\left(\mathrm{H}_{2} \mathrm{O}\right)$ (see Cohen 1989 , for a review). A particularly important microwave emission from circumstellar envelopes is that from the carbon monoxide ( $\mathrm{CO})$ molecule Observations of $\mathrm{CO}$ have been widely used to derive mass loss rates from AGB stars (Knapp et al. 1989) and these mass loss rates are probably the most accurate available.

\subsubsection{Mass Loss on the AGB}

The Reimers' mass-loss formula (Reimers 1975) has been widely used in stellar evolution calculations. It is an empirical dimensional relationship describing mass-loss in terms of the stellar parameters mass, radius, and luminosity. However, the data on which the formula is based does not include observations of very metal-poor stars, largeamplitude red variables, or carbon stars. Nevertheless, the dimensional functional form of Reimers' original formula has often been adopted, and then multiplied by a factor which is calibrated to give a desired answer (e. g., $0.2 \mathrm{M}_{\odot}$ of mass loss on the FGB-Fusi Pecci \& Renzini 1978). The multiplicative factor typically can vary from $1 / 3$ to 3 (e. g., see Boothroyd \& Sackmann 1988c, who have recently studied AGB evolution with mass loss). Unfortunately, the Reimers' formula does not seem to even approximately represent the mass loss rates observed for AGB stars (e. g., Wood 1986).

The treatment of mass-loss in stellar evolution calculations should naturally depend on the mechanism of mass loss. Observations appear to support the hypothesis that stellar pulsations are necessary for lifting the stellar atmosphere into regions where it is sufficiently cool for dust grains to form (Hearn 1990). From there, the material can be accelerated away from the surface of the star by radiation pressure (Wood 1979; Bowen 1988; Gail 1990). Quantitative measurements of mass-loss rates have been made possible by studying $\mathrm{CO}$ and $\mathrm{OH}$ emission from low-mass late-type stellar objects with shells of circumstellar material. For Mira variables and $\mathrm{OH} / \mathrm{IR}$ stars, there seems to be a good correlation of $\dot{M}$ with period (P) up $P \sim 500$ days (Wood 1986; 1990). For larger periods, the mass-loss rate seems roughly constant at the value consistent with the hypothesis that radiation pressure on dust grains drives the gas away from the star (Knapp 1986; Wood 1986; Zuckerman \& Dyck 1986). In this situation, the mass loss 
rate is given by,

$$
\dot{M}=\frac{L}{c v_{\infty}}
$$

where $\mathrm{L}$ represents stellar luminosity, $\mathrm{c}$ is the speed of light, and $v_{\infty}$ is the terminal stellar wind velocity. In the present calculations, an empirical formulation of $\dot{M}$ based on observational data is adopted for the AGB phase of evolution, rather than the Reimers' formula.

A continuous mass loss formula will not allow for the possibility that a He shell flash can somehow (for example, dynamic instability of the envelope-Tuchman, Sack, \& Barkat $1978 ; 1979)$ lead to a discrete ejection of the stellar envelope and subsequent PN formation. However, a number of stellar evolution calculations have examined the effect of such an ejection on subsequent stellar evolution. Härm and Schwarzschild (1975) used a high mass loss rate to simulate ejection of the outer envelope at the surface luminosity maximum of a He shell flash. Such an ejection mechanism leads to a PN nucleus which is burning He, rather than H, in a shell around the core. Schönberner (1983), Iben (1984) and Wood \& Faulkner (1986) have all studied the effect that the phase of the shell flash cycle, at which a star leaves the AGB, has on the subsequent evolution of the PN nuclei. From the calculations completed to-date, PNe powered by a central star initially burning $\mathrm{He}$ (i. e., the central star leaves the AGB close to a thermal pulse) take $\sim 3$ times longer to evolve to the blue part of the H-R diagram than central stars that are initially burning H (Wood \& Faulkner 1986; Schönberner 1990).

\subsection{Planetary Nebulae}

The great majority of PNe have been identified in the optical part of the spectrum, the identification being made on the basis of extended structure and emission line spectra. By 1967, 1000 Galactic objects had been classified as planetary nebula candidates (Perek \& Kohoutek 1967) and the number of known objects has now reached $\sim 1400$ (Acker et al. 1991), although some of these candidates will be mis-identifications. From kinematic studies of PNe it is estimated that there are $\sim 15000 \mathrm{PNe}$ in the Galaxy (Pottasch 1984; Maciel 1989).

Radio observations of $\mathrm{PNe}$ were first carried out in 1950 but showed little structure as the beam sizes were too large. However, with the advent of interferometric techniques the resolution of radio images has surpassed that of optical images. Radio observations have also proved useful for studying objects which suffer badly due to extinction in the 
optical regime, particularly $\mathrm{PNe}$ near the Galactic Centre. Radio images of PNe also tend to appear more symmetrical because of the reduced effects of internal extinction at these wavelengths.

The launch of the International Ultraviolet Explorer (IUE) in 1978 allowed investigation of the $\sim 1000-2500 \AA$ region of the electromagnetic spectrum. It was then possible to measure important ionization states in this spectral region and therefore tighten the theoretical constraints on photoionisation modelling of PNe (e. g., Harrington et al. 1982). Examination of the line profiles of the central stars of some objects showed them to be asymmetrical or P-Cygni in shape (e. g., Cerruti-Sola \& Perinotto 1989), a clear indication of the presence of a stellar wind.

The IR spectral region of PNe has been studied since the 1960's (see Barlow 1983, for a review). As with studies of the UV region, observation of IR emission lines aids in placing tighter constraints on photoionisation modelling and abundance determinations. The major virtue of observing in the IR is the reduced extinction. IRAS has proved invaluable in detecting young, relatively cool, dusty $\mathrm{PNe}$ which occupy a characteristic area in the IRAS 2-colour diagram (van der Veen 1989).

The study of the PNe will be greatly enhanced this decade by the launch of the Hubble Space Telescope (HST). Its superior resolution capabilities above the distorting effects of the Earth's atmosphere will allow the spatial mapping of PNe in nearby extragalactic systems. For instance, the angular diameter of a very large PN in the LMC is of the order of $1-2^{\prime \prime}$, so that most PNe will have sizes easily resolvable with HST.

\subsection{The Evolution of Planetary Nebulae}

\subsubsection{AGB stars as the Progenitors of Planetary Nebulae}

By extrapolating the evolution of PNe backwards, Shklovsky (1956) noted that the properties of the progenitor stars would resemble those of red giants. A similar deduction was reached by Abell \& Goldreich (1966) using more thorough arguments, such as (see Abell \& Goldreich 1966, and references therein):

- The galactic distribution (and kinematics) of PNe resembles that of Population II ( halo) objects found in the bulge, and old disk, of the galaxy. Therefore PNe must represent a stage of evolution of low mass stars $\left(\sim 1.2 \mathrm{M}_{\odot}\right)$ after they have 
left the main sequence.

- Based on the available data of the time, the number of stars with $M \sim 1.2 M_{\odot}$ evolving off the main sequence was of the same order as the number of $\mathrm{PNe}$ being formed. Therefore most, if not all, stars with $M \sim 1.2 M_{\odot}$ that evolve off the main sequence must pass through the PN phase.

- For solar-sized stars, the escape energy is several orders of magnitudes greater $(\sim 4)$ than the energy of a typical nebular shell expanded to infinity. These energies become comparable when stars $R \sim 400 R_{\odot}$ are considered. Therefore, the ejection of a PN shell most likely occurs from a star with a large radius.

It was noted (e. g., Rose \& Smith 1970) that, of the progenitor models available at that time, only the ones burning $\mathrm{H}$ and $\mathrm{He}$ in shell sources (i. e., AGB stars) provided luminosities in reasonable agreement with the observed luminosities of $\mathrm{PN}$ nuclei (Harmon \& Seaton 1965; Seaton 1966; O'Dell 1968). With the progenitors of PNe established as red giants, probably AGB stars, a reason for the ejection was sought, and three early suggestions were: Dynamic instabilities in the envelope (Roxburgh 1967), radiation pressure (Faulkner 1970), and thermal pulses (Rose 1968). However, none of these mechanisms are now thought to be the main source of PN ejection as originally proposed, although stellar pulsation and shell flashes do play some role in the ejection process, and radiation pressure may play a role in the most massive AGB stars (Wood \& Faulkner 1986).

\subsubsection{The Transition from AGB Star to PN}

Detailed knowledge of the actual transition from the AGB to the PN region in the $\mathrm{H}-\mathrm{R}$ diagram does not exist as there are very few observations of stars in this phase of evolution (e. g., Zijlstra et al. 1989). Theoretically, although calculations of AGB to PN evolution by Schönberner (1983) and Wood \& Faulkner (1986) indicate that the transition takes of the order of 1000 years, the mass loss rates in these calculations were contrived to make the transition rapid; the real cause and magnitude of the mass loss in the transition phase remains unknown. Consequently, the hunt for proto-planetary nebulae is a very active field of research at the present time.

Recent observations of what are believed to be post-AGB stars, or proto-planetary nebulae, may provide knowledge of the stars between the AGB and PN regimes. One class of such objects have spectra of A to G supergiants, but they also have an infrared 
excess believed to be associated with the remnants of the dust shell formed on the AGB (Parthasarathy \& Pottasch 1986; Parthasarathy 1989). Some of these objects also still have detectable $\mathrm{OH}$ maser or $\mathrm{CO}$ emission (te Lintel Hekkert, Chapman, \& Zijlstra 1992), but the central stars are not yet hot enough to ionize the circumstellar material. The fact that they are no longer obscured by circumstellar material means that either they left the AGB long ago, the mass loss rate on the AGB was small, or perhaps we are looking at the central stars along the axis of bipolar nebulae.

A PN will only form when the central star has evolved to a high enough temperature to emit sufficient UV photons and ionize the surrounding material. At this stage, the nebula is likely to be compact, dense and optically thick due to dust. Radio and IR techniques are ideally suited to the study of such optically-obscured dusty proto-planetary nebulae. In particular, radio emission from the ionized, but opticallyobscured, central region can be detected in the youngest PNe (Gathier 1987; Zijlstra et al. 1989).

\subsubsection{Evolution through the Planetary Nebula Phase}

The comprehensive set of evolutionary tracks computed by Paczyński (1971) provided, for the first time, a basis for comparing the observations of PN nuclei with theoretical evolution from the AGB, through the PN phase, and onto the white dwarf regime. Although the Paczyński (1971) models were derived from AGB stars in which He shell flashes had been suppressed, at least they covered a range in mass and showed the broad picture of luminosity and timescale variation with PN nucleus mass. The distribution of PNe in the H-R diagram could now be interpreted as a superposition of PN nuclei with different masses, and in different stages along the evolutionary tracks. The direction of evolution was more-or-less as expected from the observations of the time. The nebulae formed a sequence which showed them increasing in size and evolving from optically thick to optically thin, while the central stars evolved from low temperature and luminosity to higher temperature and luminosity, and then returned to low luminosity (Harmon \& Seaton 1965). Since Paczyński's (1971) early work, new evolutionary tracks for PN nuclei have been computed, properly taking into account helium shell flashes during the previous AGB evolution (e. g., Schonberner 1983; Wood and Faulkner 1986).

The very large stellar wind mass loss rates that occur on the AGB lead to the formation of $\mathrm{PNe}$, but during the PN phase itself there is evidence that stellar winds 
persist. The stellar winds in $\mathrm{PNe}$ lead to mass loss rates of only $\sim 10^{-7}$ to $10^{-8} \mathrm{M}_{\odot} \mathrm{yr}^{-1}$, compared to the $\sim 10^{-5}$ to $10^{-4} \mathrm{M}_{\odot} \mathrm{yr}^{-1}$ during the late AGB phases (Perinotto 1989). On the other hand, the PN winds have velocities of the order of $1000 \mathrm{~km} \mathrm{~s}^{-1}$ (e. g., Patriarchi \& Perinotto 1991), whereas wind velocities of Mira variables and OH/IR stars are only of the order of $15 \mathrm{~km} \mathrm{~s}^{-1}$. This is consistent with the general increase in expansion velocity across the H-R diagram (de Jager et al. 1988). In the calculations performed here, empirical relationships for the mass loss and terminal wind velocity were used during the PN phase.

\subsection{The Evolution of the Circumstellar Shells and Nebulae}

The discussion so far has been largely related to the evolution of PN nuclei and their likely progenitors. One of the initial aims of this thesis was to study the nebulae that resulted from the evolutionary calculations with mass loss, since these calculations provided a detailed, time-dependent history of the mass loss rate and ejection velocity from the star. Time has prevented much work in this direction, but some initial studies of circumstellar shell formation have been made. The evolution of the circumstellar shells around AGB stars and the formation of nebulae will now be discussed.

The shapes of PNe have largely been determined through broad and narrow band optical imaging and high resolution radio mapping. Generally, radio images appear more symmetrical than their optical counterparts due to the smaller effect of internal extinction in the radio spectral region. Also, the radio continuum emission arises from the more uniformly distributed electron gas (e. g., Zijlstra 1989). Narrow band CCD imaging usually concentrates on the strong optical emission lines such as [O III] $\lambda 5007 \AA$ and $\mathrm{H} \alpha \lambda 6563 \AA$ which may be more localised (i. e., clumped) than the electron gas (e.g., Balick 1987). Images at different wavelengths for the same object usually show slightly different structure because of the different ionisation zones present in the nebula. The shapes of structures seen ranges from spherical to elongated, or bipolar. In fact, roughly $50 \%$ of planetary nebulae can be considered to deviate significantly from spherical symmetry (Zuckerman \& Aller 1986; see also compilation by Chu, Jacoby, \& Arendt 1987).

Recent CCD imaging and spectroscopic studies have been expanded to search for haloes of PNe, shells of matter beyond the photoionised central regions (Balick 1987; Jacoby, Quigley, \& Africano 1987; Chu 1989, and references therein). A halo is typically 
observed at 2-6 times the radius of the main nebula, and appears to follow an $\sim \mathrm{r}^{-2}$ density law (e. g., Bässgen \& Grewing 1989). It has also been noted that they are hotter, and less metal-rich, than the main nebula (Harrington, Monk, \& Clegg 1988; Middlemass et al. 1989, 1991). These observations have important consequences for stellar evolution in terms of the total mass contained in nebulae and the episodes of mass loss experienced by the progenitors of PNe. These haloes are presumably the remnants of stellar winds that occurred during AGB evolution, before the final heavy mass loss phase (Balick et al. 1992). Why there should be a distinction between the mass loss regimes leading to the halo and main nebula in a PN is a question that the present study might hope to address.

As well as being bounded by haloes on the outside, planetary nebulae have incident on their inner edges the fast wind mentioned in section 1.4.3 which emanates from the central star. The interacting winds model (Castor, McCray, \& Weaver 1975; Kwok, Fitzgerald, \& Purton 1978; Kahn 1989) generally describes the way in which the momentum from the hot, fast wind accelerates the original, slow AGB wind material which is now the PN. This model seems to provide a good description of spherical nebulae. Recent calculations have been aimed at finding how the bipolar structure develops in many nebulae. The amplification of small initial asymmetries in the old AGB wind by the hot wind now seems to be a plausible process (Icke, Balick, \& Frank 1992; Icke et al. 1992).

\subsection{Planetary Nebulae in the Magellanic Clouds}

The obvious benefit of studying PNe in the Magellanic Clouds is that, essentially, all objects are at a common distance, where that distance can be determined independently. A similar situation exists for PNe in the Galactic Bulge, but they suffer from greater extinction along their lines-of-sight.

The disadvantage of studying PNe in the Magellanic Clouds is that their apparent sizes are $\lesssim 2$ ". Consequently, at least before the advent of the HST, it was not possible to get spatial information across the face of the nebula. Spectra of Magellanic Cloud $\mathrm{PNe}$ were integrated over the whole nebula and included the contribution from the central star. The central star spectrum could only be obtained in a spectral region where the nucleus dominated. 


\subsubsection{Observational Samples}

The first systematic searches for PNe in the Magellanic Clouds involved the detection of $\mathrm{H} \alpha$ emitting objects with no continuum on objective prism survey plates (LMC: Henize 1956; Lindsay \& Mullan 1963; SMC: Henize 1956; Lindsay 1961). The more luminous objects were resolved further, and the majority were found to be compact H II regions (LMC: Westerlund \& Smith 1964; SMC: Henize \& Westerlund 1963). Of the remaining candidates, some were positively identified as $\mathrm{PNe}$ and studied in detail (see Meatheringham \& Dopita 1991b, and references therein).

One of the main catalogues referred to today is that compiled by Sanduleak, McConnell, \& Philip (1978) based on their own objective prism observations which went to a fainter limiting magnitude than previous surveys. Apart from locating candidates reported by previous investigators, they identified an additional 22 PN candidates. A small additional number of candidates was identified by Sanduleak \& Pesch (1981) using a similar method. Spectrophotometry for the majority of these objects has been obtained by Meatheringham \& Dopita (1991a, b).

Another catalogue is that produced by Jacoby (1980). His technique was different to that mentioned above in that he performed interference filter photography over specific fields of the Magellanic Clouds. Although severely restricting his field of search, he was able to push his observations to a fainter limiting magnitude than that reached by objective prism surveys of the time. Spectrophotometry for the Jacoby sample of objects has been obtained by Boroson \& Liebert (1989).

Morgan \& Good (1985) have had recent success in identifying 10 new candidates in the SMC from medium dispersion objective prism plates. The increase in dispersion has meant that the nebulium ([O III]) lines $\lambda 4959 \AA$ and $\lambda 5007 \AA$ can be resolved from $\mathrm{H} \beta$, thus enhancing the chances of correctly identifying PNe. The technique has been extended to the LMC where several plates were taken to cover a large area of the LMC (Morgan \& Good 1992; Morgan 1992). Such a sample of objects has the advantage of covering a wide field, as opposed to the sample observed by Jacoby (1980), and measuring down to a fainter magnitude limit than previous objective prism surveys. The importance of observing faint PNe manifests itself in the constraints it imposes on the luminosity-decline phase of PN nuclei, and consequently on their mass distribution.

The large body of homogenous data being collected for PNe in the Magellanic Clouds offers an opportunity to study not only AGB-PN evolution, but also the behaviour of the kinematics and star formation processes in the Clouds. This thesis 
has contributed to the collection of spectroscopic data for Magellanic Cloud PNe and presents a kinematic study of the LMC.

\subsection{Summary of Thesis}

The overall aim of this research is to construct a self-consistent picture of the evolution from an AGB star into a PN. The research presented in this thesis can be divided into three main components:

- To study the evolution of low to intermediate mass $\left(\sim 1-5 \mathrm{M}_{\odot}\right)$ stars with mass loss from the AGB to the PN domain;

- To study the hydrodynamics of the ejected material;

- To study PNe in the Magellanic Clouds and the Galactic Centre.

The Mount Stromlo Stellar Evolution Code was used to construct a grid of evolutionary tracks covering the mass range $\sim 1$ to $5 \mathrm{M}_{\odot}$, and with abundances especially selected to represent the solar neighbourhood and the Magellanic Clouds. Over 2600000 stellar evolution models were calculated for this study. Various modifications have been made to the code, with particular emphasis on mass loss. The results of the evolutionary calculations can be used for detailed comparisons of the relative numbers of Mira variables, $\mathrm{OH} / \mathrm{IR}$ stars, non-variable AGB stars, and PNe. These results are presented in Chapter 2.

Chapter 3 presents new PN nuclei evolutionary tracks based on the progenitor models derived in the previous chapter. These tracks are unique due to the complete proper treatment of pre-PN evolution. Mass loss has also been incorporated into the PN regime following from IUE observations of PN nuclei, and the predictions from radiation driven wind theory.

Some initial studies were made of the circumstellar envelope structures that result from the time-dependent mass loss rates arising from the evolution calculations presented in chapter 2. The results are presented in Chapter 4 . These calculations were done using a hydrodynamic code in which mass was injected at an inner boundary close to the star, but far enough away that grains were assumed to have already formed. The equation of motion of the gas included a term describing the radiation force on the dust grains. 
The observational part of this thesis consisted of spectroscopic observations of a sample of predominantly faint PNe in the Magellanic Clouds. Low resolution ( 10 $\AA$ ) spectra were obtained for 70 objects in the wavelength range $\lambda \lambda 3400-7900 \AA$. The results of these observations are presented in Chapter 5.

In addition to the low resolution observations presented in Chapter 5, échelle observations (resolution $=11.5 \mathrm{~km} \mathrm{~s}^{-1}$ ) were conducted to obtain radial and expansion velocities for a sample of 10 new PNe in the outlying regions of the LMC. Combined with previously published PN data, these data allowed a re-analysis of the structure of the LMC. The results are presented in Chapter 6.

Chapter 7 presents a fitting conclusion to this thesis research by combining theory and observation to provide a theoretical calibration of the planetary nebular cosmic distance scale. Details of the calibration, and subsequent derivations of distances to some nearby galaxies and the Galactic Bulge, are presented. 


\subsection{References}

Abell, G. O., \& Goldreich, P. 1966, PASP, 78, 232

Acker, A. Stenholm, B., \& Véron, P. 1991, A\&AS, 87, 499

Balick, B. 1987, AJ, 94, 671

Balick, B., Gonzalez, G., Frank, A., \& Jacoby, G. H. 1992, ApJ,

Barlow, M. J. 1983, in IAU Symp. 103, Planetary Nebulae, ed. D. R. Flower (Dordrecht: Reidel), 105

Bässgen, M., \& Grewing, M. 1989, A\&A, 218, 273

Bedijn, P. J. 1987, A\&A, 186, 136

Bessell, M. S., Wood, P. R., Lloyd Evans, T. 1983, MNRAS, 202, 59

Blanco, V. M., McCarthy, M. F., \& Blanco, B. M. 1980, ApJ, 242, 938

Boothroyd, A. I., \& Sackmann, I. J. 1988a, ApJ, 328, 671

1988b, ApJ, 328, 641

$1988 \mathrm{c}, \mathrm{ApJ}, 328,653$

1988d, ApJ, 328, 671

Boroson, T. H., \& Liebert, J. 1989, ApJ, 339, 844

Bowen, G. H. 1988, ApJ, 329, 299

Castor, J., McCray, R., \& Weaver, J. 1975, ApJL, 200, L107

Cerruti-Sola, M., \& Perinotto, M. 1989, ApJ, 345, 339

Cohen, R. J. 1989, Rep. Progr. Phys., 52, 881

Chiosi, 1986, in Nucleosynthesis \& Chemical Evolution, eds. B. Hauck, A. Maeder, \& G. Meynet (Geneva Obseratory),

Chu, Y.-H. 1989, in IAU Symp. 131, Planetary Nebulae, ed. S. Torres-

Peimbert (Dordrecht: Kluwer), 105

Chu, Y.-H., Jacoby, G. H., Arendt, R. 1987, ApJS, 64, 529

de Jager, C., Nieuwenhuijzen, H., \& van der Hucht, K. A. 1988, A\&AS, 72, 259

Elitzur, M., Goldreich, P., \& Scoville, N. 1976, ApJ, 205, 384

Engels, D., Lewis, B. M., Schultz, G. V., Sherwood, W. A. 1983, A\&A, 124, 123

Faulkner, J. 1966, ApJ, 144, 978

Faulkner, D. J. 1970, ApJ, 162, 513

Gail, H.-P. 1990, in Reviews in Modern Astronomy, Accretion and Winds, 3, ed. G. Klare (Berlin: Springer-Verlag), 156

Gathier, R., in Late Stages of Stellar Evolution, eds. S. Kwok, \& S. R. Pottasch (Dordrecht: Reidel), 371 
Habing, H. J. 1986, in Light on Dark Matter, ed. F. P. Israel (Dordrecht: Reidel), 329 1987, in Late Stages of Stellar Evolution, eds. S. Kwok, \& S. R. Pottasch (Dordrecht: Reidel), 291

Härm, R., \& Schwarschild, M. 1975, ApJ, 200, 324

Harmon, R. J., \& Seaton, M. J. 1965, MNRAS, 132, 15

Harrington, J. P., Monk, D. J., \& Clegg, R. E. S. 1988, MNRAS,

Harrington, J. P., Seaton, M. J., Adams, S., \& Lutz, J. H. 1982, MNRAS, 199,517

Harvey, P. M., Bechis, K. B., Wilson, W. J., Ball, J. A. 1974, ApJS, 27, 331 Hearn, A. G. 1990, in From Miras to Planetary Nebulae: Which Path for Stellar Evolution?, eds. M. O. Mennessier \& A. Omont (Yvette Cedex: Editions Frontières), 121

Henize, K. G. 1956, ApJS, 2, 315

Henize, K. G, \& Westerlund, B. E. 1963, ApJ, 137, 747

Herman, J., \& Habing, H. J. 1985, A\&AS, 59, 523

Hollowell, \& Iben, I. Jr. 1989a, ApJ, 340, 966 1989b, in Evolution of Peculiar Red Giant Stars, eds. H. R.

Johnson \& B. Zuckerman (Cambridge: Cambridge Univ. Press), 227

Hughes, S. M. G. 1989, AJ, 97, 1634

Hughes, S. M. G., \& Wood, P. R. 1990, AJ, 99, 784

Hughes, S. M. G., Wood, P. R., \& Reid, N. 1991, AJ, 101, 1304

Iben, I. Jr. $1975 \mathrm{a}, \mathrm{ApJ}, 196,525$ 1975b, ApJ, 196, 549 , 1976, ApJ, 208, 165 , 1984, ApJ, 277, 333

Iben, I. Jr., \& Renzini, A. 1983, ARA\&A, 21, 271

Icke, V., Balick, B., \& Frank, A. 1992, A\&A, 253, 224

Icke, V., Mellema, G., Balick, B., Eulderink, F., \& Frank, A. 1992, Nature, 355,524

Jacoby, G. H., Quigley, R. J., \& Africano, J. L. 1987, PASP, 99, 672

Kahn, F. D. 1989, in IAU Symp. 131, Planetary Nebulae, ed. S. TorresPeimbert (Dordrecht: Reidel), 411

Kholopov, P. N. 1985, General Catalogue of Variable Stars, vols. 1-3, (4th ed.: Moscow: Nauka Publishing House). 
Kleinmann, S. G. 1989, in Evolution of Peculiar Red Giant Stars, eds. H. R. Johnson \& B. Zuckerman (Cambridge: Cambridge Univ. Press), 13

Knapp, G. R. 1986, 311, 731

Knapp, G. R, Sutin, B. M., Phillips, T. G., Ellison, B. N., Keene, J. B., Leighton, R. B., Masson, C. R., Steiger, W., Veidt, B., \& Young, K. 1989, ApJ, 336, 822

Kwok, S., Fitzgerald, P. M., Purton, C. R. 1978, ApJL, 219, L125

Lattanzio, J. 1989, in Evolution of Peculiar Red Giant Stars, eds. H. R. Johnson \& B. Zuckerman (Cambridge: Cambridge Univ. Press), 161

Lindsay, E. M. 1961, AJ, 66, 169

Lindsay, E. M., \& Mullan, D. J. 1963, Irish AJ, 6, 51

te Lintel Hekkert, P. 1991, A\&A, 248, 209

te Lintel Hekkert, P., Chapman, J. M., \& Zijlstra, A. A. 1992, ApJL, in press

Little, S. J., Little-Marenin, I. R., \& Bauer, W. H. 1987, AJ, 94, 981

Lloyd Evans, T. 1984, MNRAS, 208, 447

Maciel, W. J. 1989, in IAU Symp. 131, Planetary Nebulae, ed. S. TorresPeimbert (Dordrecht: Kluwer), 73

Merrill, P. W. 1940, Spectra of Long Period Variables, (Chicago: Univ. of Chicago Press) 1952, ApJ, 116, 21

Meatheringham, S. J., \& Dopita, M. A. 1991a, ApJS, 75, 407 ., 1991b, ApJS, 76, 1085

Middlemass, D., Clegg, R. E. S., Walsh, J. R. 1989, MNRAS, 239, 1

Middlemass, D., Clegg, R. E. S., Walsh, J. R., \& Harrington, J. P. 1991, MNRAS, 251, 284

Morgan, D. H. 1992, in preparation

Morgan, D. H., \& Good, A. R. 1985, MNRAS, 213, 491 1992, A\&AS, 92, 571

Neugebauer, G., \& Leighton, R. B. 1969, NASA SP-3047

Neugebauer, G., van Duinen, R. J., Habing, H. J., Aumann, H. H., Beintema, D. A., Bogess, N., Borgman, J., Clegg, P. E., Gillet, F. C., Hauser, M. G., Houck, J. R., Jennings, R. E., De Jong, T., Low, F. J., Marsden, P. L., Pottasch, S. R., Soifer, B. T., Walker, R. G., Emerson, J. P., Raimond, E., Rowan-Robinson, M., Wesselius, P. R., Baud, B., Beichman, C. A., Gautier, T. N., Harris, S., Miley, G. K., Olnon, F. M., Young, 
E. 1984, IRAS Catalogues and Atlases: Explanatory Supplement, Point Source Catalogue

O'Dell, C. R. 1968, in IAU Symp. 34, Planetary Nebulae, eds. D. E. Osterbrock \& C. R. O'Dell (Dordrecht: Reidel), 361

Olnon, F. M., Baud, B., Habing, H. J., de Jong, T., Harris, S., \& Pottasch, S. R. 1984, ApJL, 278, L37

Paczyński, B. 1971, Acta Astron., 21, 417

Parthasarthy, M. 1989, in Evolution of Peculiar Red Giant Stars, eds. H. R.

Johnson \& B. Zuckerman (Cambridge: Cambridge Univ. Press), 384

Parthasarathy, M., \& Pottasch, S. R. 1986, A\&A, 154, L16

Patriarchi, P., \& Perinotto, M. 1991, A\&AS, 91, 325

Perek, L., \& Kohoutek, L. 1967, Catalogue of Galactic Planetary Nebulae (Praha: Academic Press, USSR)

Perinotto, M. 1989, in IAU Symp. 131, Planetary Nebulae, ed. S. TorresPeimbert (Dordrecht: Kluwer), 293

Pottasch, S. R. 1984, Planetary Nebulae (Dordrecht: Reidel)

Reimers, D. 1975, in Problems in Stellar Atmospheres and Envelopes, ed.

B. Baschek, W. H. Kegel, \& G. Traving (Berlin: Springer), 229

Rose, W. K. 1968, in IAU Symp. 34, Planetary Nebulae, eds. D. E. Osterbrock \& C. R. O'Dell (Dordrecht: Reidel), 390

Rose, W. K., \& Smith, R. L. 1970, ApJ, 159, 903

Roxburgh, I. W. 1967, Nature, 215, 838

Sanders, R. H. 1967, ApJ, 150, 971

Sanduleak, N., MacConnell, D. J., \& Philip, A. G. D. 1978, PASP, 90, 621

Sanduleak, N., \& Pesch, P. 1981, PASP, 93, 431

Schönberner, D. 1983, ApJ, 272, 708 1990, in From Miras to Planetary Nebulae: Which Path for

Stellar Evolution?, eds. M. O. Mennessier \& A. Omont (Yvette Cedex:

Editions Frontières), 355

Schwarzschild, M. \& Härm, R. 1965, ApJ, 142, 855

Seaton, M. J. 1966, MNRAS, 132, 347

Shklovsky, I. S. 1956, Astron. Zh., 33, 315

Sivagnanam, P., Le Squeren, A. M., Foy, F., \& Tran Minh, F. 1989, A\&A, 211,341

Smith, G. H. 1987, PASP, 99, 67

Strom, S. E., \& Strom, K. M. 1971, A\&A, 14, 111 
Strom, S. E., Strom, K. M., Rood, R. T., \& Iben, I. Jr., 1970, A\&A, 8, 243

Truran, J. W., \& Iben, I. Jr. 1977, ApJ, 216, 797

Tuchman, Y., Sack, N., \& Barkat, Z. 1978a, ApJ, 219, 183 , 1979, ApJ, 234, 217

Ulrich, R. K. 1973, in Explosive Nucleosynthesis, ed. D. N. Schramm \& D.

W. Arnett (Austin: University of Texas), 139

van der Veen, W. E. C. J. 1989, A\&A, 210, 127

van Langevelde, H. J. 1992, PhD Thesis, Leiden

Weigert, A. 1966, ZsAp, 64, 395

Westerlund, B. E., Azzopardi, M., Breysacher, J., Reboiret, E. 1991, A\&AS, 91,425

Westerlund, B. E., Smith, L. F. 1964, MNRAS, 127, 449

Wilson, W. J., \& Barrett, A. H. 1968, Science, 161, 778

Wood, P. R. 1979, ApJ, 227, 220

1986, in Stellar Pulsation, Lecture Notes in Physics, No.

274, eds. A. N. Cox, W. M. Sparks, \& S. G. Starrfield (Berlin: Springer-

Verlag), 250

1990, in Confrontation Between Stellar Pulsation and Evo-

lution, ASP Conference Series, 11, eds. C. Cacciari \& G. Clementini

(Provo, Utah: Brigham Young Univ. Press), 355

Wood, P. R., Bessell, M. S., \& Fox, M. W. 1983, ApJ, 272, 99

Wood, P. R., \& Faulkner, D. J. 1986, ApJ, 307, 659

Wood, P. R., \& Zarro, D. M. 1981, ApJ, 247, 247

Zijlstra, A. A. 1989, PhD thesis, Leiden

Zijlstra, A. A., te Lintel Hekkert, P., Caswell, J. L., Ratag, M., \& Habing, H. $1989, \mathrm{~A} \& \mathrm{~A}, 217,157$

Zuckerman, B., \& Aller, L. H. 1986, ApJ, 301, 772

Zuckerman, B., \& Dyck, H. M. 1986, ApJ, 304, 394 


\section{Chapter 2}

\section{Evolution of Low and Intermediate Mass Stars to the End of the AGB with Mass Loss}

\subsection{Introduction}

Two essential features of asymptotic giant branch (AGB) evolution are helium shell flashes and mass loss. Helium shell flashes give rise to red giants such as the $\mathrm{S}$ and C stars in which the surface abundances have clearly been modified by contamination with nuclear processed material from the interior. Iben (1975) showed that this contamination was produced by the dredge-up of carbon (C) and s-process elements at helium shell flashes. Direct evidence for the occurrence of helium shell flashes is provided by the rapid observed period changes in some Mira variables (Wood \& Zarro 1981).

The obvious importance of mass loss lies in the fact that the progeny of AGB stars, the planetary nebula nuclei (PNNi) and white dwarfs, have mass distributions peaked closely around $0.6 \mathrm{M}_{\odot}$ (Weidemann \& Koester 1983; Weidemann 1990) while the mainsequence masses of these objects must have been $\gtrsim 1 \mathrm{M}_{\odot}$ (Pottasch 1984; Jura 1990). It is also clear that the mass loss between the main sequence and the white dwarf stage occurred largely on the AGB. Working back from the planetary nebula (PN) phase, the typical PN mass of $\sim 0.2 \mathrm{M}_{\odot}$ means that this amount of mass was ejected on the AGB. Renzini (1981) noted that the mean mass loss rate required in order to produce the typical PN was $\sim 3 \times 10^{-5} \mathrm{M}_{\odot} \mathrm{yr}^{-1}$ and he introduced the term superwind to describe such mass loss rates since they are much higher than the rates given by the widely-used mass loss formula of Reimers (1975). Since that time, many AGB stars (particularly infrared sources, $\mathrm{OH} / \mathrm{IR}$ stars, and Mira variables) have been found to have mass loss 
rates consistent with superwind values (e. g., Knapp \& Morris (1985) in the Galaxy, and Wood et al. (1992) in the LMC). The frequent occurrence of such objects means that the production of planetary nebulae $(\mathrm{PNe})$ by discrete ejection events such as dynamical instability of the envelope (Roxburgh 1967; Paczyński \& Ziolkowski 1968) is unlikely. However, the interaction of mass loss mechanisms with the changes in surface luminosity produced by helium shell flash cycles should still modulate the mass loss rate. It is the interplay between mass loss and evolution on the AGB that this paper sets out to address.

Few of the previous stellar evolution calculations of AGB evolution have included mass loss, and when this has been done (Schönberner 1979, 1981, 1983; Boothroyd \& Sackmann 1988a, b, c, d), the mass loss has been incorporated in the form defined by Reimers (1975). As noted above (see also Iben \& Renzini 1983; Wood 1986), this formula fails to produce mass loss at anything like the rates observed in AGB stars. In this paper, empirical data based on observations of AGB stars is used in order to derive the mass loss rate.

Given a reliable estimate of the mass loss rate for AGB stars, the study of AGB evolution yields the AGB termination luminosity, the core mass of the star at the time, the mass in the nearby circumstellar environment of the remnant star, and the phase of the shell flash cycle at which the star leaves the AGB. Since this star will evolve to become a planetary nebula nucleus (PNN), an outcome of the calculations will be PNN mass, luminosity, and phase of shell flash cycle as a function of initial mass. Combined with the evolutionary tracks of these objects, such data is capable of providing the PN luminosity function and the input required for computation of the UV flux generated by hot stars passing to the white dwarf phase. These problems will be considered in future papers. We note that extensive studies of $\mathrm{PNe}$ in the Magellanic Clouds have been carried out in recent times (Monk, Barlow, \& Clegg 1988; Boroson \& Liebert 1989; Meatheringham \& Dopita 1991a, b; Vassiliadis et al. 1992), and these studies provide a large homogeneous sample of $\mathrm{PNe}$ at a single distance with which theoretical calculations can be compared.

A totally separate problem that AGB calculations seek to address is that of $\mathrm{S}$ and $\mathrm{C}$ star formation. The large body of observational material obtained for late-M and C stars in the Magellanic Clouds (Westerlund et al. 1991, and references therein) provides a strong reference point for comparing theory with observation. However, available stellar evolution models cannot readily explain the formation of $\mathrm{C}$ stars at the low luminosities observed (see Lattanzio 1989a for a review), and the current set 
of calculations also suffer from this problem.

In this paper, we present a grid of 22 stellar evolutionary tracks, complete from the main sequence through to the termination of the AGB. Since the evolution has been followed from the main sequence, it is possible to investigate AGB properties as a function of initial stellar mass and total stellar age. The models span a range in initial mass of $0.89 \lesssim M / M_{\odot} \leq 5.0$. The abundances were chosen to be appropriate for stars in the solar neighbourhood, the LMC and the SMC.

\subsection{Computational Details}

The calculations presented in this work were made with the Mount Stromlo Stellar Structure Program (Wood \& Zarro 1981; Wood \& Faulkner 1986, 1987). Models typically contained 400 mesh points, going up to 500 at shell flashes.

Four values of the metal abundance were used during the calculations: $Z=0.016$, $Z=0.008, Z=0.004$ and $Z=0.001$. The metal abundance $Z=0.016$ corresponds to the Solar mix of Ross \& Aller (1976). Metal abundances $Z=0.008$ and $Z=0.004$ were adopted to approximate the LMC and SMC abundances, respectively, to agree with the abundance analyses of Russell \& Bessell (1989) and Russell \& Dopita (1990) which indicate that the LMC metal abundance is $\sim 1 / 2$ solar and the SMC is $\sim 1 / 4$ solar. These abundance analyses also indicate that ${ }^{14} \mathrm{~N}$ is less abundant in the LMC and SMC than in the Sun by an additional factor of $\sim 2$, and the adopted LMC and SMC mixtures have ${ }^{14} \mathrm{~N}$ abundances of $1 / 4$ solar and $1 / 16$ solar, respectively. The $Z=0.001$ mixture is simply $1 / 16$ solar, and is designed to simulate an old (low mass) population.

All evolutionary sequences were begun with a ${ }^{4} \mathrm{He}$ mass fraction of $Y=0.25$. This helium abundance is representative of the pre-galactic He abundance (Wagoner 1973; Steigman 1983) and of the HII regions in the Magellanic Clouds (Dufour 1984), and gives good fits to colour-magnitude diagrams of clusters (e. g., Buzzoni et al. 1983; Caputo, Martinez Roger, \& Paez 1987).

Opacities with appropriate values of $\mathrm{H}, \mathrm{He}, \mathrm{C}, \mathrm{N}, \mathrm{O}$, and heavier elements (in the Ross-Aller mix ratio) were generated from the Astrophysical Opacity Library of Huebner et al. (1977). A molecular opacity contribution was added to the surface opacity as described in the appendix of Bessell et al. (1989) (however, note that the term 6.56 in the equation for $C N+C O$ opacity should be 1.84 and that the terms 5.97 
and 0.005 in the equation for $\mathrm{H}_{2} \mathrm{O}+\mathrm{TiO}$ opacity should be 1.13 and 0.05 , respectively).

Convection was treated by standard mixing-length theory, with mixing-length to pressure scale height ratio $\alpha=1.6$. No overshoot (e. g., Maeder 1975; Bertelli et al. 1985; Alongi et al. 1991; see also Andersen, Nordström, \& Clausen 1990) or extramixing (Hollowell \& Iben 1988) was used. Semi-convection during He core burning has been treated explicitly using the method of Castellani et al. (1985). Another convective phenomenon that needs to be considered during the core helium burning exhaustion phase is that of breathing pulses (Castellani, Giannone, \& Renzini 1971; Sweigert \& Demarque 1973). There is some debate as to whether this phenomenon occurs in real stars. The problem has been addressed by comparing relative numbers of horizontal branch (HB) and AGB stars in globular clusters (Chiosi, Bertelli, Bressan 1987; Renzini $\&$ Fusi Pecci 1988; Caputo et al. 1989). These comparisons suggest that models which suppress breathing pulse appear best able to reproduce the observed ratios of AGB to HB stars. We have therefore suppressed breathing pulses in our calculations by stopping any mixing of helium into the central core if that mixing would lead to a rise in the central He mass fraction.

Nuclear reaction rates for hydrogen burning were taken from Harris et al. (1983) when available, and Fowler, Caughlan, \& Zimmermann (1975) otherwise. For He burning and beyond, new rates have been adopted from Caughlan \& Fowler (1988). The main difference between these new rates and those used previously is that the rate for the reaction ${ }^{12} \mathrm{C}(\alpha, \gamma){ }^{16} \mathrm{O}$ has decreased by a factor of $\sim 3$ from the value published by Caughlan et al. (1985) - the rate is lowered to approximately its 1975 value.

The primary purpose of this work is to study AGB evolution using a realistic prescription of the mass loss rate. As pointed out in the Introduction, the widely-used Reimers' (1975) rate does not predict the superwind mass loss rates required for the production of planetary nebulae (Renzini 1981) or the high mass loss rates (e. g., Knapp \& Morris 1985) observed in AGB stars, and neither does it accurately reflect the increase in mass loss during ascent of the AGB (Wood 1986).

No complete theory of mass loss from AGB stars exists at present, although mass loss is thought to be a dual process involving levitation of matter above the photosphere by large-amplitude radial pulsation followed by the formation of grains on which radiation pressure acts to drive the circumstellar material away from the star (Wood 1979; Castor 1981; Holzer \& MacGregor 1985; Bowen 1988; Hearn 1990). The involvement of pulsation in the mass loss process suggests that the mass loss rate may be a function 
of pulsation period. Empirical plots (Wood 1986, 1990a; Schild 1989) of $\log \dot{M}$ against pulsation period $\mathrm{P}$ for mass losing AGB stars show two distinct phases of mass loss. For periods less than $\sim 500$ days, the mass loss rate increases exponentially with period, while beyond $\sim 500$ days, the mass loss rate is essentially constant at superwind values of a few times $10^{-5} \mathrm{M}_{\odot} \mathrm{yr}^{-1}$. Examination of mass loss rates for AGB stars in this superwind phase shows that in the Galaxy (Knapp 1986), the LMC (Wood et al. 1992) and in the Galactic bulge (Whitelock et al. 1991), the mass loss rate lies within a factor of $\sim 2$ of the value

$$
\dot{M}=L / c v_{\text {exp }}
$$

which corresponds to a radiation pressure driven wind. In this equation, $L$ represents stellar luminosity, $c$ represents the speed of light, and $v_{\text {exp }}$ denotes the stellar wind expansion velocity far from the central star. The fact that this relation applies to a mixture of stars in the local Galactic Disk, to young upper AGB stars in the metal deficient LMC, and to a population that is probably old and metal rich in the Galactic bulge suggests that this relation applies universally (to within a factor of $\sim 2$ ).

Before the superwind phase (which starts at $P \sim 500$ days), the mass loss rate increases exponentially with $\mathrm{P}$ as noted above. In this phase, the mass loss rate used here is that given by equation $8 \mathrm{a}$ of Wood (1990a), viz.

$$
\log \dot{M}\left(M_{\odot} y r^{-1}\right)=-11.4+0.0125 P(\text { days }) .
$$

The mass loss rate applying at any time during the calculations was assumed to be the minimum of those given by equations 2.1 and 2.2 .

In order to compute the mass loss rate from equation 2.1, $v_{\exp }$ needs to be known. This was calculated from equation 9 from Wood (1990a), viz.

$$
v_{\text {exp }}\left(k m s^{-1}\right)=-13.5+0.056 P(\text { days }),
$$

with the additional constraint that $v_{\text {exp }}$ lie in the range 3.0 to $15.0 \mathrm{~km} \mathrm{~s}^{-1}$. In practice, $v_{\text {exp }}$ is almost always $15 \mathrm{~km} \mathrm{~s}^{-1}$ in the superwind phase, in agreement with the expansion velocity observed in high mass loss rate OH/IR stars (e. g., Eder, Lewis, \& Terzian 1988).

Calculation of the mass loss rate from equation 2.2 requires a knowledge of the pulsation period $P$. The computed value of $P$ clearly depends on the mode of pulsation. We adopt the arguments put forward by Wood (1990a, 1990b) and Willson (1982) and assume that Mira variables and dust enshrouded variable AGB stars are pulsating in 
the fundamental mode (see Tuchman 1991 for alternative arguments). Then $\mathrm{P}$ can be derived using the period-mass- radius relation given by equation 5 of Wood (1990a):

$$
\log P(\text { days })=-2.07+1.94 \log R / R_{\odot}-0.9 \log M / M_{\odot} .
$$

The above equations predict that stars of low mass $\left(\lesssim 2.5 M_{\odot}\right)$ will attain superwindlike mass loss rates at $P \sim 500$ days when they will become dust enshrouded and no longer optically visible. This is what is observed in low mass populations such as those in the solar vicinity, where the number density of optically visible Mira variables drops sharply between $P=425$ and 500 days (Wood \& Cahn 1977), and in the Galactic Bulge, where the long period variable (LPV) population is optically visible up to $P=500$ days (Lloyd Evans 1976; Wood \& Bessell 1983) while at longer periods it can only be seen in the infrared (Whitelock et al. 1991). However, on the upper AGB where masses are $\sim 5 M_{\odot}$ and $M_{\text {bol }} \sim-6$ to -7 , optically visible LPVs exist out to periods of $\sim 750$ days (Wood, Bessell \& Fox 1983; Hughes \& Wood 1990). In order to prevent the adopted mass loss prescription removing these observed objects, equation 2.2 was modified to delay the onset of the superwind phase in stars of mass greater than $2.5 M_{\odot}$. For these stars, the mass loss rate was derived from the equation

$$
\log \dot{M}\left(M_{\odot} y r^{-1}\right)=-11.4+0.0125\left[P(\text { days })-100\left(M / M_{\odot}-2.5\right)\right]
$$

In summary, the above AGB mass loss scheme reproduces the observed rapid exponential increase in mass loss rate with period before the onset of the superwind phase, it has a superwind phase corresponding to radiation pressure driven mass loss, and it predicts the onset of this superwind mass loss phase at pulsation periods where optically-visible LPVs disappear in the solar vicinity, the Galactic bulge and the LMC. For the purposes of this paper, the AGB mass loss phase is assumed to be complete when the envelope mass is reduced to such a small value that $\log T_{\text {eff }}$ has become hotter than a reference AGB temperature by an amount $\Delta \log T_{\text {eff }}=0.3$, where the reference AGB is given by

$$
M_{\text {bol }}=12.5 \log T_{\text {eff }}-2.925 \log M / M_{\odot}+1.453 \log Z / Z_{\odot}-47.1 \text {. }
$$

This reference AGB temperature is a fit to the AGB temperatures produced by the evolution code for stars with envelope masses sufficiently large that the star has not begun to evolve to the blue. Some preliminary sequences were run in order to derive the fit. The period of pulsation used in the mass loss equations 2.2 and 2.5 was derived assuming the star had a $T_{\text {eff }}$ given by equation 2.6 (rather than the actual $T_{\text {eff }}$ ). This 
was done so that the superwind mass loss rate would be maintained while the star evolved to the blue of the AGB, where the superwind ceased at $\Delta \log T_{\text {eff }}=0.3$.

AGB mass loss accounts for the majority of the mass lost in most low to intermediate mass stars. However, in low mass globular cluster stars, mass loss amounting to $\sim 0.2$ $\mathrm{M}_{\odot}$ is required on the first giant branch (FGB) in order to match the observed HB morphology (Fusi Pecci \& Renzini 1976; 1978; Renzini \& Fusi Pecci 1988, and references therein). (But we note here that recent results (Simon 1992) using new opacities indicate that the well established first giant branch mass loss of $\sim 0.2 M_{\odot}$ may be negligible after all!). If we adopt the Reimers' (1975) mass loss rate on the FGB, then the mass loss rate is proportional to $R / M$, and a significant amount of envelope mass is lost only for the stars of lowest $M$ (apart from the direct effect of $M$ on the Reimers' mass loss rate, lowering $M$ also gives a cooler giant branch which increases $R$ and further increases $\dot{M}$ ). For this reason, we have only considered FGB mass loss for evolutionary sequences with $M_{i}<1 \mathrm{M}_{\odot}$. The mass loss rate used was actually $1 / 3$ Reimers' (1975) rate in order to get a total mass loss of $\sim 0.2 M_{\odot}$ on the FGB.

\subsection{Results and Discussion}

All evolutionary sequences in this study were begun on the zero age main sequence (ZAMS) and went through to the end of the AGB. Although the study of AGB evolution with realistic mass loss rates is the prime motivation for these calculations, we briefly look at some properties of the stellar models prior to the AGB, and see how they compare with other recent theoretical studies.

\subsubsection{Evolution to the First Helium Shell Flash}

Figures 2.1-2.4 show the evolutionary tracks in the H-R diagram. For $M_{i} \geq 2.5$, the tracks shown end at the first helium shell flash while for $M_{i}<2.5$ the helium core burning and AGB phases have been supressed for clarity. Equivalent evolutionary points (EEPs) in each sequence have been defined in table 2.1, and evolutionary properties at these points are presented in table 2.2. The following quantities are given in the table 2.2: time elapsed from the beginning of the ZAMS, stellar effective temperature $\mathrm{T}_{\text {eff }}$, stellar luminosity $L$, total stellar mass $\mathrm{M}$, the mass of the core $\mathrm{M}_{c}^{H}$ (defined as the point where the hydrogen abundance, by mass, falls to $50 \%$ of its surface value), the mass $\Delta_{c}^{H e}$ between $\mathrm{M}_{c}^{H}$ and the helium core (defined as the point where the he- 
lium mass fraction first reaches 0.5 going outward from the centre), and the mass $\Delta_{b c e}$ between $M_{c}^{H}$ and the base of the convective envelope. All masses are in units of $M_{\odot}$.

In table 2.3, we have summarized the evolutionary lifetimes of various phases, including the AGB phase. The main sequence (MS) lifetime, $\tau_{M S}$, has been defined as the time the central hydrogen abundance drops to zero. This corresponds closely to the age quoted at point 3 in table 2.2 , but significant variation $(\sim 10 \%)$ is seen for $M_{i} \lesssim 1.0$ $\mathrm{M}_{\odot}$. Two lifetimes related to the FGB are tabulated: $\tau_{F G B}$ is calculated from the end of the MS to the point of He core ignition, while $\tau_{F G B-C}$ is the time taken to evolve up the FGB from the luminosity of the helium core burning clump to the point of He core ignition. The interval $\tau_{F G B}$ includes the time spent in the sub-giant, or $\mathrm{H}$-shell narrowing phase, which varies from $40-60 \%$ of $\tau_{F G B}$. The He core burning lifetime $\tau_{H e B}$ is defined as the time from core helium ignition to the time when He shell burning is established (point 11). The AGB lifetime is defined as the time from establishment of helium shell burning (point 11) to the time the star begins to move away from the AGB locus in the H-R diagram (point 15). Table 2.3 also lists ratios of various lifetimes, these ratios being equivalent to population ratios in the various evolutionary phases.

Between the MS and the end of the AGB, low to intermediate mass stars experience three types of dredge-up episode during which material that has undergone nuclear processing in the stellar interior is mixed to the surface by envelope convection. The first episode takes place on the FGB (Iben 1964), the second during early AGB (EAGB) evolution (Kippenhahn, Thomas, \& Weigert 1965; Weigert 1966), and the third during the helium shell flashing AGB phase (Iben 1975; Sugimoto \& Nomoto 1975). Products from the CNO cycle are transported to the stellar surface during the first and second dredge-up episodes, while the third dredge-up brings up He shell burning products $\left({ }^{12} \mathrm{C}\right.$ and s-process elements) as well. The first dredge-up occurs at point 5 in table 2.1 and the second dredge-up occurs at point 13. Initial (ZAMS) abundances and surface abundances after the first, second, and third dredge-up episodes are listed in tables 2.4-2.7 (for the third dredge-up, the abundance listed is that at the end of the AGB phase, point 15). Some detailed comments are now presented on various aspects of the pre-AGB evolution computed here.

\subsubsection{Main Sequence Evolution}

Recent studies of the main sequence and sub-giant branch evolution have been made by Sweigert, Greggio, \& Renzini (1989; hereafter SGR), Lattanzio (1991), and Castellani, 
Chieffi, \& Straniero (1992; hereafter CCS). SGR use opacities from Cox \& Stewart (1970a, b), whereas Lattanzio and CCS use opacities from Huebner et al. (1977) - the former opacities are lower for the same metal content. The value of the mixing-length $\alpha$ is approximately the same in all three studies.

Comparison of MS lifetimes from the present study with those of Lattanzio (1991) and CCS shows good agreement, generally to $3 \%$ for the lower masses and to $\sim 10 \%$ for higher masses. However, as shown clearly in figure 2.5, the ages given by the SGR models are $\sim 30 \%$ smaller than those presented here. The difference can probably be attributed to the use of the older Cox \& Stewart $(1970 \mathrm{a}, \mathrm{b})$ opacities by CCS rather than the Huebner et al. (1977) opacities. This discrepancy has been noted previously, and it is found to become more severe with increasing Z (VandenBerg \& Laskarides 1987).

\subsubsection{First Giant Branch Evolution}

Evolution along the sub-giant and lower giant branches involves the convective envelope moving inwards. For $M_{i}<2.5$, deepest penetration (first dredge-up) occurs well before core He ignition, while for the $2.5,3.5$, and $5.0 \mathrm{M}_{\odot}$ sequences, the maximum depth of the convective envelope was found to occur at He core ignition. This is essentially what was found by SGR.

A halt in evolution up the FGB occurs when the H-burning shell encounters the abundance discontinuity left behind by first dredge-up. This stalling of FGB evolution should give rise to a bump in the FGB luminosity function and such a bump has been confirmed observationally, for example in 47 Tuc (King et al. 1985; Hesser et al. 1987). However, the luminosity at which the bump is observed is lower than predicted by standard evolutionary calculations such as the present ones. The solution to this problem may be the inclusion in models of convective undershoot at the base of the convective envelope (Alongi et al. 1991). This will allow convection to penetrate deeper during dredge-up phases and the outward-burning $\mathrm{H}$-shell will therefore meet the abundance discontinuity at lower luminosities than in standard calculations.

All evolutionary sequences with $M_{i}<2.5 \mathrm{M}_{\odot}$ developed a degenerate He core while ascending the FGB. For sequences with $M_{i} \geq 2.5 \mathrm{M}_{\odot}$, non-degenerate He-core ignition terminated the FGB phase. SGR find that the transition from degenerate to nondegenerate FGB evolution occurs for $2.0<M_{i}<2.8$, depending on the composition. The stellar temperature, luminosity, and core mass, at the point of core He ignition 
for each sequence are presented in figure 2.6. We have also included the $Z=0.004$, $Y=0.2$ and 0.3 , results from SGR to show the variation with mass of these quantities through the transition from degenerate to non-degenerate FGBs.

As a final test of our calculations up to the end of the FGB, we compare our tracks in $\mathrm{H}-\mathrm{R}$ diagram with those of Lattanzio (1991) (figure 2.7). A one-to-one comparison is difficult because of the different $\mathrm{Y}$ and $\mathrm{Z}$ values used in the two studies (there is also a small difference in $\alpha$, with Lattanzio using $\alpha=1.5$ while we use $\alpha=1.6$ ). However, it is clear that our tracks evolve to cooler temperatures during the latter half of the FGB phase. This is most likely due to the inclusion of molecular opacities. On the other hand, the maximum luminosities reached at the tip of the FGB, and the ages at this point, are identical to within $1 \%$, and the core masses $M_{c}^{H}$ there agree to within $4 \%$. We also note that values of $\tau_{F G B} / \tau_{M S}$ from this study agree with those of CCS to within $\sim 10 \%$.

\subsubsection{Core Helium Burning Evolution}

Recovery from He-core ignition at the tip of the FGB is relatively rapid, for both degenerate and non-degenerate ignition scenarios, typically lasting $\sim 10^{6}$ years. Evolutionary sequences corresponding to $M_{i} \lesssim 2.5 \mathrm{M}_{\odot}$ spend a large fraction of their time undergoing core He burning $(\mathrm{CHeB})$ in a very narrow range of luminosity and temperature. They do not develop the extended blue loops characteristic of larger mass sequences (see figures 2.1-2.4). Hence, these low mass stars remain close to the locus in the H-R diagram defined by the FGB, and have come to be known as clump giants (Cannon 1970; Faulkner \& Cannon 1973). For the clump stars in the present calculations, the luminosities are similar to those of the models of Lattanzio (1991) and Seidel, Demarque, \& Weinberg (1987).

An important point to note about the present calculations is that the last $5-10 \%$ of $\mathrm{CHeB}$ is included in the calculations along with an explicit treatment of semi-convection (as opposed to letting ordinary convection mix the core as in Lattanzio's calculations). Many calculations in the past have tended to ignore this phase due to convergence difficulties associated with breathing pulses (e. g., Sweigert et al. 1987). Calculation through the final phases of core He burning increases the total $\mathrm{CHeB}$ lifetime. It also increases the final $\mathrm{O} / \mathrm{C}$ abundance ratio in the core as this ratio increase markedly as the end of $\mathrm{CHeB}$ approaches (Sweigert \& Gross 1976). A comparison of our clump burning lifetimes with those of Lattanzio (1991) (who also evolved right through the helium 
core burning stage) shows close agreement, whereas the lifetimes of Seidel, Demarque, \& Weinberg (1987) (who stopped their calculations when the central helium abundance reached $\left.X_{H e} \sim 0.1\right)$ are $\sim 30 \%$ shorter. In another comparison, values of $\tau_{H e B}$ from CCS (who evolved right through the He core burning phase) are $\sim 15 \%$ shorter than the corresponding values in this study.

The ${ }^{12} \mathrm{C}$ and ${ }^{16} \mathrm{O}$ core abundances following core He burning for our $M_{i} \lesssim 3.5 \mathrm{M}_{\odot}$ models (table 2.8) are $X_{C} \sim 0.1$ and $X_{O} \sim 0.9$, respectively. This is despite the fact that we are using the new, reduced ${ }^{12} C(\alpha, \gamma){ }^{16} \mathrm{O}$ reaction rate. The models presented by Lattanzio (1991), who used the ${ }^{12} C(\alpha, \gamma){ }^{16} \mathrm{O}$ reaction rate which is similar to the one used here, have $X_{C} \sim 0.2-0.3$ and $X_{O} \sim 0.7-0.8$. Nearly all the conversion of ${ }^{12} \mathrm{C}$ to ${ }^{16} \mathrm{O}$ occurs during the burning of the last $10 \%$ of He in the core. Our high rates of conversion are almost certainly the result of our treatment of semi-convection/breathing pulses which appears to be more efficient at prolonging the last stages of $\mathrm{CHeB}$ than most codes. Models with $M_{i} \geq 3.5 \mathrm{M}_{\odot}$ have cores with $X_{C} \sim 0.45$ and $X_{O} \sim 0.55$, similar to the abundances in Lattanzio's (1991) $5 \mathrm{M}_{\odot}$ models.

\subsubsection{The Early-AGB Phase}

The early-AGB (E-AGB) phase is the time interval from ignition of He shell burning (close to point 11 in table 2.1) to the onset of the first major helium shell flash, or thermal pulse (point 14 in table 2.1). Physically, during this phase, a helium burning shell eats away at the base of the helium-rich layer which has resulted from past $\mathrm{H}$ shell burning around the helium burning core.

Following the establishment of He shell burning, the $\mathrm{H}$ shell burning luminosity decreases. In the lower mass stars, $\mathrm{H}$ shell burning remains a significant contributor to the total luminosity while in more massive stars $\left(M_{i} \gtrsim 3.5 \mathrm{M}_{\odot}\right)$ the hydrogen burning is extinguished. The drop in $\mathrm{H}$ shell burning luminosity also causes the total luminosity to decline. This feature is not usually seen in the H-R diagram as the star traverses down and up the giant branch although in low mass, low metallicity evolutionary tracks the feature can be seen as a small loop (e. g., Castellani, Chieffi \& Pulone 1991, hereafter CCP).

The duration of the E-AGB phase in theoretical calculations depends very strongly on the treatment of the preceding $\mathrm{CHeB}$ evolution. In particular, the relative timescales of the $\mathrm{CHeB}$ and $\mathrm{E}$-AGB phases depend on the amount of mixing of He into the core through semi-convection, core overshoot and breathing pulses. Mixing in more $\mathrm{He}$ 
during core helium burning extends the $\mathrm{CHeB}$ phase at the expense of the E-AGB phase. The ratio of AGB to CHeB lifetimes in our lowest mass models is $\tau_{A G B} / \tau_{H e B} \sim$ 0.08 , which is similar to the value Caputo et al. (1989) obtained when they included breathing pulses in their models. This result gives added weight to the suggestion above that our treatment of central helium exhaustion extends this phase longer than most codes which suppress breathing pulses.

When the helium-rich layer has become thin enough at the end of the E-AGB phase, the alternating hydrogen and helium shell burning episodes characteristic of the thermally pulsing AGB phase begin. Mini-pulses are observed just prior to the first major thermal pulse, as found in other studies (e. g,. Becker \& Iben 1980; Lattanzio 1986; Boothroyd \& Sackmann 1998). We have defined the first major thermal pulse as the thermal pulse that results in shell $\mathrm{H}$ burning being extinguished (point 14 in table 2.1). The surface luminosity given at point 14 corresponds to the luminosity at $\mathrm{H}$ shell extinction.

The E-AGB phase for our models is observed to last for $\sim 10^{7}$ yrs up to $M_{i} \sim 2.5$ $\mathrm{M}_{\odot}$, dropping to $\sim 3 \times 10^{6}$ years at $3.5 \mathrm{M}_{\odot}$ and $10^{6}$ years at $5 \mathrm{M}_{\odot}$. Up to the end of the E-AGB phase, with our mass loss formalism, the total stellar mass remains essentially unchanged from its main-sequence mass (or the mass at the end of the FGB for the two sequences where FGB mass loss was included). We note that the E-AGB phase has been described in detail by Lattanzio (1986) and Becker \& Iben (1980).

\subsubsection{Surface Abundance Changes-First and Second Dredge-up}

Figures 2.8-2.9 show surface abundance enhancement factors which result from the first and second dredge-ups: these figures were constructed from data in tables 2.4-2.7. The small ${ }^{4} \mathrm{He}$ enhancements, the ${ }^{12} \mathrm{C}$ depletion by a factor of $\sim 0.7$, and the small changes in ${ }^{16} \mathrm{O}$ are consistent with past results (e. g., Becker \& Iben 1979; Lattanzio 1986; SGR). One noticeable difference between the present calculations and other calculations is the large ${ }^{14} \mathrm{~N}$ enhancement factor of up to $\sim 9$ for the lowest metallicity case. Becker \& Iben (1979) studied the mass range $3 \leq M_{i} \leq 11$ and found that ${ }^{14} \mathrm{~N}$ was only enhanced by a factor of 2.7 , at most. The other studies cited above found similar enhancement factors and, indeed, our solar metallicity case is in agreement with this result. The reason that large enhancement factors were found in the low metallicity cases studied here is that these mixtures began with a N/C ratio of half solar. Thus, for a given amount of dredged-up CN-cycled material, the $\mathrm{N}$ enhancement factor will clearly be 
greater in the present calculations.

Second dredge-up, which occurs during the early-AGB phase, is significant mostly in models of relatively large mass $\left(M_{i} \gtrsim 5 \mathrm{M}_{\odot}\right)$ where the H-burning shell is dormant (Becker \& Iben 1979). Once the base of the convective envelope penetrates the HHe discontinuity during second dredge-up, large enhancements $(\sim 20 \%)$ in ${ }^{4} \mathrm{He}$ occur (Becker \& Iben 1979). The fact that we saw no such enhancements indicates that our most massive models are, at $5 \mathrm{M}_{\odot}$, just below the mass limit where significant second dredge-up occurs. 
TABLE 2.1

\section{Equivalent Evolutionary Points}

\begin{tabular}{|c|c|}
\hline Point Number & Description \\
\hline $1 \ldots \ldots \ldots \ldots$ & Point at $1 \%$ of the main sequence lifetime. \\
\hline $2 \ldots$ & $\begin{array}{l}\text { Maximum main sequence redward extension. } \\
\text { Overall contraction of the star begins, and the mass fraction } \\
\text { of the convective core begins to decrease rapidly. }\end{array}$ \\
\hline 3 & $\begin{array}{l}\text { Maximum main sequence blueward extension; } \\
\mathrm{H} \text { nearly exhausted at centre; Core contracts further, } \\
\text { and thick H-shell burning is soon established. }\end{array}$ \\
\hline 4 . & $\begin{array}{l}\text { Luminosity minimum corresponding to the base of the FGB. } \\
\text { Envelope convection begins to move inwards. }\end{array}$ \\
\hline 5 & Maximum inward penetration of convective envelope on FGB. \\
\hline . & $\begin{array}{l}\text { Luminosity minimum corresponding to the H-shell reaching } \\
\text { the abundance discontinuity created by first dredge-up. }\end{array}$ \\
\hline 7 & Surface luminosity maximum at He-core ignition. \\
\hline 8 & $\begin{array}{l}\text { He-core luminosity minimum following establishment of He-core } \\
\text { burning. }\end{array}$ \\
\hline 9 & $\begin{array}{l}\text { H-shell luminosity minimum following establishment of He-core } \\
\text { burning. }\end{array}$ \\
\hline 10 & $\begin{array}{l}\text { Maximum horizontal branch blueward extension. } \\
\text { Only defined for models with } M_{i} \geq 2.5 \text {. }\end{array}$ \\
\hline 11 & H-shell luminosity peak at He-core exhaustion. \\
\hline 12 & $\begin{array}{l}\text { Equal } \mathrm{H} \text { and He shell burning luminosities on AGB following } \\
\text { H-shell recovery after He-shell ignition. }\end{array}$ \\
\hline 13 & Maximum inward penetration of convective envelope on AGB. \\
\hline & $\begin{array}{l}\text { First major thermal pulse; first time H-shell burning is } \\
\text { extinguished. }\end{array}$ \\
\hline $15 \ldots \ldots \ldots$ & End of AGB; $\Delta \log T_{\text {eff }}=0.3$. \\
\hline
\end{tabular}


TABLE 2.2

Equivalent Evolutionary PoInts

\begin{tabular}{|c|c|c|c|c|c|c|c|}
\hline EEP & $\begin{array}{l}\text { Time } \\
\text { (yrs) }\end{array}$ & $\log \mathrm{T}_{\text {eff }}$ & $\log \mathrm{L} / \mathrm{L}_{\odot}$ & $\mathrm{M}_{\text {total }}$ & $\mathbf{M}_{c}^{H}$ & $\Delta_{c}^{H e}$ & $\Delta_{b c e}$ \\
\hline \multicolumn{8}{|c|}{$(M, Y, Z)=(1.0,0.25,0.016)$} \\
\hline $\begin{array}{l}1 \\
2\end{array}$ & $1.0823589 \mathrm{E}+08$ & 3.741 & 0.225 & 1.0000 & & 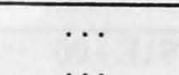 & \\
\hline 3 & $1.0508762 \mathrm{E}+10$ & 3.762 & 0.102 & 1.0000 & 0.0749170 & & $9.06 \mathrm{E}-01$ \\
\hline 5 & $1.4659747 \mathrm{E}+10$ & 3.660 & 1.238 & 1.0000 & 0.2173825 & & $3.60 \mathrm{E}-02$ \\
\hline 6 & $1.4733977 \mathrm{E}+10$ & 3.647 & 1.497 & 1.0000 & 0.2531778 & $\ldots$ & $2.42 \mathrm{E}-02$ \\
\hline 7 & $1.4813064 \mathrm{E}+10$ & 3.457 & 3.385 & 1.0000 & 0.4778179 & & $5.22 \mathrm{E}-01$ \\
\hline $\begin{array}{l}8,9 \\
10\end{array}$ & $1.4815051 \mathrm{E}+10$ & 3.662 & 1.667 & 1.0000 & 0.4778583 & & $2.08 \mathrm{E}-01$ \\
\hline 11 & $1.4954658 \mathrm{E}+10$ & 3.623 & 2.154 & 1.0000 & 0.5365303 & $2.10 \mathrm{E}-01$ & $1.06 \mathrm{E}-01$ \\
\hline 12 & $1.4966024 \mathrm{E}+10$ & 3.529 & 2.985 & 1.0000 & 0.5445179 & $5.21 \mathrm{E}-02$ & $1.74 \mathrm{E}-02$ \\
\hline 13 & $1.4966374 \mathrm{E}+10$ & 3.504 & 3.159 & 1.0000 & 0.5480492 & $4.14 \mathrm{E}-02$ & $1.12 \mathrm{E}-02$ \\
\hline 14 & $1.4966745 \mathrm{E}+10$ & 3.486 & 3.271 & 0.9999 & 0.5547234 & $3.29 \mathrm{E}-02$ & $9.21 \mathrm{E}-03$ \\
\hline 15 & $1.4967240 \mathrm{E}+10$ & 3.695 & 3.474 & 0.5712 & 0.5679647 & $2.50 \mathrm{E}-02$ & $2.92 \mathrm{E}-03$ \\
\hline \multicolumn{8}{|c|}{$(M, Y, Z)=(1.5,0.25,0.016)$} \\
\hline 1 & $2.7728901 \mathrm{E}+07$ & 3.852 & 0.623 & 1.5000 & & & \\
\hline 2 & $2.6621934 \mathrm{E}+09$ & 3.810 & 0.784 & 1.5000 & 0.1342438 & $\ldots$ & $1.37 \mathrm{E}+00$ \\
\hline 3 & $2.7412960 \mathrm{E}+09$ & 3.836 & 0.894 & 1.5000 & 0.1342438 & 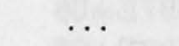 & $1.37 \mathrm{E}+00$ \\
\hline 4 & $3.1487051 \mathrm{E}+09$ & 3.714 & 0.775 & 1.5000 & 0.1771254 & $\cdots$ & $9.60 \mathrm{E}-01$ \\
\hline 5 & $3.4223587 \mathrm{E}+09$ & 3.667 & 1.593 & 1.5000 & 0.2451361 & $\ldots$ & $3.28 \mathrm{E}-02$ \\
\hline 6 & $3.4559808 \mathrm{E}+09$ & 3.653 & 1.825 & 1.5000 & 0.2771459 & $\cdots$ & $2.22 \mathrm{E}-02$ \\
\hline 7 & $3.4988641 \mathrm{E}+09$ & 3.503 & 3.376 & 1.5000 & 0.4753451 & .. & $3.09 \mathrm{E}-03$ \\
\hline 8,9 & $3.5007285 \mathrm{E}+09$ & 3.674 & 1.735 & 1.5000 & 0.4754102 & $\cdots$ & $3.38 \mathrm{E}-01$ \\
\hline 10 & & & & & & & \\
\hline 11 & $3.6383415 E+09$ & 3.614 & 2.472 & 1.5000 & 0.5523962 & $2.39 \mathrm{E}-01$ & $7.27 \mathrm{E}-02$ \\
\hline 12 & $3.6469687 \mathrm{E}+09$ & 3.550 & 3.061 & 1.5000 & 0.5445179 & $5.21 \mathrm{E}-02$ & $1.74 \mathrm{E}-02$ \\
\hline 13 & $3.6472461 \mathrm{E}+09$ & 3.529 & 3.223 & 1.5000 & 0.5598327 & $3.80 \mathrm{E}-02$ & $1.08 \mathrm{E}-02$ \\
\hline 14 & $3.6475322 \mathrm{E}+09$ & 3.517 & 3.303 & 1.5000 & 0.5657311 & $3.06 \mathrm{E}-02$ & $9.43 \mathrm{E}-03$ \\
\hline 15 & $3.6483588 \mathrm{E}+09$ & 3.640 & 3.770 & 0.6025 & 0.5998146 & $2.21 \mathrm{E}-02$ & $1.05 \mathrm{E}-03$ \\
\hline \multicolumn{8}{|c|}{$(M, Y, Z)=(2.0,0.25,0.016)$} \\
\hline 1 & $1.2628138 \mathrm{E}+07$ & 3.959 & 1.155 & 2.0000 & & & \\
\hline 2 & $1.2007735 \mathrm{E}+09$ & 3.871 & 1.298 & 2.0000 & 0.2263633 & $\ldots$ & $1.77 \mathrm{E}+00$ \\
\hline 3 & $1.2359935 \mathrm{E}+09$ & 3.919 & 1.406 & 2.0000 & 0.2263633 & $\cdots$ & $1.77 \mathrm{E}+00$ \\
\hline 4 & $1.3085564 \mathrm{E}+09$ & 3.717 & 1.147 & 2.0000 & 0.2444330 & $\cdots$ & $1.30 \mathrm{E}+00$ \\
\hline 5 & $1.3494544 \mathrm{E}+09$ & 3.671 & 1.791 & 2.0000 & 0.2651905 & $\ldots$ & $4.27 \mathrm{E}-02$ \\
\hline 6 & $1.3762669 \mathrm{E}+09$ & 3.651 & 2.093 & 2.0000 & 0.3069570 & $\ldots$ & $2.15 \mathrm{E}-02$ \\
\hline 7 & $1.4004876 \mathrm{E}+09$ & 3.540 & 3.290 & 2.0000 & 0.4596059 & $\cdots$ & $3.53 \mathrm{E}-03$ \\
\hline 8,9 & $1.4016925 \mathrm{E}+09$ & 3.687 & 1.749 & 2.0000 & 0.4596533 & 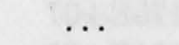 & $5.96 \mathrm{E}-01$ \\
\hline 10 & & & & & & & \\
\hline 11 & $1.5513762 \mathrm{E}+09$ & 3.629 & 2.482 & 2.0000 & 0.5578790 & $2.30 \mathrm{E}-01$ & $9.14 \mathrm{E}-02$ \\
\hline 12 & $1.5588156 \mathrm{E}+09$ & 3.568 & 3.095 & 2.0000 & 0.5619442 & $4.52 \mathrm{E}-02$ & $1.66 \mathrm{E}-02$ \\
\hline 13 & $1.5590323 \mathrm{E}+09$ & 3.551 & 3.235 & 2.0000 & 0.5646395 & $3.73 \mathrm{E}-02$ & $1.08 \mathrm{E}-02$ \\
\hline 14 & $1.5593096 \mathrm{E}+09$ & 3.538 & 3.337 & 2.0000 & 0.5705433 & $2.95 \mathrm{E}-02$ & $9.26 \mathrm{E}-03$ \\
\hline 15 & $1.5604841 \mathrm{E}+09$ & 3.622 & 3.895 & 0.6371 & 0.6332230 & $1.65 \mathrm{E}-02$ & $6.53 \mathrm{E}-04$ \\
\hline
\end{tabular}


TABLE 2.2

Equivalent Evolutionary Points-Continued

\begin{tabular}{|c|c|c|c|c|c|c|c|}
\hline$\overline{\text { EEP }}$ & $\begin{array}{l}\text { Time } \\
(\mathrm{yrs})\end{array}$ & $\log \mathrm{T}_{\text {eff }}$ & $\log \mathrm{L} / \mathrm{L}_{\odot}$ & $\mathrm{M}_{\text {total }}$ & $\overline{\mathbf{M}_{c}^{H}}$ & $\Delta_{c}^{H e}$ & $\overline{\Delta_{\text {bce }}}$ \\
\hline \multicolumn{8}{|c|}{$(M, Y, Z)=(2.5,0.25,0.016)$} \\
\hline 1 & $6.3170791 \mathrm{E}+06$ & 4.033 & 1.545 & 2.5000 & & & \\
\hline 2 & $6.0014404 \mathrm{E}+08$ & 3.939 & 1.699 & 2.5000 & 0.3079392 & $\cdots$ & $2.19 \mathrm{E}+00$ \\
\hline 3 & $6.1938939 \mathrm{E}+08$ & 3.986 & 1.799 & 2.5000 & 0.3079392 & $\ldots$ & $2.19 \mathrm{E}+00$ \\
\hline 4 & $6.4779347 \mathrm{E}+08$ & 3.717 & 1.495 & 2.5000 & 0.3241679 & $\cdots$ & $1.79 \mathrm{E}+00$ \\
\hline 5,7 & $6.6208695 \mathrm{E}+08$ & 3.644 & 2.384 & 2.5000 & 0.3333296 & $\cdots$ & $3.00 \mathrm{E}-02$ \\
\hline 6 & & & & & & $\ldots$ & \\
\hline 8 & $6.6756869 \mathrm{E}+08$ & 3.681 & 1.863 & 2.5000 & 0.3457917 & $\ldots$ & $1.86 \mathrm{E}-01$ \\
\hline 9 & $6.8423149 \mathrm{E}+08$ & 3.701 & 1.615 & 2.5000 & 0.363 & $\ldots$ & $1.16 \mathrm{E}+00$ \\
\hline 10 & $7.5737272 \mathrm{E}+08$ & 3.702 & 1.681 & 2.5000 & 0.4040086 & $\ldots$ & $1.31 \mathrm{E}+00$ \\
\hline 11 & $9.4258266 \mathrm{E}+08$ & 3.645 & 2.428 & 2.5000 & 0.5277498 & $2.26 \mathrm{E}-01$ & $1.05 \mathrm{E}-01$ \\
\hline 12 & $9.5250837 \mathrm{E}+08$ & 3.600 & 2.930 & 2.5000 & 0.5350412 & $5.57 \mathrm{E}-02$ & $2.33 \mathrm{E}-02$ \\
\hline 13 & $9.5304857 \mathrm{E}+08$ & 3.576 & 3.151 & 2.5000 & 0.5402853 & $4.14 \mathrm{E}-02$ & $1.20 \mathrm{E}-02$ \\
\hline 14 & $9.5342602 \mathrm{E}+08$ & 3.557 & 3.314 & 2.5000 & 0.5469485 & $3.49 \mathrm{E}-02$ & $8.05 \mathrm{E}-03$ \\
\hline 15 & $9.5561052 \mathrm{E}+08$ & 3.620 & 3.930 & 0.6702 & 0.6658755 & $9.29 \mathrm{E}-03$ & $6.82 \mathrm{E}-04$ \\
\hline \multicolumn{8}{|c|}{$(M, Y, Z)=(3.5,0.25,0.016)$} \\
\hline 1 & $2.2460027 \mathrm{E}+06$ & 4.135 & 2.113 & 3.5000 & & $\cdots$ & \\
\hline 2 & $2.2462697 \mathrm{E}+08$ & 4.042 & 2.287 & 3.5000 & 0.4747733 & $\ldots$ & $3.03 \mathrm{E}+00$ \\
\hline 3 & $2.3076522 \mathrm{E}+08$ & 4.087 & 2.370 & 3.5000 & 0.4747733 & ... & $3.03 \mathrm{E}+00$ \\
\hline 4 & $2.4013753 \mathrm{E}+08$ & 3.704 & 2.033 & 3.5000 & 0.4830578 & $\ldots$ & $2.63 \mathrm{E}+00$ \\
\hline 5,7 & $2.4180689 \mathrm{E}+08$ & 3.653 & 2.527 & 3.5000 & 0.4830578 & $\cdots$ & $1.05 \mathrm{E}-01$ \\
\hline 6 & & & ... & $\ldots$ & $\cdots$ & $\ldots$ & \\
\hline 8 & $2.4258479 \mathrm{E}+08$ & 3.658 & 2.470 & 3.5000 & 0.4830578 & & $1.66 \mathrm{E}-01$ \\
\hline 9 & $2.6657682 \mathrm{E}+08$ & 3.687 & 2.192 & 3.5000 & 0.5561431 & $\ldots$ & $2.23 \mathrm{E}+00$ \\
\hline 10 & $2.7791766 \mathrm{E}+08$ & 3.718 & 2.300 & 3.5000 & 0.5849319 & & $2.89 \mathrm{E}+00$ \\
\hline 11 & $3.3322698 \mathrm{E}+08$ & 3.640 & 2.760 & 3.5000 & 0.7097074 & $3.11 \mathrm{E}-01$ & $3.00 \mathrm{E}-01$ \\
\hline 12 & $3.3593669 \mathrm{E}+08$ & 3.531 & 3.769 & 3.5000 & 0.7111888 & $1.94 \mathrm{E}-02$ & $4.46 \mathrm{E}-03$ \\
\hline 13 & $3.3595107 \mathrm{E}+08$ & 3.527 & 3.801 & 3.5000 & 0.7119999 & $1.71 \mathrm{E}-02$ & $3.64 \mathrm{E}-03$ \\
\hline 14 & $3.3601989 \mathrm{E}+08$ & 3.506 & 3.952 & 3.5000 & 0.7175049 & $1.21 \mathrm{E}-02$ & $1.60 \mathrm{E}-03$ \\
\hline 15 & $3.3644693 \mathrm{E}+08$ & 3.588 & 4.143 & 0.7623 & 0.7506785 & $4.63 \mathrm{E}-03$ & $2.77 \mathrm{E}-04$ \\
\hline \multicolumn{8}{|c|}{$(M, Y, Z)=(5.0,0.25,0.016)$} \\
\hline 1 & $9.1087735 \mathrm{E}+05$ & 4.234 & 2.684 & 5.0000 & & $\cdots$ & \\
\hline 2 & $9.2859365 \mathrm{E}+07$ & 4.144 & 2.902 & 5.0000 & 0.7820981 & $\ldots$ & $4.22 \mathrm{E}+00$ \\
\hline 3 & $9.5607011 \mathrm{E}+07$ & 4.186 & 2.977 & 5.0000 & 0.7820981 & $\ldots$ & $4.22 \mathrm{E}+00$ \\
\hline 4 & $9.7821560 \mathrm{E}+07$ & 3.685 & 2.648 & 5.0000 & 0.7873003 & $\ldots$ & $3.95 \mathrm{E}+00$ \\
\hline 5,7 & $9.8185447 \mathrm{E}+07$ & 3.630 & 3.068 & 5.0000 & 0.7873003 & & $3.22 \mathrm{E}-01$ \\
\hline 6 & & ... & .. & & & $\ldots$ & \\
\hline 8 & $9.8381175 \mathrm{E}+07$ & 3.631 & 3.054 & 5.0000 & 0.7873003 & & $3.77 \mathrm{E}-01$ \\
\hline 9 & $1.0976329 \mathrm{E}+08$ & 3.654 & 2.896 & 5.0000 & 0.9160519 & $\ldots$ & $3.07 \mathrm{E}+00$ \\
\hline 10 & $1.1783318 \mathrm{E}+08$ & 3.719 & 3.039 & 5.0000 & 1.0101822 & $5.35 \mathrm{E}-01$ & $3.99 \mathrm{E}+00$ \\
\hline 11 & $1.2171955 \mathrm{E}+08$ & 3.622 & 3.214 & 5.0000 & 1.0493966 & $5.27 \mathrm{E}-01$ & $5.29 \mathrm{E}-01$ \\
\hline 13 & $1.2281935 \mathrm{E}+08$ & 3.506 & 4.195 & 5.0000 & 0.8706349 & $3.34 \mathrm{E}-02$ & $1.35 \mathrm{E}-05$ \\
\hline 12 & $1.2284353 \mathrm{E}+08$ & 3.519 & 4.099 & 5.0000 & 0.8707433 & $6.84 \mathrm{E}-03$ & $1.51 \mathrm{E}-03$ \\
\hline 14 & $1.2286490 \mathrm{E}+08$ & 3.497 & 4.254 & 5.0000 & 0.8741754 & $4.40 \mathrm{E}-03$ & $3.65 \mathrm{E}-04$ \\
\hline 15 & $1.2312734 \mathrm{E}+08$ & 3.535 & 4.513 & 0.9604 & 0.8914651 & $3.27 \mathrm{E}-03$ & $1.25 \mathrm{E}-05$ \\
\hline
\end{tabular}


TABLE 2.2

Equivalent Evolutionary Points-Continued

\begin{tabular}{|c|c|c|c|c|c|c|c|}
\hline EEP & $\begin{array}{l}\text { Time } \\
(\mathrm{yrs})\end{array}$ & $\log \mathrm{T}_{\text {eff }}$ & $\log \mathrm{L} / \mathrm{L}_{\odot}$ & $\mathrm{M}_{\text {total }}$ & $\mathrm{M}_{c}^{H}$ & $\Delta_{c}^{H e}$ & $\Delta_{b c e}$ \\
\hline \multicolumn{8}{|c|}{$(M, Y, Z)=(0.95,0.25,0.008)$} \\
\hline 1 & $1.0672458 \mathrm{E}+08$ & 3.760 & -0.196 & 0.9450 & $\cdots$ & $\cdots$ & \\
\hline $\begin{array}{l}2 \\
3\end{array}$ & $1.0370597 \mathrm{E}+10$ & & & & & & \\
\hline 4 & & $\begin{array}{c}0.102 \\
\ldots\end{array}$ & 0.191 & 0.9434 & 0.0953895 & & 01 \\
\hline 5 & $1.3432514 \mathrm{E}+10$ & 3.669 & 1.367 & 0.9395 & 0.2278509 & $\cdots$ & $3.91 \mathrm{E}-02$ \\
\hline 6 & $1.3493946 \mathrm{E}+10$ & 3.655 & 1.650 & 0.9357 & 0.2666206 & $\ldots$ & $2.54 \mathrm{E}-02$ \\
\hline 7 & $1.3556044 \mathrm{E}+10$ & 3.472 & 3.363 & 0.7223 & 0.4809210 & $\cdots$ & $3.55 \mathrm{E}-03$ \\
\hline 8 & $1.3556110 \mathrm{E}+10$ & 3.680 & 1.658 & 0.7223 & 0.4809231 & $\ldots$ & $1.56 \mathrm{E}-01$ \\
\hline 9 & $1.3556175 \mathrm{E}+10$ & 3.660 & 1.827 & 0.7223 & 0.4809231 & $\cdots$ & $9.84 \mathrm{E}-02$ \\
\hline 10 & & $\cdots$ & & & & & \\
\hline 11 & $1.3691690 \mathrm{E}+10$ & 3.617 & 2.378 & 0.7223 & 0.5264320 & $2.40 \mathrm{E}-01$ & $5.69 \mathrm{E}-02$ \\
\hline 12 & $1.3701417 \mathrm{E}+10$ & 3.556 & 2.902 & 0.7223 & 0.5322764 & $5.56 \mathrm{E}-02$ & -02 \\
\hline 13 & $1.3701873 \mathrm{E}+10$ & 3.532 & 3.072 & 0.7223 & 0.5360573 & $4.43 \mathrm{E}-02$ & $1.29 \mathrm{E}-02$ \\
\hline 14 & $1.3702261 \mathrm{E}+10$ & 3.508 & 3.236 & 0.7222 & 0.5414655 & $3.64 \mathrm{E}-02$ & $6.72 \mathrm{E}-03$ \\
\hline 15 & $1.3702831 \mathrm{E}+10$ & 3.723 & 3.496 & 0.5561 & 0.5533068 & $3.09 \mathrm{E}-02$ & $2.61 \mathrm{E}-03$ \\
\hline \multicolumn{8}{|c|}{$(M, Y, Z)=(1.0,0.25,0.008)$} \\
\hline 1 & $7.4893206 \mathrm{E}+07$ & 3.776 & -0.080 & 1.0000 & & $\cdots$ & \\
\hline 2 & & & & & & $\ldots$ & \\
\hline 3 & $7.2396011 \mathrm{E}+09$ & 3.792 & 0.221 & 1.0000 & 0.0651257 & . & $9.31 \mathrm{E}-01$ \\
\hline 4 & & & & & & .. & \\
\hline 5 & $1.0781341 \mathrm{E}+10$ & 3.674 & 1.349 & 1.0000 & 0.2253045 & $\cdots$ & $4.53 \mathrm{E}-02$ \\
\hline 6 & $1.0848979 \mathrm{E}+10$ & 3.656 & 1.694 & 1.0000 & 0.2698216 & $\ldots$ & $2.51 \mathrm{E}-02$ \\
\hline 7 & $1.0905800 \mathrm{E}+10$ & 3.497 & 3.364 & 1.0000 & 0.4801420 & $\ldots$ & $3.91 \mathrm{E}-03$ \\
\hline 8 & $1.0905873 \mathrm{E}+10$ & 3.672 & 1.800 & 1.0000 & 0.4801530 & ... & $2.30 \mathrm{E}-01$ \\
\hline 9 & $1.0905959 \mathrm{E}+10$ & 3.669 & 1.776 & 1.0000 & 0.4801530 & $\cdots$ & $1.72 \mathrm{E}-01$ \\
\hline 10 & & & & & & & \\
\hline 11 & $1.1039434 \mathrm{E}+10$ & 3.618 & 2.443 & 1.0000 & 0.5440072 & $2.83 \mathrm{E}-01$ & $7.23 \mathrm{E}-02$ \\
\hline 12 & $1.1048397 \mathrm{E}+10$ & 3.557 & 3.008 & 1.0000 & 0.5484677 & $4.96 \mathrm{E}-02$ & $1.96 \mathrm{E}-02$ \\
\hline 13 & $1.1048707 \mathrm{E}+10$ & 3.535 & 3.161 & 1.0000 & 0.5516759 & $3.98 \mathrm{E}-02$ & $1.31 \mathrm{E}-02$ \\
\hline 14 & $1.1049034 \mathrm{E}+10$ & 3.509 & 3.348 & 1.0000 & 0.5574617 & $3.21 \mathrm{E}-02$ & $6.04 \mathrm{E}-03$ \\
\hline 15 & $1.1049684 \mathrm{E}+10$ & 3.698 & 3.640 & 0.5799 & 0.5776485 & $2.64 \mathrm{E}-02$ & $1.82 \mathrm{E}-03$ \\
\hline \multicolumn{8}{|c|}{$(M, Y, Z)=(1.5,0.25,0.008)$} \\
\hline 1 & $2.4165750 \mathrm{E}+07$ & 3.899 & 0.742 & 1.5000 & & . & \\
\hline 2 & $2.3895487 \mathrm{E}+09$ & 3.847 & 0.944 & 1.5000 & 0.1469788 & $\ldots$ & $1.35 \mathrm{E}+00$ \\
\hline 3 & $2.4617621 \mathrm{E}+09$ & 3.888 & 1.044 & 1.5000 & 0.1469788 & . & $1.35 \mathrm{E}+00$ \\
\hline 4 & $2.7257484 \mathrm{E}+09$ & 3.725 & 0.943 & 1.5000 & 82438 & & $9.73 \mathrm{E}-01$ \\
\hline 5 & $2.9192523 \mathrm{E}+09$ & 3.671 & 1.773 & 1.5000 & 0.2602070 & 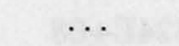 & $3.51 \mathrm{E}-02$ \\
\hline 6 & $2.9434600 \mathrm{E}+09$ & 3.660 & 1.996 & 1.5000 & 0.2949295 & .. & $2.42 \mathrm{E}-02$ \\
\hline 7 & $2.9751743 \mathrm{E}+09$ & 3.537 & 3.349 & 1.5000 & 0.4764204 & $\ldots$ & $4.00 \mathrm{E}-03$ \\
\hline 8,9 & $2.9752499 \mathrm{E}+09$ & 3.676 & 1.966 & 1.5000 & 0.4764203 & .. & $3.45 \mathrm{E}-01$ \\
\hline 10 & & & $\cdots$ & & & & \\
\hline 11 & $3.1055549 \mathrm{E}+09$ & 3.633 & 2.497 & 1.5000 & 0.5608673 & $2.42 \mathrm{E}-01$ & $9.38 \mathrm{E}-02$ \\
\hline 12 & $3.1128418 \mathrm{E}+09$ & 3.572 & 3.108 & 1.5000 & 0.5646877 & $4.42 \mathrm{E}-02$ & $1.84 \mathrm{E}-02$ \\
\hline 13 & $3.1131324 \mathrm{E}+09$ & 3.550 & 3.286 & 1.5000 & 0.5688439 & $3.37 \mathrm{E}-02$ & $1.08 \mathrm{E}-02$ \\
\hline 14 & $3.1133377 \mathrm{E}+09$ & 3.526 & 3.464 & 1.5000 & 0.5738291 & $2.90 \mathrm{E}-02$ & $3.55 \mathrm{E}-03$ \\
\hline 15 & $3.1142762 \mathrm{E}+09$ & 3.667 & 3.835 & 0.6217 & 0.6193959 & $1.94 \mathrm{E}-02$ & $9.64 \mathrm{E}-04$ \\
\hline
\end{tabular}


TABLE 2.2

Equivalent Evolutionary Points-Continued

\begin{tabular}{|c|c|c|c|c|c|c|c|}
\hline EEP & $\begin{array}{l}\text { Time } \\
\text { (yrs) }\end{array}$ & $\log \mathrm{T}_{\text {eff }}$ & $\log \mathrm{L} / \mathrm{L}_{\odot}$ & $\mathbf{M}_{\text {total }}$ & $\mathbf{M}_{c}^{H}$ & $\Delta_{c}^{H e}$ & $\Delta_{b c e}$ \\
\hline \multicolumn{8}{|c|}{$(M, Y, Z)=(2.0,0.25,0.008)$} \\
\hline 1 & $9.5767250 \mathrm{E}+06$ & 4.006 & 1.260 & 2.0000 & & & \\
\hline 2 & $9.9539337 \mathrm{E}+08$ & 3.928 & 1.445 & 2.0000 & 0.2393940 & $\ldots$ & $1.76 \mathrm{E}+00$ \\
\hline 3 & $1.0176741 \mathrm{E}+09$ & 3.972 & 1.530 & 2.0000 & 0.2393940 & . & $1.76 \mathrm{E}+00$ \\
\hline 4 & $1.0793509 \mathrm{E}+09$ & 3.724 & 1.317 & 2.0000 & 0.2581493 & & $1.32 \mathrm{E}+00$ \\
\hline 5 & $1.1163310 \mathrm{E}+09$ & 3.671 & 2.083 & 2.0000 & 0.2961773 & & $3.24 \mathrm{E}-02$ \\
\hline 6 & $1.1287013 \mathrm{E}+09$ & 3.661 & 2.247 & 2.0000 & 0.3276060 & & $2.37 \mathrm{E}-02$ \\
\hline 7 & $1.1459954 \mathrm{E}+09$ & 3.581 & 3.181 & 2.0000 & 0.4464366 & & $5.45 \mathrm{E}-03$ \\
\hline 8,9 & $1.1462945 \mathrm{E}+09$ & 3.703 & 1.807 & 2.0000 & 0.4464505 & & $8.67 \mathrm{E}-01$ \\
\hline 10 & & & & & & & \\
\hline 11 & $1.2980434 \mathrm{E}+09$ & 3.648 & 2.514 & 2.0000 & 0.5614907 & $1.94 \mathrm{E}-01$ & $1.17 \mathrm{E}-01$ \\
\hline 12 & $1.3109399 \mathrm{E}+09$ & 3.593 & 3.116 & 2.0000 & 0.5706640 & $4.38 \mathrm{E}-02$ & $1.97 \mathrm{E}-02$ \\
\hline 13 & $1.3114161 \mathrm{E}+09$ & 3.549 & 3.479 & 2.0000 & 0.5791966 & $2.82 \mathrm{E}-02$ & $5.97 \mathrm{E}-03$ \\
\hline 14 & $1.3114439 \mathrm{E}+09$ & 3.550 & 3.472 & 2.0000 & 0.5802911 & $2.83 \mathrm{E}-02$ & $2.81 \mathrm{E}-03$ \\
\hline 15 & $1.3127829 \mathrm{E}+09$ & 3.656 & 3.974 & 0.6699 & 0.6668609 & $1.30 \mathrm{E}-02$ & $5.71 \mathrm{E}-04$ \\
\hline \multicolumn{8}{|c|}{$(M, Y, Z)=(2.5,0.25,0.008)$} \\
\hline 1 & $4.7430594 \mathrm{E}+06$ & 4.078 & 1.642 & 2.5000 & & & \\
\hline 2 & $5.0391822 \mathrm{E}+08$ & 3.992 & 1.832 & 2.5000 & 0.3285348 & $\cdots$ & $2.17 \mathrm{E}+00$ \\
\hline 3 & $5.1712492 \mathrm{E}+08$ & 4.036 & 1.910 & 2.5000 & 0.3285348 & $\ldots$ & $2.17 \mathrm{E}+00$ \\
\hline 4 & $5.4148181 \mathrm{E}+08$ & 3.722 & 1.658 & 2.5000 & 0.3386571 & $\ldots$ & $1.80 \mathrm{E}+00$ \\
\hline 5,7 & $5.5051012 \mathrm{E}+08$ & 3.666 & 2.351 & 2.5000 & 0.3425060 & 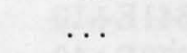 & $4.85 \mathrm{E}-02$ \\
\hline 6 & & & & & & & \\
\hline 8,9 & $5.5217487 \mathrm{E}+08$ & 3.686 & 2.058 & 2.5000 & 380 & & $1.50 \mathrm{E}-01$ \\
\hline 10 & $6.4739211 \mathrm{E}+08$ & 3.717 & 1.871 & 2.5000 & 0.4428816 & & $1.75 \mathrm{E}+00$ \\
\hline 11 & $7.7138223 \mathrm{E}+08$ & 3.659 & 2.512 & 2.5000 & 0.5673248 & $2.27 \mathrm{E}-01$ & $1.73 \mathrm{E}-01$ \\
\hline 12 & $7.8129927 \mathrm{E}+08$ & 3.604 & 3.139 & 2.5000 & 0.5732606 & $4.34 \mathrm{E}-02$ & $2.00 \mathrm{E}-02$ \\
\hline 13 & $7.8160491 \mathrm{E}+08$ & 33 & 38 & 2.5000 & 0955 & $3.15 \mathrm{E}-02$ & $1.06 \mathrm{E}-02$ \\
\hline 14 & $7.8172833 \mathrm{E}+08$ & 3.580 & 3.371 & 2.5000 & 2943 & $2.79 \mathrm{E}-02$ & $4.12 \mathrm{E}-03$ \\
\hline 15 & $7.8355523 \mathrm{E}+08$ & 3.637 & 4.027 & 0.6817 & 0.6777026 & $1.20 \mathrm{E}-02$ & $4.47 \mathrm{E}-04$ \\
\hline \multicolumn{8}{|c|}{$(M, Y, Z)=(3.5,0.25,0.008)$} \\
\hline 1 & $1.8132851 \mathrm{E}+06$ & 4.176 & 2.192 & 3.5000 & & & \\
\hline 2 & $1.9633902 \mathrm{E}+08$ & 4.089 & 2.396 & 3.5000 & 0.4902242 & & $3.01 \mathrm{E}+00$ \\
\hline 3 & $2.0095575 \mathrm{E}+08$ & 4.130 & 2.466 & 3.5000 & 0.4902242 & & $3.01 \mathrm{E}+00$ \\
\hline 4 & $2.0885055 \mathrm{E}+08$ & 3.710 & 2.190 & 3.5000 & 0.5067959 & 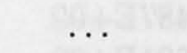 & $2.68 \mathrm{E}+00$ \\
\hline 5,7 & $2.0995032 \mathrm{E}+08$ & 3.667 & 2.581 & 3.5000 & 0.5090770 & .. & $2.37 \mathrm{E}-01$ \\
\hline 6 & & & & & & & \\
\hline 8 & $2.1059624 \mathrm{E}+08$ & 3.670 & 2.535 & 3.5000 & 0.5090770 & $\ldots$ & $3.76 \mathrm{E}-01$ \\
\hline 9 & $2.3626396 \mathrm{E}+08$ & 3.692 & 2.400 & 3.5000 & 0.6153098 & & $2.29 \mathrm{E}+00$ \\
\hline 10 & $2.6576260 \mathrm{E}+08$ & 3.755 & 2.652 & 3.5000 & 0.7414918 & $3.83 \mathrm{E}-01$ & $2.76 \mathrm{E}+00$ \\
\hline 11 & $2.7383428 \mathrm{E}+08$ & 3.658 & 2.777 & 3.5000 & 0.7709393 & $3.81 \mathrm{E}-01$ & $6.49 \mathrm{E}-01$ \\
\hline 12 & $2.7667996 \mathrm{E}+08$ & 3.556 & 3.795 & 3.5000 & 0.7717029 & $9.19 \mathrm{E}-02$ & $4.39 \mathrm{E}-03$ \\
\hline 13 & $2.7681087 \mathrm{E}+08$ & 3.529 & 4.007 & 3.5000 & 0.7717984 & $1.48 \mathrm{E}-02$ & $2.93 \mathrm{E}-03$ \\
\hline 14 & $2.7686648 \mathrm{E}+08$ & 3.520 & 4.075 & 3.5000 & 0.7774617 & $8.57 \mathrm{E}-03$ & $2.00 \mathrm{E}-04$ \\
\hline 15 & $2.7721743 \mathrm{E}+08$ & 3.612 & 4.214 & 0.8039 & 0.7936204 & $3.28 \mathrm{E}-03$ & $2.26 \mathrm{E}-04$ \\
\hline
\end{tabular}


TABLE 2.2

Equivalent Evolutionary Points-Continued

\begin{tabular}{|c|c|c|c|c|c|c|c|}
\hline$\overline{\text { EEP }}$ & $\begin{array}{l}\text { Time } \\
(y r s)\end{array}$ & $\overline{l o g} \mathrm{~T}_{\text {eff }}$ & $\log \mathrm{L} / \mathrm{L}_{\odot}$ & $\mathrm{M}_{\text {total }}$ & $\mathrm{M}_{c}^{H}$ & $\Delta_{c}^{H e}$ & $\overline{\Delta_{\text {bce }}}$ \\
\hline \multicolumn{8}{|c|}{$(M, Y, Z)=(5.0,0.25,0.008)$} \\
\hline 1 & $9.3036370 \mathrm{E}+05$ & 4.268 & 2.745 & 5.0000 & & & \\
\hline 2 & $8.3451346 \mathrm{E}+07$ & 4.185 & 2.978 & 5.0000 & 0.7983535 & & $4.20 \mathrm{E}+00$ \\
\hline 3 & $8.5688476 \mathrm{E}+07$ & 4.223 & 3.040 & 5.0000 & 0.7983535 & & $4.20 \mathrm{E}+00$ \\
\hline 4 & $8.7849826 \mathrm{E}+07$ & 3.689 & 2.769 & 5.0000 & 0.7983535 & & $3.89 \mathrm{E}+00$ \\
\hline $\begin{array}{l}5,7 \\
6\end{array}$ & $8.8099411 \mathrm{E}+07$ & 3.647 & 3.091 & 5.0000 & 0.8035130 & & $5.85 \mathrm{E}-01$ \\
\hline 8 & $8.8267086 \mathrm{E}+07$ & 3.648 & 3.078 & 5.0000 & 0.8035130 & & $6.90 \mathrm{E}-01$ \\
\hline 9 & $9.5867873 \mathrm{E}+07$ & 3.667 & 2.987 & 5.0000 & 0.8883246 & & $3.35 \mathrm{E}+00$ \\
\hline 10 & $1.0674431 \mathrm{E}+08$ & 3.861 & 3.252 & 5.0000 & 1.1041927 & $5.62 \mathrm{E}-01$ & $3.90 \mathrm{E}+00$ \\
\hline 11 & $1.0970875 \mathrm{E}+08$ & 3.635 & 3.316 & 5.0000 & 1.1553786 & $5.60 \mathrm{E}-01$ & $7.86 \mathrm{E}-01$ \\
\hline 12 & $1.1047475 \mathrm{E}+08$ & 3.533 & 4.222 & 5.0000 & 0.8955703 & $2.96 \mathrm{E}-02$ & $5.73 \mathrm{E}-06$ \\
\hline 13 & $1.1049526 \mathrm{E}+08$ & 3.545 & 4.131 & 5.0000 & 0.8956654 & $6.08 \mathrm{E}-03$ & $1.54 \mathrm{E}-03$ \\
\hline 14 & $1.1051235 \mathrm{E}+08$ & 3.530 & 4.247 & 5.0000 & 0.8984608 & $3.68 \mathrm{E}-03$ & $6.09 \mathrm{E}-05$ \\
\hline 15 & $1.1087245 \mathrm{E}+08$ & 3.587 & 4.371 & 0.9333 & 0.9104542 & $2.11 \mathrm{E}-03$ & $8.69 \mathrm{E}-05$ \\
\hline \multicolumn{8}{|c|}{$(M, Y, Z)=(0.89,0.25,0.004)$} \\
\hline 1 & $8.8645558 \mathrm{E}+07$ & 3.770 & -1.214 & 0.8900 & & & \\
\hline 2 & & . & $\ldots$ & & & & $\cdots$ \\
\hline 3 & $1.0369072 \mathrm{E}+10$ & 3.794 & 0.189 & 0.8885 & 0.0930556 & & $7.93 \mathrm{E}-01$ \\
\hline 4 & & & & & & & \\
\hline 5 & $1.3477445 \mathrm{E}+10$ & 3.677 & 1.492 & 0.8835 & 0.2399917 & & $4.13 \mathrm{E}-02$ \\
\hline 6 & $1.3527669 \mathrm{E}+10$ & 3.660 & 1.803 & 0.8785 & 0.2809609 & & $2.53 \mathrm{E}-02$ \\
\hline 7 & $1.3575956 \mathrm{E}+10$ & 3.514 & 3.338 & 0.6910 & 0.4825310 & & $4.39 \mathrm{E}-03$ \\
\hline 8 & $1.3576027 \mathrm{E}+10$ & 3.697 & 1.724 & 0.6910 & 0.4825262 & & $1.67 \mathrm{E}-01$ \\
\hline 9 & $1.3576106 \mathrm{E}+10$ & 3.685 & 1.771 & 0.6910 & 0.4825262 & 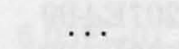 & $1.29 \mathrm{E}-01$ \\
\hline 10 & & $\cdots$ & & & & & \\
\hline 11 & $1.3705313 \mathrm{E}+10$ & 3.640 & 2.366 & 0.6910 & 0.5272300 & $2.16 \mathrm{E}-01$ & $6.46 \mathrm{E}-02$ \\
\hline 12 & $1.3715848 \mathrm{E}+10$ & 3.585 & 2.905 & 0.6910 & 0.5332964 & $5.51 \mathrm{E}-02$ & $2.12 \mathrm{E}-02$ \\
\hline 13 & $1.3716502 \mathrm{E}+10$ & 3.558 & 3.131 & 0.6909 & 0.5395323 & $3.98 \mathrm{E}-02$ & $1.25 \mathrm{E}-02$ \\
\hline 14 & $1.3716581 \mathrm{E}+10$ & 3.549 & 3.197 & 0.6909 & 0.5406812 & $3.73 \mathrm{E}-02$ & $7.90 \mathrm{E}-03$ \\
\hline 15 & $1.3717352 \mathrm{E}+10$ & 3.756 & 3.520 & 0.5604 & 0.5575691 & $3.07 \mathrm{E}-02$ & $2.68 \mathrm{E}-03$ \\
\hline \multicolumn{8}{|c|}{$(M, Y, Z)=(1.0,0.25,0.004)$} \\
\hline 1 & $6.4546402 \mathrm{E}+07$ & 3.800 & 0.027 & 1.0000 & & & \\
\hline 2 & & $\ldots$ & & $\cdots$ & $\cdots$ & & \\
\hline 3 & $6.0620636 \mathrm{E}+09$ & 3.815 & 0.349 & 1.0000 & 0.0704146 & & $9.29 \mathrm{E}-01$ \\
\hline 4 & $\cdots$ & & & & $\cdots$ & & \\
\hline 5 & $8.6692192 \mathrm{E}+09$ & 3.681 & 1.517 & 1.0000 & 0.2395904 & 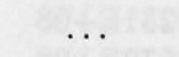 & $4.80 \mathrm{E}-02$ \\
\hline 6 & $8.7195976 \mathrm{E}+09$ & 3.663 & 1.868 & 1.0000 & 0.2875127 & & $2.62 \mathrm{E}-02$ \\
\hline 7 & $8.7611499 \mathrm{E}+09$ & 3.537 & 3.337 & 1.0000 & 0.4809482 & & $4.69 \mathrm{E}-03$ \\
\hline 8 & $8.7612331 \mathrm{E}+09$ & 3.683 & 1.881 & 1.0000 & 0.4809559 & & $2.76 \mathrm{E}-01$ \\
\hline 9 & $8.7613069 \mathrm{E}+09$ & 3.695 & 1.706 & 1.0000 & 0.4809559 & & $3.03 \mathrm{E}-01$ \\
\hline 10 & .. & $\ldots$ & $\ldots$ & & $\ldots$ & & \\
\hline 11 & $8.8890556 \mathrm{E}+09$ & 3.637 & 2.456 & 1.0000 & 0.5502444 & $2.46 \mathrm{E}-01$ & $8.72 \mathrm{E}-02$ \\
\hline 12 & $8.8965443 \mathrm{E}+09$ & 3.581 & 3.042 & 1.0000 & 0.5545926 & $4.77 \mathrm{E}-02$ & $2.09 \mathrm{E}-02$ \\
\hline 13 & $8.8969978 \mathrm{E}+09$ & 3.564 & 3.187 & 1.0000 & 0.5606416 & $3.30 \mathrm{E}-02$ & $1.48 \mathrm{E}-02$ \\
\hline 14 & $8.8970628 \mathrm{E}+09$ & 3.521 & 3.492 & 1.0000 & 0.5621834 & $3.30 \mathrm{E}-02$ & $3.99 \mathrm{E}-03$ \\
\hline 15 & $8.8979308 \mathrm{E}+09$ & 3.745 & 3.604 & 0.5952 & 0.5924512 & $1.90 \mathrm{E}-02$ & $2.55 \mathrm{E}-03$ \\
\hline
\end{tabular}


TABLE 2.2

Equivalent Evolutionary Points-Continued

\begin{tabular}{|c|c|c|c|c|c|c|c|}
\hline EEP & $\begin{array}{l}\text { Time } \\
\text { (yrs) }\end{array}$ & $\log \mathrm{T}_{\text {eff }}$ & $\log \mathrm{L} / \mathrm{L}_{\odot}$ & $\mathrm{M}_{\text {total }}$ & $\mathrm{M}_{c}^{H}$ & $\Delta_{c}^{H e}$ & $\overline{\Delta_{b c e}}$ \\
\hline \multicolumn{8}{|c|}{$(M, Y, Z)=(1.5,0.25,0.004)$} \\
\hline 1 & $1.9314620 \mathrm{E}+07$ & 3.936 & 0.822 & 1.5000 & & & \\
\hline 2 & $2.0380145 E+09$ & 3.898 & 1.056 & 1.5000 & 0.1418832 & $\ldots$ & $1.36 \mathrm{E}+00$ \\
\hline 3 & $2.0884808 \mathrm{E}+09$ & 3.936 & 1.140 & 1.5000 & 0.1532401 & $\ldots$ & $1.35 \mathrm{E}+00$ \\
\hline 4 & $2.3276715 \mathrm{E}+09$ & 3.730 & 1.093 & 1.5000 & 0.2026484 & $\ldots$ & $1.03 \mathrm{E}+00$ \\
\hline 5 & $2.4557854 \mathrm{E}+09$ & 3.685 & 1.791 & 1.5000 & 0.2627679 & 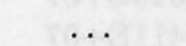 & $5.04 \mathrm{E}-02$ \\
\hline 6 & $2.4848013 E+09$ & 3.666 & 2.160 & 1.5000 & 0.3135678 & $\ldots$ & $2.50 \mathrm{E}-02$ \\
\hline 7 & $2.5084689 \mathrm{E}+09$ & 3.572 & 3.313 & 1.5000 & 0.4755255 & $\ldots$ & $5.09 \mathrm{E}-03$ \\
\hline 9 & $2.5085122 \mathrm{E}+09$ & 3.678 & 2.134 & 1.5000 & 0.4755240 & & $2.68 \mathrm{E}-01$ \\
\hline 8 & $2.5086009 \mathrm{E}+09$ & 3.696 & 1.957 & 1.5000 & 0.4755311 & & $6.00 \mathrm{E}-01$ \\
\hline 10 & & & $\cdots$ & $\cdots$ & & & \\
\hline 11 & $2.6352539 \mathrm{E}+09$ & 3.650 & 2.530 & 1.5000 & 0.5751212 & $2.38 \mathrm{E}-01$ & $1.28 \mathrm{E}-01$ \\
\hline 12 & $2.6411975 \mathrm{E}+09$ & 3.591 & 3.181 & 1.5000 & 0.5785839 & $4.06 \mathrm{E}-02$ & $1.96 \mathrm{E}-02$ \\
\hline 13 & $2.6414911 \mathrm{E}+09$ & 3.568 & 3.385 & 1.5000 & 0.5838434 & $2.91 \mathrm{E}-02$ & $1.06 \mathrm{E}-02$ \\
\hline 14 & $2.6415565 \mathrm{E}+09$ & 3.554 & 3.492 & 1.5000 & 0.5857862 & $2.74 \mathrm{E}-02$ & $3.84 \mathrm{E}-03$ \\
\hline 15 & $2.6425231 \mathrm{E}+09$ & 3.693 & 3.862 & 0.6413 & 0.6392266 & $1.33 \mathrm{E}-02$ & $1.07 \mathrm{E}-03$ \\
\hline \multicolumn{8}{|c|}{$(M, Y, Z)=(2.0,0.25,0.004)$} \\
\hline 1 & $8.7730145 \mathrm{E}+06$ & 4.042 & 1.337 & 2.0000 & & & \\
\hline 2 & $8.7068622 \mathrm{E}+08$ & 3.977 & 1.546 & 2.0000 & 0.2395849 & $\ldots$ & $1.76 \mathrm{E}+00$ \\
\hline 3 & $8.9313749 \mathrm{E}+08$ & 4.017 & 1.622 & 2.0000 & 0.2395849 & $\ldots$ & $1.76 \mathrm{E}+00$ \\
\hline 4 & $9.4893085 \mathrm{E}+08$ & 3.729 & 1.463 & 2.0000 & 0.2720292 & $\cdots$ & $1.37 \mathrm{E}+00$ \\
\hline 5 & $9.7966102 \mathrm{E}+08$ & 3.678 & 2.207 & 2.0000 & 0.3115365 & .. & $3.90 \mathrm{E}-02$ \\
\hline 6 & $9.9016644 \mathrm{E}+08$ & 3.665 & 2.413 & 2.0000 & 0.3505925 & & $2.41 \mathrm{E}-02$ \\
\hline 7 & $1.0012045 \mathrm{E}+09$ & 3.616 & 3.056 & 2.0000 & 0.4319755 & & $8.26 \mathrm{E}-03$ \\
\hline 9 & $1.0012207 \mathrm{E}+09$ & 3.672 & 2.359 & 2.0000 & 0.4319874 & & $1.44 \mathrm{E}-01$ \\
\hline 8 & $1.0015590 \mathrm{E}+09$ & 3.716 & 1.851 & 2.0000 & 0.4320113 & $\ldots$ & $1.16 \mathrm{E}+00$ \\
\hline 10 & & & & & & & \\
\hline 11 & $1.1550716 \mathrm{E}+09$ & 3.662 & 2.542 & 2.0000 & 0.5769604 & $2.39 \mathrm{E}-01$ & $1.69 \mathrm{E}-01$ \\
\hline 12 & $1.1614480 \mathrm{E}+09$ & 3.609 & 3.191 & 2.0000 & 0.5802067 & $4.03 \mathrm{E}-02$ & $2.04 \mathrm{E}-02$ \\
\hline 13 & $1.1616908 \mathrm{E}+09$ & 3.592 & 3.356 & 2.0000 & 0.5843893 & $2.97 \mathrm{E}-02$ & $1.17 \mathrm{E}-02$ \\
\hline 14 & $1.1617767 \mathrm{E}+09$ & 3.594 & 3.342 & 2.0000 & 0.5866438 & $2.69 \mathrm{E}-02$ & $6.26 \mathrm{E}-03$ \\
\hline 15 & $1.1633352 \mathrm{E}+09$ & 3.697 & 3.882 & 0.6736 & 0.6718294 & $1.02 \mathrm{E}-02$ & $9.35 \mathrm{E}-04$ \\
\hline \multicolumn{8}{|c|}{$(M, Y, Z)=(2.5,0.25,0.004)$} \\
\hline 1 & $3.9784279 \mathrm{E}+06$ & 4.112 & 1.705 & 2.5000 & & & \\
\hline 2 & $4.5137623 \mathrm{E}+08$ & 4.037 & 1.928 & 2.5000 & 0.3379968 & $\ldots$ & $2.16 \mathrm{E}+00$ \\
\hline 3 & $4.6046866 \mathrm{E}+08$ & 4.078 & 1.993 & 2.5000 & 0.3379968 & $\ldots$ & $2.16 \mathrm{E}+00$ \\
\hline 4 & $4.8190231 \mathrm{E}+08$ & 3.727 & 1.798 & 2.5000 & 0.3564580 & $\ldots$ & $1.85 \mathrm{E}+00$ \\
\hline 5,7 & $4.8785572 \mathrm{E}+08$ & 3.682 & 2.322 & 2.5000 & 0.3564580 & .. & $9.14 \mathrm{E}-02$ \\
\hline 6 & & & & & & $\ldots$ & \\
\hline 8 & $4.8946928 \mathrm{E}+08$ & 3.694 & 2.118 & 2.5000 & 0.3564580 & .. & $2.64 \mathrm{E}-01$ \\
\hline 9 & $5.2095925 \mathrm{E}+08$ & 3.716 & 1.924 & 2.5000 & 0.4172357 & & $1.58 \mathrm{E}+00$ \\
\hline 10 & $5.4672469 \mathrm{E}+08$ & 3.738 & 2.053 & 2.5000 & 0.4543532 & 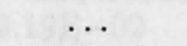 & $2.02 \mathrm{E}+00$ \\
\hline 11 & $6.5478632 \mathrm{E}+08$ & 3.670 & 2.598 & 2.5000 & 0.6339499 & $2.46 \mathrm{E}-01$ & $3.48 \mathrm{E}-01$ \\
\hline 12 & $6.5975997 \mathrm{E}+08$ & 3.596 & 3.467 & 2.5000 & 0.6360710 & $2.95 \mathrm{E}-02$ & $1.24 \mathrm{E}-02$ \\
\hline 13 & $6.5993302 \mathrm{E}+08$ & 3.568 & 3.718 & 2.5000 & 0.6426632 & $1.96 \mathrm{E}-02$ & $3.81 \mathrm{E}-03$ \\
\hline 14 & $6.5993528 \mathrm{E}+08$ & 3.574 & 3.669 & 2.5000 & 0.6427802 & $1.91 \mathrm{E}-02$ & $2.02 \mathrm{E}-03$ \\
\hline 15 & $6.6118311 \mathrm{E}+08$ & 3.650 & 4.061 & 0.6948 & 0.6909104 & $1.03 \mathrm{E}-02$ & $4.19 \mathrm{E}-04$ \\
\hline
\end{tabular}


TABLE 2.2

Equivalent Evolutionary Points-Continued

\begin{tabular}{|c|c|c|c|c|c|c|c|}
\hline EEP & $\begin{array}{l}\begin{array}{l}\text { Time } \\
(\mathrm{yrs})\end{array}\end{array}$ & $\log \mathrm{T}_{\text {eff }}$ & $\log \mathrm{L} / \mathrm{L}_{\odot}$ & $\mathrm{M}_{\text {total }}$ & $\overline{\mathrm{M}_{c}^{H}}$ & $\Delta_{c}^{H e}$ & $\overline{\Delta_{b c e}}$ \\
\hline \multicolumn{8}{|c|}{$(M, Y, Z)=(3.5,0.25,0.004)$} \\
\hline 1 & $1.6455753 \mathrm{E}+06$ & 4.206 & 2.245 & 3.5000 & & $\cdots$ & \\
\hline 2 & $1.7829387 \mathrm{E}+08$ & 4.127 & 2.469 & 3.5000 & 0.5094250 & $\ldots$ & $2.99 \mathrm{E}+00$ \\
\hline 3 & $1.8308567 \mathrm{E}+08$ & 4.165 & 2.529 & 3.5000 & 0.5094250 & .. & $2.99 \mathrm{E}+00$ \\
\hline 4 & $1.9052627 \mathrm{E}+08$ & 3.714 & 2.318 & 3.5000 & 0.5282522 & $\ldots$ & $2.69 \mathrm{E}+00$ \\
\hline 5,7 & $1.9123323 \mathrm{E}+08$ & 3.678 & 2.613 & 3.5000 & 0.5282522 & $\cdots$ & $5.59 \mathrm{E}-01$ \\
\hline 6 & & & $-\pi$ & 0 & & $\cdots$ & \\
\hline 8 & $1.9177714 \mathrm{E}+08$ & 3.681 & 2.576 & 3.5000 & 0.5282522 & $\ldots$ & $8.30 \mathrm{E}-01$ \\
\hline 9 & $2.0633681 \mathrm{E}+08$ & 3.697 & 2.487 & 3.5000 & 0.5932178 & & $2.28 \mathrm{E}+00$ \\
\hline 10 & $2.3163868 \mathrm{E}+08$ & 3.850 & 2.784 & 3.5000 & 0.7611096 & $4.13 \mathrm{E}-01$ & $2.74 \mathrm{E}+00$ \\
\hline 11 & $2.4478311 \mathrm{E}+08$ & 3.665 & 2.897 & 3.5000 & 0.8452082 & $4.09 \mathrm{E}-01$ & $7.81 \mathrm{E}-01$ \\
\hline 13 & $2.4687552 \mathrm{E}+08$ & 3.542 & 4.146 & 3.5000 & 0.8393469 & $4.23 \mathrm{E}-02$ & $2.91 \mathrm{E}-08$ \\
\hline 12 & $2.4691000 \mathrm{E}+08$ & 3.559 & 4.018 & 3.5000 & 0.8394191 & $7.79 \mathrm{E}-03$ & $2.66 \mathrm{E}-03$ \\
\hline 14 & $2.4693262 \mathrm{E}+08$ & 3.535 & 4.198 & 3.5000 & 0.8423935 & $5.35 \mathrm{E}-03$ & $6.30 \mathrm{E}-05$ \\
\hline 15 & $2.4718503 \mathrm{E}+08$ & 3.632 & 4.294 & 0.8626 & 0.8532340 & $3.90 \mathrm{E}-03$ & $1.68 \mathrm{E}-04$ \\
\hline \multicolumn{8}{|c|}{$(M, Y, Z)=(5.0,0.25,0.004)$} \\
\hline 1 & $8.9276462 \mathrm{E}+05$ & 4.294 & 2.786 & 5.0000 & & & \\
\hline 2 & $7.8838274 \mathrm{E}+07$ & 4.217 & 3.039 & 5.0000 & 0.8456958 & . & $4.15 \mathrm{E}+00$ \\
\hline 3 & $8.0597611 \mathrm{E}+07$ & 4.253 & 3.090 & 5.0000 & 0.8456958 & & $4.15 \mathrm{E}+00$ \\
\hline 4 & $8.2578966 \mathrm{E}+07$ & 3.693 & 2.885 & 5.0000 & 0.8568272 & & $3.79 \mathrm{E}+00$ \\
\hline 5,7 & $8.2754834 \mathrm{E}+07$ & 3.660 & 3.136 & 5.0000 & 0.8624293 & & $8.81 \mathrm{E}-01$ \\
\hline $\begin{array}{l}6 \\
8\end{array}$ & $8.2921553 \mathrm{E}+07$ & 3.661 & 3.125 & 5.0000 & 0.8624293 & & $1.03 \mathrm{E}+00$ \\
\hline 9 & $8.7423330 \mathrm{E}+07$ & 3.674 & 3.069 & 5.0000 & 0.8796127 & & $3.27 \mathrm{E}+00$ \\
\hline 10 & $9.7475792 \mathrm{E}+07$ & 3.976 & 3.366 & 5.0000 & 1.1651768 & $6.05 \mathrm{E}-01$ & $3.83 \mathrm{E}+00$ \\
\hline 11 & $1.0140012 \mathrm{E}+08$ & 3.649 & 3.376 & 5.0000 & 1.2582222 & $5.95 \mathrm{E}-01$ & $1.39 \mathrm{E}+00$ \\
\hline 13 & $1.0196579 \mathrm{E}+08$ & 3.557 & 4.270 & 5.0000 & 0.9252701 & $2.09 \mathrm{E}-02$ & $1.27 \mathrm{E}-07$ \\
\hline 12 & $1.0197917 \mathrm{E}+08$ & 3.569 & 4.169 & 5.0000 & 0.9253476 & $5.23 \mathrm{E}-03$ & $1.46 \mathrm{E}-03$ \\
\hline 14 & $1.0199247 \mathrm{E}+08$ & 3.558 & 4.263 & 5.0000 & 0.9276063 & $2.84 \mathrm{E}-03$ & $2.48 \mathrm{E}-04$ \\
\hline 15 & $1.0230480 \mathrm{E}+08$ & 3.593 & 4.537 & 1.0386 & 0.9409381 & $2.12 \mathrm{E}-03$ & $9.25 \mathrm{E}-07$ \\
\hline \multicolumn{8}{|c|}{$(M, Y, Z)=(1.0,0.25,0.001)$} \\
\hline 1 & $6.4035987 \mathrm{E}+07$ & 3.828 & 0.130 & 1.0000 & & $\cdots$ & $\cdots$ \\
\hline 2 & & & & & & $\ldots$ & \\
\hline 3 & $5.9620907 \mathrm{E}+09$ & 3.857 & 0.616 & 1.0000 & 0.1371808 & $\ldots$ & $8.63 \mathrm{E}-01$ \\
\hline 4 & & & & & & $\ldots$ & \\
\hline 5 & $7.0220579 \mathrm{E}+09$ & 3.695 & 1.760 & 1.0000 & 0.2658108 & $\ldots$ & $5.61 \mathrm{E}-02$ \\
\hline 6 & $7.0561029 \mathrm{E}+09$ & 3.677 & 2.144 & 1.0000 & 0.3215442 & $\ldots$ & $2.93 \mathrm{E}-02$ \\
\hline 7 & $7.0812347 \mathrm{E}+09$ & 3.606 & 3.261 & 1.0000 & 0.4797938 & $\ldots$ & $7.09 \mathrm{E}-03$ \\
\hline 8,9 & $7.0813243 \mathrm{E}+09$ & 3.703 & 1.997 & 1.0000 & 0.4798167 & $\ldots$ & $3.38 \mathrm{E}-01$ \\
\hline 10 & $\ldots$ & 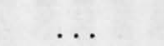 & $\ldots$ & $\ldots$ & 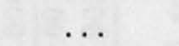 & & \\
\hline 11 & $7.2023545 \mathrm{E}+09$ & 3.671 & 2.454 & 1.0000 & 0.5568709 & $2.72 \mathrm{E}-01$ & $1.19 \mathrm{E}-01$ \\
\hline 12 & $7.2097178 \mathrm{E}+09$ & 3.627 & 3.070 & 1.0000 & 0.5603793 & $4.41 \mathrm{E}-02$ & $2.53 \mathrm{E}-02$ \\
\hline 13 & $7.2100715 \mathrm{E}+09$ & 3.612 & 3.259 & 1.0000 & 0.5651056 & $3.29 \mathrm{E}-02$ & $1.49 \mathrm{E}-02$ \\
\hline 14 & $7.2100917 \mathrm{E}+09$ & 3.614 & 3.233 & 1.0000 & 0.5654622 & $3.17 \mathrm{E}-02$ & $1.21 \mathrm{E}-02$ \\
\hline 15 & $7.2114483 \mathrm{E}+09$ & 3.771 & 3.772 & 0.6246 & 0.6228087 & $1.50 \mathrm{E}-02$ & $1.65 \mathrm{E}-03$ \\
\hline
\end{tabular}


TABLE 2.2

Equivalent Evolutionary Points-Continued

\begin{tabular}{|c|c|c|c|c|c|c|c|}
\hline EEP & $\begin{array}{l}\text { Time } \\
(\mathrm{yrs})\end{array}$ & $\log \mathrm{T}_{\text {eff }}$ & $\log \mathrm{L} / \mathrm{L}_{\odot}$ & $\mathbf{M}_{\text {total }}$ & $\mathbf{M}_{c}^{H}$ & $\Delta_{c}^{H e}$ & $\Delta_{b c e}$ \\
\hline \multicolumn{8}{|c|}{$(M, Y, Z)=(1.5,0.25,0.001)$} \\
\hline 1 & $1.1899508 \mathrm{E}+07$ & 3.979 & 0.901 & 1.5000 & & & \\
\hline 2 & & & & & & & \\
\hline 3 & $1.1507950 \mathrm{E}+09$ & 3.989 & 1.072 & 1.5000 & 0.1290499 & & $1.37 \mathrm{E}+00$ \\
\hline 4 & & & $\cdots$ & & & & \\
\hline 5 & $1.9344811 \mathrm{E}+09$ & 3.694 & 2.114 & 1.5000 & 0.3005803 & & $5.10 \mathrm{E}-02$ \\
\hline 6 & $1.9494981 \mathrm{E}+09$ & 3.680 & 2.403 & 1.5000 & 0.3506541 & . & $2.82 \mathrm{E}-02$ \\
\hline 7 & $1.9633356 \mathrm{E}+09$ & 3.631 & 3.201 & 1.5000 & 0.4676962 & & $8.25 \mathrm{E}-03$ \\
\hline 8 & $1.9633609 \mathrm{E}+09$ & 3.692 & 2.291 & 1.5000 & 0.4677025 & & $2.37 \mathrm{E}-01$ \\
\hline 9 & $1.9635570 \mathrm{E}+09$ & 3.723 & 1.963 & 1.5000 & 0.4677113 & & $8.72 \mathrm{E}-01$ \\
\hline 10 & & & & & & & \\
\hline 11 & $2.0855553 \mathrm{E}+09$ & 3.679 & 2.558 & 1.5000 & 0.5967783 & $2.42 \mathrm{E}-01$ & $1.93 \mathrm{E}-01$ \\
\hline 12 & $2.0902974 \mathrm{E}+09$ & 3.629 & 3.285 & 1.5000 & 0.5993735 & $3.45 \mathrm{E}-02$ & $1.94 \mathrm{E}-02$ \\
\hline 13 & $2.0904657 \mathrm{E}+09$ & 3.619 & 3.413 & 1.5000 & 0.6030267 & $2.61 \mathrm{E}-02$ & $1.27 \mathrm{E}-02$ \\
\hline 14 & $2.0905170 \mathrm{E}+09$ & 3.619 & 3.407 & 1.5000 & 0.6045240 & $2.40 \mathrm{E}-02$ & $7.82 \mathrm{E}-03$ \\
\hline 15 & $2.0916438 \mathrm{E}+09$ & 3.711 & 3.984 & 0.6641 & 0.6625777 & $1.54 \mathrm{E}-02$ & $6.68 \mathrm{E}-04$ \\
\hline
\end{tabular}


TABLE 2.3

Lifetimes of Major Evolutionary Phases

\begin{tabular}{|c|c|c|c|c|c|c|c|c|c|c|c|c|}
\hline$\left(\mathrm{M}_{\odot}\right)$ & $(\mathrm{Z})$ & (yrs) & $\begin{array}{c}{ }^{\mathrm{a}} \tau_{F G B-C} \\
(\mathrm{yrs})\end{array}$ & $\begin{array}{c}\tau_{F G B} \\
(\mathrm{yrs}) \\
\end{array}$ & $\begin{array}{r}\tau_{H \in B} \\
(\mathrm{yrs}) \\
\end{array}$ & $\begin{array}{l}{ }^{\mathrm{b}} \tau_{A G B} \\
(\mathrm{yrs}) \\
\end{array}$ & $\frac{\tau_{F G B}}{\tau_{M S}}$ & $\frac{\tau_{F G B-C}}{\tau_{H e B}}$ & $\frac{\tau_{H e B}}{\tau_{M S}}$ & $\frac{\tau_{A G B}}{\tau_{M S}}$ & $\frac{\tau_{A G B}}{\tau_{F G B}}$ & $\frac{\tau_{A G B}}{\tau_{H e B}}$ \\
\hline $1.0 \ldots$ & 0.016 & $1.125 \mathrm{E}+10$ & $786 \mathrm{E}+07$ & $3.563 \mathrm{E}+09$ & $1.416 \mathrm{E}+08$ & $1.258 \mathrm{E}+07$ & 0.317 & 0.409 & 0.013 & 0.001 & .004 & 0.089 \\
\hline 1.5 & 0.016 & $2.742 \mathrm{E}+09$ & $197 \mathrm{E}+07$ & $7.570 \mathrm{E}+08$ & $1.395 \mathrm{E}+08$ & $5.246 \mathrm{E}+06$ & 0.276 & 0.373 & 0.051 & 0.002 & .007 & 0.038 \\
\hline & 0.016 & $1.236 \mathrm{E}+09$ & $5.454 \mathrm{E}+07$ & $1.648 \mathrm{E}+08$ & $1.509 \mathrm{E}+08$ & $9.108 \mathrm{E}+06$ & 133 & 0.361 & 0.122 & 0.007 & 055 & 0.060 \\
\hline & 0.016 & $6.192 E+08$ & $E+07$ & +07 & 2.805 & $1.303 \mathrm{I}$ & 069 & 0.042 & & 21 & 304 & 0.046 \\
\hline 3.5 & 0.016 & +08 & 1.2 & +07 & 9.142 & $3.220 \mathrm{E}$ & 048 & 0.013 & & 0.014 & .290 & 0.035 \\
\hline 5.0 & 0.016 & 9.560 & +05 & +06 & +07 & $1.408 \mathrm{E}+05$ & 0.027 & 0.010 & 0.246 & 0.001 & 0.055 & 0.006 \\
\hline $0.945 \ldots$ & 0.008 & $1.052 \mathrm{E}+10$ & +07 & & & $1.114 \mathrm{E}$ & 0.289 & & & & 0.004 & 0.082 \\
\hline 1.0 & 0.008 & 8.12 & +07 & & & $1.025 \mathrm{E}+07$ & 0.342 & & 0.016 & & .004 & 0.077 \\
\hline 1.5 & 0.008 & +09 & +07 & +08 & & $8.721 \mathrm{E}+06$ & 0.209 & & 3 & 4 & 0.017 & 0.067 \\
\hline 2.0 & 0.008 & +09 & $E+07$ & +08 & 1.52 & $1.474 \mathrm{E}+07$ & 0.126 & & 0.149 & 0. & 15 & 0.097 \\
\hline 2.5 & 0.008 & $5.170 \mathrm{E}+08$ & $3.935 E+06$ & $3.355 E+07$ & $2.209 \mathrm{E}+08$ & $1.217 \mathrm{E}+07$ & 0.065 & 0.0 & 0.427 & 0.024 & 0.363 & .055 \\
\hline & 0.008 & +08 & +05 & +06 & +07 & 3.383 & 0.045 & 0.0 & 0.318 & 0.017 & 0.374 & 0.053 \\
\hline 5.0 & 0.008 & +07 & +05 & $2.426 \mathrm{E}+06$ & -07 & $1.150 \mathrm{E}$ & 0.028 & 0.009 & 0.252 & 0.013 & 0.474 & 0.053 \\
\hline $0.89 \ldots$ & 0.004 & 10 & 6.2 & 99 & 1.2 & $1.204 \mathrm{I}$ & 0.239 & 0.485 & 0.012 & 0.001 & 0.005 & 0.093 \\
\hline 1.0 & 0.004 & 09 & +07 & +09 & 1.279 & $8.875 E+06$ & 0.317 & 0 & 9 & 1 & 0.004 & 0.069 \\
\hline 1.5 & 0.004 & $2.088 \mathrm{E}+09$ & $3.650 \mathrm{E}+07$ & $4.202 \mathrm{E}+08$ & $1.268 \mathrm{E}+08$ & $7.269 \mathrm{E}+06$ & 0.201 & 0.288 & 0.061 & 0.003 & 0.017 & 0.057 \\
\hline 2.0 & 0.004 & $8.930 \mathrm{E}+08$ & $3.693 \mathrm{E}+07$ & $1.082 \mathrm{E}+08$ & $1.539 \mathrm{E}+08$ & $8.264 \mathrm{E}+06$ & 0.121 & 0.240 & 0.172 & 0.009 & 0.076 & 0.054 \\
\hline $2.5 \ldots$ & 0.004 & $4.604 \mathrm{E}+08$ & $4.482 \mathrm{E}+06$ & $2.745 \mathrm{E}+07$ & $1.669 \mathrm{E}+08$ & $6.397 \mathrm{E}+06$ & 0.060 & 0.02 & 0.363 & 0.014 & 0.233 & 0.038 \\
\hline 3.5 & 0.004 & $1.844 \mathrm{E}+08$ & $4.182 \mathrm{E}+05$ & $6.868 \mathrm{E}+06$ & $5.355 \mathrm{E}+07$ & $2.402 \mathrm{E}+06$ & & & 0.290 & 0.013 & 0.350 & 0.045 \\
\hline 5.0 & 0.004 & $8.058 \mathrm{E}+07$ & & & & $9.205 \mathrm{E}+05$ & & & 0.231 & 0.011 & 0.422 & 0.049 \\
\hline 1.0 & 0.001 & $5.737 \mathrm{E}+09$ & $3.436 \mathrm{E}+07$ & & $1.211 \mathrm{E}+08$ & $9.094 \mathrm{E}+06$ & 0.234 & 0.284 & 0.021 & 0.002 & 0.007 & 0.075 \\
\hline $1.5 \ldots$ & 0.001 & $1.603 \mathrm{E}+09$ & $3.633 \mathrm{E}+07$ & $3.606 \mathrm{E}+08$ & $1.222 \mathrm{E}+08$ & $6.088 \mathrm{E}+06$ & 0.225 & 0.297 & 0.076 & 0.004 & 0.017 & 0.050 \\
\hline
\end{tabular}

a $\tau_{F G B-C}$ is defined as the time between the HB clump luminosity on the FGB and core He ignition at the end of the FGB.

b $\tau_{A G B}$ represents the total AGB lifetime. 
TABLE 2.4

Abundances After Major Dredge-up Episodes: $Z=0.016$

\begin{tabular}{|c|c|c|c|c|c|c|c|}
\hline $\begin{array}{l}\text { Dredge-up } \\
\text { Episode }\end{array}$ & ${ }^{1} \mathrm{H}$ & ${ }^{3} \mathrm{He}$ & ${ }^{4} \mathrm{He}$ & ${ }^{12} \mathrm{C}$ & ${ }^{4} \mathrm{~N}$ & ${ }^{16} \mathrm{O}$ & $Z>8$ \\
\hline Initial & $7.34 \mathrm{E}-1$ & $0.00 \mathrm{E}-0$ & $.50 \mathrm{E}-1$ & $3.49 \mathrm{E}-3$ & $8.49 \mathrm{E}-4$ & $7.71 \mathrm{E}-3$ & $3.95 \mathrm{E}-3$ \\
\hline \multicolumn{8}{|c|}{$M_{i}=1.0 M_{\odot}$} \\
\hline 1st & $7.16 \mathrm{E}-1$ & $6.90 \mathrm{E}-4$ & $2.67 \mathrm{E}-1$ & $3.30 \mathrm{E}-3$ & $07 \mathrm{E}-3$ & $71 \mathrm{E}-3$ & $3.95 \mathrm{E}-3$ \\
\hline 2nd & $7.17 \mathrm{E}-1$ & $6.86 \mathrm{E}-4$ & $2.67 \mathrm{E}-1$ & $3.30 \mathrm{E}-3$ & $1.07 \mathrm{E}-3$ & $7.71 \mathrm{E}-3$ & $3.95 \mathrm{E}-3$ \\
\hline $3 \mathrm{rd}$ & $7.17 \mathrm{E}-1$ & $6.85 \mathrm{E}-4$ & $2.67 \mathrm{E}-1$ & $3.30 \mathrm{E}-3$ & $1.07 \mathrm{E}-3$ & $7.71 \mathrm{E}-3$ & $3.95 \mathrm{E}-3$ \\
\hline \multicolumn{8}{|c|}{$M_{i}=1.5 M_{\odot}$} \\
\hline 1 st & $7.19 \mathrm{E}-1$ & $30 \mathrm{E}-4$ & $2.64 \mathrm{E}-1$ & $2.65 \mathrm{E}-3$ & $1.82 \mathrm{E}-3$ & $7.71 \mathrm{E}-3$ & $3.95 \mathrm{E}-3$ \\
\hline 2nd & $7.19 \mathrm{E}-1$ & $5.56 \mathrm{E}-4$ & $2.64 \mathrm{E}-1$ & $2.65 \mathrm{E}-3$ & $1.83 \mathrm{E}-3$ & $7.71 \mathrm{E}-3$ & $3.95 \mathrm{E}-3$ \\
\hline 3 rd & $7.19 \mathrm{E}-1$ & $5.55 \mathrm{E}-4$ & $2.64 \mathrm{E}-1$ & $2.65 \mathrm{E}-1$ & $1.83 \mathrm{E}-3$ & $7.71 \mathrm{E}-3$ & $3.95 \mathrm{E}-3$ \\
\hline \multicolumn{8}{|c|}{$M_{i}=2.0 M_{\odot}$} \\
\hline 1st & $7.25 \mathrm{E}-1$ & $38 \mathrm{E}-4$ & $2.59 \mathrm{E}-1$ & $2.39 \mathrm{E}-3$ & $14 \mathrm{E}-3$ & $71 \mathrm{E}-3$ & $3.95 \mathrm{E}-3$ \\
\hline $2 \mathrm{r}$ & $7.25 \mathrm{E}-1$ & $3.36 \mathrm{E}-4$ & $2.59 \mathrm{E}-1$ & $2.39 E-3$ & $2.14 \mathrm{E}-3$ & $7.71 \mathrm{E}-3$ & $3.95 \mathrm{E}-3$ \\
\hline 3 rd & $7.25 \mathrm{E}-1$ & $3.36 \mathrm{E}-4$ & $2.59 \mathrm{E}-1$ & $2.38 \mathrm{E}-3$ & $2.15 \mathrm{E}-3$ & $7.71 \mathrm{E}-3$ & $3.95 \mathrm{E}-3$ \\
\hline \multicolumn{8}{|c|}{$M_{i}=2.5 M_{\odot}$} \\
\hline & $7.28 \mathrm{E}-1$ & $2.11 \mathrm{E}-4$ & $2.56 \mathrm{E}-1$ & $2.29 \mathrm{E}-3$ & $2.29 \mathrm{E}-3$ & $7.66 \mathrm{E}-3$ & $3.95 \mathrm{E}-3$ \\
\hline & $7.28 \mathrm{E}-1$ & $12 E-4$ & $2.56 \mathrm{E}-1$ & $2.29 \mathrm{E}-3$ & $2.29 \mathrm{E}-3$ & $7.66 \mathrm{E}-3$ & $3.95 \mathrm{E}-3$ \\
\hline$\ldots$ & $7.28 \mathrm{E}-1$ & $2.11 \mathrm{E}-4$ & $2.56 \mathrm{E}-1$ & $2.29 \mathrm{E}-3$ & $2.29 \mathrm{E}-3$ & $7.66 \mathrm{E}-3$ & $3.95 \mathrm{E}-3$ \\
\hline \multicolumn{8}{|c|}{$M_{i}=3.5 M_{\odot}$} \\
\hline 1st & $7.28 \mathrm{E}-1$ & $1.02 \mathrm{E}-4$ & $2.56 \mathrm{E}-1$ & $2.25 \mathrm{E}-3$ & $2.42 \mathrm{E}-3$ & $7.56 \mathrm{E}-3$ & $3.95 \mathrm{E}-3$ \\
\hline & $7.28 \mathrm{E}-1$ & $1.02 \mathrm{E}-4$ & $2.56 \mathrm{E}-1$ & $2.24 \mathrm{E}-3$ & $2.44 \mathrm{E}-3$ & $7.56 \mathrm{E}-3$ & $3.95 \mathrm{E}-3$ \\
\hline $3 \mathrm{rd}$ & $7.28 \mathrm{E}-1$ & $1.02 \mathrm{E}-4$ & $2.56 \mathrm{E}-1$ & $2.39 \mathrm{E}-3$ & $2.45 \mathrm{E}-3$ & $7.56 \mathrm{E}-3$ & $3.95 \mathrm{E}-3$ \\
\hline \multicolumn{8}{|c|}{$M_{i}=5.0 M_{\odot}$} \\
\hline 1 st & $7.32 \mathrm{E}-1$ & $5.17 \mathrm{E}-5$ & $2.51 \mathrm{E}-1$ & $2.33 \mathrm{E}-3$ & $2.25 \mathrm{E}-3$ & $7.65 \mathrm{E}-3$ & $3.95 \mathrm{E}-3$ \\
\hline & $7.30 \mathrm{E}-1$ & $18 \mathrm{E}-5$ & $2.53 \mathrm{E}-1$ & $2.30 \mathrm{E}-3$ & $2.34 \mathrm{E}-3$ & $7.59 \mathrm{E}-3$ & $3.95 \mathrm{E}-3$ \\
\hline 3 rd & $7.30 \mathrm{E}-1$ & $4.93 E-5$ & $2.57 \mathrm{E}-1$ & $3.12 \mathrm{E}-3$ & $2.46 \mathrm{E}-3$ & $7.59 \mathrm{E}-3$ & $3.96 \mathrm{E}-3$ \\
\hline
\end{tabular}


TABLE 2.5

Abundances After Major Dredge-up Episodes: $Z=0.008$

\begin{tabular}{|c|c|c|c|c|c|c|c|}
\hline $\begin{array}{l}\text { Dredge-up } \\
\text { Episode }\end{array}$ & ${ }^{1} \mathrm{H}$ & ${ }^{3} \mathrm{He}$ & ${ }^{4} \mathrm{He}$ & ${ }^{12} \mathrm{C}$ & ${ }^{14} \mathrm{~N}$ & ${ }^{16} \mathrm{O}$ & $4>$ \\
\hline Initial & $7.42 \mathrm{E}-1$ & $0.00 \mathrm{E}-0$ & $2.50 \mathrm{E}-1$ & $1.74 \mathrm{E}-3$ & $2.12 \mathrm{E}-4$ & $3.85 \mathrm{E}-3$ & $1.99 \mathrm{E}-3$ \\
\hline \multicolumn{8}{|c|}{$M_{i}=0.95 M_{\odot}$} \\
\hline 1st & $7.31 \mathrm{E}-1$ & $6.71 \mathrm{E}-4$ & $2.61 \mathrm{E}-1$ & $1.72 \mathrm{E}-3$ & $2.41 \mathrm{E}-4$ & $3.85 \mathrm{E}-3$ & $1.99 \mathrm{E}-3$ \\
\hline 2nd & $7.31 \mathrm{E}-1$ & $6.37 \mathrm{E}-4$ & $2.61 \mathrm{E}-1$ & $1.71 \mathrm{E}-3$ & $2.48 \mathrm{E}-4$ & $3.85 \mathrm{E}-3$ & $1.99 \mathrm{E}-3$ \\
\hline 3 rd & $7.31 \mathrm{E}-1$ & $6.30 \mathrm{E}-4$ & $2.61 \mathrm{E}-1$ & $1.70 \mathrm{E}-3$ & $2.56 \mathrm{E}-4$ & $3.85 \mathrm{E}-3$ & $1.99 \mathrm{E}-3$ \\
\hline \multicolumn{8}{|c|}{$M_{i}=1.0 M_{\odot}$} \\
\hline 1st & $7.29 \mathrm{E}-1$ & $6.70 \mathrm{E}-4$ & $2.63 \mathrm{E}-1$ & $1.67 \mathrm{E}-3$ & $2.94 \mathrm{E}-4$ & $3.85 \mathrm{E}-3$ & $1.99 \mathrm{E}-3$ \\
\hline 2nd & $7.29 \mathrm{E}-1$ & $6.52 E-4$ & $2.63 \mathrm{E}-1$ & $1.66 \mathrm{E}-3$ & $3.04 \mathrm{E}-4$ & $3.85 E-3$ & $1.99 \mathrm{E}-3$ \\
\hline$\ldots$ & $7.29 \mathrm{E}-1$ & $6.51 \mathrm{E}-4$ & $2.63 \mathrm{E}-1$ & $1.66 \mathrm{E}-3$ & $3.05 E-4$ & $3.85 E-3$ & $1.99 \mathrm{E}-3$ \\
\hline \multicolumn{8}{|c|}{$M_{i}=1.5 M_{\odot}$} \\
\hline 1st & $7.29 \mathrm{E}-1$ & $5.48 \mathrm{E}-4$ & $2.63 \mathrm{E}-1$ & $1.36 \mathrm{E}-3$ & $6.60 \mathrm{E}-4$ & $3.85 \mathrm{E}-3$ & $1.99 \mathrm{E}-3$ \\
\hline 2nd & $7.29 \mathrm{E}-1$ & $5.38 \mathrm{E}-4$ & $2.63 \mathrm{E}-1$ & $1.35 \mathrm{E}-3$ & $6.70 \mathrm{E}-4$ & $3.85 \mathrm{~F}$ & $1.99 \mathrm{E}-3$ \\
\hline $3 \mathrm{rd}$ & $7.29 \mathrm{E}-1$ & $5.38 \mathrm{E}-4$ & $2.63 \mathrm{E}-1$ & $1.35 E-3$ & $6.71 \mathrm{E}-4$ & $3.85 E-3$ & $1.99 \mathrm{E}-3$ \\
\hline \multicolumn{8}{|c|}{$M_{i}=2.0 M_{\odot}$} \\
\hline 1st & $7.32 \mathrm{E}-1$ & $3 E-4$ & $2.59 \mathrm{E}-1$ & $1.15 E-3$ & $07 \mathrm{E}-4$ & -3 & E-3 \\
\hline & $7.33 \mathrm{E}-1$ & $3.11 \mathrm{E}-4$ & $2.60 \mathrm{E}-1$ & $1.15 \mathrm{E}-3$ & $9.09 \mathrm{E}-4$ & 3.85 & $E-3$ \\
\hline $3 \mathrm{rd}$ & $7.33 \mathrm{E}-1$ & $3.10 \mathrm{E}-4$ & $2.60 \mathrm{E}-1$ & $1.14 \mathrm{E}-3$ & $9.13 \mathrm{E}-4$ & $3.85 \mathrm{E}-3$ & $1.99 \mathrm{E}-3$ \\
\hline \multicolumn{8}{|c|}{$M_{i}=2.5 M_{\odot}$} \\
\hline $1 \mathrm{st}$ & $7.35 \mathrm{E}-1$ & $1.96 \mathrm{E}-4$ & $2.57 \mathrm{E}-1$ & $1.09 \mathrm{E}-3$ & $1.02 \mathrm{E}-3$ & $3.80 \mathrm{E}-3$ & $1.99 \mathrm{E}-3$ \\
\hline 2 & $7.35 \mathrm{E}-1$ & $1.96 \mathrm{E}-4$ & $2.57 \mathrm{E}-1$ & $1.09 \mathrm{E}-3$ & $1.02 \mathrm{E}-3$ & $3.80 \mathrm{E}-3$ & $1.99 \mathrm{E}-3$ \\
\hline $3 \mathrm{rd}$ & $7.35 \mathrm{E}-1$ & $1.94 \mathrm{E}-4$ & $2.60 \mathrm{E}-1$ & $2.02 \mathrm{E}-3$ & $1.05 \mathrm{E}-3$ & $3.79 \mathrm{E}-3$ & $1.99 \mathrm{E}-3$ \\
\hline \multicolumn{8}{|c|}{$M_{i}=3.5 M_{\odot}$} \\
\hline $1 \mathrm{st}$ & $7.40 \mathrm{E}-1$ & $1.00 \mathrm{E}-4$ & $2.52 \mathrm{E}-1$ & $1.11 \mathrm{E}-3$ & $9.67 \mathrm{E}-4$ & $3.83 \mathrm{E}-3$ & $1.99 \mathrm{E}-3$ \\
\hline & $7.40 \mathrm{E}-1$ & $9.80 \mathrm{E}-5$ & $2.52 \mathrm{E}-1$ & $1.09 \mathrm{E}-3$ & $1.00 \mathrm{E}-3$ & $3.82 \mathrm{E}-3$ & $1.99 \mathrm{E}-3$ \\
\hline 3 rd & $7.39 \mathrm{E}-1$ & $9.77 \mathrm{E}-5$ & $2.55 \mathrm{E}-1$ & $1.84 \mathrm{E}-3$ & $1.03 \mathrm{E}-3$ & $3.81 \mathrm{E}-3$ & $1.99 \mathrm{E}-3$ \\
\hline \multicolumn{8}{|c|}{$M_{i}=5.0 M_{\odot}$} \\
\hline & $7.42 \mathrm{E}-1$ & $5.22 \mathrm{E}-5$ & $2.50 \mathrm{E}-1$ & $1.17 \mathrm{E}-3$ & $8.77 \mathrm{E}-4$ & $3.85 \mathrm{E}-3$ & $1.99 \mathrm{E}-3$ \\
\hline & $7.38 \mathrm{E}-1$ & $4.98 \mathrm{E}-5$ & $2.54 \mathrm{E}-1$ & $1.10 \mathrm{E}-3$ & $1.02 \mathrm{E}-3$ & $3.76 \mathrm{E}-3$ & $1.99 \mathrm{E}-3$ \\
\hline 3 rd & $7.34 \mathrm{E}-1$ & $3.23 \mathrm{E}-5$ & $2.60 \mathrm{E}-1$ & $2.92 \mathrm{E}-3$ & $1.81 \mathrm{E}-3$ & $3.80 \mathrm{E}-3$ & $2.00 \mathrm{E}-3$ \\
\hline
\end{tabular}


TABLE 2.6

Abundances After Major Dredge-up Episodes: $Z=0.004$

\begin{tabular}{|c|c|c|c|c|c|c|c|}
\hline $\begin{array}{l}\text { Dredge-up } \\
\text { Episode }\end{array}$ & ${ }^{1} \mathrm{H}$ & ${ }^{3} \mathrm{He}$ & ${ }^{4} \mathrm{He}$ & ${ }^{12} \mathrm{C}$ & ${ }^{14} \mathrm{~N}$ & ${ }^{16} \mathrm{O}$ & $Z>8$ \\
\hline Initial $\ldots$ & $7.46 \mathrm{E}-1$ & $0.00 \mathrm{E}-0$ & $2.50 \mathrm{E}-1$ & $8.71 \mathrm{E}-4$ & $5.31 \mathrm{E}-5$ & $1.93 \mathrm{E}-3$ & $9.90 \mathrm{E}-4$ \\
\hline \multicolumn{8}{|c|}{$M_{i}=0.89 M_{\odot}$} \\
\hline 1st & $7.35 \mathrm{E}-1$ & $6.18 \mathrm{E}-4$ & $2.60 \mathrm{E}-1$ & $8.60 \mathrm{E}-4$ & $6.62 \mathrm{E}-5$ & $1.93 \mathrm{E}-3$ & $9.90 \mathrm{E}-4$ \\
\hline 2nd & $7.35 \mathrm{E}-1$ & $5.45 \mathrm{E}-4$ & $2.60 \mathrm{E}-1$ & $8.23 \mathrm{E}-4$ & $1.09 \mathrm{E}-4$ & $1.93 \mathrm{E}-3$ & $9.90 \mathrm{E}-4$ \\
\hline 3 rd & $7.35 \mathrm{E}-1$ & $5.42 \mathrm{E}-4$ & $2.61 \mathrm{E}-1$ & $8.17 \mathrm{E}-4$ & $1.16 \mathrm{E}-4$ & $1.93 \mathrm{E}-3$ & $9.90 \mathrm{E}-4$ \\
\hline \multicolumn{8}{|c|}{$M_{i}=1.0 M_{\odot}$} \\
\hline 1st & $7.34 \mathrm{E}-1$ & $6.41 \mathrm{E}-4$ & $2.62 \mathrm{E}-1$ & $8.28 \mathrm{E}-4$ & $1.02 \mathrm{E}-4$ & $1.93 \mathrm{E}-3$ & $9.90 \mathrm{E}-4$ \\
\hline 2nd & $7.34 \mathrm{E}-1$ & $6.14 \mathrm{E}-4$ & $2.62 \mathrm{E}-1$ & $8.19 \mathrm{E}-4$ & $1.14 \mathrm{E}-4$ & $1.93 \mathrm{E}-3$ & $9.90 \mathrm{E}-4$ \\
\hline 3 rd & $7.34 \mathrm{E}-1$ & $6.04 \mathrm{E}-4$ & $2.62 \mathrm{E}-1$ & $8.06 \mathrm{E}-4$ & $1.28 \mathrm{E}-4$ & $1.93 \mathrm{E}-3$ & $9.90 \mathrm{E}-4$ \\
\hline \multicolumn{8}{|c|}{$M_{i}=1.5 M_{\odot}$} \\
\hline 1st & $7.32 \mathrm{E}-1$ & $5.00 \mathrm{E}-4$ & $2.63 \mathrm{E}-1$ & $6.52 \mathrm{E}-4$ & $3.09 \mathrm{E}-4$ & $1.93 \mathrm{E}-3$ & $9.90 \mathrm{E}-4$ \\
\hline 2nd & $7.32 \mathrm{E}-1$ & $4.89 \mathrm{E}-4$ & $2.63 \mathrm{E}-1$ & $6.45 E-4$ & $3.17 E-4$ & $1.93 \mathrm{E}-3$ & $9.90 \mathrm{E}-4$ \\
\hline $3 \mathrm{rd}$ & $7.32 \mathrm{E}-1$ & $4.86 \mathrm{E}-4$ & $2.63 \mathrm{E}-1$ & $6.42 \mathrm{E}-4$ & $3.20 \mathrm{E}-4$ & $1.93 \mathrm{E}-3$ & $9.90 \mathrm{E}-4$ \\
\hline \multicolumn{8}{|c|}{$M_{i}=2.0 M_{\odot}$} \\
\hline 1st & $7.35 \mathrm{E}-1$ & $2.90 \mathrm{E}-4$ & $2.61 \mathrm{E}-1$ & $5.52 \mathrm{E}-4$ & $4.29 \mathrm{E}-4$ & $1.93 \mathrm{E}-3$ & $9.90 \mathrm{E}-4$ \\
\hline 2nd & $7.35 \mathrm{E}-1$ & $2.87 \mathrm{E}-4$ & $2.61 \mathrm{E}-1$ & $5: 50 \mathrm{E}-4$ & $4.31 \mathrm{E}-4$ & $1.93 \mathrm{E}-3$ & $9.90 \mathrm{E}-4$ \\
\hline $3 \mathrm{rd}$ & $7.33 \mathrm{E}-1$ & $2.83 \mathrm{E}-4$ & $2.63 \mathrm{E}-1$ & $1.36 \mathrm{E}-3$ & $4.52 \mathrm{E}-4$ & $1.93 \mathrm{E}-3$ & $9.90 \mathrm{E}-4$ \\
\hline \multicolumn{8}{|c|}{$M_{i}=2.5 M_{\odot}$} \\
\hline 1st & $7.38 \mathrm{E}-1$ & $1.82 \mathrm{E}-4$ & $2.58 \mathrm{E}-1$ & $5.24 \mathrm{E}-4$ & $4.89 \mathrm{E}-4$ & $1.89 \mathrm{E}-3$ & $9.90 \mathrm{E}-4$ \\
\hline 2nd & $7.38 \mathrm{E}-1$ & $1.82 \mathrm{E}-4$ & $2.58 \mathrm{E}-1$ & $5.20 \mathrm{E}-4$ & $4.94 \mathrm{E}-4$ & $1.89 \mathrm{E}-3$ & $9.90 \mathrm{E}-4$ \\
\hline $3 \mathrm{rd}$ & $7.33 \mathrm{E}-1$ & $1.80 \mathrm{E}-4$ & $2.64 \mathrm{E}-1$ & $2.12 \mathrm{E}-3$ & $5.25 \mathrm{E}-4$ & $1.89 \mathrm{E}-3$ & $9.90 \mathrm{E}-4$ \\
\hline \multicolumn{8}{|c|}{$M_{i}=3.5 M_{\odot}$} \\
\hline 1st & $7.45 \mathrm{E}-1$ & $1.05 \mathrm{E}-4$ & $2.51 \mathrm{E}-1$ & $5.97 \mathrm{E}-4$ & $3.73 \mathrm{E}-4$ & $1.93 \mathrm{E}-3$ & $9.90 \mathrm{E}-4$ \\
\hline 2nd & $7.43 \mathrm{E}-1$ & $9.82 \mathrm{E}-5$ & $2.53 \mathrm{E}-1$ & $5.44 \mathrm{E}-4$ & $4.47 \mathrm{E}-4$ & $1.92 \mathrm{E}-3$ & $9.90 \mathrm{E}-4$ \\
\hline $3 \mathrm{rd}$ & $7.42 \mathrm{E}-1$ & $9.75 \mathrm{E}-5$ & $2.55 \mathrm{E}-1$ & $1.41 \mathrm{E}-3$ & $4.96 \mathrm{E}-4$ & $1.92 \mathrm{E}-3$ & $9.90 \mathrm{E}-4$ \\
\hline \multicolumn{8}{|c|}{$M_{i}=5.0 M_{\odot}$} \\
\hline 1st & $7.46 \mathrm{E}-1$ & $5.59 \mathrm{E}-5$ & $2.50 \mathrm{E}-1$ & $6.44 \mathrm{E}-4$ & $3.18 \mathrm{E}-4$ & $1.93 \mathrm{E}-3$ & $9.90 \mathrm{E}-4$ \\
\hline 2nd & $7.42 \mathrm{E}-1$ & $4.90 \mathrm{E}-5$ & $2.54 \mathrm{E}-1$ & $5.33 \mathrm{E}-4$ & $4.74 \mathrm{E}-4$ & $1.90 \mathrm{E}-3$ & $9.90 \mathrm{E}-4$ \\
\hline 3rd $\ldots \ldots$ & $7.39 \mathrm{E}-1$ & $2.18 \mathrm{E}-5$ & $2.59 \mathrm{E}-1$ & $1.76 \mathrm{E}-3$ & $1.39 E-3$ & $1.86 \mathrm{E}-3$ & $9.90 \mathrm{E}-4$ \\
\hline
\end{tabular}


TABLE 2.7

Abundances After Major Dredge-up Episodes: $Z=0.001$

\begin{tabular}{lccccccc}
\hline $\begin{array}{l}\text { Dredge-up } \\
\text { Episode }\end{array}$ & ${ }^{1} \mathrm{H}$ & ${ }^{3} \mathrm{He}$ & ${ }^{4} \mathrm{He}$ & ${ }^{12} \mathrm{C}$ & ${ }^{14} \mathrm{~N}$ & ${ }^{16} \mathrm{O}$ & $\mathrm{Z}>8$ \\
\hline Initial $\ldots$ & $7.49 \mathrm{E}-1$ & $0.00 \mathrm{E}-0$ & $2.50 \mathrm{E}-1$ & $2.18 \mathrm{E}-4$ & $5.31 \mathrm{E}-5$ & $4.82 \mathrm{E}-4$ & $2.50 \mathrm{E}-4$ \\
\hline \multicolumn{7}{c}{$M_{i}=1.0 M_{\odot}$} \\
\hline 1st $\ldots \ldots$ & $7.37 \mathrm{E}-1$ & $5.70 \mathrm{E}-4$ & $2.61 \mathrm{E}-1$ & $2.02 \mathrm{E}-4$ & $7.18 \mathrm{E}-5$ & $4.82 \mathrm{E}-4$ & $2.50 \mathrm{E}-4$ \\
2nd $\ldots \ldots$ & $7.37 \mathrm{E}-1$ & $5.25 \mathrm{E}-4$ & $2.61 \mathrm{E}-1$ & $1.95 \mathrm{E}-4$ & $8.02 \mathrm{E}-5$ & $4.82 \mathrm{E}-4$ & $2.50 \mathrm{E}-4$ \\
3rd $\ldots \ldots$. & $7.37 \mathrm{E}-1$ & $5.07 \mathrm{E}-4$ & $2.61 \mathrm{E}-1$ & $1.88 \mathrm{E}-4$ & $8.79 \mathrm{E}-5$ & $4.81 \mathrm{E}-4$ & $2.50 \mathrm{E}-4$ \\
\hline \multicolumn{7}{c}{$M_{i}=1.5 M_{\odot}$} \\
\hline 1st $\ldots \ldots$. & $7.32 \mathrm{E}-1$ & $4.11 \mathrm{E}-4$ & $2.66 \mathrm{E}-1$ & $1.51 \mathrm{E}-4$ & $1.32 \mathrm{E}-4$ & $4.82 \mathrm{E}-4$ & $2.50 \mathrm{E}-4$ \\
2nd $\ldots \ldots$ & $7.32 \mathrm{E}-1$ & $3.92 \mathrm{E}-4$ & $2.86 \mathrm{E}-1$ & $1.47 \mathrm{E}-4$ & $1.36 \mathrm{E}-4$ & $4.82 \mathrm{E}-4$ & $2.50 \mathrm{E}-4$ \\
3rd $\ldots \ldots$. & $7.27 \mathrm{E}-1$ & $3.84 \mathrm{E}-4$ & $2.70 \mathrm{E}-1$ & $1.56 \mathrm{E}-4$ & $1.47 \mathrm{E}-4$ & $4.97 \mathrm{E}-4$ & $2.50 \mathrm{E}-4$ \\
\hline
\end{tabular}

TABLE 2.8

Central $\left({ }^{12} \mathrm{C},{ }^{16} \mathrm{O}\right)$ Abundances on the E-AGB

\begin{tabular}{|c|c|c|c|c|}
\hline $\mathrm{M} / \mathrm{M}_{\odot}$ & $\mathrm{Z}=0.016$ & $\mathrm{Z}=0.008$ & $\mathrm{Z}=0.004$ & $\mathrm{Z}=0.001$ \\
\hline $0.89 \ldots$ & $\ldots$ & & $(0.17,0.82)$ & \\
\hline $0.95 \ldots$ & $\ldots$ & $(0.13,0.86)$ & $\ldots$ & \\
\hline $1.0 \ldots$ & $(0.08,0.90)$ & $(0.09,0.90)$ & $(0.10,0.89)$ & $(0.11,0.88)$ \\
\hline $1.5 \ldots$ & $(0.07,0.91)$ & $(0.09,0.90)$ & $(0.06,0.92)$ & $(0.04,0.95)$ \\
\hline $2.0 \ldots$ & $(0.04,0.94)$ & $(0.06,0.92)$ & $(0.05,0.94)$ & . \\
\hline $2.5 \ldots$ & $(0.06,0.91)$ & $(0.05,0.93)$ & $(0.02,0.97)$ & $\ldots$ \\
\hline $3.5 \ldots$ & $(0.01,0.98)$ & $(0.41,0.58)$ & $(0.44,0.55)$ & $\ldots$ \\
\hline $5.0 \ldots$ & $(0.47,0.51)$ & $(0.47,0.52)$ & $(0.47,0.52)$ & $\ldots$ \\
\hline
\end{tabular}




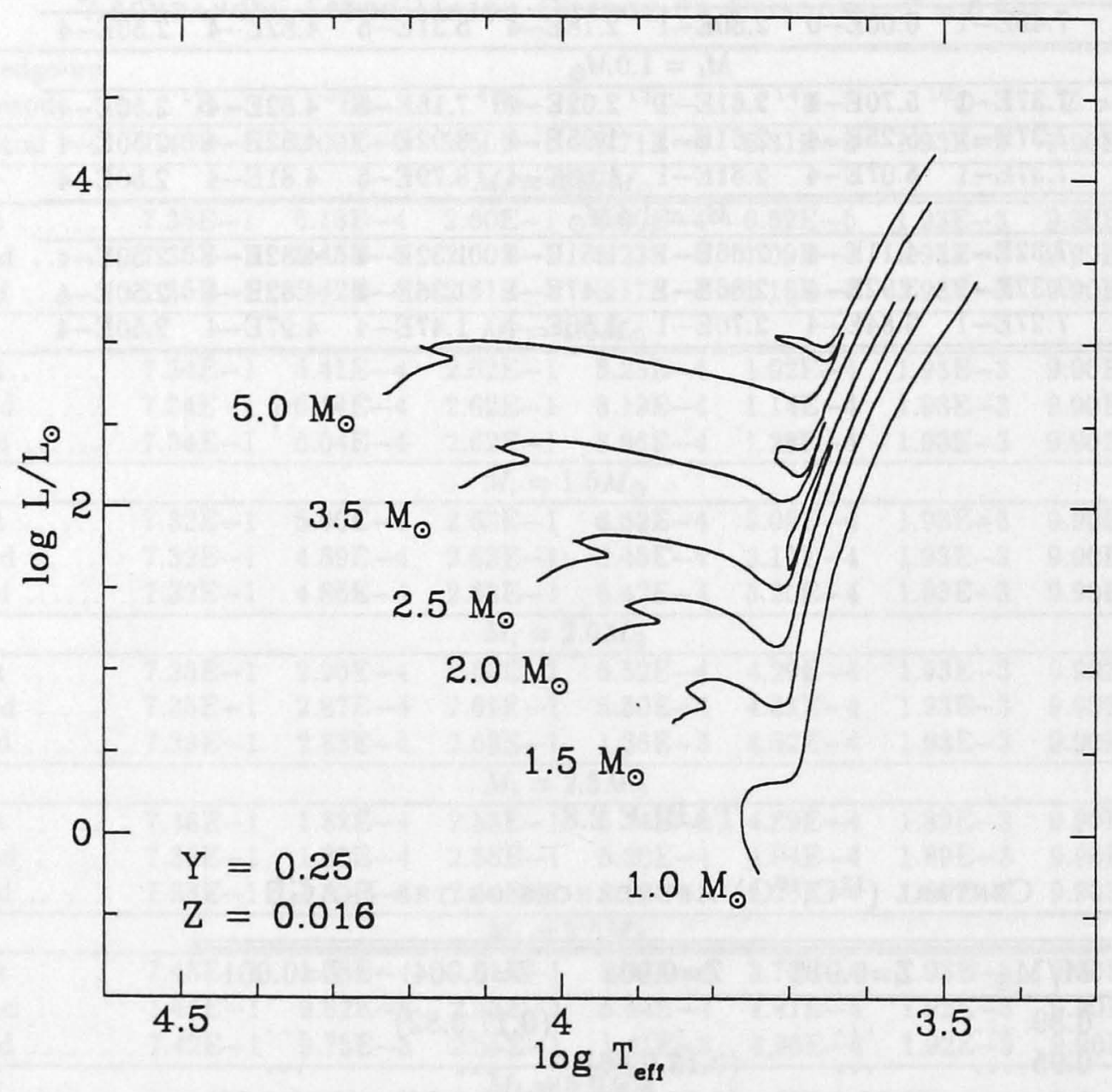

Figure 2.1: H-R diagram for evolutionary sequences calculated in this study with (Y,Z) $=(0.25,0.016)$. 


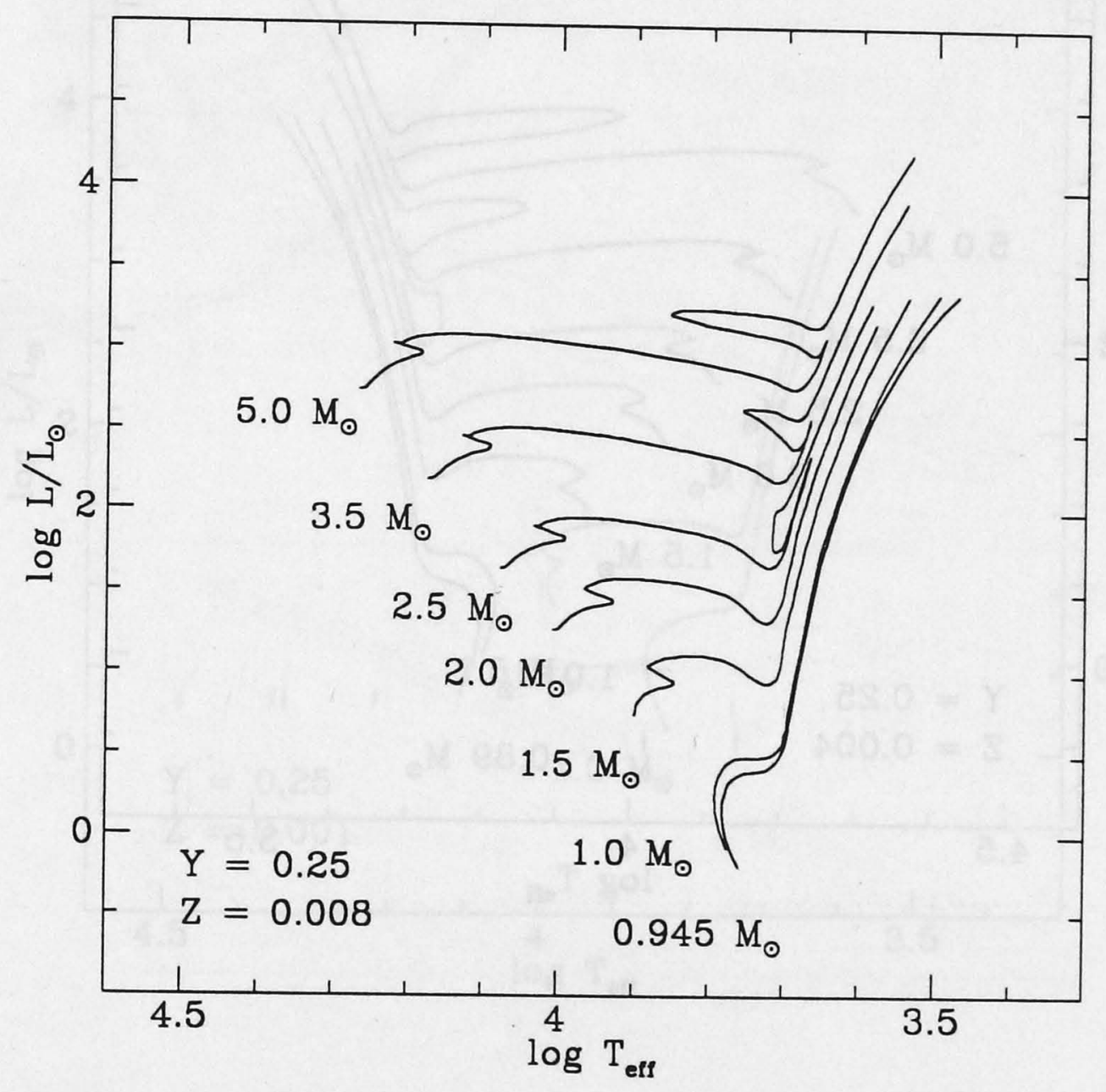

Figure 2.2: Same as figure 2.1 for $(Y, Z)=(0.25,0.008)$. 


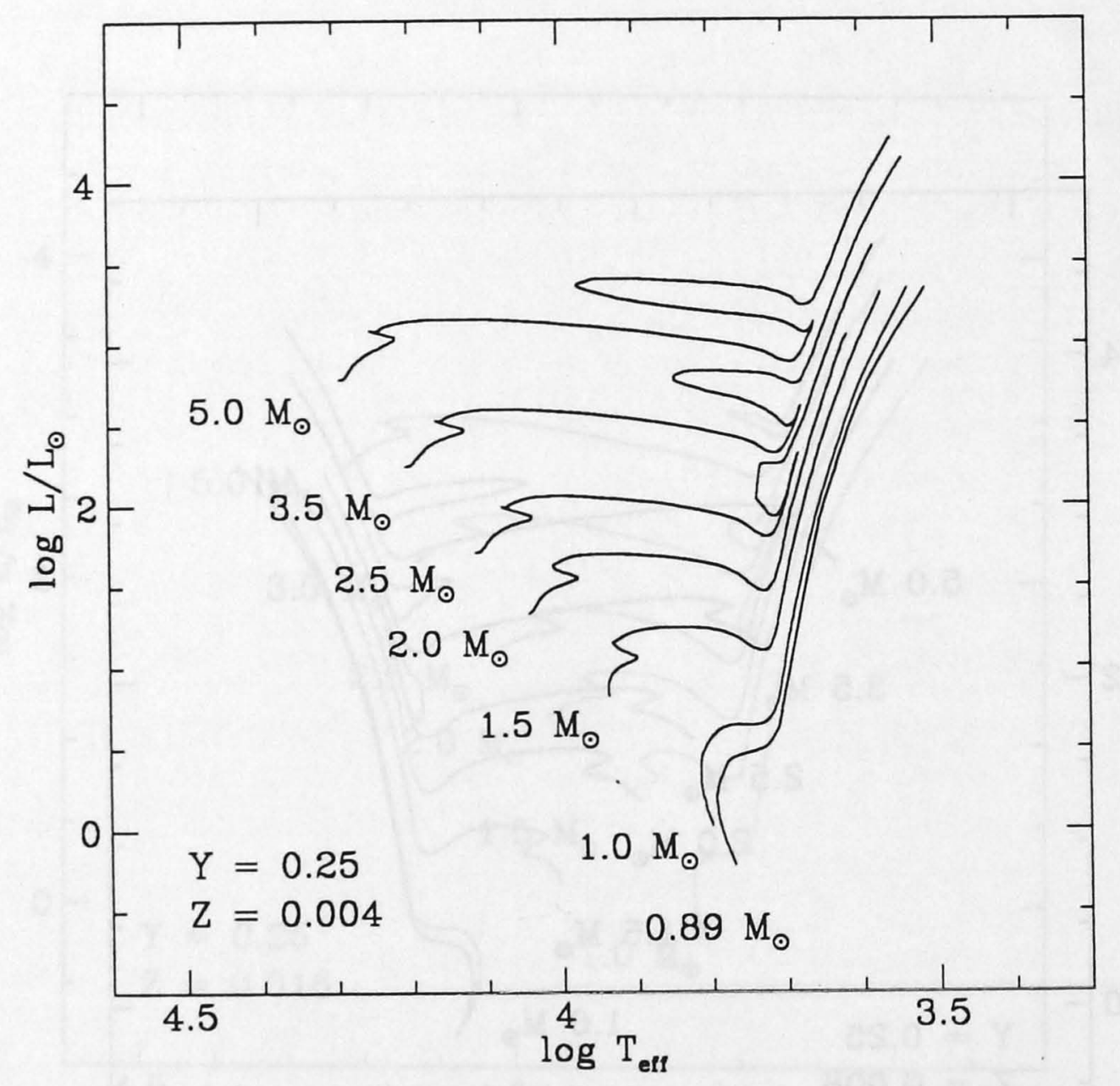

Figure 2.3: Same as figure 2.1 for $(\mathrm{Y}, \mathrm{Z})=(0.25,0.004)$. 


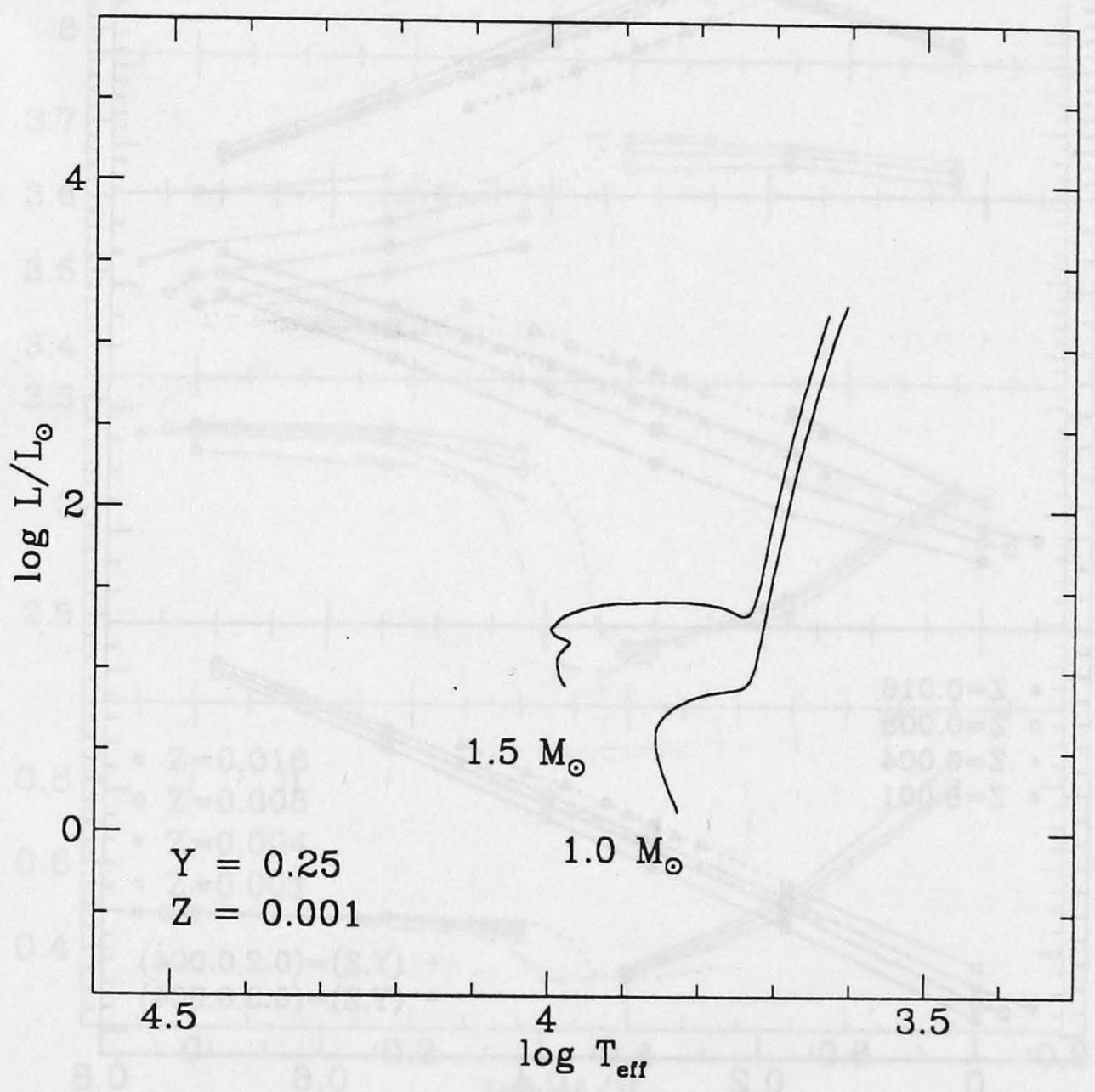

Figure 2.4: Same as figure 2.1 for $(Y, Z)=(0.25,0.001)$. 


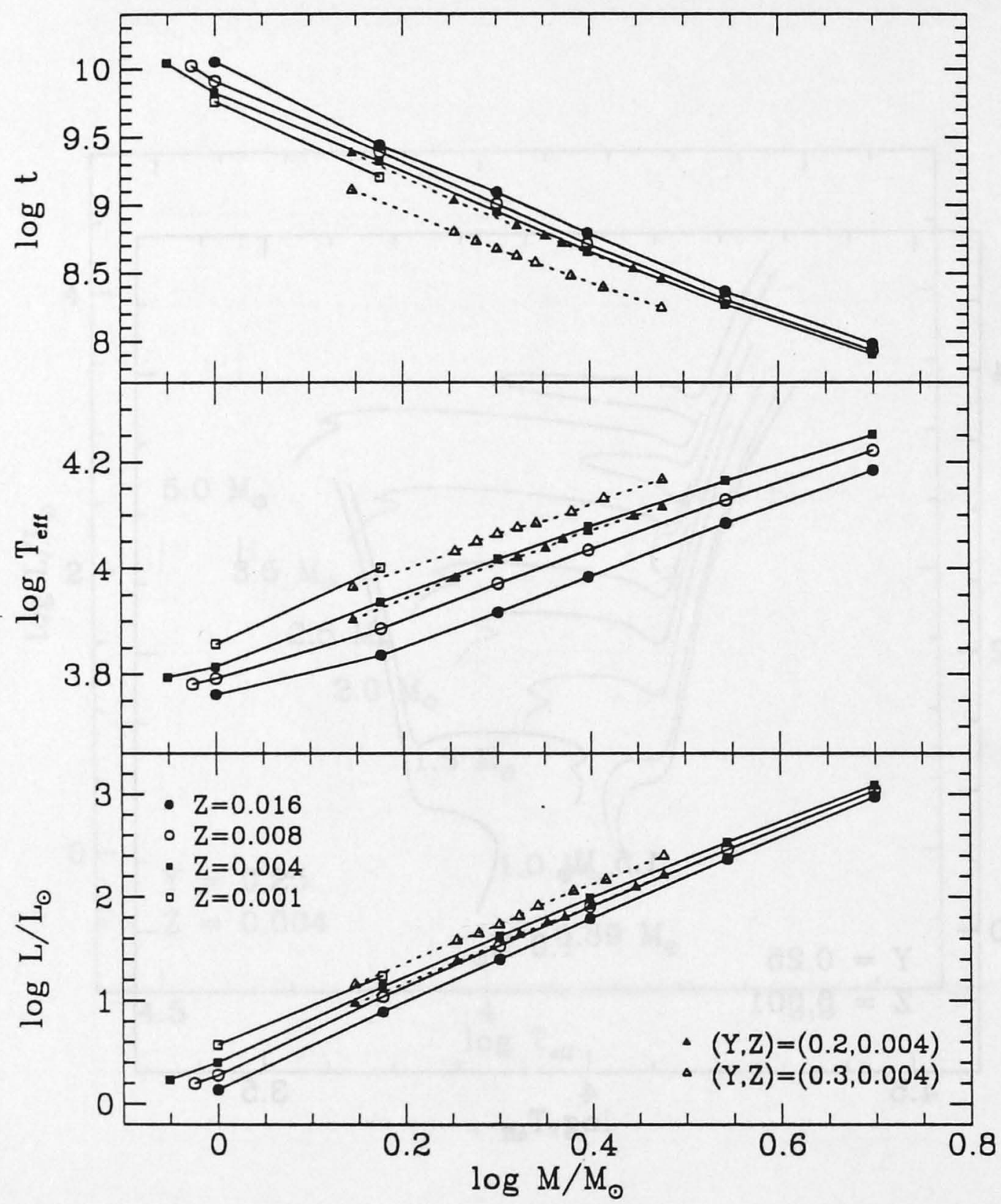

Figure 2.5: Age $(\mathrm{t})$, luminosity $(\mathrm{L})$, and effective temperature $\left(\mathrm{T}_{\text {eff }}\right)$ at the main sequence turn-off for each evolutionary sequence. The dotted lines represent results taken from SGR for their $Z=0.004, Y=0.2$ and 0.3 evolutionary sequences. 


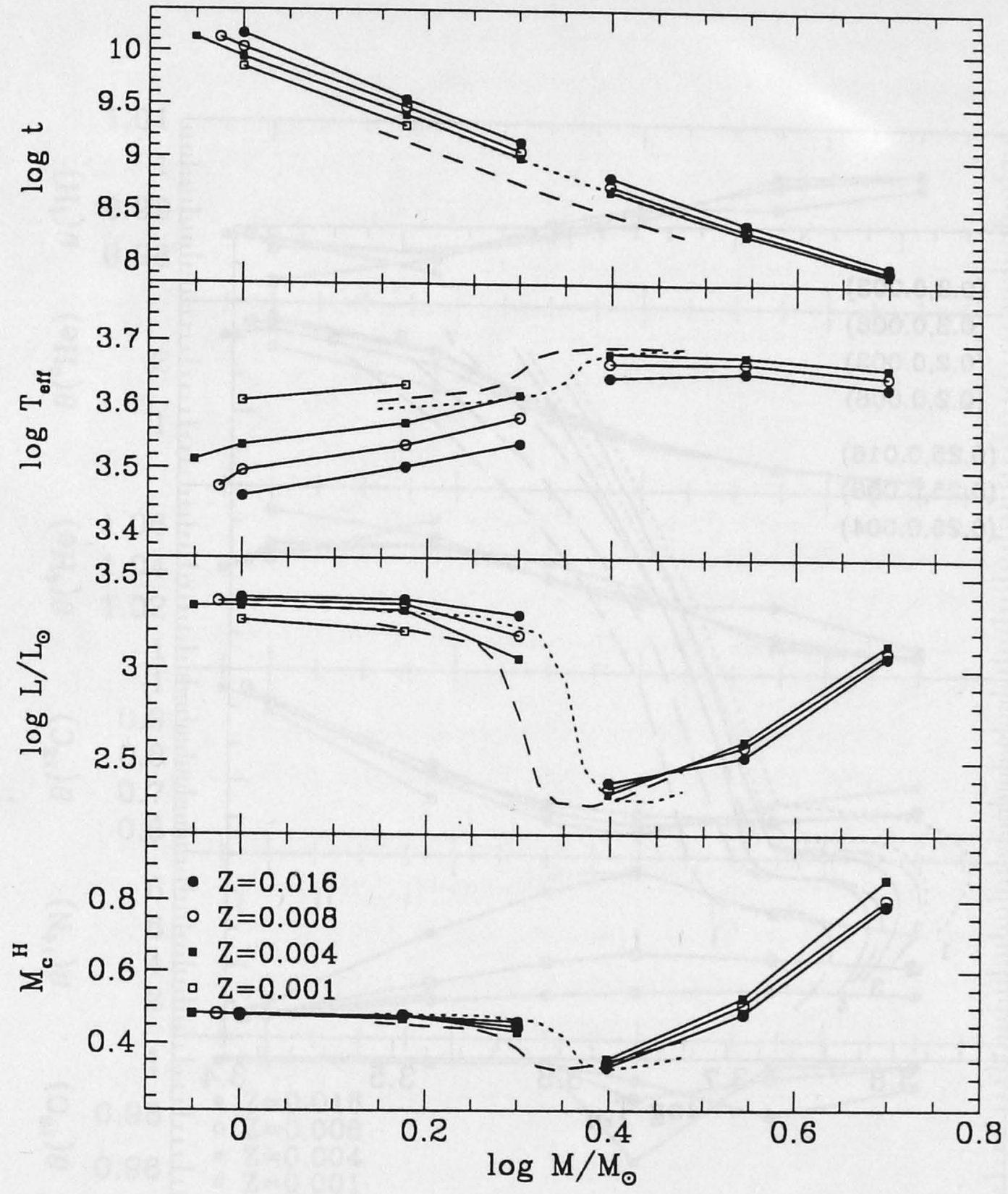

Figure 2.6: The age, stellar temperature, luminosity, and core mass, as a function of initial mass, at the point of He core ignition for each evolutionary sequence. Points corresponding to degenerate and non-degenerate He core ignition are linked by separate solid lines. The dotted curves correspond to the $Z=0.004, Y=0.2$ and 0.3 sequences of SGR. 


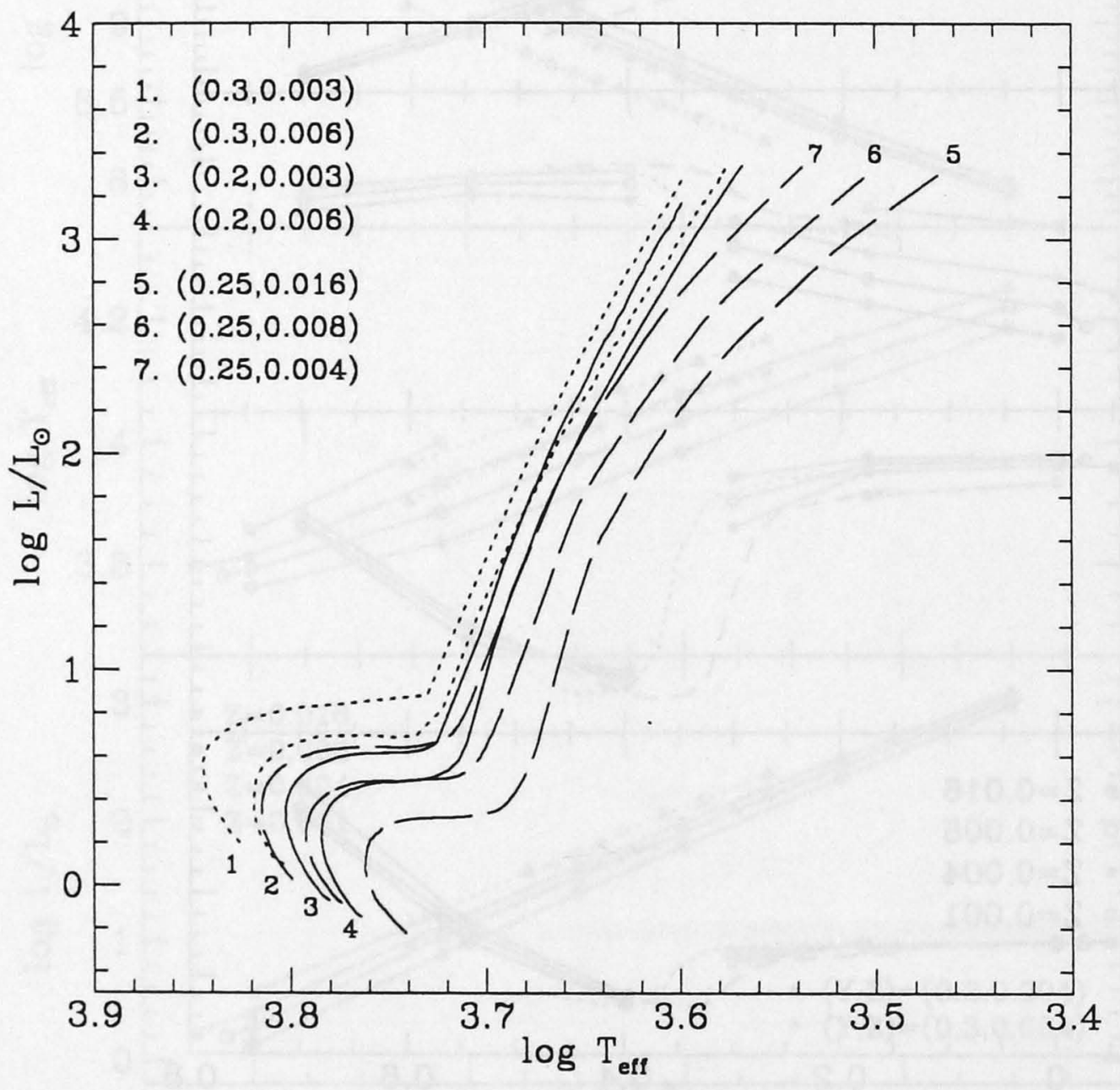

Figure 2.7: Comparison of $1 M_{\odot}$ evolutionary tracks from this study (dashed curves 5, 6, and 7) with those of Lattanzio (1991) (dotted curves 1 and 2; solid curves 3 and 4). 


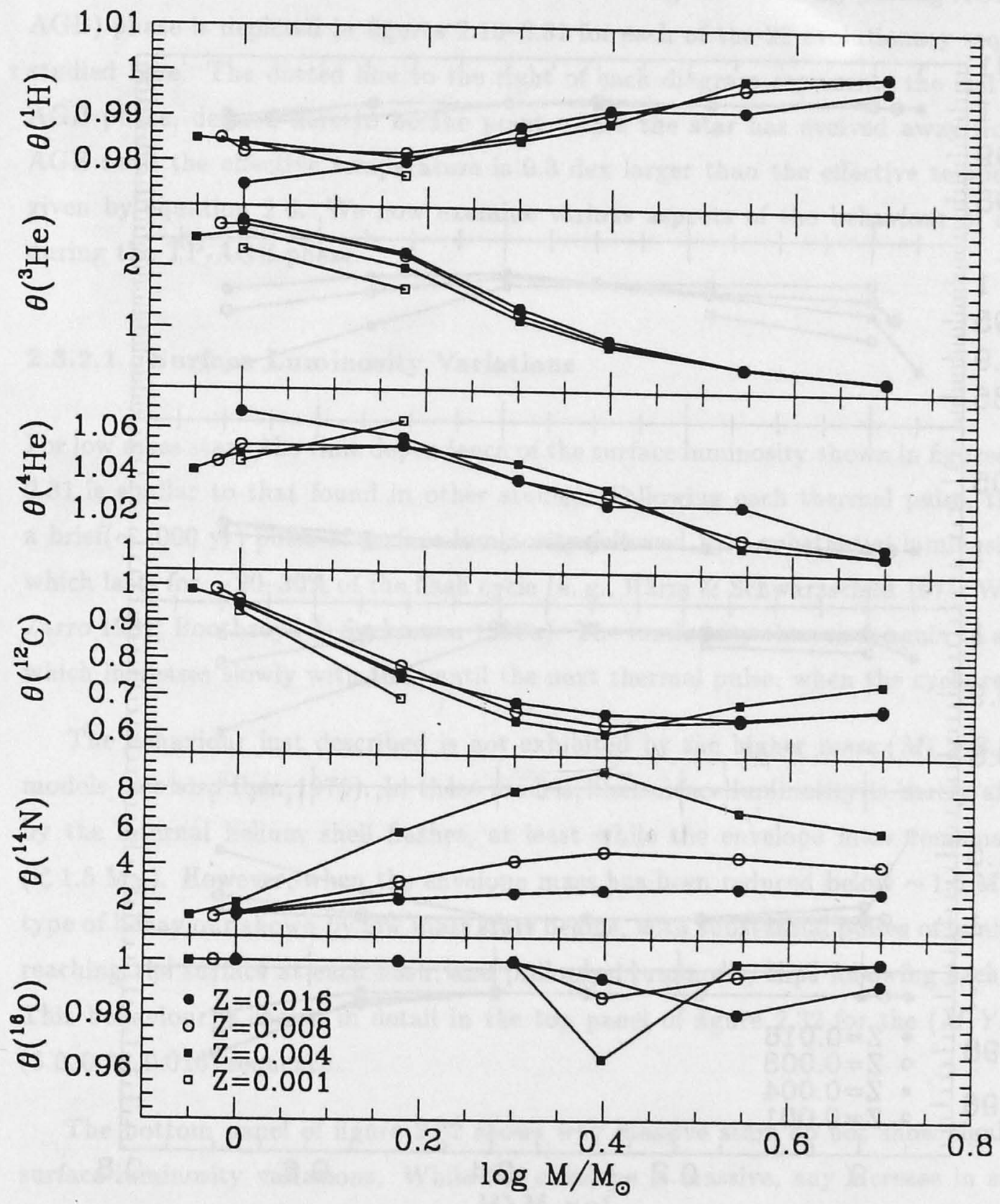

Figure 2.8: The ratio $\theta$ of surface abundance after first dredge-up to the initial, mainsequence abundance, except for ${ }^{3} \mathrm{He}$ where $\theta\left({ }^{3} \mathrm{He}\right)$ is 4000 times the ${ }^{3} \mathrm{He}$ abundance (the initial ${ }^{3} \mathrm{He}$ abundance is zero). 


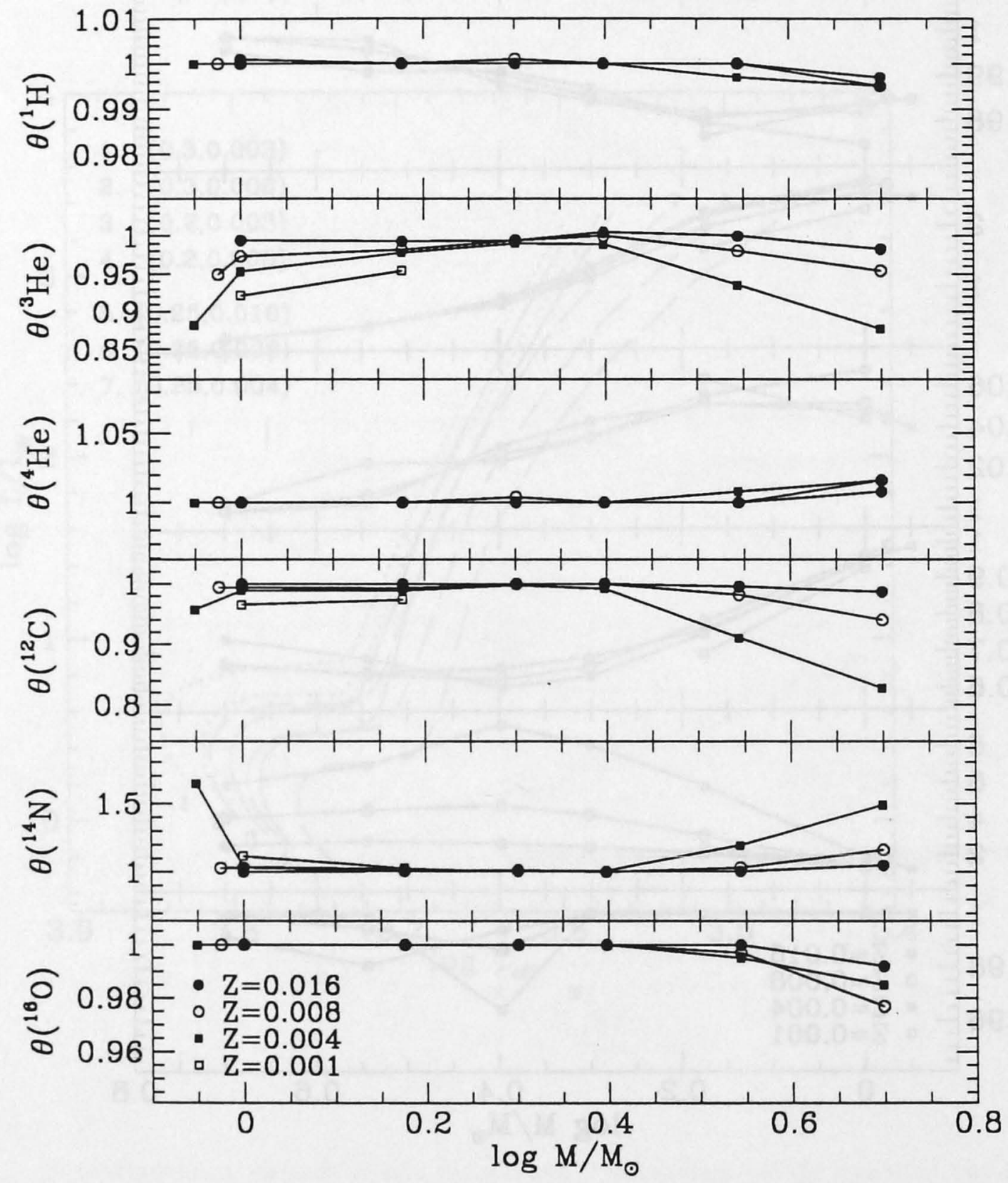

Figure 2.9: The ratio $\theta$ of surface abundance after second dredge-up to that after first dredge-up. 


\subsubsection{AGB Evolution with Mass Loss: The Thermally Pulsing AGB Phase}

The variation with time of luminosity, effective temperature, wind expansion velocity, total mass, mass loss rate and pulsation period during the thermally pulsing AGB (TPAGB) phase is depicted in figures $2.10-2.31$ for each of the 22 evolutionary sequences studied here. The dotted line to the right of each diagram represents the end of the AGB phase, defined here to be the point where the star has evolved away from the AGB until the effective temperature is 0.3 dex larger than the effective temperature given by equation 2.6. We now examine various aspects of the behaviour of models during the TP-AGB phase.

\subsubsection{Surface Luminosity Variations}

For low mass stars, the time dependence of the surface luminosity shown in figures 2.102.31 is similar to that found in other studies. Following each thermal pulse, there is a brief $(\sim 1000 \mathrm{yr})$ pulse of surface luminosity followed by a substantial luminosity dip which lasts for $\sim 20-30 \%$ of the flash cycle (e. g., Härm \& Schwarzschild 1975; Wood \& Zarro 1981; Boothroyd \& Sackmann 1988a). The luminosity then rises again to a value which increases slowly with time until the next thermal pulse, when the cycle repeats.

The behaviour just described is not exhibited by the higher mass $\left(M_{i} \geq 3.5 \mathrm{M}_{\odot}\right)$ models (see also Iben 1975). In these models, the surface luminosity is barely affected by the internal helium shell flashes, at least while the envelope mass remains large $\left(\gtrsim 1.5 \mathrm{M}_{\odot}\right)$. However, when the envelope mass has been reduced below $\sim 1.5 \mathrm{M}_{\odot}$, the type of behaviour shown by low mass stars begins, with substantial pulses of luminosity reaching the surface at each flash, and prolonged luminosity dips following each flash. This behaviour is shown in detail in the top panel of figure 2.32 for the $(M, Y, Z)=$ $(5.0,0.25,0.016)$ sequence.

The bottom panel of figure 2.32 shows why massive stars do not show significant surface luminosity variations. While the envelope is massive, any increase in surface luminosity due to a shell flash causes envelope convection to eat inwards, giving rise to the sawtooth variation in $\mathrm{M}_{c}^{H}$ (the base of the convective envelope is essentially coincident with the line representing the hydrogen core in figure 2.32). More importantly, the inward advance of the envelope convection cools the He burning shell which is extinguished before it can burn a significant mass of helium. Thus there is only a small amount of energy produced during the shell flash and the surface luminosity barely 
changes.

\subsubsection{Luminosity-Core Mass Relations}

Paczyński (1970) first showed that there was a linear relation between luminosity and core mass for AGB stars and this has been confirmed by various calculations since then (Iben 1977; Wood \& Zarro 1981; Lattanzio 1986; Boothroyd \& Sackmann 1988b). As shown by Boothroyd \& Sackmann (1988b), a proper comparison of the available luminosity-core mass $\left(\mathrm{L}-\mathrm{M}_{c}^{H}\right)$ relations in the literature is not a trivial matter, although there is general agreement between the relations derived by different authors. At least that was the situation until Blöcker \& Schönberner (1991) noted that the $\mathrm{L}-\mathrm{M}_{c}^{H}$ relation may not hold for stars with $M_{i} \geq 7 \mathrm{M}_{\odot}$. These authors found that their $7 \mathrm{M}_{\odot}$ star had a luminosity well above that expected from standard luminosity-core mass relations for low mass stars and they attributed this result to the fact that their $7 \mathrm{M}_{\odot}$ star had deep envelope convection. Our $5 \mathrm{M}_{\odot}$ models have core masses similar to those of Blöcker \& Schönberner (1991) and deep envelope convection. However, our models are only slightly more luminous than the canonical $\mathrm{L}-\mathrm{M}_{c}^{H}$ relation and do not show the large over-luminosities exhibited by the Blöcker \& Schönberner (1991) models. However, there is no doubt that deep envelope convection does cause some increase in luminosity for a given core mass since the $5 \mathrm{M}_{\odot}$ sequences all show a clear decrease in luminosity with time as the envelope mass is reduced by mass loss from $\sim 4 \mathrm{M}_{\odot}$ to $\sim 1.5 \mathrm{M}_{\odot}$. At this point, a radiative layer separates the $\mathrm{H}$ burning shell from the envelope convection and the core burning luminosity becomes independent of the envelope parameters (Wood \& Zarro 1981).

\subsubsection{Interpulse Timescales and TP-AGB Lifetimes}

For each of the evolutionary sequences presented in this study, figures 2.33-2.39 show the variation in $\tau_{T P}$, the time between thermal pulses, $\mathrm{m}_{T P}$, the change in total mass between thermal pulses (in $\mathrm{M}_{\odot}$ ), and $\tau_{R}$, the recovery time defined as the time from the beginning of a $\mathrm{He}$ shell flash to the time when the $\mathrm{H}$ shell luminosity recovers to be equal to the He shell luminosity.

The lower mass $\left(M_{i} \lesssim 2 \mathrm{M}_{\odot}\right)$ sequences show the well-known initial increase in $\tau_{T P}$ as the flashes approach full amplitude, followed by the steady decline resulting from the increase in core mass. Comparing the $Z=0.001 \tau_{T P}-\mathrm{M}_{c}^{H}$ relation from Boothroyd \& Sackmann $(1988 \mathrm{c})$ with our $(M, Z)=(1.5,0.001)$ sequence, we note that our results 
have slightly larger values of $\mathrm{M}_{c}^{H}\left(\sim 0.01 \mathrm{M}_{\odot}\right)$ for a given value of $\tau_{T P}$.

The more massive models show a completely different behaviour, with $\tau_{T P}$ increasing continuously until the onset of heavy mass loss. But mass loss itself does not affect $\tau_{T P}$-it is the disappearance of deep envelope convection that causes $\tau_{T P}$ to decrease (see the 3.5 and $5 \mathrm{M}_{\odot}$ series). Note the large increase in $\tau_{R}$ at this time also, a result of the change in the nature of the surface luminosity variations detailed above. The decrease in the inter-pulse duration caused by lowering of the envelope mass clearly causes a significant decrease in the amount of mass lost per cycle during the superwind phase.

The total TP-AGB lifetime (readily seen in figures $2.33-2.39$ is largely determined by the point of onset of the superwind phase, and it changes in a rather complicated way with both $\mathrm{M}$ and $\mathrm{Z}$. For the lower mass $\left(M_{i} \lesssim 2 \mathrm{M}_{\odot}\right)$ stars, the AGB lifetime increases with decreasing metallicity due to the fact that low metallicity stars have warmer giant branches and need to evolve to higher luminosities in order to reach the superwind phase (which starts when $P \sim 500$ days). At 2.5 and $3.5 \mathrm{M}_{\odot}$, the metallicity dependence is reversed, with high metallicity sequences lasting the longest. This is due to the higher starting luminosity for lower metallicity sequences at these masses. At $5 \mathrm{M}_{\odot}$, the TP-AGB lifetime has a maximum value at intermediate $(Z=0.008)$ metallicities.

All metallicities show the same basic variation of total TP-AGB lifetime with mass. The lifetime increases from $\sim 5$ to $\sim 17 \times 10^{5}$ years as $\mathrm{M}$ increase from 1 to $2.5 \mathrm{M}_{\odot}$ but for heavier masses $\left(3.5\right.$ and $\left.5 \mathrm{M}_{\odot}\right)$ the TP-AGB lifetime drops dramatically to $\sim 2.4 \times 10^{5}$ years. This drop-off in AGB lifetimes for the mass range $2.5 \lesssim M_{i} \lesssim 5.0$ might offer an explanation for the apparent paucity of objects observed in the LMC on the upper AGB (e. g., Reid \& Mould 1985, 1991; Wood, Bessell, \& Paltoglou 1985).

A feature of particular interest regarding AGB stars with deep envelope convection is their slow rate of evolution up the AGB. It has long been known (e. g., Wood 1974) that the linear relation between AGB core mass and luminosity for low mass AGB stars implies a mean rate of evolution up the AGB of $\sim 1 \mathrm{mag}$ per $10^{6}$ years. But implicit in this result is the assumption that the energy produced by AGB stars comes from burning hydrogen in a shell at the edge of the stellar core, and that the core mass increases continuously with time due to conversion of $\mathrm{H}$ to $\mathrm{He}$. However, recall that figure 2.32 shows that the core mass for stars with deep envelope convection does not increase continuously with time. In fact, there is essentially no increase in core 
mass with time because envelope convection eats inward at each shell flash to replenish almost completely the layer that burnt its hydrogen in the preceding quiescent phase. Consequently, there is only a very slow increase in luminosity with time on the AGB for such stars. Indeed, when the mass loss rate becomes large enough to cause a significant decrease in envelope mass, upper AGB stars actually evolve down the AGB!

Finally, we note that our most massive models did not encounter the radiation pressure ejection mechanism proposed by Wood \& Faulkner (1986). The radiation pressure associated with the thermal pulses was shown by these authors to be sufficient to eject the remaining stellar envelope for $M_{c}^{H} \gtrsim 0.86 \mathrm{M}_{\odot}$ and total mass $M_{i} \lesssim 2$ $\mathrm{M}_{\odot}$. Our models of initial mass $5 \mathrm{M}_{\odot}$ satisfy these limits during their final shell flashes but they managed (with difficulty!) to hold together. The limiting core mass for the radiation pressure mechanism to operate seems to be slightly greater than that estimated by Wood \& Faulkner (1986). 
TABLE 2.9

AGB Tip Luminosities

\begin{tabular}{|c|c|c|c|c|}
\hline Point & $\mathrm{t}$ & $\log L / L_{\odot}$ & $\log T_{\text {eff }}$ & $\mathrm{M}_{\text {Total }}$ \\
\hline \multicolumn{5}{|c|}{$(M, Z)=(1.0,0.016)$} \\
\hline 1 & -10189 & 3.345 & 3.474 & 0.9999 \\
\hline 2 & 68919 & 3.149 & 3.505 & 0.9998 \\
\hline $3 \ldots \ldots$ & 275451 & 3.514 & 3.442 & 0.9951 \\
\hline \multicolumn{5}{|c|}{$(M, Z)=(1.5,0.016)$} \\
\hline 1 & -10396 & 3.393 & 3.504 & 1.5000 \\
\hline 2 & 56332 & 3.215 & 31 & 1.5000 \\
\hline $3 \ldots \ldots$ & 576263 & 3.709 & 3.451 & 1.4819 \\
\hline \multicolumn{5}{|c|}{$(M, Z)=(2.0,0.016)$} \\
\hline 1 & -11235 & 3.413 & 3.528 & 2.0000 \\
\hline $2 \ldots \ldots$ & 55749 & 3.226 & 3.551 & 2.0000 \\
\hline $3 \ldots \ldots$ & 975715 & 3.860 & 3.457 & 1.9244 \\
\hline \multicolumn{5}{|c|}{$(M, Z)=(2.5,0.016)$} \\
\hline & -13247 & 3.360 & 3.552 & 2.5000 \\
\hline $2 \ldots \ldots$ & 10697 & & 3.582 & 2.5000 \\
\hline $3 \ldots \ldots$ & 2002867 & 3.958 & 3.464 & 2.3740 \\
\hline \multicolumn{5}{|c|}{$(M, Z)=(3.5,0.016)$} \\
\hline & -3134 & 3.969 & 3.503 & 3.5000 \\
\hline 2 & 1831 & 3. & 3.528 & 3.5000 \\
\hline $3 \ldots \ldots$ & 264550 & 4.159 & 3.472 & 3.4323 \\
\hline \multicolumn{5}{|c|}{$(M, Z)=(5.0,0.016)$} \\
\hline & -904 & 4.263 & 3.496 & 5.0000 \\
\hline $2 \ldots \ldots$ & 421 & 4.168 & 3.509 & 5.0000 \\
\hline $3 \ldots \ldots$ & 135071 & 4.387 & 3.471 & 4.8463 \\
\hline
\end{tabular}


TABLE 2.9-Continued

AGB Luminosities

\begin{tabular}{|c|c|c|c|c|}
\hline Point & $\mathrm{t}$ & $\log L / L_{\odot}$ & $\log T_{\text {eff }}$ & $\mathrm{M}_{\text {Total }}$ \\
\hline \multicolumn{5}{|c|}{$(M, Z)=(0.95,0.008)$} \\
\hline 1 & -16101 & 3.228 & 3.510 & 0.7222 \\
\hline 2 & 8037 & 3.060 & 3.536 & 0.7222 \\
\hline $3 \ldots \ldots$ & 463412 & 3.469 & 3.471 & 0.6973 \\
\hline \multicolumn{5}{|c|}{$(M, Z)=(1.0,0.008)$} \\
\hline 1 & -9695 & 3.350 & 3.508 & 1.0000 \\
\hline & 4292 & 3.129 & 3.541 & 1.0000 \\
\hline $3 \ldots \ldots$ & 436776 & 3.589 & 3.466 & 0.9903 \\
\hline \multicolumn{5}{|c|}{$(M, Z)=(1.5,0.008)$} \\
\hline 1. & -6420 & 3.483 & 3.523 & 1.5000 \\
\hline 2 . & 6767 & 3.216 & 3.560 & 1.5000 \\
\hline & 777627 & 3.802 & 3.468 & 1.4369 \\
\hline \multicolumn{5}{|c|}{$(M, Z)=(2.0,0.008)$} \\
\hline 1. & -11021 & 3.521 & 3.543 & 2.0000 \\
\hline 2 . & 9211 & 3.249 & 3.577 & 2.0000 \\
\hline $3 \ldots \ldots$ & 1112609 & 3.928 & 3.482 & 1.9563 \\
\hline \multicolumn{5}{|c|}{$(M, Z)=(2.5,0.008)$} \\
\hline & -8700 & 3.488 & 3.565 & 2.5000 \\
\hline $2 \ldots \ldots$ & 6674 & 3.252 & 3.593 & 2.5000 \\
\hline $3 \ldots \ldots$ & 1646394 & 4.027 & 3.479 & 2.3914 \\
\hline \multicolumn{5}{|c|}{$(M, Z)=(3.5,0.008)$} \\
\hline 1 & -2549 & 4.077 & 3.520 & 3.5000 \\
\hline 2 . & 1125 & 3.935 & 3.539 & 3.5000 \\
\hline 3. & 237655 & 4.235 & 3.486 & 3.3396 \\
\hline \multicolumn{5}{|c|}{$(M, Z)=(5.0,0.008)$} \\
\hline 1. & -954 & 4.280 & 3.525 & 5.0000 \\
\hline $2 \ldots \ldots$ & 293 & 4.201 & 3.536 & 5.0000 \\
\hline $3 \ldots \ldots$ & 213525 & 4.454 & 3.493 & 4.9309 \\
\hline
\end{tabular}


TABLE 2.9-Continued

AGB Luminosities

\begin{tabular}{|c|c|c|c|c|}
\hline Point & $\mathrm{t}$ & $\log L / L_{\odot}$ & $\log T_{\text {eff }}$ & $\mathrm{M}_{\text {Total }}$ \\
\hline \multicolumn{5}{|c|}{$(M, Z)=(0.89,0.004)$} \\
\hline 1 . & -16761 & 3.189 & 3.549 & 0.6909 \\
\hline & 4152 & 3.041 & 3.571 & 0.6909 \\
\hline $3 \ldots \ldots$ & 628585 & 3.492 & 3.508 & 0.6817 \\
\hline \multicolumn{5}{|c|}{$(M, Z)=(1.0,0.004)$} \\
\hline $1 \ldots \ldots$ & -9059 & 3.388 & 3.536 & 1.0000 \\
\hline $2 \ldots \ldots$ & 11885 & 3.134 & 3.570 & 1.0000 \\
\hline $3 \ldots \ldots$ & 712060 & 3.685 & 3.489 & 0.9677 \\
\hline \multicolumn{5}{|c|}{$(M, Z)=(1.5,0.004)$} \\
\hline $1 \ldots \ldots$ & -8837 & 3.498 & 3.554 & 1.5000 \\
\hline $2 \ldots \ldots$ & 6008 & 3.265 & 3.582 & 1.5000 \\
\hline $3 \ldots \ldots$ & 848930 & 3.864 & 3.495 & 1.3979 \\
\hline \multicolumn{5}{|c|}{$(M, Z)=(2.0,0.004)$} \\
\hline & -9436 & 3.457 & 3.581 & 2.0000 \\
\hline $2 \ldots \ldots$ & 2955 & 3.263 & 3.602 & 2.0000 \\
\hline$\ldots$ & 1412224 & 3.997 & 3.501 & 1.9255 \\
\hline \multicolumn{5}{|c|}{$(M, Z)=(2.5,0.004)$} \\
\hline$\cdots$ & -5842 & 3.717 & 3.568 & 2.5000 \\
\hline & 3997 & 3.511 & 3.592 & 2.5000 \\
\hline 3 . & 1119559 & 4.083 & 3.503 & 2.3807 \\
\hline \multicolumn{5}{|c|}{$(M, Z)=(3.5,0.004)$} \\
\hline 1 & -1418 & 4.182 & 3.537 & 3.5000 \\
\hline$\ldots \ldots$ & 659 & 4.078 & 3.550 & 3.5000 \\
\hline $3 \ldots \ldots$ & 146616 & 4.312 & 3.511 & 3.4256 \\
\hline \multicolumn{5}{|c|}{$(M, Z)=(5.0,0.004)$} \\
\hline & -1046 & 4.300 & 3.553 & 5.0000 \\
\hline & 191 & 4.242 & 3.560 & 5.0000 \\
\hline $3 \ldots \ldots$ & 203636 & 4.484 & 3.520 & 4.9094 \\
\hline
\end{tabular}


TABLE 2.9-Continued

AGB Luminosities

\begin{tabular}{lrrrr}
\hline \hline Point & $\mathrm{t}$ & $\log L / L_{\odot}$ & $\log T_{\text {eff }}$ & $\mathrm{M}_{\text {Total }}$ \\
\hline \multicolumn{5}{c}{$(M, Z)=(1.0,0.001)$} \\
\hline $1 \ldots \ldots$. & -15369 & 3.265 & 3.611 & 1.0000 \\
$2 \ldots \ldots \ldots$ & 87049 & 3.141 & 3.621 & 1.0000 \\
$3 \ldots \ldots$. & 1197920 & 3.774 & 3.557 & 0.9532 \\
\hline \multicolumn{5}{c}{$(M, Z)=(1.5,0.001)$} \\
\hline $1 \ldots \ldots$. & -7982 & 3.476 & 3.613 & 1.5000 \\
$2 \ldots \ldots$ & 3677 & 3.319 & 3.625 & 1.5000 \\
$3 \ldots \ldots$ & 1059832 & 3.918 & 3.601 & 1.3987 \\
\hline
\end{tabular}

Notes-1. Maximum luminosity reached during the E-AGB phase; 2. Minimum luminosity reached during the TP-AGB phase; 3 . Maximum queiscent luminosity reached during the TP-AGB phase before the onset of the superwind. 


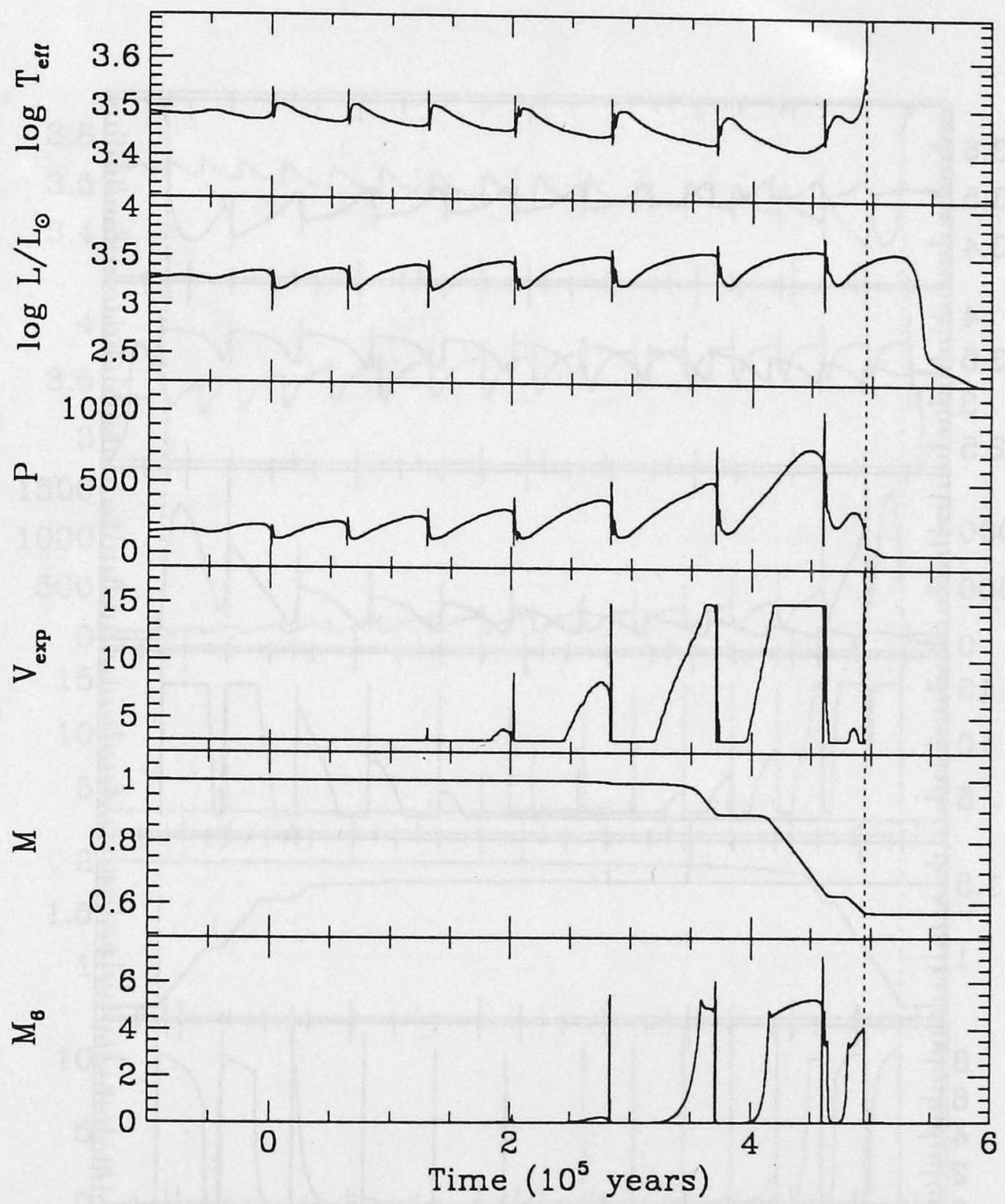

Figure 2.10: Time dependence of effective temperature, luminosity, pulsation period (days), wind expansion velocity $\left(\mathrm{km} \mathrm{s}^{-1}\right)$, mass $\left(\mathrm{M}_{\odot}\right)$, mass loss rate $\left(\mathrm{M}_{6}\right.$ : in units of $\left.10^{-6} \mathrm{M}_{\odot} \mathrm{yr}^{-1}\right)$ during the TP-AGB phase of a star with $(M, Y, Z)=(1.0,0.25,0.016)$. The abscissa represents the time after the first major thermal pulse. The dotted vertical line at right represents the end of the AGB phase, as defined in the text. 


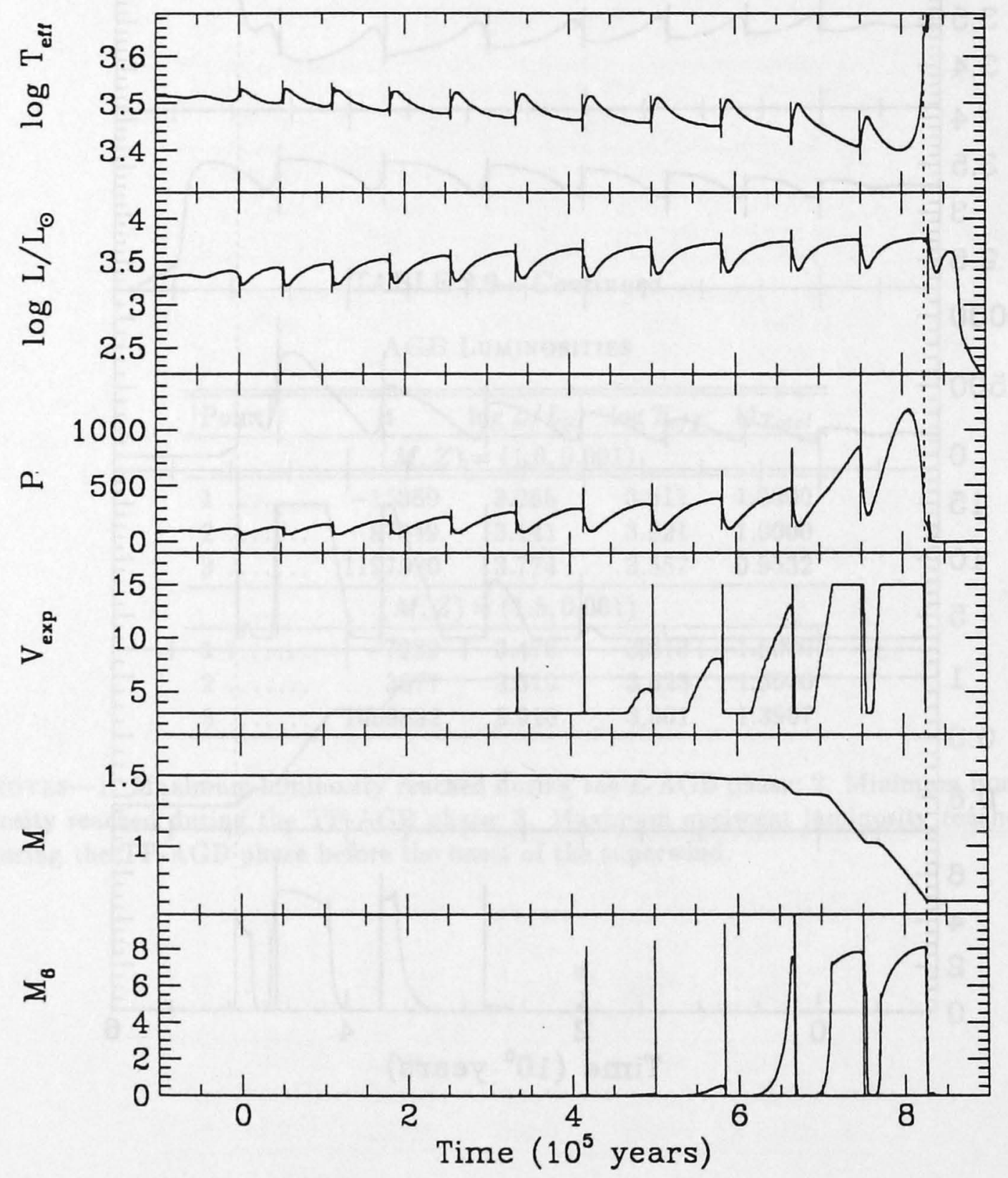

Figure 2.11: Same as figure 2.10, except for $(M, Y, Z)=(1.5,0.25,0.016)$. 


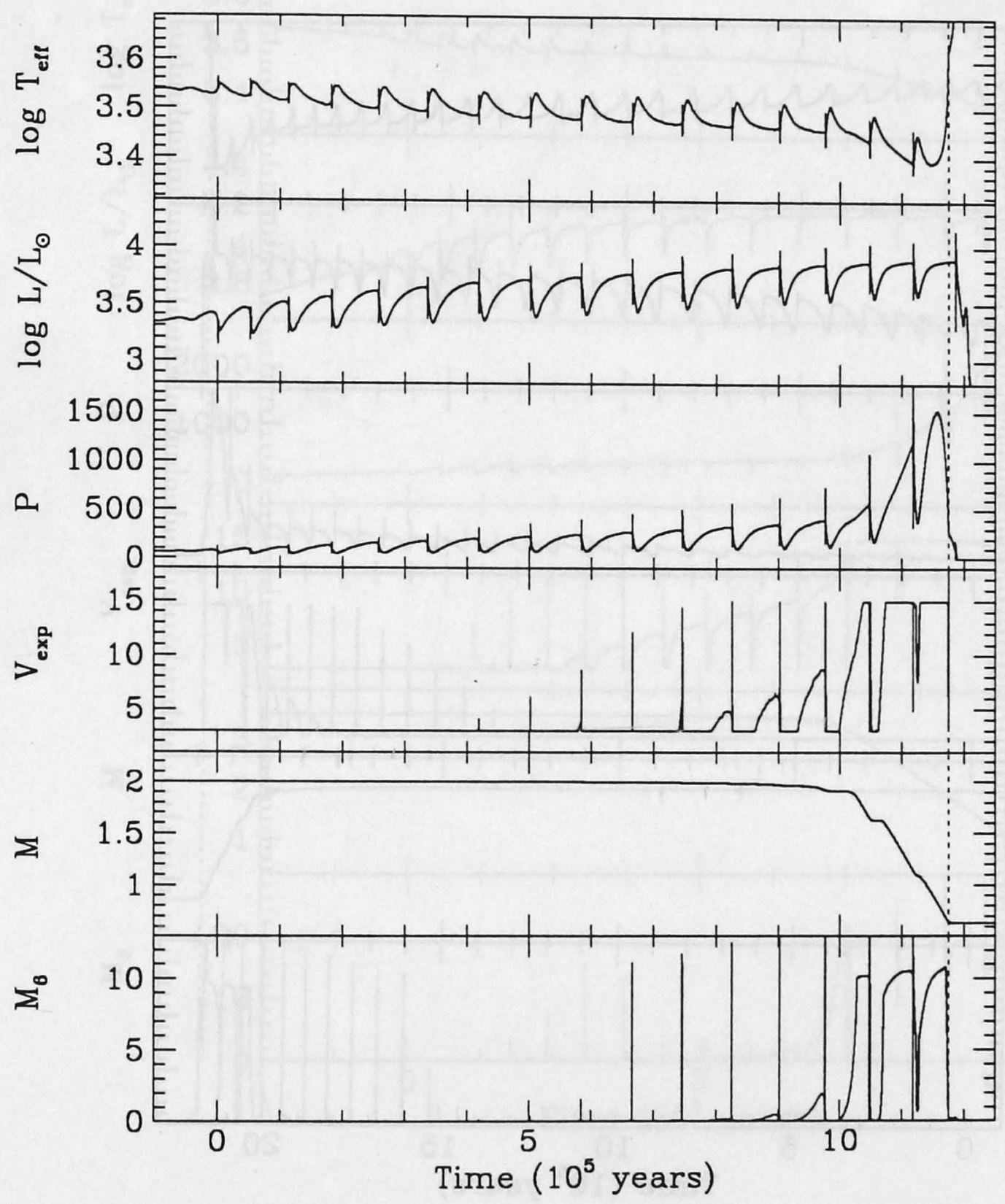

Figure 2.12: Same as figure 2.10, except for $(M, Y, Z)=(2.0,0.25,0.016)$. 


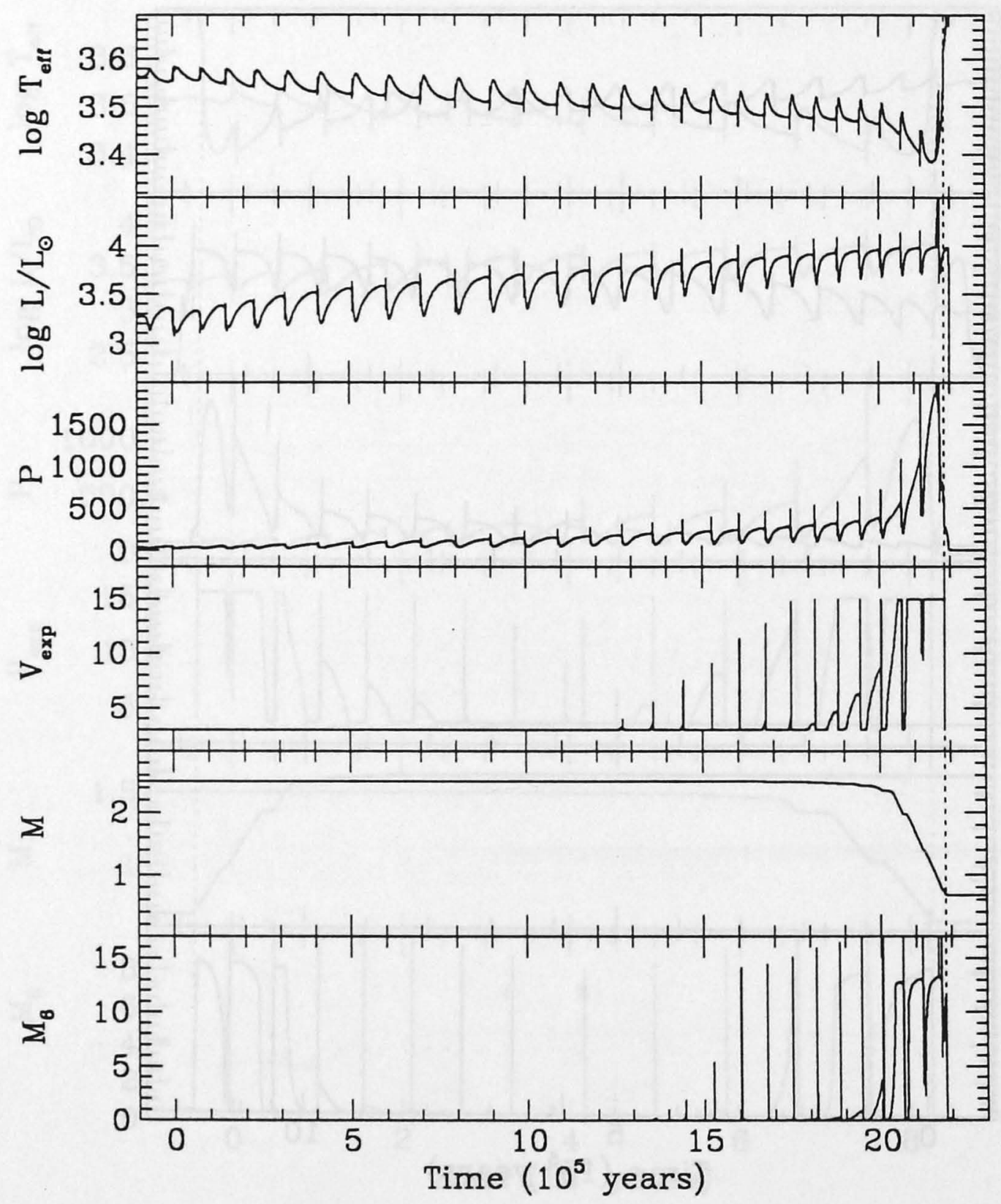

Figure 2.13: Same as figure 2.10, except for $(M, Y, Z)=(2.5,0.25,0.016)$. 


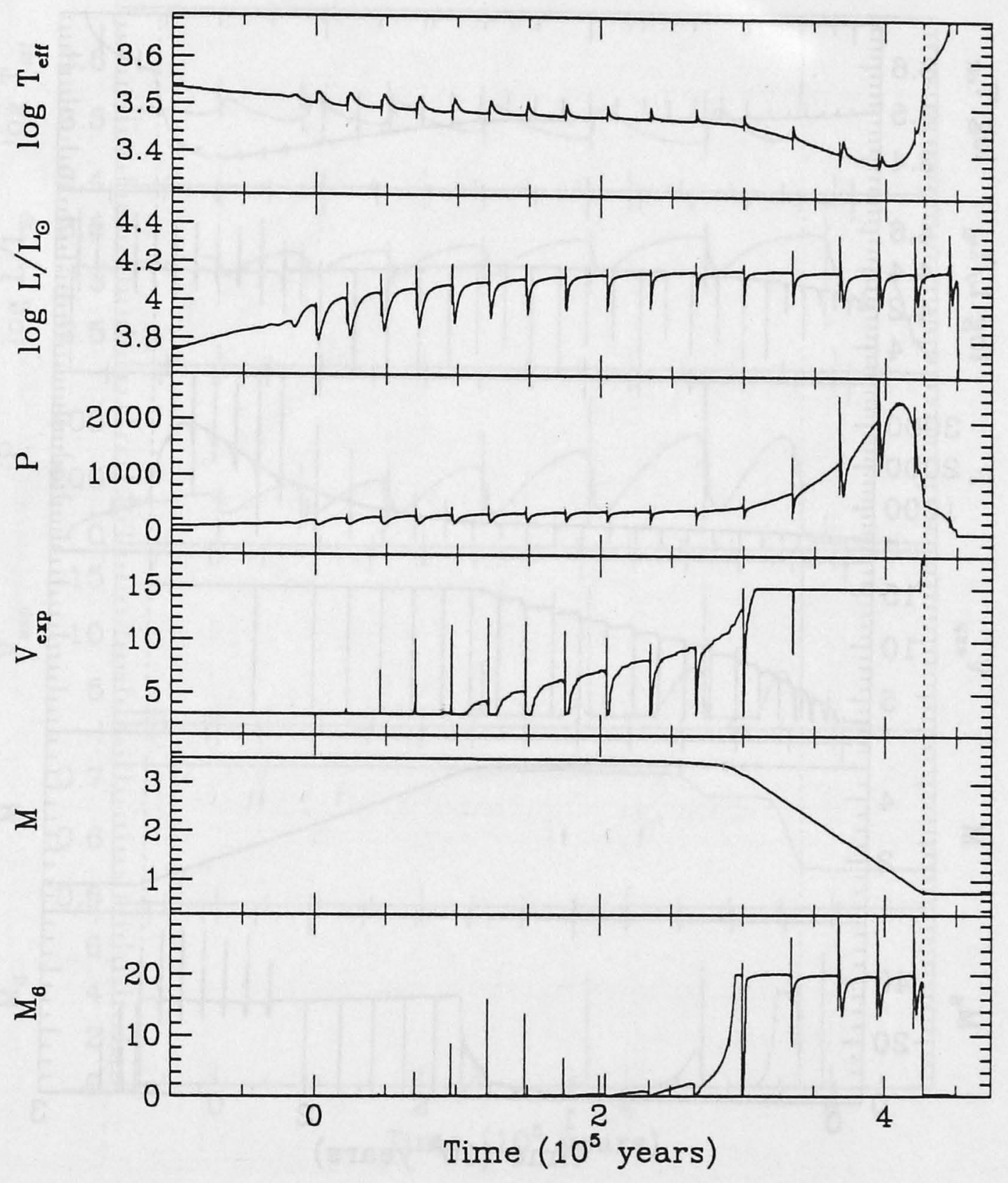

Figure 2.14: Same as figure 2.10, except for $(M, Y, Z)=(3.5,0.25,0.016)$. 


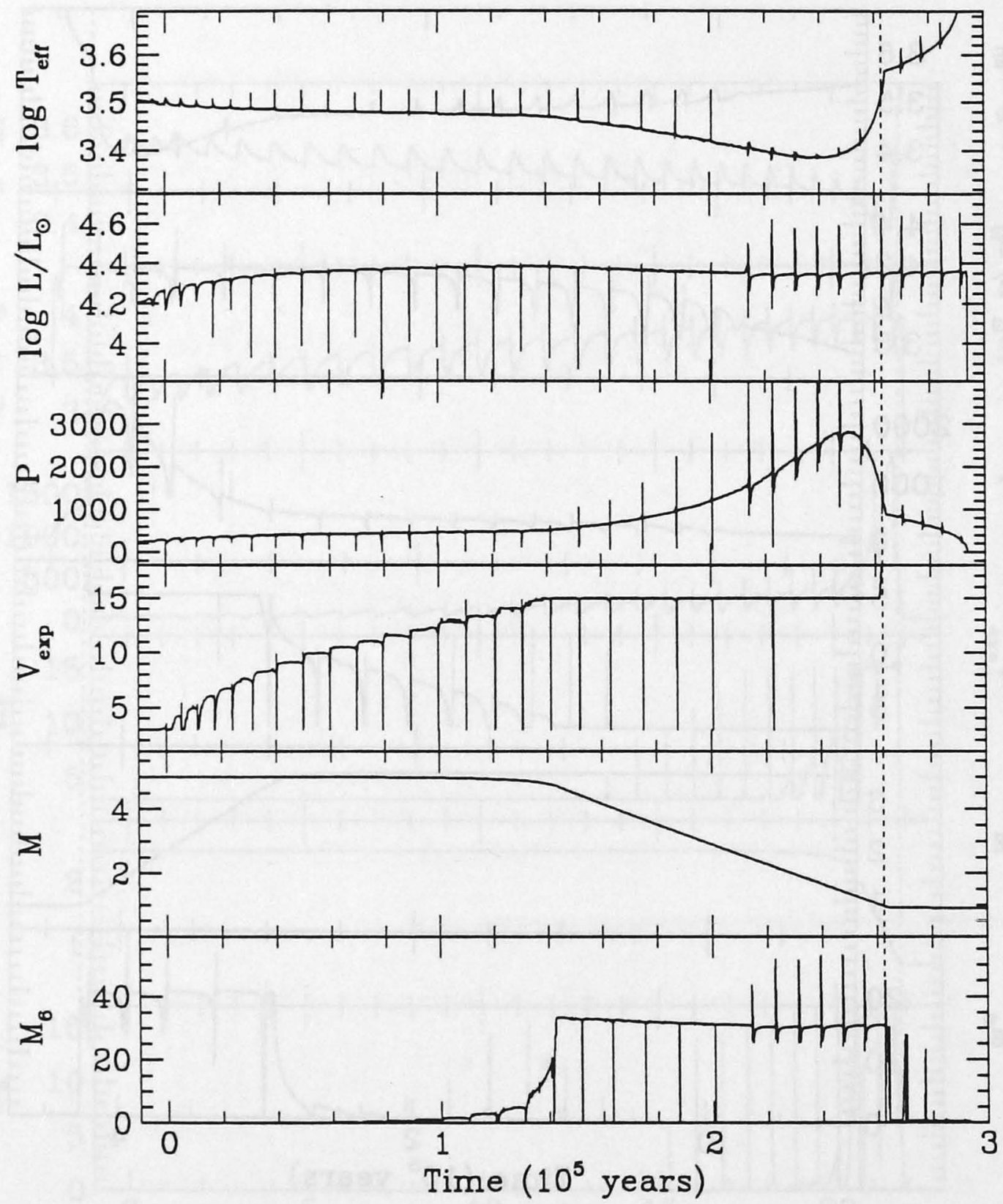

Figure 2.15: Same as figure 2.10, except for $(M, Y, Z)=(5.0,0.25,0.016)$. 


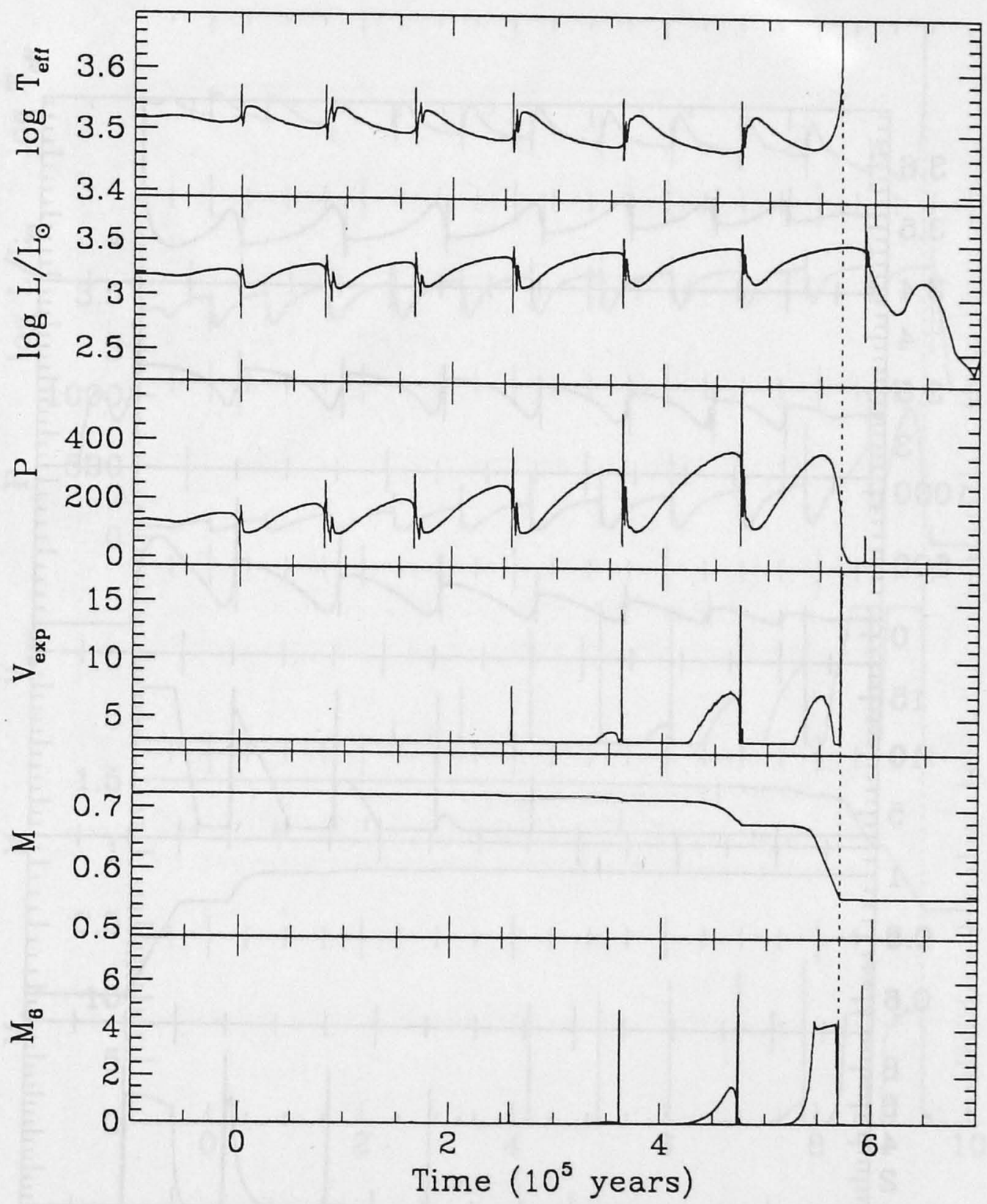

Figure 2.16: Same as figure 2.10, except for $(M, Y, Z)=(0.945,0.25,0.008)$. 


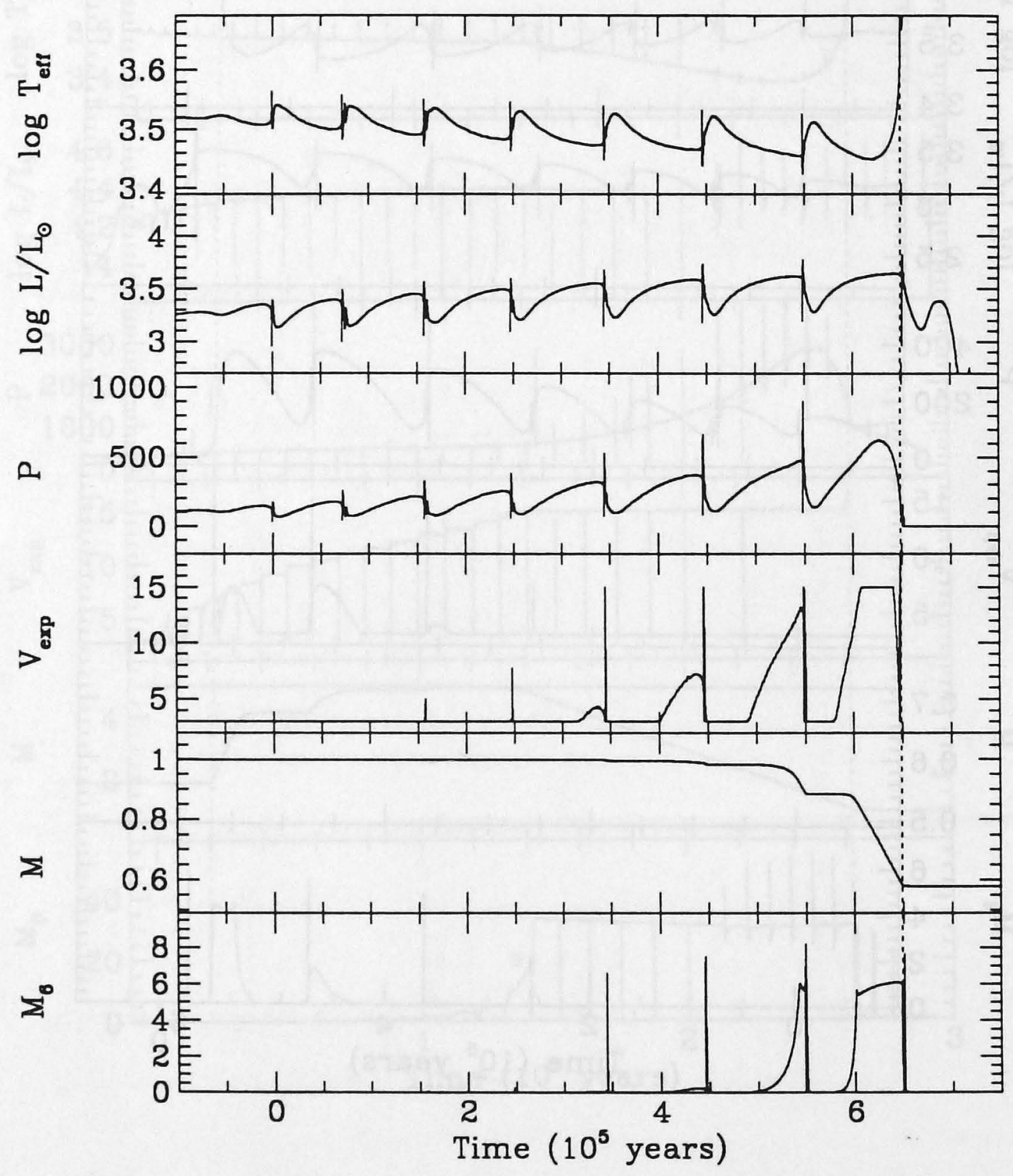

Figure 2.17: Same as figure 2.10, except for $(M, Y, Z)=(1.0,0.25,0.008)$. 


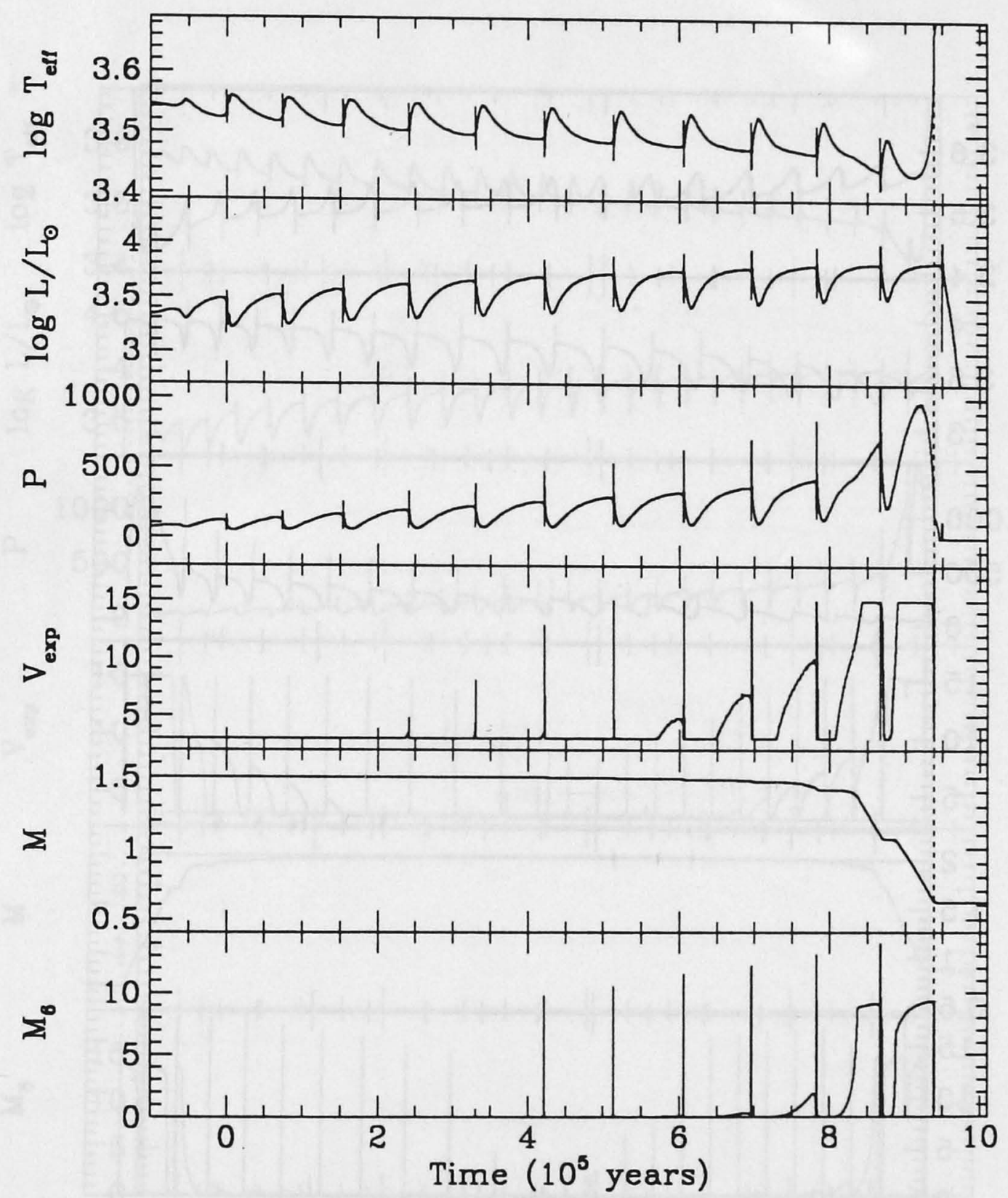

Figure 2.18: Same as figure 2.10, except for $(M, Y, Z)=(1.5,0.25,0.008)$. 


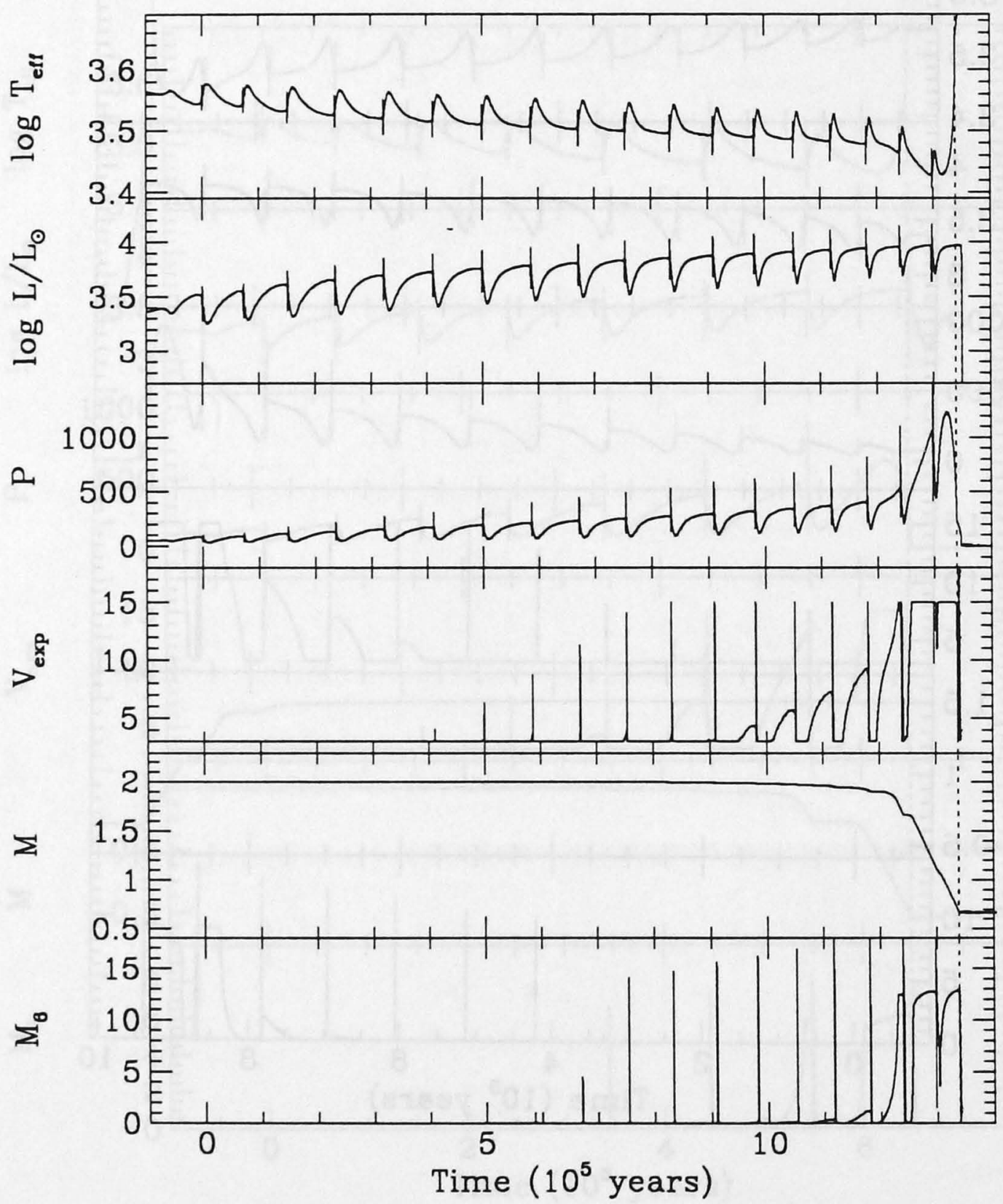

Figure 2.19: Same as figure 2.10, except for $(M, Y, Z)=(2.0,0.25,0.008)$. 


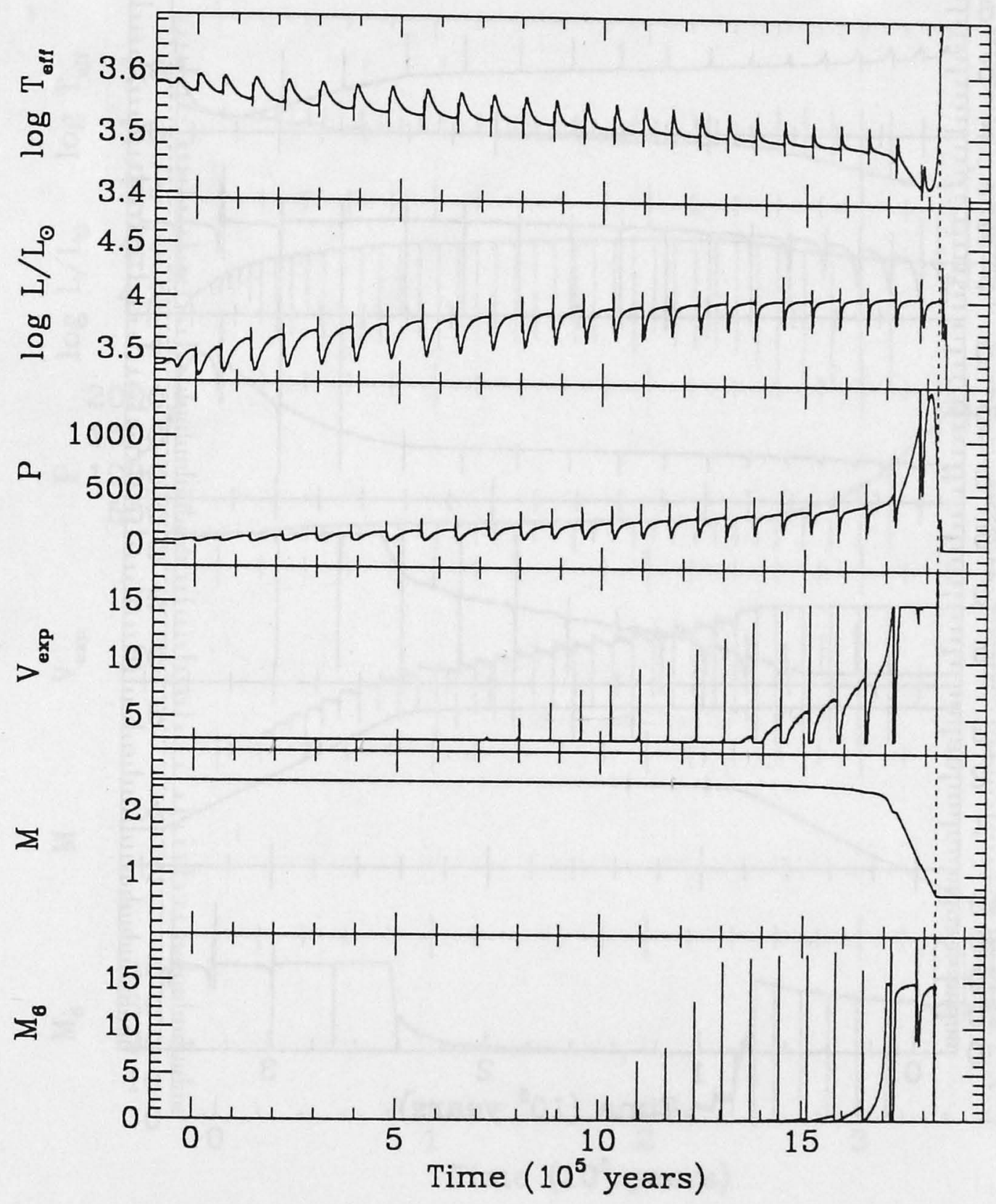

Figure 2.20: Same as figure 2.10, except for $(M, Y, Z)=(2.5,0.25,0.008)$. 


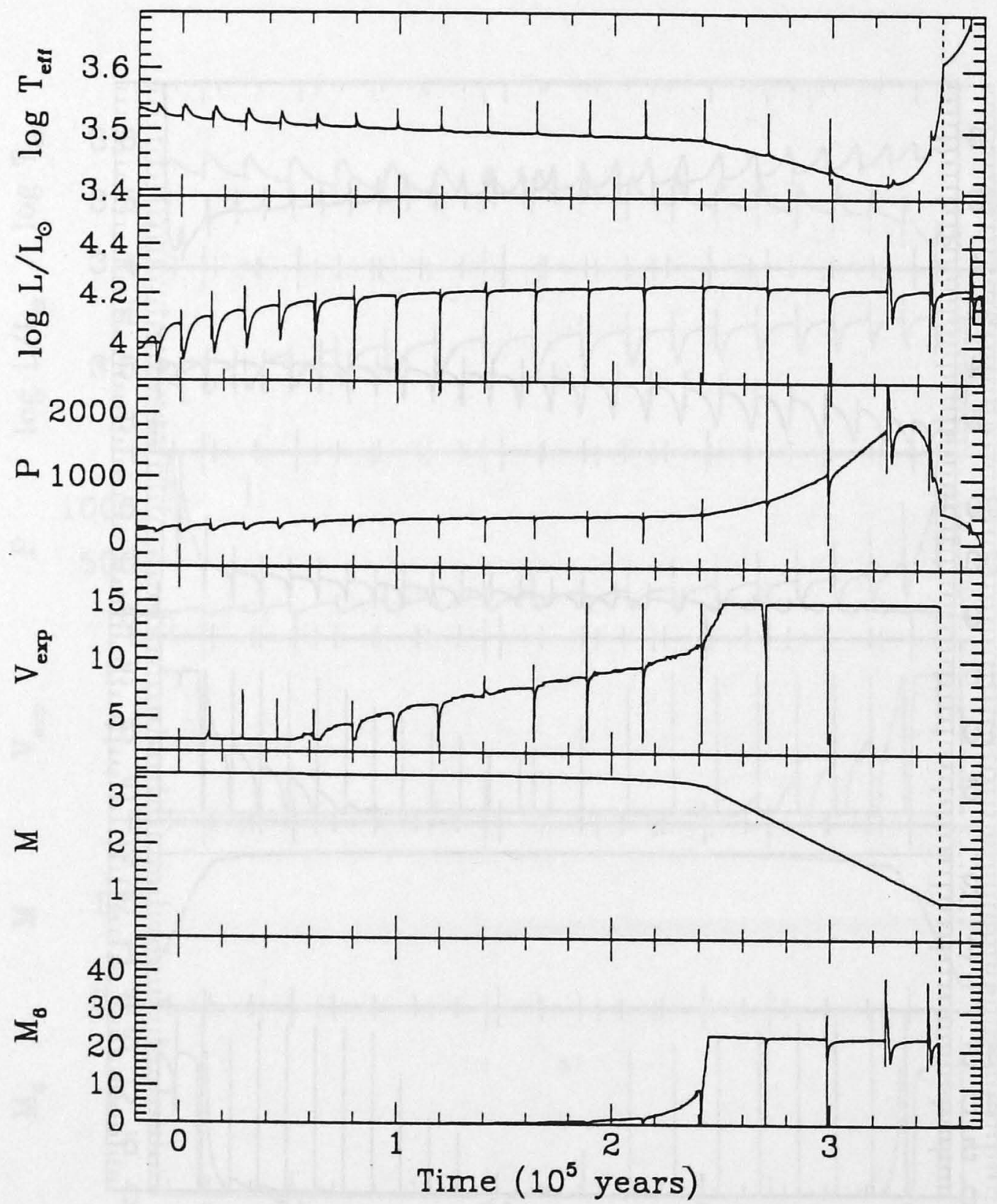

Figure 2.21: Same as figure 2.10, except for $(M, Y, Z)=(3.5,0.25,0.008)$. 


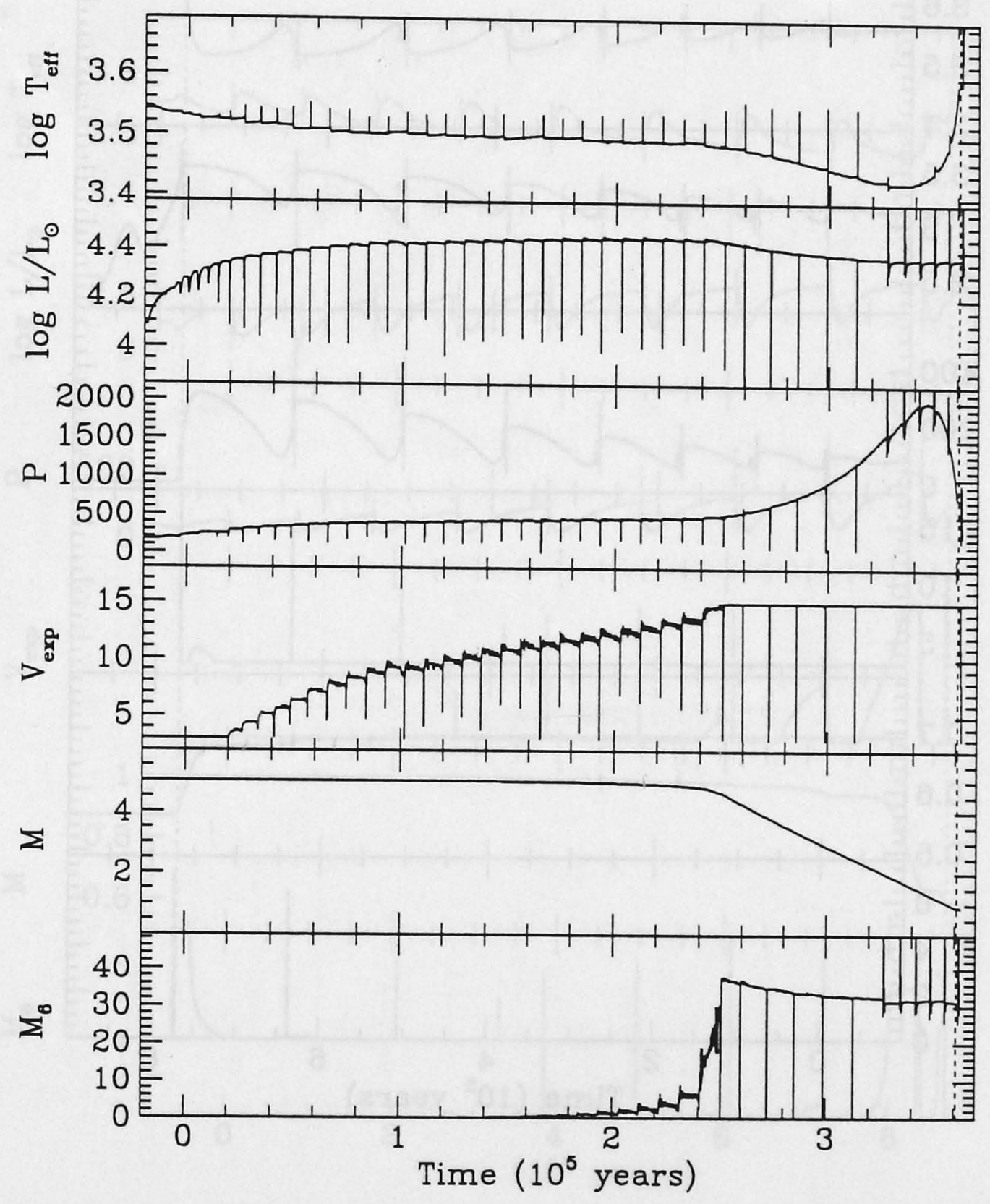

Figure 2.22: Same as figure 2.10, except for $(M, Y, Z)=(5.0,0.25,0.008)$. 


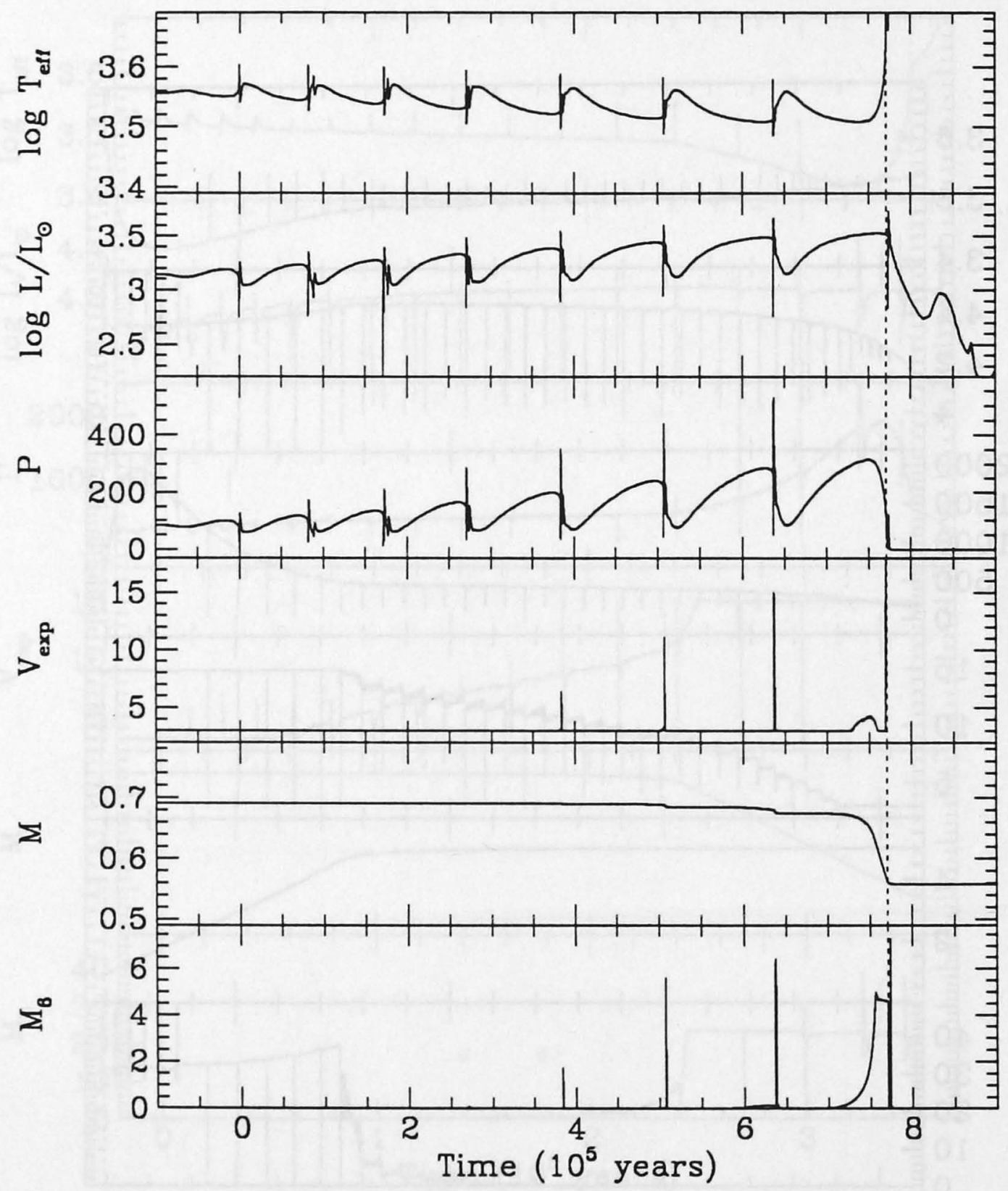

Figure 2.23: Same as figure 2.10, except for $(M, Y, Z)=(0.89,0.25,0.004)$. 


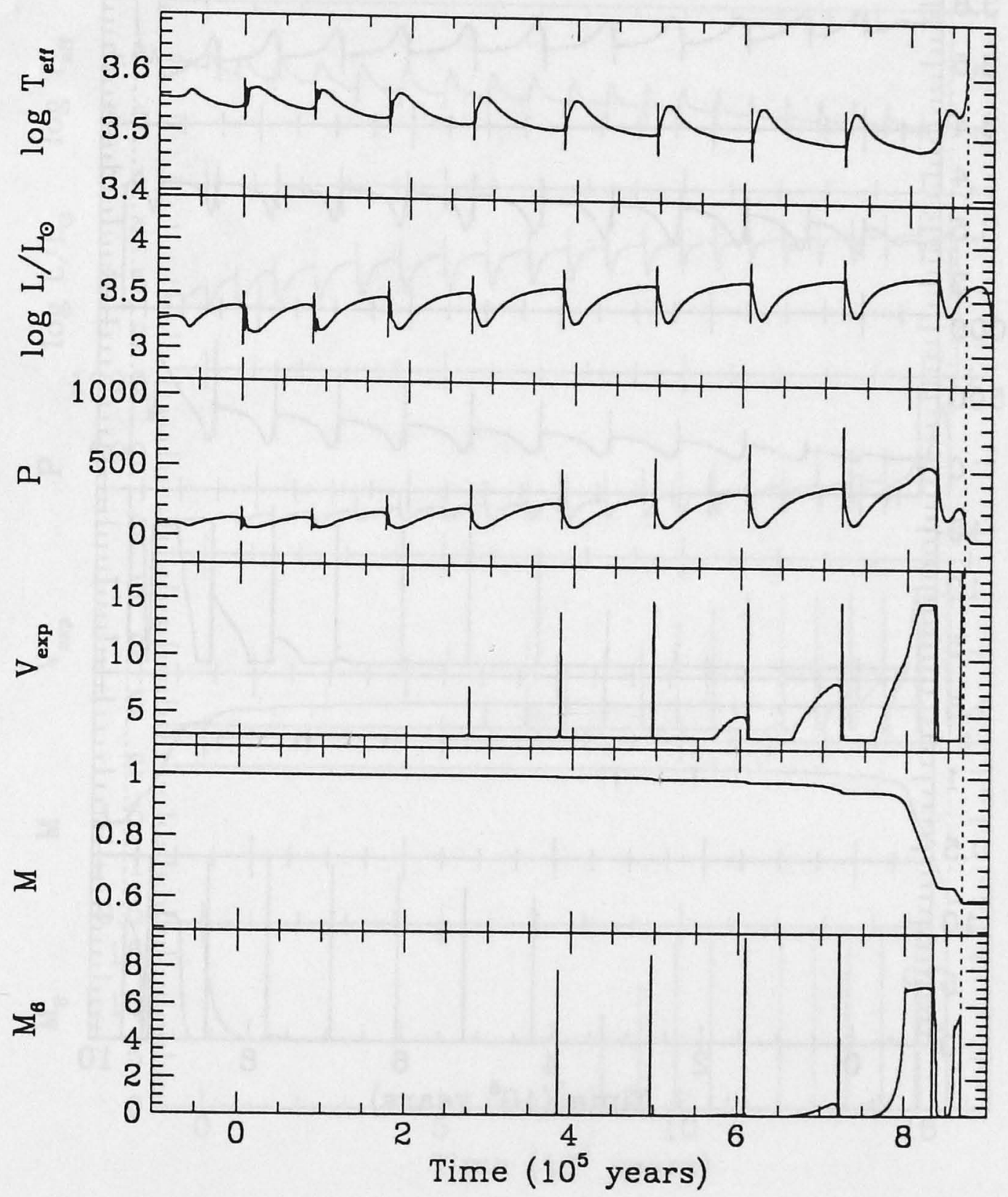

Figure 2.24: Same as figure 2.10, except for $(M, Y, Z)=(1.0,0.25,0.004)$. 


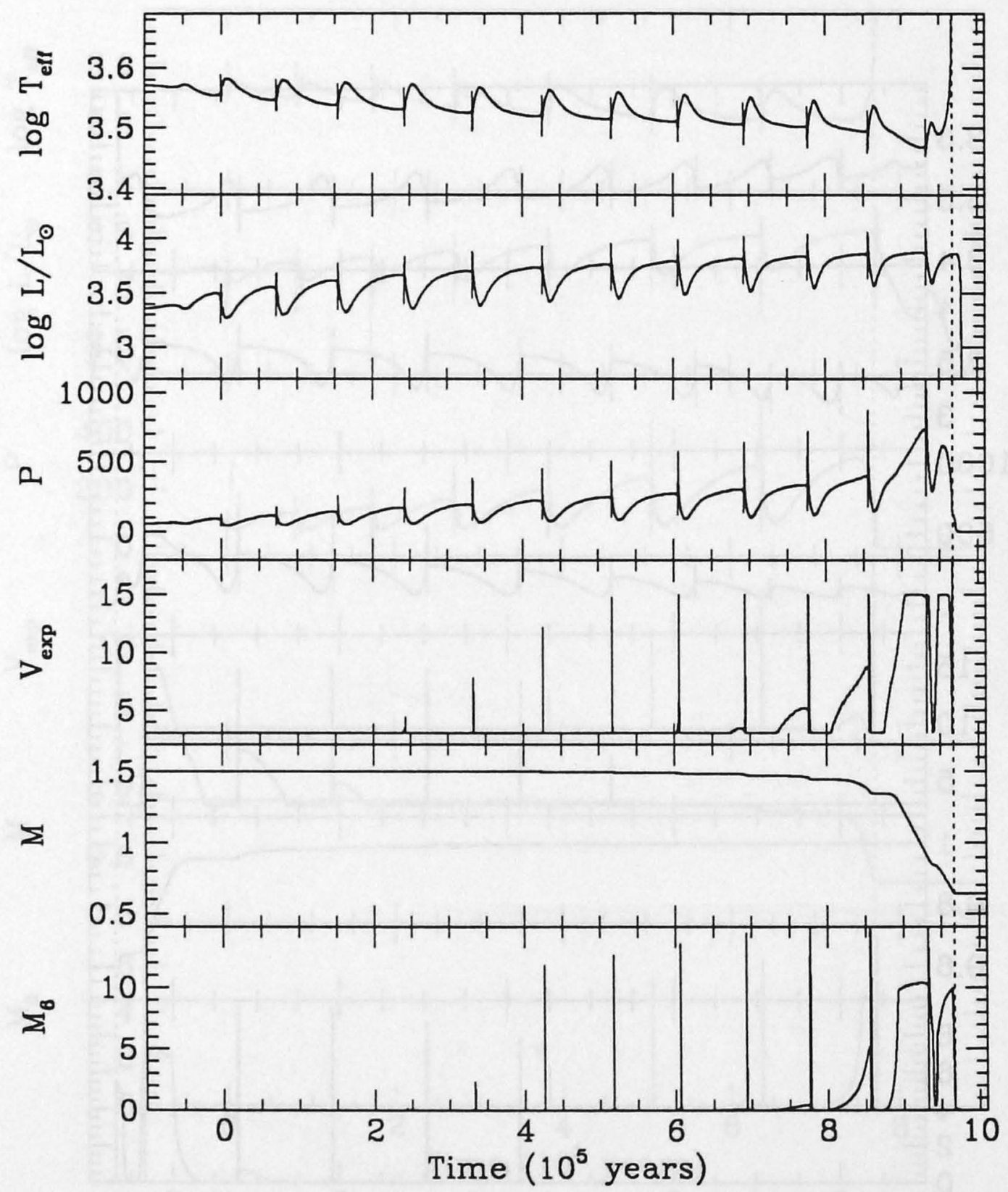

Figure 2.25: Same as figure 2.10 , except for $(M, Y, Z)=(1.5,0.25,0.004)$. 


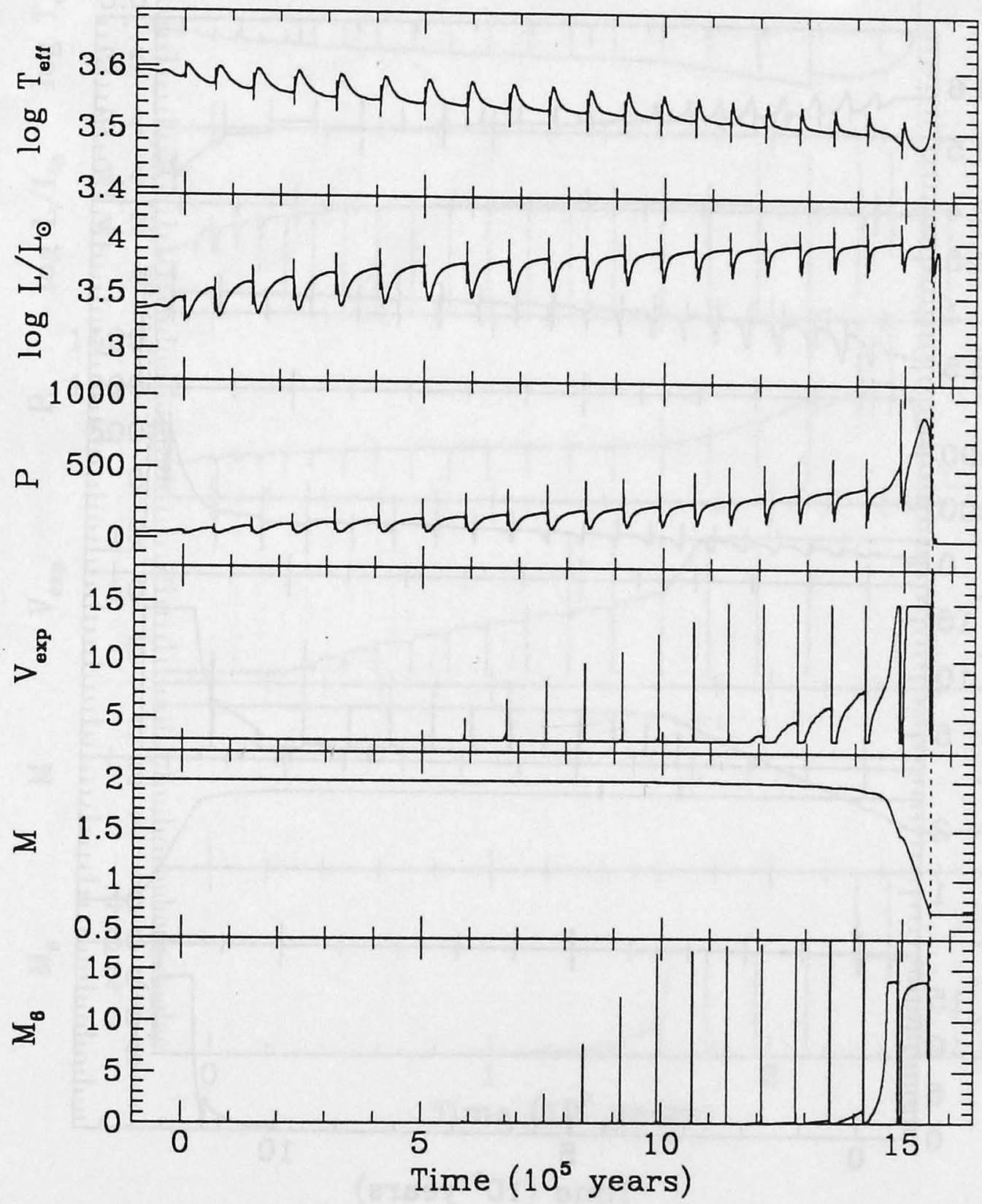

Figure 2.26: Same as figure 2.10, except for $(M, Y, Z)=(2.0,0.25,0.004)$. 


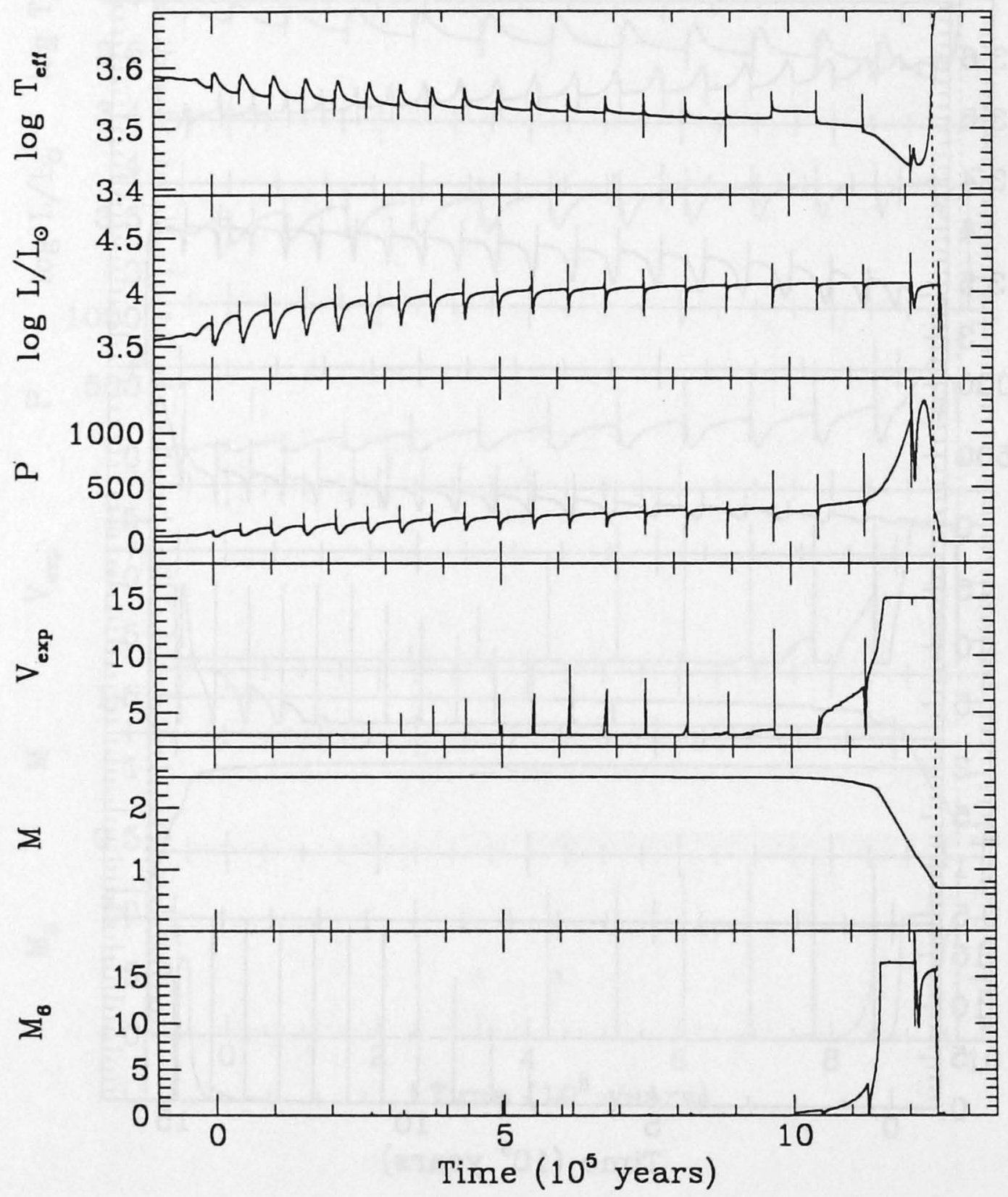

Figure 2.27: Same as figure 2.10, except for $(M, Y, Z)=(2.5,0.25,0.004)$. 


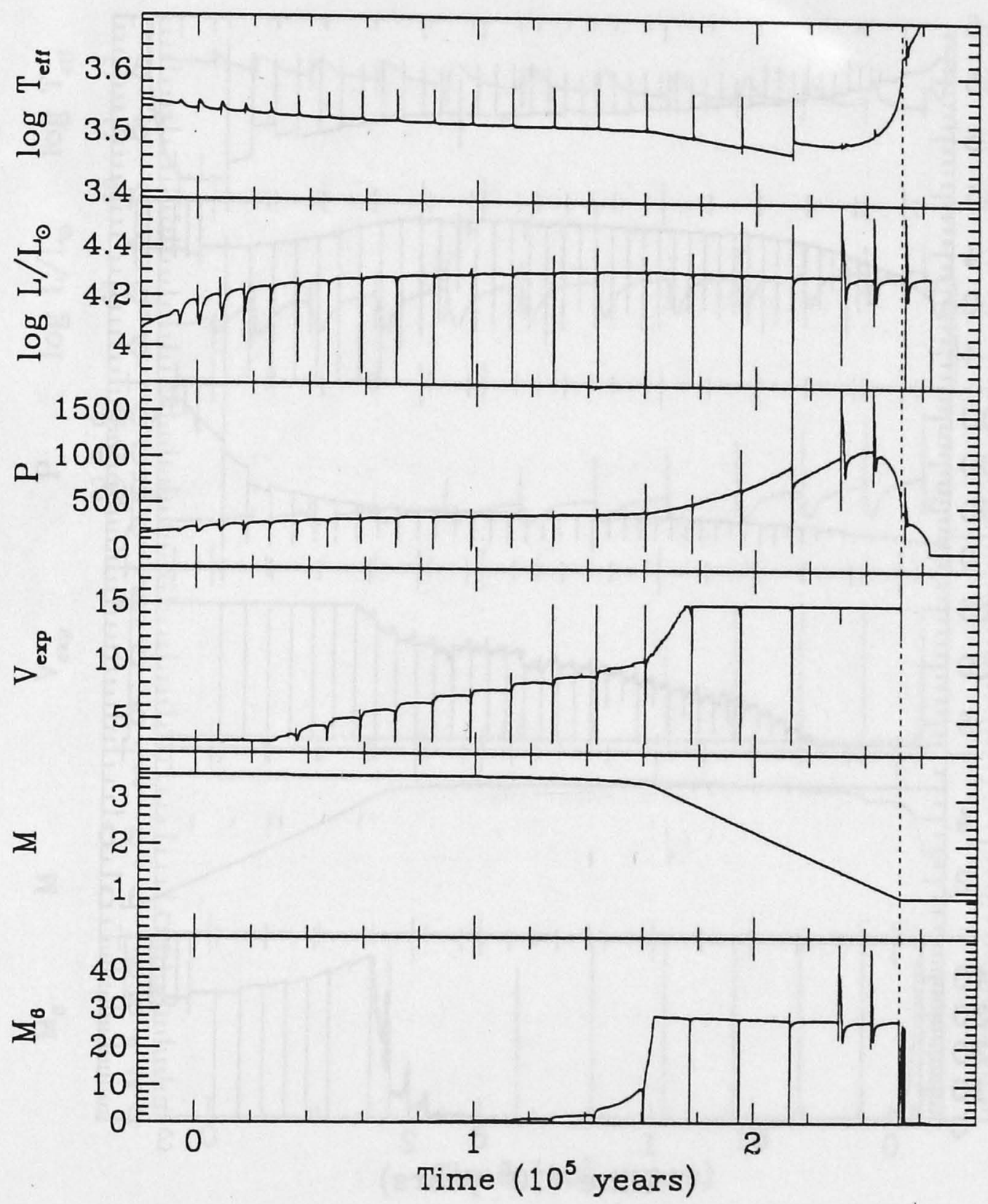

Figure 2.28: Same as figure 2.10, except for $(M, Y, Z)=(3.5,0.25,0.004)$. 


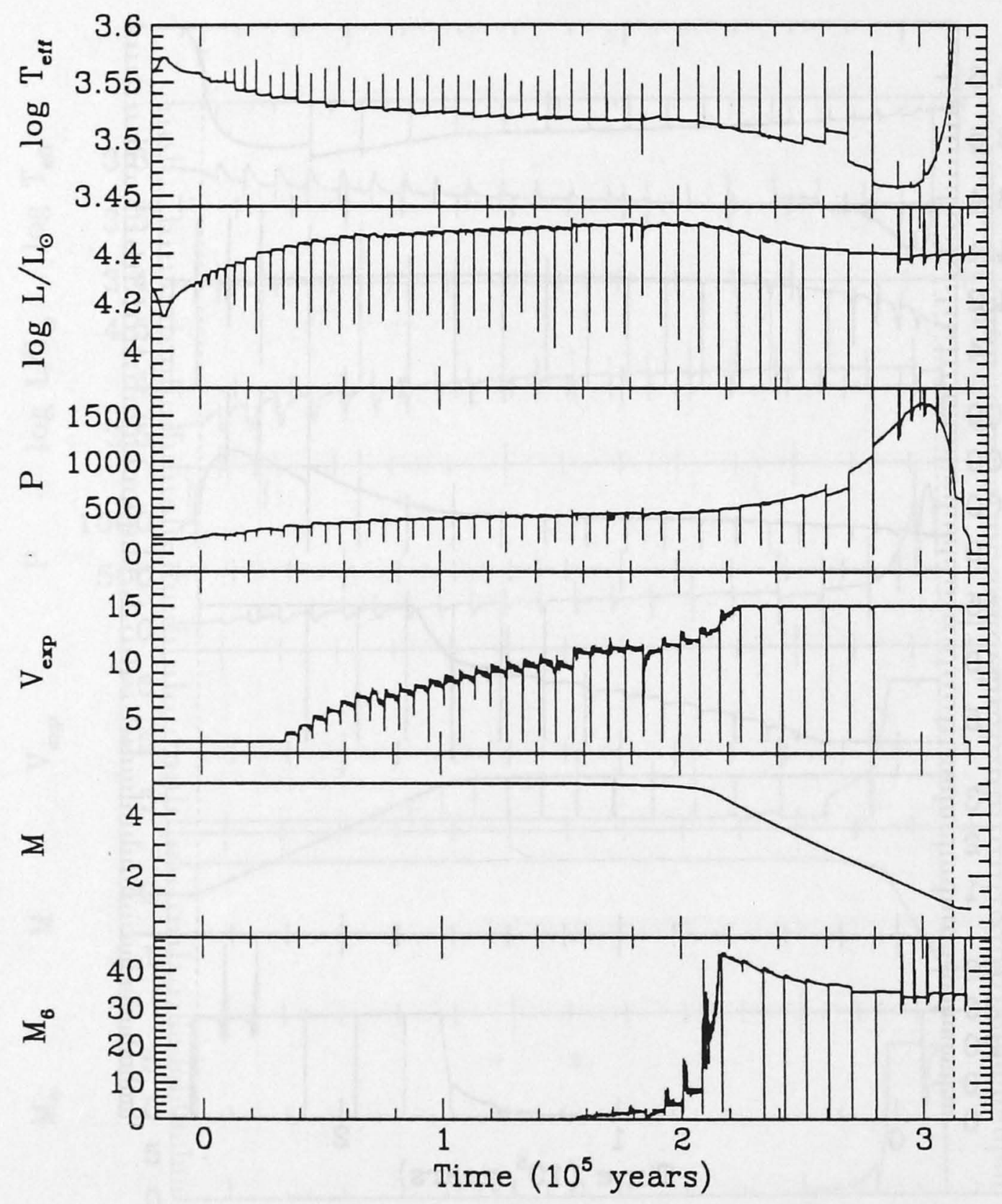

Figure 2.29: Same as figure 2.10, except for $(M, Y, Z)=(5.0,0.25,0.004)$. 


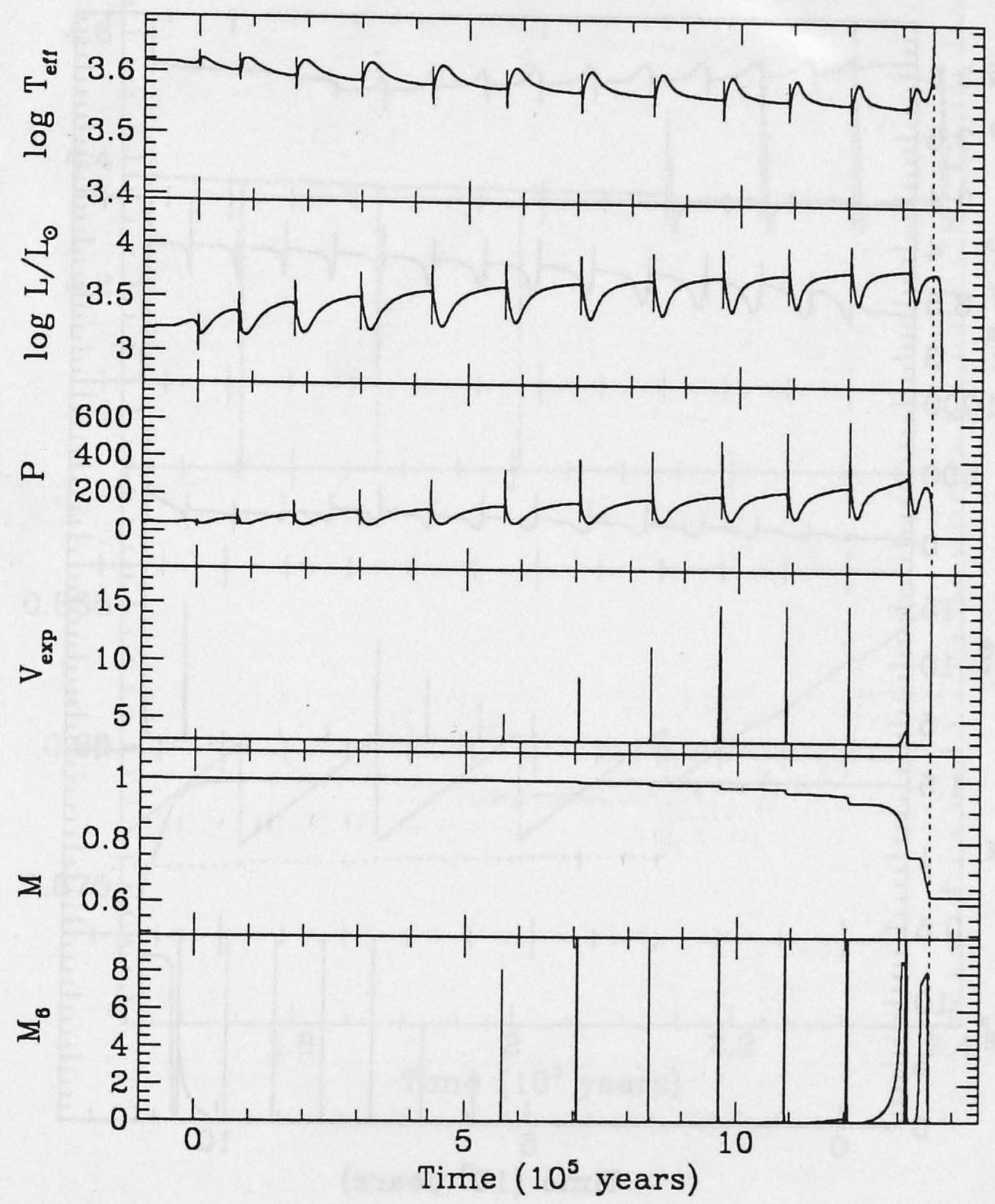

Figure 2.30: Same as figure 2.10, except for $(M, Y, Z)=(1.0,0.25,0.001)$. 


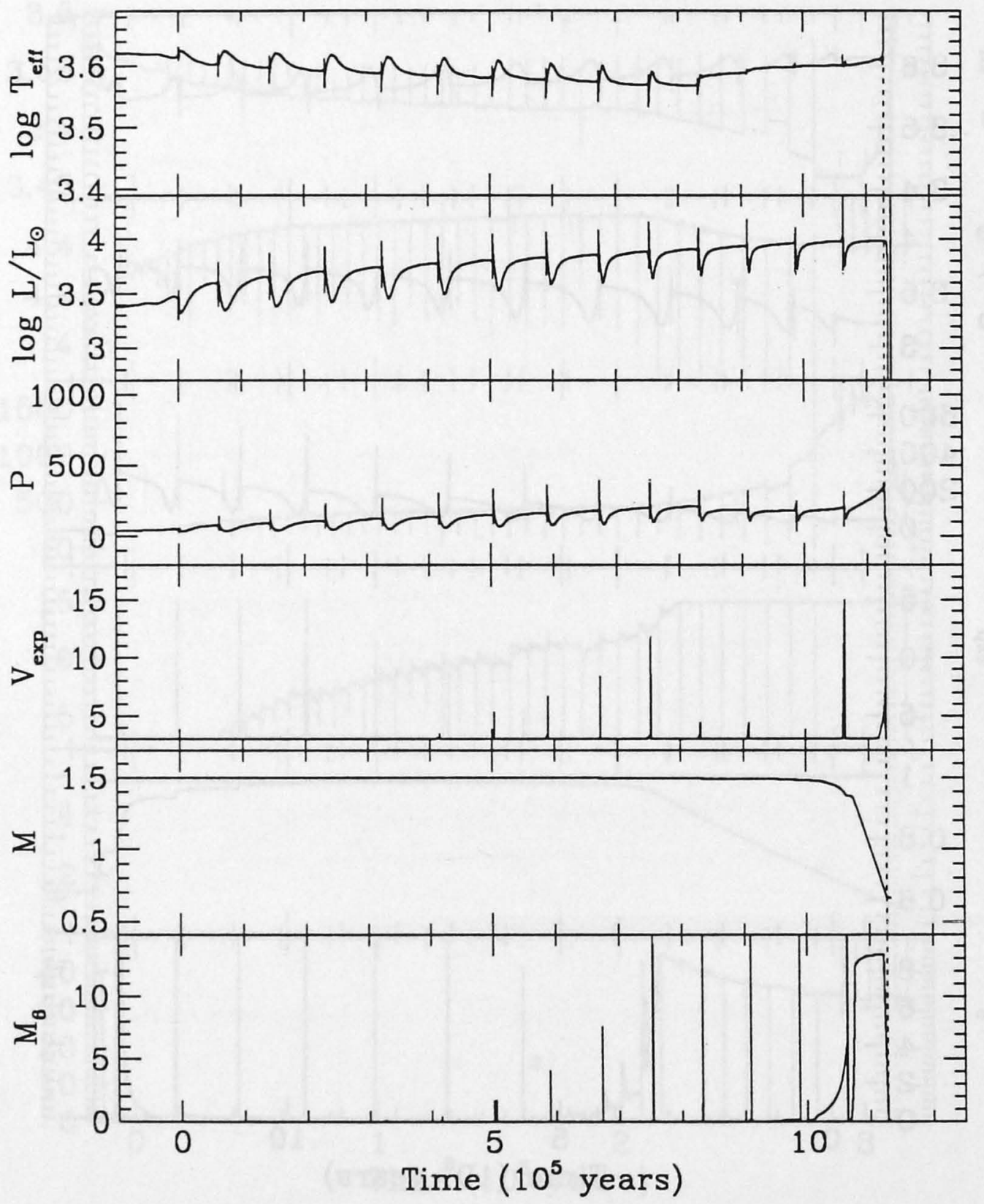

Figure 2.31: Same as figure 2.10, except for $(M, Y, Z)=(1.5,0.25,0.001)$. 


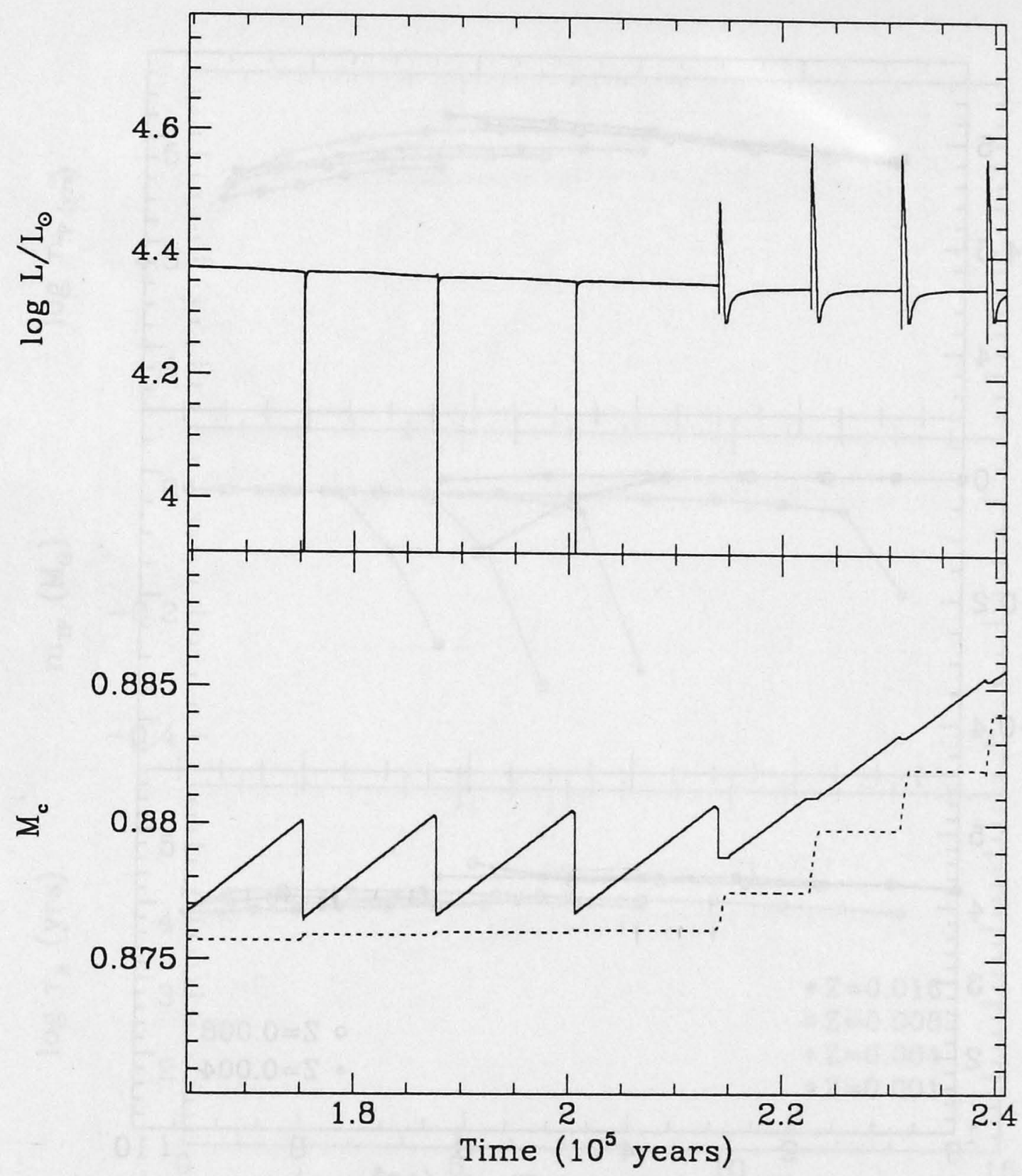

Figure 2.32: The time dependence of surface luminosity (top panel) and core mass (lower panel) for the $(M, Y, Z)=(5.0,0.25,0.016)$ evolutionary sequence. In the lower panel, the solid line represents the hydrogen core mass and the dotted line represents the helium core mass. The change in flash cycle characteristics with time is due to the decrease in envelope mass. The abscissa represents the time since the first thermal pulse and the first pulse shown is the 22nd. 


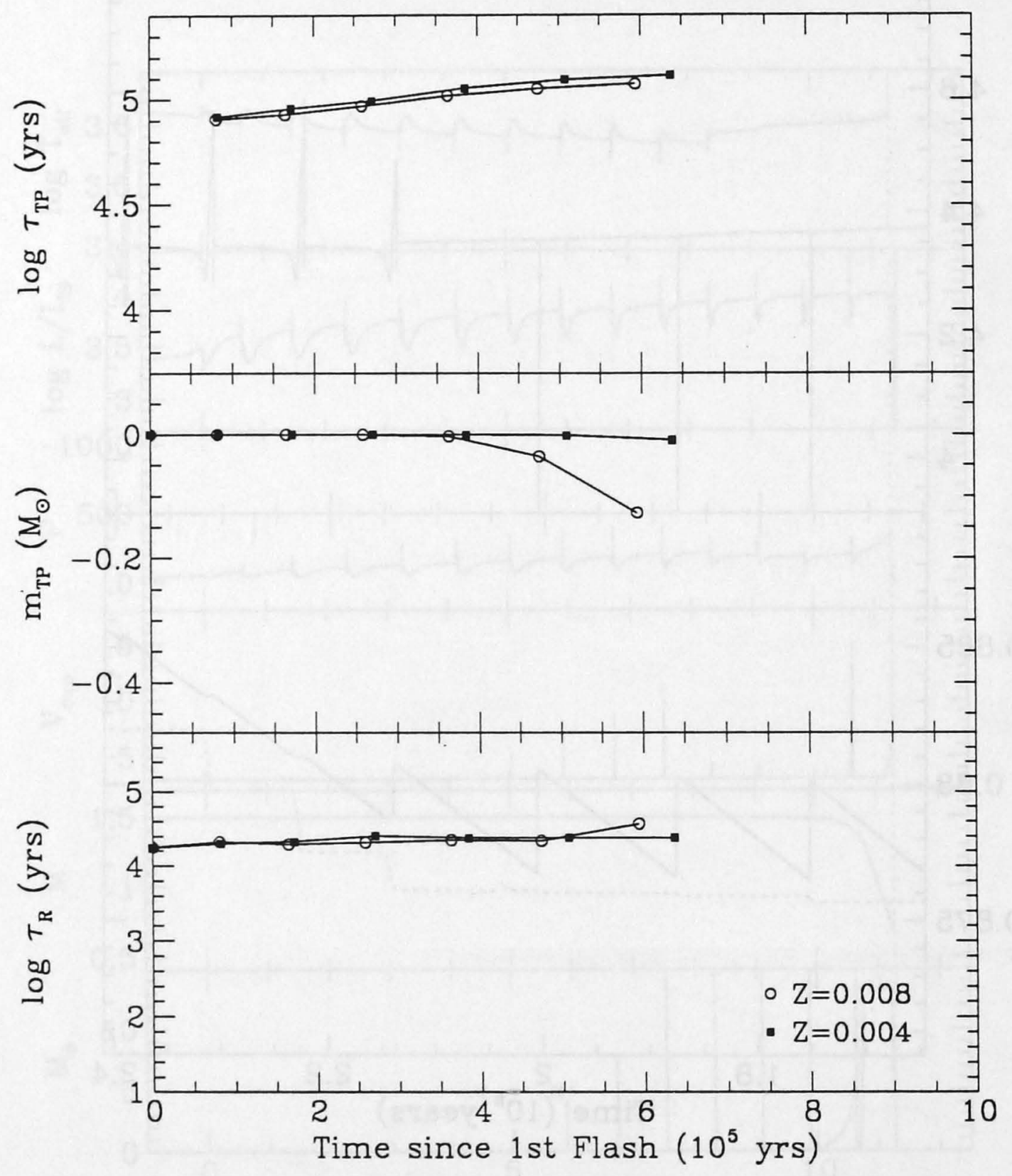

Figure 2.33: The inter-pulse duration $\left(\tau_{T P}\right)$, the change in total stellar mass per cycle $\left.\mathrm{m}_{T P}\right)$, and He shell flash recovery time $\left(\tau_{R}\right)$, for the $0.89(Z=0.004)$ and 0.945 $(Z=0.008) \mathrm{M}_{\odot}$ evolutionary sequences plotted against time since the first helium shell flash. 


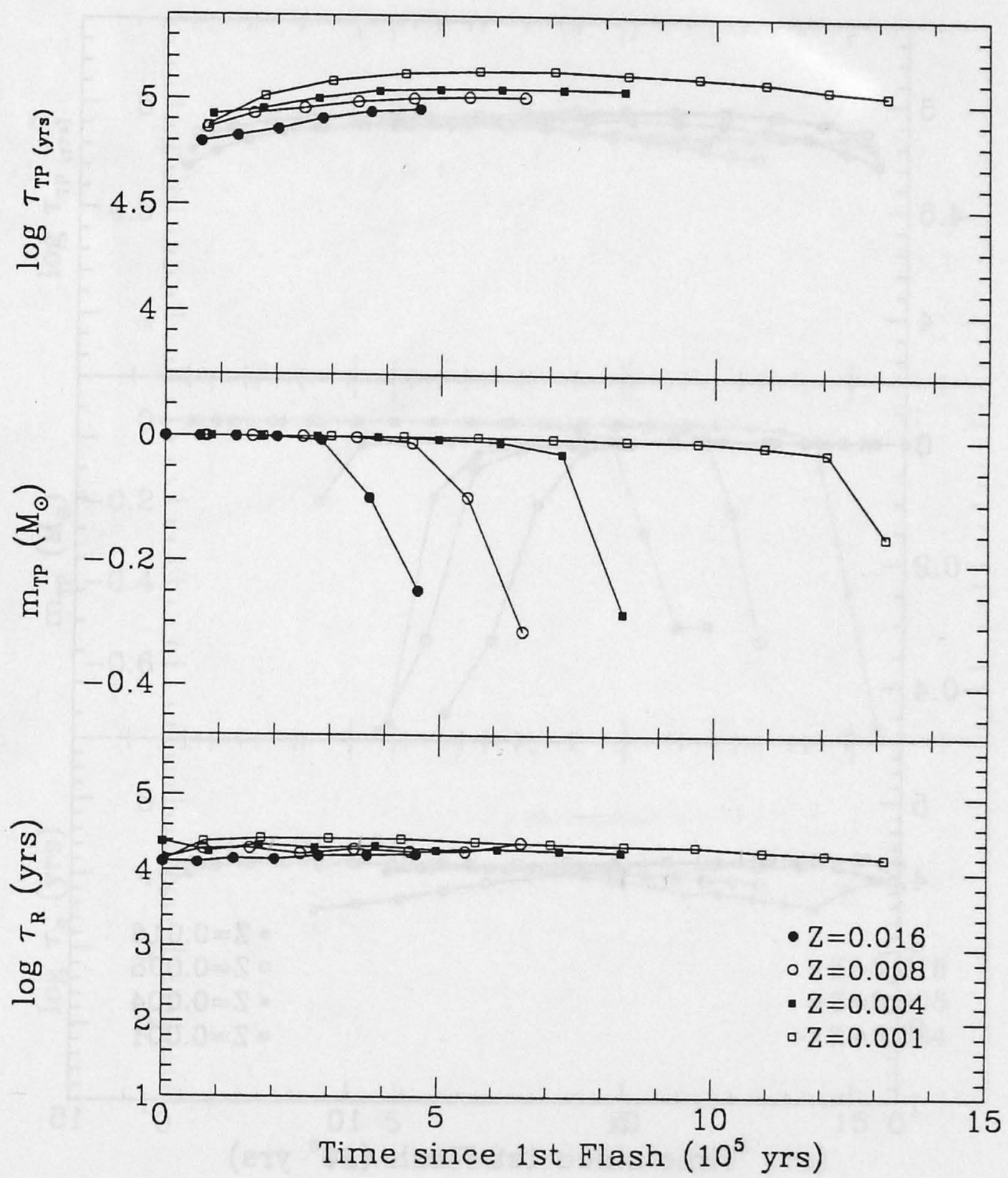

Figure 2.34: Same as figure 2.33, except for $1.0 \mathrm{M}_{\odot}$ evolutionary sequences. 


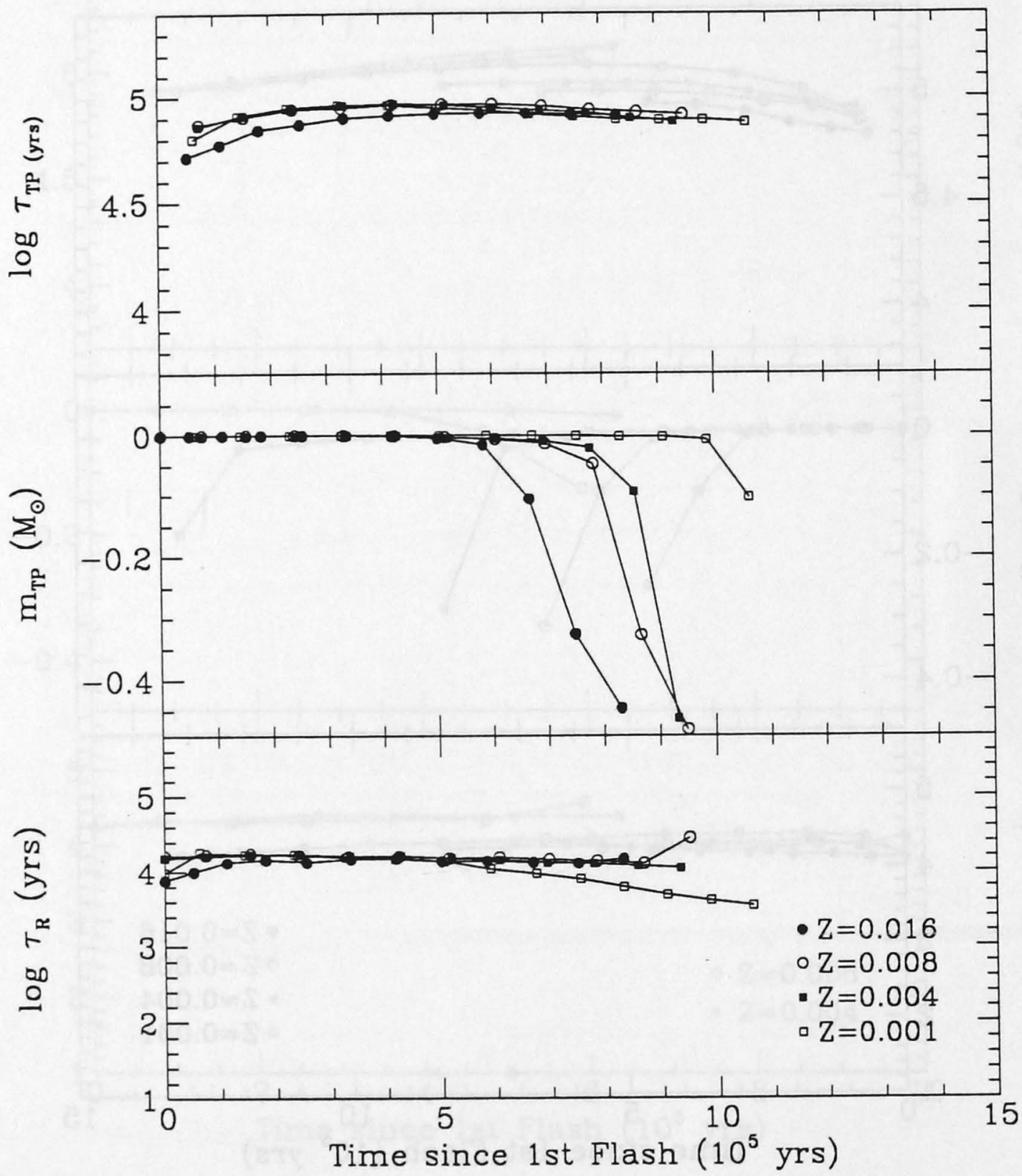

Figure 2.35: Same as figure 2.33 , except for $1.5 \mathrm{M}_{\odot}$ evolutionary sequences. 


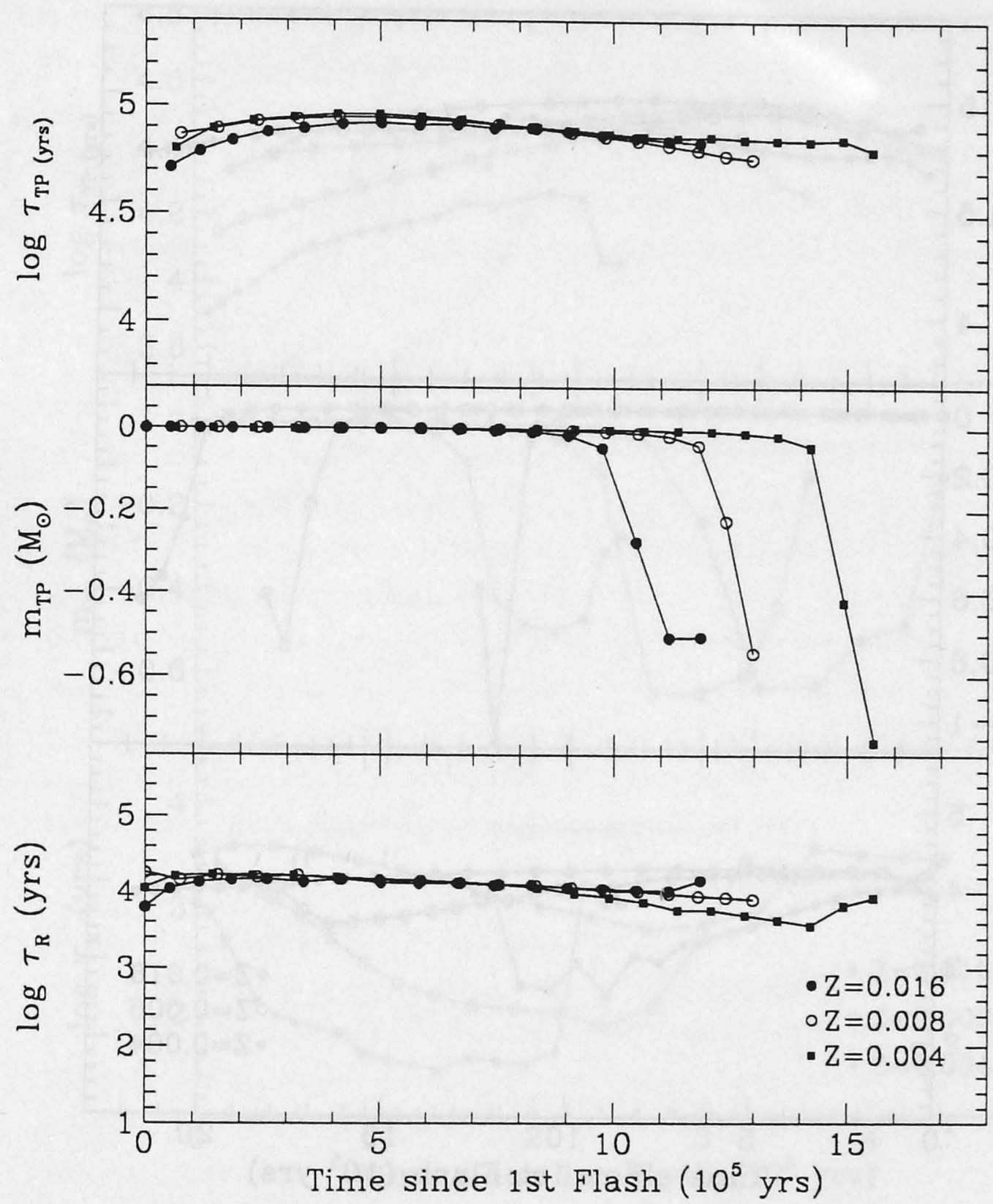

Figure 2.36: Same as figure 2.33 , except for $2.0 \mathrm{M}_{\odot}$ evolutionary sequences. 


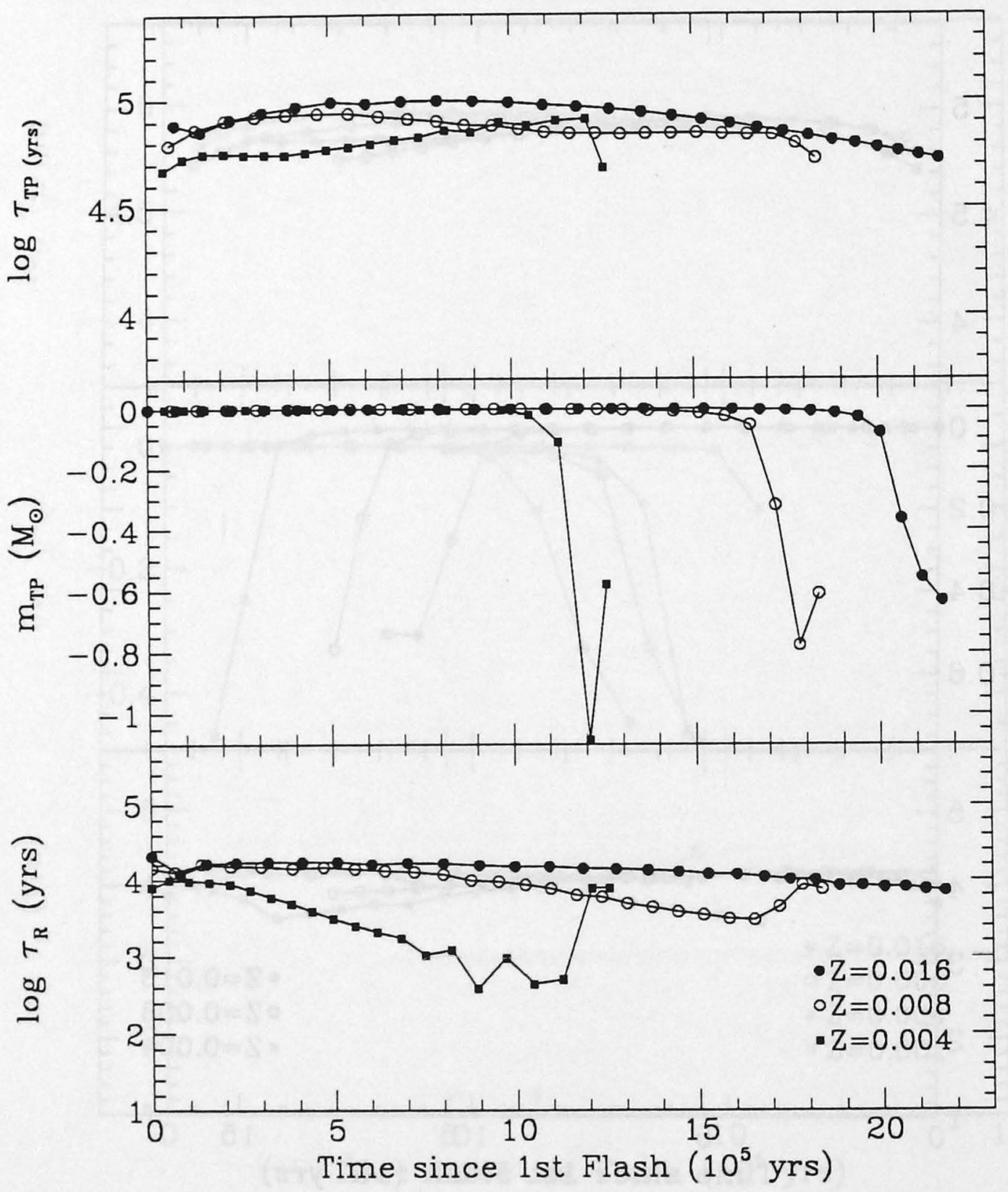

Figure 2.37: Same as figure 2.33 , except for $2.5 \cdot \mathrm{M}_{\odot}$ evolutionary sequences. 


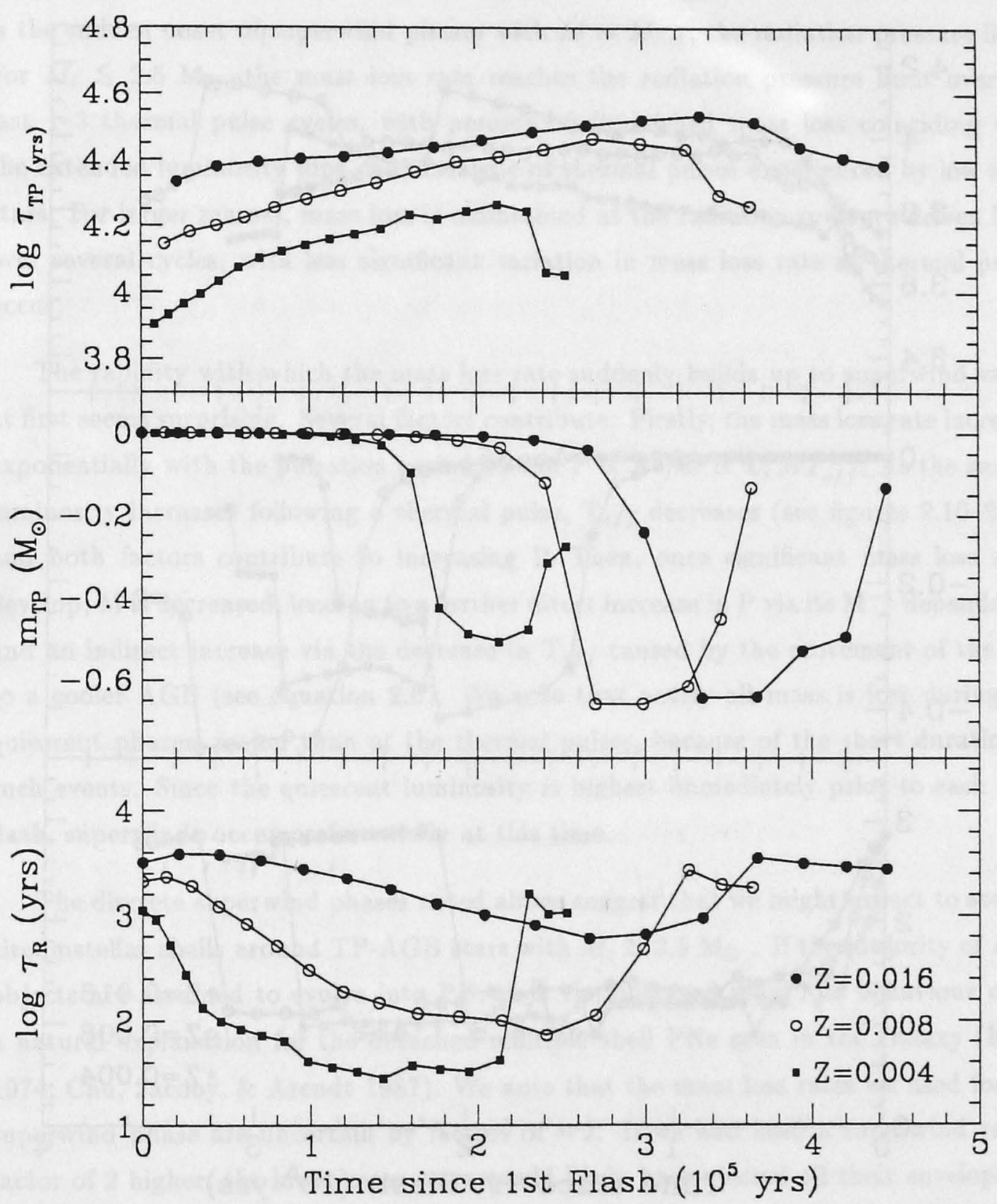

Figure 2.38: Same as figure 2.33, except for $3.5 \mathrm{M}_{\odot}$ evolutionary sequences. 


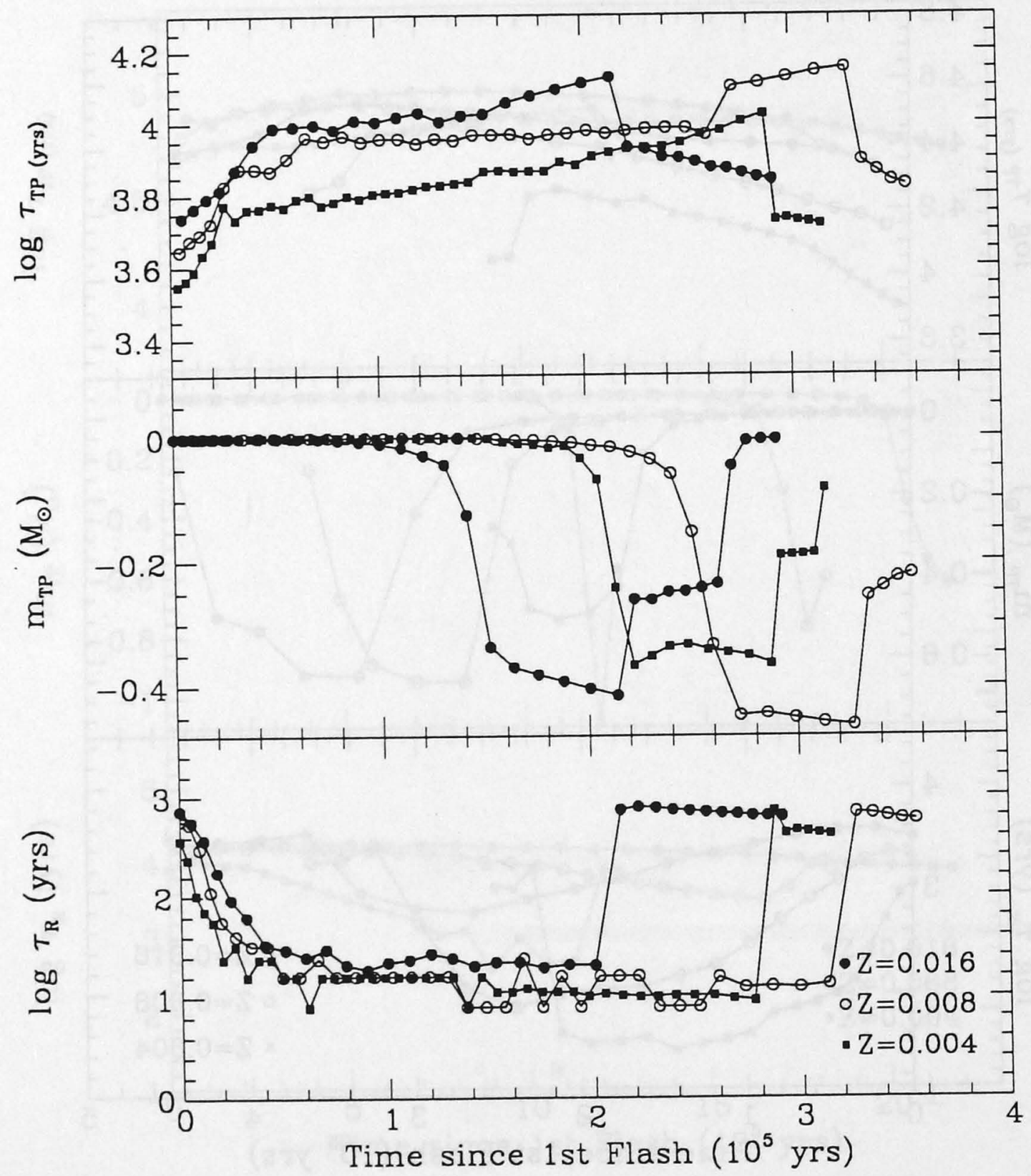

Figure 2.39: Same as figure 2.33 , except for $5.0 \mathrm{M}_{\odot}$ evolutionary sequences. 


\subsubsection{The Superwind Mass Loss Phase}

A striking feature of the mass loss behaviour shown in figures 2.10-2.31 and 2.33-2.39 is the sudden onset of superwind phases with $\dot{M} \sim \dot{M}_{\text {lim }}$, the radiation pressure limit. For $M_{i} \lesssim 2.5 \mathrm{M}_{\odot}$, the mass loss rate reaches the radiation pressure limit over the last 1-3 thermal pulse cycles, with periods of diminished mass loss coinciding with the extended luminosity dips characteristic of thermal pulses experienced by low mass stars. For larger masses, mass loss is maintained at the radiation pressure driven limit over several cycles, with less significant variation in mass loss rate as thermal pulses occur.

The rapidity with which the mass loss rate suddenly builds up to superwind values at first seems surprising. Several factors contribute. Firstly, the mass loss rate increases exponentially with the pulsation period $\mathrm{P}$, and $P \propto R^{2} / M \propto L / M T_{\text {eff }}^{4}$. As the surface luminosity increases following a thermal pulse, $\mathrm{T}_{\text {eff }}$ decreases (see figures 2.10-2.31), and both factors contribute to increasing P. Then, once significant mass loss rates develop, $\mathrm{M}$ is decreased, leading to a further direct increase in $\mathrm{P}$ via its $\mathrm{M}^{-1}$ dependence, and an indirect increase via the decrease in $\mathrm{T}_{\text {eff }}$ caused by the movement of the star to a cooler AGB (see equation 2.6). We note that nearly all mass is lost during the quiescent phases, rather than at the thermal pulses, because of the short duration of such events. Since the quiescent luminosity is highest immediately prior to each shell flash, superwinds occur preferentially at this time.

The discrete superwind phases noted above suggest that we might expect to see 1-3 circumstellar shells around TP-AGB stars with $M_{i} \lesssim 2.5 \mathrm{M}_{\odot}$. If the majority of AGB objects are assumed to evolve into PN, then the observed mass loss behaviour offers a natural explanation for the detached multiple shell PNe seen in the Galaxy (Kaler 1974; Chu, Jacoby, \& Arendt 1987). We note that the mass loss rates we used for the superwind phase are uncertain by factors of $\sim 2$. If we had used a superwind rate a factor of 2 higher, the lower mass stars would likely have ejected all their envelopes in one flash cycle and multiple shell objects would only be produced by the more massive stars.

Finally, we compare various mass loss processes and formulae during the TP-AGB evolution for the $(M, Y, Z)=(1.5,0.25,0.008)$ evolutionary sequence (figure 2.40$)$. As well as the adopted mass loss rates, we show the Reimers' rate, the rate of consumption of the envelope by $\mathrm{H}$ shell burning, and radiation pressure driven limit mass loss rate. The adopted mass loss rate exceeds the Reimers' rate only over the last 4 thermal pulse 
cycles. For the case depicted in figure 2.40 , the radiation pressure limit is reached only during the last 2 thermal pulses, for a $\sim 5 \times 10^{4}$ years per pulse.

\subsubsection{AGB Tip Luminosities}

A strong test of our adopted mass loss formalism is to see if the maximum luminosities we predict for AGB stars of various masses agree with the observed maximum luminosities observed in Magellanic Cloud clusters by Frogel, Mould \& Blanco (1990). In table 2.9, we list for each of the evolutionary sequences, the maximum luminosity during the E-AGB phase, and the maximum and minimum luminosities of optically-visible TP-AGB stars during quiescent evolution. Only the quiescent phase has been considered in order to exclude the brief luminosity excursions associated with shell flashes. Optically-visible stars are assumed to be those where the mass loss rate is less than the radiation pressure driven limit (i. e., the superwind rate). In figure 2.41 , each of these luminosities is plotted against initial mass.

The solid line in figure 2.41 shows the maximum luminosity reached by AGB stars in LMC clusters from Frogel, Mould \& Blanco (1990). The line is a linear fit through the upper points in their figure 14, and their SWB type has been converted to initial mass using their table 3. We have adopted their LMC distance modulus of 18.3. It is clear that for $M_{i} \lesssim 3.5 \mathrm{M}_{\odot}$, there is excellent agreement between our estimated tip luminosities and those observed, a result that suggests that our adopted mass loss rates are realistic. At $5 \mathrm{M}_{\odot}$, we predict higher luminosities than observed. In fact, the observed luminosities are similar to the maximum E-AGB luminosities at this mass. Given the rapid decrease in AGB lifetime noted above from stars more massive than $\sim 3.5 \mathrm{M}_{\odot}$, we suspect that the low observed maximum luminosity at these masses is simply due to small sample statistics. We certainly know that AGB stars exist with luminosities up to $\mathrm{M}_{b o l} \sim-7$ (Wood, Bessell, \& Fox 1983).

\subsubsection{Third Dredge-up - M, S and C stars}

The abundances at the end of the TP-AGB phase, relative to those at the beginning of this phase (i. e., at the end of the 2nd dredge-up phase), are plotted in figure 2.42. Clearly, some ${ }^{12} \mathrm{C}$ enhancement has occurred due to third dredge-up events at helium shell flashes: if efficient enough, third dredge-up leads to carbon star formation. The amount of ${ }^{12} \mathrm{C}$ enhancement increases strongly with metallicity and mass as found in other studies (e. g., Wood 1981; Lattanzio 1987; Boothroyd \& Sackmann 1988d). The 
metallicity dependence of third dredge-up is supported observationally by the fact that the ratio of $\mathrm{C}$ to $\mathrm{M}$ stars increase from the Galactic bulge to the LMC to the SMC, a sequence of decreasing metallicity (Blanco, Blanco, \& McCarthy 1978).

Most stellar evolution calculations fail to predict $\mathrm{C}$ stars at the observed luminosities (see Lattanzio 1989a for a review; Westerlund et al. 1991 and Frogel, Mould \& Blanco 1990 for recent observations). Where dredge-up has been obtained in the past (Iben 1975; Wood 1981; Lattanzio 1987, 1989b; Boothroyd \& Sackmann 1988d), it has only been when mass is relatively large $\left(M_{i} \geq 1.5 \mathrm{M}_{\odot}\right)$ and if the stars have a metal abundance appropriate for the Galaxy or Magellanic Clouds . Even then, the lowest luminosity carbon stars that result are $\sim 0.5$ mag brighter than the lowest observed luminosities at masses $\gtrsim 1.5 \mathrm{M}_{\odot}$ (comparing data in Frogel, Mould, \& Blanco 1990 with results in Lattanzio $1989 \mathrm{~b}$ ).

Two ways of obtaining $\mathrm{C}$ stars readily are to decrease the metal abundance (the last three sets of authors cited above obtained dredge-up at $Z=0.001$ ) or to increase the mixing-length parameter $\alpha$. By using both $Z=0.001$ and $\alpha=3$, Boothroyd \& Sackmann 1988d) obtained dredge-up in a $0.81 \mathrm{M}_{\odot}$ star at $\mathrm{M}_{b o l} \sim-3.6$, similar to the luminosity of the faintest $\mathrm{C}$ stars in old clusters in the SMC and LMC (Frogel, Mould, $\&$ Blanco 1990). However, the use of such large values of $\alpha$ is questionable: $\alpha \sim 1.5$ appears to reproduce observed stellar effective temperatures during many stages of evolution prior to the AGB (CCS; CCP; VandenBerg 1991).

We have not been able to reproduce the $\mathrm{C}$ star distribution observed in the SMC and LMC. For LMC abundances, we found dredge-up only for $M_{i} \geq 2.5 \mathrm{M}_{\odot}$ while for the SMC abundances we find dredge-up only for $M_{i} \geq 2.0 \mathrm{M}_{\odot}$ (see figure 2.42). In figure 2.41, the thick dashed line indicates the transition luminosity from $\mathrm{M}$ to $\mathrm{C}$ star in LMC clusters, as given in table 3 of Frogel, Mould \& Blanco (1990). This line indicates that $\mathrm{C}$ stars form almost as soon as shell flashes start at the end of the E- AGB (see also Lattanzio 1991). Clearly, all dredge-up calculations need some new physics or additional process to produce the observed $\mathrm{C}$ stars. The new, larger OPAL opacities (Rogers \& Iglesias 1992) might help, as would envelope undershoot (Alongi et al. 1990).

\subsubsection{Hot-Bottom Burning in Massive AGB Stars}

The other notable abundance change in figure 2.42 is a large ${ }^{14} \mathrm{~N}$ enhancement in the 5 $\mathrm{M}_{\odot}$ stars with SMC and LMC abundance. This is caused by the so-called hot-bottom 
burning (HBB) (Iben 1973; Sackmann, Smith, \& Despain 1974; Scalo, Despain, \& Ulrich 1975) during quiescent evolution. HBB occurs when deep envelope convection cycles envelope material through the upper parts of the $\mathrm{H}$ burning shell where ${ }^{12} \mathrm{C}$ is converted to ${ }^{14} \mathrm{~N}$. The enhanced ${ }^{7} \mathrm{Li}$ abundances which have been observed in luminous $\left(M_{b o l} \gtrsim-6\right)$ Magellanic Cloud AGB stars are attributed to HBB and the beryllium transport mechanism (Cameron \& Fowler 1971). In this process, ${ }^{7} \mathrm{Be}$ is formed from ${ }^{3} \mathrm{He}$ produced primarily during the main sequence phase and ${ }^{7} \mathrm{Be}$ is transported rapidly to cooler regions by convection where ${ }^{7} \mathrm{Li}$ is produced via the reaction ${ }^{7} \mathrm{Be}\left(\beta^{-}, \gamma\right)^{7} \mathrm{Li}$. Our calculations do predict the occurrence of HBB in luminous AGB stars but we do not treat the processes involved in detail so we can not make detailed comparisons with observations. The processes of nucleosynthesis involved are best examined in a separate calculation using input from the evolution calculations.

Type I planetary nebulae (Peimbert 1978) show both $\mathrm{N}(N / O \geq 1)$ and He $\left(N(H e) / N(H) \geq 0.14\right.$ or $\left.\mathrm{X}_{H e} \geq 0.36\right)$ enhancements and are thought to come from relatively massive AGB stars with $M \gtrsim 2.4 \mathrm{M}_{\odot}$ (Peimbert \& Serrano 1980). These enriched nebulae are continually being found in the Galaxy (Kaler, Shaw, \& Kwitter 1990) and in the Magellanic Clouds (Meatheringham \& Dopita 1991a, b). We have managed to reproduce significant $\mathrm{N}$ enchancement during the first, second and third dredge-up episodes, but the associated He enhancement are small by comparison with those in type I planetary nebulae. Once again there seems to be a need to make dredge-up go deeper, this time in order to bring up more He at 2nd dredge-up. It has been shown (Kaler \& Jacoby 1989: Kaler, Shaw, \& Kwitter 1990) that the $N$ enhancement rises very steeply for core masses greater than $\sim 0.8 \mathrm{M}_{\odot}$. Indeed, this is the approximate value of $\mathrm{M}_{c}^{H}$ seen in our $5.0 \mathrm{M}_{\odot}$ models. The final $\mathrm{N} / \mathrm{O}$ ratios in our $5 \mathrm{M}_{\odot}$ models are $0.37,0.54$, and 0.85 for $Z=0.016, Z=0.008$, and $Z=0.004$, respectively. This is still less than observed in type I PN and indicates insufficient dredge-up.

There is also a deficiency in our calculations whose correction might help to produce higher $\mathrm{N} / \mathrm{O}$ values in our more massive stars. The deficiency, which only occurs during $\mathrm{HBB}$, is that for the time steps used, the $\mathrm{CN}$ cycle is assumed to come into equilibrium in the convective regions of the $\mathrm{H}$ burning shell, so that conversion of $\mathrm{C}$ to $\mathrm{N}$ is limited to that obtained by converting the local matter to equilibrium abundance ratios. Since fresh envelope material is continuously cycled through the shell by convection, more $\mathrm{C}$ to $\mathrm{N}$ conversion could occur, the exact amount depending on the relative burning and convective turnover times. That problem will be studied in future. Note, however, that the helium production will hardly be affected by these changes. 


\subsubsection{The Initial-Final Mass Relation}

The final core masses at which the sequences depart from the AGB are plotted against initial mass in figure 2.43. We have taken the core mass $\mathrm{M}_{c}^{H}$ from point 15 in table 2.2 to be the final mass, as this mass will be very close to the mass of the remnant white dwarf. Some of the stars at point 15 in the tables still have substantial $\mathrm{H}$ envelopes which will be lost in the transition to the planetary nebula region of the H-R diagram.

Also shown in the figure is the observational calibration of the initial mass-final (i. e., white dwarf) mass relation from Weidemann \& Koester (1983) and Weidemann (1987). There is clearly a large amount of scatter in the observational calibration, but our theoretical calibration to lie $\sim 0.1 \mathrm{M}_{\odot}$ above the observational calibration. The core mass at the beginning of the TP-AGB phase seems to be in better agreement with the observational calibration. Our theoretical initial-final mass relation is determined essentially by our adopted mass loss formalism and we have noted above that the agreement between the theoretical and observational maximum AGB luminosities in the Magellanic Clouds, suggests that our adopted mass loss rates may be close to reality. Alternatively, the current observational initial-final mass relation implies the lack of existence of TP-AGB stars, whereas we know such stars (e. g., C stars, S stars) definitely exist. We therefore believe our theoretical initial-final mass relation is not seriously compromised by the lack of agreement with the observed initial mass-white dwarf mass relation.

\subsection{Summary}

The results of stellar evolution calculations from the main sequence through to the end of the AGB phase with mass loss have been presented. The mass loss rates used are based on empirical determinations for AGB stars. It is shown that typical low mass AGB stars naturally suffer one or more superwind phases of mass loss towards the end of the AGB, these superwind phases occurring towards the end of the quiescent phases of helium shell flash cycles. The mass loss rates produced by the superwind are similar to those required for PN production, and the occurrence of multiple superwind phases can account for the existence of multiple shell PNe. A comparison of the maximum AGB luminosities predicted by the models with the maximum luminosities reached by Magellanic Cloud stars shows excellent agreement between theory and observation.

The initial mass-final mass relation resulting from our calculations predicts white dwarf masses $\sim 0.1 \mathrm{M}_{\odot}$ larger, at a given initial mass, than those in current observational 
calibrations of the initial mass-white dwarf mass relation. 


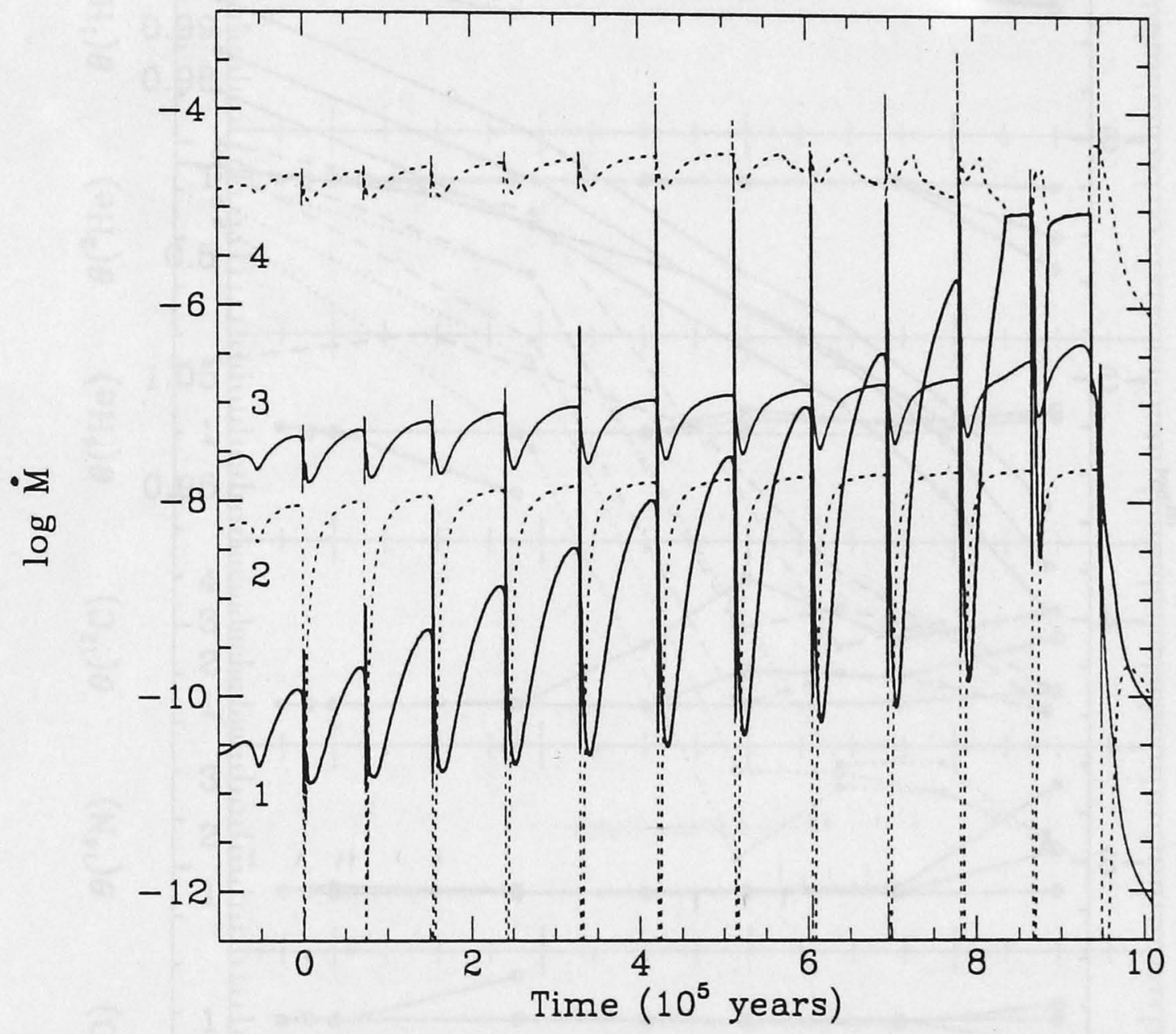

Figure 2.40: Mass loss behaviour for the $(M, Y, Z)=(1.5,0.25,0.008)$ evolutionary sequence. Curve 1 depicts the mass loss rate applied in the stellar evolution calculation; curve 2 represents mass lost via $H$ burning; curve 3 represents the canonical Reimers' mass loss rate with $\eta=1 / 3$; and curve 4 represents the radiation pressure driven limit mass loss rate. 


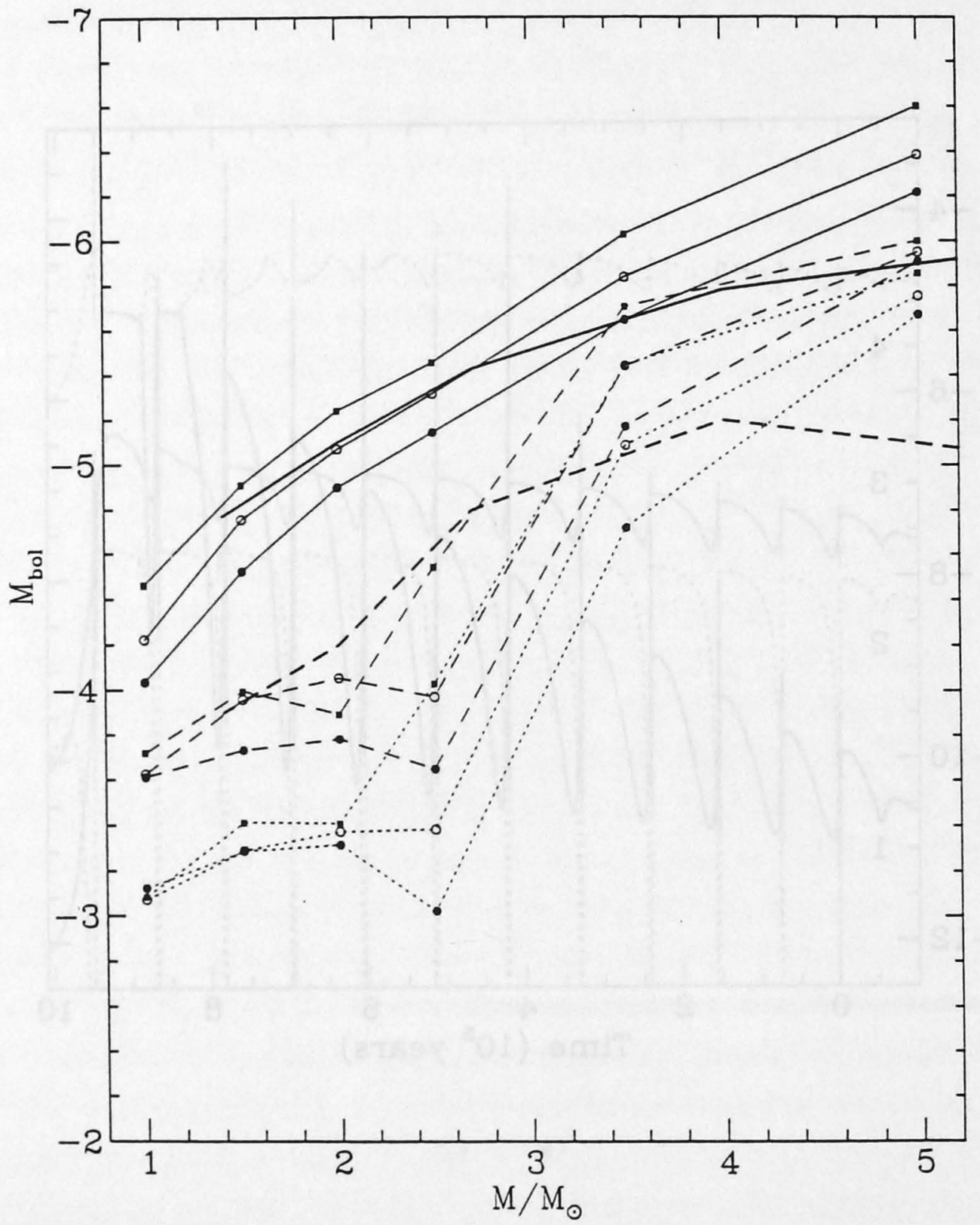

Figure 2.41: The luminosity at the tip of the optically-visible AGB (thin solid lines), the maximum luminosity on the E-AGB (thin dashed lines), and the minimim luminosity on the TP-AGB (dotted lines) plotted as a function of the initial mass. Filled circles correspond to $Z=0.016$, open circles to $Z=0.008$, and squares to $Z=004$. Also shown is the luminosity at the tip of LMC cluster AGBs (thick solid line) and the transition luminosity from $\mathrm{M}$ to $\mathrm{C}$ stars in LMC clusters (thick dashed line), from the data in Frogel, Mould and Blanco (1990). 


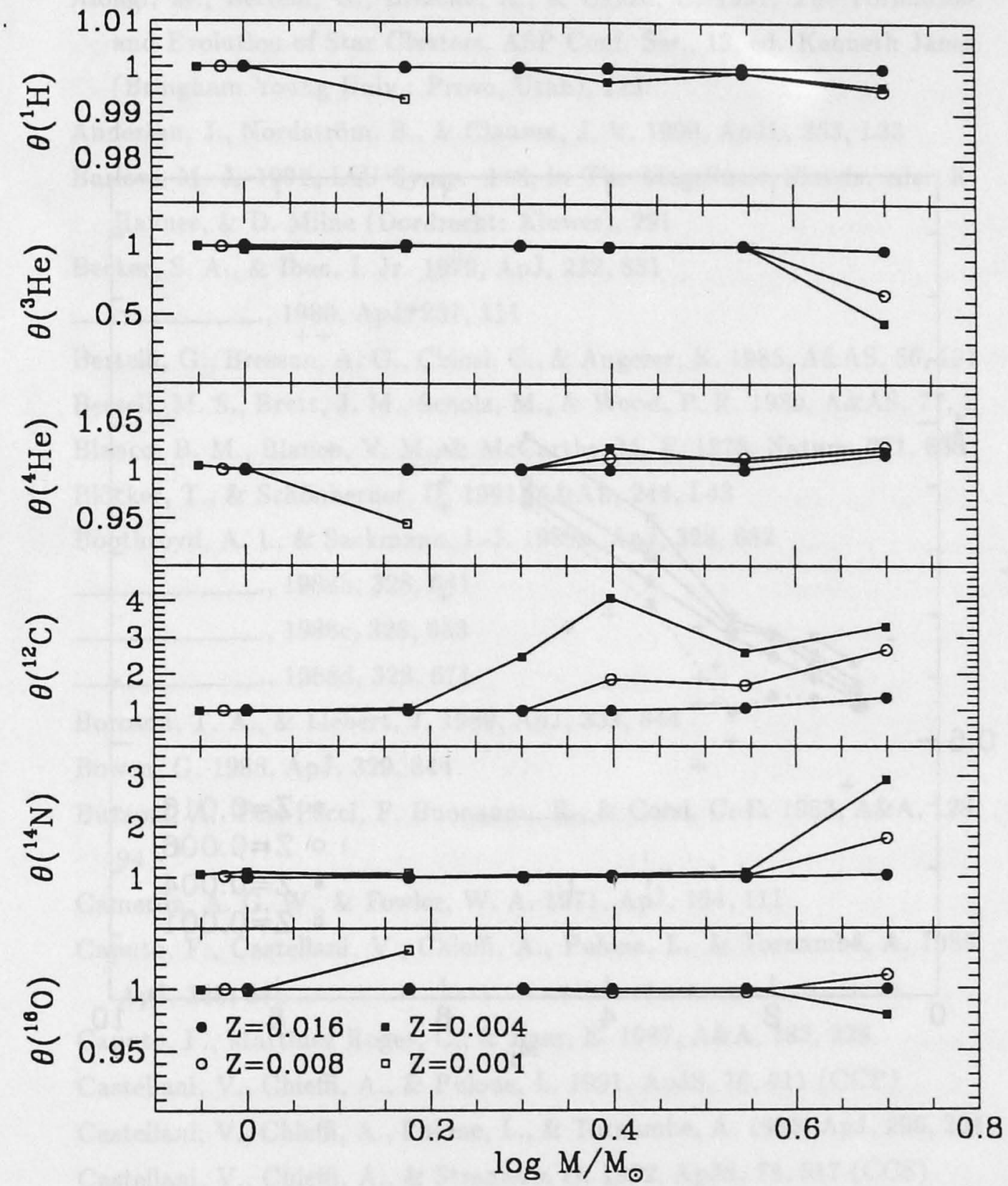

Figure 2.42: Abundances at the end of the TP-AGB relative to the abundances after second dredge-up plotted against $\log \mathrm{M} / \mathrm{M}_{\odot}$. 


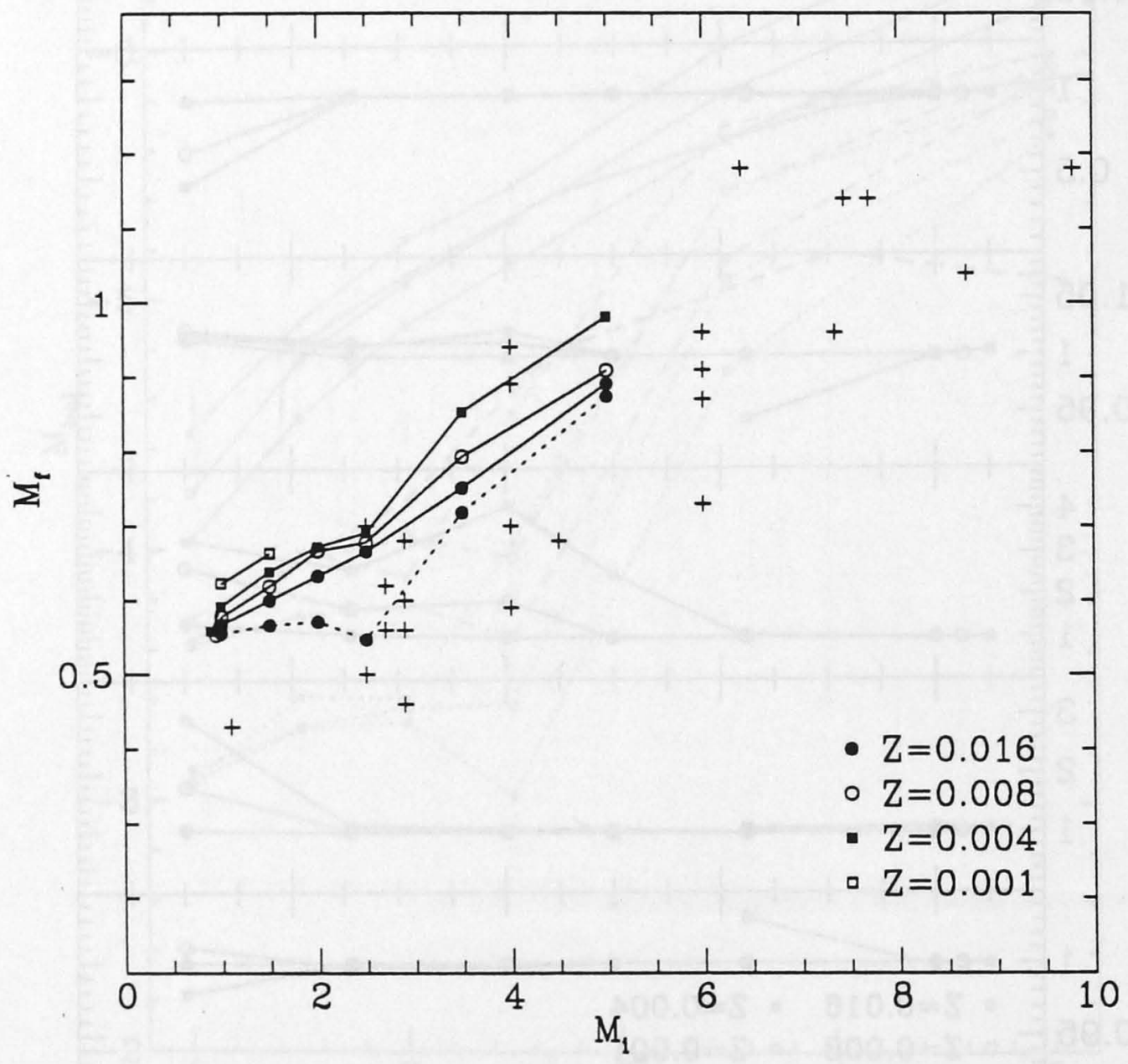

Figure 2.43: The final stellar remnant mass after AGB mass loss plotted as a function of the initial mass (solid curves). The dashed line represents the core mass at the first helium shell flash as a function of the initial mass for the $Z=0.016$ calculations. Also shown $(+)$ is the observed relation given by Weidemann \& Koester (1983) and Weidemann (1987). 


\subsection{References}

Alongi, M., Bertelli, G., Bressan, A., \& Chiosi, C. 1991, The Formation and Evolution of Star Clusters, ASP Conf. Ser., 13, ed. Kenneth Janes

(Bringham Young Univ.: Provo, Utah), 223

Andersen, J., Nordström, B., \& Clausen, J. V. 1990, ApJL, 363, L33

Barlow, M. J. 1991, IAU Symp. 148, in The Magellanic Clouds, eds. R.

Haynes, \& D. Milne (Dordrecht: Kluwer), 291

Becker, S. A., \& Iben, I. Jr. 1979, ApJ, 232, 831 ., 1980, ApJ, 237, 111

Bertelli, G., Bressan, A. G., Chiosi, C., \& Angerer, K. 1985, A\&AS, 66, 191

Bessell, M. S., Brett, J. M., Scholz, M., \& Wood, P. R. 1989, A\&AS, 77, 1

Blanco, B. M., Blanco, V. M., \& McCarthy, M. F. 1978, Nature, 271, 638

Blöcker, T., \& Schönberner, D. 1991, A\&AL, 244, L43

Boothroyd, A. I., \& Sackmann, I.-J. 1988a, ApJ, 328, 632 ., $1988 \mathrm{~b}, 328,641$ , $1988 \mathrm{c}, 328,653$ , 1988d, 328, 671

Boroson, T. A., \& Liebert, J. 1989, ApJ, 339, 844

Bowen, G. 1988, ApJ, 329, 844

Buzzoni, A., Fusi-Pecci, F. Buonanno, R., \& Corsi, C. E. 1983, A\&A, 128, 94

Cameron, A. G. W., \& Fowler, W. A. 1971, ApJ, 164, 111

Caputo, F., Castellani, V., Chieffi, A., Pulone, L., \& Tornambé, A. 1989, ApJ, 340, 241

Caputo, F., Martinez Roger, C., \& Paez, E. 1987, A\&A, 183, 228

Castellani, V., Chieffi, A., \& Pulone, L. 1991, ApJS, 76, 911 (CCP)

Castellani, V., Chieffi, A., Pulone, L., \& Tornambe, A. 1985, ApJ, 296, 204

Castellani, V., Chieffi, A., \& Straniero, O. 1992, ApJS, 78, 517 (CCS)

Castellani, V., Giannone, P., \& Renzini, A. 1971, Ap\&SS, 10, 360

Castor, J. I. 1981, in Physical Processes in Red Giants, eds. I. Iben \& A. Renzini (Reidel: Dordrecht), 285

Caughlan, G. R., Fowler, W., Harris, M., \& Zimmerman, B. 1985, Atomic Data \& Nuclear Data Tables, 32, 197

Caughlan, G. R., \& Fowler, W. A. 1988, Atomic Data \& Nuclear Data Tables, 40, 283 
Chiosi, C., Bertelli, G., \& Bressan, A. 1987, in Late Stages of Stellar Evolution, eds. S. Kwok \& S. R. Pottasch (Dordrecht: Reidel), 213

Chu, Y.-H., Jacoby, G. H., \& Arendt, R. 1987, ApJS, 64, 529

Cox, A. N., \& Stewart, J. N. 1970a, ApJS, 19, 243 ., 1970b, ApJS, 19, 261

Dufour, R.J. 1984, in IAU Symp. 108, Structure and Evolution of the Magellanic Clouds, eds. S. van den Bergh \& K. S. de Boer (Reidel: Dordrecht), 353

Eder, J., Lewis, B. M. and Terzian, Y. 1988, ApJS, 66, 183

Fowler, W. A., Caughlan, G. R., \& Zimmermann, B. A. 1975, ARA\&A, 13, 69

Frogel, J. A., Mould, J., \& Blanco, V. M. 1990, ApJ, 352, 96

Fusi Pecci, F., \& Renzini, A. 1976, A\&A, 46, 447 , 1978, in The HR Diagram, eds. A. G. D. Philip, D. S.

Hayes (Dordrecht: Reidel), 225

Härm, R., \& Schwarzschild, M. 1975, ApJ, 200, 324

Harris, M. J., Fowler, W. A., Caughlan, G. R., \& Zimmermann, B. A. 1983, ARA\&A, 21, 165

Hearn, A. G. 1990, in From Miras to Planetary Nebulae: Which Path for Stellar Evolution?, eds. M. O. Mennessier \& A. Omont (Yvette Cedex: Editions Frontières), 121

Hesser, J. E., Harris, W. E., VandenBerg, D. A., Allwright, J. W. B., Shott, P., \& Stetson, P. B. 1987, PASP, 99, 739

Hollowell, D., \& Iben, I. Jr. 1988, ApJL, 333, L25

Holzer, T. E., \& MacGregor, K. B. 1985, in Mass Loss from Red Giants, eds. M. Morris \& B. Zuckerman (Reidel: Dordrecht), 229

Huebner, W. F., Merts, A. L., Magee, N. H. Jr., \& Argo, M. F. 1977, Astrophysical Opacity Library, Los Alamos Scientific Laboratory, LA6760-M

Hughes, S. M. G., \& Wood, P. R. 1990, AJ, 99, 784

Iben, I. Jr. 1964, ApJ, 140, 1631 ., 1973, ApJ, 185, 209 , 1975, ApJ, 196, 525 1977, ApJ, 217, 788

Iben, I. Jr., \& Renzini, A. 1983, ARA\&A, 21, 271 
Jura, M. 1990, in From Miras to Planetary Nebulae: Which Path for Stellar Evolution?, eds. M. O. Mennessier \& A. Omont (Yvette Cedex: Editions Frontières), 67

Kaler, J. B. 1974, AJ, 79, 594

Kaler, J. B., \& Jacoby, G. H. 1989, ApJ, 345, 871

Kaler, J. B., Shaw, R. A., \& Kwitter, K. B. 1990, ApJ, 359, 392

King, C. R., Da Costa, G. S., \& Demarque, P. 1985, ApJ, 299, 674

Kippenhahn, R., Thomas, H.-C., \& Weigert, A. 1965, ZsAp, 61, 241

Knapp, G. R. 1986, ApJ, 311, 731

Knapp, G. R. and Morris, M. 1985, ApJ, 292, 640

Lattanzio, J. C. 1986, ApJ, 311, 708 ., 1987, 313, L15 1989a, in Evolution of Peculiar Red Giants, eds. H. R.

Johnson \& B. Zuckerman (Cambridge: University Press), 161

$\begin{array}{r}\text {., 1989b, ApJ, 344, L25 } \\ \hline \text {., 1991, ApJS, 76, } 215\end{array}$

Lloyd Evans, T. 1976, MNRAS, 174, 169 ., 1984, MNRAS, 208, 447

Maeder, A. 1975, A\&A, 40, 303

Meatheringham, S. J., \& Dopita, M. A. 1991a, ApJS, 75, 407 , 1991b, ApJS, 76, 1085

Monk, D. J., Barlow, M. J., Clegg, R. E. S. 1988, MNRAS, 234, 583

Paczyński, B. 1970, A\&A, 6, 426

Paczyński, B., \& Ziolkowski, J. 1968, Acta Astr., 18, 255.

Peimbert, M. 1978, in IAU Symp. 76, Planetary Nebulae: Observations and Theory, ed. Y. Terzian (Dordrecht: Reidel), 215

Peimbert, M., \& Serrano, A. 1980, Rev. Mexicana A\&A, 5, 9

Pottasch, S. R. 1984, Planetary Nebulae (Reidel: Dordrecht, p.31.

Reid, N., \& Mould, J. R. 1985, ApJ, 299, 236 , 1991, in IAU Symp. 148, The Magellanic Clouds, eds. R.

Haynes, \& D. Milne (Dordrecht: Kluwer), 363

Renzini, A. 1981, in Physical Processes in Red Giants, eds. I. Iben Jr. \& A. Renzini (Dordrecht: Reidel), 431

Renzini, A., \& Fusi Pecci, F. 1988, ARA\&A, 26, 199

Rogers, F. J., \& Iglesias, C. A. 1992, ApJS, 79, 507

Ross, J. E., \& Aller, L. H. 1976, Science, 191, 1223 
Roxburgh, I. W. 1967, Nature, 215, 838

Russell, S. C., \& Bessell, M. S. 1989, ApJS, 70, 865

Russell, S. C., \& Dopita, M. A. 1990, ApJS, 74, 93

Sackmann, I.-J., Smith, R. L., Despain, K. H. 1974, ApJ, 187, 555

Scalo, J. M., Despain, K. H., \& Ulrich, R. K. 1975, ApJ, 196, 805

Schild, H. 1989, MNRAS, 240, 63

Schönberner, D. 1979, A\&A, 79, 108 , 1981, A\&A, 103, 119 , 1983, ApJ, 272, 708

Seidel, E., Demarque, P., \& Weinberg, D. 1987, ApJS, 63, 917

Simon, N. 1992, ApJ, 387, 162

Steigman, G. 1983, in Primordial Helium, eds. P. A. Shaver, D. Kunth, \& K. Kjär (ESO), 13

Sugimoto, D., \& Nomoto, R. 1975, PASJ, 27, 197

Sweigert, A. V., \& Demarque, P. 1972, A\&A, 20, 445

Sweigert, A. V., Greggio, L., \& Renzini, A. 1989, ApJS, 69, 911 (SGR)

Sweigert, A. V., \& Gross, P. G. 1976, ApJS, 32, 367

Truran, J. W., \& Iben, I. Jr. 1977, ApJ, 216, 797

Tuchman, Y. 1991, ApJ, 383, 779

VandenBerg, D. A. 1991,in The Formation and Evolution of Star Clusters, ASP Conf. Ser., 13, ed. Kenneth Janes (Bringham Young Univ.: Provo, Utah), 183

VandenBerg, D. A., \& Laskarides, P. G. 1987, ApJS, 64, 103

Vassiliadis, E., Dopita, M. A., Morgan, D. H., \& Bell, J. F. 1992, ApJS, in press

Wagoner, R. V. 1973, ApJ, 179, 343

Weidemann, V. 1987, A\&A, 188, 74 , 1990, ARA\&A, 28, 103

Weidemann, V., \& Koester, D. 1983, A\&A, 121, 77

Westerlund, B. E., Azzopardi, M., Breysacher, J., \& Rebeirot, E. 1991, A\&AS, 91, 425

Whitelock, P., Feast, M. \& Catchpole, R. 1991, MNRAS, 248, 276

Willson, L. A. 1982, in Pulsations in Classical and Cataclysmic Variables, eds. J. P. Cox, \& C. J. Hansen (Boulder: JILA), 284

Wood, P. R. 1974, ApJ, 190, 609 , 1979, ApJ, 227, 220 
1981, in Physical Processes in Red Giants, eds. I. Iben \&

A. Renzini (Dordrecht: Reidel), 135 ., 1986, in Stellar Pulsation, eds. A. N. Cox, W. M. Sparkes,

\& S. G. Starrfield (Berlin: Springer-Verlag), 250 1990a, in From Miras to Planetary Nebulae: Which Path

for Stellar Evolution?, eds. M. O. Mennessier \& A. Omont (Yvette Cedex: Editions Frontières), 67

1990b, in Confrontation Between Stellar Pulsation and

Evolution, eds. C. Cacciari and G. Clementini, ASP Conference Series, 11,355

Wood, P. R. \& Bessell, M. S. 1983, ApJ, 265, 748

Wood, P. R., Bessell, M. S. \& Fox, M.W. 1983, ApJ, 272, 99

Wood, P. R., Bessell, M. S., \& Paltoglou, G. 1985, ApJ, 290, 477

Wood, P. R., Bessell, M. S., \& Whiteoak, J. B. 1986, ApJL, 306, L81

Wood, P. R., \& Cahn, J. H. 1977, ApJ, 211, 499

Wood, P. R., \& Faulkner, D. J. 1986, ApJ, 307, 659

., 1987, Proc. Astr. Soc. Australia, 7, 75

Wood, P. R., Whiteoak, J. B., Hughes, S. M. G., Bessell, M. S., Gardner, F. F., \& Hyland, A. R. 1992, ApJ, in press

Wood, P. R., \& Zarro, D. M. 1981, ApJ, 247, 247 


\section{Chapter 3}

\section{Post-AGB Evolution of Low to Intermediate Mass Stars.}

\subsection{Introduction}

The study of post-AGB evolution by Paczyński (1971) was one of the first to show that the observational Harmon-Seaton evolutionary sequence of planetary nebulae (PNe) (Harmon \& Seaton 1965) could be interpreted as a superposition of theoretical evolutionary sequences of planetary nebula nuclei (PNNi) with different masses, and therefore, different ages. In the past 20 years, progress in the development of new evolutionary sequences for $\mathrm{PNNi}$ has been slow due to the realisation that the preceding AGB phase of evolution must be properly considered (Schönberner 1981). The AGB phase of evolution is characterized by repeated helium shell flash events (Schwarzschild \& Härm 1965; Weigert 1966) which are computationally intensive, and thus have often been suppressed (e. g., Paczyński 1971; Härm \& Schwarzschild 1975; Harpaz \& Kovetz 1981).

An accurate description of the AGB and planetary nebula nucleus (PNN) phases is necessary to derive the star formation history of systems, such as the Magellanic Clouds and Galactic Bulge, using the recently obtained luminosity-limited samples of PNe (e. g., Vassiliadis et al. 1992). It is also necessary for studies of galactic evolution as the AGB and PNN evolutionary phases contribute a significant portion to the total bolometric luminosity of a galaxy (Renzini \& Buzzoni 1986), and specifically, the PNNi contribute to the total observed UV flux.

The calculations most commonly referred to in the literature are those of Paczyński (1971), Schönberner $(1979 ; 1981 ; 1983)$ and Wood \& Faulkner $(1986$, hereafter WF86). 
The initial models used by Paczyński were constructed from a flash-suppressed AGB sequence that had hydrogen-rich envelopes added to the desired core masses. The resultant models were quite far from the AGB when calculations were begun, and no further mass loss was incorporated. Despite the artificial nature of these models, they are still widely referred to because they are the only calculations available which span the large range in core mass $0.6-1.2 \mathrm{M}_{\odot}$. The Schönberner $(1979 ; 1981)$ calculations were one of the first to properly consider the AGB phase of evolution, with mass loss defined by a Reimers (1975) -type rate. However, recent observations have shown that observed mass loss rates can exceed the Reimers rate by several orders of magnitude. High mass loss rates were employed by Schönberner (1983) at the end of the AGB but for only two initial core masses. The WF86 calculations span a larger range in core mass $\left(0.60-0.89 \mathrm{M}_{\odot}\right)$ than the Schönberner calculations $\left(0.546-0.644 \mathrm{M}_{\odot}\right)$, and examine the effects of high mass loss rates. They also conducted a systematic study of the effect on PNN evolution of the phase of the helium flash cycle at which PN ejection was assumed to occur (see also Iben 1984). The main criticism of the WF86 calculations is that all their PNN tracks were produced from a single initial model of 2 $\mathrm{M}_{\odot}$ which had various amounts of mass instantaneously stripped to produce the initial PNN models (see Schönberner 1989).

The initial models used in this study were taken from Vassiliadis \& Wood (1992, hereafter VW92). The models were calculated complete from the main sequence phase through to the end of the AGB phase with empirically calibrated mass loss rates (refer to VW92 for details). Since the VW92 calculations have mass loss rates close to those observed, the initial state of the starting models should be a good representation of reality. In particular, the number of shell flashes experienced on the AGB should be reasonably correct. Thus the calculations offer the opportunity to derive the initial masses of the stars from which PNe may arise.

The present calculations concern the post-AGB evolution of low and intermediate mass stars. If mass loss occurs in this phase it will have a critical effect on PNN evolution rates (compare the different mass loss schemes employed in WF86). Certainly, many PNNi are observed to be losing mass (e. g., Perinotto 1989), although not all have been observed to have winds. Mèndez et al. (1988) have shown that objects close to their Eddington luminosity limit are more likely to possess winds. A strong correlation also exists between the observed terminal wind velocities and effective temperatures of PNNi (Heap 1986; see Grewing 1989, for a review), which is expected from radiationdriven wind theory (Pauldrach et al. 1988). Typical stellar wind velocities are of the 
order of $1000 \mathrm{~km} \mathrm{~s}^{-1}$. The corresponding mass loss rates can be typically 3 orders of magnitude less than that experienced near the end of the AGB phase (Cerruti-Sola \& Perinotto 1989; Perinotto 1989).

The transition from the AGB to the PN phase involves a change from a slow, dense wind, to a fast, thin wind (Kwok et al. (1978); Kwok 1982; Khan 1989). The uncertainty in the transition mechanism manifests itself as an uncertainty in the transition time from the AGB to the PN phase. In theoretical calculations conducted in the past, transition times were contrived to be of the order of 1000 yrs (e. g., Schönberner 1983; WF86; Schönberner 1989). Hydrodynamical models of PNe (e. g., Schmidt-Voigt \& Köppen 1987; Kahn \& Breitschwerdt 1990) rely on such calculations to describe the central star evolution. Examination of the dynamical ages of PNe indicate that transition times can vary from $10^{2}$ to $10^{4}$ yrs (Sabbadin 1986; Dopita et al. 1987; Ratag 1990), and a one-to-one correspondence between observed and theoretical evolutionary timescales through this phase is difficult to establish (Renzini 1989; McCarthy et al. 1990; Schönberner 1990).

In recent years, objects exhibiting spectra of $\mathrm{A}$ to $\mathrm{F}$ supergiants, while also having a large infrared excess presumably associated with a cool circumstellar dust shell, have been identified (e. g., Pottasch \& Partharasathy 1988; Hrivnak, Kwok, \& Volk 1989). Such objects are referred to as Pre- (or Proto) PNe because it is believed the dust shell formed on the AGB, and is now optically thin so that the central star can be seen. Thus, they presumably represent a stage of evolution between the AGB and the PN phases. More observations of such objects may provide tighter constraints on the theoretical description of the transitional phase (e. g., Trams et al. 1989, Waelkens et al. 1990 , Hu et al. 1990).

Finally, we note that there have been several studies attempting to relate the incidence of hydrogen $(\mathrm{H})$ and helium (He) burning PNNi, to the observed ratio of DA (i. e., H-rich) to non-DA white dwarfs (Iben 1984, 1987; WF86; Schönberner 1986; Renzini 1989 , and references therein). These studies indicate that He burning PNNi may constitute $\lesssim 25 \%$ of the total PN population. The mass distributions derived for white dwarf (WD) stars and PNNi are approximately the same which supports the suggestion of an evolutionary link between these two groups of objects (see Weidemann 1990, and references therein). There is a large range in WD stellar properties (particularly, in abundance) but no detailed unambiguous relationship has yet been made between the various subclasses of WD stars and particular categories of PNNi. Additional processes following the PN phase may be responsible for many WD stellar properties (see Liebert 
1989, and references therein; Bergeron et al. 1990), which are beyond the scope of the work presented here.

In this work we examine the evolution of the PNNi that result from the stellar evolutionary calculations presented in VW92. Consequently, the calculations also span a range in metallicity pertinent to the study of PNNi in the Galaxy and the Magellanic Clouds. No previous calculations have considered the effect of abundance on the evolution of post-AGB stars, though the matter has been discussed in the context of galactic evolution models (e. g., Brocato et al. 1989). We adopt a semi-empirical prescription of the mass loss rate in the PN phase based on the recent developments in radiation pressure driven stellar wind theory and on observed mass loss rates available in the literature. The occurrence of $\mathrm{H}$ and He burning evolutionary tracks is examined, though little effort is made to control the phase at which the AGB is terminated.

\subsection{Computational Details}

The Mount Stromlo Stellar Structure program was used to calculate the PN evolutionary sequences presented in this paper. The program is the same as that described in VW92. Additional modifications were made to incorporate mass loss in the post-AGB regime.

As with AGB evolution, mass loss is a crucial factor controlling timescales beyond the AGB. Although many estimates of mass loss rates have been made in the AGB and PN phases, there is little data pertaining to the phase of transition from AGB star to PN. In the present calculations, we have used a mass loss formula involving the terminal stellar wind velocity, $v_{\infty}$, which is moderately well known across the H-R diagram, and the ratio of the mass loss rate to the radiation pressure driven mass loss limit, $L /\left(c v_{\infty}\right)$.

\subsubsection{Terminal Wind Velocity}

Theory predicts that $v_{\infty}$ will increase with the stellar surface escape velocity, $v_{e s c}$. As a PNN evolves along a constant luminosity post-AGB evolutionary track towards higher values of $\mathrm{T}_{\text {eff }}$, its radius decreases and $v_{\text {esc }}$ subsequently increases (Abott 1982; Friend \& Abott 1986; Kudritzki et al. 1987; Kudritzki et al. 1989). We have used $v_{\infty}$ and $\log \mathrm{T}_{\text {eff }}$ values for PNe compiled by Pauldrach et al. (1988), and assumed $M=0.6$ $\mathrm{M}_{\odot}$ for all PNNi, implying $\log L / L_{\odot}=3.79$ (from the $0.6 \mathrm{M}_{\odot}$ tracks of WF86), to 
produce a plot of $v_{\infty} / v_{\text {esc }}$ against $\mathrm{T}_{\text {eff }}$ (figure 3.1). In this figure, $v_{\text {esc }}$ is defined by $v_{e s c}^{2}=2 G M / R$ rather than $v_{e s c}^{2}=2 G M(1-\Gamma) / R$, where $\Gamma$ is the ratio of the stellar luminosity to the Eddington luminosity. The use of individual masses (Méndez et al. $1988)$ and the latter definition of $v_{\text {esc }}$, causes $v_{\infty} / v_{\text {esc }}$ to change by less than $10 \%$ from the values shown. Heap (1986) presents wind velocities for many of the objects listed in Pauldrach et al. which result in values of $v_{\infty} / v_{\text {esc }}$ approximately $25 \%$ larger, and with greater scatter, than those presented by Pauldrach et al.

Predicted values of $v_{\infty}$ from radiation pressure driven wind theory (table $3 \mathrm{a}$, Pauldrach et al. 1988) are also indicated in figure 3.1. Given the uncertainty in $v_{\infty}$ and $\dot{\mathrm{M}}$ determinations for $\mathrm{PNNi}$, we have only shown the predictions given for PNNi with normal $\mathrm{He}$ content corresponding to the four masses defining the theoretical model grid used. Similarly, the decrease in $v_{\infty}$ and $\dot{M}$ with metallicity that is observed (cf. Kudritzki et al. 1987) is comparatively small compared to the uncertainty in these quantities, and is not included explicitly in the calculations.

The AGB stars from which PNe evolve lie at low values of $\mathrm{T}_{\text {eff }}$ in figure 3.1. $\mathrm{OH} / \mathrm{IR}$ stars, which represent a stage of evolution near the end of the AGB phase (e .g., Habing et al. 1989), can be characterized with the following parameters: $v_{\infty}=15 \mathrm{~km} \mathrm{~s}^{-1}$ (Eder, Lewis, \& Terzian 1988; Sivagnanam et al. 1989), $M \sim 0.6 \mathrm{M}_{\odot}, \log T_{\text {eff }}=3.48$, and $\log L / L_{\odot}=3.79$ (WF86). From these parameters, $v_{\text {esc }} \sim 30 \mathrm{~km} \mathrm{~s}^{-1}$ and $v_{\infty} / v_{\text {esc }} \sim$ 0.5. The fiducial point corresponding to the $\mathrm{OH} / \mathrm{IR}$ stellar parameters given above is indicated in figure 3.1 .

Between the PNe and AGB stars, recent observations of A-F supergiants with infrared excesses may prove useful. There are more such objects known now than when we began our calculations, but their evolutionary status is still not definite. Two such objects from de Jager et al. (1988) are plotted in figure 3.1. We have included data for B supergiants (Snow \& Morton 1976), which are slightly cooler than the O-type PNNi.

In figure 3.1 , there is a clear increase of $v_{\infty} / v_{\text {esc }}$ with $\mathrm{T}_{\text {eff }}$. We adopt a linear relationship between $\log \mathrm{T}_{\text {eff }}$ and $\log \left(v_{\infty} / v_{\text {esc }}\right)$ for the temperature range between the AGB and the hottest PNe (cf. figure 9, Abott 1982), but at a steeper slope than predicted by Pauldrach et al. from radiation-driven wind theory, for the $\mathrm{PNe}$ alone. A linear fit to the data presented in figure 3.1 yields,

$$
\log \frac{v_{\infty}}{v_{\text {esc }}}=-2.0+0.52 \log T_{\text {eff }},
$$

with a correlation coefficient of $\sim 0.7$. This fit is shown in figure 3.1 and is used in the calculations. 


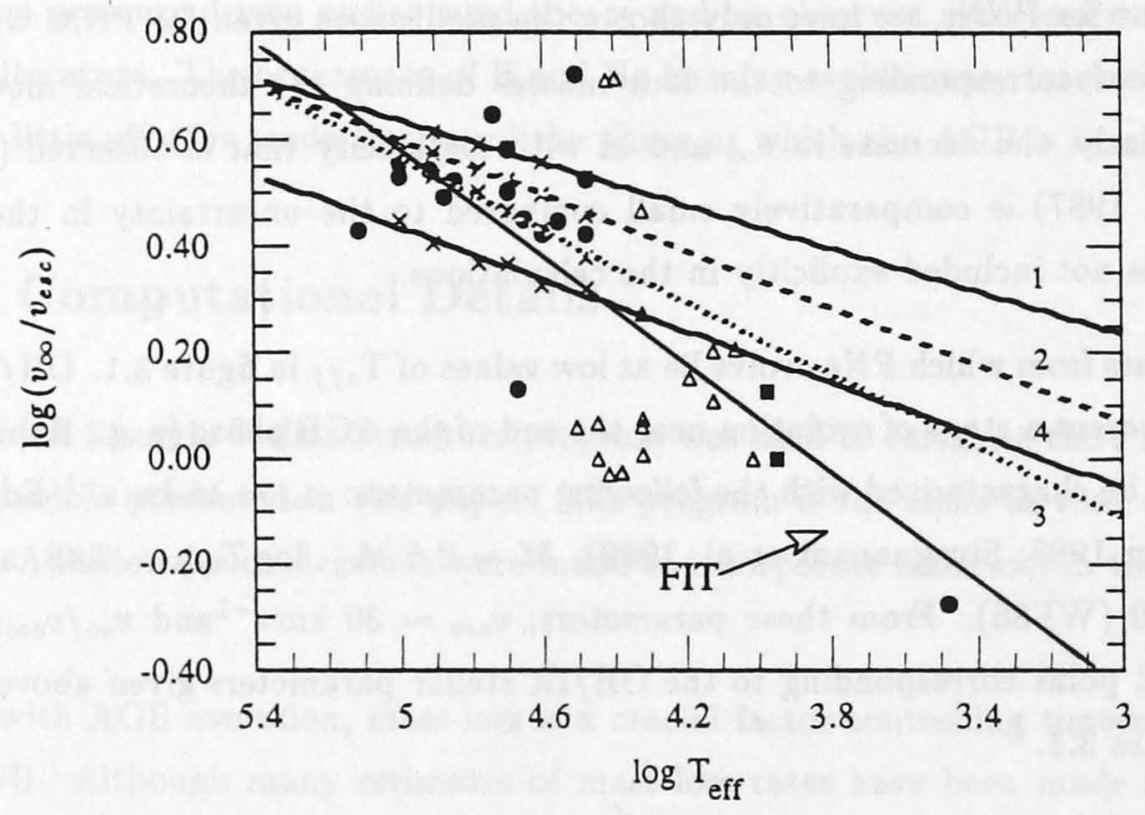

Figure 3.1: The observed terminal wind velocity, relative to the escape velocity at the stellar surface, as a function of the stellar effective temperature for Galactic PNe. The lines marked with crosses $(x)$ and denoted as $1,2,3$, and 4, represent the theoretical predictions of Pauldrach et al. (1988) for PNNi with masses $0.546,0.565,0.644$, and $1.0 \mathrm{M}_{\odot}$, respectively, which we have extended to cooler temperatures representative of the AGB. Filled circles denote PNe (Pauldrach et al. 1988), except for the point at $\log T_{\text {eff }} \sim 3.5$, which represents a mean position for OH/IR stars as defined in the text. Open triangles represent B stars (Snow \& Morton 1976), and filled squares represent A-F supergiants (de Jager et al. 1988). 


\subsubsection{Mass Loss Rate}

ERrATUM-page 118-119.

Different

page 119, line 16: Include ...It is important to note that one does not want to make a fit to the PN data alone, as it is over a very limited temperature range. The radiation-driven wind models merely define an initial slope. It is granted that based on the available observations there is no clear correlation, such as that exhibited between $\dot{M}$ and luminosity for massive $O B$ Pop. I stars. There is an J0 for any eview). Igh to the mit which on field is additional problem of constraining $\dot{M}$ at the point of departure from the $A G B$ : One can either have $\dot{M} \sim \dot{M}_{\text {limit }}^{A G B}$ just before a helium shell flash, or $\dot{M} \ll \dot{M}_{\text {limit }}^{A G B}$ just after.

Although not indicated, current observational data may have an order of magnitude difference in $\dot{M}$ for the same object measured with different techniques. If $\dot{M}$ is increased by a factor of 10 , the rate of evolution, particulary through the constant luminosity phase, will increase. In the models presented in this study, the explicit mass loss is already superceded by the mass lost due to $H$ burning by the time $\log T_{\text {eff }} \sim 4.5$, by a factor of $\sim 2.5$. For low mass models, this remains true even once evolution begins down the cooling track. For high mass models $\left(M_{i} \geq 2.5 M_{\odot}\right)$, the corresponding factor is $\leq 2.5$. In contrast, the Reimer's rate is an order of magnitude smaller than the mass loss rate used here. As figure 3.2 shows, current observations are inadequate to constrain the mass loss rate to a desired accuracy.

Perinotto
wn are the
ical model
tight cor-
enormous
ip,
ope to the
would not
5 mass loss
from PNNi.

In VW92 we constrained the mass loss rate for the preceding AGB phase of evolution such that $\dot{M} \lesssim \dot{M}_{\text {limit }}^{A G B}$. There does not appear to be any calibrating data for $\dot{M}$ between the AGB and PN phases, and we have adopted equation 3.3 to give $\dot{M}$ in all post-AGB phases. The transition point at which the AGB mass loss was stopped, and the mass loss rate described above was adopted, occurred when $\log \mathrm{T}_{\text {ef } f}$ increased from a fiducial AGB temperature by $0.3 \mathrm{dex}$ (VW92). Initial tests with $\Delta \log T_{\text {eff }}=0.05$ indicated that such a value was too small: the switch over to the lower PN mass loss rate was too early, resulting in the evolution taking at least $10^{5}$ years through the transition region. This is in conflict with dust shell models matched to the observed infrared flux distribution of non-pulsating (and thus, presumably, post-AGB) OH/IR objects (Kwok et al. 1989, and references therein) which indicate that heavy mass loss must have ceased by the time when the star reaches $\sim 5000 \mathrm{~K}$. We finally adopted $\Delta \log T_{\text {eff }}=0.3$ as 


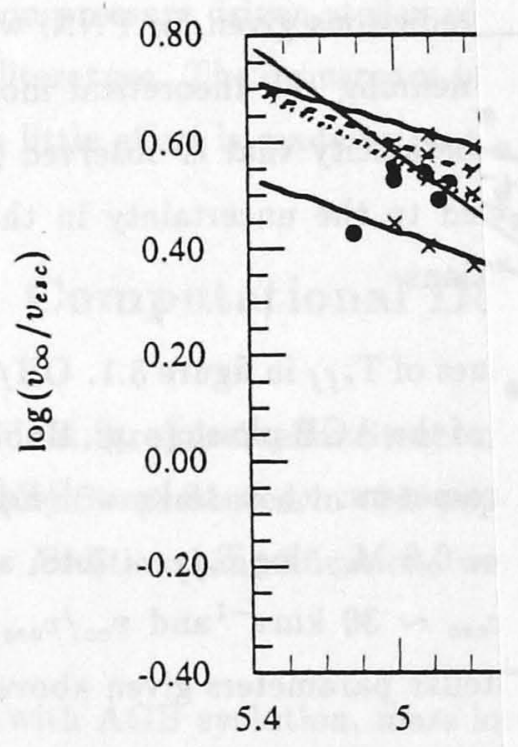

Figure 3.1: The observed terminal wind velocity, relative to the escape velocity at the stellar surface, as a function of the stellar effective temperature for Galactic PNe. The lines marked with crosses $(x)$ and denoted as $1,2,3$, and 4, represent the theoretical predictions of Pauldrach et al. (1988) for PNNi with masses $0.546,0.565,0.644$, and $1.0 \mathrm{M}_{\odot}$, respectively, which we have extended to cooler temperatures representative of the AGB. Filled circles denote PNe (Pauldrach et al. 1988), except for the point at $\log T_{\text {eff }} \sim 3.5$, which represents a mean position for OH/IR stars as defined in the text. Open triangles represent B stars (Snow \& Morton 1976), and filled squares represent A-F supergiants (de Jager et al. 1988). 


\subsubsection{Mass Loss Rate}

Large uncertainties exist in the determination of mass loss rates for PNNi. Different investigators can arrive at mass loss rates which differ by factors of 10-100 for any particular object (Cerruti-Sola \& Perinotto 1989; see Perinotto 1989, for a review).

Now that we can prescribe the behaviour of $v_{\text {exp }}$ from the AGB through to the PN phase, we can determine the radiation pressure driven mass loss rate limit which corresponds to the situation where all the momentum of the stellar radiation field is passed to a wind,

$$
\dot{M}_{\text {limit }}=\frac{L}{c v_{\infty}}
$$

where $L$ represents the stellar luminosity and $c$ represents the speed of light.

Mass loss rates (relative to $\dot{\mathrm{M}}_{\text {limit }}$ ) from the literature (see compilations by Perinotto 1989 and Hutsemékers \& Surdej 1989) are depicted in figure 3.2. Also shown are the theoretically predicted mass loss rates for the four given masses of the theoretical model grid of Pauldrach et al. (see their table 3a). The models define a relatively tight correlation between $\log \dot{\mathrm{M}} / \dot{\mathrm{M}}_{\text {limit }}$ and $\log \mathrm{T}_{\text {eff }}$ which approximately bisects the enormous scatter of the observational data. We have adopted a mean linear relationship,

$$
\log \frac{\dot{M}}{\dot{M}_{\text {limit }}}=-3.92+0.67 \log T_{\text {eff }},
$$

to describe the mass loss rate in the PN regime, approximately equal in slope to the model data of Pauldrach et al. Given the enormous observational scatter, it would not be surprising if factors other than radiation pressure were at work producing mass loss from PNNi.

In VW92 we constrained the mass loss rate for the preceding AGB phase of evolution such that $\dot{M} \lesssim \dot{M}_{\text {limit }}^{A G B}$. There does not appear to be any calibrating data for $\dot{M}$ between the AGB and PN phases, and we have adopted equation 3.3 to give $\dot{M}$ in all post-AGB phases. The transition point at which the AGB mass loss was stopped, and the mass loss rate described above was adopted, occurred when $\log \mathrm{T}_{\text {eff }}$ increased from a fiducial AGB temperature by $0.3 \mathrm{dex}$ (VW92). Initial tests with $\Delta \log T_{\text {eff }}=0.05$ indicated that such a value was too small: the switch over to the lower PN mass loss rate was too early, resulting in the evolution taking at least $10^{5}$ years through the transition region. This is in conflict with dust shell models matched to the observed infrared flux distribution of non-pulsating (and thus, presumably, post-AGB) OH/IR objects (Kwok et al. 1989, and references therein) which indicate that heavy mass loss must have ceased by the time when the star reaches $\sim 5000 \mathrm{~K}$. We finally adopted $\Delta \log T_{\text {eff }}=0.3$ as 


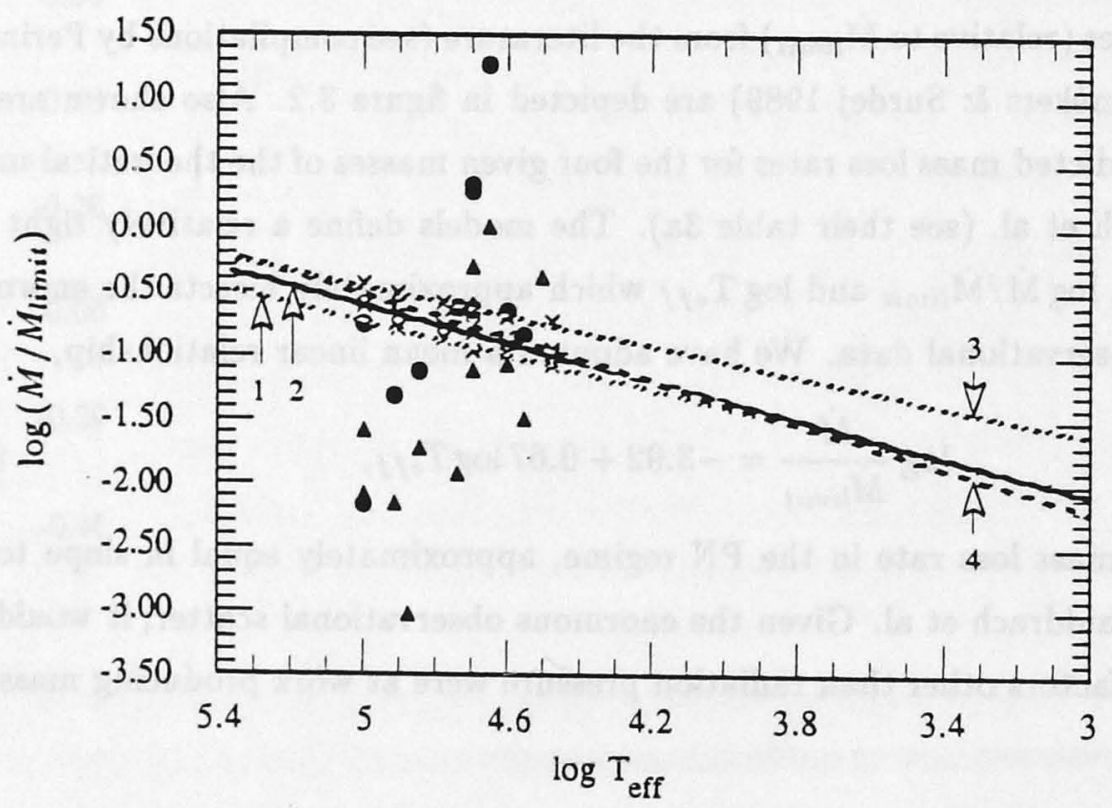

Figure 3.2: The observed mass loss rate, relative to the radiation pressure driven limit, as a function of stellar effective temperature. The lines are theoretical predictions of Pauldrach et al. (1988), as in figure 3.1. Filled triangles represent data taken from Hutsemékers \& Surdej (1989), and filled circles represent data taken from Perinotto (1989, and references therein). 
being more appropriate. From examination of the model data in VW92, it is seen that the transition point of our calculations occurred in the range $3.5 \lesssim \log T_{\text {eff }} \lesssim 3.7$, for the corresponding range in initial mass of $5 \geq M_{i} \gtrsim 1$.

\subsection{Results}

\subsubsection{The Calculations}

We have calculated 27 PNN evolutionary tracks from the 22 AGB sequences presented in VW92, where 18 of the PNN sequences are $\mathrm{H}$ burning and 9 are He burning. The additional 5 tracks ( $2 \mathrm{H}$ burning and $3 \mathrm{He}$ burning) were computed by slightly varying the applied AGB mass loss rate over the final 2-3 thermal pulses to achieve the desired phase of departure, $\phi$, from the AGB. In this paper, we define $\phi$ to be the phase of the shell flash cycle at the transition point where the AGB mass loss rate is terminated. The change in $\dot{M}$ was administered in this fashion to minimize the effects on the thermal equilibrium of the models (cf. Schönberner 1989). Changes in the applied mass loss rate were of the order of $10 \%$. The remaining 22 evolutionary sequences followed the AGB mass loss prescription given in VW92. The variation in $\phi$ seen between the different sequences is a natural consequence of the mass loss behaviour during the AGB and transition phases.

We have divided the PNN evolutionary tracks into $\mathrm{H}$ and He burning by their morphology in the Hertzsprung-Russell (H-R) diagram. The starting point characteristics and the fading times for each sequence are tabulated in tables 3.1 and 3.2 for the $\mathrm{H}$ and He burning sequences, respectively. The tracks begin at $\log T_{\text {eff }}=4$, and are grouped according to metallicity, $Z=0.016,0.008,0.004$, and 0.001 , respectively (figures 3.3-3.9). The tracks are also presented in tabular form in table 3.3: 45 points are listed down to $\log L / L_{\odot} \sim-1$. This is simply an arbitrary starting point for comparing relative timescales of evolution.

We have defined a transition time, $\mathrm{t}_{t r}$ (cf. Renzini 1989; Schönberner 1990), which denotes the time elapsed from the point where the PN mass loss formalism is activated (movement off the AGB by $\Delta \log T_{\text {eff }}=0.3$ ), to the point where $\log T_{\text {eff }}=4$. The transition time is extremely uncertain as it is determined by the essentially unknown mass loss rate in the transition domain. Values for $t_{t r}$ are listed in tables 3.1 and 3.2 for each evolutionary sequence. This quantity has primarily been included to serve as a link between the AGB calculations presented in VW92, and the PNN calculations 
here.

It can be seen that $t_{t r}$ varies markedly between 3000 and 20000 years, with no obvious correlation with $\mathrm{M}_{i}$. In addition to the mass loss rate, $t_{t r}$ seems to be affected by the value of $\phi$ at which $\Delta \log T_{\text {eff }}=0.3$ is encountered. At the point of transition, $\dot{\mathrm{M}}$ instantaneously falls from a typical high AGB mass loss rate of the order of $10^{-5}$ $\mathrm{M}_{\odot} \mathrm{yr}^{-1}$ to a value determined by to a typical PNN mass loss rate of the order of $10^{-7}$ $\mathrm{M}_{\odot} \mathrm{yr}^{-1}$.

The situation becomes slightly more complex for He-burning tracks. Characteris-

tic fast ( $\sim 5000$ years), blue ( $\log T_{\text {eff }} \lesssim 4.6$ ) loops (cf. Iben 1984; WF86) can occur which result in evolution back towards the AGB for an additional period of time. If $\Delta \log T_{\text {eff }}<0.3$ results, then the high AGB mass loss rate may be reinstated, resulting in an increased rate of evolution as the envelope mass decreases more rapidly. We also note that the inter-pulse time of the highest mass models towards the later stages of the thermal pulsing AGB phase ( $\sim 6000$ years), can be at least a factor of 2 smaller than the quoted transition times. Thus, one or more thermal pulses can be experienced through the transition region. The envelope mass is still large enough that these helium shell flashes do not produce fast blue loops in the H-R diagram.

Finally, we draw attention to the $(M, Z)=(0.95,0.008)$ and $(0.89,0.004)$ evolutionary sequences, constructed specifically for producing post-AGB models with ages equivalent to the age of the Universe ( 13-15 Gyrs). Recall that a Reimers-type mass loss was included during the first giant branch phase of evolution for these two evolutionary sequences (see VW92 for details). This will obviously decrease the amount of mass lost on the AGB. The total stellar masses at the beginning of thermal pulsing AGB phase were 0.722 and $0.691 \mathrm{M}_{\odot}$ for the $M_{i}=0.95$ and $0.89 \mathrm{M}_{\odot}$ sequences, respectively.

\subsubsection{Comparison with Previous Calculations}

WF86 present ages with respect to $\log T_{\text {eff }}=4$ and so a direct comparison can be made of the relative timescales, particularly for the $Z=0.016$ sequences with $M_{i}=1.5,3.5$, and $5.0 \mathrm{M}_{\odot}$ : these are the only results we have that are close enough to the metallicity and core mass values presented by WF86. As pointed out by these authors, their $\phi=0.25,0.5$, and 0.75 calculations are very similar, though ages can vary by a factor as large as 2 along any of these tracks for $t \lesssim 30000$ years. From the data they present in their table 1 , the dispersion in $\log L / L_{\odot}$ caused by $\phi$ varying from 0.25 to 0.75 for 
TABLE 3.1

H-BURNING TRACKS

\begin{tabular}{|c|c|c|c|c|c|c|c|c|}
\hline \multirow[b]{2}{*}{$\begin{array}{c}\mathbf{M}_{i} \\
\left(\mathbf{M}_{\odot}\right)\end{array}$} & \multirow[b]{2}{*}{${ }^{a} N_{T P}$} & \multicolumn{3}{|c|}{$\log T_{e f f}=4$} & \multirow[b]{2}{*}{$\begin{array}{c}b_{t_{t r}} \\
\left(10^{3} y r s\right) \\
\end{array}$} & \multicolumn{3}{|c|}{${ }^{\mathrm{d}} \log \mathrm{L} / \mathrm{L}_{\odot}$} \\
\hline & & $\begin{array}{c}\text { time } \\
\text { (Gyrs) }\end{array}$ & $\log \mathrm{L} / \mathrm{L}_{\odot}$ & $\begin{array}{c}\mathrm{M} \\
\left(\mathrm{M}_{\odot}\right)\end{array}$ & & $\begin{array}{l}+10^{4} \\
(\mathrm{yrs})\end{array}$ & $\begin{array}{r}+3 \times 10^{4} \\
(\mathrm{yrs})\end{array}$ & $\begin{array}{l}+10^{5} \\
(\mathrm{yrs})\end{array}$ \\
\hline \multicolumn{9}{|c|}{$\mathrm{Z}=0.016 \mathrm{Z}_{\odot}$} \\
\hline $1.0 \ldots$ & 7 & 14.967 & 3.54 & 0.569 & 13.3 & 3.56 & 3.20 & 2.07 \\
\hline $1.5^{\mathrm{c}} \ldots$ & 11 & 3.648 & 3.74 & 0.597 & 8.6 & 3.43 & 2.17 & 1.77 \\
\hline $2.0^{c} \ldots$ & 16 & 1.560 & 3.89 & 0.633 & 11.6 & 2.12 & 1.96 & 1.62 \\
\hline $2.5 \ldots$ & 28 & 0.956 & 3.98 & 0.677 & 12.2 & 2.15 & 1.90 & 1.54 \\
\hline 3.5 & 16 & 0.336 & 4.14 & 0.754 & 22.7 & 2.20 & 1.83 & 1.41 \\
\hline $5.0 \ldots$ & 29 & 0.123 & 4.36 & 0.900 & 31.1 & 1.97 & 1.64 & 1.22 \\
\hline \multicolumn{9}{|c|}{$\mathrm{Z}=0.008 \mathrm{Z}_{\odot}$} \\
\hline 1.5 & 11 & 3.114 & 3.88 & 0.620 & 11.4 & 3.37 & 2.50 & 1.78 \\
\hline 2.0 & 18 & 1.312 & 3.98 & 0.668 & 8.1 & & . & \\
\hline 3.5 & 17 & 0.277 & 4.22 & 0.797 & 17.5 & 2.06 & 1.71 & 1.31 \\
\hline $5.0 \ldots$ & 42 & 0.111 & 4.37 & 0.912 & 5.0 & 1.84 & 1.49 & 1.04 \\
\hline \multicolumn{9}{|c|}{$\mathrm{Z}=0.004 \mathrm{Z}_{\odot}$} \\
\hline $1.0 \ldots$ & $\overline{9}$ & 8.898 & 3.64 & 0.593 & 6.5 & 3.66 & 2.26 & 1.82 \\
\hline $1.5 \ldots$ & 12 & 2.643 & 3.87 & 0.640 & 5.0 & 2.19 & 1.94 & 1.60 \\
\hline $2.0 \ldots$ & 21 & 1.163 & 3.92 & 0.672 & 8.0 & 2.41 & 1.96 & 1.48 \\
\hline $2.5 \ldots$ & 20 & 0.661 & 4.15 & 0.692 & 10.0 & 2.62 & 1.89 & 1.35 \\
\hline $3.5 \ldots$ & 19 & 0.247 & 4.30 & 0.855 & 10.0 & 1.91 & 1.62 & 1.24 \\
\hline $5.0 \ldots$ & 47 & 0.102 & 4.40 & 0.943 & 8.4 & 1.80 & 1.46 & 1.02 \\
\hline \multicolumn{9}{|c|}{$\mathrm{Z}=0.001 \mathrm{Z}_{\odot}$} \\
\hline 1.0 & 12 & 7.211 & 3.78 & 0.623 & 1.7 & 3.71 & 1.97 & 1.62 \\
\hline 1.5 & 14 & 2.092 & 3.99 & 0.663 & 2.9 & $\cdots$ & $\ldots$ & $\ldots$ \\
\hline
\end{tabular}

a $\mathrm{N}_{T P}$ denotes the number of thermal pulses experienced on the AGB prior to $\Delta \log T_{\text {eff }}=0.3$.

${ }^{b} t_{t r}$ denotes the elapsed time from $\Delta \log T_{\text {eff }}=0.3$ to $\log T_{\text {eff }}=4$.

${ }^{c}$ Artificially enhanced and/or diminished mass loss rates employed on the AGB.

${ }^{d}$ Values of $\log \mathrm{L} / \mathrm{L}_{\odot}$ when the indicated time intervals have elapsed since the time when $\log T_{\text {eff }}=4$. 
TABLE 3.2

He-BURNING TRACKS

\begin{tabular}{|c|c|c|c|c|c|c|c|c|}
\hline \multirow[b]{2}{*}{$\begin{array}{c}\mathbf{M}_{i} \\
\left(\mathbf{M}_{\odot}\right)\end{array}$} & \multirow[b]{2}{*}{${ }^{\mathrm{a}} \mathrm{N}_{T P}$} & \multicolumn{3}{|c|}{$\log \mathrm{T}_{\text {eff }}=4$} & \multirow[b]{2}{*}{$\begin{array}{c}{ }^{b} t_{t r} \\
\left(10^{3} y r s\right)\end{array}$} & \multicolumn{3}{|c|}{${ }^{d} \log \mathrm{L} / \mathrm{L}_{\odot}$} \\
\hline & & $\begin{array}{c}\text { time } \\
\text { (Gyrs) }\end{array}$ & $\log \mathrm{L} / \mathrm{L}_{\odot}$ & $\begin{array}{c}\mathbf{M} \\
\left(\mathrm{M}_{\odot}\right)\end{array}$ & & $\begin{array}{l}+10^{4} \\
(\mathrm{yrs})\end{array}$ & $\begin{array}{r}+3 \times 10^{4} \\
(\mathrm{yrs}) \\
\end{array}$ & $\begin{array}{l}+10^{5} \\
(\mathrm{yrs})\end{array}$ \\
\hline \multicolumn{9}{|c|}{$\mathrm{Z}=0.016 \mathrm{Z}_{\odot}$} \\
\hline $1.0^{c}$. & 7 & 14.967 & 3.53 & 0.567 & 5.5 & 3.15 & 3.34 & 2.20 \\
\hline 1.5 & 11 & 3.648 & 3.61 & 0.600 & 7.6 & 3.38 & 3.50 & 1.87 \\
\hline 2.0 & 16 & 1.560 & 3.84 & 0.634 & 13.0 & 3.34 & 2.43 & 1.71 \\
\hline \multicolumn{9}{|c|}{$\mathrm{Z}=0.008 \mathrm{Z}_{\odot}$} \\
\hline 0.95 & 6 & 13.703 & 3.50 & 0.554 & 22.3 & 3.13 & 2.88 & 2.35 \\
\hline 1.0 & 7 & 11.049 & 3.60 & 0.578 & 3.5 & 3.22 & 3.36 & 2.07 \\
\hline $1.5^{\mathrm{c}}$. & 11 & 3.114 & 3.79 & 0.626 & 3.4 & 3.37 & 2.57 & 1.74 \\
\hline $2.0^{c} \ldots$ & 18 & 1.312 & 3.92 & 0.669 & 16.3 & 3.69 & 2.17 & 1.60 \\
\hline $2.5 \ldots$ & 25 & 0.784 & 3.95 & 0.679 & 10.3 & 2.93 & 2.03 & 1.48 \\
\hline \multicolumn{9}{|c|}{$\mathrm{Z}=0.004 \mathrm{Z}_{\odot}$} \\
\hline 0.89 & 7 & 13.717 & 3.62 & 0.558 & 3.2 & 3.20 & 2.85 & 2.37 \\
\hline
\end{tabular}

${ }^{a} \mathrm{~N}_{T P}$ denotes the number of thermal pulses experienced on the AGB prior to $\Delta \log T_{\text {eff }}=0.3$.

${ }^{b} t_{t r}$ denotes the elapsed time from $\Delta \log T_{\text {eff }}=0.3$ to $\log T_{\text {eff }}=4$.

${ }^{c}$ Artificially enhanced and/or diminished mass loss rates employed on the AGB.

${ }^{d}$ Values of $\log \mathrm{L} / \mathrm{L}_{\odot}$ when the indicated time intervals have elapsed since the time when $\log T_{\text {eff }}=4$. 
their H-burning PNN tracks is $\lesssim 0.07, \lesssim 0.03$, and $\lesssim 0.03$, at $+10000,+30000$, and +100000 years, respectively. Our $M \sim 0.6$ and $0.89 \mathrm{M}_{\odot}$ PNN tracks are consistently fainter at the three listed times by $0.1-0.3 \mathrm{dex}$ in $\log L / L_{\odot}$. The apparent quicker evolution is most likely due to the inclusion of mass loss during the PN phase in this study: WF86 stopped mass $\operatorname{loss}$ for $\log T_{\text {eff }}>3.8$. Our $M \sim 0.76$ PNN track appears equivalent to that presented by WF86, given the scatter in $\log L / L_{\odot}$ with $\phi$ quoted above. Comparing $\log L / L_{\odot}$ values at the starting point $\log T_{\text {eff }}=4$, we find that our results are $\sim 0.04$ dex fainter for the two smaller core masses considered. There is no difference in $\log \mathrm{L} / \mathrm{L}_{\odot}$ for the $M \sim 0.89 \mathrm{M}_{\odot}$ PNN tracks.

We now compare the $0.6 \mathrm{M}_{\odot}$ PNN tracks of the present work, Schönberner (1981), Iben (1984), WF86, and Blöcker \& Schönberner (1990). The time spent on the horizontal, constant luminosity, portion of the PNN track from $\log T_{\text {eff }}=4$ for WF86 is $\sim 12000$ years. The corresponding values from the Schönberner (figure 3a), Iben (figure 1), and Blöcker \& Schönberner (figure 1) studies are $\sim 7000, \sim 18000$, and $\sim 6000$ years, respectively. Our PNN track stays at a constant luminosity for $\sim 8000$ years, comparable to the Schönberner and Blöcker \& Schönberner results. This is most likely due to the fact that these calculations include some form of mass loss into the post-AGB regime whereas WF86 and Iben (in the case quoted) do not.

With regard to the fading timescales, we have already pointed out that our calculations are $\sim 0.2$ dex fainter in $\log L / L_{\odot}$ at +30000 years than the WF86 calculations; $\log L / L_{\odot}=2.17$, compared to 2.35 , respectively. The corresponding values from the Schönberner, Iben, and Blöcker \& Schönberner studies are $\log L / L_{\odot}=1.9,1.8$, and 2.1 , respectively.

Despite the similarity between the $M \sim 0.6$ PNN tracks of this study and that of Blöcker \& Schönberner, the situation is markedly different for the $M \sim 0.84 \mathrm{M}_{\odot}$ PNN track they present. This track has $\log L / L_{\odot} \sim 2.3$ at +30000 years while our calculations indicate that we should have $\log L / L_{\odot} \sim 1.72$, from interpolation between the results at $M=0.75$ and $0.90 \mathrm{M}_{\odot}$, at the same time. The higher rate of fading of our $Z=0.016$ sequence is consistent with the lower metallicity PNN sequences.

We have only one He burning PNN sequence with $Z=0.016$ which has a mass that can be matched with an equivalent sequence in WF86. The rate of evolution for the $M=0.6 \mathrm{M}_{\odot}$ PNN tracks are approximately the same up to 10000 years. In this case, this is equivalent to the point where the $\mathrm{H}$-shell reignites. During the $\mathrm{H}$-shell burning phase, the evolution of our PNN sequence is slower. It took $\sim 36000$ years to reach the 
luminosity of the WF86 sequence at +30000 years, although both PNN tracks reached $\log L / L_{\odot} \sim 1.87$ at +100000 years. This result highlights the diversity of behaviour possible for the He Burning PNNi as a result of slightly different phases of departure from the AGB. The slightly different masses of $\mathrm{H}$ left to burn on the star can alter the lifetimes in the PNN phase. If a flash occurs when the star is still a relatively cool $\mathrm{PNN}$, then there will be considerable $\mathrm{H}$ left to burn when the $\mathrm{H}$ shell reignites, and the PNN lifetime will be prolonged.

The sensitivity of He burning PNN tracks to $\phi$ has been noted in previous studies (Iben 1984, 1987; WF86, figure 5). The majority of the helium shell flashes experienced by our PNN models occurred prior to $\log T_{\text {eff }}=3.7$, and they exhibited behaviour similar to that depicted in figure 6 of Iben (1984). In one case, the $M=2 \mathrm{M}_{\odot} \mathrm{He}$ burning PNN sequence (figure 3.7) experienced a He shell flash at $\log T_{\text {eff }}=3.82$, when the $\mathrm{H}$ envelope mass was small, and the subsequent reignition of shell $\mathrm{H}$ burning occurred later than was the case for the other He burning tracks. Relatively little time is spent burning $\mathrm{H}$ after the shell flash in this evolutionary sequence.

We point out that the $(M, Z)=(2.0,0.004) \mathrm{H}$ burning PNN track presented in figure 3.5 , is recovering from a helium shell flash and its luminosity subsequently increases as it evolves during the high luminosity phase. Also note that two of the H-burning PNN tracks presented, $(M, Z)=(2,0.008)$ and $(M, Z)=(1.5,0.001)$, end prematurely due to mixing flash events. This has been seen in some of the previous calculations of Schönberner, Iben, and WF86. We did not follow the subsequent evolution, but a procedure to bypass the mixing produced by the flash (Iben et al. 1983) has shown that the star evolves back towards the AGB. The loops observed for the low mass He burning PNN tracks at $\log T_{\text {eff }} \sim 4.5$ correspond to the secondary luminosity maximum characteristic of helium shell flashes in low mass stars (e. g., Iben 1982).

Finally, we note that the $M_{i}=5 \mathrm{M}_{\odot}$ sequences produce core masses of $\sim 0.9 \mathrm{M}_{\odot}$ which are just on the limit above which radiation pressure will blow off the envelope during a helium shell flash (WF86). Even during quiescent $\mathrm{H}$ burning there exist PNNi that have luminosities near the Eddington limit. They will probably have high mass loss rates in the PN regime, possibly higher than implied by our calculations because we have neglected the $(1-\Gamma)$ factor in our definition of $v_{e s c}$. Thus the evolutionary timescales given here may be too long. 


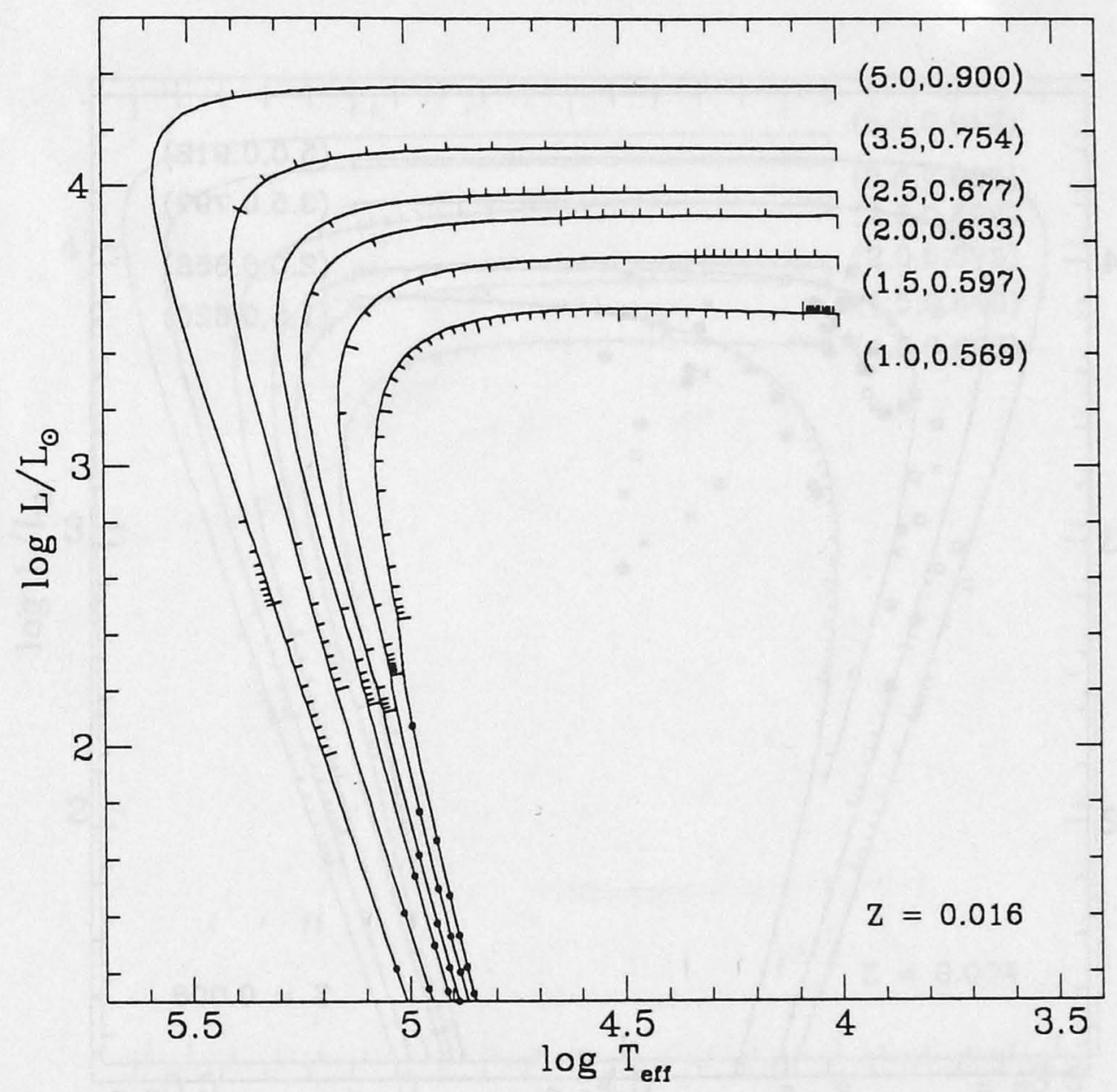

Figure 3.3: $\mathrm{H}$ burning PNN evolutionary tracks for $Z=0.016$. Each track is labelled with its corresponding values of initial (i .e., main sequence) mass, and core mass at $\log T_{\text {eff }}=4$. Tick marks on the upper (or left) side of the PNN tracks are in steps of 100 years, and only continue until $t=1000$ years is reached. Tick marks on the under (or right) side of the PN tracks are in steps of 1000 years. The filled circles on the cooling portions of the PN tracks represent time steps of 100000 years, the first point in each case beginning at $t=100000$ years. 


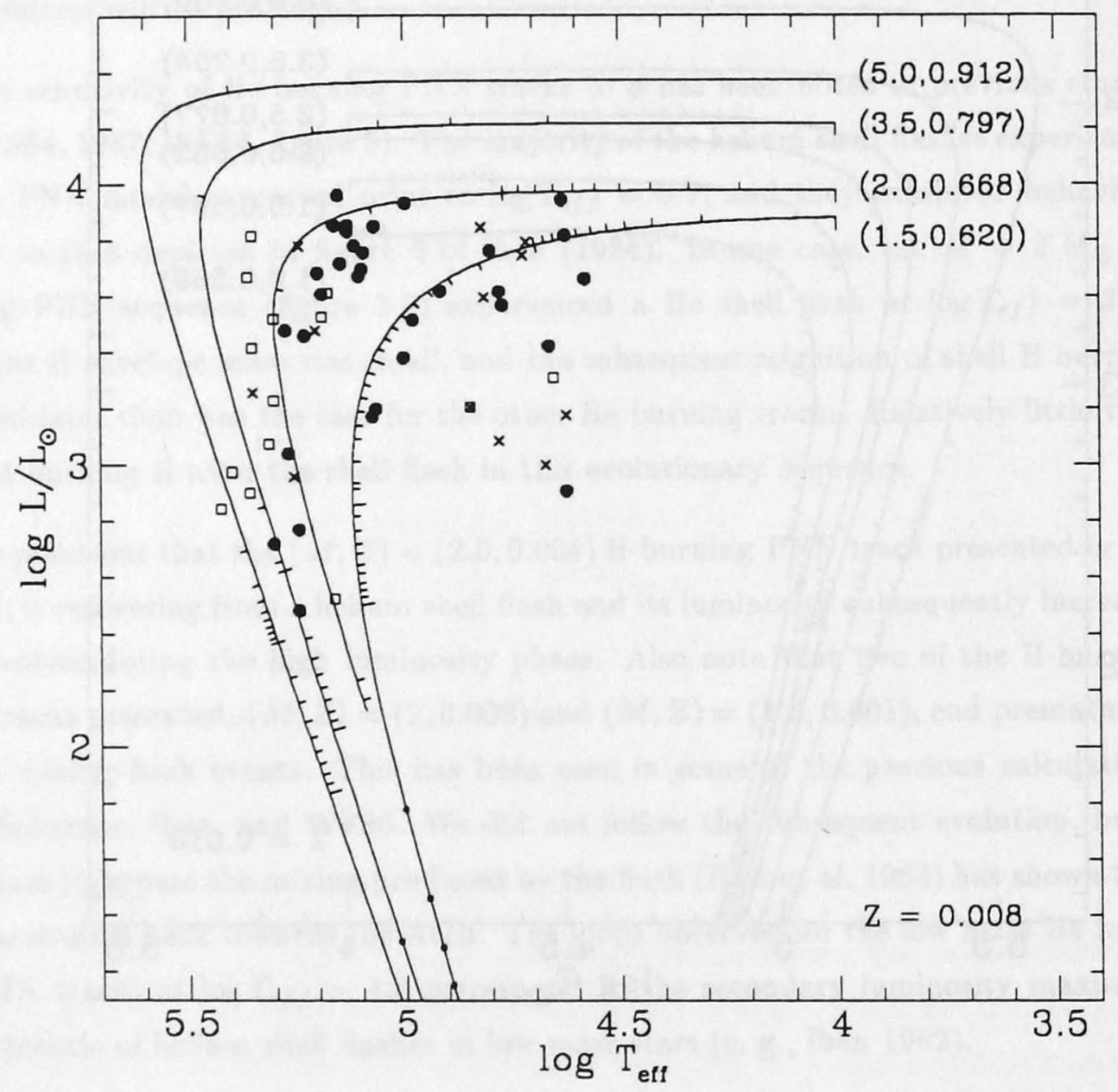

Figure 3.4: Same as figure 3.3, except for $Z=0.008$. Also shown is data for LMC PNe taken from Dopita \& Meatheringham (1991a, b): the filled circles represent optically thick objects, the open squares represent type I PNe, and the crosses represent optically thin objects. 


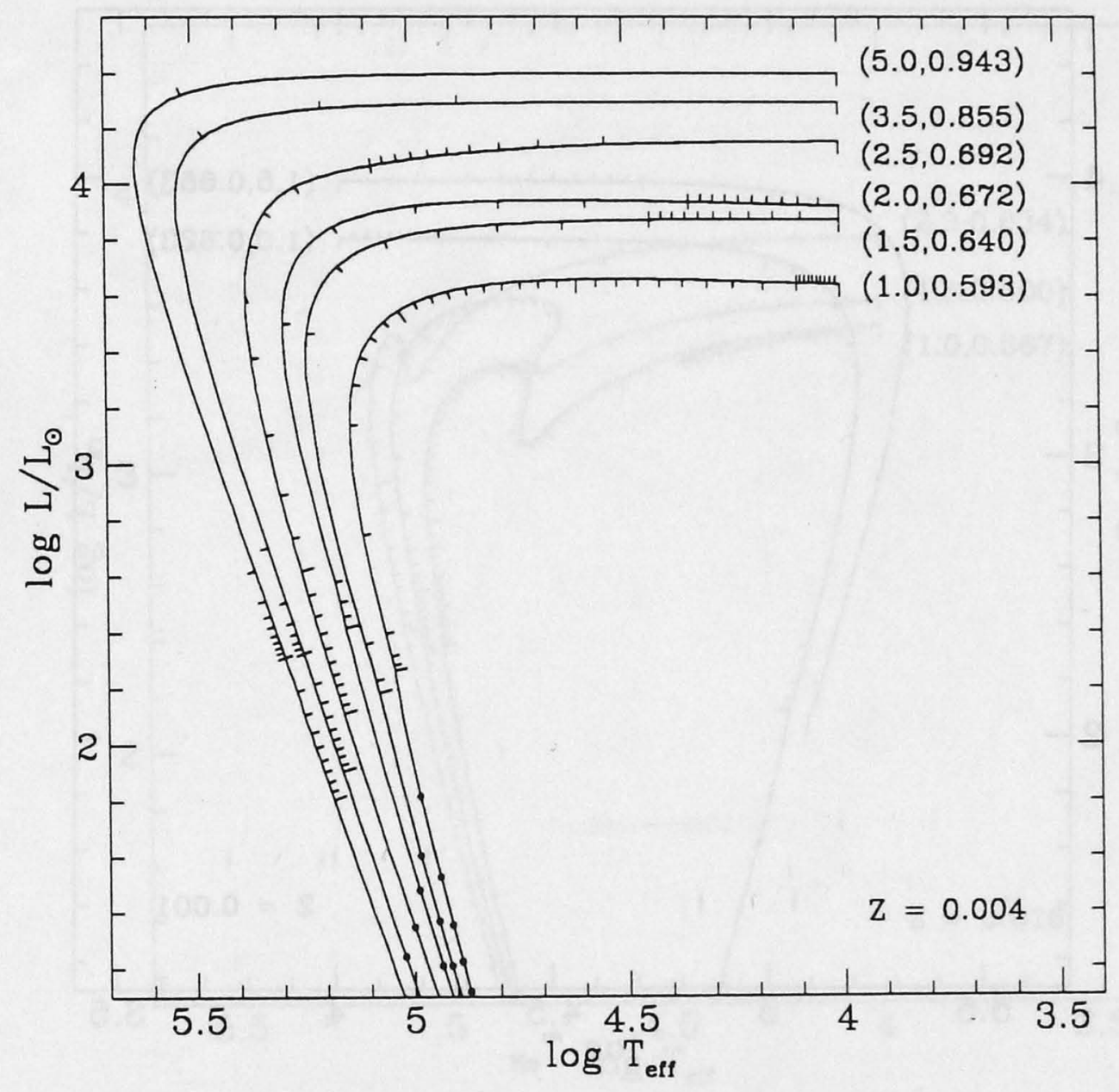

Figure 3.5: Same as figure 3.3, except for $Z=0.004$. 


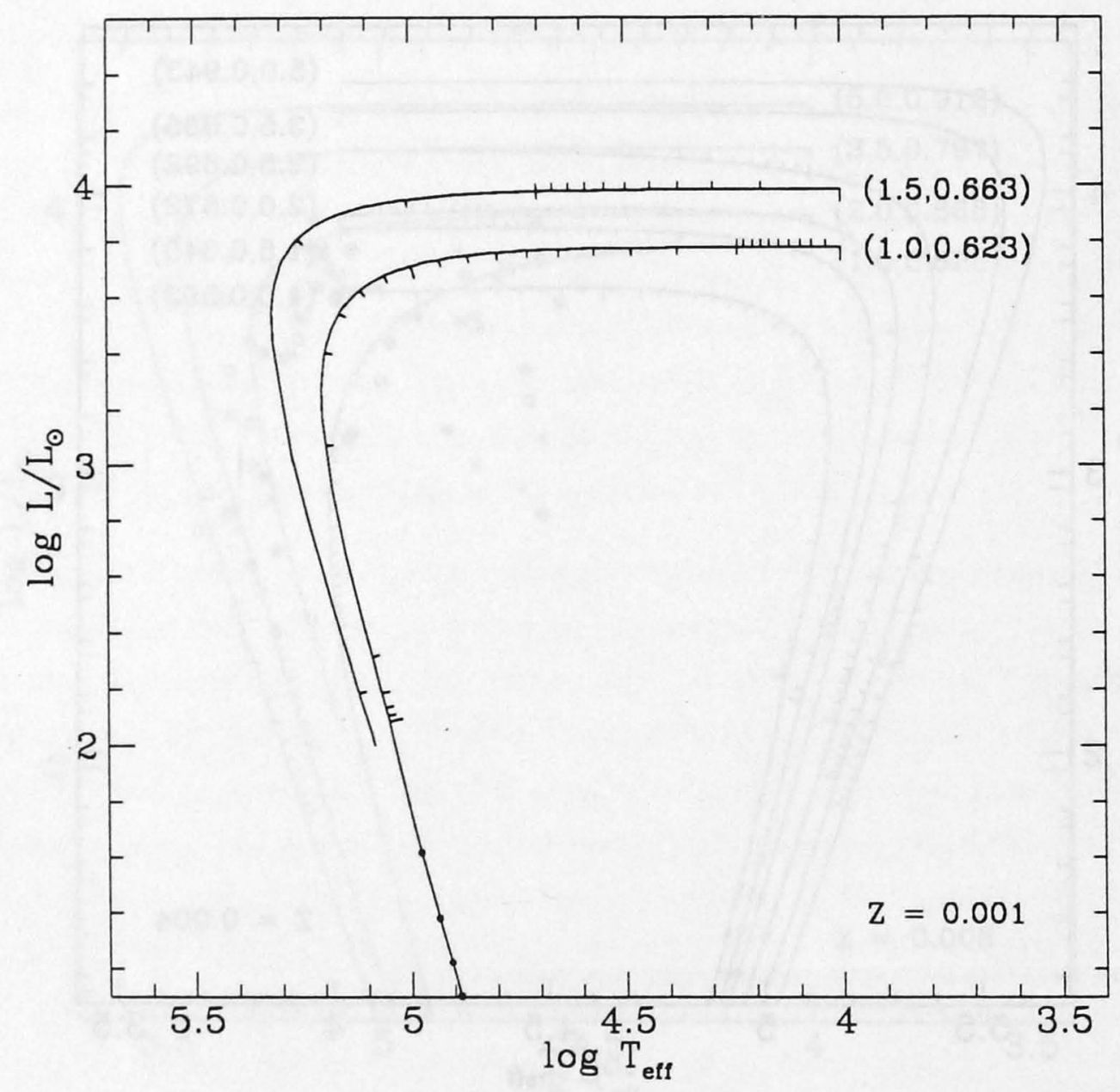

Figure 3.6: Same as figure 3.3, except for $Z=0.001$. 


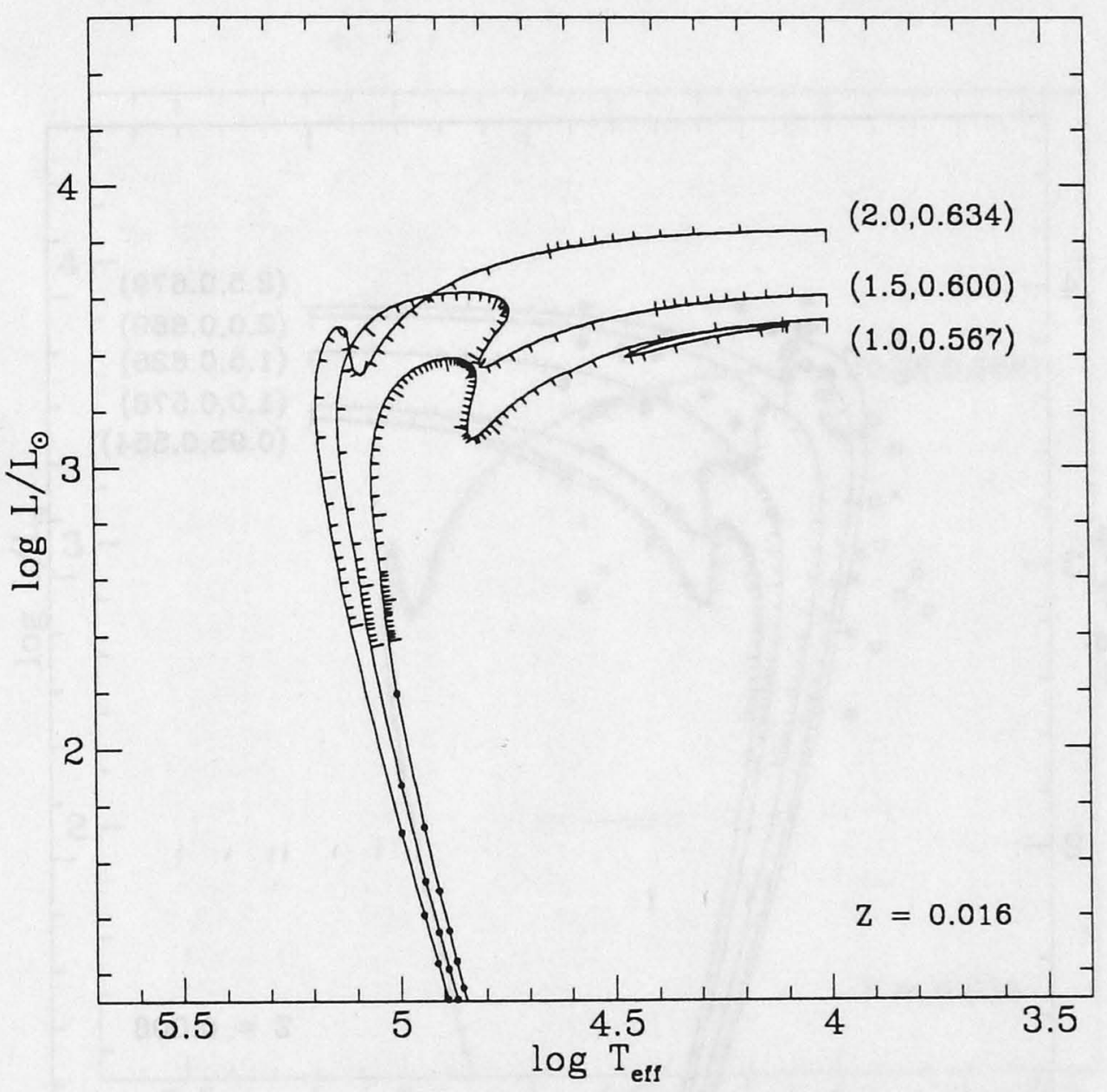

Figure 3.7: Same as figure 3.3, except for He burning PNNi. 


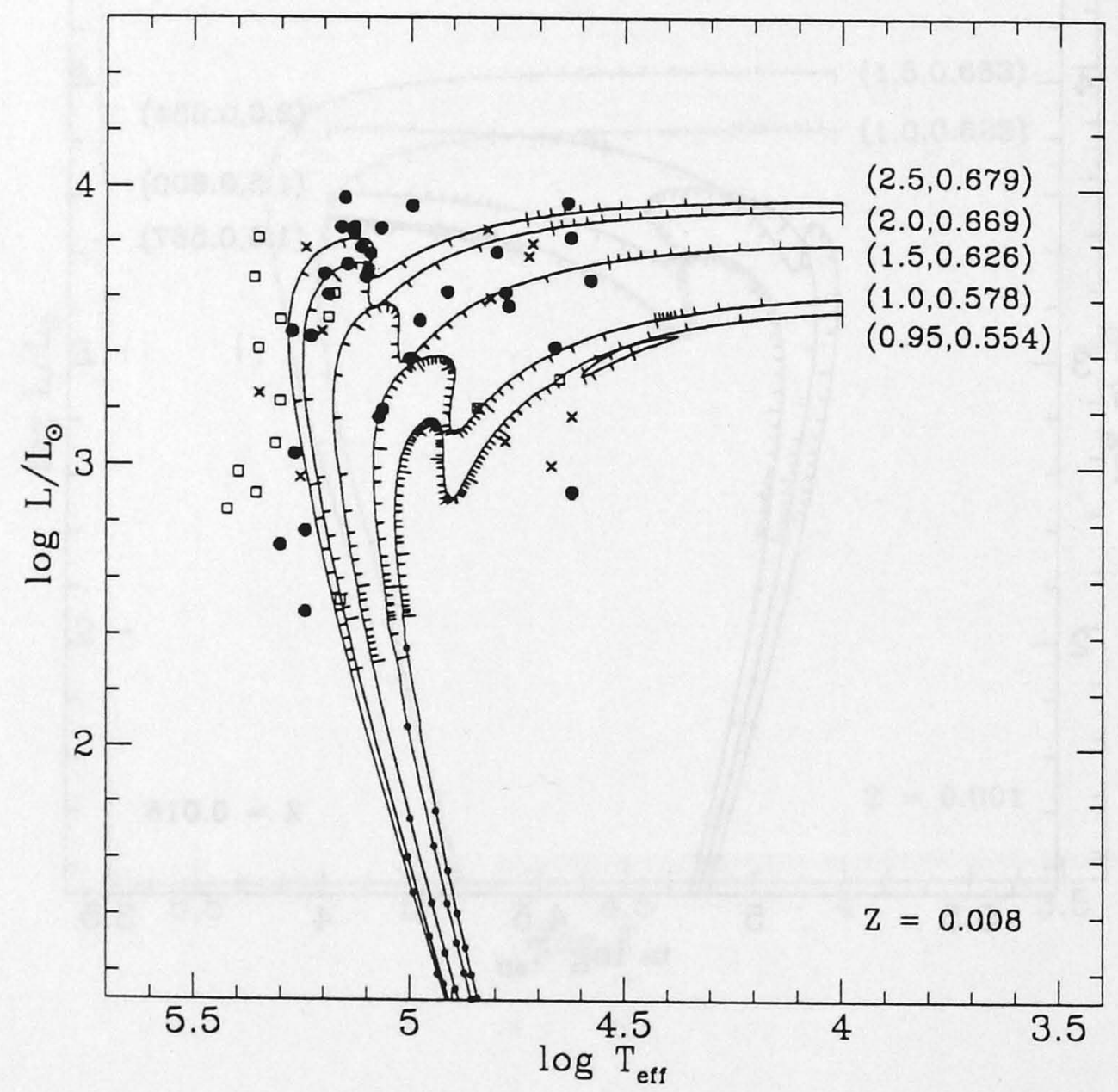

Figure 3.8: Same as figure 3.7, except for $Z=0.008$. Data for the LMC PNe is also shown. Point types are as defined as in figure 3.4 . 


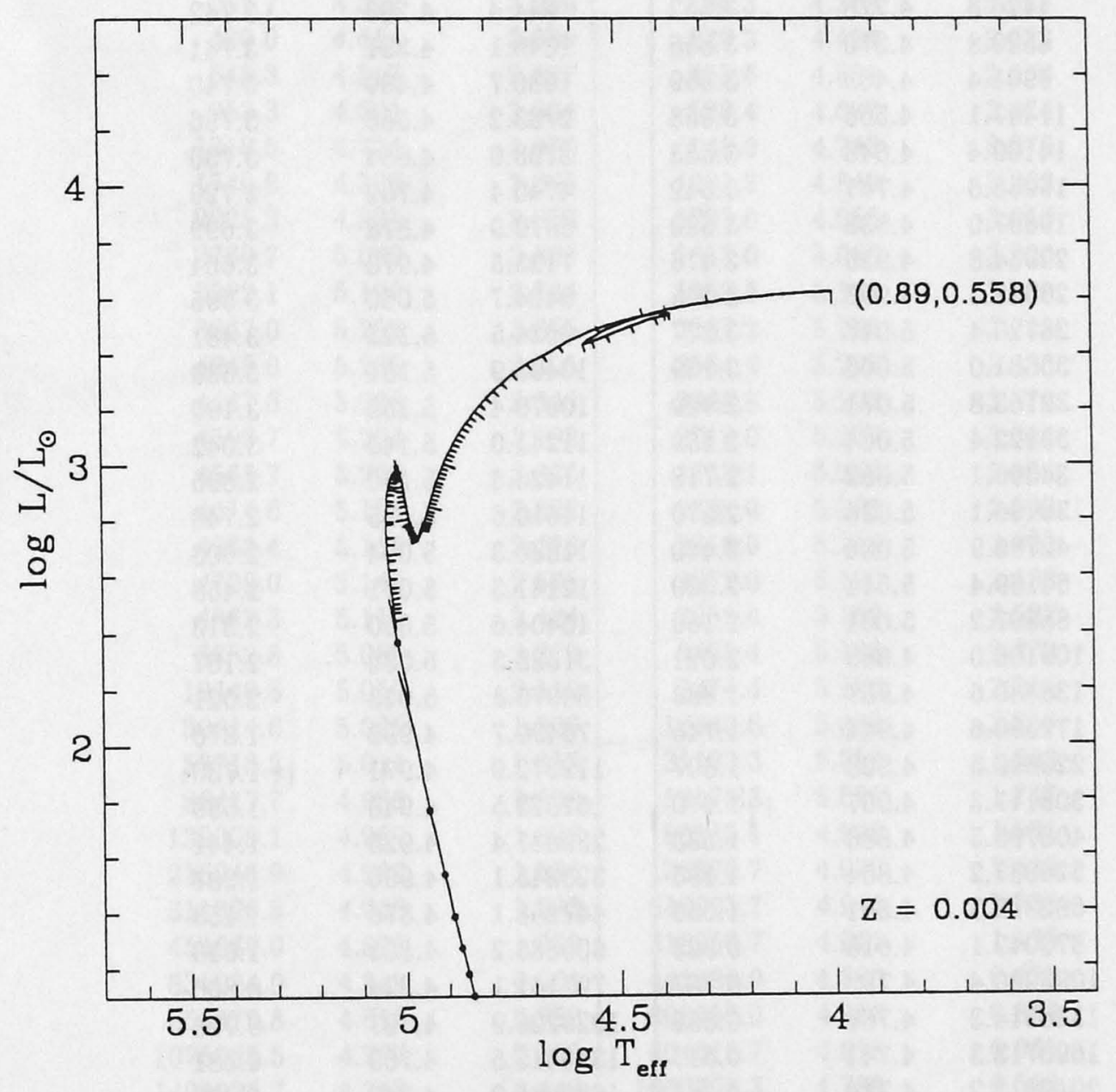

Figure 3.9: Same as figure 3.7, except for $Z=0.004$. 
TABLE 3.3

H Burning PNN Evolutionary Models

\begin{tabular}{|c|c|c|c|c|c|}
\hline $\begin{array}{c}\text { time }^{\mathrm{a}} \\
(\mathrm{yrs})\end{array}$ & $\overline{\log T_{\text {eff }}}$ & $\log L / L_{\odot}$ & $\begin{array}{l}\text { time } \\
\text { (yrs) }\end{array}$ & $\overline{l o g} T_{\text {eff }}$ & $\log L / L_{\odot}$ \\
\hline \multicolumn{3}{|c|}{$(M, Y, Z)=(1.0,0.25,0.016)$} & \multicolumn{3}{|c|}{$(M, Y, Z)=(1.5,0.25,0.016)$} \\
\hline 0.0 & 4.000 & 3.541 & 0.0 & 4.000 & 3.743 \\
\hline 1137.0 & 4.093 & 3.544 & 196.7 & 4.098 & 3.743 \\
\hline 2620.4 & 4.185 & 3.548 & 461.7 & 4.195 & 3.743 \\
\hline 4426.8 & 4.278 & 3.552 & 844.4 & 4.293 & 3.742 \\
\hline 6529.8 & 4.370 & 3.556 & 1349.1 & 4.391 & 3.741 \\
\hline 8901.4 & 4.463 & 3.559 & 1980.7 & 4.489 & 3.740 \\
\hline 11407.1 & 4.556 & 3.558 & 2763.2 & 4.586 & 3.736 \\
\hline 14109.4 & 4.648 & 3.553 & 3703.9 & 4.684 & 3.730 \\
\hline 16955.6 & 4.741 & 3.542 & 4740.4 & 4.782 & 3.720 \\
\hline 19897.0 & 4.832 & 3.520 & 5879.9 & 4.878 & 3.699 \\
\hline 22964.8 & 4.920 & 3.476 & 7123.5 & 4.973 & 3.664 \\
\hline 26018.3 & 4.996 & 3.395 & 8424.7 & 5.060 & 3.595 \\
\hline 28720.4 & 5.047 & 3.277 & 9624.5 & 5.123 & 3.481 \\
\hline 30681.0 & 5.066 & 3.139 & 10493.9 & 5.151 & 3.339 \\
\hline 32153.8 & 5.071 & 2.999 & 10979.4 & 5.153 & 3.190 \\
\hline 33222.4 & 5.064 & 2.858 & 11241.0 & 5.145 & 3.042 \\
\hline 34306.1 & 5.052 & 2.718 & 11425.8 & 5.130 & 2.895 \\
\hline 36136.1 & 5.038 & 2.579 & 11610.6 & 5.115 & 2.748 \\
\hline 42786.9 & 5.024 & 2.440 & 11830.3 & 5.094 & 2.603 \\
\hline 64169.4 & 5.014 & 2.300 & 12141.3 & 5.071 & 2.458 \\
\hline 85597.2 & 5.001 & 2.160 & 15404.6 & 5.050 & 2.313 \\
\hline 109106.0 & 4.985 & 2.021 & 31528.5 & 5.032 & 2.167 \\
\hline 135830.6 & 4.967 & 1.883 & 50975.8 & 5.013 & 2.021 \\
\hline 172390.6 & 4.947 & 1.745 & 76499.7 & 4.992 & 1.876 \\
\hline 225690.5 & 4.928 & 1.607 & 112372.9 & 4.971 & 1.730 \\
\hline 303117.3 & 4.907 & 1.470 & 162522.5 & 4.948 & 1.586 \\
\hline 406795.5 & 4.886 & 1.333 & 232037.4 & 4.925 & 1.441 \\
\hline 526837.2 & 4.864 & 1.196 & 325845.1 & 4.900 & 1.297 \\
\hline 682812.5 & 4.841 & 1.059 & 447258.1 & 4.876 & 1.153 \\
\hline 870042.1 & 4.818 & 0.923 & 605885.2 & 4.851 & 1.010 \\
\hline 1093980.4 & 4.793 & 0.787 & 797141.1 & 4.824 & 0.866 \\
\hline 1346514.3 & 4.767 & 0.652 & 1029226.9 & 4.797 & 0.723 \\
\hline 1693713.3 & 4.741 & 0.517 & 1318415.5 & 4.769 & 0.581 \\
\hline 2040912.2 & 4.714 & 0.381 & 1686085.9 & 4.740 & 0.439 \\
\hline 2503246.6 & 4.687 & 0.247 & 2053756.3 & 4.710 & 0.297 \\
\hline 3067785.3 & 4.659 & 0.112 & 2598416.8 & 4.681 & 0.155 \\
\hline 3632324.0 & 4.631 & -0.022 & 3321404.5 & 4.650 & 0.014 \\
\hline 4492096.0 & 4.602 & -0.156 & 4044392.2 & 4.620 & -0.127 \\
\hline 5625840.4 & 4.573 & -0.290 & 4976635.9 & 4.589 & -0.269 \\
\hline 7114025.5 & 4.544 & -0.424 & 6260000.8 & 4.558 & -0.410 \\
\hline 8878766.6 & 4.514 & -0.557 & 8123094.4 & 4.527 & -0.550 \\
\hline 11212719.2 & 4.484 & -0.691 & 10916214.3 & 4.495 & -0.691 \\
\hline 14844281.0 & 4.454 & -0.824 & 14418407.6 & 4.463 & -0.832 \\
\hline 20330772.2 & 4.425 & -0.957 & 20817510.5 & 4.431 & -0.972 \\
\hline 28560245.3 & 4.394 & -1.091 & 29644981.1 & 4.399 & -1.113 \\
\hline
\end{tabular}

a Time since $\log T_{\text {eff }}=4$ 
TABLE 3.3-Continued

H Burning PNN Evolutionary Models

\begin{tabular}{|c|c|c|c|c|c|}
\hline $\begin{array}{l}\text { time }^{\mathrm{a}} \\
\text { (yrs) }\end{array}$ & $\log T_{\text {eff }}$ & $\log L / L_{\odot}$ & $\begin{array}{l}\text { time } \\
\text { (yrs) }\end{array}$ & $\log T_{\text {eff }}$ & $\log L / L_{\odot}$ \\
\hline \multicolumn{3}{|c|}{$(M, Y, Z)=(2.0,0.25,0.016)$} & \multicolumn{3}{|c|}{$(M, Y, Z)=(2.5,0.25,0.016)$} \\
\hline 0.0 & 4.000 & 3.892 & 0.0 & 4.000 & 3.979 \\
\hline 54.6 & 4.103 & 3.891 & 27.7 & 4.106 & 3.979 \\
\hline 135.1 & 4.207 & 3.891 & 72.4 & 4.212 & 3.979 \\
\hline 257.7 & 4.310 & 3.890 & 136.3 & 4.318 & 3.978 \\
\hline 420.0 & 4.414 & 3.889 & 229.3 & 4.424 & 3.977 \\
\hline 648.3 & 4.517 & 3.887 & 353.6 & 4.531 & 3.976 \\
\hline 953.3 & 4.621 & 3.884 & 526.4 & 4.637 & 3.974 \\
\hline 1310.5 & 4.724 & 3.879 & 745.6 & 4.743 & 3.970 \\
\hline 1740.8 & 4.828 & 3.869 & 1001.3 & 4.849 & 3.963 \\
\hline 2225.3 & 4.931 & 3.853 & 1293.6 & 4.955 & 3.950 \\
\hline 2760.7 & 5.032 & 3.823 & 1617.0 & 5.060 & 3.926 \\
\hline 3322.1 & 5.128 & 3.764 & 1958.5 & 5.161 & 3.880 \\
\hline 3867.0 & 5.202 & 3.655 & 2297.2 & 5.246 & 3.783 \\
\hline 4245.0 & 5.235 & 3.505 & 2554.4 & 5.290 & 3.636 \\
\hline 4447.6 & 5.238 & 3.348 & 2666.8 & 5.292 & 3.474 \\
\hline 4506.7 & 5.224 & 3.192 & 2710.0 & 5.277 & 3.314 \\
\hline 4565.7 & 5.209 & 3.036 & 2753.1 & 5.263 & 3.154 \\
\hline 4614.6 & 5.188 & 2.882 & 2785.0 & 5.241 & 2.997 \\
\hline 4663.4 & 5.166 & 2.728 & 2816.9 & 5.218 & 2.839 \\
\hline 4728.0 & 5.140 & 2.575 & 2872.0 & 5.190 & 2.683 \\
\hline 4862.3 & 5.112 & 2.424 & 2952.4 & 5.162 & 2.527 \\
\hline 5352.8 & 5.085 & 2.272 & 3455.4 & 5.133 & 2.372 \\
\hline 12140.5 & 5.059 & 2.119 & 7374.4 & 5.106 & 2.216 \\
\hline 30014.6 & 5.035 & 1.966 & 16840.6 & 5.080 & 2.059 \\
\hline 53718.3 & 5.011 & 1.813 & 31123.3 & 5.053 & 1.903 \\
\hline 88417.7 & 4.986 & 1.660 & 52973.3 & 5.026 & 1.747 \\
\hline 139008.1 & 4.960 & 1.508 & 86565.1 & 4.998 & 1.591 \\
\hline 216046.9 & 4.933 & 1.356 & 136670.7 & 4.970 & 1.435 \\
\hline 311326.8 & 4.906 & 1.203 & 210993.7 & 4.941 & 1.280 \\
\hline 455666.9 & 4.878 & 1.052 & 316250.7 & 4.911 & 1.125 \\
\hline 631024.0 & 4.849 & 0.901 & 441389.9 & 4.880 & 0.970 \\
\hline 853479.8 & 4.819 & 0.750 & 600350.0 & 4.850 & 0.816 \\
\hline 1075935.5 & 4.789 & 0.599 & 804613.7 & 4.818 & 0.661 \\
\hline 1403225.7 & 4.758 & 0.449 & 1066752.3 & 4.786 & 0.508 \\
\hline 1831408.3 & 4.726 & 0.299 & 1408844.9 & 4.753 & 0.354 \\
\hline 2259590.8 & 4.694 & 0.149 & 1750937.5 & 4.719 & 0.201 \\
\hline 2907667.3 & 4.662 & 0.000 & 2277908.1 & 4.686 & 0.048 \\
\hline 3794554.8 & 4.629 & -0.150 & 3004074.7 & 4.652 & -0.105 \\
\hline 4681442.3 & 4.596 & -0.299 & 3730241.2 & 4.618 & -0.258 \\
\hline 6182142.4 & 4.563 & -0.448 & 4972807.2 & 4.583 & -0.411 \\
\hline 8026525.0 & 4.528 & -0.597 & 6708427.3 & 4.548 & -0.563 \\
\hline 11477834.3 & 4.494 & -0.745 & 9173037.9 & 4.513 & -0.716 \\
\hline 16298104.8 & 4.460 & -0.894 & 13101555.2 & 4.478 & -0.868 \\
\hline 23621410.4 & 4.426 & -1.043 & 19576784.1 & 4.442 & -1.020 \\
\hline 35709206.0 & 4.391 & -1.191 & 30825111.3 & 4.407 & -1.172 \\
\hline
\end{tabular}


TABLE 3.3-Continued

H Burning PNN Evolutionary Models

\begin{tabular}{|c|c|c|c|c|c|}
\hline $\begin{array}{l}\text { time }^{\mathrm{a}} \\
(\mathrm{yrs})\end{array}$ & $\overline{\log T_{\text {eff }}}$ & $\log L / L_{\odot}$ & $\begin{array}{l}\text { time } \\
\text { (yrs) } \\
\end{array}$ & $\overline{l o g} T_{\text {eff }}$ & $\overline{l o g} L / L_{\odot}$ \\
\hline \multicolumn{3}{|c|}{$(M, Y, Z)=(3.5,0.25,0.016)$} & \multicolumn{3}{|c|}{$(M, Y, Z)=(5.0,0.25,0.016)$} \\
\hline 0.0 & 4.000 & 4.138 & 0.0 & 4.000 & 4.360 \\
\hline 11.7 & 4.111 & 4.138 & 3.3 & 4.121 & 4.360 \\
\hline 28.6 & 4.223 & 4.138 & 5.7 & 4.242 & 4.360 \\
\hline 51.8 & 4.334 & 4.138 & 7.6 & 4.363 & 4.360 \\
\hline 87.1 & 4.445 & 4.138 & 10.2 & 4.484 & 4.360 \\
\hline 131.2 & 4.557 & 4.137 & 14.1 & 4.604 & 4.359 \\
\hline 199.4 & 4.668 & 4.136 & 20.3 & 4.725 & 4.359 \\
\hline 287.1 & 4.779 & 4.134 & 28.8 & 4.846 & 4.357 \\
\hline 390.5 & 4.891 & 4.130 & 40.3 & 4.967 & 4.355 \\
\hline 507.9 & 5.002 & 4.123 & 54.5 & 5.088 & 4.351 \\
\hline 633.3 & 5.113 & 4.107 & 71.1 & 5.209 & 4.343 \\
\hline 769.1 & 5.222 & 4.078 & 89.5 & 5.329 & 4.328 \\
\hline 905.4 & 5.324 & 4.011 & 109.4 & 5.448 & 4.294 \\
\hline 1017.3 & 5.385 & 3.868 & 129.8 & 5.551 & 4.199 \\
\hline 1067.2 & 5.390 & 3.699 & 141.7 & 5.581 & 4.020 \\
\hline 1095.4 & 5.379 & 3.531 & 145.5 & 5.567 & 3.838 \\
\hline 1123.7 & 5.367 & 3.362 & 147.5 & 5.542 & 3.658 \\
\hline 1144.7 & 5.342 & 3.197 & 149.4 & 5.510 & 3.480 \\
\hline 1176.6 & 5.315 & 3.033 & 151.7 & 5.476 & 3.304 \\
\hline 1350.5 & 5.285 & 2.869 & 155.5 & 5.440 & 3.128 \\
\hline 2167.6 & 5.258 & 2.705 & 167.1 & 5.403 & 2.953 \\
\hline 3675.3 & 5.230 & 2.541 & 219.5 & 5.366 & 2.778 \\
\hline 5916.2 & 5.200 & 2.378 & 603.0 & 5.330 & 2.602 \\
\hline 9861.7 & 5.170 & 2.215 & 1580.1 & 5.294 & 2.427 \\
\hline 15707.3 & 5.139 & 2.052 & 3512.1 & 5.257 & 2.252 \\
\hline 25191.2 & 5.108 & 1.890 & 6870.5 & 5.220 & 2.076 \\
\hline 40498.1 & 5.076 & 1.727 & 12915.8 & 5.182 & 1.902 \\
\hline 65429.8 & 5.044 & 1.565 & 23535.6 & 5.145 & 1.727 \\
\hline 103948.2 & 5.011 & 1.403 & 40411.7 & 5.107 & 1.552 \\
\hline 158505.2 & 4.978 & 1.242 & 67786.6 & 5.069 & 1.377 \\
\hline 232510.2 & 4.944 & 1.080 & 103747.8 & 5.030 & 1.203 \\
\hline 331783.2 & 4.910 & 0.919 & 153402.3 & 4.991 & 1.029 \\
\hline 470037.0 & 4.876 & 0.758 & 221025.9 & 4.952 & 0.855 \\
\hline 641139.4 & 4.840 & 0.597 & 314620.9 & 4.912 & 0.681 \\
\hline 812241.8 & 4.805 & 0.436 & 432909.6 & 4.872 & 0.508 \\
\hline 1073081.8 & 4.769 & 0.276 & 591289.4 & 4.832 & 0.334 \\
\hline 1427191.8 & 4.733 & 0.116 & 812814.0 & 4.791 & 0.161 \\
\hline 1836416.4 & 4.696 & -0.044 & 1121548.9 & 4.750 & -0.012 \\
\hline 2380532.7 & 4.659 & -0.204 & 1537543.1 & 4.710 & -0.185 \\
\hline 3175457.9 & 4.622 & -0.364 & 2091488.0 & 4.669 & -0.359 \\
\hline 4409527.1 & 4.585 & -0.523 & 3048361.7 & 4.628 & -0.531 \\
\hline 7011900.6 & 4.547 & -0.683 & 5469434.6 & 4.586 & -0.704 \\
\hline 10265329.8 & 4.510 & -0.842 & 11638798.4 & 4.545 & -0.877 \\
\hline 16790830.7 & 4.473 & -1.002 & 22917624.6 & 4.503 & -1.050 \\
\hline 28473393.6 & 4.434 & -1.161 & 40699832.6 & 4.461 & -1.222 \\
\hline
\end{tabular}


TABLE 3.3-Continued

H Burning PNN Evolutionary Models

\begin{tabular}{|c|c|c|c|c|c|}
\hline $\begin{array}{l}\text { time }^{\mathrm{a}} \\
(\mathrm{yrs})\end{array}$ & $\log T_{\text {eff }}$ & $\overline{l o g} L / L_{\odot}$ & $\begin{array}{l}\text { time } \\
\text { (yrs) }\end{array}$ & $\overline{\log T_{\text {eff }}}$ & $\overline{\log L / L_{\odot}}$ \\
\hline \multicolumn{3}{|c|}{$(M, Y, Z)=(1.5,0.25,0.008)$} & \multicolumn{3}{|c|}{$(M, Y, Z)=(2.0,0.25,0.008)$} \\
\hline 0.0 & 4.000 & 3.880 & 0.0 & 4.000 & 3.977 \\
\hline 17.7 & 4.092 & 3.876 & 42.1 & 4.114 & 3.977 \\
\hline 44.2 & 4.183 & 3.871 & 108.3 & 4.228 & 3.976 \\
\hline 83.5 & 4.275 & 3.865 & 205.7 & 4.342 & 3.976 \\
\hline 144.4 & 4.367 & 3.855 & 344.1 & 4.455 & 3.975 \\
\hline 256.3 & 4.458 & 3.842 & 529.1 & 4.569 & 3.973 \\
\hline 462.6 & 4.549 & 3.825 & 778.2 & 4.683 & 3.969 \\
\hline 759.1 & 4.639 & 3.801 & 1082.0 & 4.797 & 3.964 \\
\hline 1192.7 & 4.728 & 3.768 & 1425.7 & 4.910 & 3.953 \\
\hline 1920.4 & 4.815 & 3.723 & 1811.7 & 5.024 & 3.932 \\
\hline 3113.4 & 4.897 & 3.661 & 2219.3 & 5.134 & 3.892 \\
\hline 4951.5 & 4.971 & 3.579 & 2634.6 & 5.233 & 3.805 \\
\hline 7493.8 & 5.032 & 3.474 & 2961.6 & 5.288 & 3.654 \\
\hline 10514.7 & 5.074 & 3.350 & 3127.0 & 5.298 & 3.481 \\
\hline 13459.8 & 5.102 & 3.217 & 3170.2 & 5.284 & 3.309 \\
\hline 16031.6 & 5.113 & 3.078 & 3213.5 & 5.270 & 3.137 \\
\hline 18539.5 & 5.117 & 2.939 & 3241.8 & 5.244 & 2.969 \\
\hline 21331.4 & 5.115 & 2.799 & 3276.0 & 5.216 & 2.801 \\
\hline 24548.5 & 5.110 & 2.660 & 3320.6 & 5.187 & 2.633 \\
\hline 29219.3 & 5.100 & 2.521 & 3427.9 & 5.156 & 2.466 \\
\hline 35963.7 & 5.087 & 2.383 & 3729.7 & 5.125 & 2.300 \\
\hline 45333.4 & 5.071 & 2.246 & 5848.1 & 5.089 & 2.135 \\
\hline 56619.7 & 5.051 & 2.109 & $\ldots$ & $\cdots$ & $\cdots$ \\
\hline 70472.5 & 5.030 & 1.973 & $\cdots$ & $\ldots$ & $\cdots$ \\
\hline 89790.2 & 5.009 & 1.838 & $\cdots$ & $\cdots$ & $\cdots$ \\
\hline 116686.0 & 4.987 & 1.702 & $\cdots$ & $\cdots$ & $\cdots$ \\
\hline 161415.0 & 4.964 & 1.567 & $\cdots$ & $\cdots$ & $\cdots$ \\
\hline 219508.3 & 4.941 & 1.432 & $\ldots$ & $\ldots$ & $\cdots$ \\
\hline 296065.3 & 4.917 & 1.297 & $\ldots$ & $\cdots$ & $\cdots$ \\
\hline 395438.0 & 4.893 & 1.162 & $\ldots$ & $\cdots$ & $\cdots$ \\
\hline 520758.9 & 4.868 & 1.028 & $\cdots$ & $\ldots$ & $\cdots$ \\
\hline 675801.9 & 4.843 & 0.894 & $\ldots$ & $\cdots$ & $\cdots$ \\
\hline 862986.3 & 4.817 & 0.760 & $\cdots$ & $\cdots$ & $\cdots$ \\
\hline 1092802.3 & 4.790 & 0.626 & $\ldots$ & $\cdots$ & $\cdots$ \\
\hline 1378477.9 & 4.763 & 0.493 & $\cdots$ & $\cdots$ & $\cdots$ \\
\hline 1742760.2 & 4.735 & 0.360 & $\cdots$ & $\cdots$ & $\cdots$ \\
\hline 2107042.4 & 4.707 & 0.227 & $\cdots$ & $\cdots$ & $\cdots$ \\
\hline 2548029.9 & 4.678 & 0.095 & $\cdots$ & $\cdots$ & $\cdots$ \\
\hline 3114143.6 & 4.650 & -0.038 & $\cdots$ & $\cdots$ & $\cdots$ \\
\hline 3870769.2 & 4.620 & -0.170 & $\cdots$ & $\cdots$ & $\cdots$ \\
\hline 4627394.7 & 4.591 & -0.302 & $\cdots$ & $\cdots$ & $\cdots$ \\
\hline 5890419.5 & 4.561 & -0.434 & $\cdots$ & $\cdots$ & $\cdots$ \\
\hline 7397708.1 & 4.531 & -0.566 & $\cdots$ & $\cdots$ & $\cdots$ \\
\hline 10087160.9 & 4.502 & -0.698 & $\cdots$ & $\cdots$ & $\cdots$ \\
\hline 13808257.0 & 4.472 & -0.830 & $\ldots$ & $\ldots$ & $\ldots$ \\
\hline
\end{tabular}


TABLE 3.3-Continued

H Burning PNN Evolutionary Models

\begin{tabular}{|c|c|c|c|c|c|}
\hline $\begin{array}{c}\text { time }^{\mathrm{a}} \\
(\mathrm{yrs})\end{array}$ & $\log T_{\text {eff }}$ & $\log L / L_{\odot}$ & $\begin{array}{l}\text { time } \\
\text { (yrs) } \\
\end{array}$ & $\log T_{\text {eff }}$ & $\log L / L_{\odot}$ \\
\hline \multicolumn{3}{|c|}{$(M, Y, Z)=(3.5,0.25,0.008)$} & \multicolumn{3}{|c|}{$(M, Y, Z)=(5.0,0.25,0.008)$} \\
\hline 0.0 & 4.000 & 4.216 & 0.0 & 4.000 & 4.370 \\
\hline 7.4 & 4.115 & 4.216 & 3.0 & 4.112 & 4.370 \\
\hline 16.5 & 4.230 & 4.216 & 5.4 & 4.224 & 4.370 \\
\hline 29.2 & 4.346 & 4.215 & 7.6 & 4.336 & 4.370 \\
\hline 47.2 & 4.461 & 4.215 & 10.3 & 4.448 & 4.370 \\
\hline 72.7 & 4.576 & 4.214 & 13.6 & 4.560 & 4.370 \\
\hline 110.6 & 4.691 & 4.213 & 19.2 & 4.672 & 4.369 \\
\hline 160.1 & 4.806 & 4.211 & 26.3 & 4.784 & 4.369 \\
\hline 219.9 & 4.921 & 4.208 & 36.0 & & 4.367 \\
\hline 288.7 & 5. & 201 & 47.3 & 09 & .365 \\
\hline 363.2 & 5.151 & 189 & 61.2 & 5.121 & 4.361 \\
\hline 440.6 & 5.265 & 4.164 & 75.7 & 5.233 & 4.353 \\
\hline 520.3 & 5.374 & .106 & 91.6 & 44 & 4.339 \\
\hline 591.1 & 5.449 & 972 & 108.3 & & 4.310 \\
\hline 626.8 & 5.465 & & 125.3 & & 4.233 \\
\hline 637.1 & & & 137.5 & & 1.077 \\
\hline 645.3 & 5.425 & 454 & 141.6 & 3 & 3.907 \\
\hline 653.5 & 5 & 282 & 143.5 & 3 & 3.739 \\
\hline 664.9 & 5. & 13 & .8 & & 3.574 \\
\hline 685.7 & 5.339 & 944 & 146.0 & 14 & 3.410 \\
\hline 756.8 & & 777 & 147.6 & & 3.247 \\
\hline 1105.3 & 5.2 & 608 & 151.1 & 17 & 3.085 \\
\hline 2338.3 & 5.242 & 2.440 & 159.4 & 5.412 & 2.923 \\
\hline 4766.6 & 5.210 & 2.272 & 181.9 & 5.377 & 2.761 \\
\hline 8936.3 & 5.176 & 2.104 & 274.3 & & 2.599 \\
\hline 15261.9 & 5.143 & 1.936 & 664.2 & 5.2 & 2.437 \\
\hline 25234.6 & 5.108 & 1.769 & 1736.8 & 73 & 2.274 \\
\hline 44546.9 & 5.074 & 1.602 & 0.0 & 8 & 2.112 \\
\hline 69891.5 & 5.038 & 1.435 & 6802.2 & 5.203 & 1.950 \\
\hline 113353.2 & 5.003 & 1.268 & 11935.0 & 5.167 & 1.789 \\
\hline 179104.0 & 4.968 & 1.101 & 20075.9 & 5.131 & 1.627 \\
\hline 263447.2 & 4.932 & 0.935 & 31952.6 & 5.096 & 1.465 \\
\hline 375219.8 & 4.895 & 0.768 & 50296.6 & 5.060 & 1.304 \\
\hline 523545.2 & 4.859 & 0.602 & 78524.1 & 5.023 & 1.143 \\
\hline 721871.2 & & 0.437 & 116726.2 & 4.986 & 0.982 \\
\hline 920197.2 & 4.783 & 0.271 & 171263.2 & 4.950 & 0.821 \\
\hline 1244622.7 & 4.746 & 0.105 & 238856.1 & 4.912 & 0.660 \\
\hline 1704246.6 & 4.707 & -0.060 & 343327.1 & 4.875 & 0.499 \\
\hline 2390467.8 & 4.669 & -0.225 & 478183.3 & 4.837 & 0.338 \\
\hline 3461404.1 & 4.630 & -0.390 & 662662.7 & 4.800 & 0.178 \\
\hline 4920564.0 & 4.591 & -0.555 & 911372.5 & 4.762 & 0.017 \\
\hline 7234475.8 & 4.552 & -0.720 & 1205689.9 & 4.724 & -0.143 \\
\hline 12660443.6 & 4.513 & -0.885 & 1674051.1 & 4.685 & -0.303 \\
\hline 23154814.2 & 4.473 & -1.049 & 2422995.8 & 4.647 & -0.464 \\
\hline 35828109.2 & 4.434 & -1.214 & 3959814.2 & 4.609 & -0.624 \\
\hline
\end{tabular}


TABLE 3.3-Continued

H Burning PNN Evolutionary Models

\begin{tabular}{|c|c|c|c|c|c|}
\hline $\begin{array}{c}\text { time }^{\mathrm{a}} \\
(\mathrm{yrs})\end{array}$ & $\overline{\log T_{\text {eff }}}$ & $\log L / L_{\odot}$ & $\begin{array}{l}\text { time } \\
\text { (yrs) }\end{array}$ & $\overline{\log T_{\text {eff }}}$ & $\overline{\log L / L_{\odot}}$ \\
\hline \multicolumn{3}{|c|}{$(M, Y, Z)=(1.0,0.25,0.004)$} & \multicolumn{3}{|c|}{$(M, Y, Z)=(1.5,0.25,0.004)$} \\
\hline 0.0 & 4.000 & 3.641 & 0.0 & 4.000 & 3.870 \\
\hline 943.5 & 4.095 & 3.645 & 116.5 & 4.101 & 3.870 \\
\hline 2147.9 & 4.191 & 3.649 & 286.6 & 4.203 & 3.870 \\
\hline 3612.6 & 4.286 & 3.653 & 533.5 & 4.304 & 3.870 \\
\hline 5307.7 & 4.381 & 3.657 & 871.4 & 4.405 & 3.869 \\
\hline 7172.4 & 4.477 & 3.660 & 1290.0 & 4.506 & 3.867 \\
\hline 9190.7 & 4.572 & 3.660 & 1807.0 & 4.608 & 3.865 \\
\hline 11276.6 & 4.667 & 3.656 & 2424.1 & 4.709 & 3.860 \\
\hline 13515.8 & 4.763 & 3.649 & 3113.1 & 4.810 & 3.852 \\
\hline 15739.6 & 4.857 & 3.631 & 3847.6 & 4.911 & 3.838 \\
\hline 18025.9 & 4.950 & 3.599 & 4641.1 & 5.010 & 3.811 \\
\hline 20318.5 & 5.036 & 3.535 & 5449.1 & 5.106 & 3.761 \\
\hline 22367.8 & 5.099 & 3.427 & 6235.0 & 5.186 & 3.667 \\
\hline 23914.9 & 5.132 & 3.290 & 6826.8 & 5.229 & 3.528 \\
\hline 24859.2 & 5.138 & 3.145 & 7154.8 & 5.237 & 3.374 \\
\hline 25475.7 & 5.135 & 3.000 & 7266.0 & 5.227 & 3.221 \\
\hline 25780.3 & 5.122 & 2.857 & 7377.2 & 5.216 & 3.068 \\
\hline 26056.4 & 5.105 & 2.714 & 7452.6 & 5.197 & 2.916 \\
\hline 26377.7 & 5.086 & 2.571 & 7528.0 & 5.178 & 2.765 \\
\hline 26818.2 & 5.066 & 2.429 & 7615.9 & 5.154 & 2.615 \\
\hline 28862.6 & 5.045 & 2.288 & 7784.1 & 5.128 & 2.466 \\
\hline 45388.5 & 5.029 & 2.145 & 8180.7 & 5.101 & 2.318 \\
\hline 66622.2 & 5.011 & 2.002 & 11163.8 & 5.076 & 2.169 \\
\hline 92583.9 & 4.990 & 1.861 & 23124.7 & 5.052 & 2.019 \\
\hline 130017.3 & 4.969 & 1.719 & 42160.4 & 5.027 & 1.869 \\
\hline 175818.3 & 4.946 & 1.578 & 73582.8 & 5.003 & 1.720 \\
\hline 247816.1 & 4.924 & 1.437 & 114768.0 & 4.978 & 1.570 \\
\hline 343488.4 & 4.901 & 1.296 & 180163.5 & 4.952 & 1.422 \\
\hline 466533.7 & 4.877 & 1.156 & 261617.7 & 4.925 & 1.273 \\
\hline 637505.7 & 4.852 & 1.016 & 370824.7 & 4.898 & 1.124 \\
\hline 838732.6 & 4.826 & 0.876 & 514748.1 & 4.870 & 0.976 \\
\hline 1059747.3 & 4.799 & 0.737 & 697135.5 & 4.841 & 0.829 \\
\hline 1322625.9 & 4.772 & 0.598 & 927734.3 & 4.811 & 0.682 \\
\hline 1649042.9 & 4.743 & 0.459 & 1224424.0 & 4.781 & 0.535 \\
\hline 2067460.8 & 4.715 & 0.321 & 1521113.6 & 4.750 & 0.388 \\
\hline 2485878.8 & 4.686 & 0.182 & 1954736.8 & 4.719 & 0.241 \\
\hline 3112823.3 & 4.657 & 0.044 & 2451834.7 & 4.687 & 0.095 \\
\hline 3836771.6 & 4.627 & -0.093 & 3086190.2 & 4.655 & -0.051 \\
\hline 4774958.3 & 4.597 & -0.231 & 3955887.7 & 4.622 & -0.197 \\
\hline 6083001.2 & 4.566 & -0.369 & 4825585.2 & 4.590 & -0.343 \\
\hline 7992644.0 & 4.536 & -0.506 & 6394720.0 & 4.557 & -0.489 \\
\hline 10353314.5 & 4.506 & -0.644 & 8786817.0 & 4.524 & -0.634 \\
\hline 13742416.8 & 4.474 & -0.781 & 12216611.3 & 4.490 & -0.780 \\
\hline 20349465.9 & 4.443 & -0.918 & 17551734.4 & 4.457 & -0.925 \\
\hline 29517532.8 & 4.411 & -1.055 & 26739238.4 & 4.423 & -1.071 \\
\hline
\end{tabular}


TABLE 3.3-Continued

H Burning PNN Evolutionary Models

\begin{tabular}{|c|c|c|c|c|c|}
\hline $\begin{array}{c}\operatorname{time}^{\mathrm{a}} \\
(\mathrm{yrs})\end{array}$ & $\overline{l o g} T_{\text {eff }}$ & $\log L / L_{\odot}$ & $\begin{array}{l}\text { time } \\
\text { (yrs) }\end{array}$ & $\log T_{\text {eff }}$ & $\log L / L_{\odot}$ \\
\hline \multicolumn{3}{|c|}{$(M, Y, Z)=(2.0,0.25,0.004)$} & \multicolumn{3}{|c|}{$(M, Y, Z)=(2.5,0.25,0.004)$} \\
\hline 0.0 & 4.000 & 3.918 & 0.0 & 4.000 & 4.152 \\
\hline 226.3 & 4.105 & 3.922 & 8.5 & 4.109 & 4.151 \\
\hline 518.9 & 4.209 & 3.927 & 20.0 & 4.217 & 4.150 \\
\hline 876.5 & 4.314 & 3.931 & 36.8 & 4.326 & 4.148 \\
\hline 1266.3 & 4.419 & 3.935 & 62.0 & 4.435 & 4.145 \\
\hline 1697.7 & 4.523 & 3.938 & 100.8 & 4.543 & 4.141 \\
\hline 2193.6 & 4.628 & 3.940 & 165.7 & 4.652 & 4.134 \\
\hline 2705.2 & 4.733 & 3.939 & 271.2 & 4.760 & 4.125 \\
\hline 3243.6 & 4.838 & 3.936 & 429.3 & 4.868 & 4.110 \\
\hline 3788.7 & 4.942 & 3.927 & 665.4 & 4.976 & 4.091 \\
\hline 4330.2 & 5.046 & 3.906 & 998.7 & 5.084 & 4.066 \\
\hline 4858.6 & 5.147 & 3.863 & 1499.6 & 5.190 & 4.028 \\
\hline 5366.7 & 5.234 & 3.775 & 2315.6 & 5.289 & 3.961 \\
\hline 5754.3 & 5.282 & 3.633 & 3572.9 & 5.355 & 3.830 \\
\hline 5980.0 & 5.292 & 3.474 & 4701.9 & 5.376 & 3.668 \\
\hline 6093.9 & 5.285 & 3.315 & 5511.1 & 5.375 & 3.502 \\
\hline 6148.2 & 5.267 & 3.158 & 6138.0 & 5.362 & 3.338 \\
\hline 6207.6 & 5.245 & 3.003 & 6724.4 & 5.344 & 3.175 \\
\hline 6289.8 & 5.222 & 2.847 & 7392.1 & 5.321 & 3.014 \\
\hline 6529.9 & 5.196 & 2.693 & 8210.5 & 5.295 & 2.853 \\
\hline 7752.0 & 5.172 & 2.538 & 9394.1 & 5.268 & 2.693 \\
\hline 11049.6 & 5.147 & 2.383 & 11089.1 & 5.239 & 2.533 \\
\hline 16107.9 & 5.123 & 2.228 & 13376.9 & 5.210 & 2.374 \\
\hline 23641.9 & 5.096 & 2.074 & 17088.3 & 5.179 & 2.215 \\
\hline 33164.5 & 5.069 & 1.920 & 22405.3 & 5.148 & 2.057 \\
\hline 49812.7 & 5.042 & 1.766 & 30664.3 & 5.116 & 1.899 \\
\hline 72527.1 & 5.013 & 1.613 & 41352.8 & 5.084 & 1.741 \\
\hline 112148.9 & 4.983 & 1.460 & 58888.5 & 5.050 & 1.584 \\
\hline 164338.4 & 4.954 & 1.307 & 82592.4 & 5.017 & 1.427 \\
\hline 237237.9 & 4.924 & 1.154 & 123810.6 & 4.983 & 1.270 \\
\hline 338898.2 & 4.893 & 1.002 & 178499.8 & 4.950 & 1.112 \\
\hline 476836.1 & 4.862 & 0.849 & 255611.1 & 4.916 & 0.955 \\
\hline 659443.6 & 4.831 & 0.698 & 364813.5 & 4.881 & 0.799 \\
\hline 899807.0 & 4.798 & 0.546 & 516259.3 & 4.846 & 0.642 \\
\hline 1140170.5 & 4.766 & 0.394 & 720776.5 & 4.811 & 0.485 \\
\hline 1505320.6 & 4.733 & 0.243 & 995410.5 & 4.775 & 0.329 \\
\hline 1999771.0 & 4.700 & 0.092 & 1368221.7 & 4.740 & 0.173 \\
\hline 2494221.4 & 4.666 & -0.059 & 1741032.9 & 4.704 & 0.017 \\
\hline 3151065.2 & 4.632 & -0.209 & 2332956.4 & 4.668 & -0.139 \\
\hline 4306931.1 & 4.597 & -0.360 & 3109869.7 & 4.632 & -0.295 \\
\hline 5953696.0 & 4.563 & -0.510 & 4141244.5 & 4.595 & -0.451 \\
\hline 8411310.6 & 4.528 & -0.661 & 5750572.7 & 4.559 & -0.606 \\
\hline 11561732.4 & 4.494 & -0.811 & 8199415.3 & 4.522 & -0.762 \\
\hline 17691030.6 & 4.459 & -0.961 & 12435816.7 & 4.485 & -0.918 \\
\hline 26551698.0 & 4.424 & -1.112 & 19600968.0 & 4.448 & -1.073 \\
\hline
\end{tabular}


TABLE 3.3-Continued

H Burning PNN Evolutionary Models

\begin{tabular}{|c|c|c|c|c|c|}
\hline $\begin{array}{c}\operatorname{time}^{\mathrm{a}} \\
(\mathrm{yrs})\end{array}$ & $\log T_{\text {eff }}$ & $\log L / L_{\odot}$ & $\begin{array}{l}\text { time } \\
\text { (yrs) }\end{array}$ & $\log T_{\text {eff }}$ & $\log L / L_{\odot}$ \\
\hline \multicolumn{3}{|c|}{$(M, Y, Z)=(3.5,0.25,0.004)$} & \multicolumn{3}{|c|}{$(M, Y, Z)=(5.0,0.25,0.004)$} \\
\hline 0.0 & 4.000 & 4.296 & 0.0 & 4.000 & 4.399 \\
\hline 4.4 & 4.116 & 4.296 & 3.2 & 4.111 & 4.399 \\
\hline 10.4 & 4.231 & 4.295 & 5.5 & 4.223 & 4.399 \\
\hline 17.1 & 4.347 & 4.295 & 7.8 & 4.334 & 4.399 \\
\hline 26.0 & 4.462 & 4.295 & 9.9 & 4.445 & 4.399 \\
\hline 38.7 & 4.578 & 4.295 & 13.0 & 4.556 & 4.398 \\
\hline 57.5 & 4.693 & 4.294 & 17.8 & 4.668 & 4.398 \\
\hline 82.1 & 4.809 & 4.292 & 24.0 & 4.779 & 4.398 \\
\hline 111.9 & 4.924 & 4.289 & 31.7 & 4.890 & 4.396 \\
\hline 146.4 & 5.040 & 4.285 & 41.5 & 5.002 & 4.395 \\
\hline 184.5 & 5.155 & 4.277 & 52.5 & 5.113 & 4.391 \\
\hline 224.3 & 5.271 & 4.262 & 64.6 & 5.224 & 4.385 \\
\hline 265.3 & 5.384 & 4.228 & 77.1 & 5.335 & 4.374 \\
\hline 307.0 & 5.487 & 4.148 & 90.1 & 5.446 & 4.353 \\
\hline 335.4 & 5.532 & 3.986 & 103.8 & 5.552 & 4.302 \\
\hline 342.6 & 5.520 & 3.811 & 116.0 & 5.621 & 4.169 \\
\hline 349.7 & 5.507 & 3.637 & 120.7 & 5.623 & 4.000 \\
\hline 353.6 & 5.479 & 3.466 & 122.5 & 5.605 & 3.833 \\
\hline 357.5 & 5.451 & 3.296 & 123.7 & 5.579 & 3.668 \\
\hline 362.9 & 5.418 & 3.127 & 124.9 & 5.549 & 3.505 \\
\hline 372.1 & 5.384 & 2.959 & 126.3 & 5.517 & 3.343 \\
\hline 389.0 & 5.350 & 2.791 & 128.3 & 5.484 & 3.181 \\
\hline 423.4 & 5.315 & 2.623 & 131.7 & 5.450 & 3.020 \\
\hline 586.1 & 5.279 & 2.456 & 141.2 & 5.415 & 2.859 \\
\hline 1243.5 & 5.243 & 2.289 & 175.0 & 5.379 & 2.698 \\
\hline 3871.2 & 5.209 & 2.121 & 297.8 & 5.344 & 2.538 \\
\hline 8397.4 & 5.173 & 1.954 & 708.4 & 5.309 & 2.377 \\
\hline 16605.4 & 5.138 & 1.786 & 1787.3 & 5.274 & 2.216 \\
\hline 32229.2 & 5.103 & 1.619 & 3846.4 & 5.239 & 2.056 \\
\hline 57142.6 & 5.067 & 1.452 & 7382.1 & 5.204 & 1.895 \\
\hline 90017.9 & 5.031 & 1.285 & 12811.0 & 5.168 & 1.734 \\
\hline 145476.6 & 4.994 & 1.118 & 21460.9 & 5.133 & 1.574 \\
\hline 215352.0 & 4.957 & 0.951 & 34887.5 & 5.097 & 1.414 \\
\hline 306781.1 & 4.920 & 0.785 & 56236.9 & 5.061 & 1.254 \\
\hline 428207.0 & 4.882 & 0.618 & 86229.2 & 5.024 & 1.094 \\
\hline 592080.2 & 4.845 & 0.452 & 123028.6 & 4.987 & 0.934 \\
\hline 755953.4 & 4.807 & 0.286 & 180864.9 & 4.950 & 0.774 \\
\hline 1027694.6 & 4.768 & 0.120 & 254314.5 & 4.913 & 0.614 \\
\hline 1422991.1 & 4.729 & -0.045 & 352464.0 & 4.876 & 0.455 \\
\hline 1939710.3 & 4.690 & -0.211 & 487730.4 & 4.838 & 0.295 \\
\hline 2726019.3 & 4.651 & -0.376 & 672243.6 & 4.801 & 0.136 \\
\hline 4051508.5 & 4.612 & -0.542 & 919383.1 & 4.763 & -0.023 \\
\hline 6986859.5 & 4.573 & -0.707 & 1220395.0 & 4.725 & -0.183 \\
\hline 13354308.3 & 4.533 & -0.872 & 1694149.9 & 4.687 & -0.342 \\
\hline 23490067.6 & 4.494 & -1.037 & 2580437.2 & 4.648 & -0.501 \\
\hline
\end{tabular}


TABLE 3.3-Continued

H Burning PNN Evolutionary Models

\begin{tabular}{|c|c|c|c|c|c|}
\hline $\begin{array}{c}\text { time }^{\mathrm{a}} \\
(\mathrm{yrs})\end{array}$ & $\log T_{\text {eff }}$ & $\log L / L_{\odot}$ & $\begin{array}{l}\text { time } \\
(\mathrm{yrs})\end{array}$ & $\log T_{\text {eff }}$ & $\log L / L_{\odot}$ \\
\hline \multicolumn{3}{|c|}{$(M, Y, Z)=(1.0,0.25,0.001)$} & \multicolumn{3}{|c|}{$(M, Y, Z)=(1.5,0.25,0.001)$} \\
\hline 0.0 & 4.000 & 3.776 & 0.0 & 4.000 & 3.985 \\
\hline 328.2 & 4.098 & 3.776 & 53.5 & 4.114 & 3.985 \\
\hline 775.9 & 4.196 & 3.776 & 137.7 & 4.228 & 3.984 \\
\hline 1370.9 & 4.295 & 3.776 & 257.5 & 4.342 & 3.983 \\
\hline 2147.8 & 4.393 & 3.776 & 430.7 & 4.455 & 3.982 \\
\hline 3101.5 & 4.491 & 3.774 & 656.8 & 4.569 & 3.980 \\
\hline 4253.8 & 4.589 & 3.773 & 950.4 & 4.683 & 3.977 \\
\hline 5501.1 & 4.688 & 3.767 & 1288.5 & 4.797 & 3.972 \\
\hline 6871.2 & 4.786 & 3.759 & 1664.6 & 4.911 & 3.962 \\
\hline 8317.1 & 4.883 & 3.744 & 2069.1 & 5.024 & 3.944 \\
\hline 9779.6 & 4.980 & 3.716 & 2485.3 & 5.135 & 3.908 \\
\hline 11285.0 & 5.072 & 3.663 & 2904.1 & 5.238 & 3.833 \\
\hline 12704.1 & 5.148 & 3.568 & 3246.7 & 5.302 & 3.689 \\
\hline 13827.7 & 5.191 & 3.434 & 3409.0 & 5.312 & 3.517 \\
\hline 14550.7 & 5.203 & 3.285 & 3459.0 & 5.298 & 3.345 \\
\hline 14800.1 & 5.194 & 3.137 & 3509.1 & 5.284 & 3.173 \\
\hline 15049.5 & 5.185 & 2.988 & 3544.3 & 5.262 & 3.003 \\
\hline 15202.9 & 5.167 & 2.841 & 3579.5 & 5.239 & 2.833 \\
\hline 15356.3 & 5.150 & 2.693 & 3617.0 & 5.210 & 2.666 \\
\hline 15514.9 & 5.128 & 2.548 & 3665.7 & 5.181 & 2.498 \\
\hline 15740.2 & 5.104 & 2.403 & 3778.7 & 5.148 & 2.332 \\
\hline 16394.4 & 5.079 & 2.258 & 4055.2 & 5.115 & 2.167 \\
\hline 19054.0 & 5.054 & 2.113 & 4796.6 & 5.082 & 2.001 \\
\hline 31747.3 & 5.032 & 1.968 & $\ldots$ & $\ldots$ & $\cdots$ \\
\hline 52644.0 & 5.009 & 1.822 & $\ldots$ & $\ldots$ & $\ldots$ \\
\hline 85692.8 & 4.986 & 1.677 & $\ldots$ & $\ldots$ & $\ldots$ \\
\hline 136428.6 & 4.961 & 1.533 & $\cdots$ & .. & $\ldots$ \\
\hline 200335.4 & 4.936 & 1.388 & $\ldots$ & $\ldots$ & $\ldots$ \\
\hline 285935.9 & 4.910 & 1.244 & $\cdots$ & $\ldots$ & $\cdots$ \\
\hline 421110.9 & 4.884 & 1.100 & $\cdots$ & $\cdots$ & $\cdots$ \\
\hline 568703.4 & 4.857 & 0.956 & $\cdots$ & $\cdots$ & $\cdots$ \\
\hline 744582.6 & 4.829 & 0.812 & $\ldots$ & $\cdots$ & $\cdots$ \\
\hline 964233.1 & 4.800 & 0.669 & $\ldots$ & $\ldots$ & $\ldots$ \\
\hline 1244129.5 & 4.771 & 0.527 & $\ldots$ & $\ldots$ & ... \\
\hline 1606161.6 & 4.742 & 0.384 & $\ldots$ & $\ldots$ & $\ldots$ \\
\hline 1968193.8 & 4.712 & 0.242 & $\ldots$ & $\ldots$ & $\cdots$ \\
\hline 2425509.1 & 4.681 & 0.100 & $\cdots$ & $\cdots$ & $\cdots$ \\
\hline 3029092.9 & 4.651 & -0.042 & $\ldots$ & $\cdots$ & $\cdots$ \\
\hline 3856672.0 & 4.620 & -0.184 & $\ldots$ & $\cdots$ & $\cdots$ \\
\hline 4968514.8 & 4.588 & -0.326 & $\ldots$ & $\ldots$ & $\cdots$ \\
\hline 6487932.4 & 4.556 & -0.467 & $\ldots$ & $\ldots$ & $\ldots$ \\
\hline 8669595.7 & 4.524 & -0.608 & $\ldots$ & $\ldots$ & $\cdots$ \\
\hline 11882791.3 & 4.491 & -0.750 & $\ldots$ & $\ldots$ & $\cdots$ \\
\hline 17798530.0 & 4.459 & -0.891 & $\ldots$ & $\ldots$ & $\ldots$ \\
\hline 25939029.6 & 4.427 & -1.032 & $\ldots$ & $\ldots$ & $\ldots$ \\
\hline
\end{tabular}


TABLE 3.4

He Burning PNN Evolutionary Models

\begin{tabular}{|c|c|c|c|c|c|}
\hline $\begin{array}{c}\text { time }^{a} \\
(y r s)\end{array}$ & $\log T_{\text {eff }}$ & $\log L / L_{\odot}$ & $\begin{array}{l}\text { time } \\
\text { (yrs) }\end{array}$ & $\log T_{\text {eff }}$ & $\log L / L_{\odot}$ \\
\hline \multicolumn{3}{|c|}{$(M, Y, Z)=(1.0,0.25,0.016)$} & \multicolumn{3}{|c|}{$(M, Y, Z)=(1.5,0.25,0.016)$} \\
\hline 0.0 & 4.000 & 3.525 & 0.0 & 4.000 & 3.612 \\
\hline 30.9 & 4.107 & 3.512 & 144.5 & 4.106 & 3.604 \\
\hline 76.0 & 4.213 & 3.495 & 354.8 & 4.212 & 3.593 \\
\hline 143.3 & 4.318 & 3.468 & 679.6 & 4.318 & 3.576 \\
\hline 244.9 & 4.422 & 3.425 & 1150.7 & 4.423 & 3.553 \\
\hline 639.6 & 4.316 & 3.447 & 1876.8 & 4.527 & 3.519 \\
\hline 742.4 & 4.210 & 3.476 & 3025.8 & 4.628 & 3.472 \\
\hline 877.0 & 4.105 & 3.499 & 4837.8 & 4.726 & 3.408 \\
\hline 1191.3 & 4.211 & 3.493 & 17224.3 & 4.737 & 3.569 \\
\hline 1358.3 & 4.317 & 3.472 & 23267.4 & 4.838 & 3.618 \\
\hline 1571.6 & 4.422 & 3.440 & 26498.9 & 4.944 & 3.602 \\
\hline 1917.0 & 4.524 & 3.391 & 29163.1 & 5.042 & 3.539 \\
\hline 3664.6 & 4.622 & 3.325 & 31236.5 & 5.106 & 3.411 \\
\hline 6229.1 & 4.714 & 3.242 & 32743.6 & 5.132 & 3.254 \\
\hline 11186.6 & 4.795 & 3.135 & 33806.3 & 5.136 & 3.093 \\
\hline 26162.7 & 4.811 & 3.296 & 34577.9 & 5.124 & 2.932 \\
\hline 41399.5 & 4.901 & 3.384 & 35485.0 & 5.109 & 2.773 \\
\hline 48667.8 & 4.994 & 3.304 & 38817.5 & 5.093 & 2.613 \\
\hline 53680.0 & 5.044 & 3.160 & 45517.5 & 5.076 & 2.453 \\
\hline 57319.5 & 5.058 & 2.998 & 53818.4 & 5.056 & 2.295 \\
\hline 60022.7 & 5.055 & 2.836 & 66639.3 & 5.035 & 2.136 \\
\hline 63126.2 & 5.045 & 2.674 & 83922.1 & 5.012 & 1.979 \\
\hline 70885.2 & 5.034 & 2.512 & 110374.7 & 4.988 & 1.821 \\
\hline 83456.3 & 5.021 & 2.351 & 154685.4 & 4.964 & 1.664 \\
\hline 100917.4 & 5.004 & 2.190 & 218535.2 & 4.938 & 1.507 \\
\hline 123893.1 & 4.984 & 2.030 & 298372.0 & 4.912 & 1.350 \\
\hline 156369.0 & 4.963 & 1.870 & 418053.0 & 4.886 & 1.194 \\
\hline 205739.4 & 4.942 & 1.711 & 572912.6 & 4.857 & 1.038 \\
\hline 273240.8 & 4.918 & 1.552 & 766766.4 & 4.829 & 0.882 \\
\hline 371703.7 & 4.894 & 1.394 & 997695.9 & 4.799 & 0.727 \\
\hline 514045.9 & 4.870 & 1.235 & 1289019.6 & 4.769 & 0.573 \\
\hline 693994.4 & 4.843 & 1.077 & 1653517.0 & 4.738 & 0.418 \\
\hline 917881.0 & 4.816 & 0.920 & 2119396.4 & 4.705 & 0.264 \\
\hline 1179026.5 & 4.787 & 0.763 & 2644822.2 & 4.673 & 0.110 \\
\hline 1483518.9 & 4.758 & 0.607 & 3309034.4 & 4.640 & -0.043 \\
\hline 1904158.1 & 4.728 & 0.451 & 4204095.9 & 4.606 & -0.197 \\
\hline 2422390.9 & 4.696 & 0.296 & 5462318.9 & 4.572 & -0.350 \\
\hline 2940623.7 & 4.664 & 0.140 & 7306226.9 & 4.538 & -0.503 \\
\hline 3739476.4 & 4.631 & -0.015 & 9589210.2 & 4.504 & -0.655 \\
\hline 4652079.9 & 4.599 & -0.170 & 12936111.0 & 4.469 & -0.808 \\
\hline 5870408.9 & 4.565 & -0.324 & 19605223.3 & 4.434 & -0.961 \\
\hline 7886541.7 & 4.531 & -0.478 & 29021204.3 & 4.399 & -1.113 \\
\hline 10417032.1 & 4.497 & -0.632 & 41634214.4 & 4.364 & -1.266 \\
\hline 13952707.7 & 4.463 & -0.787 & 62786688.0 & 4.329 & -1.418 \\
\hline 19484298.8 & 4.428 & -0.941 & 86481772.1 & 4.293 & -1.570 \\
\hline
\end{tabular}


TABLE 3.4-Continued

He Burning PNN Evolutionary Models

\begin{tabular}{|c|c|c|c|c|c|}
\hline $\begin{array}{c}\text { time }^{\mathrm{a}} \\
(\mathrm{yrs})\end{array}$ & $\overline{\log T_{\text {eff }}}$ & $\log L / L_{\odot}$ & $\begin{array}{l}\text { time } \\
\text { (yrs) }\end{array}$ & $\overline{\log T_{\text {eff }}}$ & $\log L / L_{\odot}$ \\
\hline \multicolumn{3}{|c|}{$(M, Y, Z)=(2.0,0.25,0.016)$} & \multicolumn{3}{|c|}{$(M, Y, Z)=(2.5,0.25,0.016)$} \\
\hline 0.0 & 4.000 & 3.841 & 0.0 & 4.000 & 3.944 \\
\hline 43.4 & 4.109 & 3.838 & 56.1 & 4.115 & 3.944 \\
\hline 115.8 & 4.217 & 3.832 & 146.0 & 4.230 & 3.944 \\
\hline 227.4 & 4.326 & 3.824 & 284.8 & 4.346 & 3.944 \\
\hline 386.8 & 4.435 & 3.811 & 472.9 & 4.461 & 3.944 \\
\hline 636.0 & 4.543 & 3.791 & 732.1 & 4.576 & 3.943 \\
\hline 1055.0 & 4.650 & 3.764 & 1062.8 & 4.691 & 3.941 \\
\hline 1740.8 & 4.755 & 3.722 & 1441.7 & 4.806 & 3.935 \\
\hline 2886.7 & 4.856 & 3.661 & 1879.5 & 4.921 & 3.923 \\
\hline 4712.6 & 4.949 & 3.575 & 2354.5 & 5.036 & 3.900 \\
\hline 7202.2 & 5.025 & 3.456 & 2846.6 & 5.146 & 3.848 \\
\hline 14850.6 & 5.130 & 3.500 & 3325.7 & 5.234 & 3.736 \\
\hline 17230.7 & 5.178 & 3.351 & 3662.2 & 5.274 & 3.572 \\
\hline 18484.3 & 5.184 & 3.186 & 3791.3 & 5.268 & 3.396 \\
\hline 19533.3 & 5.177 & 3.020 & 3871.8 & 5.252 & 3.223 \\
\hline 21235.4 & 5.163 & 2.856 & 3952.4 & 5.237 & 3.049 \\
\hline 23580.4 & 5.146 & 2.693 & 4025.1 & 5.212 & 2.878 \\
\hline 27285.1 & 5.125 & 2.530 & 4177.7 & 5.183 & 2.708 \\
\hline 32345.2 & 5.102 & 2.368 & 5599.6 & 5.156 & 2.538 \\
\hline 39662.4 & 5.078 & 2.207 & 10354.0 & 5.130 & 2.367 \\
\hline 51318.3 & 5.053 & 2.046 & 17069.2 & 5.103 & 2.196 \\
\hline 69123.0 & 5.028 & 1.885 & 28827.7 & 5.075 & 2.026 \\
\hline 97210.5 & 5.000 & 1.725 & 46120.6 & 5.046 & 1.857 \\
\hline 142019.0 & 4.973 & 1.564 & 73031.8 & 5.016 & 1.688 \\
\hline 209514.1 & 4.944 & 1.405 & 115256.4 & 4.985 & 1.518 \\
\hline 294463.4 & 4.916 & 1.245 & 180095.7 & 4.954 & 1.350 \\
\hline 419979.6 & 4.887 & 1.085 & 277273.2 & 4.922 & 1.181 \\
\hline 594271.0 & 4.856 & 0.927 & 415456.3 & 4.889 & 1.013 \\
\hline 807518.0 & 4.824 & 0.768 & 588016.9 & 4.856 & 0.845 \\
\hline 1078445.0 & 4.792 & 0.610 & 810180.1 & 4.821 & 0.678 \\
\hline 1349372.1 & 4.760 & 0.452 & 1101756.7 & 4.786 & 0.511 \\
\hline 1755019.6 & 4.726 & 0.294 & 1393333.4 & 4.751 & 0.344 \\
\hline 2298920.7 & 4.693 & 0.137 & 1849288.4 & 4.714 & 0.178 \\
\hline 2842821.8 & 4.659 & -0.020 & 2468216.1 & 4.678 & 0.012 \\
\hline 3710269.6 & 4.624 & -0.177 & 3330842.5 & 4.640 & -0.154 \\
\hline 4726494.4 & 4.589 & -0.334 & 4193468.9 & 4.603 & -0.320 \\
\hline 6141162.4 & 4.554 & -0.490 & 5734642.2 & 4.565 & -0.486 \\
\hline 8291105.5 & 4.518 & -0.647 & 8094032.8 & 4.528 & -0.652 \\
\hline 12092309.5 & 4.482 & -0.803 & 11895480.3 & 4.489 & -0.817 \\
\hline 17124109.6 & 4.446 & -0.959 & 17916844.4 & 4.450 & -0.982 \\
\hline 26835620.6 & 4.410 & -1.115 & 27753840.6 & 4.412 & -1.147 \\
\hline 40246523.4 & 4.373 & -1.271 & 44184529.9 & 4.373 & -1.312 \\
\hline 58756285.3 & 4.336 & -1.427 & 65555573.2 & 4.334 & -1.477 \\
\hline 85885653.9 & 4.299 & -1.583 & 92580241.8 & 4.295 & -1.642 \\
\hline$\ldots$ & $\ldots$ & $\ldots$ & & $\ldots$ & $\ldots$ \\
\hline
\end{tabular}


TABLE 3.4-Continued

He Burning PNN Evolutionary Models

\begin{tabular}{|c|c|c|c|c|c|}
\hline $\begin{array}{c}\text { time }^{\mathrm{a}} \\
(\mathrm{yrs})\end{array}$ & $\log T_{\text {eff }}$ & $\log L / L_{\odot}$ & $\begin{array}{l}\text { time } \\
\text { (yrs) }\end{array}$ & $\log T_{\text {eff }}$ & $\log L / L_{\odot}$ \\
\hline \multicolumn{3}{|c|}{$(M, Y, Z)=(0.95,0.25,0.008)$} & \multicolumn{3}{|c|}{$(M, Y, Z)=(1.0,0.25,0.008)$} \\
\hline 0.0 & 4.000 & 3.550 & 0.0 & 4.000 & 3.600 \\
\hline 32.6 & 4.098 & 3.540 & 46.4 & 4.103 & 3.590 \\
\hline 79.5 & 4.197 & 3.526 & 117.3 & 4.205 & 3.576 \\
\hline 146.1 & 4.294 & 3.506 & 239.7 & 4.307 & 3.555 \\
\hline 238.1 & 4.391 & 3.477 & 876.1 & 4.408 & 3.527 \\
\hline 365.1 & 4.486 & 3.434 & 1624.2 & 4.508 & 3.492 \\
\hline 527.8 & 4.573 & 3.363 & 2697.8 & 4.606 & 3.443 \\
\hline 979.1 & 4.478 & 3.407 & 4524.9 & 4.698 & 3.374 \\
\hline 1224.9 & 4.386 & 3.459 & 7393.6 & 4.783 & 3.285 \\
\hline 1776.2 & 4.483 & 3.433 & 12334.7 & 4.855 & 3.173 \\
\hline 2170.8 & 4.576 & 3.382 & 27675.2 & 4.897 & 3.316 \\
\hline 2930.6 & 4.660 & 3.303 & 40729.9 & 4.996 & 3.358 \\
\hline 6437.1 & 4.741 & 3.216 & 46463.1 & 5.061 & 3.236 \\
\hline 11271.9 & 4.807 & 3.106 & 49702.5 & 5.081 & 3.083 \\
\hline 18630.9 & 4.861 & 2.980 & 52107.7 & 5.083 & 2.926 \\
\hline 42903.3 & 4.921 & 3.099 & 54467.7 & 5.075 & 2.770 \\
\hline 66086.4 & 5.014 & 3.049 & 58632.8 & 5.062 & 2.615 \\
\hline 73034.5 & 5.033 & 2.902 & 67042.5 & 5.050 & 2.459 \\
\hline 77961.4 & 5.032 & 2.752 & 70225.2 & 5.029 & 2.306 \\
\hline 82180.2 & 5.023 & 2.602 & 89841.0 & 5.014 & 2.152 \\
\hline 91442.8 & 5.014 & 2.453 & 110150.4 & 4.994 & 1.998 \\
\hline 103488.9 & 5.000 & 2.304 & 142186.0 & 4.972 & 1.845 \\
\hline 112895.1 & 4.983 & 2.156 & 185486.3 & 4.950 & 1.692 \\
\hline 143428.7 & 4.968 & 2.008 & 245226.8 & 4.927 & 1.540 \\
\hline 169460.8 & 4.949 & 1.860 & 335067.4 & 4.903 & 1.388 \\
\hline 223544.0 & 4.931 & 1.713 & 468693.5 & 4.878 & 1.236 \\
\hline 295100.2 & 4.912 & 1.566 & 624086.2 & 4.853 & 1.084 \\
\hline 394290.3 & 4.890 & 1.419 & 831484.7 & 4.826 & 0.933 \\
\hline 519722.6 & 4.868 & 1.273 & 1068266.7 & 4.798 & 0.782 \\
\hline 683215.5 & 4.844 & 1.127 & 1356753.8 & 4.769 & 0.632 \\
\hline 877004.0 & 4.820 & 0.982 & 1698862.2 & 4.740 & 0.482 \\
\hline 1116843.0 & 4.794 & 0.837 & 2130250.4 & 4.710 & 0.333 \\
\hline 1417184.6 & 4.767 & 0.692 & 2688835.7 & 4.679 & 0.183 \\
\hline 1770820.7 & 4.740 & 0.548 & 3433557.8 & 4.647 & 0.034 \\
\hline 2212831.6 & 4.712 & 0.404 & 4178280.0 & 4.616 & -0.115 \\
\hline 2710816.6 & 4.683 & 0.260 & 5172869.8 & 4.584 & -0.263 \\
\hline 3319805.9 & 4.653 & 0.117 & 6876659.3 & 4.551 & -0.412 \\
\hline 4111728.3 & 4.623 & -0.026 & 9258227.7 & 4.518 & -0.560 \\
\hline 5182263.5 & 4.593 & -0.169 & 12198856.1 & 4.484 & -0.708 \\
\hline 6252798.7 & 4.563 & -0.312 & 16435138.1 & 4.451 & -0.856 \\
\hline 7996678.7 & 4.532 & -0.454 & 24762950.9 & 4.417 & -1.004 \\
\hline 10486114.5 & 4.500 & -0.596 & 36245231.7 & 4.384 & -1.151 \\
\hline 13659826.1 & 4.469 & -0.739 & 52634927.3 & 4.350 & -1.299 \\
\hline 18946080.0 & 4.437 & -0.881 & 73418094.5 & 4.315 & -1.446 \\
\hline 27171975.3 & 4.405 & -1.023 & $\ldots$ & $\ldots$ & $\ldots$ \\
\hline
\end{tabular}


TABLE 3.4-Continued

He Burning PNN Evolutionary Models

\begin{tabular}{|c|c|c|c|c|c|}
\hline $\begin{array}{c}\text { time }^{\mathrm{a}} \\
\text { (yrs) }\end{array}$ & $\overline{l o g} T_{\text {eff }}$ & $\log L / L_{\odot}$ & $\begin{array}{l}\text { time } \\
\text { (yrs) }\end{array}$ & $\overline{\log T_{\text {eff }}}$ & $\overline{\log L / L_{\odot}}$ \\
\hline \multicolumn{3}{|c|}{$(M, Y, Z)=(1.5,0.25,0.008)$} & \multicolumn{3}{|c|}{$(M, Y, Z)=(2.0,0.25,0.008)$} \\
\hline 0.0 & 4.000 & 3.792 & 0.0 & 4.000 & 3.918 \\
\hline 72.0 & 4.108 & 3.787 & 44.1 & 4.113 & 3.915 \\
\hline 178.3 & 4.216 & 3.780 & 113.2 & 4.225 & 3.910 \\
\hline 341.3 & 4.323 & 3.769 & 218.5 & 4.338 & 3.903 \\
\hline 601.3 & 4.431 & 3.753 & 380.1 & 4.450 & 3.891 \\
\hline 999.1 & 4.537 & 3.730 & 630.3 & 4.563 & 3.875 \\
\hline 1642.9 & 4.643 & 3.697 & 1019.9 & 4.674 & 3.850 \\
\hline 2662.6 & 4.746 & 3.648 & 1602.5 & 4.784 & 3.813 \\
\hline 4251.7 & 4.844 & 3.579 & 2477.0 & 4.891 & 3.758 \\
\hline 6569.7 & 4.932 & 3.485 & 3691.8 & 4.990 & 3.676 \\
\hline 10130.6 & 5.008 & 3.368 & 5525.7 & 5.077 & 3.566 \\
\hline 14913.2 & 5.026 & 3.530 & 8943.6 & 5.127 & 3.719 \\
\hline 19976.9 & 5.132 & 3.502 & 10574.5 & 5.216 & 3.614 \\
\hline 21723.0 & 5.169 & 3.348 & 11378.3 & 5.245 & 3.448 \\
\hline 22812.2 & 5.174 & 3.184 & 11863.7 & 5.244 & 3.277 \\
\hline 23688.9 & 5.166 & 3.020 & 12370.5 & 5.231 & 3.106 \\
\hline 24961.8 & 5.152 & 2.858 & 13141.4 & 5.212 & 2.937 \\
\hline 27426.7 & 5.137 & 2.695 & 14845.5 & 5.192 & 2.769 \\
\hline 31650.7 & 5.118 & 2.534 & 17179.1 & 5.169 & 2.601 \\
\hline 37096.8 & 5.096 & 2.373 & 20632.4 & 5.145 & 2.433 \\
\hline 45285.0 & 5.073 & 2.212 & 25789.9 & 5.119 & 2.266 \\
\hline 56953.4 & 5.049 & 2.053 & 33935.3 & 5.091 & 2.100 \\
\hline 74441.5 & 5.023 & 1.893 & 46790.4 & 5.063 & 1.934 \\
\hline 104178.6 & 4.997 & 1.734 & 67638.7 & 5.034 & 1.768 \\
\hline 150645.3 & 4.970 & 1.575 & 100796.3 & 5.003 & 1.603 \\
\hline 212302.3 & 4.943 & 1.416 & 152320.8 & 4.973 & 1.438 \\
\hline 316631.7 & 4.914 & 1.258 & 232864.0 & 4.942 & 1.273 \\
\hline 445461.6 & 4.885 & 1.100 & 333199.3 & 4.910 & 1.108 \\
\hline 609229.8 & 4.856 & 0.942 & 487980.3 & 4.878 & 0.944 \\
\hline 818261.6 & 4.825 & 0.785 & 680526.6 & 4.844 & 0.780 \\
\hline 1086638.8 & 4.793 & 0.628 & 929046.5 & 4.810 & 0.617 \\
\hline 1435162.0 & 4.761 & 0.471 & 1177566.5 & 4.776 & 0.453 \\
\hline 1783685.1 & 4.728 & 0.315 & 1552809.1 & 4.741 & 0.290 \\
\hline 2310012.7 & 4.695 & 0.159 & 2061361.4 & 4.705 & 0.128 \\
\hline 2915630.0 & 4.661 & 0.003 & 2569913.6 & 4.669 & -0.035 \\
\hline 3699486.5 & 4.627 & -0.153 & 3392019.2 & 4.632 & -0.197 \\
\hline 4791798.3 & 4.592 & -0.308 & 4524218.7 & 4.596 & -0.359 \\
\hline 6398986.8 & 4.557 & -0.464 & 6217526.7 & 4.558 & -0.521 \\
\hline 8858144.7 & 4.522 & -0.619 & 8833619.2 & 4.521 & -0.683 \\
\hline 11991434.0 & 4.487 & -0.774 & 12249887.1 & 4.484 & -0.845 \\
\hline 17961546.1 & 4.451 & -0.929 & 19224016.5 & 4.447 & -1.007 \\
\hline 26561673.5 & 4.415 & -1.083 & 31758215.8 & 4.409 & -1.168 \\
\hline 39554675.3 & 4.379 & -1.238 & 48392349.2 & 4.370 & -1.330 \\
\hline 57801848.6 & 4.343 & -1.393 & 69341118.1 & 4.332 & -1.491 \\
\hline 83172271.7 & 4.306 & -1.547 & 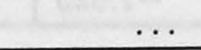 & $\ldots$ & $\cdots$ \\
\hline
\end{tabular}


TABLE 3.4-Continued

He Burning PNN Evolutionary Models

\begin{tabular}{|c|c|c|c|c|c|}
\hline $\begin{array}{c}\text { time }^{\mathrm{a}} \\
(\mathrm{yrs})\end{array}$ & $\log T_{\text {eff }}$ & $\log L / L_{\odot}$ & $\begin{array}{l}\text { time } \\
\text { (yrs) }\end{array}$ & $\overline{\log T_{\text {eff }}}$ & $\overline{l o g} L / L_{\odot}$ \\
\hline \multicolumn{3}{|c|}{$(M, Y, Z)=(2.5,0.25,0.008)$} & \multicolumn{3}{|c|}{$(M, Y, Z)=(0.89,0.25,0.004)$} \\
\hline 0.0 & 4.000 & 3.954 & 0.0 & 4.000 & 3.624 \\
\hline 37.1 & 4.115 & 3.952 & 23.4 & 4.096 & 3.616 \\
\hline 97.8 & 4.230 & 3.947 & 55.5 & 4.192 & 3.603 \\
\hline 185.1 & 4.345 & 3.941 & 100.4 & 4.288 & 3.584 \\
\hline 322.3 & 4.459 & 3.932 & 158.8 & 4.383 & 3.558 \\
\hline 531.8 & 4.574 & 3.916 & 231.6 & 4.475 & 3.517 \\
\hline 859.3 & 4.688 & 3.894 & 341.4 & 4.563 & 3.454 \\
\hline 1334.2 & 4.801 & 3.859 & 680.2 & 4.471 & 3.499 \\
\hline 2000.5 & 4.910 & 3.808 & 882.8 & 4.379 & 3.545 \\
\hline 2924.3 & 5.014 & 3.732 & 1338.5 & 4.474 & 3.522 \\
\hline 6462.7 & 5.116 & 3.813 & 1649.9 & 4.566 & 3.478 \\
\hline 7658.2 & 5.221 & 3.743 & 2361.7 & 4.651 & 3.409 \\
\hline 8334.2 & 5.273 & 3.587 & 4790.9 & 4.734 & 3.335 \\
\hline 8683.2 & 5.279 & 3.412 & 8298.6 & 4.806 & 3.236 \\
\hline 8961.8 & 5.269 & 3.238 & 13742.4 & 4.865 & 3.121 \\
\hline 9367.7 & 5.251 & 3.065 & 21104.5 & 4.909 & 2.990 \\
\hline 10339.6 & 5.230 & 2.893 & 29995.7 & 4.941 & 2.851 \\
\hline 11892.5 & 5.207 & 2.722 & 56701.1 & 5.004 & 2.962 \\
\hline 13970.3 & 5.182 & 2.551 & 73527.8 & 5.039 & 2.825 \\
\hline 17362.7 & 5.155 & 2.381 & 79732.8 & 5.037 & 2.679 \\
\hline 22437.7 & 5.127 & 2.212 & 85713.8 & 5.029 & 2.532 \\
\hline 30302.8 & 5.097 & 2.043 & 94501.5 & 5.015 & 2.387 \\
\hline 42639.5 & 5.066 & 1.874 & 102590.9 & 4.999 & 2.242 \\
\hline 58836.0 & 5.035 & 1.706 & 124429.6 & 4.985 & 2.097 \\
\hline 87653.1 & 5.003 & 1.538 & 149733.3 & 4.968 & 1.953 \\
\hline 134188.1 & 4.970 & 1.370 & 189194.2 & 4.950 & 1.809 \\
\hline 197080.9 & 4.937 & 1.203 & 242363.8 & 4.931 & 1.665 \\
\hline 306381.6 & 4.903 & 1.036 & 313419.2 & 4.909 & 1.522 \\
\hline 448745.7 & 4.869 & 0.869 & 407412.7 & 4.888 & 1.379 \\
\hline 638257.6 & 4.834 & 0.702 & 544139.3 & 4.866 & 1.236 \\
\hline 827769.5 & 4.799 & 0.535 & 714917.3 & 4.842 & 1.094 \\
\hline 1131836.8 & 4.763 & 0.369 & 904759.2 & 4.818 & 0.952 \\
\hline 1539671.3 & 4.726 & 0.204 & 1167673.2 & 4.792 & 0.810 \\
\hline 2098066.2 & 4.690 & 0.038 & 1473480.1 & 4.766 & 0.669 \\
\hline 2656461.1 & 4.653 & -0.128 & 1811994.7 & 4.739 & 0.529 \\
\hline 3607628.8 & 4.615 & -0.293 & 2216384.8 & 4.711 & 0.388 \\
\hline 4766649.1 & 4.577 & -0.458 & 2724351.4 & 4.683 & 0.248 \\
\hline 6973606.1 & 4.539 & -0.623 & 3386677.7 & 4.654 & 0.108 \\
\hline 10163527.4 & 4.500 & -0.788 & 4049004.1 & 4.625 & -0.032 \\
\hline 15641625.4 & 4.463 & -0.953 & 5077756.2 & 4.595 & -0.171 \\
\hline 24868990.4 & 4.424 & -1.118 & 6429663.7 & 4.565 & -0.311 \\
\hline 40486891.5 & 4.385 & -1.282 & 8260848.8 & 4.535 & -0.450 \\
\hline 60951078.4 & 4.346 & -1.447 & 10692180.4 & 4.504 & -0.589 \\
\hline 86142377.5 & 4.307 & -1.611 & 13700809.9 & 4.474 & -0.729 \\
\hline & & & 18803422.7 & 4.442 & -0.867 \\
\hline
\end{tabular}




\subsection{Discussion}

\subsubsection{The Transition Time}

As was noted at the beginning of this investigation, previous theoretical studies of this nature contrived the transition times to be of the order of $\sim 5000$ years by manipulating mass loss rates (e. g., Schönberner 1983; WF86). This was primarily done to agree with the observed dynamical ages (nebular radius divided by expansion velocity) seen in PNe. However, observations of Magellanic PNe have shown that the nebular expansion accelerates during the early, constant luminosity phase of evolution, and then proceeds at constant velocity as the PNN fades (Dopita et al. 1987; Dopita \& Meatheringham 1990). This means that dynamical ages represent only a lower limit to the elapsed time since departure from the AGB. There is no way of telling whether the transition times of our calculations provide a realistic description of the evolution away from the AGB. However, it is interesting to note that the observations imply a wide range in dynamical ages $\sim 10^{2}-10^{5}$ years (Sabbadin 1986), similar to what is seen for our calculations. There are no obvious correlations observed between, for instance, nebular age with stellar luminosity (Pottasch \& Acker 1989), as one might expect given the random phase of departure from the AGB. However, we note that there are considerable uncertainties associated with defining unambiguous dynamical ages (cf. Renzini 1989). Ideally, in order to constrain the transition time, one requires a hydrodynamic code to examine the behaviour of the ejected material in conjunction with the known evolution of the central star (e. g., Kahn \& Breitschwerdt 1990; Marten \& Schönberner 1991).

\subsubsection{PNN Tracks at High Core Mass}

High mass PNN ( $M \gtrsim 0.8 \mathrm{M}_{\odot}$ ) evolve so rapidly that we only expect to see PNe with large nuclei at low luminosity (Renzini 1979; Schönberner \& Weidemann 1983; Shaw 1989). For the $\mathrm{H}$ burning $\mathrm{PNNi}$ studied here with $M \sim 0.9 \mathrm{M}_{\odot}$, evolution only slows down when $\log L / L_{\odot} \sim 1.8$ to 2.0 (this corresponds to an age of $10^{4}$ years since

$\log T_{\text {eff }}=4$ ). Furthermore, the higher the PNN mass, the lower is $\log \mathrm{L} / \mathrm{L}_{\odot}$ at ages of $\sim 10^{4}$ years, although this effect is not large for $M \gtrsim 0.65 \mathrm{M}_{\odot}$ (see figures 3.3-3.9).

However, the recent calculations of Blöcker \& Schönberner (1990) show a high mass track, $M=0.836 \mathrm{M}_{\odot}$, which is still quite luminous $\left(\log L / L_{\odot} \sim 2.5\right)$ after evolving for $10^{4}$ years. Most importantly, they find that the higher the PNN mass, the higher is $\log \mathrm{L} / \mathrm{L}_{\odot}$ at $t=10^{4}$ years. Consequently, they conclude that the least 
luminous PNe that are observed correspond to low mass nuclei, rather than high mass nuclei as previously thought. The results of Blöcker \& Schönberner conflict with the present results (and those of WF86 and Paczyński 1971) in predicting higher residual luminosities for higher mass tracks. Clearly, the source of this discrepancy needs further investigation.

Type I (Peimbert 1978) PNe in the Magellanic Clouds offer a good opportunity to compare observations and theory. Spectrophotometric observations, and subsequent photoionisation modelling, consistently places these objects in the H-R plane where $M>0.7 \mathrm{M}_{\odot}$ (see figure 8, Dopita, Jacoby, \& Vassiliadis 1992; figure 3, Dopita \& Meatheringham 1991). These objects are believed to be the progeny of higher mass AGB stars that have undergone significant 3rd dredge up, and possibly, envelope burning (Peimbert \& Serrano 1980; Kaler \& Jacoby 1990). The type I objects in the LMC uniformly span a wide range in luminosity $\left(\log L / L_{\odot} \sim 2.5\right.$ to4). Comparison with the present $\mathrm{H}$ burning PNN tracks (figure 3.4), or those of Blöcker \& Schönberner, shows that this interval is one of very rapid evolution. It seems unlikely that type I PNe would be found so frequently in this interval if their nuclei were primarily burning $\mathrm{H}$. A glance at figure 3.8 shows that He burning PNN spend several thousands of years in the interval $\log L / L_{\odot} \sim 2.5$ to 4.0 where the LMC type I PNe exist. It thus seems that the observed type I PNe enter the PN regime as He burners. $\mathrm{H}$ burning equivalents may also exist but they would quickly fade to $\log L / L_{\odot}<2.5$. Finally, we note that the radiation pressure ejection mechanism of WF 86 forces the more massive AGB stars to leave the AGB at a helium shell flash. This mechanism may therefore explain the existence of type I PNe with He burning central stars.

Whether the more massive PNe of type I enter the PN domain preferentially as $\mathrm{He}$ burners, or whether selection effects (high luminosity) mean that we are seeing just the He burners while missing many massive, hydrogen burning $\mathrm{PNe}$ at $\log L / L_{\odot}<2$, can be addressed by examining the death rate of these objects. The immediate precursors of type I PNe are most likely the massive $\left(M_{i} \sim 5 M_{\odot}\right)$, luminous $\left(-6>M_{b o l}>-7\right)$, dust-enshrouded, pulsating IRAS sources throwing off their envelopes at the end of the AGB. Wood et al. (1992) have observed 8 such objects known in the LMC and their mass loss rates are $\sim 10^{-4} \mathrm{M}_{\odot} \mathrm{yr}^{-1}$. If they lose $\sim 4 \mathrm{M}_{\odot}$ of envelope during the mass loss process, their lifetimes in this phase will be $\sim 4 \times 10^{4} \mathrm{yr}$ and, in the whole LMC, there will be a death rate of $\sim 1$ object per 5000 years.

The immediate precursors of the mass-losing upper AGB stars themselves are the optically visible long period variables (Wood, Bessell \& Fox 1983; Hughes \& Wood 
1990). There are approximately 30 such objects in the LMC with $-6>M_{\text {bol }}>-7$, $P>500$ days, and $M_{i} \sim 5 M_{\odot}$. The lifetime of such LPVs is not well known, but Hughes \& Wood (1990) estimate a lifetime of $\sim 5 \times 10^{4} \mathrm{yr}$ for less massive LPVs. Given that the rate of evolution up the AGB for the massive AGB stars is very slow $(\sim 0.01$ mag per $5 \times 10^{4} \mathrm{yr}$ ) due to the fact that the core mass is prevented from increasing by dredge-up at He shell flashes (VW92), it is hard to see how the lifetime of these stars could be shorter than $5 \times 10^{4} \mathrm{yr}$. Adopting this lifetime, we find a total deathrate for these objects throughout the LMC of $\sim 1$ object per 2000 years.

From the above two results, we would expect the birth rate of type I PNe in the LMC to be 1 per $\sim 2000-5000$ years. Looking at figure 3.4, we see that there are 8 type I PNe with $2.8<\log L / L_{\odot}<3.8$. If these objects are $\mathrm{H}$ burners, then the lifetime in this phase is $\sim 1000$ years even with a core mass as low as $0.67 \mathrm{M}_{\odot}$, and $<100$ years for a more realistic core mass of $\sim 0.9 \mathrm{M}_{\odot}$, corresponding to $M_{b o l} \sim-6$ on the AGB. These lifetimes requires a death rate of at least $\sim 1$ object per 250 years, and more likely 1 per 12 years, on the upper AGB to feed the type I PN population in the LMC. This is clearly at variance with the observed AGB death rate. On the other hand, a glance at figure 3.8 shows that He burning PNe spend much more time at high luminosities. Unfortunately, we do not have a massive $\left(\sim 0.9 \mathrm{M}_{\odot}\right)$ He burning PNN, but the most massive He burning nuclei at 0.67 and $0.68 \mathrm{M}_{\odot}$ spend $\sim 4000$ years above $\log L / L_{\odot}=2.8, \sim 4$ times longer than the equivalent $\mathrm{H}$ burning nuclei. If the type $\mathrm{I}$ PNe have lifetimes above $\log L / L_{\odot}=2.8$ of 4000 years, their birth rate is $\sim 1$ per 500 years, closer to the AGB death rate. Of course, the He burning lifetime at $0.9 \mathrm{M}_{\odot}$ will be shorter than at $0.68 \mathrm{M}_{\odot}$ so that a significant discrepancy still exists between the death rate of upper AGB stars with $M_{b o l}<-6$ and the birth rate of type I PNe.

Since the death rates of both the dust enshrouded and optically visible upper AGB stars seem to agree reasonably well, we do not think that there are great errors in the death rates of these stars. The most likely solution to the above birth/death rate discrepancy is to let type I PNe come from a wider range of the LPV population than assumed. The most luminous type I PNe have $\log L / L_{\odot} \sim 3.8\left(M_{b o l} \sim-4.75\right)$. If we assume that all LPVs with luminosities down to this level can produce type I PNe, then the number of stars contributing to the type I PN population goes up by a factor of $\sim 3.5$, the mean core mass comes down closer to $\sim 0.68 \mathrm{M}_{\odot}$, and there is reasonable agreement between the death rate of LPVs with $M_{b o l}<-4.75$ and the birth rate of type I PNe under the assumption that they are He burners. Using the results of VW92, the low mass limit for production of type I PNe in this scenario is $\sim 2 \mathrm{M}_{\odot}$, reasonably 
close to the lower mass limit of $\sim 2.4 \mathrm{M}_{\odot}$ for type I PNNi given by Peimbert \& Serrano (1980).

\subsubsection{The Case For He Burning PNNi}

Although, as noted above, some PNNi appear to be He burning, it has generally been believed over the past decade that the majority of PNNi are burning $\mathrm{H}$ when they depart the AGB (Iben 1984; Schönberner 1981, 1984, 1986; Schönberner \& Weidemann 1983). PNNi undergoing He burning have been invoked to explain the existence of nonDA WD stars (Iben 1984, 1987; Schönberner 1986; WF86); it is assumed that PNNi that leave the AGB at, or near, a He shell flash will proceed to form non-DA WD stars. Iben estimated that there is an $\sim 20 \%$ chance to produce a He burning central star, this being simply the ratio of the $\mathrm{He}$ to $\mathrm{H}$ burning lifetimes over a He shell flash cycle. The occurrence of DA and non-DA WD stars does not appear related to the stellar mass as the mass distributions of DA and non-DA WDs are approximately equal (see Weidemann 1990 , for a review).

From the 27 PNN evolutionary calculations presented in this study it was noted that the lower mass models appeared more efficient at producing He burning PNN evolutionary tracks. The reason for this is associated with the interaction of the mass loss rate on the AGB, $\dot{\mathrm{M}}_{A G B}$, with the phase of the helium flash shell cycle, $\phi$. Looking at the last couple of flashes for any of the low mass sequences in VW92, $\dot{\mathrm{M}}_{A G B}$ rises to its radiation pressure driven limit only for $0.8 \lesssim \phi \lesssim 1.0$ where the surface luminosity is largest. Since the envelope is most likely to be completely dissipated during phases of high mass loss rate, we expect the star to evolve from the AGB during the last part of the $\mathrm{H}$ burning phase of the shell flash cycle. Such a star is likely to suffer a shell flash during the subsequent post-AGB evolution.

At higher masses, the helium shell flash recovery time is relatively short compared to the interpulse time, primarily due to the presence of deep envelope convection. Even when the deep envelope convection begins to recede towards the final thermal pulses on the AGB, the helium flash recovery time is still shorter than is observed in the low mass models (see also Wood \& Zarro 1981). Consequently, $\dot{\mathrm{M}}_{A G B}$, remains at a high value for a greater fraction of the interpulse time, and a star is likely to leave the AGB over a wider range of $\phi$ values than for a low mass PNN.

Galactic Bulge (GB) PNe seem to indicate that low mass stars produce He burning PNNi. A significant proportion of PNe in the Galactic Bulge have luminosities that fall 
below that of the $0.546 \mathrm{M}_{\odot}$ PNN track of Schönberner (1983). The rate of evolution of this track in the PN regime is so slow that the PN would dissipate before the PNN would evolve very far in $\log T_{\text {eff }}$. This has been noticed by several authors in recent years (Mèndez et al. 1988; Gathier \& Pottasch 1989; Pottasch \& Acker 1989). Ratag (1990), and coworkers, present an exhaustive list of arguments to show that the majority of GB PNe must be optically thick. Therefore, the luminosities are not being underestimated because of flux escaping from the nebulae. These authors go on to suggest that $\mathrm{He}$ burning PNNi may help explain the existence of the observed low luminosity PNe, and possibly their dynamical ages, but they query whether the He burning scenario is a common phenomenon. Our models seem to suggest that this is indeed the case for low mass stars $\left(M_{i} \lesssim 1 \mathrm{M}_{\odot}\right)$.

\subsubsection{Luminosity Functions}

As pointed out by Renzini \& Buzzoni (1986), an AGB/PNN population can contribute significantly to the total bolometric luminosity of a galaxy. The contribution of energy as a function of luminosity is indicated for the $Z=0.016 \mathrm{H}$ burning and $Z=0.008 \mathrm{He}$ burning PNN evolutionary tracks in figures 3.10 and 3.11 , respectively. The information displayed has been taken directly from the information presented in table 3.3. These plots clearly show the rapid drop in energy for the $\mathrm{H}$ burning PNN tracks from the high, constant luminosity phase to $\log L / L_{\odot} \sim 2.7$.

Ideally, the next step to be undertaken with the work presented in this paper is to convolve our theoretical luminosity functions for each mass with an assumed initial mass function and star formation rate (cf. Shaw 1989). This would allow comparisons to be made between the theoretical and observed luminosity functions of stellar systems. A similar exercise can be carried out considering the UV fluxes in order to estimate the PNN contribution to the UV excess seen in elliptical galaxies (Brocato et al. 1989). 


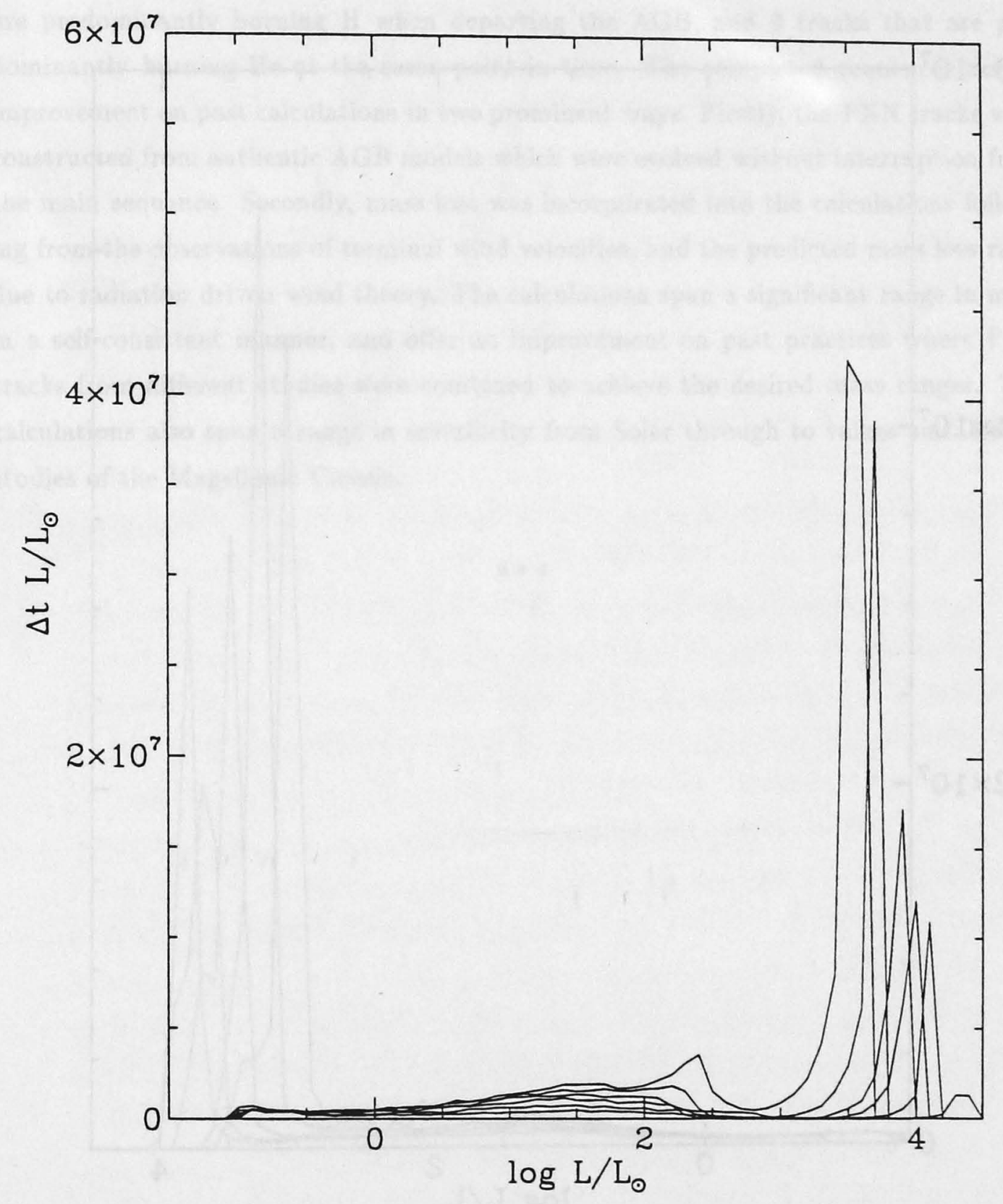

Figure 3.10: The total energy output (in $\mathrm{L}_{\odot} \mathrm{yr}$ ) per unit of 0.1 in $\log \mathrm{L} / \mathrm{L}_{\odot}$ for the $Z=0.016 \mathrm{H}$ burning PNN evolutionary tracks. The peaks correspond to tracks of increasing mass as one looks at higher luminosities. 


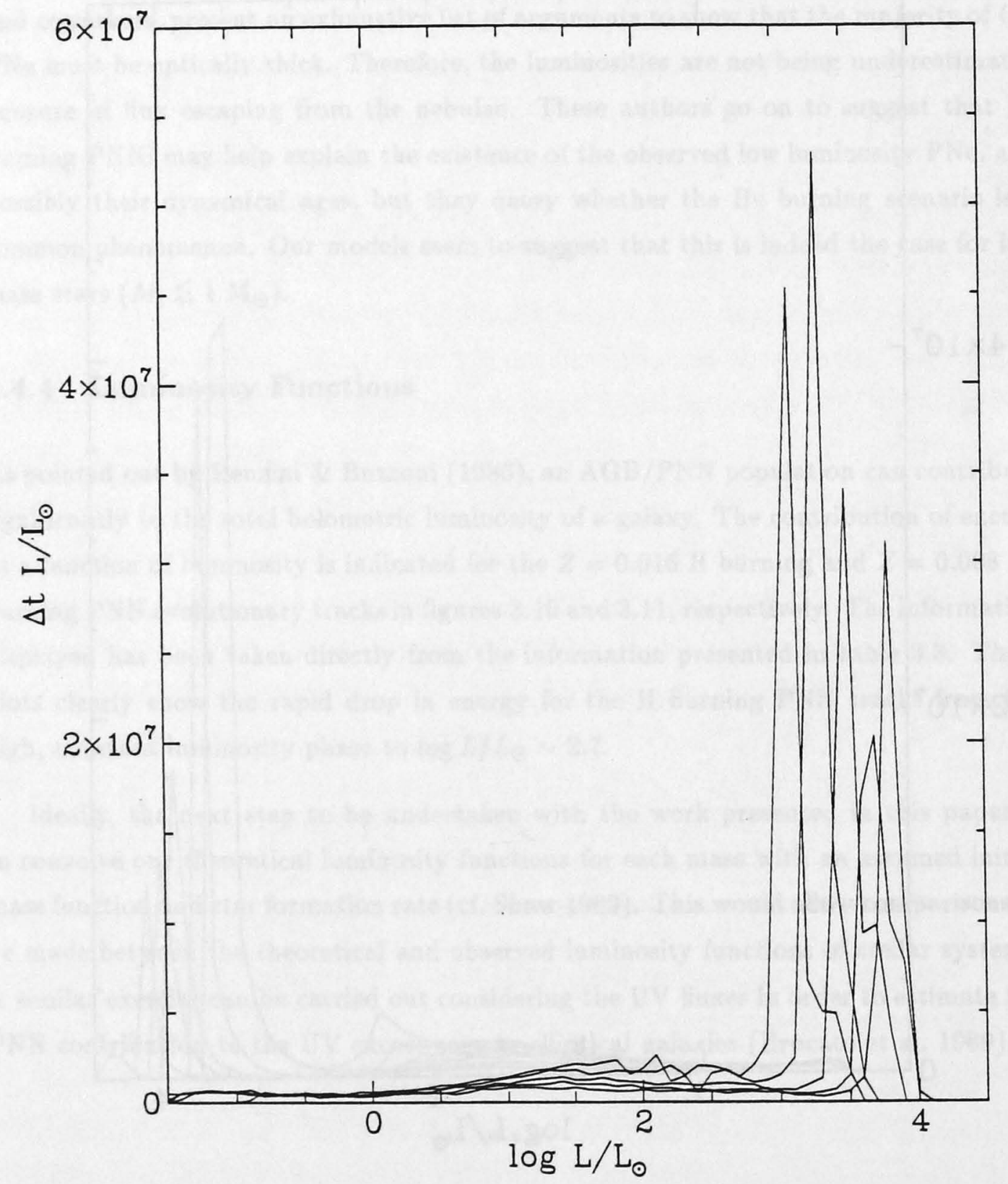

Figure 3.11: Same as figure 3.10, except for the $Z=0.008$ He burning PNN evolutionary tracks. 


\subsection{Summary}

We have presented a grid of PNN evolutionary tracks, consisting of 18 tracks which are predominantly burning $\mathrm{H}$ when departing the AGB, and 9 tracks that are predominantly burning $\mathrm{He}$ at the same point in time. The completed sequences are an improvement on past calculations in two prominent ways. Firstly, the PNN tracks were constructed from authentic AGB models which were evolved without interruption from the main sequence. Secondly, mass loss was incorporated into the calculations following from the observations of terminal wind velocities, and the predicted mass loss rates due to radiation driven wind theory. The calculations span a significant range in mass in a self-consistent manner, and offer an improvement on past practices where PNN tracks from different studies were combined to achieve the desired mass ranges. The calculations also span a range in metallicity from Solar through to values suitable for studies of the Magellanic Clouds. 


\subsection{References}

Abott, D. C. 1982, ApJ, 259, 282

Bergeron, P., Wesemael, F., Fontaine, G., \& Liebert, J. 1990, ApJ, 351, L21

Blöcker, T., \& Schönberner, D. 1990, A\&AL, 240, L11

Brocato, E., Matteucci, F., Mazzitelli, I., \& Tornambè, A. 1989, Ap\&SS, 156,159

Cerruti-Sola, M., \& Perinotto, M. 1989, ApJ, 345, 339

de Jager, C., Nieuwenhuijzen, H., \& van der Hucht, K. A. 1988, A\&AS, 72, 259

Dopita, M. A., Jacoby, G. H., \& Vassiliadis, E. 1992, ApJ, 389, 27 (Chapter 7)

Dopita, M. A., \& Meatheringham, S. J. 1990, ApJ, 357, 140 ., 1991, 377, 480

Dopita, M. A., Meatheringham, S. J., Wood, P.R., Webster, B. L., Morgan, D. H., \& Ford, H. C. 1987, ApJ, 315, L107

Eder, J., Lewis, B. M., \& Terzian, Y. 1988, ApJS, 66, 183

Friend, D. B., \& Abott, D. C. 1986, ApJ, 311, 701

Gathier, R., \& Pottasch, S. R. 1989, A\&A, 209, 369

Grewing, M. 1989, in IAU Symp. 131, Planetary Nebulae, ed. S. TorresPeimbert (Dordrecht: Kluwer), 241

Härm, R., \& Schwarschild, M. 1975, ApJ, 200, 324

Harpaz, A., \& Kovetz, A. 1981, A\&A, 93200

Heap, S. R. 1986, in New Insights in Astrophysics, Eight Years of IUE, ESA SP-263, 291

Hrivnak, B. J., Kwok, S., \& Volk, K. M. 1989, ApJ, 346, 265

$\mathrm{Hu}$, J. Y., de Jong, T., \& Slijkhuis, S. 1990, in From Miras to Planetary Nebulae: Which Path for Stellar Evolution?, eds. M. O. Mennessier \& A. Omont (Yvette Cedex: Editions Frontières), 487

Hughes, S. M. G., \& Wood, P. R. 1990, AJ, 99, 784

Hutsemékers, D., \& Surdej, J. 1989, A\&A, 219, 237

Iben, I. Jr. 1984, ApJ, 277, 333 , 1987, in Late Stages of Stellar Evolution, eds. S. Kwok, \&

S. R. Pottasch (Dordrecht: Reidel), 175

Iben, I. Jr., Kaler, J. B., Truran, J. W., \& Renzini, A. 1983, ApJ, 264, 605

Kaler, J. B., \& Jacoby, G. H. 1990, ApJ, 362, 491

Khan, F. D., \& Breitschwerdt, D. 1990, MNRAS, 242, 505 
Kudritzki, R. P., Pauldrach, A., \& Puls, J. 1987, A\&A, 173, 293

Kudritzki, R. P., Pauldrach, A., Puls, J., \& Abott, D. C. 1989, A\&A, 219, 205

Kwok, S. 1982, ApJ, 258, 280

Kwok, S., Purton, C. R., \& Fitzgerald, P. M. 1978, ApJL, 219, L125

Kwok, S., Volk, K. M., \& Hrivnak, B. J. 1989, ApJ, 346, 265

Liebert, J. 1989, in IAU Symp. 131, Planetary Nebulae, ed. S. TorresPeimbert (Dordrecht: Kluwer), 545

Marten, H., \& Schönberner, D. 1991, A\&A, 248, 590

McCarthy, J. K., Mould, J. R., Mendez, R. H., Kudritzki, R. P., Husfeld, D., Herrero, A., \& Groth, H. G. 1990, ApJ, 351, 230

Méndez, R. H., Kudritzki, R. P., Herrero, A., Husfeld, D., Groth, H. G. 1988, A\&A, 190, 113

Paczyński, B. 1971, Acta Astron., 21, 417

Pauldrach, A., Puls, J., Kudritzki, R. P., Méndez, R. H., \& Heap, S. R. 1988, A\&A, 207, 123

Peimbert, M. 1978, in IAU Symp. 76, Planetary Nebulae: Observations and Theory, ed. Y. Terzian (Dordrecht: Reidel), 215

Peimbert, M., \& Serrano, A. 1980, Rev. Mexicana Astron. Astrof., 5, 9

Perinotto, M. 1989, in IAU Symposium 131, Planetary Nebulae, ed. S. Torres-Peimbert (Dordrecht: Kluwer), 293

Pottasch, S. R., \& Acker, A. A. 1989, A\&A, 221, 123

Pottasch, S. R., \& Partharasathy, M. 1988, A\&A, 192, 182

Ratag, M. A. 1990, PhD Thesis, Groningen

Reimers, D. 1975, in Problems in Stellar Atmospheres and Envelopes, ed. B. Baschek, W. H. Kegel, \& G. Traving (Berlin: Springer), 229

Renzini, A. 1979, in Stars and Star Systems, ed. B. E. Westerlund (Dordrecht: Reidel), 155 1989 , in IAU Symp. 131, Planetary Nebulae, ed. S. Torres-

Peimbert (Dordrecht: Kluwer), 391

Renzini, A., \& Buzzoni, A. 1986, in Spectral Evolution of Galaxies, eds. C Chiosi, \& A. Renzini (Dordrecht: Reidel), 195

Sabbadin, F. 1986, A\&AS, 64, 579

Schmidt-Voigt, M., \& Köppen, J. 1987, A\&A, 174, 223

Schönberner, D. 1979, A\&A, 79, 108 1981, A\&A, 103, 119 

1983, ApJ, 272, 708

1986, A\&A, 169, 189

1989, in IAU Symposium 131, Planetary Nebulae, ed. S.

Torres-Peimbert (Dordrecht: Kluwer), 463

1990, in From Miras to Planetary Nebulae: Which Path for

Stellar Evolution?, eds. M. O. Mennessier \& A. Omont (Yvette Cedex:

Editions Frontières), 355

Schönberner, D., \& Weidemann, V. 1983, in IAU Symp. 103, Planetary

Nebulae, ed. D. R. Fowler (Dordrecht: Reidel), 359

Schwarzschild, M. \& Härm, R. 1965, ApJ, 142, 855

Shaw, R. A. 1989, in IAU Symp. 131, Planetary Nebulae, ed. S. Torres-

Peimbert (Dordrecht: Kluwer), 473

Sivagnanam, P., Le Squeren, A. M., Foy, F., \& Tran Minh, F. 1989, A\&A, 211,341

Snow, \& Morton, 1976, ApJS, 32, 429

Trams, N. R., Waters, L. B. F. M., Waelkens, C., Lamers, H. J. G. L. M., \& van der Veen, W. E. C. J. 1989, A\&AL, 218, L1

Vassiliadis, E., \& Wood, P. R. 1992, ApJ, submitted (VW92; Chapter 2)

Vassiliadis, E., Dopita, M. A., Morgan, D. H., \& Bell, J. F. 1992, ApJS, in press

Waelkens, C., Engelsman, E., Waters, L. B. F. M., \& van der Veen, W. E. C. J 1990, in From Miras to Planetary Nebulae: Which Path for Stellar Evolution?, eds. M. O. Mennessier \& A. Omont (Yvette Cedex: Editions Frontières), 470

Weidemann, V. 1990, AR\&A, 28, 103

Weigert, A. 1966, ZsAp, 64, 395

Wood, P. R., Bessell, M. S., \& Fox, M. W. 1983, ApJ, 272, 99

Wood, P. R., \& Faulkner, D. J. 1986, ApJ, 307, 659 (WF86)

Wood, P. R., Whiteoak, J. B., Hughes, S. M. G., Bessell, M. S., Gardner, F. F., \& Hyland, A. R. 1992, ApJ, in press

Wood, P. R., \& Zarro, D. M. 1981, ApJ, 247, 247

Zuckerman, B., \& Dyck, H. M. 1986, ApJ, 304, 394 


\section{Chapter 4}

\section{The Dynamical Evolution of Circumstellar Shells Surrounding Helium Shell Flashing AGB Stars}

\subsection{Introduction}

In a recent set of stellar evolution calculations, Vassiliadis and Wood (1992) have evolved a set of low and intermediate mass stars up the AGB with the inclusion of mass loss. The mass loss rate during these calculations was derived from an empirical formula (Wood 1990) based on the observed correlation of mass loss rate with pulsation period for Mira variables, $\mathrm{OH} / \mathrm{IR}$ stars and dust-enshrouded carbon stars. The stellar evolution calculations show that the combination of mass loss and helium shell flashes on the AGB leads naturally to intervals of very high mass loss rate (i. e., a superwind phase) interspersed with periods of low to intermediate mass loss rate.

The purpose of this paper is to examine the structure of the circumstellar envelopes produced by the time-varying mass loss rate and luminosity of an AGB star undergoing helium shell flashes. Clearly, the circumstellar shell will be affected by the modulation of the mass loss rate from the central star but, in addition, if radiation pressure on grains in the stellar wind is an important factor in driving mass loss flows from AGB stars, then the large luminosity excursions experienced by AGB stars undergoing shell flashes will also affect the circumstellar shells via the variation of the radiation pressure force. In this study, we examine the shell produced around a star of initial main sequence mass $1.0 \mathrm{M}_{\odot}$ with initial abundances $(Y, Z)=(0.25,0.008)$, appropriate for a star in 
the LMC.

\subsection{Numerical Methods}

Because of the long time interval ( $\sim 10^{5}$ years) over which the circumstellar shell development must be studied, as well as the large ratio of outer to inner radii of the circumstellar shell region, an implicit hydrodynamic code was required for the present calculations. The code used was a modified version of the spherically-symmetric, implicit, Eulerian code described by Wood (1979).

The inner and outer boundaries were chosen to be at $10^{14} \mathrm{~cm}$ and $3 \times 10^{18} \mathrm{~cm}$, respectively, and 1000 mesh points were distributed throughout this region at constant intervals in $\log R$. The inner boundary conditions correspond to the requirement that matter be injected into the base of the circumstellar envelope at a mass loss rate $\dot{M}$, with velocity $v$ and temperature $T$, where each of these quantities was obtained as a function of time from the stellar evolution calculations. In the region from the stellar photospheric boundary to $10^{14} \mathrm{~cm}$, the temperature $T$ (of grains and gas) was assumed to be given by

$$
L=8 \pi \sigma R^{2} T^{4} .
$$

Grains were assumed to form at a temperature of $1300 \mathrm{~K}$ (see Tielens 1990 for a discussion), corresponding to radius $R_{g} \sim 6 \times 10^{13} \mathrm{~cm}$, or $\sim 4 R_{*}$, for the star studied here. It was assumed that at $R=R_{g}$, the velocity of the flow was equal to the sound speed $c_{g}$, and that between $R_{g}$ and $10^{14} \mathrm{~cm}$ the velocity was given by the energy conservation law,

$$
\frac{1}{2} v^{2}+\left(\frac{\gamma}{\gamma-1}\right) \frac{P}{\rho}+(\alpha-1) \frac{G M_{*}}{R}=\text { constant },
$$

where $\gamma=\frac{5}{3}$ and $\alpha$ is the ratio of the outward radiative acceleration of the gas to the gravity. These equations define the temperature and velocity at which the gas passes the inner boundary of the region being studied in detail. The density at the inner boundary was defined by the requirement that,

$$
\dot{M}=4 \pi R^{2} \rho v .
$$

The above equations break down when the luminosity and mass loss rate drop suddenly at a helium shell flash. In this situation, the relatively dense matter in the circumstellar shell falls back onto the central star. The onset of infall occurs when the velocity at the inner boundary given by equation 4.2 drops to zero. At this time, 
transmitting boundary conditions of the type given by equation A12 of Wood (1979) are adopted in place of the conditions described above. These boundary conditions allow matter to flow freely in through the inner boundary.

When the star's luminosity recovers after the helium shell flash, matter from the central star is once again able to reach the inner boundary (i.e. the velocity given by equation 4.2 is positive). When the ram pressure, $P+\rho v^{2}$, of this outflowing material is sufficient to overcome that of the infalling matter, the inner boundary conditions revert to the first set described above.

The radiation pressure force, $f$, per unit mass of envelope material was assumed constant in the flow region, and it is given by,

$$
f=\frac{\kappa L}{4 \pi r^{2} c}
$$

where $\kappa$ is the grain opacity per unit mass of circumstellar matter. For spherical grains of radius $a$, density $\rho_{g}$, absorption efficiency $Q_{a b s}$ and a grain mass fraction $X_{g}$ in the circumstellar material, the opacity is given by

$$
\kappa=\frac{3}{4 a} \frac{Q_{a b s} X_{g}}{\rho_{g}}
$$

Adopting $a=0.1 \mu \mathrm{m}, Q_{a b s}=0.04, \rho_{g}=3 \mathrm{gm} \mathrm{cm}^{-3}$, and $X_{g}=4.5 \times 10^{-3}$, gives $\kappa=4.5 \mathrm{~cm}^{2} \mathrm{gm}^{-1}$. The value of $Q_{a b s}$ is obtained from Draine (1985) and was selected to be appropriate for astronomical silicates at $\lambda=1 \mu \mathrm{m}$ where the flux from a central star with $T_{\text {eff }}=3000 \mathrm{~K}$ will peak. The value of $X_{g}$ was obtained assuming essentially complete condensation of $\mathrm{MgFeSiO}_{4}$ grains. The opacity value derived above gives flow velocities far from the star in good agreement with the observed flow velocities in $\mathrm{OH} / \mathrm{IR}$ stars with thick circumstellar shells in the LMC (Wood et al. 1991; Wood et al. 1992) and Galaxy (Eder, Lewis, \& Terzian 1988).

No sources of heat input or loss were included in the flow, which was assumed to be adiabatic. The calculations of Goldreich and Scoville (1976) show that adiabatic cooling is the dominant factor controlling the flow temperature at $R \gtrsim 10^{16} \mathrm{~cm}$ from the star and that neglect of radiative cooling processes nearer the star causes temperatures to be overestimated. However, since the flow is highly supersonic most of the time, the thermal pressure (and temperature) should not play a significant role in the dynamics of the circumstellar shell. 


\subsection{Results and Discussion}

In figure 4.1 , the time dependence of the mass loss rate and luminosity of the central star is shown. The mass-loss rate runs away (i. e., a superwind develops) during the latter part of each shell flash cycle, near the end of the AGB phase of evolution. Full details are given in Vassiliadis and Wood (1992). The circumstellar shell development studied here covers the last two shell flash cycles shown in figure 4.1, starting before the first superwind phase, including the shell flash which terminates that superwind phase, and covering most of the second superwind phase. The existence of more than one superwind phase immediately suggests that multiple shells can develop around AGB stars.

Snapshots of the structure of the circumstellar envelope at a number of phases in the helium shell flash cycle are depicted in figure 4.2. At the end of the first superwind phase (case 0 ), a relatively smooth circumstellar shell with an approximately constant velocity of expansion of $\sim 16 \mathrm{~km} \mathrm{~s}^{-1}$ and density law $\rho \sim R^{-2}$ exists. When the helium shell flash occurs, the luminosity, radiation pressure force and mass loss rate drop briefly then go up for $\sim 1000$ years. The enhanced luminosity, and the increased radiation pressure force and mass loss rate, drive an outflow with velocity $v \sim 21 \mathrm{~km} \mathrm{~s}^{-1}$ into the base of the existing circumstellar envelope (case 1). In this situation, we should see evidence for winds at two velocities in the $1612 \mathrm{MHz}$ profiles of $\mathrm{OH} / \mathrm{IR}$ stars, although such objects should be rare $(\sim 1 \%$ of $\mathrm{OH} / \mathrm{IR}$ stars $)$ because of the relative timescales involved ( $\sim 1000$ years for the luminosity peak and $\sim 10^{5}$ years for the helium shell flash cycle). The objects IRAS $15520+0533$ in Eder, Lewis, and Terzian (1988) and IRAS 17253-2824 in te Lintel Hekkert (1990) show just such structure, and these two objects represent $\sim 1 \%$ of the objects in the surveys. These two objects should currently be undergoing helium shell flashes, and should be monitored to check for variability.

The large and prolonged luminosity dip following a shell flash event leads to a drop in the mass-loss rate initially and ultimately to matter falling back onto the central star. At point 2 in figure 4.2, a hollow shell has clearly formed. Such hollow shells are well known following the IRAS mission. Early after the cessation of mass loss, the central star will still be obscured and the resulting object will appear as a non-variable star at the high mass loss end of the sequence of Mira variables and $\mathrm{OH} / \mathrm{IR}$ stars in the IRAS two-colour diagram (Olnon et al. 1984; Bedijn 1987). As the circumstellar shell expands the objects will follow loops in the IRAS two-colour diagram on which many objects, mostly carbon stars, are found (Willems \& de Jong 1988; Chan \& Kwok 


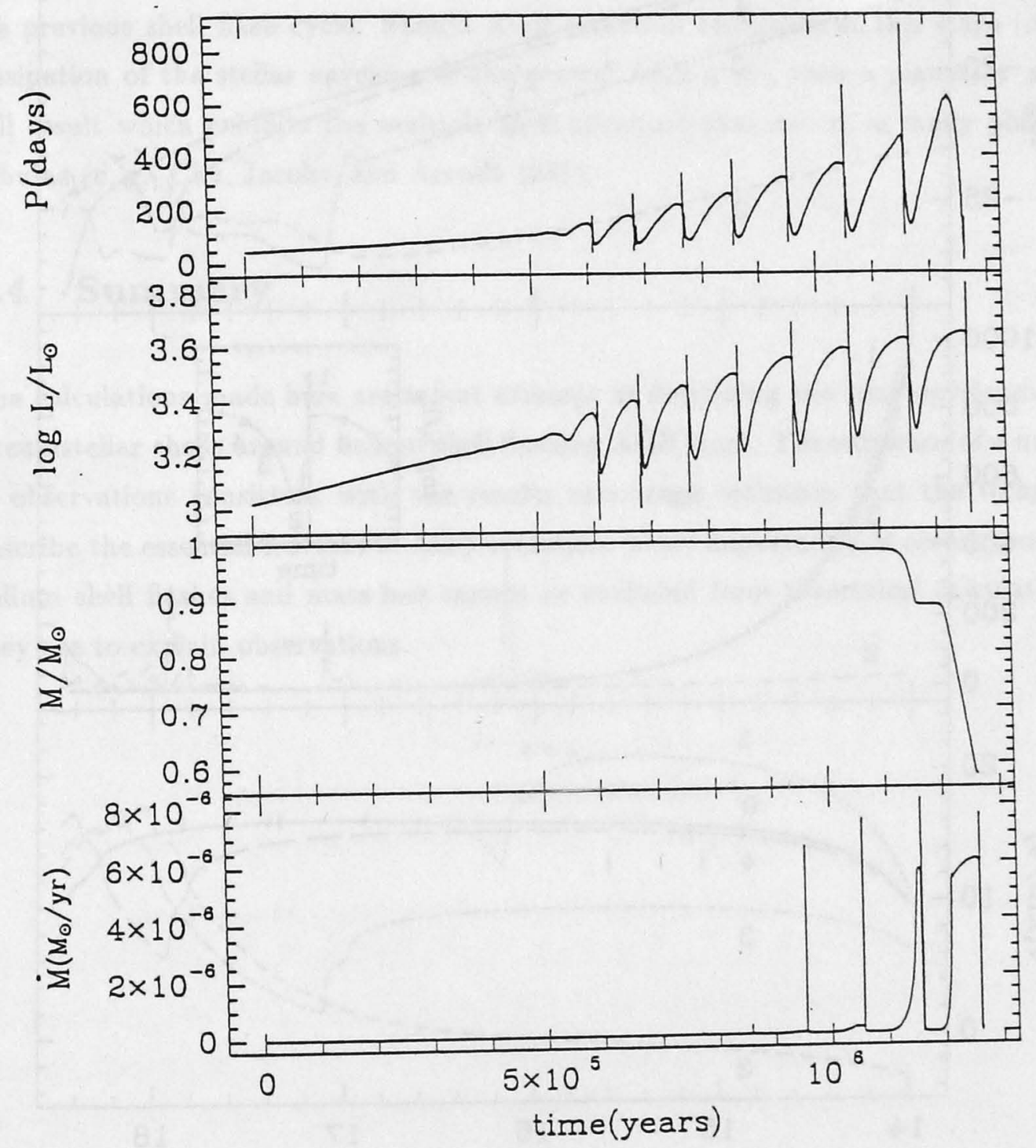

Figure 4.1: The pulsation period $P$, luminosity $L$, mass $M$, and mass loss rate $\dot{M}$ plotted against time on the AGB for a $1 \mathrm{M}_{\odot}$ star with LMC $(Z=0.008)$ abundances. 


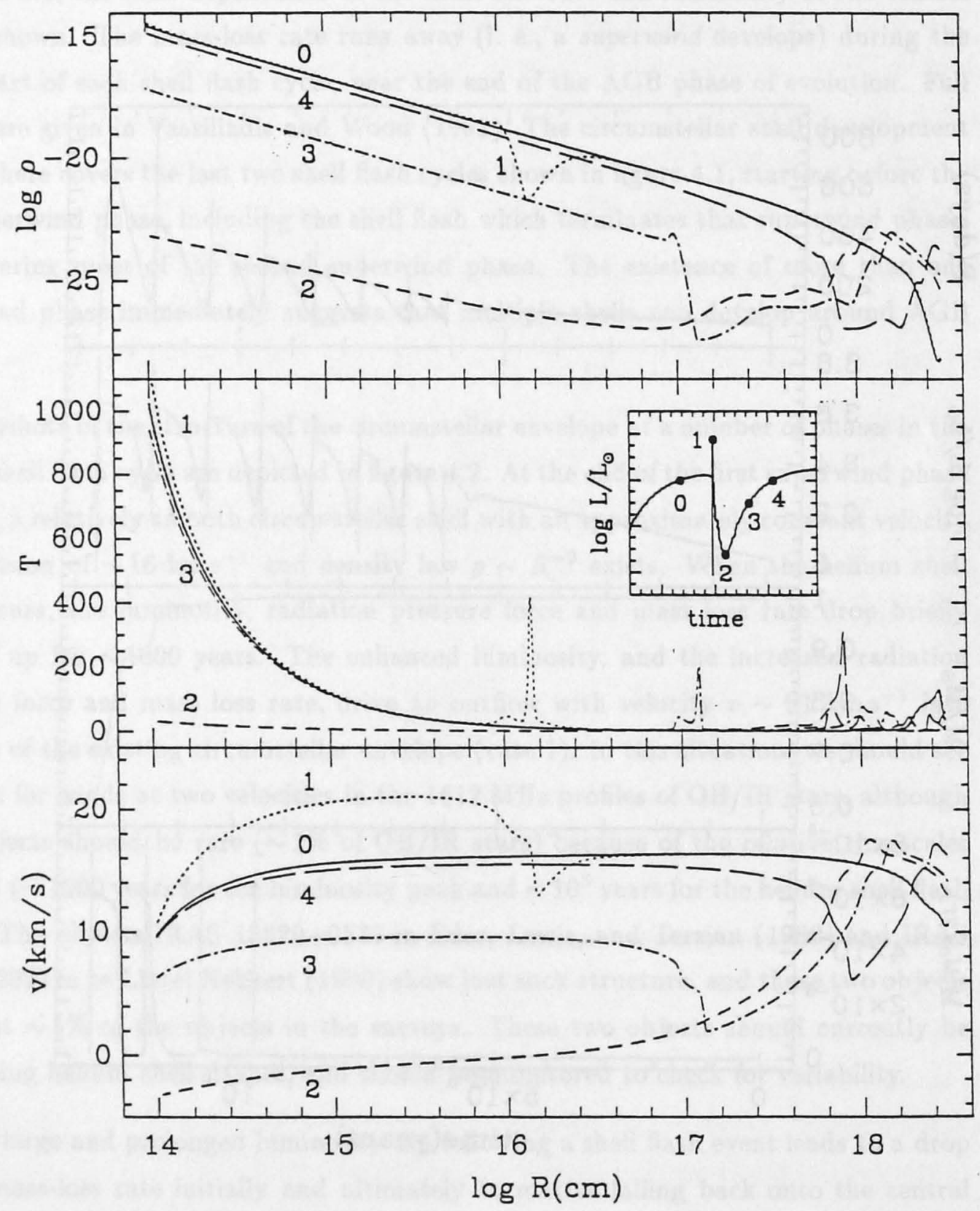

Figure 4.2: Density $\rho$, temperature $T$, and velocity $v$ of material in the stellar wind at five different times during the evolution through a helium shell flash cycle (inset). 
1988).

As the luminosity rises slowly during the recovery from the shell flash, mass loss from the central star begins again (cases 3 and 4 in figure 4.2) and a new shell begins to form. At this stage, the central shell is surrounded by an extensive halo of lower density, which is in turn surrounded by a denser shell left over from the superwind phase of the previous shell flash cycle. Should AGB evolution terminate at this stage (due to dissipation of the stellar envelope of the central AGB star), then a planetary nebula will result which exhibits the multiple shell structure that occurs in many planetary nebulae (e. g., Chu, Jacoby, and Arendt 1987).

\subsection{Summary}

The calculations made here are a first attempt at describing the time development of circumstellar shells around helium shell flashing AGB stars. The existence of a number of observations consistent with the results encourage optimism that the techniques describe the essential features of AGB evolution. Most importantly, it seems clear that helium shell flashes and mass loss cannot be excluded from theoretical calculations if they are to explain observations. 


\subsection{References}

Bedijn, P. J. 1987, A\&A, 186, 136

Chan, S. J., \& Kwok, S. 1988, ApJ, 334, 362

Chu, Y.-H., Jacoby, G. H., \& Arendt, R. 1987, ApJS, 64, 529

Draine, B.T. 1985, ApJS, 57, 587

Eder, J., Lewis, B. M., \& Terzian, Y. 1988, ApJS, 66, 183

Goldreich, P. \& Scoville, N. 1976, ApJ, 205, 144

Olnon, F. M., Baud, B., Habing, H. J., de Jong, T., Harris, S., \& Pottasch,

S. R. 1984, ApJL, 278, L41

te Lintel Hekkert, P. 1990, PhD thesis, Leiden

Tielens, A. G. G. M. 1990, in From Miras to Planetary Nebulae: Which Path for Stellar Evolution?, eds. M. O. Mennessier \& A. Omont (Editions Frontières), 186

Willems, F. J., \& de Jong, T. 1988, A\&A, 196, 173

Vassiliadis, E., \& Wood, P. R. 1992, in preparation (See Chapter 2)

Wood, P. R. 1979, ApJ, 227, 220 1990, in From Miras to Planetary Nebulae: Which Path for Stellar Evolution?, eds. M. O. Mennessier \& A. Omont (Editions Frontières), 67

Wood, P. R., Bessell, M. S., Hughes, S. M. G., Hyland, A. R., Whiteoak, J. B., Gardner, F. F., \& Otrupcek, R. E. 1991, in IAU Symp. 148, The Magellanic Clouds, eds. R. Haynes, \& D. Milne (Dordrecht: Kluwer), 386

Wood, P. R., Whiteoak, J. B., Hughes, S. M. G., Bessell, M. S., Gardner, F. F. \& Hyland, A.R. 1992, ApJ, in press. 


\section{Chapter 5}

\section{Optical Spectroscopy of Magellanic Cloud Planetary Nebulae}

\subsection{Introduction}

Planetary Nebulae (PNe) are believed to originate from low to intermediate mass stars ( 1-8 $\mathrm{M}_{\odot}$ ) which undergo some mass loss process as they ascend the asymptotic giant branch. As the majority of the stellar envelope is expelled as a circumstellar shell, the stellar nucleus evolves to hotter temperatures, thus becoming able to ionise the surrounding ejecta (see Aller 1989 for a recent review).

The study of planetary nebula (PN) shells offers an important tool for studying the chemical enrichment of the ejected material. Firstly, such a study gives insight into the precursor evolution. It is believed that as asymptotic giant branch stars evolve through their thermal pulse cycle, CNO and s-process material is dredged-up to the surface (Wood, Bessell, \& Fox 1983; Gallino et al. 1988; Hollowell \& Iben 1989). Thus, spectroscopic measurements of PNe will allow constraints to be set on precursor evolution. Secondly, the PNe eventually disperse and enrich the interstellar medium. This has obvious consequences for star formation.

Optical spectroscopy also allows the physical conditions of the PNe to be determined. Specific line ratios can be used to derive nebular temperatures and densities. With the aid of photoionisation modelling, this information can be combined to yield stellar parameters which are vital in constructing a Hertzsprung-Russell (H-R) diagram for this class of objects (Dopita \& Meatheringham 1991a; b). 
This technique has noi been successful using Galactic PNe because of the uncertainty associated with determining the distance to each object (Pottasch 1984). In recent years this problem has been circumvented by only looking at PNe in the Galactic Bulge, whose distance is determined independently (Acker et al. 1991). The obvious hindrance to this particular study is the high external extinction generally seen towards the Galactic Center.

The next samples of objects which have independently determined distances lie in the Magellanic Clouds. These objects are still bright enough that suitable spectra can be recorded in a reasonable amount of time for a relatively large number of objects, and small angular sizes allow integrated spectra to be obtained from the whole nebula (Wood, Bessell, \& Dopita 1986; Wood et al. 1987).

This is the third paper in this series (Meatheringham \& Dopita 1991a, hereafter Paper I; 1991b, hereafter Paper II) detailing optical spectral observations of PNe in the Magellanic Clouds. The previous papers contain emission-line measurements in the range $\lambda \lambda 3300-7800 \AA$ for $60 \mathrm{LMC}$ and $17 \mathrm{SMC}$ PNe, primarily from the compilation of Sanduleak, MacConnell, \& Philip (1978, hereafter SMP). In this paper, we have observed a further sample from the remaining, unobserved SMP list, and concentrated the majority of our efforts on new PN candidates identified from recent UK Schmidt objective prism surveys (Morgan \& Good 1992; Morgan 1992). We have also obtained spectra for the small sample of SMC PNe published by Morgan \& Good (1985). Emission-line measurements are presented for 52 objects in the LMC and 18 in the SMC. This brings our total number of published PN optical spectra up to 112 in the $\mathrm{LMC}$ and 35 in the SMC.

The other major sample of PNe is that of Jacoby (1980). He searched for PN candidates to fainter limiting magnitudes than other samples but only in restricted regions. Optical spectroscopy for the Jacoby objects has been obtained by Boroson \& Liebert (1989). There is also some overlap of the Jacoby sample with the objects listed by SMP. The virtue of the Morgan \& Good (1992) PNe is that they go to fainter limiting magnitudes than the SMP compilation, and cover a much wider field than that of Jacoby (1980). In this respect, they are more complete down to a limiting magnitude and are well suited to complement the measurements made in Papers I and II.

Self-consistent photoionisation modelling has been carried out for the PNe published in Papers I and II. The PNe presented in this paper will also be modelled in a similar fashion and published at a later date. It is hoped that with the inclusion of this data, a 
$\mathrm{H}-\mathrm{R}$ diagram can be constructed which enables one to follow the evolution well down the fading portions of the PN evolutionary tracks $\left(\sim 100 \mathrm{~L}_{\odot}\right)$.

\subsection{Observations}

\subsubsection{The Samples}

A small sample of objects from the SMP listing were observed to supplement the work on that compilation begun in Papers I and II. Table 5.1 provides a cross-reference listing containing other common names for this particular sub-sample of SMP objects. Two entries also exist for two objects in the Morgan \& Good (1985) sample which is discussed further, below. In all, the SMP objects correspond to 14 out of the 52 LMC objects, and 2 out of the $18 \mathrm{SMC}$ objects reported in this paper.

As was noted in Papers I and II, the only source of published coordinates for the majority of the SMP sample was the original SMP paper. These coordinates have been shown to be accurate to only $1^{\prime}$ in some cases. We present a revised list of coordinates for these objects in tables 5.2 which are accurate to \pm 2 ".

The remaining PNe reported in this paper belong to the recent work of Morgan \& Good (1992), and other work in progress by these investigators. In addition to these new candidates, 13 SMC PNe were also observed (Morgan \& Good 1985). For all of these objects accurate coordinates had been determined from sky survey plates. The coordinates and finding charts are to be found in the papers noted above.

\subsubsection{Spectrophotometry}

All observations were carried out using the Double Beam Spectrograph (DBS) (Rodgers, Conroy, \& Bloxham 1988) on the Australian National University's 2.3 meter Advanced Technology Telescope, located at Siding Spring Observatory. The instrument splits incoming light, using a dichroic mirror, into separate blue and red arms. Photon Counting Arrays (PCA) (Rodgers et al. 1988) were used to image each beam onto a pair of EEV-CCDs, giving $11 \mu \mathrm{m}$ square pixels. (Note that before 1990 January, Fairchild 222 CCDs were used, giving 15 (dispersion axis) $\times 18 \mu \mathrm{m}$ pixels). The blue and red arms used S-20 and Ga-As photocathode tubes, respectively, to give the desired spectral sensitivity in each beam. The $4 \mathrm{CCDs}$, in conjunction with the 300 line $\mathrm{mm}^{-1}$ gratings, gave a total usable spectral coverage of $\lambda \lambda 3400-7900 \AA$ at $\sim 10 \AA$ resolution. 
TABLE 5.1

Cross-Reference Name List

\begin{tabular}{lll}
\hline \hline SMP Designation & Other Names \\
\hline LMC-SMP4 $\ldots$. & $\ldots$ \\
LMC-SMP18 $\ldots$ & L95 \\
LMC-SMP21 $\ldots$ & N97, LM1-16, WS7 \\
LMC-SMP40 $\ldots$ & WS16 \\
LMC-SMP41 $\ldots$ & LM1-26 \\
LMC-SMP84 $\ldots$ & N212, LM1-53, WS36 \\
LMC-SMP95 $\ldots$ & $\ldots$ \\
LMC-SMP98 $\ldots$ & LM1-62, WS41, M57_1 \\
LMC-SMP100 $\ldots$ & $\ldots$ \\
LMC-SMP101 $\ldots$ & LM1-65, M57_1A \\
LMC-SMP102 & $\ldots$ & $\ldots$ \\
LMC-MA04 & $\ldots$ & S-104 \\
& & \\
SMC-SMP16 & $\ldots$ & N42, Ln179, J14, M84 \\
SMC-SMP17 & $\ldots$ & N44, Ln191 \\
\hline
\end{tabular}

${ }^{a}$ Key to Designations.-N: Henize 1956; Ln: Lindsay 1961; L: Lindsay 1963; LM1: Lindsay \& Mullin 1963, Table 1; WS: Westerlund \& Smith 1964; J: Jacoby 1980; S: Sanduleak 1984. 
TABLE 5.2

Coordinate List for SMP Objects

\begin{tabular}{|c|c|c|}
\hline SMP Designation & $\alpha_{(\mathrm{J} 2}$ & ${ }^{\delta}$ \\
\hline LMC-SMP4 & 044322 & -713009 \\
\hline LMC-SMP9 & 045024 & -681316 \\
\hline LMC-SMP10 & 045109 & -684901 \\
\hline LMC-SMP18 & 050342 & -700646 \\
\hline LMC-SMP21 & 050453 & -683930 \\
\hline LMC-SMP40 & 051215 & -662258 \\
\hline LMC-SMP41 ... & 051327 & -703334 \\
\hline LMC-SMP84 & 053653 & -715336 \\
\hline LMC-SMP85 ... & 054031 & -661733 \\
\hline LMC-SMP95 ... & 060145 & -675600 \\
\hline LMC-SMP98 ... & 061735 & -731236 \\
\hline LMC-SMP100 & 062254 & -720739 \\
\hline LMC-SMP101 .. & 062340 & -691037 \\
\hline LMC-SMP102 .. & 062934 & -680326 \\
\hline SMC-SMP16 & 005127 & -722613 \\
\hline SMC-SMP17 ... & 005157 & -712444 \\
\hline
\end{tabular}


The observations were collected over four separate observing runs: 1989 November, 1989 December, 1990 January, and 1990 November. As most objects were fainter than those examined in Papers I \& II, exposure times were $~ 2000$ seconds. Line intensities were measured to $\sim 10 \%$ of $\mathrm{H} \beta$ in the worst cases. There were instances where strong emission-line objects were observed, resulting in saturation for the brightest lines. In these instances, a long exposure ( $\sim 2000$ seconds) was made to bring up the faint lines as best as possible, and then a short ( $\sim 500$ seconds), defocussed exposure was made to measure the Balmer decrement and to obtain unsaturated measurements of other bright lines. The virtue of defocussed observations is that the same signal is spread over more rows of the CCD detectors, thus avoiding saturation.

Every three to four exposures of PN candidates were bracketed by observations of flux standards, selected from L745-46A, W485A, van Maanen II, Feige 110 (Oke 1974; Stone \& Baldwin 1983).

\subsubsection{H $\beta$ Photometry}

The absolute fluxes of a majority of the objects observed with the DBS were obtained using the new focal-reducing camera on the 2.3 meter telescope. This camera, operating at $\mathrm{f} / 18$, has a focal plane scale of $4.964^{\prime \prime} \mathrm{mm}^{-1}$. The detector is a thinned, backside-illuminated, Tektronix CCD with $1024 \times 1024$ pixels, giving an image scale of $0.596^{\prime \prime}$ pixel $^{-1}$. The $\mathrm{H} \alpha$ line emission was isolated using a $90 \mathrm{~mm}$ diameter Bausch \& Lomb interference filter with a bandpass of $16 \AA$ FWHM. This was tilted in the $f / 18$ Nasmyth beam at an angle of $7^{\circ}$ to tune the filter to the mean wavelength of $\mathrm{H} \alpha$ emission from the Large Magellanic Cloud. Observations of 26 objects were carried out over two photometric nights 1992 January 2-4. LMC-SMP76 was used as a primary flux standard and was observed every hour. Secondary flux standards were LMC-SMP6, LMC-SMP21, LMC-SMP83, LMC-SMP89, and LMC-SMP97. H $\alpha$ fluxes for these objects were calculated from the $\mathrm{H} \beta$ photometry of Meatheringham, Dopita, \& Morgan (1988) and using the spectrophotometric measurements of Meatheringham \& Dopita (1991a; b). Typical exposure times were 100 seconds for flux standards, and 300-600 seconds for the program objects. The $\mathrm{H} \alpha$ values measured for the program objects were converted to $\mathrm{H} \beta$ values using the spectrophotometry presented in this paper.

Additionally, for one night in the 1990 January observing run we were fortunate to experience a photometric night of good seeing, typically $\lesssim 1^{\prime \prime}$. Using the same DBS setup described in the previous section, with a slit width of $\sim 4^{\prime \prime}$, a series of short 
exposures (150 to 300 seconds) was carried out for the sole purpose of determining the absolute flux in the $\mathrm{H} \beta$ line. Every three exposures were bracketed by 300 second exposures of SMP 14, treated as the flux standard for this series of observations. Previous $\mathrm{H} \beta$ flux determinations have been made for this object by Wood et al. (1987) and Meatheringham, Dopita, \& Morgan (1988).

\subsection{Data Reduction}

\subsubsection{General Procedure}

The reduction of these data followed the basic principles outlined in Papers I and II. Consequently, this section will only note the major points and differences with earlier procedures.

All data presented in this study were reduced using the KPNO IRAF package, version 2.9.1. Qualitative investigation showed that the flat-fielding process increased the noise in a small sample of spectra which were subsequently summed into one dimensional form. The reason for the increase in noise appears to be due to the observed flat-field pattern being a function of the level of illumination. This is an intrinsic property for the type of photon counting detector being used. The detector also suffers from moiré fringing between the EEV-CCD, the fibre bundle, and the image tube. Although this fringing is much less in amplitude than with the Fairchild 222 chip, it is less stable with respect to temperature variations and spectrograph orientation. Consequently, no data were flat-fielded.

One dimensional spectra were created by simply summing together the signal rows. Sky backgrounds were averaged above and below the signal rows and subtracted. The approximate same rows were summed in $\mathrm{He}-\mathrm{Ar}$ arc exposures to minimise any pixel shift along the spatial axis of the detector. The one dimensional forms of these arc exposures were then used to wavelength calibrate all other spectra. At least one arc exposure was made per night. The estimated pixel shift during observing, due to the drop in temperature during the night, and rotation of the instrument about the Nasmyth focus, corresponds to $\sim 0.5$ pixels.

The conversion of raw counts to flux units (ergs $\mathrm{cm}^{-2} \mathrm{~s}^{-1}$ ) was accomplished by observing stars with known flux distributions. Of the stars mentioned in section 5.2.2, only one did not appear to have a list of tabulated calibration magnitudes against wavelength within the IRAF package. The appropriate list for van Maanan II was 
created from data available in the literature (Oke 1974). Comparison of the observed flux distributions and the calibration magnitudes yielded a sensitivity curve for each such observation. Standards were observed at least twice a night. The resultant sensitivity curves were averaged together for each observing run and divided through the corresponding PNe spectra.

At this stage, the PNe spectra could be used to measure line fluxes. However some of these objects exhibit the presence of a continuum, especially when looking towards the blue spectral end. Rather than fitting a local continuum with a non-zero gradient each time a line is measured, the continuum was fitted across the whole spectrum and then subtracted. This leaves a pure emission line spectrum.

Line fluxes were measured by fitting gaussian profiles to all emission lines. In some instances where blending of lines occurred, multiple gaussian fits were constrained by each fit having identical FWHM.

\subsubsection{Saturation of Bright Lines}

The majority of the objects presented in this paper were sufficiently faint that only a single exposure was required to record emission lines. Such exposures were typically a maximum of 2000 seconds. However, some of the objects, especially those with an SMP designation, clearly saturated in the bright lines ([0 III] $\lambda 5007 \AA$ and/or $\mathrm{H} \alpha \lambda 6563 \AA$ ). The finite time needed to record a photon event means that photon events are lost in such instances of high count rates. The resulting saturated line profile appears fainter in the final spectrum than its true intensity.

To circumvent this problem, we followed the procedure as set out in Paper II, utilizing narrow slit, defocussed short exposures. Narrow slit observations are normally subject to atmospheric dispersion and wavelength dependent-seeing effects. However, by defocussing the incoming light to the point where it is distributed over several arc-seconds (comparable to the slit width used for normal, focussed observations) on the detector, the dispersion and seeing effects should cancel once a one-dimensional spectrum is extracted. Also, the spectrograph design ensures that the vignetting in the camera is $<5 \%$, and as a consequence, any wavelength dependent optical effects from defocussing are negligible.

The reduction procedure is exactly the same as that described in section 5.3.1. In addition to obtaining measurements of the previously saturated lines, an unsaturated 
measurement of $\mathrm{H} \alpha \lambda 6563 \AA$ allows a better calculation of the Balmer decrement, and subsequent derivation of the reddening towards the PN. No observations were made through neutral density filters as in Paper I.

\subsubsection{H $\beta$ Photometry}

The CCD images were debiased, flat-fielded, corrected for atmospheric extinction and calibrated in absolute flux using the IRAF package. Absolute fluxes were obtained by aperture photometry using the DAOphot task within IRAF. The photometric reproducibility was found to be better than $3 \%$. However the absolute calibration is considerably less certain as the secondary standards were found to have a mean square deviation of $25 \%$. By assuming that the theoretical $\mathrm{H} \alpha / \mathrm{H} \beta$ ratio was 2.94 for any object, and using the reddening value determined from the spectrophotometry, a value for the observed intensity ratio was obtained. This value was then used to convert the measured $\mathrm{H} \alpha$ flux for an object to a $\mathrm{H} \beta$ flux. For negative reddening values, the observed intensity ratio was assumed to be equal to the theoretical value.

No attempt has been made to correct for a nebular or stellar continuum contribution. However, since the equivalent width of $\mathrm{H} \alpha$ is typically larger than $500 \AA$ this contribution should be negligible. For the objects observed in common with Meatheringham, Dopita, and Morgan (1988), the two flux scales agree to within a standard deviation of \pm 0.02 dex.

The DBS spectra specifically taken for the purpose of determining the absolute $\mathrm{H} \beta$ flux during the 1990 January observing run were reduced in exactly the same way as the spectra taken for relative spectrophotometry. Once the $\mathrm{H} \beta$ flux was determined from each of the spectra by fitting a gaussian profile to the $\mathrm{H} \beta$ signal, all measurements were put on the scale defined by the $\mathrm{H} \beta$ strength of LMC-SMP14. This PN has been observed by other authors who consistently find a value of $\log \mathrm{F}(\mathrm{H} \beta)=-13.685 \pm$ 0.005 (Wood et al. 1987; Meatheringham, Dopita, \& Morgan 1988).

The absolute fluxes obtained by this method appeared generally unreliable with a standard deviation with respect to the imaging photometry of 0.2 dex. Combined with the fact that the flux scale defined by our imager results corresponds well with the scale defined by Meatheringham, Dopita, and Morgan (1988), we have presented only the results obtained with the imager. Note that not all objects were reobserved with the imager due to a lack of time. We have retained the DBS $\mathrm{H} \beta$ flux measurements for the following objects: LMC-MA3, LMC-MA10, SMC-MG8, SMC-MG9, and SMC-MG12. 


\subsubsection{Detector Efficiency and Accuracy}

The observations in this paper were obtained during a time when the DBS PCA detectors were in a slow state of physical decay. The extent of this degradation was only evident when all reductions had been completed. A comparison between the unsaturated lines in the saturated and unsaturated spectra does not appear to be a precise linear function, but slightly parabolic. The observed non-linearity suggests that saturation may have occurred at lower count rates previously assumed to be safe for observations. Combined with the low signal-to-noise ratio for most lines, this has made in-depth analysis of the observations relatively more difficult than in Papers I and II. However, with respect to photoionisation modelling of the PNe and deriving central star parameters, preliminary calculations indicate the presented emission line measurements to be adequate.

A quantitative estimate of the error in each line measurement is difficult to produce due to the wide variation between absolute flux and exposure times between different objects. From a small subsample of objects mentioned in this paper, Table 5.3 provides an estimate of errors over wide ranges of wavelength and intensity. The wavelength ranges are defined to map good and poor regions of detector response: The region $<3700 \AA$ corresponds to the fall-off in sensitivity of the S-20 photocathode, and the beginning of relatively high atmospheric extinction; the range $\lambda \lambda 5400-6000 \AA$ corresponds to the other edge of the S-20 photocathode spectral response, and the rise in response of the Ga-As detector; and finally, the region $>7200 \AA$ defines the region where the night-sky $\mathrm{OH}$ molecular line emission becomes stronger. Other isolated night-sky lines which were identified, in order of decreasing brightness, were [O I] $\lambda 5577 \AA$, [O I] $\lambda 6300 \AA$, [O I] $\lambda 6364 \AA$, and [N I] $\lambda 5199 \AA$. The first of these was the brightest by far. The remaining three lines inhibited the measurement of faint nebular features at each of the respective wavelengths. Any measurements quoted for features at these wavelengths may be subject to slightly larger sky subtraction errors.

It should be noted that the line intensities are all relative to $\mathrm{H} \beta$. Consequently, the reader should keep in mind that the errors presented in Table 5.3 should be considered in unison with the absolute $\mathrm{H} \beta$ flux measurements. In general, bright $\mathrm{H} \beta$ sources ( $\log \mathrm{H} \beta \sim-12.5$ ergs.cm ${ }^{-2} . \mathrm{s}^{-1}$ ) will allow reasonable line flux determinations to as small as $2 \%$ of $\mathrm{H} \beta$, whereas faint $\mathrm{H} \beta$ sources $\left(\log \mathrm{H} \beta \sim-13.8 \mathrm{ergs.cm}{ }^{-2} . \mathrm{s}^{-1}\right)$ usually only register a sparse number of emission lines $\sim 20-30 \%$ of $\mathrm{H} \beta$. 
TABLE 5.3

Estimated Errors in Line Intensities

\begin{tabular}{lccccc}
\hline \hline \multirow{2}{*}{$\begin{array}{l}\text { Relative Intensity } \\
(\mathrm{H} \beta=100)\end{array}$} & $<3700 \AA$ & $3700-5400 \AA$ & $5400-6000 \AA$ & $6000-7200 \AA$ & $>7200 \AA$ \\
\cline { 2 - 6 } & $>50 \%$ & $>30 \%$ & $>50 \%$ & $>40 \%$ & $>50 \%$ \\
$3-15 \ldots \ldots \ldots \ldots \ldots$ & 30 & 20 & 30 & 30 & 30 \\
$15-30 \ldots \ldots \ldots \ldots$ & 20 & 15 & 20 & 20 & 20 \\
$>30 \ldots \ldots \ldots \ldots$ & $<20$ & $<10$ & $<20$ & $<15$ & $<20$ \\
\hline
\end{tabular}




\subsection{Results}

A complete list of all emission line fluxes measured for $52 \mathrm{LMC}$ and $18 \mathrm{SMC} \mathrm{PNe}$ is presented in table 5.4. The ordering of the objects within the table is arranged as follows: 14 LMC-SMP objects (SMP), 20 LMC-MG objects (Morgan \& Good 1992), 18 LMC-MA objects (Morgan 1992), 2 SMC-SMP objects (SMP), 1 SMC-SP object (Sanduleak \& Pesch 1981), 13 SMC-MG objects (Morgan \& Good 1985), and 2 SMCMA objects (Morgan 1992). A small subsample of objects had emission-lines in addition to ones noted in table 5.4. Identifications and intensities for these supplementary features are presented in table 5.5. Identifications for these supplementary features are not definite. A compilation of absolute $\mathrm{H} \beta$ fluxes observed in previous studies, together with values derived in this study, are given when available at the bottom of each object column in table 5.4 .

\subsubsection{Reddening determinations}

The reddening of each $\mathrm{PN}$, due to internal absorption, and absorption along the line of sight, can be determined from the observed Balmer decrement. Based on the signalto-noise ratio inherent to these data, derivation of this decrement is limited to lines $\mathrm{H} \alpha$ through to $\mathrm{H} \delta$ in most cases. In previous studies by other authors, the procedure has been to correct spectra to the case $\mathrm{B}$ theoretical $\mathrm{H} \alpha / \mathrm{H} \beta$ intensity ratio of 2.85 , assuming canonical values for the electron temperature and density of $10^{4} \mathrm{~K}$ and $10^{4}$ $\mathrm{cm}^{-3}$, respectively (Brocklehurst 1971). As pointed out in Paper I, this procedure ignores the large range in both density and temperature for Magellanic Cloud PNe. High electron temperatures, as usually seen for Magellanic Cloud PNe, suggest the $\mathrm{H} \alpha / \mathrm{H} \beta$ intensity ratio should be 2.85 , or even less. Photoionisation modelling of the observations presented in Papers I and II indicated a range in the theoretical $\mathrm{H} \alpha / \mathrm{H} \beta$ intensity ratio from 2.85 to 2.98 . These larger values are due to contributions from collisional excitation in partially ionized regions. Following from the photoionisation modelling results mentioned above, we have adopted the theoretical Balmer decrement, relative to $\mathrm{H} \beta$, to be $(\mathrm{H} \alpha: \mathrm{H} \beta: \mathrm{H} \gamma: \mathrm{H} \delta)=(2.94: 1.00: 0.465: 0.256)$.

These ratios were compared with their observed counterparts to determine the reddening constant, c, in the expression $I_{c}=I_{0} 10^{c f(\lambda)}$, where $I_{0}$ and $I_{c}$ denote the observed and theoretical line intensities, respectively, and $f(\lambda)$ represents the reddening curve as given by Whitford (1958). An intensity weighted mean of the three reddening determinations for each object was adopted as the final value for c. From a subsample 
of 32 objects the 1- $\sigma$ deviation between dereddened and theoretical $\mathrm{H} \alpha$ values was 0.1 . For $\mathrm{H} \gamma$ and $\mathrm{H} \delta$, the deviation is $\gtrsim 0.2$. As a matter of record, if one were to use a least-square-fit or equal-weight average technique to combine the three reddening determinations, the 1- $\sigma$ deviations vary from 0.2 to 0.4 over the three lines.

It can be seen that some of the reddening values noted in table 5.4 are negative, which is clearly unphysical. Sometimes a large variation is seen in reddening values calculated from the $\mathrm{H} \gamma$ and $\mathrm{H} \delta$ lines. This discrepancy may simply be due to observational errors quoted earlier. In particular, the higher members of the Balmer series have much poorer photon statistics compared to $\mathrm{H} \alpha$, and this is taken into account by adopting an intensity weighted mean for the final reddening constant. This also helps to partly resolve the problem of having short baselines between $\mathrm{H} \beta$, and $\mathrm{H} \gamma$ and $\mathrm{H} \delta$, respectively. It should also be noted that the resolution of our observations was not sufficient to deblend the H $\delta$ Balmer line from N III 4097 and $4103 \AA$ emission. The intensity of the N III lines can be up to $15 \%$ of the total line flux measured at the position of $\mathrm{H} \delta$. Consequently, the extinction calculated from the blended $\mathrm{H} \delta$ and $\mathrm{N}$ III line can be significantly underestimated. However, its effect is still quite prominent. Our other major concern is a result of the saturation problems mentioned in section 5.3.4. $\mathrm{H} \alpha$ will give a negative reddening constant if it is saturated. Indeed, this may be the case in some of our measurements. In these objects, if the reddening values derived from the $\mathrm{H} \gamma$ and $\mathrm{H} \delta$ lines are consistent with respect to themselves, then $\mathrm{H} \alpha$ is denoted as being saturated. When $\mathrm{H} \gamma$ and $\mathrm{H} \delta$ failed to assist in the decision, $\mathrm{H} \alpha$ was included in calculating an intensity-weighted reddening constant. Because of this procedure, it was difficult to discern if any PNe exhibited atypical Balmer decrements.

Due to the problems noted in section 5.3.4, and arguments presented above, we have decided to present raw line flux measurements and their reddening-corrected values for each object. The reddening value is also given so the reader can work freely between the two columns of data. The effects of atmospheric dispersion were minimal, as described in section 5.3.2, and no further corrections were made to the final measurements.

From measurements presented in Papers I and II, average values for the reddening constant for the LMC and SMC are $c_{L M C}=0.28$ and $c_{S M C}=0.26$, respectively. Using the intensity-weighted average method, we estimate the error in our reddening determinations to be of the order of 0.2 dex. This being the case, we find 12 objects with $\mathrm{c} \lesssim-0.2:$ LMC-SMP102, LMC-MG01, LMC-MG37, LMC-MG74, LMC-MA07, LMC-MA15, SMC-SMP16, SMC-SMP17, SMC-MG03, SMC-MG08, SMC-MG13, and SMC-MA1. 
TABLE 5.4

UnCoRrected and CoRrected Line Intensities

\begin{tabular}{|c|c|c|c|c|c|c|c|}
\hline \multirow{2}{*}{$\begin{array}{c}\lambda \\
(\AA)\end{array}$} & \multirow[b]{2}{*}{ Identification } & \multicolumn{2}{|c|}{ LMC-SMP4 } & \multicolumn{2}{|c|}{ LMC-SMP9 } & \multicolumn{2}{|c|}{ LMC-SMP10 } \\
\hline & & $I_{\circ}$ & $\mathrm{I}_{\mathrm{c}}$ & $\mathrm{I}_{\mathrm{o}}$ & $I_{c}$ & $\mathrm{I}_{\mathrm{o}}$ & $I_{c}$ \\
\hline 3346 & {$[\mathrm{NeV}] \ldots \ldots$} & $\cdots$ & $\cdots$ & & & $\ldots$ & $\cdots$ \\
\hline 3426 & {$[\mathrm{NeV}] \ldots \ldots \ldots \ldots$} & & & 10.7 & 12.1 & $\ldots$ & \\
\hline 3444 & OIII $\ldots \ldots \ldots \ldots$ & 7.5 & 8.2 & 5.9 & 6.7 & & \\
\hline 3727 & [OII] doublet & 11.6 & 12.4 & 262.5 & 288.1 & 71.5 & 73.5 \\
\hline 3749 & HeII, H12 & $\ldots$ & .. & ... & $\ldots$ & 3.7 & 3.8 \\
\hline 3759 & OIII. & $\ldots$ & $\ldots$ & $\ldots$ & $\ldots$ & $\ldots$ & $\ldots$ \\
\hline 3771 & H11, He II, O III.... & $\ldots$ & .. & $\ldots$ & 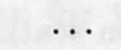 & 4.4 & 4.5 \\
\hline 3798 & В10 $\ldots \ldots \ldots \ldots \ldots$ & $\ldots$ & $\ldots$ & $\ldots$ & $\ldots$ & 5.0 & 5.1 \\
\hline 3834 & He II, H9 & 6.1 & 6.5 & 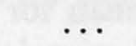 & . & 8.8 & 9.1 \\
\hline 3869 & {$[\mathrm{NeIII}]$} & 83.2 & 88.1 & 93.8 & 101.6 & 91.1 & 93.3 \\
\hline 3889 & H8, HeI.. & 16.9 & 17.9 & 22.0 & 23.8 & 25.4 & 26.0 \\
\hline 3969 & {$[\mathrm{Ne} \mathrm{III}], \mathrm{H} \epsilon \ldots \ldots \ldots$} & 38.2 & 40.2 & 46.7 & 50.2 & 50.6 & 51.6 \\
\hline 4026 & $\mathrm{HeI} \ldots \ldots \ldots \ldots \ldots$ & $\cdots$ & $\cdots$ & $\cdots$ & $\ldots$ & $\cdots$ & $\cdots$ \\
\hline 4069 & [S II] doublet ....... & $\ldots$ & & & & $\ldots$ & \\
\hline 4102 & $\mathrm{H} \delta, \mathrm{N}$ III $\ldots \ldots \ldots \ldots$ & 24.0 & 25.1 & 25.9 & 27.5 & 30.7 & 31.2 \\
\hline 4340 & $\ldots \ldots \ldots$ & 43.1 & 44.3 & 48.3 & 50.3 & 55.6 & 56.3 \\
\hline 4363 & [O III] & 15.8 & 16.2 & 13.0 & 13.6 & 19.2 & 19.4 \\
\hline 4472 & HeI $\ldots \ldots \ldots \ldots \ldots$ & $\ldots$ & $\cdots$ & 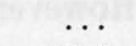 & & 6.2 & 6.3 \\
\hline 4686 & He II .... & 38.1 & 38.5 & 39.5 & 40.0 & 5.0 & 5.0 \\
\hline 4713 & [ArIV], $\mathrm{HeI},[\mathrm{NeIV}]$ & $\cdots$ & $\cdots$ & $\cdots$ & $\cdots$ & 4.4 & 4.4 \\
\hline 4725 & {$[\mathrm{NeIV}] \ldots \ldots \ldots \ldots$} & $\ldots$ & $\ldots$ & $\ldots$ & 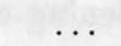 & .. & .. \\
\hline 4740 & ........ & $\ldots$ & $\ldots$ & $\ldots$ & ... & 3.0 & 3.0 \\
\hline 4959 & [OIII]. & 357.3 & 355.5 & 266.2 & 264.4 & 266.7 & 266.3 \\
\hline 5007 & [O III $]$ & 1046.9 & 1039.2 & Satur & & Satu & \\
\hline 5199 & {$[\mathrm{NI}] \ldots$} & $\cdots$ & $\cdots$ & $\cdots$ & $\cdots$ & $\cdots$ & $\cdots$ \\
\hline 5412 & He II $\ldots \ldots \ldots \ldots \ldots$ & $\cdots$ & $\cdots$ & $\cdots$ & $\cdots$ & $\cdots$ & $\cdots$ \\
\hline 5876 & n......... & 6.4 & 6.1 & 11.0 & 10.3 & 11.4 & 11.2 \\
\hline 6300 & n... & $\ldots$ & $\ldots$ & $\ldots$ & $\ldots$ & $\ldots$ & $\ldots$ \\
\hline 6312 & [S III] & $\cdots$ & $\cdots$ & $\cdots$ & $\cdots$ & $\ldots$ & $\cdots$ \\
\hline 6364 & {$[\mathrm{OI}]$} & & & & & & \\
\hline 6548 & [N II] & & .. & 55.8 & 50.3 & 8.2 & 7.9 \\
\hline 6563 & $\ldots \ldots+\cdots$ & 309.5 & 287.5 & 340.0 & 306.7 & 337.2 & 327.1 \\
\hline 6583 & n...... & $\cdots$ & . & 225.8 & 203.5 & 96.3 & 93.4 \\
\hline 6678 & He I $\ldots \ldots \ldots \ldots \ldots$ & 5.5 & 5.0 & $\cdots$ & . & 5.8 & 5.6 \\
\hline 6717 & $\ldots \ldots \ldots \ldots$ & $\cdots$ & $\cdots$ & 25.1 & 22.5 & 4.2 & 4.1 \\
\hline 6731 & [S II] & 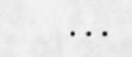 & & 38.8 & 34.7 & 6.3 & 6.1 \\
\hline 7006 & {$[\mathrm{ArV}] \ldots \ldots \ldots \ldots$} & $\cdots$ & $\cdots$ & .. & . & . & $\cdots$ \\
\hline 7065 & HeI.... & 2.2 & 2.0 & 5.2 & 4.6 & 4.5 & 4.3 \\
\hline 7136 & {$[\mathrm{ArIII}] \ldots \ldots \ldots \ldots$} & 5.7 & 5.2 & 17.7 & 15.5 & 14.5 & 14.0 \\
\hline 7320 & {$[\mathrm{OII}] \ldots \ldots \ldots \ldots$} & .. & .. & 16.1 & 14.0 & $\cdots$ & .. \\
\hline 7330 & {$[\mathrm{OII}] \ldots \ldots \ldots \ldots$} & ... & & 16.2 & 14.2 & $\cdots$ & \\
\hline 7751 & {$[$ Ar III $] \ldots \ldots \ldots \ldots$} & $\cdots$ & $\cdots$ & $\cdots$ & & - & \\
\hline & & & .0 .10 & & .0 .14 & 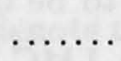 & $\ldots 0.04$ \\
\hline $\mathrm{T}_{\mathrm{e}}([\mathrm{C}$ & III])(K) $\ldots \ldots$ & 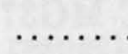 & 14000 & & .15000 & $\ldots$ & .18000 \\
\hline $\mathrm{n}_{\mathrm{e}}([\mathrm{S}$ & I] $)\left(\mathrm{cm}^{-3}\right) \ldots \ldots \ldots \ldots$ & ...... & $\ldots$. & $\ldots \ldots$ & $\ldots 4000$ & ...... & $\ldots 4200$ \\
\hline E.C. & 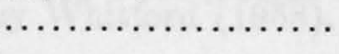 & $\ldots \ldots$ & $\ldots 6.5$ & $\ldots \ldots \ldots$ & $\ldots 6.5$ & ........ & $\ldots 4.6$ \\
\hline $\begin{array}{l}\mathbf{F}(\mathrm{H} \\
\mathrm{Com}\end{array}$ & $\cdots$ & $\begin{array}{r}-13.52^{\mathrm{a}} \\
\ldots \ldots \ldots\end{array}$ & $\begin{array}{l}-13.46^{\mathrm{f}} \\
\text { pt. thin }\end{array}$ & $-13.34^{\mathrm{a}}$ & $\begin{array}{c}-13.38^{\mathrm{b}} \\
\text { Type I }\end{array}$ & $-13.14^{\mathrm{a}}$ & $\begin{array}{l}-13.15^{\mathrm{b}} \\
\text {. Cont. }\end{array}$ \\
\hline
\end{tabular}


TABLE 5.4-Continued

UnCORRECTED AND CORRECTED LINE INTENSITIES

\begin{tabular}{|c|c|c|c|c|c|c|c|}
\hline \multirow{2}{*}{$\begin{array}{c}\lambda \\
(\hat{\AA})\end{array}$} & \multirow[b]{2}{*}{ Identification } & \multicolumn{2}{|c|}{ LMC-SMP18 } & \multicolumn{2}{|c|}{$\begin{array}{l}\text { LMC-SMP21 } \\
\end{array}$} & \multicolumn{2}{|c|}{ 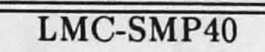 } \\
\hline & & $\mathrm{I}_{\circ}$ & $\mathbf{I}_{\mathrm{c}}$ & $\mathrm{I}_{\circ}$ & $\mathrm{I}_{\mathrm{c}}$ & $\mathrm{I}_{\mathrm{o}}$ & $\mathrm{I}_{\mathrm{c}}$ \\
\hline 3346 & {$[\mathrm{Ne} \mathrm{V}]$} & $\cdots$ & $\ldots$ & 50.1 & 54.0 & & \\
\hline 3426 & {$[\mathrm{Ne} \mathrm{V}]$} & 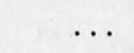 & . & 122.3 & 131.2 & 24.2 & 32.1 \\
\hline 3444 & OIII & .. & $\ldots$ & 22.9 & 24.5 & .. & \\
\hline 3727 & [O II] doublet ....... & $\cdots$ & $\ldots$ & 126.0 & 133.0 & 259.0 & 322.9 \\
\hline 3749 & He II, H12 & $\cdots$ & $\cdots$ & $\cdots$ & $\cdots$ & . & \\
\hline 3759 & O III & $\cdots$ & $\cdots$ & $\cdots$ & $\cdots$ & $\cdots$ & $\cdots$ \\
\hline 3771 & H11, He II, O III.... & $\cdots$ & $\cdots$ & $\cdots$ & $\ldots$ & $\cdots$ & $\cdots$ \\
\hline 3798 & $\ldots \ldots \ldots+\cdots$ & $\cdots$ & $\cdots$ & 3.7 & 3.9 & .. & \\
\hline 3834 & He II, H9 . & $\cdots$ & $\ldots$ & 8.0 & 8.4 & $\ldots$ & .. \\
\hline 3869 & [Ne III] & 53.6 & 62.9 & 96.6 & 101.2 & $\ldots$ & ... \\
\hline 3889 & H8, He I. & 12.9 & 15.1 & 24.7 & 25.9 & 13.6 & 16.4 \\
\hline 3969 & {$[\mathrm{Ne} \mathrm{III}], \mathrm{H} \epsilon \ldots \ldots \ldots$} & 26.4 & 30.5 & 51.0 & 53.2 & 52.1 & 61.7 \\
\hline 4026 & HeI............. & $\ldots$ & $\cdots$ & . & $\ldots$ & $\ldots$ & \\
\hline 4069 & [S II] doublet & $\ldots$ & $\ldots$ & 12.5 & 13.0 & 5.1 & 5.9 \\
\hline 4102 & $\mathrm{H} \delta, \mathrm{N}$ III $\ldots \ldots \ldots \ldots$ & 20.9 & 23.6 & 26.8 & 27.8 & 28.6 & 33.0 \\
\hline 4340 & $\mathrm{H} \gamma \ldots \ldots \ldots \ldots \ldots$ & 44.2 & 47.9 & 47.1 & 48.2 & 51.4 & 56.6 \\
\hline 4363 & [O III] & 18.0 & 19.5 & 35.6 & 36.5 & 18.6 & 20.3 \\
\hline 4472 & . $\ldots \ldots \ldots \ldots$ & 8.2 & 8.7 & 5.2 & 5.3 & 3.4 & 3.6 \\
\hline 4686 & ….... & $\cdots$ & $\cdots$ & 52.7 & 53.1 & 60.3 & 62.2 \\
\hline 4713 & ], He I, [NeIV] & $\ldots$ & $\ldots$ & 12.2 & 12.3 & $\ldots$ & $\ldots$ \\
\hline 4725 & {$[\mathrm{Ne}$ IV $] \ldots \ldots \ldots \ldots$} & $\cdots$ & $\cdots$ & $\cdots$ & $\cdots$ & $\cdots$ & $\ldots$ \\
\hline 4740 & {$[$ Ar IV] $\ldots \ldots \ldots \ldots$} & $\cdots$ & $\cdots$ & 10.7 & 10.8 & $\cdots$ & $\cdots$ \\
\hline 4959 & {$[\mathrm{OIII}] \ldots \ldots \ldots \ldots \ldots$} & 278.7 & 274.8 & 283.1 & 281.9 & 305.7 & 300.7 \\
\hline 5007 & $\ldots \ldots+\cdots$ & Satu & ated & 798.6 & 793.7 & 704.6 & 687.5 \\
\hline 5199 & {$[\mathrm{~N} \mathrm{I}$} & $\ldots$ & ... & 31.4 & 31.0 & 3.8 & 3.6 \\
\hline 5412 & $\mathrm{He}$ & $\ldots$ & $\ldots$ & 5.4 & 5.3 & 6.1 & 5.5 \\
\hline 5876 & 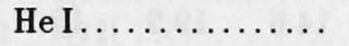 & 16.2 & 14.2 & 12.2 & 11.7 & 9.4 & 8.0 \\
\hline 6300 & $\ldots \ldots \ldots$ & $\ldots$ & $\ldots$ & 29.2 & 27.7 & 17.1 & 13.8 \\
\hline 6312 & $\ldots \ldots \ldots$ & $\ldots$ & $\ldots$ & 5.7 & 5.4 & $\ldots$ & $\ldots$ \\
\hline 6364 & $\ldots \ldots \ldots$ & $\ldots$ & $\ldots$ & 17.5 & 16.6 & 6.9 & 5.5 \\
\hline 6548 & [N II] & $\ldots$ & $\ldots$ & 199.3 & 187.8 & 45.6 & 35.8 \\
\hline 6563 & n....... & Satu & ated & 320.7 & 301.9 & 421.7 & 330.4 \\
\hline 6583 & [N II] & $\ldots$ & ‥ & 565.3 & 532.0 & 146.2 & 114.3 \\
\hline 6678 & . & $\ldots$ & $\ldots$ & 4.9 & 4.6 & 4.5 & 3.5 \\
\hline 17 & n... & $\ldots$ & $\cdots$ & 28.6 & 26.8 & 19.0 & 14.6 \\
\hline 6731 & …...... & $\ldots$ & $\ldots$ & 41.6 & 39.0 & 19.8 & 15.2 \\
\hline 7006 & $\ldots \ldots \ldots$ & $\ldots$ & $\ldots$ & 5.7 & 5 & .. & .. \\
\hline 7065 & $\ldots \ldots \ldots+\cdots$ & $\ldots$ & $\ldots$ & 9.2 & 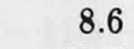 & 3.0 & 2.2 \\
\hline 7136 & ......... & $\cdots$ & $\cdots$ & 25.6 & 23.7 & 19.3 & 14.3 \\
\hline 7320 & ……... & $\cdots$ & $\cdots$ & 15.2 & 14.1 & 12.5 & 9.1 \\
\hline 7330 & $\ldots \ldots+\cdots \cdots$ & .. & $\ldots$ & 13.0 & 12.0 & 2.9 & 2.1 \\
\hline 7751 & {$[$ Ar III $] \ldots \ldots \ldots \ldots$} & $\cdots$ & $\cdots$ & 7.4 & 6.8 & $\cdots$ & $\cdots$ \\
\hline & $\cdots$ & & $\ldots 0.27$ & & . . 0.08 & & 0.32 \\
\hline $\mathrm{T}_{\mathrm{e}}([\mathrm{O}$ & $\mathrm{III}])(\mathrm{K}) \quad \ldots \ldots \ldots \ldots$ & ...... & .17000 & & .26000 & & 19000 \\
\hline $\mathbf{n}_{\mathrm{e}}([\mathrm{S}]$ & I] $)\left(\mathrm{cm}^{-3}\right) \ldots \ldots \ldots \ldots$ & $\ldots$. & $\ldots$ & & .4800 & .. & .3000 \\
\hline E.C. & $\ldots \ldots$ & $\cdots$ & ...3.6 & & $\ldots 7.3$ & $\ldots \ldots$ & $\ldots 7.8$ \\
\hline $\mathbf{F}(\mathbf{H} \beta)$ & & $-13.30^{\mathrm{a}}$ & $-13.36^{b}$ & $-12.81^{\mathrm{a}}$ & $-12.78^{d}$ & $-13.44^{\mathrm{a}}$ & $-13.25^{b}$ \\
\hline & & & & $\begin{array}{l}-12.76^{\circ} \\
-12.69^{\mathrm{c}}\end{array}$ & $-12.78^{e}$ & & \\
\hline Con & & & Cont. & & Type I & & \\
\hline
\end{tabular}


TABLE 5.4-Continued

UNCORRECTED AND CORRECTED LINE INTENSITIES

\begin{tabular}{|c|c|c|c|c|c|c|c|}
\hline \multirow{2}{*}{$\begin{array}{c}\lambda \\
(\AA) \\
(\AA)\end{array}$} & \multirow[b]{2}{*}{ Identification } & \multicolumn{2}{|c|}{ LMC-SMP41 } & \multicolumn{2}{|c|}{ "LMC-SMP84 } & \multicolumn{2}{|c|}{ LMC-SMP85 } \\
\hline & & $\mathrm{I}_{\mathrm{o}}$ & $I_{c}$ & $I_{\circ}$ & $I_{c}$ & $\mathrm{I}_{\circ}$ & $I_{c}$ \\
\hline 3346 & {$[\mathrm{Ne} \mathrm{V}]$} & $\ldots$ & & & & & \\
\hline 3426 & {$[\mathrm{Ne} \mathrm{V}]$} & $\ldots$ & $\ldots$ & $\ldots$ & $\ldots$ & $\ldots$ & $\ldots$ \\
\hline 3444 & OIII & 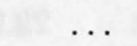 & 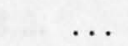 & $\ldots$ & $\ldots$ & $\ldots$ & $\ldots$ \\
\hline 3727 & [O II] doublet & 102.8 & 95.3 & 25.9 & 27.1 & 47.9 & 48.8 \\
\hline 3749 & He II, H12 & $\ldots$ & $\ldots$ & 3.6 & 3.8 & 1.7 & 1.8 \\
\hline 3759 & O III $\ldots . . .$. & $\ldots$ & $\ldots$ & $\ldots$ & $\ldots$ & $\ldots$ & $\ldots$ \\
\hline 3771 & H11, He II, O III.... & $\ldots$ & $\ldots$ & 2.7 & 2.8 & 1.5 & 1.5 \\
\hline 3798 & n.w. & $\ldots$ & $\ldots$ & 4.2 & 4.4 & 2.3 & 2.3 \\
\hline 3834 & He II, H9 & $\ldots$ & $\ldots$ & 7.6 & 7.9 & 4.8 & 4.8 \\
\hline 3869 & [Ne III] & 100.6 & 94.2 & 40.5 & 42.1 & 12.2 & 12.4 \\
\hline 3889 & H8, He I. . & 15.8 & 14.8 & 20.0 & 20.8 & 12.6 & 12.8 \\
\hline 3969 & {$[\mathrm{Ne} \mathrm{III}], \mathrm{H} \epsilon \ldots \ldots \ldots$} & 48.2 & 45.5 & 30.3 & 31.4 & 22.6 & 22.9 \\
\hline 4026 & $\mathrm{He} \mathrm{I} \ldots \ldots \ldots \ldots \ldots$ & $\ldots$ & $\ldots$ & 3.7 & 3.8 & 2.6 & 2.6 \\
\hline 4069 & [S II] doublet & $\ldots$ & $\ldots$ & $\ldots$ & $\ldots$ & $\ldots$ & $\ldots$ \\
\hline 4102 & H $\delta$, N III . & 28.5 & 27.1 & 28.2 & 29.1 & 32.4 & 32.7 \\
\hline 4340 & $\mathrm{H} \gamma \ldots$ & 52.6 & 50.9 & 46.3 & 47.2 & 55.8 & 56.2 \\
\hline 4363 & [O III] & 25.9 & 25.1 & 10.6 & 10.9 & 7.7 & 7.8 \\
\hline 4472 & HeI. & 2.8 & 2.7 & 6.0 & 6.1 & 7.4 & 7.5 \\
\hline 4686 & He II & 60.9 & 60.2 & $\ldots$ & $\ldots$ & $\ldots$ & $\ldots$ \\
\hline 4713 & [Ar IV], HeI, [Ne IV] & $\ldots$ & $\ldots$ & 2.7 & 2.7 & $\ldots$ & $\ldots$ \\
\hline 4725 & {$[\mathrm{Ne} I \mathrm{IV}] \ldots \ldots \ldots \ldots$} & $\ldots$ & $\ldots$ & $\ldots$ & $\ldots$ & $\ldots$ & $\ldots$ \\
\hline 4740 & {$[\mathrm{ArIV}] \ldots \ldots \ldots \ldots$} & & & $\ldots$ & $\ldots$ & $\ldots$ & $\ldots$ \\
\hline 4959 & [O III]. & 321.0 & 322.8 & 225.6 & 224.8 & 117.7 & 117.6 \\
\hline 5007 & n...... & 1006.0 & 1014.6 & 651.6 & 648.2 & 345.2 & 344.5 \\
\hline 5199 & {$[\mathrm{~N} \mathrm{I}]$} & $\ldots$ & $\cdots$ & $\cdots$ & $\ldots$ & $\ldots$ & $\ldots$ \\
\hline 5412 & $\ldots \ldots+\cdots$ & $\ldots$ & $\ldots$ & $\ldots$ & $\ldots$ & $\ldots$ & $\ldots$ \\
\hline 5876 & n... & $\cdots$ & $\ldots$ & 15.1 & 14.6 & 19.9 & 19.7 \\
\hline 6300 & ........ & $\ldots$ & $\ldots$ & $\ldots$ & $\ldots$ & $\ldots$ & $\ldots$ \\
\hline 6312 & $\ldots \ldots \ldots$ & $\ldots$ & $\ldots$ & $\ldots$ & .. & $\ldots$ & $\cdots$ \\
\hline 6364 & {$[\mathrm{OI}]$} & $\ldots$ & $\ldots$ & $\ldots$ & $\ldots$ & $\ldots$ & $\ldots$ \\
\hline 6548 & …... & 35.3 & 38.4 & $\ldots$ & $\ldots$ & 17.1 & 16.8 \\
\hline 6563 & ......... & 282.6 & 307.5 & 317.2 & 301.5 & 335.7 & 329.1 \\
\hline 6583 & {$[\mathrm{~N} \mathrm{II}]$} & 125.3 & 136.5 & $\ldots$ & $\ldots$ & 46.9 & 46.0 \\
\hline 6678 & $\ldots \ldots \ldots$ & $\ldots$ & $\ldots$ & 4.7 & 4.4 & 6.1 & 6.0 \\
\hline 6717 & {$[\mathrm{~S} \mathrm{II}]$} & 9.3 & 10.2 & $\cdots$ & $\ldots$ & 0.3 & 0.3 \\
\hline 6731 & [S II] & 8.5 & 9.3 & $\ldots$ & $\ldots$ & 0.9 & 0.9 \\
\hline 7006 & {$[\mathrm{ArV}] \ldots \ldots \ldots \ldots$} & $\ldots$ & $\ldots$ & $\ldots$ & $\ldots$ & $\ldots$ & $\ldots$ \\
\hline 7065 & ......... & $\ldots$ & $\ldots$ & 9.6 & 9.1 & 18.5 & 18.0 \\
\hline 7136 & {$[\mathrm{Ar} I \mathrm{III}] \ldots \ldots \ldots \ldots$} & 15.6 & 17.3 & 10.3 & 9.7 & 16.0 & 15.6 \\
\hline 7320 & {$[\mathrm{OII}] \ldots \ldots \ldots \ldots$} & $\ldots$ & $\ldots$ & 1.9 & 1.8 & 28.7 & 27.9 \\
\hline 7330 & ........... & .. & $\ldots$ & 1.1 & 1.1 & 20.7 & 20.2 \\
\hline 7751 & {$[$ Ar III $] \ldots \ldots \ldots \ldots$} & $\cdots$ & $\cdots$ & 3.6 & 3.3 & 4.8 & 4.7 \\
\hline & $\ldots \ldots \ldots$ & $\ldots$ & $\ldots-0.11$ & & $\ldots 0.07$ & & $\ldots 0.03$ \\
\hline $\mathrm{T}_{\mathrm{e}}([\mathrm{O}$ & III] $)(\mathrm{K})$ & $\ldots$ & 18000 & $\ldots$. & 14000 & & 17000 \\
\hline $\mathrm{n}_{\mathrm{e}}([\mathrm{S}]$ & II] $)\left(\mathrm{cm}^{-3}\right) \ldots \ldots \ldots$ & 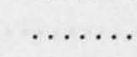 & $\ldots 1700$ & & & $\ldots$ & $>10^{5}$ \\
\hline E.C. & 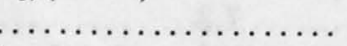 & & $\ldots 7.7$ & &. .2 .9 & & $\ldots 1.6$ \\
\hline $\mathrm{F}(\mathrm{H} \beta$ & ......... & ... & $-13.33^{\mathrm{a}}$ & & $12.63^{\mathrm{b}}$ & & $12.42^{\mathrm{b}}$ \\
\hline & & & & & & & \\
\hline
\end{tabular}


TABLE 5.4-Continued

UnCorrected and Corrected Line Intensities

\begin{tabular}{|c|c|c|c|c|c|c|c|}
\hline \multirow{2}{*}{$\begin{array}{c}\lambda \\
(\AA)\end{array}$} & \multirow[b]{2}{*}{ Identification } & \multicolumn{2}{|c|}{ LMC-SMP95 } & \multicolumn{2}{|c|}{ LMC-SMP98 } & \multicolumn{2}{|c|}{$\overline{\text { LMC-SMP100 }}$} \\
\hline & & $\mathrm{I}_{\circ}$ & $\mathrm{I}_{\mathrm{c}}$ & $\mathrm{I}_{\mathrm{o}}$ & $\mathrm{I}_{\mathrm{c}}$ & I。 & $I_{c}$ \\
\hline 3346 & {$[\mathrm{NeV}]$} & $\ldots$ & ... & & & & \\
\hline 3426 & {$[\mathrm{NeV}]$} & $\ldots$ & & 4.1 & 5.7 & 4.3 & 4.5 \\
\hline 3444 & OIII & & & 7.6 & 10.7 & 8.9 & 9.2 \\
\hline 3727 & [O II] doublet & 295.1 & 390.5 & 36.9 & 48.1 & 35.6 & 35.6 \\
\hline 3749 & He II, H12 & $\cdots$ & $\ldots$ & $\cdots$ & & $\cdots$ & $\cdots$ \\
\hline 3759 & O III ....... & $\ldots$ & $\ldots$ & $\ldots$ & $\ldots$ & & .. \\
\hline 3771 & H11, He II, O III.... & .. & $\ldots$ & 1.9 & 2.4 & .. & $\ldots$ \\
\hline 3798 & H10 & $\ldots$ & $\cdots$ & 3.2 & 4.1 & 5.1 & 5.2 \\
\hline 3834 & He II, H9 & $\ldots$ & 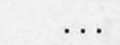 & 5.5 & 7.0 & 7.0 & 7.2 \\
\hline 3869 & [Ne III] & 85.2 & 108.5 & 89.0 & 112.0 & 73.7 & 75.4 \\
\hline 3889 & H8, He I. . & . & $\ldots$ & 15.0 & 18.8 & 17.0 & 17.3 \\
\hline 3969 & [Ne III], H $\epsilon \ldots \ldots \ldots$ & 41.1 & 51.0 & 35.7 & 43.8 & 38.0 & 38.8 \\
\hline 4026 & HeI... & $\ldots$ & $\ldots$ & 1.6 & 1.9 & 1.4 & 1.4 \\
\hline 4069 & [S II] doublet & $\cdots$ & $\ldots$ & 2.8 & 3.3 & 3.0 & 3.0 \\
\hline 4102 & $\mathrm{H} \delta, \mathrm{N}$ III . & 24.7 & 29.6 & 22.5 & 26.7 & 27.5 & 28.0 \\
\hline 4340 & $\mathrm{H} \gamma \ldots$ & 42.2 & 47.7 & 39.8 & 44.7 & 49.7 & 50.3 \\
\hline 4363 & [O III $]$ & 12.7 & 14.3 & 19.2 & 21.4 & 17.5 & 17.7 \\
\hline 4472 & He I. & $\ldots$ & $\ldots$ & 4.0 & 4.3 & 3.3 & 3.4 \\
\hline 4686 & He II. & 28.6 & 29.7 & 20.0 & 20.7 & 43.6 & 43.7 \\
\hline 4713 & [Ar IV], He I, [Ne IV] & $\cdots$ & $\cdots$ & 2.8 & 2.9 & 5.8 & 5.8 \\
\hline 4725 & [Ne IV] & $\cdots$ & $\cdots$ & $\ldots$ & $\cdots$ & $\cdots$ & \\
\hline 4740 & {$[\mathrm{Ar} I V]$} & $\ldots$ & & 2.9 & 3.0 & 2.9 & 2.9 \\
\hline 4959 & [O III] & 344.2 & 337.0 & 393.8 & 386.0 & 363.4 & 362.6 \\
\hline 5007 & [O III] & \multicolumn{2}{|c|}{ Saturated } & \multicolumn{2}{|c|}{ Saturated } & \multicolumn{2}{|c|}{ Saturated } \\
\hline 5199 & [N I] & $\ldots$ & $\cdots$ & & & & \\
\hline 5412 & HeII. & $\ldots$ & $\ldots$ & 1.8 & 1.7 & 4.0 & 4.0 \\
\hline 5876 & He I. & $\ldots$ & $\ldots$ & 12.9 & 10.6 & 7.4 & 7.3 \\
\hline 6300 & [O I] & $\ldots$ & $\ldots$ & 8.8 & 6.8 & $\cdots$ & $\ldots$ \\
\hline 6312 & [S III] & $\ldots$ & $\ldots$ & 2.6 & 2.0 & $\ldots$ & $\cdots$ \\
\hline 6364 & [O I] & .. & $\ldots$ & 2.9 & 2.2 & $\ldots$ & ... \\
\hline 6548 & [N II & 87.4 & 64.2 & 9.8 & 7.3 & 5.2 & 5.1 \\
\hline 6563 & $\mathrm{H} \alpha$ & 413.9 & 303.4 & 390.8 & 291.2 & 316.7 & 307.5 \\
\hline 6583 & [N II] & 300.5 & 219.7 & 65.2 & 48.4 & 58.1 & 56.3 \\
\hline 6678 & HeI. & & ... & 6. & 4.5 & 4.4 & 4.3 \\
\hline 6717 & [S II] & 33.4 & 24.0 & 3.8 & 2.8 & 3.7 & 3.6 \\
\hline 6731 & [S II] & 38.0 & 27.2 & 6.9 & 5.0 & 4.3 & 4.2 \\
\hline 7006 & {$[\mathrm{Ar} \mathrm{V}]$} & $\ldots$ & $\ldots$ & $\ldots$ & $\ldots$ & & \\
\hline 7065 & $\mathrm{He}$ & . & $\ldots$ & 14.8 & 10.3 & 3.6 & 3.5 \\
\hline 7136 & [Ar III] & 32.3 & 22.0 & 16.3 & 11.3 & 11.1 & 10.7 \\
\hline 7320 & [O II] & $\ldots$ & $\ldots$ & 12.0 & 8.2 & $\ldots$ & .. \\
\hline 7330 & {$[\mathrm{O}$ I] } & $\ldots$ & $\ldots$ & 10.9 & 7.4 & $\ldots$ & ... \\
\hline 7751 & [Ar III] & $\ldots$ & $\ldots$ & 3.7 & 2.4 & $\cdots$ & $\cdots$ \\
\hline & \multirow{2}{*}{\multicolumn{2}{|c|}{ …… 0.41}} & \multicolumn{2}{|c|}{$\ldots \ldots \ldots 0.39$} & \\
\hline \multicolumn{2}{|c|}{$\mathrm{T}_{\mathrm{e}}([\mathrm{O} \mathrm{III}])(\mathrm{K})}$. & & & \multicolumn{2}{|r|}{15000} & .15000 & \\
\hline \multicolumn{2}{|c|}{$\mathrm{n}_{\mathrm{e}}([\mathrm{S} \mathrm{II}])\left(\mathrm{cm}^{-3}\right)$} & \multicolumn{2}{|c|}{ …...... 3100} & \multicolumn{2}{|c|}{$\ldots 7300$} & \multicolumn{2}{|r|}{.. 3200} \\
\hline \multirow{2}{*}{\multicolumn{2}{|c|}{$F(H \beta)$}} & & $\ldots 6.0$ & & ..5.5 & & $\ldots 6.7$ \\
\hline & & $-13.39^{\mathrm{a}}$ & $-13.46^{f}$ & $\ldots \ldots$ & $12.52^{f}$ & $-12.86^{b}$ & $-12.83^{f}$ \\
\hline Co & & $46^{\circ}$ & De I? & & & & \\
\hline
\end{tabular}


TABLE 5.4-Continued

UnCorrected and CoRrected Line Intensities

\begin{tabular}{|c|c|c|c|c|c|c|c|}
\hline \multirow{2}{*}{$\begin{array}{c}\lambda \\
(\AA)\end{array}$} & \multirow[b]{2}{*}{ Identification } & \multicolumn{2}{|c|}{ LMC-SMP101 } & \multicolumn{2}{|c|}{ LMC-SMP102 } & \multicolumn{2}{|c|}{ LMC-MG01 } \\
\hline & & $\mathrm{I}_{\circ}$ & $\mathrm{I}_{\mathrm{c}}$ & $\mathrm{I}_{0}$ & $\mathrm{I}_{\mathrm{c}}$ & $\mathrm{I}_{\circ}$ & $I_{c}$ \\
\hline 3346 & {$[\mathrm{Ne} \mathrm{V}]$} & & $\cdots$ & $\ldots$ & $\ldots$ & $\cdots$ & \\
\hline 3426 & {$[\mathrm{Ne} \mathrm{V}]$} & & 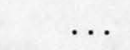 & 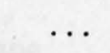 & & & \\
\hline 3444 & OIII & 10.3 & 10.8 & & & & \\
\hline 3727 & [O II $]$ doublet & 31.6 & 32.6 & 15.2 & 14.7 & 470.8 & 464.0 \\
\hline 3749 & He II, H12 & $\cdots$ & $\cdots$ & $\cdots$ & 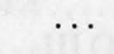 & & \\
\hline 3759 & O III ... & $\ldots$ & $\cdots$ & $\cdots$ & . & & \\
\hline 3771 & H11, He II, O III.... & 3.1 & 3.2 & . & $\cdots$ & & \\
\hline 3798 & $\mathrm{H} 10$. & 4.1 & 4.2 & $\cdots$ & $\cdots$ & & \\
\hline 3834 & He II, Hs & $\begin{array}{r}4.4 \\
80 ?\end{array}$ & 4.6 & & & & 740 \\
\hline $\begin{array}{l}3869 \\
3889\end{array}$ & $\begin{array}{l}{[\mathrm{Ne} \mathrm{II}]} \\
\mathrm{H} 8, \mathrm{H}\end{array}$ & $\begin{array}{l}80.2 \\
15.0\end{array}$ & $\begin{array}{l}82.4 \\
15.4\end{array}$ & $\begin{array}{l}44.2 \\
15.9\end{array}$ & $\begin{array}{l}43.1 \\
15.5\end{array}$ & 75.8 & 74.9 \\
\hline 3969 & {$[\mathrm{Ne} I I I], \mathrm{H} \epsilon$. } & 41.0 & 42.1 & 30.0 & 29.4 & 38.0 & 37.6 \\
\hline 4026 & HeI. & & $\cdots$ & 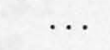 & $\cdots$ & & \\
\hline 4069 & [S II] doublet & & & & $\cdots$ & & \\
\hline 4102 & $\mathrm{H} \delta, \mathrm{N} \mathrm{I}$ & 25.5 & 26.1 & 25.1 & 24.6 & 26.0 & 25.8 \\
\hline 4340 & $\mathrm{H} \gamma$. & 47.4 & 48.0 & 51.0 & 50.4 & 46.1 & 45.8 \\
\hline 4363 & [O III] & 18.0 & 18.3 & 16.5 & 16.4 & 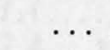 & 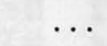 \\
\hline 4472 & $\mathrm{He}$ I. & 2.1 & 2.1 & & & & \\
\hline 4686 & He II . & 67.1 & 67.4 & 75.1 & 74.8 & & \\
\hline 4713 & [Ar IV], He I, [Ne IV] & $\cdots$ & $\cdots$ & $\cdots$ & $\cdots$ & & \\
\hline 4725 & {$[N$} & $\cdots$ & . & 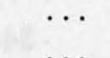 & *. & & \\
\hline 4740 & {$\left[\mathrm{~A}_{1}\right.$} & & & & & & \\
\hline 4959 & [O III] & $\begin{array}{r}373.6 \\
10170\end{array}$ & $\begin{array}{r}372.7 \\
1014.2\end{array}$ & $\begin{array}{l}244.0 \\
658.3\end{array}$ & $\begin{array}{l}45.1 \\
60.3\end{array}$ & $\begin{array}{l}238.0 \\
667.6\end{array}$ & $\begin{array}{l}238.2 \\
668.6\end{array}$ \\
\hline $\begin{array}{l}5007 \\
5199\end{array}$ & [N I] & 1017.9 & $\begin{array}{r}014.2 \\
\ldots\end{array}$ & $\ldots$ & $\ldots$ & & \\
\hline 5412 & $\mathrm{He}$ & 6.7 & 6.6 & 5.9 & 6.0 & $\ldots$ & $\cdots$ \\
\hline 5876 & $\mathrm{He}$ & 4.9 & 4.7 & 5.8 & 5.9 & .. & . \\
\hline 00 & {$[\mathrm{O}$} & $\cdots$ & $\cdots$ & $\cdot$ & $\cdots$ & $\cdots$ & \\
\hline 6312 & {$[\mathrm{~S} \mathrm{III}]$} & $\cdots$ & & & . & & \\
\hline 64 & {$[\mathrm{OI}]$} & & $\cdots$ & . & . & $\ldots$ & $\ldots$ \\
\hline 48 & {$[\mathrm{~N}$ II $]$} & & & $\ldots$ & $\cdots$ & 40.7 & 41.3 \\
\hline 6563 & $\mathrm{H} \alpha$ & 309.6 & 298.7 & 293.0 & 302.0 & 288.0 & 292.6 \\
\hline 83 & {$[\mathrm{~N}$} & & & $\ldots$ & $\ldots$ & 181.1 & 184.1 \\
\hline 6678 & HeI. & 3.7 & 3.6 & .. & $\cdots$ & $\ldots$ & .. \\
\hline 6717 & [S II] & 3.8 & 3.7 & $\ldots$ & $\cdots$ & 41.8 & 42.5 \\
\hline 6731 & [S II] & 3.3 & 3.2 & $\cdots$ & ... & 32.2 & 32.7 \\
\hline 7006 & {$[\mathrm{ArV}]$} & & & . & & & \\
\hline 7065 & $\mathrm{He}$ & 3.3 & 3.2 & 3.5 & 3.7 & & \\
\hline 7136 & {$[\mathrm{Ar}]$} & 10.1 & 9.6 & 6.5 & 6.8 & 23.0 & 23.5 \\
\hline 7320 & [O II] & 1.9 & 1.8 & $\cdots$ & $\cdots$ & $\cdots$ & $\cdots$ \\
\hline 7330 & [O II] & 1.8 & 1.7 & $\cdots$ & $\cdots$ & & \\
\hline 7751 & {$[$ Ar III $] \ldots$} & & & $\cdots$ & & & \\
\hline & & & .0 .05 & &.-0.40 & &.-0.21 \\
\hline $\mathrm{T}_{\mathrm{e}}([\mathrm{O}$ & III])(K). & & 15000 & & 17000 & & \\
\hline $\mathrm{n}_{\mathrm{e}}([\mathrm{S} \mathrm{I}$ & I]) $\left(\mathrm{cm}^{-3}\right)$ & & 900 & & & & \\
\hline E.C. . & & & 8.1 & & .8 .5 & . & .3 .0 \\
\hline $\begin{array}{l}\mathrm{F}(\mathrm{H} \beta) \\
\mathrm{Comm}\end{array}$ & & $-12.89^{b}$ & $-12.88^{f}$ & & $3.22^{\mathrm{b}}$ & & \\
\hline Comm & & $\ldots$. & t. thin & $\ldots \mathrm{O}$ & thin & & \\
\hline
\end{tabular}


TABLE 5.4-Continued

UnCorRected AND CoRrected Line Intensities

\begin{tabular}{|c|c|c|c|c|c|c|c|}
\hline \multirow{2}{*}{$\begin{array}{c}\lambda \\
(\AA)\end{array}$} & \multirow[b]{2}{*}{ Identification } & \multicolumn{2}{|c|}{ LMC-MG02 } & \multicolumn{2}{|c|}{ "LMC-MG03 } & \multicolumn{2}{|c|}{ LMC-MG07 } \\
\hline & & $\mathrm{I}_{0}$ & $\mathrm{I}_{\mathrm{c}}$ & $\mathrm{I}_{\circ}$ & $\mathrm{I}_{\mathrm{c}}$ & $\mathrm{I}_{\mathrm{o}}$ & $I_{c}$ \\
\hline 3346 & {$[\mathrm{Ne} \mathrm{V}]$} & $\ldots$ & $\ldots$ & & & $\ldots$ & $\ldots$ \\
\hline 3426 & {$[\mathrm{Ne} \mathrm{V}]$} & $\ldots$ & $\cdots$ & & . & $\ldots$ & \\
\hline 3444 & OIII & & $\ldots$ & ( & $\ldots$ & $\ldots$ & $\ldots$ \\
\hline 3727 & [O II] doublet & 389.3 & 376.9 & 430.5 & 491.5 & 358.7 & 328.8 \\
\hline 3749 & He II, H12 & $\ldots$ & $\ldots$ & $\ldots$ & $\ldots$ & & 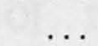 \\
\hline 3759 & O III . & $\ldots$ & $\ldots$ & $\ldots$ & $\ldots$ & & \\
\hline 3771 & H11, He II, O III.... & ... & $\ldots$ & $\ldots$ & $\ldots$ & $\ldots$ & . \\
\hline 3798 & H10 & $\ldots$ & $\ldots$ & $\ldots$ & $\ldots$ & $\ldots$ & \\
\hline 3834 & He II, H9 $\ldots \ldots \ldots$. & $\ldots$ & $\ldots$ & $\ldots$ & $\ldots$ & $\ldots$ & .. \\
\hline 3869 & {$[\mathrm{Ne}$ III $] \ldots$} & 108.8 & 105.8 & 96.1 & 107.8 & 102.9 & 95.5 \\
\hline 3889 & H8, He I. & $\ldots$ & $\ldots$ & $\ldots$ & $\ldots$ & 15.3 & 14.2 \\
\hline 3969 & {$[\mathrm{Ne}$ III $], \mathrm{H} \epsilon \ldots \ldots \ldots$} & 42.7 & 41.7 & 27.5 & 30.4 & 46.5 & 43.5 \\
\hline 4026 & He I.............. & $\ldots$ & $\ldots$ & $\ldots$ & $\ldots$ & $\ldots$ & . \\
\hline 4069 & [S II] doublet & $\ldots$ & $\ldots$ & $\ldots$ & $\ldots$ & $\ldots$ & $\cdot$ \\
\hline 4102 & H $\delta$, N III . . & 21.0 & 20.6 & 24.5 & 26.7 & 26.7 & 25.3 \\
\hline 4340 & $\mathrm{H} \gamma$. & 48.1 & 47.4 & 43.2 & 45.8 & 55.3 & 53.3 \\
\hline 4363 & [O III] & .. & $\ldots$ & 8.2 & 8.7 & 13.4 & 12.9 \\
\hline 4472 & HeI. & $\ldots$ & $\ldots$ & $\ldots$ & $\ldots$ & $\ldots$ & $\ldots$ \\
\hline 4686 & He II . & 23.4 & 23.3 & $\ldots$ & $\ldots$ & 13.5 & 13.3 \\
\hline 4713 & [Ar IV], He I, [Ne IV] & $\cdots$ & $\cdots$ & $\cdots$ & $\cdots$ & $\ldots$ & $\cdots$ \\
\hline 4725 & {$[\mathrm{NeIV}]$} & $\ldots$ & $\ldots$ & $\ldots$ & $\ldots$ & $\ldots$ & $\cdots$ \\
\hline 4740 & {$[$ Ar IV $] \ldots \ldots \ldots \ldots$} & $\ldots$ & $\ldots$ & $\ldots$ & $\ldots$ & $\ldots$ & $\ldots$ \\
\hline 4959 & [O III $]$ & 300.7 & 301.4 & 219.7 & 217.6 & 319.1 & 321.2 \\
\hline 5007 & [O III $]$ & 802.5 & 805.4 & 597.8 & 589.0 & 888.9 & 897.6 \\
\hline 5199 & {$[\mathrm{~N} \mathrm{I}]$. } & $\ldots$ & $\ldots$ & $\ldots$ & $\cdots$ & .. & $\cdots$ \\
\hline 5412 & He II $\ldots \ldots \ldots \ldots \ldots$ & $\ldots$ & $\ldots$ & $\ldots$ & $\ldots$ & $\cdots$ & ... \\
\hline 5876 & HeI $\ldots \ldots \ldots \ldots \ldots$ & $\ldots$ & $\ldots$ & $\ldots$ & $\ldots$ & $\ldots$ & ... \\
\hline 6300 & $\ldots \ldots+\cdots$ & $\cdots$ & $\ldots$ & $\ldots$ & $\ldots$ & $\ldots$ & $\cdots$ \\
\hline 6312 & $\ldots \ldots \ldots \ldots$ & $\ldots$ & $\ldots$ & $\ldots$ & $\cdots$ & $\cdots$ & $\cdots$ \\
\hline 6364 & n........ & $\ldots$ & $\ldots$ & $\ldots$ & $\ldots$ & 26.3 & 28.6 \\
\hline 6548 & [N II $]$ & 45.4 & 47.0 & 50.1 & 43.3 & 52.0 & 57.2 \\
\hline 6563 & $\mathrm{H} \alpha$ & 276.8 & 286.9 & Satu & ated & 281.5 & 309.9 \\
\hline 6583 & {$[\mathrm{~N} \mathrm{II}]$} & 204.7 & 212.2 & 161.2 & 139.0 & 178.8 & 197.0 \\
\hline 6678 & n....... & $\ldots$ & $\ldots$ & $\ldots$ & $\ldots$ & $\ldots$ & $\ldots$ \\
\hline 6717 & {$[\mathrm{~S} \mathrm{II}]$} & $\cdots$ & $\ldots$ & 22.5 & 19.2 & 17.5 & 19.4 \\
\hline 6731 & [S II] & $\ldots$ & $\cdots$ & 21.4 & 18.3 & 33.7 & 37.4 \\
\hline 7006 & {$[\mathrm{Ar} V] \ldots \ldots \ldots \ldots$} & $\ldots$ & $\ldots$ & $\ldots$ & $\cdots$ & $\cdots$ & $\cdots$ \\
\hline 7065 & HeI. & $\cdots$ & .. & $\cdots$ & $\cdots$ & $\cdots$ & .. \\
\hline 7136 & {$[\mathrm{Ar} \mathrm{III}] \ldots \ldots \ldots \ldots$} & $\ldots$ & $\ldots$ & 18.5 & 15.4 & $\ldots$ & ... \\
\hline 7320 & {$[\mathrm{O} \mathrm{II}] \ldots \ldots \ldots \ldots$} & $\ldots$ & $\ldots$ & $\ldots$ & $\ldots$ & $\cdots$ & .. \\
\hline 7330 & [O II $]$ & $\ldots$ & ... & $\cdots$ & $\ldots$ & $\ldots$ & .. \\
\hline 7751 & {$[$ Ar III $] \ldots \ldots \ldots \ldots$} & $\cdots$ & ․ & $\cdots$ & $\cdots$ & $\cdots$ & $\cdots$ \\
\hline & & $\ldots$. &.-0.05 & $\ldots$ & 0.19 & ... &.-0.13 \\
\hline $\mathrm{T}_{\mathrm{e}}([\mathrm{O}$ & III])(K) & ... & ....... & $\ldots$ & 14000 & $\cdots$ & 13000 \\
\hline$n_{e}([S]$ & I]) $\left(\mathrm{cm}^{-3}\right)$ & & & & .1900 & $\ldots$ & $\sim 10^{5}$ \\
\hline E.C. & & $\ldots$ & $\ldots 5.6$ & $\ldots$ & $\ldots 2.7$ & $\ldots$ & ...5.1 \\
\hline $\mathbf{F}(\mathbf{H} \beta$ & .............. & $\ldots \ldots$ & ...... & $\ldots$ & $\cdots \cdots$ & . & $\cdots$ \\
\hline Comn & ent $\ldots \ldots \ldots \ldots \ldots$ & $\ldots$. & I I? & & & & \\
\hline
\end{tabular}


TABLE 5.4-Continued

UnCorrected AND CoRrected Line InTensities

\begin{tabular}{|c|c|c|c|c|c|c|c|}
\hline \multirow{2}{*}{$\begin{array}{c}\lambda \\
(\AA) \\
(\AA 346\end{array}$} & \multirow[b]{2}{*}{ Identification } & \multicolumn{2}{|c|}{ LMC-MG20 } & \multicolumn{2}{|c|}{ LMC-MG21 } & \multicolumn{2}{|c|}{ LMC-MG29 } \\
\hline & & $I_{o}$ & $\mathrm{I}_{\mathrm{c}}$ & $\mathrm{I}_{\circ}$ & $I_{c}$ & $\mathrm{I}_{\mathrm{o}}$ & $\mathrm{I}_{\mathrm{c}}$ \\
\hline & {$[\mathrm{Ne} \mathrm{V}]$} & - & $\cdots$ & . & & & \\
\hline 3426 & {$[\mathrm{Ne} \mathrm{V}]$} & & $\cdots$ & $\cdots$ & & 15.2 & 18.6 \\
\hline 3444 & OIII & & $\ldots$ & & & & \\
\hline 3727 & [O II] doublet & 348.9 & 412.1 & & & 199.3 & 232.3 \\
\hline 3749 & He II, H12 & $\cdots$ & & & & & 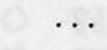 \\
\hline 3759 & O III & 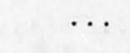 & 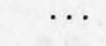 & 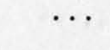 & & & 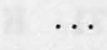 \\
\hline 3771 & H11, & .. & $\cdots$ & .. & $\cdots$ & .. & .. \\
\hline 3798 & H10 & & $\cdots$ & & & & . \\
\hline 3834 & $\mathrm{He}$ II, H & - & 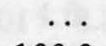 & & & & .. \\
\hline 3869 & [Ne III] & 144.5 & 166.8 & 24.0 & 22.3 & 80.1 & 91.4 \\
\hline 3889 & H8, & & .. & 7. & & 11.7 & 13.3 \\
\hline 969 & {$[1$} & 56.7 & 64.5 & 22.6 & 21.1 & 35.1 & 39.5 \\
\hline 4026 & & $\cdots$ & $\cdots$ & $\cdots$ & . & $\cdots$ & $\cdots$ \\
\hline 4069 & [S II & $\cdots$ & $\ldots$ & & & $\ldots$ & \\
\hline 4102 & $\mathbf{H} \delta$, & 21.7 & 24.1 & 24.0 & 22 & 21. & 24.2 \\
\hline 340 & & 36.1 & 38. & 49 & & 44 & 47.5 \\
\hline 63 & {$[0$} & $\ldots$ & $\cdots$ & 9.1 & 8.8 & 16.6 & 17.7 \\
\hline 72 & & $\ldots$ & $\ldots$ & $\ldots$ & & & $\ldots$ \\
\hline 4686 & $\mathrm{He}$ & 89.8 & 91.9 & 112.1 & 110.7 & 66.7 & 68.2 \\
\hline 4713 & $\mathrm{I},[\mathrm{Ne} \mathrm{IV}]$ & $\cdots$ & .. & $\cdots$ & & $\cdots$ & $\cdots$ \\
\hline 25 & {$[\mathrm{r}$} & $\cdots$ & $\cdots$ & . & & $\cdots$ & $\cdots$ \\
\hline 10 & & $\because=$ & 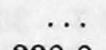 & $\cdots$ & $\because$ &.. & $\ldots$ \\
\hline $9 y$ & {$[\mathrm{O}$} & 31.7 & 28.8 & 26.4 & 27.2 & 312.2 & 308.6 \\
\hline 07 & {$[\mathrm{O}$} & 622.2 & 010.8 & 351.1 & 54.5 & \multicolumn{2}{|c|}{ Saturated } \\
\hline 99 & {$[\mathrm{~N}$} & . & & & & $\cdots$ & $\cdots$ \\
\hline 12 & & $\cdots$ & . & $\cdots$ & . & . & $\cdots$ \\
\hline & & $\cdots$ & .. & .. & $\cdots$ & $\cdots$ & $\cdots$ \\
\hline & & $\cdots$ & . & . & $\cdots$ & . & . \\
\hline & & & & . & & . & * \\
\hline & & $\cdots$ & & & & & \\
\hline & & 71.7 & 309.0 & 1 & & 43.8 & 37.0 \\
\hline 63 & I & 325.5 & 270.8 & 264.6 & 291.0 & \multicolumn{2}{|c|}{ Saturated } \\
\hline 83 & {$[\mathrm{~N}]$} & 1111.5 & 923.2 & $\ldots$ & $\cdots$ & 156.0 & 131.5 \\
\hline 78 & & 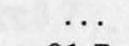 & $\ldots$ & $\cdots$ & $\cdots$ & . & . \\
\hline 17 & {$[\mathrm{~S}$} & 91. & 75.3 & $\cdots$ & $\cdots$ & 25.1 & 20.9 \\
\hline & [S I & 54.5 & 44.7 & $\cdots$ & $\cdots$ & 26.4 & 22.0 \\
\hline & & $\cdots$ & & . & $\cdots$ & $\cdots$ & $\cdots$ \\
\hline & & & & $\cdots$ & & $\ldots$ & . \\
\hline & & 21. & 17.2 & $\cdots$ & $\cdots$ & 17.3 & 14.0 \\
\hline & & 12. & 10.1 & $\cdots$ & $\cdots$ & $\cdots$ & $\cdots$ \\
\hline 7330 & {$[\mathrm{O} I \mathrm{II}]$} & 15.6 & 12.3 & $\ldots$ & $\cdots$ & $\cdots$ & $\cdots$ \\
\hline 7751 & [Ar III] & $\cdots$ & ' & $\cdots$ & 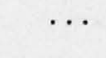 & $\cdots$ & 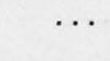 \\
\hline \multirow{2}{*}{\multicolumn{2}{|c|}{$\begin{array}{l}\mathrm{c} \ldots \ldots \ldots \ldots \ldots \\
\mathrm{T}_{e}([\mathrm{OIII}])(\mathrm{K}) .\end{array}$}} & \multicolumn{2}{|c|}{$\ldots \ldots \ldots .0 .24$} & \multicolumn{2}{|c|}{$\ldots \ldots \ldots-0.13$} & \multicolumn{2}{|c|}{...... 0.22} \\
\hline \multirow{2}{*}{\multicolumn{2}{|c|}{$\mathrm{n}_{\mathrm{e}}([\mathrm{S} \mathrm{II}])\left(\mathrm{cm}^{-3}\right)$}} & \multirow{2}{*}{\multicolumn{2}{|c|}{${ }^{\cdots}+\ldots+\cdots, \cdots$}} & \multicolumn{2}{|c|}{$\begin{array}{l}\ldots \ldots \ldots \ldots \ldots \\
\ldots \ldots \ldots \ldots \ldots\end{array}$} & \multirow{2}{*}{\multicolumn{2}{|c|}{$\begin{array}{l}\ldots \ldots+16000 \\
\ldots \ldots \ldots 2900\end{array}$}} \\
\hline & & \multirow{2}{*}{\multicolumn{2}{|c|}{ …...... 9.4}} & \multicolumn{2}{|c|}{ …... 10.5} & & \\
\hline \multicolumn{2}{|l|}{$\mathrm{F}(\mathrm{H} \beta)$} & & & & & \multicolumn{2}{|c|}{$\ldots \ldots \ldots 8.1$} \\
\hline \multicolumn{2}{|c|}{ Comment } & \multicolumn{2}{|c|}{...... Type I } & $\ldots \mathrm{O}_{\mathrm{F}}$ & thin & & \\
\hline
\end{tabular}


TABLE 5.4-Continued

UnCorrected and Corrected Line Intensities

\begin{tabular}{|c|c|c|c|c|c|c|c|}
\hline \multirow{2}{*}{$\begin{array}{c}\lambda \\
(\AA)\end{array}$} & \multirow[b]{2}{*}{ Identification } & \multicolumn{2}{|c|}{ LMC-MG31 } & \multicolumn{2}{|c|}{ "LMC-MG35 } & \multicolumn{2}{|c|}{ LMC-MG37 } \\
\hline & & $I_{0}$ & $I_{c}$ & $I_{0}$ & $\mathrm{I}_{\mathrm{c}}$ & $\mathrm{I}_{\mathrm{o}}$ & $\mathrm{I}_{\mathrm{c}}$ \\
\hline & & $\ldots$ & & & & & \\
\hline 3426 & {$[\mathrm{e} V]$} & 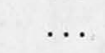 & 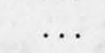 & 186.5 & 197.8 & & \\
\hline 444 & OIII & & & & & & \\
\hline 3727 & [O II] & 296.7 & 427.4 & 33.9 & 35.5 & & \\
\hline 3749 & He II, & $\ldots$ & $\ldots$ & $\ldots$ & & & \\
\hline 9 & & $\ldots$ & $\ldots$ & $\ldots$ & $\ldots$ & & \\
\hline 71 & 1 & $\ldots$ & $\ldots$ & $\ldots$ & $\ldots$ & & \\
\hline 98 & H10 & $\cdots$ & $\ldots$ & $\ldots$ & $\ldots$ & & \\
\hline 34 & & & & & & & \\
\hline 869 & [I & 82.1 & 112.5 & 40.5 & 42.1 & 27.8 & 22.5 \\
\hline 389 & $\mathrm{H}$ & 16.7 & 22.7 & 0 & 10 & & \\
\hline 69 & {$[\mathrm{~N}$} & 36.8 & 48.7 & 28.5 & 29.5 & 27.0 & 22.4 \\
\hline 26 & $\mathrm{He}$ & $\ldots$ & $\ldots$ & .. & .. & & \\
\hline 69 & [S II & & & & $\ldots$ & 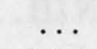 & \\
\hline 02 & $\mathrm{H} \delta$, & & & 19.4 & 20.0 & 30.8 & 26.3 \\
\hline 340 & $\mathrm{H}$ & 39.2 & 45.9 & 49.9 & 50.9 & 61.5 & 55.2 \\
\hline 4363 & [O II & $\cdots$ & $\cdots$ & 25.8 & 26.3 & & \\
\hline 2 & & & & & & & \\
\hline 686 & & $\cdots$ & $\ldots$ & 61.0 & 61.4 & & \\
\hline 4713 & $\mathrm{I},[\mathrm{N}$ & $\ldots$ & . & $\ldots$ & $\ldots$ & & \\
\hline 5 & {$[\mathrm{~N}$} & .. & & & $\ldots$ & $\ldots$ & \\
\hline 40 & & & & & $\cdots$ & .. & \\
\hline 59 & {$[\mathrm{O}$} & 298.8 & 290.8 & 89.4 & 89.1 & 32.1 & 236.4 \\
\hline 007 & [O I & 817.4 & 784.8 & 254.3 & 253.0 & 653.1 & 671.4 \\
\hline 99 & {$[\mathrm{~N} \mathrm{I}$} & $\cdots$ & $\cdots$ & $\cdots$ & $\ldots$ & $\cdots$ & \\
\hline & & 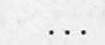 & & & $\ldots$ & $\ldots$ & \\
\hline 76 & & ... & $\ldots$ & $\cdots$ & $\ldots$ & .. & \\
\hline 00 & {$[\mathrm{C}$} & 42.3 & 29.7 & $\ldots$ & $\ldots$ & 16. & 20.4 \\
\hline & & $\cdots$ & $\ldots$ & $\ldots$ & $\ldots$ & 24.7 & 31.4 \\
\hline 4 & {$[\mathrm{O}$} & & & ... & $\cdots$ & 17.4 & 22.2 \\
\hline & {$[\mathrm{N}]$} & 71.3 & 47.7 & & & & \\
\hline & $\mathrm{H}$ & 437.8 & 292.4 & 309.0 & 293.9 & 240.5 & 316.2 \\
\hline 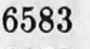 & {$[\mathrm{N}$} & 252.1 & 167.8 & 32.2 & 30.6 & & \\
\hline 78 & $\mathrm{He}$ & & & ... & $\cdots$ & .. & \\
\hline 6717 & {$[\mathrm{~S}$} & 24.3 & 15 & & .. & . & \\
\hline 6731 & {$[\mathrm{~S}$} & 33.8 & 21.9 & & .. & 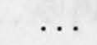 & \\
\hline & & & & & & $\cdots$ & \\
\hline & & & & 3.8 & 3.6 & $\cdots$ & \\
\hline & & 26.2 & 15.9 & &. & $\cdots$ & \\
\hline & {$[\mathrm{OI}$} & $\cdots$ & $\cdots$ & 8 & 7.5 & $\cdots$ & \\
\hline 30 & {$[0$} & & $\cdots$ & 2.0 & 1.9 & .. & \\
\hline 7751 & [Ar III] & $\cdots$ & $\cdots$ & $\cdots$ & $\cdots$ & $\cdots$ & 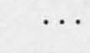 \\
\hline & ene & $\ldots$ & 0.53 & & & .. & -0.36 \\
\hline $\mathrm{T}_{\mathrm{e}}([\mathrm{O}$ & III] $)($ & & & & 73000 & $\cdots$ & … \\
\hline$n_{e}([S$ & $\ldots$ & & .. & & - & $\ldots$ & . \\
\hline E.C. & .. & & .3 .5 & &. .7 .7 & +5 & .3 .0 \\
\hline Comn & & & & Op & in? & & \\
\hline
\end{tabular}


TABLE 5.4-Continued

UnCorrected AND Corrected Line InTENSITIES

\begin{tabular}{|c|c|c|c|c|c|c|c|}
\hline \multirow{2}{*}{$\begin{array}{c}\lambda \\
(\AA) \\
\end{array}$} & \multirow[b]{2}{*}{ Identification } & \multicolumn{2}{|c|}{ LMC-MG39 } & \multicolumn{2}{|c|}{ LMC-MG44 } & \multicolumn{2}{|c|}{ LMC-MG45 } \\
\hline & & $\mathrm{I}_{\mathrm{o}}$ & $I_{c}$ & $\mathrm{I}_{\mathrm{o}}$ & $\mathrm{I}_{\mathrm{c}}$ & $\mathrm{I}_{\mathrm{o}}$ & $I_{c}$ \\
\hline & {$[\mathrm{NeV}] \ldots \ldots \ldots \ldots$} & $\cdots$ & $\cdots$ & $\cdots$ & $\cdots$ & & $\cdots$ \\
\hline 3426 & {$[\mathrm{NeV}]$} & & & & & $\cdots$ & \\
\hline 3444 & OIII & & $\ldots$ & $\cdots$ & & & \\
\hline 3727 & [O II] doublet ....... & 31.2 & 47.2 & $\cdots$ & $\cdots$ & 7.6 & 14.0 \\
\hline 3749 & He II, H12. & $\cdots$ & $\cdots$ & $\cdots$ & .. & $\cdots$ & $\cdots$ \\
\hline 3759 & O III $\ldots \ldots \ldots \ldots$ & $\cdots$ & $\cdots$ & $\cdots$ & $\cdots$ & $\ldots$ & $\cdots$ \\
\hline 3771 & H11, He II, O III.... & $\cdots$ & ‥ & $\cdots$ & & $\cdots$ & $\cdots$ \\
\hline 3798 & $\mathrm{H}_{10} \ldots \ldots \ldots \ldots \ldots$ & $\cdots$ & $\cdots$ & $\cdots$ & $\cdots$ & $\cdots$ & \\
\hline 3834 & He II, H9 ......... & $\cdots$ & $\cdots$ & $\cdots$ & & 4.7 & 8.2 \\
\hline 3869 & [Ne III $]$ & $\cdots$ & $\cdots$ & 41.8 & 49.5 & 79.7 & 135.6 \\
\hline 3889 & $\mathrm{H} 8, \mathrm{HeI}$. & 4.0 & 5.7 & & & 11.8 & 19.9 \\
\hline 3969 & {$[\mathrm{Ne}$ III $], \mathrm{H} \epsilon \ldots \ldots \ldots$} & 10.8 & 14.8 & 24.7 & 28.7 & 37.1 & 59.5 \\
\hline 4026 & HeI............. & $\cdots$ & $\cdots$ & 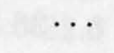 & $\cdots$ & $\cdots$ & $\cdots$ \\
\hline 4069 & [S II] doublet & & $\ldots$ & $\cdots$ & $\ldots$ & $\cdots$ & \\
\hline 4102 & $\mathrm{H} \delta, \mathrm{N}$ III $\ldots \ldots \ldots \ldots$ & 18.6 & 24.2 & 22.8 & 25.9 & 19.8 & 29.5 \\
\hline 4340 & 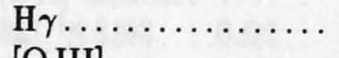 & 39.7 & 47.4 & 42.5 & 46.3 & 40.9 & 53.4 \\
\hline 4363 & w..... & $\cdots$ & $\cdots$ & 15.5 & 16.8 & 21.5 & 27.8 \\
\hline 4472 & He I... & $\cdots$ & $\cdots$ & $\cdots$ & $\because$ & 4.3 & 5.3 \\
\hline 4686 & He II. & $\cdots$ & $\cdots$ & 74.8 & 77.0 & 16.1 & 17.5 \\
\hline 4713 & [Ar IV], He I, [Ne IV] & $\cdots$ & $\cdots$ & $\cdots$ & $\cdots$ & $\cdots$ & $\cdots$ \\
\hline 4725 & {$[\mathrm{Ne} I \mathrm{IV}] \ldots \ldots \ldots \ldots$} & 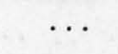 & $\cdots$ & $\cdots$ & 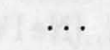 & & $\because$ \\
\hline 4740 & {$[$ Ar IV] $\ldots \ldots \ldots \ldots$} & $\because$ & $\cdots$ & & & 3.0 & 3.2 \\
\hline 4959 & {$[\mathrm{O}$ III] $\ldots \ldots \ldots \ldots$} & 34.6 & 33.6 & 255.6 & 251.9 & 503.4 & 480.7 \\
\hline 5007. & {$[\mathrm{O} I I I], \ldots \ldots \ldots \ldots$} & 106.6 & 101.8 & 758.5 & 742.1 & 1577.7 & 1472.7 \\
\hline 5199 & {$[\mathrm{~N} \mathrm{I}] \ldots \ldots \ldots \ldots \ldots$} & $\cdots$ & $\cdots$ & $\cdots$ & $\cdots$ & $\cdots$ & $\cdots$ \\
\hline 5412 & He II .............. & $\cdots$ & $\ldots$ & $\cdots$ & $\cdots$ & $\cdots$ & $\cdots$ \\
\hline 5876 & $\mathrm{He} \mathrm{I} \ldots \ldots \ldots \ldots \ldots$ & 10.0 & 7.5 & $\cdots$ & $\cdots$ & 22.0 & 14.2 \\
\hline 6300 & $\ldots \ldots \ldots$ & $\cdots$ & $\cdots$ & $\cdots$ & $\cdots$ & $\cdots$ & $\cdots$ \\
\hline 6312 & {$[\mathrm{~S} \mathrm{IIII}] \ldots \ldots \ldots \ldots$} & $\cdots$ & $\cdots$ & $\cdots$ & . & & $\ldots$ \\
\hline 6364 & {$[\mathrm{OI}] \ldots \ldots \ldots \ldots \ldots$} & $\cdots$ & $\cdots$ & $\cdots$ & $\cdots$ & 6.9 & 3.7 \\
\hline 6548 & {$[\mathrm{~N} \mathrm{II}]$} & 15.1 & 9.6 & $\ldots$ & $\cdots$ & 14.7 & 7.5 \\
\hline 6563 & n... & Satu & ated & Satu & ated & 627.5 & 317.6 \\
\hline 6583 & [N II] & 57.2 & 36.1 & $\cdots$ & $\cdots$ & 80.5 & 40.5 \\
\hline 6678 & ........ & $\cdots$ & $\cdots$ & $\cdots$ & $\cdots$ & 8.5 & 4.2 \\
\hline 6717 & $\ldots \ldots \ldots$ & $\cdots$ & $\cdots$ & $\cdots$ & $\cdots$ & $\cdots$ & $\cdots$ \\
\hline 6731 & [S II $]$ & $\cdots$ & $\cdots$ & .. & $\cdots$ & . & $\cdots$ \\
\hline 7006 & {$[\mathrm{ArV}]$} & $\ldots$ & $\ldots$ & $\cdots$ & *. & 2 & \\
\hline 7065 & He I... & 12.3 & 7.0 & $\cdots$ & $\cdots$ & 32.5 & 14.2 \\
\hline 7136 & [Ar III] & 11.9 & 6.8 & $\cdots$ & $\cdots$ & 27.8 & 12.0 \\
\hline 7320 & [O II] & 69.6 & 38.3 & $\cdots$ & $\cdots$ & 23.6 & 9.6 \\
\hline 7330 & [O II $]$ & 52.9 & 29.1 & $\cdots$ & $\cdots$ & 18.3 & 7.5 \\
\hline 7751 & [Ar III] & $\cdots$ & $\cdots$ & $\cdots$ & $\cdots$ & 13.8 & 5.1 \\
\hline & \multicolumn{2}{|c|}{ ….... 0.60} & \multirow{2}{*}{\multicolumn{2}{|c|}{$\begin{array}{l}\ldots \ldots \ldots 0.28 \\
\ldots \ldots \ldots 17000\end{array}$}} & \multicolumn{2}{|c|}{ n...... 0.90} \\
\hline \multicolumn{2}{|c|}{$\mathrm{T}_{\mathrm{e}}([\mathrm{OIII}])(\mathrm{K})$} & 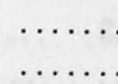 & & & & & ....... 15000 \\
\hline \multicolumn{2}{|c|}{$\begin{array}{l}\mathrm{n}_{\mathrm{e}}([\mathrm{S} \mathrm{II}])\left(\mathrm{cm}^{-3}\right) \\
\text { E.C. } \ldots \ldots \ldots \ldots \ldots\end{array}$} & & .0 .5 & & 8.6 & \multicolumn{2}{|c|}{$\ldots \ldots+\cdots \cdots$} \\
\hline \multicolumn{2}{|c|}{$\begin{array}{l}\mathrm{F}(\mathrm{H} \beta) \ldots \\
\text { Comment }\end{array}$} & & $\ldots$ & .... & & & \\
\hline Comn & & & Cont. & $\ldots O p$ & thin & \multicolumn{2}{|c|}{ Opt. thin? } \\
\hline
\end{tabular}


TABLE 5.4-Continued

UnCorRected AND CoRrected Line Intensities

\begin{tabular}{|c|c|c|c|c|c|c|c|}
\hline \multirow{2}{*}{$\begin{array}{c}\lambda \\
(\AA)\end{array}$} & \multirow[b]{2}{*}{ Identification } & \multicolumn{2}{|c|}{$\begin{array}{l}\text { LMC-MG46 } \\
\end{array}$} & \multicolumn{2}{|c|}{ "LMC-MG63 } & \multicolumn{2}{|c|}{ "LMC-MG66 } \\
\hline & & $I_{o}$ & $\mathrm{I}_{\mathrm{c}}$ & $\mathrm{I}_{0}$ & $I_{c}$ & $\mathrm{I}_{\mathrm{o}}$ & $\mathrm{I}_{\mathrm{c}}$ \\
\hline 3346 & {$[\mathrm{NeV}] \ldots \ldots$} & $\ldots$ & $\cdots$ & $\ldots$ & & & \\
\hline 3426 & {$[\mathrm{Ne} \mathrm{V}]$} & $\ldots$ & $\ldots$ & $\ldots$ & $\ldots$ & & \\
\hline 3444 & OIII ... & 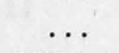 & $\ldots$ & $\ldots$ & $\ldots$ & & \\
\hline 3727 & [O II] doublet & 177.7 & 204.6 & 565.2 & 581.4 & 320.1 & 443.1 \\
\hline 3749 & He II, H12 ........ & $\ldots$ & $\ldots$ & $\cdots$ & $\ldots$ & $\cdots$ & . \\
\hline 3759 & O III ............. & $\cdots$ & $\cdots$ & $\cdots$ & $\cdots$ & & \\
\hline 3771 & H11, He II, O III.... & $\cdots$ & $\cdots$ & $\cdots$ & $\cdots$ & & \\
\hline 3798 & $\mathrm{H} 10 \ldots \ldots \ldots \ldots$ & .. & $\cdots$ & $\cdots$ & $\cdots$ & $\cdots$ & \\
\hline 3834 & He II, H9 $\ldots \ldots \ldots \ldots$ & $\ldots$ & $\ldots$ & $\ldots$ & $\ldots$ & 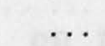 & \\
\hline 3869 & [Ne III] & 82.5 & 93.3 & 61.6 & 63.1 & 67.8 & 89.9 \\
\hline 3889 & H8, He I.... & 16.6 & 18.7 & $\cdots$ & $\ldots$ & $\ldots$ & 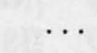 \\
\hline 3969 & {$[\mathrm{Ne} \mathrm{III}], \mathrm{H} \epsilon .$.} & 46.2 & 51.5 & $\cdots$ & $\cdots$ & $\cdots$ & \\
\hline 4026 & HeI......... & $\ldots$ & $\ldots$ & $\ldots$ & $\cdots$ & $\cdots$ & \\
\hline 4069 & [S II] doublet ...... & $\ldots$ & $\ldots$ & $\cdots$ & $\ldots$ & $\cdots$ & \\
\hline 4102 & H $\delta$, N III ........... & 23.3 & 25.6 & $\ldots$ & $\ldots$ & $\ldots$ & .. \\
\hline 4340 & $\mathrm{H} \gamma \ldots$ & 43.7 & 46.5 & 46.6 & 47.2 & 45.2 & 52.0 \\
\hline 4363 & [O III] & 14.5 & 15.4 & $\cdots$ & $\cdots$ & $\cdots$ & \\
\hline 4472 & He I..... & $\ldots$ & $\ldots$ & $\cdots$ & $\cdots$ & $\cdots$ & \\
\hline 4686 & He II. & 80.1 & 81.8 & $\ldots$ & $\ldots$ & $\ldots$ & \\
\hline 4713 & [Ar IV], He I, [Ne IV] & $\cdots$ & $\ldots$ & $\cdots$ & $\cdots$ & $\cdots$ & \\
\hline 4725 & {$[\mathrm{Ne} I V] \ldots \ldots \ldots \ldots$} & $\ldots$ & $\ldots$ & $\ldots$ & $\ldots$ & $\ldots$ & \\
\hline 4740 & {$[$ Ar IV $] \ldots \ldots \ldots \ldots$} & & & $\cdots$ & $\ldots$ & & \\
\hline 4959 & {$[\mathrm{OIII}] \ldots$} & 205.8 & 203.7 & 210.6 & 210.2 & 253.4 & 247.2 \\
\hline 5007 & [O III] & Satur & rated & 562.7 & 560.9 & 720.9 & 695.1 \\
\hline 5199 & {$[\mathrm{~N} \mathrm{I}]$. } & $\ldots$ & $\ldots$ & $\ldots$ & $\ldots$ & $\cdots$ & 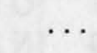 \\
\hline 5412 & He II. . & $\ldots$ & $\ldots$ & $\ldots$ & $\ldots$ & $\ldots$ & \\
\hline 5876 & He I... & $\cdots$ & $\cdots$ & $\cdots$ & $\cdots$ & $\cdots$ & . \\
\hline 6300 & {$[\mathrm{OI}] \ldots \ldots \ldots \ldots \ldots$} & $\ldots$ & $\ldots$ & $\ldots$ & $\ldots$ & $\cdots$ & \\
\hline 6312 & {$[\mathrm{~S} \mathrm{IIII}] \ldots \ldots \ldots \ldots$} & $\ldots$ & $\ldots$ & $\ldots$ & $\ldots$ & $\cdots$ & \\
\hline 6364 & {$[\mathrm{OI}] \ldots \ldots, \ldots, \ldots$} & $\ldots$ & $\ldots$ & $\ldots$ & $\ldots$ & $\ldots$ & \\
\hline 6548 & {$[\mathrm{~N} \mathrm{II}]$. } & 203.5 & 174.0 & 44.1 & 42.7 & 52.5 & 36.7 \\
\hline 6563 & $\mathrm{H} \alpha \ldots$ & Satu & rated & 305.0 & 295.7 & 441.2 & 307.6 \\
\hline 6583 & {$[\mathrm{~N} \mathrm{II}]$} & 532.8 & 454.4 & 142.8 & 138.4 & 185.0 & 128.6 \\
\hline 6678 & HeI.. & & $\ldots$ & $\ldots$ & $\ldots$ & $\cdots$ & \\
\hline 6717 & {$[\mathrm{~S} \mathrm{II}]$} & 44.3 & 37.5 & 54.0 & 52.2 & 20.6 & 14.0 \\
\hline 6731 & [S II] & 33.1 & 27.9 & 54.0 & 52.2 & 20.6 & 14.0 \\
\hline 7006 & {$[\mathrm{Ar} V] \ldots \ldots \ldots \ldots$} & $\cdots$ & $\cdots$ & $\ldots$ & $\ldots$ & $\cdots$ & \\
\hline 7065 & HeI........ & 8.3 & 6.9 & $\ldots$ & .. & .. & \\
\hline 7136 & [Ar III] & 21.9 & 18.0 & $\ldots$ & $\cdots$ & .. & \\
\hline 7320 & {$[\mathrm{OII}] \ldots \ldots \ldots \ldots$} & $\cdots$ & $\cdots$ & $\cdots$ & $\cdots$ & $\cdots$ & \\
\hline 7330 & {$[\mathrm{OII}] \ldots \ldots \ldots \ldots$} & $\cdots$ & $\cdots$ & $\cdots$ & $\cdots$ & $\cdots$ & \\
\hline 7751 & {$[$ Ar III $] \ldots$.} & & & $\cdots$ & $\cdots$ & & \\
\hline & & $\cdots$ & $\ldots 0.21$ & ... & .0 .04 & ... & 0.48 \\
\hline $\mathrm{T}_{\mathrm{e}}([\mathrm{O}$ & III] $)(\mathrm{K})$ & $\ldots \ldots$ & 17000 & $\cdots$ & $\cdots \cdots$ & $\cdots$ & * \\
\hline $\mathrm{n}_{\mathrm{e}}([\mathrm{S}$ & I]) $\left(\mathrm{cm}^{-3}\right)$ & $\ldots \ldots$ & $<300$ & & & & \\
\hline E.C. & $\ldots \ldots \ldots \ldots$ & $\ldots \ldots$ & $\ldots 8.6$ & $\cdots$ & $\ldots 2.5$ & .. & .3 .1 \\
\hline $\mathrm{F}(\mathbf{H} \beta$ & $\ldots \ldots \ldots \ldots$ & $\cdots$ & $\ldots \ldots$ & $\cdots$ & $-14.95^{f}$ & $\cdots$ & $\cdots \cdots$ \\
\hline Comr & ient . & $\ldots . \mathrm{T}$ & ype I? & $\ldots \ldots$ & $\cdots$ & $\cdots \cdots$ & \\
\hline
\end{tabular}


TABLE 5.4-Continued

UNCORRECTED AND CoRRected Line InTENSITIES

\begin{tabular}{|c|c|c|c|c|c|c|c|}
\hline \multirow{3}{*}{$\begin{array}{c}\begin{array}{c}\lambda \\
(\AA)\end{array} \\
3346\end{array}$} & \multirow[b]{2}{*}{ Identification } & \multicolumn{2}{|c|}{ LMC-MG74 } & \multicolumn{2}{|c|}{$\begin{array}{l}\text { LMC-MG83 } \\
\end{array}$} & \multicolumn{2}{|c|}{$\overline{\text { LMC-MG84 }}$} \\
\hline & & $I_{o}$ & $I_{c}$ & $\mathrm{I}_{\mathrm{o}}$ & $\mathrm{I}_{\mathrm{c}}$ & $\mathrm{I}_{\mathrm{o}}$ & $\mathrm{I}_{c}$ \\
\hline & {$[\mathrm{Ne} \mathrm{V}] \ldots \ldots \ldots \ldots$} & $\cdots$ & $\cdots$ & & & & \\
\hline 3426 & {$[\mathrm{NeV}] \ldots \ldots \ldots \ldots$} & .. & & 114.7 & 167.3 & & \\
\hline 3444 & OIII & & & & & & \\
\hline 3727 & [O II] doublet ....... & 140.9 & 137.9 & 554.7 & 743.1 & 340.9 & 333.0 \\
\hline 3749 & He II, H12 & $\cdots$ & $\cdots$ & $\cdots$ & . & & \\
\hline 3759 & O III . & $\cdots$ & $\ldots$ & $\cdots$ & & & \\
\hline 3771 & H11, He II, O III.... & 4.1 & 4.0 & $\cdots$ & & & \\
\hline 3798 & $\mathrm{H}_{10} \ldots \ldots \ldots \ldots \ldots$ & $\ldots$ & $\ldots$ & .. & & & \\
\hline $\begin{array}{l}3834 \\
3869\end{array}$ & $\begin{array}{l}\text { He II, H9 } \\
\text { [Ne III] . }\end{array}$ & $\begin{array}{l}2.8 \\
8.9\end{array}$ & $\begin{array}{l}2.8 \\
8.7\end{array}$ & 123.2 & 158.6 & 65.0 & 63.7 \\
\hline 3889 & H8, He I. & 10.4 & 10.2 & 22.1 & 28.3 & 22.9 & 22.4 \\
\hline 3969 & {$[\mathrm{Ne}$ III $], \mathrm{H} \epsilon \ldots \ldots \ldots$} & 15.2 & 14.9 & 47.5 & 59.4 & 36.4 & 35.8 \\
\hline 4026 & He I. & & & & & & \\
\hline 4069 & [S II] doublet & & & & & & \\
\hline 4102 & $\mathrm{H} \delta, \mathrm{N}$ III & 23.9 & 23.6 & & & 31.1 & 30.6 \\
\hline 4340 & $\mathrm{H} \gamma \ldots$ & 47.4 & 46.9 & 65.8 & 74.7 & 54.0 & 53.5 \\
\hline 4363 & [O III] & & & 78.3 & 88.4 & & \\
\hline 4472 & HeI.. & 5.8 & 5.8 & $\cdots$ & & & $\cdots$ \\
\hline 4686 & He II $\ldots \ldots \ldots \ldots \ldots$ & $\cdots$ & $\cdots$ & 170.4 & 177.6 & . & \\
\hline 4713 & [ArIV], HeI, [NeIV] & $\cdots$ & $\cdots$ & $\cdots$ & . & $\cdots$ & \\
\hline 4725 & {$[\mathrm{Ne}$ IV] $] \ldots \ldots \ldots \ldots$} & & & 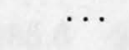 & & . & 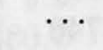 \\
\hline 4740 & [ArIV] & & & $\ddot{x}$ & & & \\
\hline 4959 & [O III] & 74.8 & 74.9 & 604.0 & 590.9 & 191.9 & 192.3 \\
\hline 5007 & [O III] & 207.5 & 208.0 & 1596.0 & 1544.7 & 531.0 & 532.4 \\
\hline 5199 & [N I] & 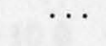 & & $\cdots$ & $\cdots$ & $\cdots$ & \\
\hline 5412 & $\ldots \ldots \ldots$ & & & $\cdots$ & $\cdots$ & $\cdots$ & \\
\hline 5876 & He I....... & 11.6 & 11.8 & $\cdots$ & $\cdots$ & $\cdots$ & . \\
\hline $\begin{array}{l}6300 \\
6312\end{array}$ & $\begin{array}{l}{[\mathrm{OI}] \ldots \ldots} \\
{[\mathrm{S} \mathrm{III}]}\end{array}$ & $\cdots$ & $\cdots$ & $\cdots$ & $\cdots$ & $\cdots$ & .. \\
\hline $\begin{array}{l}0312 \\
6364\end{array}$ & 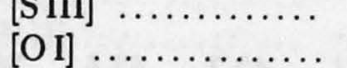 & 4.7 & 4.8 & $\cdots$ & $\cdots$ & $\cdots$ & 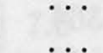 \\
\hline 6548 & [N II] & 29.6 & 30.3 & 378.2 & 274.3 & 35.8 & 36.7 \\
\hline 6563 & $\mathrm{H} \alpha$ & 284.6 & 291.5 & 406.3 & 294.1 & 311.3 & 319.5 \\
\hline 6583 & {$[\mathrm{~N} \mathrm{II}]$} & 96.5 & 98.8 & 1167.7 & 842.5 & 150.8 & 154.8 \\
\hline 6678 & HeI.. & 5.1 & 5.2 & $\ldots$ & $\ldots$ & $\ldots$ & \\
\hline 6717 & [S II] & 12.3 & 12.6 & .. & $\ldots$ & 46.8 & 48.1 \\
\hline 6731 & ........ & 12.5 & 12.9 & $\ldots$ & $\cdots$ & 40.4 & 41.5 \\
\hline 7006 & {$[\mathrm{Ar} \mathrm{V}]$} & & $\cdots$ & $\ldots$ & $\ldots$ & & \\
\hline 7065 & HeI... & 4.1 & 4.2 & $\ldots$ & ... & & . \\
\hline 7136 & [Ar III] & 9.0 & 9.3 & 61.1 & 40.9 & 12.7 & 13.1 \\
\hline 7320 & $\ldots \ldots \ldots \ldots$ & 3.2 & 3.6 & $\cdots$ & $\cdots$ & $\cdots$ & \\
\hline 7330 & $\ldots+\ldots \ldots$ & 3.5 & 3.6 & $\cdots$ & $\cdots$ & & \\
\hline 7751 & [Ar III] . & . & & & & & \\
\hline & & $\cdots$ & -0.31 & & .0 .43 & .. & -0.03 \\
\hline $\mathrm{T}_{\mathrm{e}}([\mathrm{O}$ & ........ & $\cdots$ & & & 29000 & & \\
\hline $\begin{array}{l}\mathrm{n}_{\mathrm{e}}([\mathrm{S} \\
\text { E.C. }\end{array}$ & $\left(\mathrm{cm}^{-3}\right) \ldots \ldots \ldots$ & & & & & & \\
\hline E.C. & $\ldots \ldots \ldots \ldots+\ldots, \ldots$ & & 0.9 & & 14.2 & & .2 .4 \\
\hline $\begin{array}{l}\mathrm{F}(\mathrm{H} / \beta \\
\mathrm{Comn}\end{array}$ & $\cdots$ & & Cont. & & $\begin{array}{l}-13.98^{1} \\
\text { Type I }\end{array}$ & & \\
\hline & & & & & & & \\
\hline
\end{tabular}


TABLE 5.4-Continued

UnCorrected and Corrected Line Intensities

\begin{tabular}{|c|c|c|c|c|c|c|c|}
\hline \multirow{2}{*}{$\begin{array}{c}\lambda \\
(\AA)\end{array}$} & \multirow[b]{2}{*}{ Identification } & \multicolumn{2}{|c|}{$\overline{\text { LMC-MG86 }}$} & \multicolumn{2}{|c|}{ LMC-MA01 } & \multicolumn{2}{|c|}{ LMC-MA02 } \\
\hline & & I。 & $I_{c}$ & $\mathrm{I}_{0}$ & $I_{c}$ & $\mathrm{I}_{\mathrm{o}}$ & $I_{c}$ \\
\hline 3346 & {$[\mathrm{Ne} \mathrm{V}] \ldots \ldots$} & & & & & & \\
\hline 3426 & {$[\mathrm{NeV}]$.} & & $\ldots$ & $\ldots$ & & & \\
\hline 3444 & OIII & & & 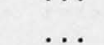 & 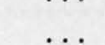 & & \\
\hline 3727 & [O II] doublet & 507.6 & 810.0 & 208.1 & 323.2 & 331.6 & 394.4 \\
\hline 3749 & He II, H12 ........ & $\ldots$ & $\ldots$ & $\ldots$ & $\ldots$ & $\ldots$ & $\ldots$ \\
\hline 3759 & O III .............. & $\ldots$ & $\ldots$ & $\ldots$ & $\ldots$ & $\ldots$ & \\
\hline 3771 & H11, He II, O III.... & $\ldots$ & $\ldots$ & $\ldots$ & $\ldots$ & $\cdots$ & \\
\hline 3798 & …… & $\ldots$ & $\ldots$ & $\ldots$ & $\ldots$ & $\ldots$ & \\
\hline 3834 & He II, H9 & $\ldots$ & $\ldots$ & & $\ldots$ & $\ldots$ & 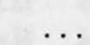 \\
\hline 3869 & [Ne III] & $\ldots$ & $\ldots$ & 68.6 & 100.3 & 51.3 & 59.6 \\
\hline 3889 & H8, He I. & $\ldots$ & $\ldots$ & & . & & \\
\hline 3969 & {$[\mathrm{Ne}$ III $], \mathrm{H} \epsilon \ldots$} & $\ldots$ & $\ldots$ & 40.1 & 56.3 & 27.4 & 31.3 \\
\hline 4026 & HeI......... & $\ldots$ & $\ldots$ & $\ldots$ & $\ldots$ & $\ldots$ & $\ldots$ \\
\hline 4069 & [S II] doublet ....... & $\ldots$ & $\ldots$ & & & $\ldots$ & $\cdots$ \\
\hline 4102 & H $\delta$, N III . & $\ldots$ & $\ldots$ & 22.7 & 30.2 & $\ldots$ & 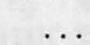 \\
\hline 4340 & $\mathrm{H} \gamma \ldots \ldots$ & .. & $\ldots$ & 39.7 & 48.1 & 44.3 & 47.7 \\
\hline 4363 & [O III] & $\ldots$ & $\ldots$ & $\ldots$ & $\ldots$ & $\cdots$ & . \\
\hline 4472 & HeI. & $\ldots$ & $\ldots$ & $\ldots$ & $\ldots$ & $\cdots$ & $\cdots$ \\
\hline 4686 & He II. & $\ldots$ & $\ldots$ & $\ldots$ & $\ldots$ & $\ldots$ & $\cdots$ \\
\hline 4713 & [Ar IV], He I, [Ne IV] & $\ldots$ & $\ldots$ & $\cdots$ & $\cdots$ & $\ldots$ & $\ldots$ \\
\hline 4725 & [NeIV]. & $\ldots$ & $\ldots$ & $\ldots$ & $\cdots$ & $\cdots$ & $\cdots$ \\
\hline 4740 & [ArIV] .. & $\ldots$ & $\ldots$ & $\ldots$ & $\ldots$ & $\ldots$ & \\
\hline 4959 & [O III $]$. & 278.9 & 269.3 & 322.4 & 312.0 & 204.8 & 202.2 \\
\hline 5007 & [O III] & 880.7 & 835.9 & 994.2 & 946.5 & 602.0 & 590.5 \\
\hline 5199 & {$[\mathrm{~N} \mathrm{I}]$} & $\ldots$ & $\cdots$ & $\ldots$ & $\ldots$ & $\ldots$ & $\ldots$ \\
\hline 5412 & He II. & & & $\ldots$ & $\ldots$ & $\cdots$ & $\cdots$ \\
\hline 5876 & HeI. & 100.3 & 71.8 & $\ldots$ & $\ldots$ & 15.3 & 13.5 \\
\hline 6300 & {$[\mathrm{OI}]$} & $\ldots$ & $\ldots$ & $\ldots$ & $\cdots$ & $\cdots$ & . \\
\hline 6312 & [S III] & $\ldots$ & $\ldots$ & $\ldots$ & $\ldots$ & $\ldots$ & \\
\hline 6364 & [O I] & $\ldots$ & $\ldots$ & $\ldots$ & & $\cdots$ & \\
\hline 6548 & {$[\mathrm{~N}$ II $] \ldots \ldots \ldots \ldots \ldots$} & 126.8 & 75.9 & 114.3 & 70.5 & $\ldots$ & $\ldots$ \\
\hline 6563 & $\mathrm{H} \alpha$ & 493.0 & 294.0 & 497.4 & 305.6 & 359.9 & 297.1 \\
\hline 6583 & {$[\mathrm{~N} \mathrm{II}]$} & 215.7 & 128.0 & 379.2 & 231.9 & 180.5 & 148.8 \\
\hline 6678 & HeI. & $\ldots$ & $\ldots$ & $\cdots$ & & & $\cdots$ \\
\hline 6717 & [S II] & $\ldots$ & $\ldots$ & 34.5 & 20.5 & 42.7 & 34.8 \\
\hline 6731 & [S II] & $\ldots$ & .. & 18.3 & 10.8 & 35.8 & 29.1 \\
\hline 7006 & {$[\mathrm{Ar} \mathrm{V}]$. } & $\cdots$ & .. & $\ldots$ & $\cdots$ & $\cdots$ & . \\
\hline 7065 & He I............. & $\cdots$ & $\cdots$ & $\cdots$ & $\ldots$ & $\ldots$ & $\cdots$ \\
\hline 7136 & {$[\mathrm{Ar} I \mathrm{III}] \ldots \ldots \ldots \ldots$} & $\ldots$ & $\ldots$ & $\ldots$ & $\ldots$ & $\ldots$ & ... \\
\hline 7320 & {$[\mathrm{OII}] \ldots \ldots \ldots \ldots$} & $\ldots$ & $\ldots$ & $\ldots$ & $\ldots$ & $\cdots$ & ... \\
\hline 7330 & [O II] & $\ldots$ & $\cdots$ & $\ldots$ & $\cdots$ & $\cdots$ & .. \\
\hline 7751 & {$[$ Ar III $] \ldots \ldots \ldots \ldots$} & $\cdots$ & $\cdots$ & $\cdots$ & $\cdots$ & *. & $\cdots$ \\
\hline & \multicolumn{2}{|c|}{$\ldots \ldots 0.68$} & \multicolumn{2}{|c|}{$\ldots \ldots 0.64$} & \multicolumn{2}{|c|}{$\ldots \ldots \ldots 0.25$} \\
\hline \multirow{2}{*}{\multicolumn{2}{|c|}{$\mathrm{T}_{e}([\mathrm{O} \mathrm{III}])(\mathrm{K})$}} & \multicolumn{2}{|c|}{........... } & \multicolumn{2}{|c|}{$\ldots \ldots \ldots \ldots$} & \multicolumn{2}{|c|}{$\ldots \ldots \ldots \ldots$} \\
\hline \multirow{2}{*}{\multicolumn{2}{|c|}{$\mathrm{n}_{\mathrm{e}}([\mathrm{S} \mathrm{II}])\left(\mathrm{cm}^{-3}\right)$}} & \multicolumn{2}{|c|}{........... } & \multirow{2}{*}{\multicolumn{2}{|c|}{$\ldots \ldots+4$}} & & \\
\hline & & $\ldots \ldots$ & $\ldots 3.8$ & & & & .2 .7 \\
\hline \multicolumn{2}{|c|}{$F(H \beta)$} & \multicolumn{2}{|c|}{$\ldots \ldots-14.37^{f}$} & \multirow{2}{*}{\multicolumn{2}{|c|}{$\ldots \cdots-14.28^{\mathrm{f}}$}} & . & $13.95^{\mathrm{f}}$ \\
\hline Comr & ler & . & $\cdots$ & & & $\cdots \cdot$ & … \\
\hline
\end{tabular}


TABLE 5.4-Continued

UNCORRECTED AND CORRECTED LINE INTENSITIES

\begin{tabular}{|c|c|c|c|c|c|c|c|}
\hline \multirow{2}{*}{$\begin{array}{c}\lambda \\
(\AA)\end{array}$} & \multirow[b]{2}{*}{ Identification } & \multicolumn{2}{|c|}{ LMC-MA03 } & \multicolumn{2}{|c|}{ LMC-MA04 } & \multicolumn{2}{|c|}{ LMC-MA05 } \\
\hline & & $I_{0}$ & $\mathrm{I}_{c}$ & I。 & $I_{c}$ & $I_{0}$ & $\mathrm{I}_{\mathrm{c}}$ \\
\hline 3346 & {$[\mathrm{Ne} \mathrm{V}] \ldots \ldots$} & 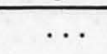 & $\cdots$ & & & & \\
\hline 3426 & {$[\mathrm{Ne} \mathrm{V}]$} & & 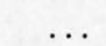 & 43.4 & 49.3 & & \\
\hline 3444 & OIII & & & 6.7 & 7.6 & & \\
\hline 3727 & {$[\mathrm{OII}] \mathrm{d}$} & 329.0 & 382.4 & 25.9 & 28.6 & 725.8 & 805.0 \\
\hline 3749 & HeII, H12. & $\ldots$ & $\ldots$ & $\ldots$ & $\ldots$ & $\ldots$ & \\
\hline 759 & O III & $\ldots$ & $\ldots$ & $\ldots$ & $\ldots$ & & \\
\hline 771 & H11, He II, O III.... & $\ldots$ & $\ldots$ & $\cdots$ & $\ldots$ & $\cdots$ & \\
\hline 98 & H10 & $\ldots$ & $\ldots$ & $\ldots$ & $\ldots$ & $\cdots$ & \\
\hline 34 & $\mathrm{He} \mathrm{I}$ & $\ldots$ & $\ldots$ & 3.8 & 4.1 & & \\
\hline 69 & {$[\mathrm{~N}$} & 86.5 & 98.5 & 42.4 & 46.2 & 73.0 & 79.9 \\
\hline 389 & $\mathrm{H} \varepsilon$ & & & 10.8 & 11.7 & $\cdots$ & $\ldots$ \\
\hline 969 & [Ne III $], \mathrm{H} \epsilon$ & 33.6 & 37.7 & 25.2 & 27.2 & .. & $\cdots$ \\
\hline 026 & HeI. & $\ldots$ & $\ldots$ & $\ldots$ & $\cdots$ & & \\
\hline 69 & [S II] d & & & $\ldots$ & & & \\
\hline 2 & & 19.1 & 21.1 & 23.6 & 25.2 & 34.3 & 36.7 \\
\hline 40 & $\mathrm{H} \gamma$ & 50.6 & 54.0 & 8.8 & 0.9 & 70.3 & 73.5 \\
\hline 63 & {$[\mathrm{O} I$} & $\cdots$ & $\ldots$ & 14.3 & 14.9 & $\cdots$ & .. \\
\hline 4472 & HeI... & 10.8 & 11.3 & $\cdots$ & . & . & \\
\hline 4686 & He II & $\ldots$ & $\ldots$ & 83.1 & 84.2 & $\cdots$ & $\cdots$ \\
\hline 4713 & [Ar IV], He I, [Ne IV] & $\cdots$ & $\ldots$ & 8.0 & 8.1 & $\ldots$ & $\cdots$ \\
\hline 25 & ....... & $\cdots$ & $\ldots$ & $\ldots$ & $\ldots$ & $\ldots$ & .. \\
\hline 40 & ..... & .. & & 4.5 & 4.6 & 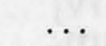 & 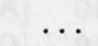 \\
\hline 59 & [O I & 81.2 & 278.1 & 212.2 & 10.6 & 03.7 & 202.1 \\
\hline 5007 & [O II] & 774.4 & 761.5 & 640.6 & 633.6 & 589.2 & 582.4 \\
\hline 99 & [N I & $\cdots$ & $\cdots$ & $\ldots$ & $\cdots$ & $\ldots$ & $\ldots$ \\
\hline 2 & $\mathrm{He}$ & & & 6.9 & 6.6 & $\ldots$ & \\
\hline 6 & $\mathrm{H}$ & 100.3 & 71.8 & $\ldots$ & $\ldots$ & $\ldots$ & \\
\hline 6300 & {$[\mathrm{C}$} & $\ldots$ & $\cdots$ & $\cdots$ & $\ldots$ & $\cdots$ & \\
\hline . & ...... & $\ldots$ & .. & $\cdots$ & $\cdots$ & $\cdots$ & . \\
\hline 64 & {$[\mathrm{O}$} & & $\cdots$ & $\cdots$ & $\cdots$ & $\cdots$ & 0 \\
\hline 8 & {$[\mathrm{~N}$} & 73.7 & 62.5 & $\ldots$ & $\ldots$ & 26.1 & 23.3 \\
\hline 3 & H & 358.3 & 303.4 & 339.2 & 304.2 & 329.6 & 293.9 \\
\hline 6583 & {$[\mathrm{~N}$} & 318.8 & 269.5 & $\cdots$ & $\ldots$ & 122.9 & 109.5 \\
\hline 6678 & $\mathrm{He}$ & & & 4.1 & 3.6 & & \\
\hline 6717 & [S & 38.8 & 32 & $\ldots$ & $\ldots$ & 45.8 & 40.5 \\
\hline 6731 & [S II] & 33.8 & 28.2 & $\ldots$ & $\cdots$ & 41.8 & 36.9 \\
\hline 7006 & {$[\mathrm{ArV}]$.} & $\ldots$ & $\ldots$ & $\ldots$ & $\ldots$ & ... & .. \\
\hline 70 & & $\ldots$ & $\ldots$ & $\ldots$ & . & & \\
\hline 7136 & {$[\mathrm{Ar}$} & 25.5 & 20.8 & 8.8 & 7.6 & & \\
\hline 7320 & [O I & $\cdots$ & $\cdots$ & $\cdots$ & .. & & \\
\hline 7330 & [O II $]$ & $\cdots$ & $\cdots$ & $\cdots$ & .. & & \\
\hline 7751 & [Ar III] .. & & & $\cdots$ & & & \\
\hline & & .. & 0.22 & $\cdots$ & 0.14 & . . & 0.15 \\
\hline $\mathrm{T}_{\mathrm{e}}([\mathrm{C}$ & III])( & .... & $\cdots$ & $\cdots$ & 17000 & & \\
\hline $\mathrm{n}_{\mathrm{e}}([\mathrm{S}$ & ]) $(\mathrm{cr}$ & & & & & & \\
\hline E.C. & & & ..3.4 & & .9 .0 & & \\
\hline $\mathrm{F}(\mathrm{H} \beta$ & 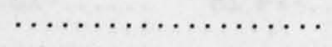 & & $-14.1^{8}$ & $\ldots$ & $13.21^{f}$ & & $22^{f}$ \\
\hline Comr & & & Type I & & Cont. & & \\
\hline
\end{tabular}


TABLE 5.4-Continued

UnCorrected and Corrected Line Intensities

\begin{tabular}{|c|c|c|c|c|c|c|c|}
\hline \multirow{2}{*}{$\begin{array}{c}\lambda \\
(\AA)\end{array}$} & \multirow[b]{2}{*}{ Identification } & \multicolumn{2}{|c|}{ LMC-MA06 } & \multicolumn{2}{|c|}{ "LMC-MA07 } & \multicolumn{2}{|c|}{ 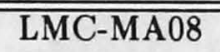 } \\
\hline & & $I_{0}$ & $I_{c}$ & $I_{\circ}$ & $I_{c}$ & $\mathrm{I}_{0}$ & $\mathrm{I}_{\mathrm{c}}$ \\
\hline & {$[\mathrm{NeV}] \ldots \ldots$} & $\cdots$ & $\ldots$ & .. & .. & & \\
\hline 426 & {$[\mathrm{Ne} \mathrm{V}]$} & ... & .. & $\ldots$ & & & \\
\hline 3444 & OIII & & & & & & \\
\hline 3727 & [O II] & 350.0 & 353.8 & 404.7 & 359.7 & 347.2 & 369.7 \\
\hline 49 & He II, & $\ldots$ & $\cdots$ & $\cdots$ & $\ldots$ & & \\
\hline 3759 & $\mathrm{O}$ & $\ldots$ & $\ldots$ & $\ldots$ & $\ldots$ & & \\
\hline 71 & H & $\ldots$ & $\cdots$ & $\ldots$ & $\cdots$ & & \\
\hline 98 & & $\cdots$ & $\ldots$ & $\ldots$ & $\ldots$ & & \\
\hline 34 & & $\ldots$ & . & $\ldots$ & .. & +0 & 30 \\
\hline 3869 & & 81.7 & 82.5 & 58.4 & 52.7 & 85.9 & 90.7 \\
\hline 3889 & & 21.4 & 6 & 5.4 & 1 & 4.4 & 36.3 \\
\hline 3969 & & 37.0 & 37.3 & 24.8 & 22.6 & 47.3 & 49.6 \\
\hline 4026 & & $\ldots$ & $\cdots$ & $\ldots$ & $\ldots$ & $\cdots$ & .. \\
\hline 4069 & & & & .. & 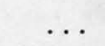 & & \\
\hline 4102 & $\mathrm{H} \delta, \mathrm{I}$ & 29.4 & 9.6 & 25.6 & 23.7 & 26.2 & 27.3 \\
\hline 4340 & & 50.2 & 0. & 47.9 & 45.5 & 41.3 & 42.4 \\
\hline 63 & & 18.1 & 18.2 & & & & \\
\hline 72 & & $\ldots$ & 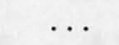 & 7.0 & 6.7 & & \\
\hline 4686 & & 62.5 & 62.6 & $\cdots$ & $\ldots$ & 23.3 & 23.5 \\
\hline 4713 & {$[A$} & $\cdots$ & $\cdots$ & $\cdots$ & $\cdots$ & & \\
\hline 4725 & & $\ldots$ & $\ldots$ & $\ldots$ & $\ldots$ & $\cdots$ & \\
\hline 10 & & & & & $\cdots$ & . & \\
\hline 9 & & 9.1 & 9 & 1.7 & 3.3 & 96.6 & 95.2 \\
\hline 7 & & 748.7 & 747.8 & 15.2 & 522.0 & 806.9 & 801.3 \\
\hline & & $\cdots$ & $\cdots$ & $\ldots$ & $\cdots$ & $\cdots$ & $\cdots$ \\
\hline 2 & & $\ldots$ & $\cdots$ & & & $\cdots$ & \\
\hline 6 & & $\cdots$ & $\cdots$ & 23.4 & 25.5 & . & \\
\hline 300 & & $\cdots$ & $\cdots$ & $\cdots$ & $\cdots$ & . & \\
\hline 2 & & $\cdots$ & $\cdots$ & $\cdots$ & $\cdots$ & . & - \\
\hline 64 & & & ... & 等 & · & $\because$. & \\
\hline 6548 & & 8.7 & & 19.5 & 22.2 & 31.1 & 29.0 \\
\hline 6563 & & & & 252.9 & 288.2 & 306.6 & 286.0 \\
\hline 3 & {$[\mathrm{~N}$} & 599.4 & 592.2 & 62.0 & 70.7 & 129.3 & 120.6 \\
\hline 8 & & & & & & & \\
\hline & {$[\mathbf{S}$} & 39.0 & 38. & 34.0 & 39. & 23.7 & 22.0 \\
\hline 731 & {$[\mathbf{S}$} & 30.8 & 30.4 & 28.9 & 33.3 & 17.6 & 16.4 \\
\hline 7006 & & $\cdots$ & $\cdots$ & $\cdots$ & . & . . & \\
\hline & & .. & $\cdots$ & & .. & ... & \\
\hline 7136 & & $\cdots$ & $\ldots$ & 11.5 & 13.5 & . & $\cdots$ \\
\hline 732 & & $\cdots$ & $\cdots$ & $\cdots$ & $\cdots$ & $\cdots$ & $\cdots$ \\
\hline 7330 & & $\cdots$ & $\cdots$ & $\cdots$ & $\cdots$ & $\cdots$ & .. \\
\hline 7751 & [Ar III] & $\cdots$ & $\cdots$ & $\cdots$ & $\cdots$ & - & 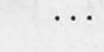 \\
\hline & & $\cdots$ & 0.02 & . & -0.17 & & 0.09 \\
\hline $\mathrm{T}_{\mathrm{e}}([\mathrm{O}$ & th & $\ldots$ & 17000 & r & & & \\
\hline $\mathrm{n}_{\mathrm{e}}([$ & II) & & & & & & \\
\hline E.C. & & & .7 .8 & & $\ldots 2.3$ & & .5. \\
\hline $\mathrm{F}(\mathrm{H} \beta$ & & $\cdots$ & $13.58^{f}$ & . & $3.90^{\mathrm{t}}$ & & $1.02^{f}$ \\
\hline$C$ & thes & $\cdots \cdots$ & Type I & & r & & \\
\hline
\end{tabular}


TABLE 5.4-Continued

UnCORRECTED AND CORRECTED Line INTENSITIES

\begin{tabular}{|c|c|c|c|c|c|c|c|}
\hline \multirow{2}{*}{$\begin{array}{c}\lambda \\
(\AA)\end{array}$} & \multirow[b]{2}{*}{ Identification } & \multicolumn{2}{|c|}{$\begin{array}{l}\text { LMC-MA09 } \\
\end{array}$} & \multicolumn{2}{|c|}{ LMC-MA10 } & \multicolumn{2}{|c|}{ LMC-MA11 } \\
\hline & & $I_{0}$ & $I_{c}$ & $I_{0}$ & $I_{c}$ & $I_{0}$ & $\mathrm{I}_{\mathrm{c}}$ \\
\hline 3346 & {$[\mathrm{Ne} \mathrm{V}]$} & $\cdots$ & $\cdots$ & $\cdots$ & $\cdots$ & & $\cdots$ \\
\hline 3426 & {$[\mathrm{Ne} \mathrm{V}]$} & .. & $\cdots$ & & 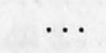 & & $\cdots$ \\
\hline 3444 & OIII & & $\ldots$ & & & & \\
\hline 3727 & [O II] doublet . & 422.4 & 400.7 & 282.4 & 462.0 & 217.9 & 393.5 \\
\hline 3749 & He II, H12 & $\cdots$ & $\cdots$ & $\cdots$ & $\cdots$ & $\cdots$ & $\cdots$ \\
\hline 3759 & O III . & $\ldots$ & $\ldots$ & $\cdots$ & $\cdots$ & ... & \\
\hline 3771 & H11, He II, O III.... & $\ldots$ & $\cdots$ & $\cdots$ & $\cdots$ & $\cdots$ & \\
\hline 3798 & $\ldots \ldots \ldots \ldots$ & $\ldots$ & $\ldots$ & $\ldots$ & $\ldots$ & .. & .. \\
\hline 3834 & He II, H9 & 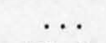 & $\ldots$ & & .. & .. & \\
\hline 3869 & {$[\mathrm{Ne}$} & 58.4 & 55.8 & 100.3 & 153.4 & $\ldots$ & ... \\
\hline 3889 & $\mathrm{H} 8, \mathrm{He} \mathrm{I}$. & 16.2 & 15.5 & 14.6 & 22.1 & $\cdots$ & .. \\
\hline 3969 & [Ne III], H $\epsilon$. & 32.8 & 31.5 & 35.4 & 51.7 & & \\
\hline 4026 & $\ldots \ldots \ldots$ & $\ldots$ & $\ldots$ & $\ldots$ & .. & .. & $\ldots$ \\
\hline 4069 & [S II] doublet & & & $\cdots$ & & $\ldots$ & \\
\hline 4102 & $\mathrm{H} \delta, \mathrm{N}$ & 26.1 & 25.3 & 17.7 & 24.4 & & \\
\hline 4340 & $\mathrm{H} \gamma$. & 49.5 & 48.4 & 38.2 & 47.3 & 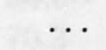 & \\
\hline 4363 & [O III $]$ & $\cdots$ & $\cdots$ & 14.7 & 18.0 & & \\
\hline 4472 & HeI. & & & & & $\ldots$ & \\
\hline 4686 & He II & 8.4 & 8.3 & 67.8 & 72.7 & $\cdots$ & \\
\hline 4713 & , $\mathrm{HeI},[\mathrm{NeIV}]$ & $\cdots$ & $\cdots$ & 9.7 & 10.3 & $\cdots$ & \\
\hline 4725 & {$[\mathrm{NeIV}] \ldots \ldots \ldots \ldots$} & $\cdots$ & $\ldots$ & $\cdots$ & $\cdots$ & . & \\
\hline 4740 & [ArIV] & & & & . & & \\
\hline 4959 & {$[\mathrm{O}$ III $] \ldots \ldots \ldots \ldots$} & 222.7 & 223.5 & 221.1 & 213.1 & 346.4 & 331.4 \\
\hline 5007 & [O III] & Satu & ated & Satu & ated & 979.0 & 916.4 \\
\hline 5199 & {$[\mathrm{NI}]$} & .. & $\cdots$ & 15.5 & 13.7 & $\cdots$ & $\cdots$ \\
\hline 5412 & He II. & $\ldots$ & $\cdots$ & $\cdots$ & $\cdots$ & $\cdots$ & \\
\hline 5876 & $\mathrm{HeI}$ & $\cdots$ & $\cdots$ & $\cdots$ & .. & $\cdots$ & $\cdots$ \\
\hline 6300 & [OI] & $\cdots$ & $\cdots$ & $\cdots$ & $\cdots$ & $\cdots$ & · \\
\hline 6312 & [S III] & $\cdots$ & $\cdots$ & $\cdots$ & $\ldots$ & $\cdots$ & $\cdots$ \\
\hline 6364 & {$[\mathrm{OI}]$} & $\ldots$ & $\cdots$ & $\cdots$ & $\cdots$ & $\ldots$ & $\cdots$ \\
\hline 6548 & {$[\mathrm{~N} \mathrm{II}]$} & 38.6 & 40.9 & 245.7 & 143.1 & & \\
\hline 6563 & $\mathrm{H} \alpha$ & 281.4 & 298.3 & Satu & ated & 565.1 & 294.0 \\
\hline 6583 & ……… & 172.3 & 182.7 & 696.6 & 402.1 & 280.7 & 145.2 \\
\hline 6678 & HeI. & & & & & .. & \\
\hline 6717 & [S II] & 39.6 & 42.1 & 61.9 & 34.5 & . & \\
\hline 6731 & [S II] & 26.8 & 28.5 & 47.0 & 26.2 & & \\
\hline 7006 & {$[\mathrm{ArV}]$. } & .. & $\cdots$ & $\cdots$ & $\cdots$ & . & .. \\
\hline 7065 & HeI... & $\cdots$ & 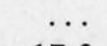 & $\cdots$ & . & $\cdots$ & . \\
\hline 7136 & [Ar III] & 16.5 & 17.8 & 19.0 & 9.7 & $\cdots$ & . \\
\hline 7320 & [O II $]$ & $\cdots$ & .. & . & $\cdots$ & & \\
\hline 7330 & [O II $]$ & $\cdots$ & . & & .. & & \\
\hline 7751 & {$[$ Ar III $] \ldots \ldots \ldots \ldots$} & $\cdots$ & $\cdots$ & .. & & 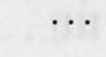 & \\
\hline & & $\cdots$ &.-0.08 & & 0.72 & .. & 0.86 \\
\hline $\mathrm{T}_{\mathrm{e}}([\mathrm{O}$ & III])(K) & & & & 20000 & & \\
\hline $\mathrm{n}_{\mathrm{e}}([\mathrm{S}$ & I] $)\left(\mathrm{cm}^{-3}\right)$ & & $<300$ & & & & \\
\hline E.C. & & & $\ldots 4.8$ & & $\ldots 8.4$ & .. & .4 .1 \\
\hline $\mathrm{F}(\mathrm{H} \beta$ & .. & $\ldots$. & $13.77^{f}$ & & $-13.6^{\mathrm{g}}$ & ... & $-14.41^{f}$ \\
\hline Comr & ient & & & & Гуре I & & \\
\hline
\end{tabular}


TABLE 5.4-Continued

UnCorRected and CoRrected Line InTENSITIES

\begin{tabular}{|c|c|c|c|c|c|c|c|}
\hline \multirow{3}{*}{\begin{tabular}{c}
$\lambda$ \\
$(\AA)$ \\
\multirow{2}{*}{$(\AA)$}
\end{tabular}} & \multirow[b]{2}{*}{ Identification } & \multicolumn{2}{|c|}{$\begin{array}{l}\text { LMC-MA12 } \\
\end{array}$} & \multicolumn{2}{|c|}{ "LMC-MA13 } & \multicolumn{2}{|c|}{ LMC-MA14 } \\
\hline & & $\mathrm{I}_{\mathrm{o}}$ & $\mathrm{I}_{\mathrm{c}}$ & $I_{0}$ & $\mathrm{I}_{\mathrm{c}}$ & $\mathrm{I}_{\circ}$ & $\mathrm{I}_{\mathrm{c}}$ \\
\hline & {$[\mathrm{Ne} \mathrm{V}]$} & & & 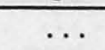 & $\ldots$ & & \\
\hline 3426 & {$[\mathrm{Ne} \mathrm{V}]$} & & & $\cdots$ & & & \\
\hline 444 & OIII & & & 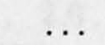 & $\ldots$ & & \\
\hline 3727 & [O II $]$ & 253.2 & 287.4 & 233.4 & 321.4 & 265.1 & 266.5 \\
\hline 3749 & He II, & & & $\cdots$ & & & \\
\hline 3759 & O III. & & ... & $\ldots$ & $\ldots$ & & \\
\hline 71 & H & $\ldots$ & $\ldots$ & $\ldots$ & $\ldots$ & & \\
\hline 98 & & & $\ldots$ & 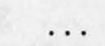 & & & \\
\hline 834 & & & 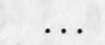 & & 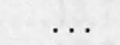 & 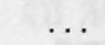 & \\
\hline 3869 & & 72.5 & 80.9 & 85.7 & 113.0 & 102.4 & 102.9 \\
\hline 3889 & & 10.8 & 12.0 & 14.7 & 19.2 & & \\
\hline 3969 & & 33.7 & 37.2 & & 42.3 & & \\
\hline 4026 & & $\ldots$ & $\ldots$ & $\ldots$ & $\ldots$ & $\ldots$ & \\
\hline 4069 & & & $\ldots$ & $\ldots$ & & $\cdots$ & \\
\hline 102 & & 20.8 & 22.6 & 19.8 & 24.4 & 30. & 30.7 \\
\hline 340 & & 43.1 & 45.5 & & 26 & 56.8 & 56.9 \\
\hline 4363 & [O II & 11.1 & 11.7 & 17.5 & 20.0 & 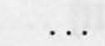 & \\
\hline 4472 & & & & & & & \\
\hline 4686 & & 19.4 & 19.7 & 74.5 & 78.0 & 55.1 & 55.1 \\
\hline 4713 & {$[\mathrm{Ne}]$} & $\ldots$ & $\ldots$ & $\ldots$ & $\ldots$ & $\ldots$ & $\ldots$ \\
\hline 4725 & & & & & & $\ldots$ & \\
\hline 4740 & & & & $\cdots$ & & . & \\
\hline 9 & & 4.0 & 71.4 & 2.4 & 6 & 14.9 & 214.8 \\
\hline 5007 & & 793.2 & 782.0 & 573.3 & 553.1 & 581.3 & 581.0 \\
\hline 5199 & {$[\mathrm{~N}]$} & $\cdots$ & $\ldots$ & $\ldots$ & $\ldots$ & $\ldots$ & $\cdots$ \\
\hline 12 & & $\ldots$ & $\ldots$ & $\ldots$ & $\ldots$ & $\ldots$ & \\
\hline 6 & & & & & $\ldots$ & $\ldots$ & $\cdots$ \\
\hline 6300 & & $\ldots$ & $\ldots$ & $\ldots$ & $\ldots$ & $\ldots$ & $\cdots$ \\
\hline 6312 & & & & $\ldots$ & $\ldots$ & $\ldots$ & 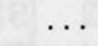 \\
\hline & & & & & .. & .. & \\
\hline 6548 & & 57.3 & 49.9 & 272.9 & 191.8 & 251.3 & 249.8 \\
\hline 6563 & H & 329.4 & 286.2 & Satu & rated & 29.5 & 327.5 \\
\hline 6583 & {$[\mathrm{~N}$} & 184.4 & 160.1 & 788.8 & 551.2 & 880.8 & 875.3 \\
\hline & & & $\ldots$ & & & & \\
\hline & & 23.5 & 20.2 & 4 & 33 & 32 & 32.7 \\
\hline 6731 & [S & 15.9 & 13.6 & 40.5 & 27.7 & 33.3 & 33.1 \\
\hline & & $\cdots$ & $\ldots$ & $\ldots$ & $\ldots$ & $\ldots$ & $\cdots$ \\
\hline 7065 & $\mathrm{He}$ & $\ldots$ & ( & & 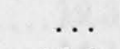 & 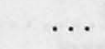 & \\
\hline 7136 & {$[\mathrm{Ar}$} & 17.4 & 14.6 & 26.1 & 16.8 & ... & \\
\hline 732 & [O I & $\cdots$ & $\cdots$ & $\cdots$ & $\cdots$ & $\cdots$ & \\
\hline 73 & & & & $\cdots$ & $\cdots$ & $\cdots$ & .. \\
\hline 7751 & {$[\mathrm{ArI}]$} & & & $\cdots$ & & & \\
\hline & & & 0.19 & & & ‥ & 0.01 \\
\hline $\mathrm{T}_{\mathrm{e}}($ & $111]$ (1) & & 14000 & & .22000 & & $\ldots$ \\
\hline $\mathbf{n}_{\mathbf{e}}([\mathbf{S}$ & I] $)\left(\mathrm{cm}^{-3}\right) \ldots \ldots$ & & $<300$ & & .800 & . . & $\cdots$ \\
\hline E.C. & 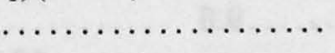 & . & $\ldots 5.4$ & & $\ldots 8.6$ & $\ldots$ & .7 .4 \\
\hline $\mathrm{F}(\mathrm{H} \beta$ & & & $14.10^{f}$ & & $\begin{array}{l}-13.88^{f} \\
\text { Type I }\end{array}$ & & $\begin{array}{l}4.11^{t} \\
\text { e I? }\end{array}$ \\
\hline & & & & & & & \\
\hline
\end{tabular}


TABLE 5.4-Continued

Uncorrected and Corrected Line Intensities

\begin{tabular}{|c|c|c|c|c|c|c|c|}
\hline \multirow{2}{*}{$\begin{array}{c}\lambda \\
(\AA)\end{array}$} & \multirow[b]{2}{*}{ Identification } & \multicolumn{2}{|c|}{ LMC-MA15 } & \multicolumn{2}{|c|}{ LMC-MA16 } & \multicolumn{2}{|c|}{ LMC-MA17 } \\
\hline & & $I_{o}$ & $\mathrm{I}_{\mathrm{c}}$ & $\mathrm{I}_{0}$ & $\mathrm{I}_{\mathrm{c}}$ & $\mathrm{I}_{\mathrm{o}}$ & $\mathrm{I}_{\mathrm{c}}$ \\
\hline 3346 & {$[\mathrm{NeV}] \ldots \ldots$} & $\cdots$ & $\cdots$ & & & $\cdots$ & \\
\hline 3426 & {$[\mathrm{Ne} \mathrm{V}]$} & $\ldots$ & $\ldots$ & 72.7 & 68.5 & $\ldots$ & .. \\
\hline 3444 & OIII & $\ldots$ & - & 6.2 & 5.9 & 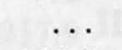 & 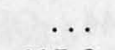 \\
\hline 3727 & [O II] doublet & 160.3 & 142.4 & 75.3 & 71.9 & 291.7 & 415.3 \\
\hline 3749 & He II, H12 & 3.6 & 3.2 & $\cdots$ & $\cdots$ & $\cdots$ & $\ldots$ \\
\hline 3759 & OIII. & $\ldots$ & 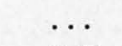 & $\ldots$ & & $\cdots$ & $\cdots$ \\
\hline 3771 & H11, HeII, O III. & 1.9 & 1.7 & 3.8 & 3.6 & & $\cdots$ \\
\hline 3798 & H10 & 3.7 & 3.3 & 3.1 & 3.0 & $\cdots$ & $\ldots$ \\
\hline 3834 & He II, H9 & 5.6 & 5.0 & 5.5 & 5.3 & $\cdots$ & $\cdots$ \\
\hline 3869 & [Ne III] & & & 60.0 & 57.6 & $\ldots$ & \\
\hline 3889 & H8, He I. . & 16.6 & 15.0 & 14.3 & 13.7 & 9.9 & 13.3 \\
\hline 3969 & {$[\mathrm{Ne}$ III $], \mathrm{H} \epsilon \ldots$} & 20.4 & 18.6 & 33.6 & 32.5 & 8.4 & 11.0 \\
\hline 4026 & HeI.. & $\cdots$ & $\cdots$ & $\cdots$ & $\cdots$ & $\cdots$ & $\cdots$ \\
\hline 4069 & [S II] doublet & .. & $\ldots$ & ... & & 7.8 & 9.9 \\
\hline 4102 & $\mathrm{H} \delta, \mathrm{I}$ & 34.5 & 32.0 & 31.0 & 30.1 & 19.5 & 24.5 \\
\hline 4340 & $\ldots \ldots$ & 59.5 & 56.6 & 57.4 & 56.3 & 49.0 & 57.2 \\
\hline 4363 & [O III] & 5.1 & 4.9 & 19.1 & 18.7 & 9.0 & 10.5 \\
\hline 4472 & HeI... & 4.7 & 4.5 & $\ldots$ & $\ldots$ & $\ldots$ & $\cdots$ \\
\hline 4686 & HeII $\ldots \ldots \ldots \ldots \ldots$ & $\cdots$ & $\ldots$ & 96.2 & 95.5 & $\cdots$ & .. \\
\hline 4713 & [Ar IV], He I, [Ne IV] & $\ldots$ & $\ldots$ & 8.2 & 8.2 & $\ldots$ & $\cdots$ \\
\hline 4725 & {$[\mathrm{NeIV}]$} & $\cdots$ & $\ldots$ & & 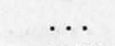 & $\ldots$ & $\ldots$ \\
\hline 4740 & [Ar IV] & $\ldots$ & $\ldots$ & 5.7 & 5.7 & $\ldots$ & $\cdots$ \\
\hline 4959 & [O III] . & 75.7 & 76.4 & 285.9 & 286.9 & 181.1 & 176.4 \\
\hline 5007 & [O III] & 198.8 & 201.4 & Satu & ated & 498.5 & 479.2 \\
\hline 5199 & {$[\mathrm{~N} \mathrm{I}]$} & ... & $\cdots$ & & & ... & $\ldots$ \\
\hline 5412 & He II. & $\cdots$ & $\ldots$ & 5.8 & 5.9 & .. & \\
\hline 5876 & $\mathrm{He}$ & 13.5 & 14.7 & & $\cdots$ & 15.6 & 12.1 \\
\hline 6300 & {$[0$} & $\ldots$ & $\ldots$ & $\ldots$ & $\ldots$ & .. & $\ldots$ \\
\hline 6312 & [S III] & $\ldots$ & ... & $\ldots$ & $\ldots$ & $\ldots$ & $\ldots$ \\
\hline 6364 & $\ldots \ldots$ & & . & $\ldots$ & $\ldots$ & & \\
\hline 6548 & [N II] & 17.6 & 20.1 & 5.6 & 5.9 & 66.7 & 45.2 \\
\hline 6563 & $\mathrm{H} \alpha$ & 288.4 & 328.8 & 309.1 & 325.1 & 469.3 & 317.5 \\
\hline 6583 & {$[\mathrm{~N} \mathrm{I}$} & 68.3 & 78.0 & 48.7 & 51.2 & 239.8 & 161.7 \\
\hline 6678 & $\mathrm{He}$ & 3.4 & 3.9 & .. & 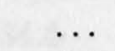 & 11.7 & 7.8 \\
\hline 6717 & {$[\mathrm{~S} \mathrm{II}]$} & 2.0 & 2.3 & 4.3 & 4.6 & 11.5 & 7.6 \\
\hline 6731 & [SII] . & 4.0 & 4.6 & 5.0 & 5.2 & 26.3 & 17.3 \\
\hline 7006 & {$[\mathrm{Ar} \mathrm{V}]$} & & & $\cdots$ & $\ldots$ & & \\
\hline 7065 & $\mathrm{He}$ & 8.2 & 9.6 & .. & .. & 22.8 & 14.2 \\
\hline 7136 & {$[\mathrm{Ar} I$} & 7.3 & 8.6 & 8.6 & 9.2 & 19.7 & 12.1 \\
\hline 7320 & [O II] & 13.7 & 16.3 & .. & $\ldots$ & 74.3 & 44.5 \\
\hline 7330 & {$[\mathrm{O} I \mathrm{II}] \ldots$} & 9.5 & 11.3 & $\ldots$ & $\ldots$ & 58.0 & 34.7 \\
\hline 7751 & [Ar III $] \ldots$ & 2.4 & 2.9 & .. & 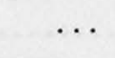 & . & $\cdots$ \\
\hline & $\ldots$ & & $\ldots-0.17$ & & $\ldots-0.07$ & & 0.52 \\
\hline $\mathrm{T}_{e}($ & $\mathrm{III}])(\mathrm{K})$ & & .17000 & & .17000 & & 15000 \\
\hline $\mathrm{n}_{\mathrm{e}}([\mathrm{S})$ & II] $)\left(\mathrm{cm}^{-3}\right) \ldots \ldots \ldots$ & & .20600 & & $\ldots 3400$ & & 59450 \\
\hline E.C. & & & $\ldots 0.9$ & $\ldots$ & $\ldots 9.6$ & & $\ldots 2.2$ \\
\hline $\mathbf{F}(\mathrm{H} \beta$ & & - & $-12.65^{f}$ & & $x^{2}+2$ & & $-13.56^{\mathrm{f}}$ \\
\hline Cor & & & & & & $\ldots w$ & t. \\
\hline
\end{tabular}


TABLE 5.4-Continued

UnCorrected and Corrected Line Intensities

\begin{tabular}{|c|c|c|c|c|c|c|c|}
\hline \multirow{2}{*}{$\begin{array}{l}\lambda \\
(\AA)\end{array}$} & \multirow[b]{2}{*}{ Identification } & \multicolumn{2}{|c|}{ 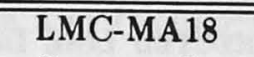 } & \multicolumn{2}{|c|}{ SMC-SMP16 } & \multicolumn{2}{|c|}{$\overline{\text { SMC-SMP17 }}$} \\
\hline & & $\mathrm{I}_{\mathrm{O}}$ & $\mathrm{I}_{\mathrm{c}}$ & $I_{0}$ & $\mathrm{I}_{\mathrm{c}}$ & $\mathrm{I}_{\mathrm{o}}$ & $\mathrm{I}_{\mathrm{c}}$ \\
\hline & {$[\mathrm{Ne} \mathrm{V}] \ldots \ldots$} & 28.2 & 30.1 & & $\ldots$ & & \\
\hline 3426 & $\ldots \ldots \ldots \ldots$ & 54.2 & 57.6 & & & & \\
\hline 3444 & $\ldots \ldots \ldots \ldots$ & 15.2 & 16.1 & & & $\ldots$ & \\
\hline 3727 & [O II] doublet ...... & 83.5 & 87.6 & 130.9 & 109.7 & 33.6 & 18.9 \\
\hline 3749 & He II, H12 $\ldots$ & 6.5 & 6.8 & 2.0 & 1.7 & 1.6 & 0.9 \\
\hline 3759 & O III. & 3.8 & 4.0 & & $\ldots$ & $\ldots$ & $\ldots$ \\
\hline 3771 & H11, He II, O III.... & 2.0 & 2.1 & 2.5 & 2.1 & 2.1 & 1.2 \\
\hline 3798 & $\mathrm{H} 10 \ldots \ldots \ldots \ldots$ & 4.4 & 4.6 & 3.5 & 3.0 & 5.0 & 2.9 \\
\hline 3834 & He II, H9 & 6.0 & 6.2 & 6.5 & 5.5 & 8.1 & 4.8 \\
\hline 3869 & [Ne III] & 80.1 & 83.5 & & & 79.6 & 48.4 \\
\hline 3889 & H8, He I. & 15.1 & 15.7 & 15.9 & 13.7 & 24.8 & 15.3 \\
\hline 3969 & {$[\mathrm{Ne} I \mathrm{III}], \mathrm{H} \epsilon \ldots \ldots .$.} & 43.0 & 44.6 & 19.8 & 17.3 & 46.5 & 29.8 \\
\hline 4026 & $\ldots \ldots \ldots$ & 2.0 & 2.1 & $\ldots$ & $\ldots$ & 2.3 & 1.5 \\
\hline 4069 & [S II] doublet ...... & 4.3 & 4.5 & $\ldots$ & $\ldots$ & $\ldots$ & $\ldots$ \\
\hline 4102 & H $\delta, \quad$ N III $\ldots \ldots \ldots . .$. & 24.0 & 24.8 & 33.1 & 29.5 & 36.5 & 25.2 \\
\hline 4340 & $\mathrm{H} \gamma$. & 44.2 & 45.1 & 60.3 & 55.9 & 60.1 & 46.8 \\
\hline 4363 & [O III] & 17.4 & 17.8 & & & 15.1 & 11.9 \\
\hline 4472 & ........ & 4.4 & 4.5 & 4.7 & 4.5 & 7.8 & 6.5 \\
\hline 4686 & He II . & 47.7 & 48.0 & $\ldots$ & $\ldots$ & $\ldots$ & $\ldots$ \\
\hline 4713 & [Ar IV], He I, [Ne IV] & 4.0 & 4.0 & $\ldots$ & $\cdots$ & $\cdots$ & $\cdots$ \\
\hline 4725 & {$[\mathrm{Ne} I \mathrm{~V}]$} & 2.1 & 2.1 & $\ldots$ & $\ldots$ & $\ldots$ & $\ldots$ \\
\hline 4740 & [Ar IV] & 3.3 & 3.3 & & $\cdots$ & $\ldots$ & $\ldots$ \\
\hline 4959 & [O III $]$. & 338.0 & 336.8 & 60.2 & 61.0 & 276.6 & 288.8 \\
\hline 5007 & [O III] & 1013.1 & 1007.7 & 157.6 & 160.8 & \multicolumn{2}{|c|}{ Saturated } \\
\hline 5199 & $\ldots \ldots \ldots$ & $\ldots$ & $\ldots$ & $\ldots$ & $\ldots$ & $\ldots$ & $\ldots$ \\
\hline 5412 & $\ldots \ldots+\cdots$ & 4.5 & 4.4 & $\ldots$ & ... & $\ldots$ & $\cdots$ \\
\hline 5876 & ........ & 9.4 & 9.1 & 10.4 & 11.8 & 15.7 & 23.7 \\
\hline 6300 & $\ldots \ldots \ldots$ & $\ldots$ & $\cdots$ & $\ldots$ & $\ldots$ & $\ldots$ & $\ldots$ \\
\hline 6312 & [S III] & $\ldots$ & $\ldots$ & $\ldots$ & $\cdots$ & $\ldots$ & $\cdots$ \\
\hline 6364 & [O I]. & 3.7 & 3.6 & $\ldots$ & $\ldots$ & $\ldots$ & $\ldots$ \\
\hline 6548 & $\ldots \ldots \ldots$ & 32.4 & 30.8 & 14.9 & 18.2 & $\ldots$ & 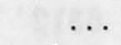 \\
\hline 6563 & $\ldots \ldots$ & 304.6 & 289.1 & 265.8 & 323.2 & \multicolumn{2}{|c|}{ Saturated } \\
\hline 6583 & {$[\mathrm{~N} \mathrm{II}]$} & 80.5 & 76.4 & 56.2 & 68.5 & 35.1 & 66.8 \\
\hline 6678 & HeI.............. & 4.2 & 4.0 & 4.6 & 5.7 & 5.2 & 10.2 \\
\hline 6717 & {$[\mathrm{~S} \mathrm{II}] \ldots \ldots \ldots \ldots$} & 4.4 & 4.2 & 1.3 & 1.6 & 0.4 & 0.7 \\
\hline 6731 & $\ldots \ldots \ldots \ldots$ & 10.0 & 9.4 & 3.1 & 3.9 & 1.7 & 3.4 \\
\hline 7006 & {$[\mathrm{Ar} \mathrm{V}] \ldots \ldots \ldots \ldots$} & 1.2 & 1.2 & 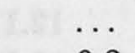 & $\cdots$ & $\ldots$ & $\cdots$ \\
\hline 7065 & HeI... & 7.8 & 7.3 & 9.2 & 11.7 & 11.4 & 24.6 \\
\hline 7136 & {$[$ Ar III $] \ldots \ldots \ldots \ldots$} & 13.4 & 12.6 & 4.0 & 5.1 & 6.7 & 14.7 \\
\hline 7320 & {$[\mathrm{OII}] \ldots \ldots \ldots \ldots$} & 15.3 & 14.3 & 15.0 & 19.3 & 4.4 & 10.2 \\
\hline 7330 & {$[\mathrm{O}$ II $] \ldots \ldots \ldots \ldots$} & 12.1 & 11.3 & 10.9 & 14.1 & 1.6 & 3.8 \\
\hline 7751 & {$[$ Ar III $] \ldots \ldots \ldots \ldots$} & 3.1 & 2.9 & . & $\ldots$ & $\cdots$ & \\
\hline & \multicolumn{2}{|c|}{$\ldots \ldots \ldots+0.07$} & \multicolumn{2}{|c|}{$\ldots \ldots \ldots-0.26$} & \multirow{2}{*}{\multicolumn{2}{|c|}{ …... -0.84}} \\
\hline \multirow{2}{*}{\multicolumn{2}{|c|}{ 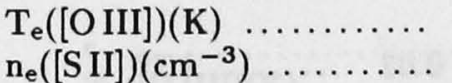 }} & \multicolumn{2}{|c|}{....... 15000} & 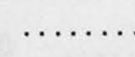 & 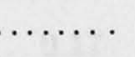 & ....... 14000 & \\
\hline & & $\ldots \ldots$ &.$>10^{5}$ & & & ........ & $>10^{5}$ \\
\hline \multicolumn{2}{|c|}{ E.C. .......... } & $\ldots \ldots$ & $\ldots 7.0$ & ........ & $\ldots 0.7$ & \multicolumn{2}{|c|}{ „.......... 3.7} \\
\hline \multirow{2}{*}{\multicolumn{2}{|c|}{$\mathrm{F}(\mathrm{H} \beta)$}} & & $-12.67^{f}$ & $-12.72^{\mathrm{a}}$ & $-12.75^{c}$ & $-12.61^{\mathrm{a}}$ & $-12.54^{c}$ \\
\hline & & & & $-12.70^{\mathrm{b}}$ & & $12.49^{\circ}$ & $-12.50^{e}$ \\
\hline
\end{tabular}


TABLE 5.4-Continued

UNCORRECTED AND CORRECTED LINE INTENSITIES

\begin{tabular}{|c|c|c|c|c|c|c|c|}
\hline \multirow{2}{*}{$\begin{array}{c}\lambda \\
(\AA)\end{array}$} & \multirow[b]{2}{*}{ Identification } & \multicolumn{2}{|c|}{ SMC-SP34 } & \multicolumn{2}{|c|}{ SMC-MG01 } & \multicolumn{2}{|c|}{ SMC-MG02 } \\
\hline & & $I_{0}$ & $I_{c}$ & $I_{0}$ & $I_{c}$ & $I_{0}$ & $I_{c}$ \\
\hline & {$[\mathrm{NeV}]$} & & $\ldots$ & $\ldots$ & $\ldots$ & & \\
\hline 3426 & {$[\mathrm{Ne} \mathrm{V}]$} & $\ldots$ & $\ldots$ & $\ldots$ & & & \\
\hline 3444 & OIII & $\ldots$ & $\ldots$ & $\ldots$ & $\ldots$ & 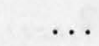 & 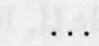 \\
\hline 3727 & [O II] doublet & 187.2 & 184.2 & 251.9 & 255.6 & 15.7 & 35.4 \\
\hline 3749 & He II, H12 & $\ldots$ & $\ldots$ & .. & . & .. & $\ldots$ \\
\hline 3759 & O III .... & $\ldots$ & $\ldots$ & $\ldots$ & $\ldots$ & $\ldots$ & $\ldots$ \\
\hline 3771 & H11, He II, O III. & $\ldots$ & $\ldots$ & $\ldots$ & $\ldots$ & .. & .. \\
\hline 3798 & H10 & $\ldots$ & $\ldots$ & $\ldots$ & $\ldots$ & $\cdots$ & $\ldots$ \\
\hline 3834 & He II, H9 & $\ldots$ & $\ldots$ & $\ldots$ & $\ldots$ & & 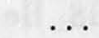 \\
\hline 3869 & [Ne III] & 35.0 & 34.6 & $\ldots$ & $\ldots$ & 20.7 & 41.7 \\
\hline 3889 & H8, He I & 16.3 & 16.1 & $\ldots$ & $\ldots$ & & 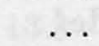 \\
\hline 3969 & {$[\mathrm{Ne} \mathrm{III}], \mathrm{H} \epsilon \ldots$} & 26.6 & 26.3 & $\ldots$ & $\ldots$ & 16.5 & 30.8 \\
\hline 4026 & HeI.. & $\ldots$ & $\ldots$ & $\ldots$ & $\ldots$ & 9.9 & 17.7 \\
\hline 4069 & [S II] doublet & $\ldots$ & $\ldots$ & $\ldots$ & $\ldots$ & 9.6 & 16.7 \\
\hline 4102 & H $\delta$, N III & 22.3 & 22.0 & 28.4 & 28.7 & 8.7 & 14.8 \\
\hline 4340 & $\mathrm{H} \gamma$. & 49.5 & 49.1 & 56.4 & 56.8 & 21.6 & 30.8 \\
\hline 4363 & [O III] & 9.5 & 9.4 & $\ldots$ & $\ldots$ & $\ldots$ & $\ldots$ \\
\hline 4472 & HeI. & $\ldots$ & $\ldots$ & $\ldots$ & $\ldots$ & $\ldots$ & $\ldots$ \\
\hline 4686 & HeII. & 10.6 & 10.6 & .. & $\cdots$ & .. & $\cdots$ \\
\hline 4713 & [Ar IV], $\mathrm{He}$ I, [Ne IV] & $\cdots$ & $\cdots$ & $\cdots$ & $\cdots$ & $\cdots$ & $\cdots$ \\
\hline 4725 & [NeIV] & ... & $\ldots$ & $\ldots$ & $\ldots$ & $\cdots$ & $\ldots$ \\
\hline 4740 & [ArIV] & $\ldots$ & $\ldots$ & $\ldots$ & $\ldots$ & $\ldots$ & $\ldots$ \\
\hline 4959 & [O III] & 147.9 & 148.1 & 218.3 & 218.1 & 207.1 & 194.8 \\
\hline 5007 & [O III $]$ & 410.4 & 411.2 & 625.9 & 624.9 & 599.6 & 547.5 \\
\hline 5199 & [N I] & $\ldots$ & $\ldots$ & $\ldots$ & $\ldots$ & $\ldots$ & $\ldots$ \\
\hline 5412 & HeII. & $\ldots$ & .. & $\ldots$ & $\cdots$ & $\cdots$ & $\cdots$ \\
\hline 5876 & $\mathrm{HeI}$. & 9.9 & 10.0 & $\ldots$ & $\cdots$ & $\ldots$ & $\ldots$ \\
\hline 6300 & {$[\mathrm{OI}]$} & . & $\ldots$ & $\ldots$ & $\ldots$ & $\cdots$ & $\ldots$ \\
\hline 6312 & [S III] & $\cdots$ & $\ldots$ & $\ldots$ & $\ldots$ & $\ldots$ & $\ldots$ \\
\hline 6364 & {$[\mathrm{O} I]$} & $\ldots$ & $\ldots$ & $\ldots$ & $\ldots$ & $\ldots$ & $\ldots$ \\
\hline 6548 & [N II] & 16.6 & 16.9 & $\ldots$ & $\ldots$ & $\ldots$ & $\ldots$ \\
\hline 6563 & $\mathrm{H} \alpha$ & Satu & ated & 329.3 & 324.0 & 564.5 & 229.6 \\
\hline 6583 & [N II] & 65.5 & 66.7 & 70.7 & 69.5 & $\ldots$ & $\ldots$ \\
\hline 6678 & $\mathrm{HeI}$ & $\cdots$ & 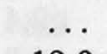 & $\cdots$ & $\ldots$ & ... & $\ldots$ \\
\hline 6717 & {$[\mathrm{~S} \mathrm{II}]$} & 12.1 & 12.3 & $\ldots$ & $\cdots$ & $\cdots$ & $\ldots$ \\
\hline 6731 & [S II] & 19.2 & 19.5 & $\ldots$ & $\cdots$ & $\cdots$ & $\ldots$ \\
\hline 7006 & {$[\mathrm{ArV}]$} & $\cdots$ & $\cdots$ & $\cdots$ & $\cdots$ & & $\ldots$ \\
\hline 7065 & $\mathrm{He} \mathrm{I}$ & .. & $\ldots$ & $\ldots$ & & 32.4 & 10.9 \\
\hline 7136 & [Ar III] & $\cdots$ & $\cdots$ & $\ldots$ & $\ldots$ & 41.3 & 13.5 \\
\hline 7320 & [O II] & 10.0 & 10.2 & $\cdots$ & $\cdots$ & $\cdots$ & $\ldots$ \\
\hline 7330 & [O II] & 6.2 & 6.3 & $\cdots$ & $\cdots$ & $\cdots$ & $\ldots$ \\
\hline 7751 & [Ar III] & $\cdots$ & $\cdots$ & 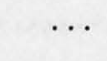 & 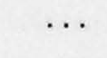 & $\cdots$ & $\cdots$ \\
\hline & & & .0 .02 & .. & 0.02 & & 1.19 \\
\hline $\mathrm{T}_{\mathrm{e}}([\mathrm{O}$ & III])(K) . & & 17000 & & & & \\
\hline $\mathrm{n}_{\mathrm{e}}([\mathrm{S}]$ & I]) $\left(\mathrm{cm}^{-3}\right)$ & & 4600 & & & & \\
\hline E.C. & ..... & & ...4.9 & & .2 .8 & $\ldots$ & .2 .5 \\
\hline $\begin{array}{l}\mathrm{F}(\mathrm{H} \beta \\
\text { Comn }\end{array}$ & ient $\ldots \ldots \ldots \ldots \ldots \ldots \ldots \ldots$ & & & & $27^{\mathrm{a}}$ & & $\begin{array}{l}13.97^{\circ} \\
\text { Cont. }\end{array}$ \\
\hline
\end{tabular}


TABLE 5.4-Continued

UnCorrected and Corrected Line Intensities

\begin{tabular}{|c|c|c|c|c|c|c|c|}
\hline \multirow{2}{*}{$\begin{array}{c}\lambda \\
(\AA)\end{array}$} & \multirow[b]{2}{*}{ Identification } & \multicolumn{2}{|c|}{ SMC-MG03 } & \multicolumn{2}{|c|}{ SMC-MG04 } & \multicolumn{2}{|c|}{ SMC-MG05 } \\
\hline & & $\mathrm{I}_{\circ}$ & $\mathrm{I}_{\mathrm{c}}$ & $\mathrm{I}_{\mathrm{o}}$ & $I_{c}$ & $\mathrm{I}_{\mathrm{o}}$ & $\mathrm{I}_{\mathrm{c}}$ \\
\hline 3346 & {$[\mathrm{NeV}]$} & & & .. & & $\ldots$ & \\
\hline 3426 & $\mathrm{NeV}$ ] & $\ldots$ & $\ldots$ & $\ldots$ & $\ldots$ & $\ldots$ & \\
\hline 3444 & III & ... & $\cdots$ & $\ldots$ & $\ldots$ & 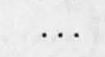 & \\
\hline 3727 & {$[\mathrm{O} I \mathrm{II}] \mathrm{d}$} & $\ldots$ & $\ldots$ & $\ldots$ & $\ldots$ & $\ldots$ & .. \\
\hline 3749 & He II, & $\cdots$ & . & $\cdots$ & $\cdots$ & . & \\
\hline 3759 & & ... & $\ldots$ & $\ldots$ & $\ldots$ & $\ldots$ & \\
\hline 71 & UI & $\ldots$ & $\ldots$ & $\ldots$ & $\ldots$ & $\ldots$ & \\
\hline 98 & & $\ldots$ & $\ldots$ & $\ldots$ & $\ldots$ & $\ldots$ & \\
\hline 34 & & & & $\ldots$ & $\ldots$ & $\ldots$ & . \\
\hline 69 & & 26.5 & 22.2 & $\ldots$ & $\ldots$ & $\ldots$ & \\
\hline 89 & & 19.1 & & & $\ldots$ & $\ldots$ & \\
\hline 69 & & 28.5 & & & $\ldots$ & 18.4 & 18.0 \\
\hline 26 & & $\cdots$ & $\ldots$ & $\ldots$ & $\ldots$ & $\ldots$ & \\
\hline 9 & & & & $\ldots$ & $\ldots$ & 1 & 14.3 \\
\hline 02 & & 35.8 & .3 & $\ldots$ & 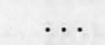 & 25 . & 25.4 \\
\hline 4340 & & 66.9 & 1.8 & 36.8 & 38.7 & 51.0 & 50.5 \\
\hline 4363 & & $\ldots$ & $\cdots$ & $\ldots$ & ... & $\cdots$ & 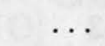 \\
\hline 4472 & & $\ldots$ & $\cdots$ & $\ldots$ & $\ldots$ & . & \\
\hline 4686 & & $\ldots$ & $\ldots$ & $\ldots$ & $\ldots$ & 95.5 & 95.1 \\
\hline 4713 & $\mathrm{I},[\mathrm{Ne}$ IV] & $\ldots$ & $\cdots$ & $\cdots$ & $\cdots$ & $\cdots$ & $\cdots$ \\
\hline 5 & & $\ldots$ & $\ldots$ & $\ldots$ & $\ldots$ & $\ldots$ & \\
\hline 0 & & & & $\because$ & - & 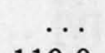 & \\
\hline 9 & & 90.6 & 6 & 2.9 & 0.5 & 19.0 & 119.3 \\
\hline 5 & & 516.0 & 528.2 & 778.4 & 768.3 & 357.0 & 358.0 \\
\hline 9 & {$[\mathrm{~N}$} & $\cdots$ & $\cdots$ & ... & $\ldots$ & $\cdots$ & $\cdots$ \\
\hline 2 & & $\cdots$ & $\ldots$ & $\ldots$ & $\cdots$ & $\cdots$ & . \\
\hline & & $\cdots$ & $\cdots$ & $\cdots$ & $\cdots$ & $\cdots$ & $\cdots$ \\
\hline & & $\ldots$ & $\ldots$ & $\ldots$ & $\cdots$ & $\cdots$ & $\cdots$ \\
\hline 6. & & $\ldots$ & $\cdots$ & $\cdots$ & $\cdots$ & $\cdots$ & $\cdots$ \\
\hline 6 & & $\cdots$ & $\cdots$ & $\cdots$ & $\cdots$ & $\cdots$ & .. \\
\hline & & & & $\ldots$ & & .. & \\
\hline 3 & & 268.6 & 338.2 & 310.8 & 273.1 & 294.9 & 303.6 \\
\hline 3 & & $\cdots$ & $\cdots$ & $\cdots$ & $\cdots$ & $\cdots$ & $\cdots$ \\
\hline 0 & & $\cdots$ & $\cdots$ & $\cdots$ & $\cdots$ & $\cdots$ & $\cdots$ \\
\hline & & $\cdots$ & $\cdots$ & $\cdots$ & $\cdots$ & $\cdots$ & . \\
\hline & & $\cdots$ & $\cdots$ & $\cdots$ & $\cdots$ & $\cdots$ & $\cdots$ \\
\hline & & $\cdots$ & $\cdots$ & $\cdots$ & $\cdots$ & $\cdots$ & $\cdots$ \\
\hline & & $\cdots$ & $\cdots$ & $\cdots$ & $\cdots$ & . & . \\
\hline 71 & & .. & . & $\cdots$ & $\cdots$ & $\cdots$ & \\
\hline 7 & & $\cdots$ & . & . & . & $\cdots$ & $\cdots$ \\
\hline 7330 & & & $\cdots$ & $\cdots$ & & $\cdots$ & $\cdots$ \\
\hline 7751 & [Ar III] & & & 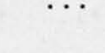 & & . & \\
\hline & & & $0.3^{3}$ & & 0.17 & & -0.04 \\
\hline $\mathrm{T}_{\mathrm{e}}$ & & & & & & . & $\cdots$ \\
\hline $\mathrm{n}_{\mathrm{e}}($ & -3) & & & & & & .6 \\
\hline $\begin{array}{l}\text { E.C. } \\
\text { F(H } \beta\end{array}$ & & & $13.63^{\mathrm{a}}$ & & $14.15^{\mathrm{a}}$ & $\cdots$ & $13.81^{\mathrm{a}}$ \\
\hline & & & Cont. & & Cont. & $\ldots \mathrm{O}_{\mathrm{F}}$ & t. thin \\
\hline
\end{tabular}


TABLE 5.4-Continued

UnCorrected AND Corrected Line Intensities

\begin{tabular}{|c|c|c|c|c|c|c|c|}
\hline \multirow{2}{*}{$\begin{array}{c}\lambda \\
(\AA)\end{array}$} & \multirow[b]{2}{*}{ Identification } & \multicolumn{2}{|c|}{ SMC-MG06 } & \multicolumn{2}{|c|}{ SMC-MG07 } & \multicolumn{2}{|c|}{ SMC-MG08 } \\
\hline & & $\mathrm{I}_{\mathrm{o}}$ & $\mathrm{I}_{\mathrm{c}}$ & $I_{\circ}$ & $\mathrm{I}_{\mathrm{c}}$ & $\mathrm{I}_{\mathrm{o}}$ & $\mathrm{I}_{\mathrm{c}}$ \\
\hline 3346 & {$[\mathrm{Ne} \mathrm{V}]$} & . & 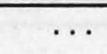 & - & 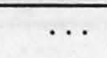 & & \\
\hline 3426 & {$[\mathrm{Ne} \mathrm{V}]$} & & $\cdots$ & & & & \\
\hline 3444 & OIII & $\cdots$ & $\ldots$ & & & $\cdots$ & \\
\hline 3727 & [O II] doublet ....... & $\ldots$ & $\ldots$ & 276.8 & 317.8 & 424.9 & 379.0 \\
\hline 3749 & He II, H12 & $\cdots$ & $\cdots$ & .. & $\ldots$ & $\ldots$ & .. \\
\hline 3759 & O III ....... & $\ldots$ & $\ldots$ & .. & $\ldots$ & .. & \\
\hline 3771 & H11, He II, O III.... & $\ldots$ & $\ldots$ & $\ldots$ & $\ldots$ & $\ldots$ & $\cdots$ \\
\hline 3798 & H10 & $\ldots$ & $\ldots$ & $\ldots$ & $\ldots$ & $\ldots$ & \\
\hline 3834 & He II, H9 & $\ldots$ & $\ldots$ & $\ldots$ & $\ldots$ & $\ldots$ & $\ldots$ \\
\hline 3869 & [Ne III] & $\ldots$ & $\ldots$ & 45.4 & 51.1 & $\ldots$ & $\ldots$ \\
\hline 3889 & $\mathrm{H} 8, \mathrm{HeI}$. & $\ldots$ & $\ldots$ & 25.0 & 28.1 & 21.4 & 19.4 \\
\hline 3969 & {$[\mathrm{Ne} \mathrm{III}], \mathrm{H} \epsilon \ldots$} & $\cdots$ & $\ldots$ & 32.0 & 35.5 & 13.1 & 12.0 \\
\hline 4026 & He I. & $\ldots$ & $\ldots$ & $\ldots$ & $\ldots$ & $\ldots$ & ... \\
\hline 4069 & [S II] doublet & $\ldots$ & $\ldots$ & & $\ldots$ & & $\ldots$ \\
\hline 4102 & $\mathrm{H} \delta, \mathrm{N}$ & & & & & 25.4 & 23.6 \\
\hline 4340 & $\mathrm{H} \gamma$. & 40.2 & 42.4 & 43.8 & 46.5 & 49.5 & 47.1 \\
\hline 4363 & [O III] & $\ldots$ & $\ldots$ & $\ldots$ & $\ldots$ & $\ldots$ & $\ldots$ \\
\hline 4472 & HeI. & $\ldots$ & $\ldots$ & $\ldots$ & $\ldots$ & $\ldots$ & $\ldots$ \\
\hline 4686 & $\mathrm{HeI}$ & 114.1 & 116.1 & $\ldots$ & $\ldots$ & 28.5 & 28.0 \\
\hline 4713 & [Ar IV], $\mathrm{HeI},[\mathrm{Ne} I V]$ & $\cdots$ & $\cdots$ & ... & $\cdots$ & $\cdots$ & $\cdots$ \\
\hline 4725 & {$[\mathrm{NeIV}]$} & $\ldots$ & $\ldots$ & $\ldots$ & $\ldots$ & $\ldots$ & $\ldots$ \\
\hline 4740 & {$[\mathrm{ArIV}]$} & & $\cdots$ & $\cdots$ & & & \\
\hline 4959 & [O III] & 136.0 & 134.8 & 161.0 & 159.3 & 35.4 & 35.7 \\
\hline 5007 & {$[\mathrm{O} \mathrm{III}] \ldots \ldots \ldots \ldots$} & 372.6 & 367.5 & 487.5 & 480.1 & 100.2 & 101.4 \\
\hline 5199 & $\ldots \ldots \ldots \ldots$ & $\ldots$ & $\ldots$ & $\cdots$ & $\cdots$ & $\cdots$ & $\cdots$ \\
\hline 5412 & He II. & $\ldots$ & $\ldots$ & . & $\ldots$ & $\ldots$ & $\ldots$ \\
\hline 5876 & HeI. & $\ldots$ & $\ldots$ & 100.3 & 71.8 & $\ldots$ & $\ldots$ \\
\hline 6300 & {$[\mathrm{OI}]$} & 40.8 & 36.2 & $\cdots$ & $\cdots$ & $\cdots$ & $\cdots$ \\
\hline 6312 & $\ldots \ldots \ldots \ldots$ & $\ldots$ & $\ldots$ & $\ldots$ & $\ldots$ & $\ldots$ & $\ldots$ \\
\hline 6364 & {$[\mathrm{OI}] \ldots$} & $\cdots$ & $\ldots$ & . & $\ldots$ & & $\ldots$ \\
\hline 6548 & {$[\mathrm{~N} \mathrm{II}]$} & .. & .. & 31.3 & 26.9 & 29.7 & 33.7 \\
\hline 6563 & $\mathrm{H} \alpha$ & 325.1 & 283.3 & Satu & ated & 257.3 & 292.0 \\
\hline 6583 & {$[\mathrm{~N} \mathrm{II}]$} & $\cdots$ & $\cdots$ & 72.3 & 62.1 & 116.6 & 132.5 \\
\hline 6678 & HeI $\ldots \ldots \ldots \ldots \ldots$ & $\ldots$ & $\ldots$ & $\ldots$ & $\ldots$ & $\ldots$ & $\ldots$ \\
\hline 6717 & {$[\mathrm{SII}]$} & $\ldots$ & $\ldots$ & $\ldots$ & $\ldots$ & 5.9 & 6.7 \\
\hline 6731 & {$[\mathrm{SII}]$} & $\cdots$ & $\cdots$ & $\cdots$ & $\cdots$ & 17.5 & 20.1 \\
\hline 7006 & {$[\mathrm{ArV}]$} & $\ldots$ & $\ldots$ & $\ldots$ & $\ldots$ & $\ldots$ & $\ldots$ \\
\hline 7065 & HeI.... & $\cdots$ & $\ldots$ & $\cdots$ & $\ldots$ & 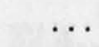 & $\ldots$ \\
\hline 7136 & [Ar III] & $\cdots$ & $\cdots$ & $\cdots$ & $\cdots$ & $\cdots$ & $\cdots$ \\
\hline 7320 & {$[\mathrm{OII}] \ldots \ldots \ldots \ldots$} & $\ldots$ & $\ldots$ & $\ldots$ & $\ldots$ & 9.4 & 11.1 \\
\hline 7330 & [O II $]$ & $\cdots$ & $\cdots$ & $\cdots$ & $\cdots$ & 6.9 & 8.1 \\
\hline 7751 & {$[$ Ar III $]$} & $\cdots$ & $\cdots$ & $\cdots$ & . & $\cdots$ & $\cdots$ \\
\hline & & .... & 0.18 & $\ldots$ & .0 .20 & &.-0.17 \\
\hline $\mathrm{T}_{\mathrm{e}}([\mathrm{C}$ & III] $)(\mathrm{K}$ & $\ldots$ & $\ldots$ & $\ldots$ & $\ldots$ & & $\ldots \ldots$ \\
\hline $\mathrm{n}_{\mathrm{e}}([\mathrm{S}$ & [I] $)\left(\mathrm{cm}^{-3}\right)$ & 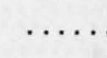 & . & & . & . & $>10^{5}$ \\
\hline E.C. & & & . 10.8 & $\cdots$ & .2 .1 & & .5 .9 \\
\hline $\mathrm{F}(\mathrm{H} \beta$ & & $\ldots \ldots$ & $-13.87^{\mathrm{a}}$ & $\ldots$ & $13.71^{\mathrm{a}}$ & & $-13.5^{\mathrm{g}}$ \\
\hline Com? & $\ldots .$. & $\ldots \mathrm{O}$ & t. thin & & & 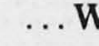 & cont. \\
\hline
\end{tabular}


TABLE 5.4-Continued

UnCorrected and Corrected Line InTensities

\begin{tabular}{|c|c|c|c|c|c|c|c|}
\hline \multirow{2}{*}{$\begin{array}{c}\lambda \\
(\AA)\end{array}$} & \multirow[b]{2}{*}{ Identification } & \multicolumn{2}{|c|}{ SMC-MG09 } & \multicolumn{2}{|c|}{ SMC-MG10 } & \multicolumn{2}{|c|}{ SMC-MG11 } \\
\hline & & $\mathrm{I}_{\mathrm{o}}$ & $\mathrm{I}_{\mathrm{c}}$ & $I_{0}$ & $\mathrm{I}_{\mathrm{c}}$ & $\mathrm{I}_{0}$ & $\mathrm{I}_{\mathrm{c}}$ \\
\hline & {$[\mathrm{Ne} \mathrm{V}]$} & $\ldots$ & $\cdots$ & $\cdots$ & & $\ldots$ & \\
\hline 3426 & {$[\mathrm{Ne} \mathrm{V}]$} & $\ldots$ & $\ldots$ & $\ldots$ & $\ldots$ & $\ldots$ & $\ldots$ \\
\hline 3444 & III & & & $\ldots$ & $\ldots$ & & $\ldots$ \\
\hline 3727 & [O II] & 17.5 & 27.9 & 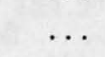 & $\ldots$ & 321.5 & 296.1 \\
\hline 3749 & He II & $\cdots$ & $\cdots$ & & & & \\
\hline 3759 & O III . & $\ldots$ & .. & $\cdots$ & $\ldots$ & $\ldots$ & $\ldots$ \\
\hline 71 & III .... & $\ldots$ & $\cdots$ & $\ldots$ & $\ldots$ & $\ldots$ & $\ldots$ \\
\hline 98 & & $\ldots$ & $\ldots$ & 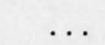 & $\ldots$ & $\ldots$ & \\
\hline 34 & & & $\ldots$ & $\cdots$ & $\ldots$ & $\ldots$ & ... \\
\hline 69 & & 20.5 & 30.6 & & $\ldots$ & 66.9 & 62.3 \\
\hline 89 & & 8.0 & 11.8 & & & 29.3 & 27.3 \\
\hline 3969 & & 16.1 & 23.1 & 23.3 & 22.1 & & $\ldots$ \\
\hline 4026 & & $\ldots$ & $\ldots$ & & .. & $\ldots$ & $\ldots$ \\
\hline 4069 & & $\ldots$ & ${ }^{2}$ & & 11. & .. & $\ldots$ \\
\hline 02 & & 19.8 & 26.7 & & 18. & 34.6 & 32.8 \\
\hline 4340 & & 42.2 & 01. & 40 & 43. & 51.1 & 49.3 \\
\hline 4363 & & 10.1 & 12.3 & $\cdots$ & $\cdots$ & $\cdots$ & $\ldots$ \\
\hline 4472 & & $\ldots$ & $\cdots$ & & & $\cdots$ & $\ldots$ \\
\hline 86 & & $\ldots$ & $\ldots$ & 106.5 & 105.4 & $\cdots$ & $\cdots$ \\
\hline 13 & V] & $\cdots$ & $\ldots$ & .. & $\ldots$ & $\ldots$ & $\cdots$ \\
\hline 4725 & & $\ldots$ & $\ldots$ & $\ldots$ & .. & $\ldots$ & $\cdots$ \\
\hline 40 & & & & & $\cdots$ & $\cdots$ & . \\
\hline 59 & & 163.2 & 157.6 & 77.8 & 78.2 & .7 & 261.3 \\
\hline 07 & {$[0$} & Satu & ated & 244.1 & 246.0 & 743.5 & 750.4 \\
\hline 5199 & & $\ldots$ & $\ldots$ & $\ldots$ & $\ldots$ & $\ldots$ & $\ldots$ \\
\hline 2 & & $\ldots$ & $\ldots$ & $\ldots$ & $\ldots$ & $\ldots$ & $\ldots$ \\
\hline & & 20.2 & 14.5 & $\ldots$ & $\ldots$ & $\ldots$ & $\cdots$ \\
\hline & & $\cdots$ & $\cdots$ & $\cdots$ & $\cdots$ & $\cdots$ & $\cdots$ \\
\hline & & $\cdots$ & $\ldots$ & $\ldots$ & $\ldots$ & $\ldots$ & $\ldots$ \\
\hline & & $\cdots$ & $\cdots$ & $\cdots$ & $\ldots$ & $\ldots$ & .. \\
\hline 6548 & & ... & $\cdots$ & & & 7.6 & 63.0 \\
\hline 6563 & & 515.8 & 308.5 & 252.0 & 272.5 & 5.1 & 312.2 \\
\hline 6583 & & $\ldots$ & $\ldots$ & $\ldots$ & $\cdots$ & 116.5 & 127.7 \\
\hline 78 & & $\ldots$ & $\ldots$ & $\ldots$ & .. & .. & ... \\
\hline 17 & & $\cdots$ & $\cdots$ & $\cdots$ & $\cdots$ & $\cdots$ & .. \\
\hline 6 & & $\cdots$ & $\cdots$ & $\cdots$ & $\cdots$ & $\cdots$ & $\cdots$ \\
\hline 7 & & & & . & $\cdots$ & $\cdots$ & $\cdots$ \\
\hline & & .1 & 10.8 & & ( & $\cdots$ & $\cdots$ \\
\hline 6 & & 18.5 & 9. & $\cdots$ & $\cdots$ & $\cdots$ & . \\
\hline 7320 & & 19.2 & 9. & $\cdots$ & $\cdots$ & $\cdots$ & * \\
\hline 7330 & {$[0$} & 7.6 & 3.9 & $\cdots$ & $\cdots$ & $\cdots$ & . \\
\hline 7751 & [Ar III] & 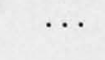 & $\cdots$ & $\cdots$ & $\cdots$ & $\cdots$ & $\cdots$ \\
\hline & & & & $\ldots$ & -0.10 & & 0.12 \\
\hline $\mathrm{T}_{\mathrm{e}}($ & & & 19000 & 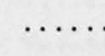 & & & \\
\hline $\mathrm{n}_{\mathrm{e}}([\mathrm{S}$ & & & & & & & \\
\hline E.C. & & & ..1.9 & & 10.2 & & .3 .4 \\
\hline $\mathrm{F}(\mathrm{H} \beta)$ & & & $12.8^{\mathrm{g}}$ & $\cdots$ & $13.72^{\mathrm{a}}$ & . & $09^{a}$ \\
\hline Comn & 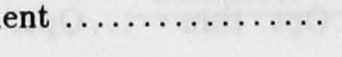 & & 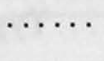 & 8 & thin & & \\
\hline
\end{tabular}


TABLE 5.4-Continued

UnCorrected and CoRrected Line Intensities

\begin{tabular}{|c|c|c|c|c|c|c|c|}
\hline \multirow{2}{*}{$\begin{array}{c}\lambda \\
(\AA)\end{array}$} & \multirow[b]{2}{*}{ Identification } & \multicolumn{2}{|c|}{ SMC-MG12 } & \multicolumn{2}{|c|}{ SMC-MG13 } & \multicolumn{2}{|c|}{ SMC-MA01 } \\
\hline & & $\mathrm{I}_{\mathrm{o}}$ & $I_{c}$ & $\mathrm{I}_{\mathrm{o}}$ & $I_{c}$ & $I_{\circ}$ & $I_{c}$ \\
\hline 3346 & {$[\mathrm{NeV}]$. } & $\cdots$ & 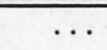 & . & $\cdots$ & & \\
\hline 3426 & {$[\mathrm{Ne} \mathrm{V}] \ldots \ldots \ldots \ldots \ldots$} & $\ldots$ & $\ldots$ & $\ldots$ & $\ldots$ & . & $\ldots$ \\
\hline 3444 & OIII & $\ldots$ & $\ldots$ & $\ldots$ & $\ldots$ & $\cdots$ & $\cdots$ \\
\hline 3727 & [O II] doublet & 54.6 & 66.0 & $\ldots$ & $\ldots$ & ... & $\ldots$ \\
\hline 3749 & He II, H12 & $\ldots$ & $\ldots$ & $\ldots$ & $\ldots$ & & $\ldots$ \\
\hline 3759 & OIII. & $\ldots$ & $\ldots$ & $\ldots$ & $\ldots$ & .. & $\ldots$ \\
\hline 3771 & H11, He II, O III.... & $\ldots$ & $\ldots$ & $\ldots$ & $\ldots$ & .. & $\ldots$ \\
\hline 3798 & H10 & $\ldots$ & $\ldots$ & $\ldots$ & $\ldots$ & .. & $\ldots$ \\
\hline 3834 & He II, H9 & & $\ldots$ & & 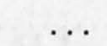 & 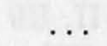 & 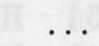 \\
\hline 3869 & [Ne III $]$ & 47.4 & 55.9 & 23.4 & 18.4 & 59.6 & 46.9 \\
\hline 3889 & H8, HeI. & 22.9 & 26.9 & & & 21.7 & 17.1 \\
\hline 3969 & {$[\mathrm{Ne}$ III $], \mathrm{H} \epsilon \ldots \ldots \ldots$} & 37.4 & 43.2 & 33.0 & 26.6 & 39.0 & 31.5 \\
\hline 4026 & HeI.......... & $\ldots$ & $\ldots$ & $\ldots$ & $\ldots$ & & .. \\
\hline 4069 & [SII] doublet & & & $\ldots$ & $\ldots$ & & \\
\hline 4102 & H $\delta$, N III & 19.0 & 21.4 & 38.1 & 31.8 & 24.8 & 20.7 \\
\hline 4340 & $\mathrm{H} \gamma$. & 44.6 & 48.4 & 64.9 & 57.5 & 69.3 & 61.4 \\
\hline 4363 & [O III] & 14.6 & 15.8 & 13.5 & 12.1 & $\ldots$ & $\ldots$ \\
\hline 4472 & $\mathrm{HeI}$. & 10.1 & 10.8 & .. & 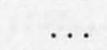 & & $\ldots$ \\
\hline 4686 & HeII. & 59.4 & 61.1 & 121.1 & 116.4 & .. & ... \\
\hline 4713 & [Ar IV], HeI, [Ne IV] & $\ldots$ & $\ldots$ & $\ldots$ & $\ldots$ & $\ldots$ & $\ldots$ \\
\hline 4725 & {$[\mathrm{NeIV}] \ldots \ldots \ldots \ldots$} & $\ldots$ & $\ldots$ & $\ldots$ & $\ldots$ & .. & $\ldots$ \\
\hline 4740 & {$[\mathrm{Ar} I V]$. } & $\therefore$ & L & & & 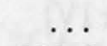 & ... \\
\hline 4959 & {$[\mathrm{O}$ III $] \ldots \ldots \ldots \ldots$} & 92.8 & 91.5 & 106.9 & 109.1 & 236.6 & 241.6 \\
\hline 5007 & [O III $]$ & 260.6 & 255.1 & 290.0 & 299.2 & 755.8 & 779.6 \\
\hline 5199 & {$[\mathrm{NI}]$. } & 8.0 & 7.6 & $\ldots$ & $\ldots$ & $\ldots$ & $\ldots$ \\
\hline 5412 & He II. & $\ldots$ & $\ldots$ & $\ldots$ & $\ldots$ & $\ldots$ & $\ldots$ \\
\hline 5876 & HeI.. & $\ldots$ & .. & $\cdots$ & $\cdots$ & $\ldots$ & ... \\
\hline 6300 & ........ & $\cdots$ & $\cdots$ & $\ldots$ & $\ldots$ & .. & ... \\
\hline 6312 & [S III] & $\cdots$ & $\ldots$ & $\ldots$ & $\ldots$ & $\ldots$ & $\ldots$ \\
\hline 6364 & [O I] & & & $\cdots$ & $\ldots$ & $\cdots$ & .. \\
\hline 6548 & [N II] & 155.5 & 126.2 & .. & $\ldots$ & $\ldots$ & $\cdots$ \\
\hline 6563 & $\mathrm{H} \alpha$ & 359.1 & 291.1 & 242.5 & 330.7 & 234.4 & 318.7 \\
\hline 6583 & [N II] & 468.1 & 378.1 & $\ldots$ & $\ldots$ & .. & $\cdots$ \\
\hline 6678 & ........ & & & $\ldots$ & $\ldots$ & $\ldots$ & $\ldots$ \\
\hline 6717 & {$[\mathrm{~S} \mathrm{II}]$} & 11.7 & 9.4 & $\ldots$ & $\ldots$ & $\ldots$ & $\ldots$ \\
\hline 6731 & {$[\mathrm{SII}] \ldots \ldots \ldots \ldots \ldots$} & 24.8 & 19.8 & $\ldots$ & $\ldots$ & $\ldots$ & $\ldots$ \\
\hline 7006 & {$[\mathrm{Ar} \mathrm{V}] \ldots \ldots \ldots \ldots$} & $\cdots$ & . & $\ldots$ & $\ldots$ & $\ldots$ & $\ldots$ \\
\hline 7065 & $\ldots \ldots$ & 13.3 & 10.3 & $\ldots$ & $\ldots$ & $\ldots$ & $\ldots$ \\
\hline 7136 & ......... & . & $\cdots$ & $\ldots$ & $\cdots$ & $\ldots$ & . \\
\hline 7320 & [O II] & 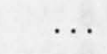 & . & $\cdots$ & $\cdots$ & ... & .. \\
\hline 7330 & [O II $]$ & .. & . & $\cdots$ & . & . & . \\
\hline 7751 & {$[$ Ar III $] \ldots$} & .. & .. & $\cdots$ & $\cdots$ & $\cdots$ & $\because$ \\
\hline & & & 0.28 & $\ldots$ & .. -0.41 & $\ldots$ &.-0.41 \\
\hline $\mathrm{T}_{\mathrm{e}}([\mathrm{C}$ & III])(K) & $\ldots$ & .34000 & $\cdots$ & .23000 & & 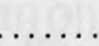 \\
\hline $\mathrm{n}_{\mathrm{e}}([\mathrm{S}$ & I]) $\left(\mathrm{cm}^{-3}\right)$. & $\ldots$ & .36900 & & & & \\
\hline E.C. & & & $\ldots 7.7$ & & 10.0 & & $\ldots 3.1$ \\
\hline $\mathbf{F}(\mathbf{H}$ & & 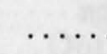 & $-13.7^{8}$ & $\ldots$ & $-13.51^{\mathrm{a}}$ & & ...... \\
\hline Com & & .. & Type I & $\ldots \mathrm{O}$ & thin & .. $\mathrm{O}$ & t. thin \\
\hline
\end{tabular}


TABLE 5.4-Continued

\section{UnCorrected and Corrected Line Intensities}

\begin{tabular}{|c|c|c|c|}
\hline \multirow{2}{*}{$\begin{array}{c}\lambda \\
(\AA)\end{array}$} & \multirow[b]{2}{*}{ Identification } & \multicolumn{2}{|c|}{ SMC-MA02 } \\
\hline & & $\mathrm{I}_{\circ}$ & $\mathrm{I}_{\mathrm{c}}$ \\
\hline 3346 & {$[\mathrm{Ne} \mathrm{V}]$} & & \\
\hline 3426 & {$[\mathrm{Ne} \mathrm{V}]$} & & \\
\hline 3444 & OIII .. & $\ldots$ & $\cdots$ \\
\hline 3727 & [O II] doublet ....... & 181.5 & 433.1 \\
\hline 3749 & He II, H12 & $\cdots$ & . \\
\hline 3759 & O III .... & $\ldots$ & $\ldots$ \\
\hline 3771 & H11, He II, O III.... & .. & . \\
\hline 3798 & H10 & $\cdots$ & $\cdots$ \\
\hline 3834 & He II, H9 & & . \\
\hline 3869 & {$[\mathrm{Ne} \mathrm{III}]$} & 58.3 & 123.5 \\
\hline 3889 & H8, He I. & & \\
\hline 3969 & {$[\mathrm{Ne} \mathrm{III}], \mathrm{H} \epsilon .$.} & 23.5 & 45.9 \\
\hline 4026 & $\mathrm{HeI} \ldots .$. & $\cdots$ & $\cdots$ \\
\hline 4069 & [S II] doublet & & $\ldots$ \\
\hline 4102 & $\mathrm{H} \delta, \mathrm{N}$ III & 12.8 & 22.5 \\
\hline 4340 & $\mathrm{H} \gamma$. & 33.4 & 48.8 \\
\hline 4363 & [O III $]$ & $\cdots$ & $\cdots$ \\
\hline 4472 & HeI $\ldots \ldots \ldots \ldots \ldots$ & ... & $\cdots$ \\
\hline 4686 & HeII. & 40.3 & 45.6 \\
\hline 4713 & [Ar IV], He I, [Ne IV] & $\cdots$ & $\cdots$ \\
\hline 4725 & {$[$ NeIV]...$\ldots \ldots \ldots$} & $\cdots$ & . \\
\hline 4740 & {$[$ Ar IV] $\ldots \ldots \ldots \ldots$} & 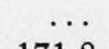 & \\
\hline 4959 & {$[\mathrm{O} \mathrm{III}] \ldots \ldots \ldots \ldots$} & 171.8 & 161.0 \\
\hline 5007 & [O III $]$ & 506.2 & 459.3 \\
\hline 5199 & {$[\mathrm{NI}]$} & $\cdots$ & $\cdots$ \\
\hline 5412 & He II & .. & $\cdots$ \\
\hline 5876 & HeI.. & $\cdots$ & . \\
\hline 6300 & {$[\mathrm{OI}]$} & - & $\cdots$ \\
\hline 6312 & [S III] & - & . \\
\hline 6364 & {$[\mathrm{OI}]$} & $\ldots$ & 105 \\
\hline 6548 & {$[\mathrm{~N} \mathrm{II}]$} & 2.2 & 10.0 \\
\hline 6563 & .... & Salt & \\
\hline 6583 & [N II] & 100.3 & 38.0 \\
\hline 6678 & HeI........... & $\cdots$ & $\cdots$ \\
\hline 6717 & [S II] & $\cdots$ & $\cdots$ \\
\hline 6731 & {$[\mathrm{~S} \mathrm{II}]$} & . & . \\
\hline 7006 & {$[\mathrm{ArV}]$} & $\cdots$ & \\
\hline 7065 & HeI... & & \\
\hline 7136 & [ArIII] & & \\
\hline 7320 & [OII] & $\cdots$ & \\
\hline $\begin{array}{l}7330 \\
7751\end{array}$ & {$\left[\begin{array}{lll}{[\mathrm{OII}]} \\
{[\mathrm{Ar} I \mathrm{III}]}\end{array}\right.$} & $\cdots$ & \\
\hline 7751 & [Arili] & & \\
\hline & ....... & $\cdots$ & . . 1.27 \\
\hline $\mathrm{T}_{e}([\mathrm{C}$ & II] $)(\mathrm{K})$ & $\cdots$ & \\
\hline $\mathrm{n}_{\mathrm{e}}([\mathrm{S}$ & $\left(\mathrm{cm}^{-3}\right) \ldots \ldots \ldots \ldots$ & & \\
\hline E.C. & $\ldots \ldots \ldots \ldots$ & & ..6.8 \\
\hline $\begin{array}{l}\mathrm{F}(\mathrm{H} / \\
\mathrm{Com}\end{array}$ & & $\ldots$ & Cont. \\
\hline
\end{tabular}

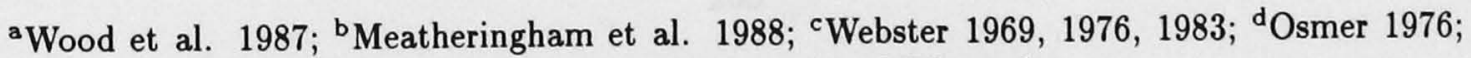
${ }^{\mathrm{e}}$ Aller 1983 ; ${ }^{\mathrm{f}}$ This study-Imager results; ${ }^{\mathrm{g}}$ This study-DBS results. 
TABLE 5.5

SuPplementary Line LIST

\begin{tabular}{|c|c|c|c|c|}
\hline Object & $\begin{array}{c}\lambda \\
(\AA)\end{array}$ & Identification & $\overline{\overline{\mathrm{I}_{0}}}$ & $\overline{\overline{\mathrm{I}_{c}}}$ \\
\hline LMC-SMP21 $\ldots \ldots \ldots$ & 7155 & {$[$ Fe II $] \ldots \ldots$} & 4.9 & 4.5 \\
\hline LMC-SMP40 ..... & 7155 & {$[\mathrm{Fe} \mathrm{II}]$. } & 7.3 & 5.4 \\
\hline LMC-SMP41 ...... & 7155 & [Fe II $]$. & 6.3 & 7.0 \\
\hline LMC-SMP85 & 7237 & [ArIV]. & 1.6 & 1.6 \\
\hline & 7281 & HeI. & 1.7 & 1.7 \\
\hline LMC-MG46 $\ldots \ldots \ldots$ & 3466 & {$[\mathrm{~N} \mathrm{I}] ?$} & 30.0 & 35.9 \\
\hline LMC-MG74 $\ldots \ldots \ldots$ & 3909 & [S II] ? & 1.4 & 1.4 \\
\hline SMC-SMP16 & 4267 & C II & 3.0 & 2.7 \\
\hline SMC-MG02 & 4416 & {$[\mathrm{Fe} \mathrm{II}]$} & 5.8 & 7.8 \\
\hline SMC-MG08 & 4647 & C III, O II ... & 125.4 & 123.0 \\
\hline & 5696 & C III . & 39.8 & 42.7 \\
\hline SMC-MG11 & 4388 & He I.. & 24.6 & 23.8 \\
\hline$\ldots \ldots \ldots \ldots \ldots \ldots \ldots$ & 6950 & & 33.3 & 37.0 \\
\hline n.m.w.m. & 7237 & {$[$ Ar IV] ...... } & 88.2 & 99.1 \\
\hline
\end{tabular}


From the sample presented here we find average values of the reddening constant of $c_{L M C}=0.17$ and $c_{S M C}=0.07$, respectively. These correspond to $E(B-V)=0.12$ and $0.05 \mathrm{mag}$, respectively, and are therefore intermediate between the reddenings found in the first sample and the values of $\mathrm{E}(\mathrm{B}-\mathrm{V})$ found for stars in the LMC, 0.074-0.11 (Caldwell \& Coulson 1986), and the value of 0.02 given for the SMC (Gascoigne 1969; see also Bessell 1991). The reason for this appears to lie with the existence of dust in these PNe. The sample observed in Papers I and II is generally composed of brighter, denser, and younger $\mathrm{PNe}$, in which obscuration by internal dust is more important. As the nebula expands, this obscuration would be expected to become progressively less important.

Three sample spectra are presented in figures 5.1-5.3. In particular, in SMC-MG04 only 5 emission lines are detected.

\subsubsection{Nebular Diagnostics}

The emission line measurements presented in table 5.4 permit us to derive various physical properties of the PNe (Osterbrock 1989). In particular, we calculate the electron temperature using the $\left[\mathrm{O}\right.$ III] line ratio, $\left(I_{\lambda 4959}+I_{\lambda 5007}\right) / I_{\lambda 4363}$, and the electron density using the $[\mathrm{S} \mathrm{II}]$ line ratio, $\left(I_{\lambda 6717} / I_{\lambda 6731}\right)$. The resolution was too low to allow separation of the [O II]doublet at $\lambda \lambda 3727-3729 \AA$, and therefore make a secondary density determination. Also, a secondary temperature determination could not be made using the [N II] line ratio because [N II] $\lambda 5755 \AA$ was positioned in a region of very low spectral response.

The [O III] electron temperature was calculated in the zero-density limit and is given in table 5.4. The accuracy of the temperature determination was mainly determined by the relatively faint [O III] line at $\lambda 4363 \AA$. The density determination is also affected by poor photon statistics of the [S II] lines at $\lambda 6717$ and $\lambda 6731 \AA$. The excitation class for each object has been calculated using equations 2.1a-2.1b from Dopita and Meatheringham (1990).

We estimate typical errors in the [O III] electron temperature, [S II] electron density, and the excitation class to be $20 \%, 20-30 \%$, and $10 \%$, respectively. 


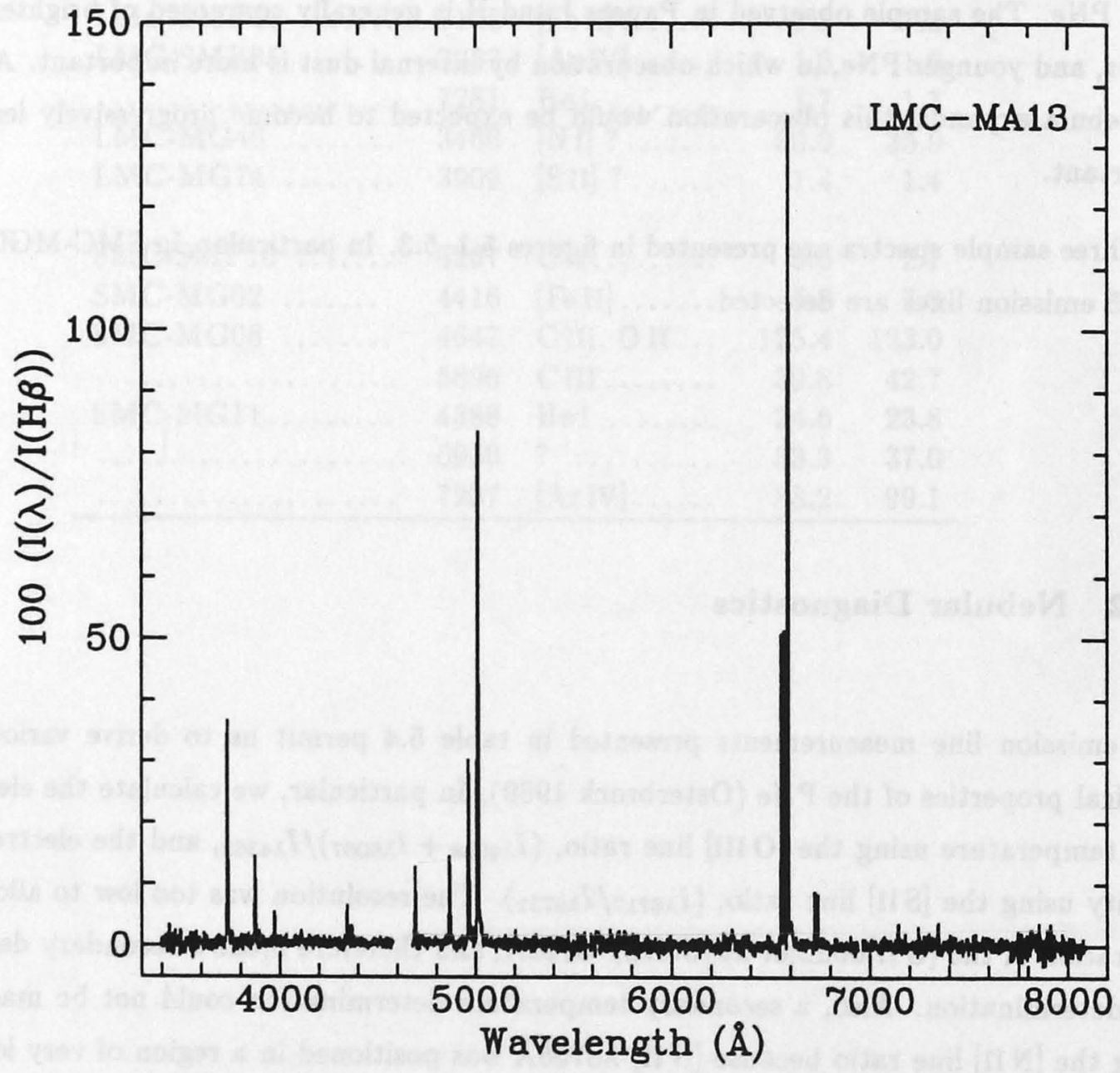

Figure 5.1: Flux calibrated, but not dereddened, composite DBS spectrum of LMCMA13. This object represents a typical Type I object with very strong [N II] $\lambda 6583 \AA$ emission, and He II $\lambda 4686 \AA$ comparable in strength to $\mathrm{H} \beta$. Note also the clear detection of the [S II] doublet at $\lambda \lambda 6717-6731 \AA$. 


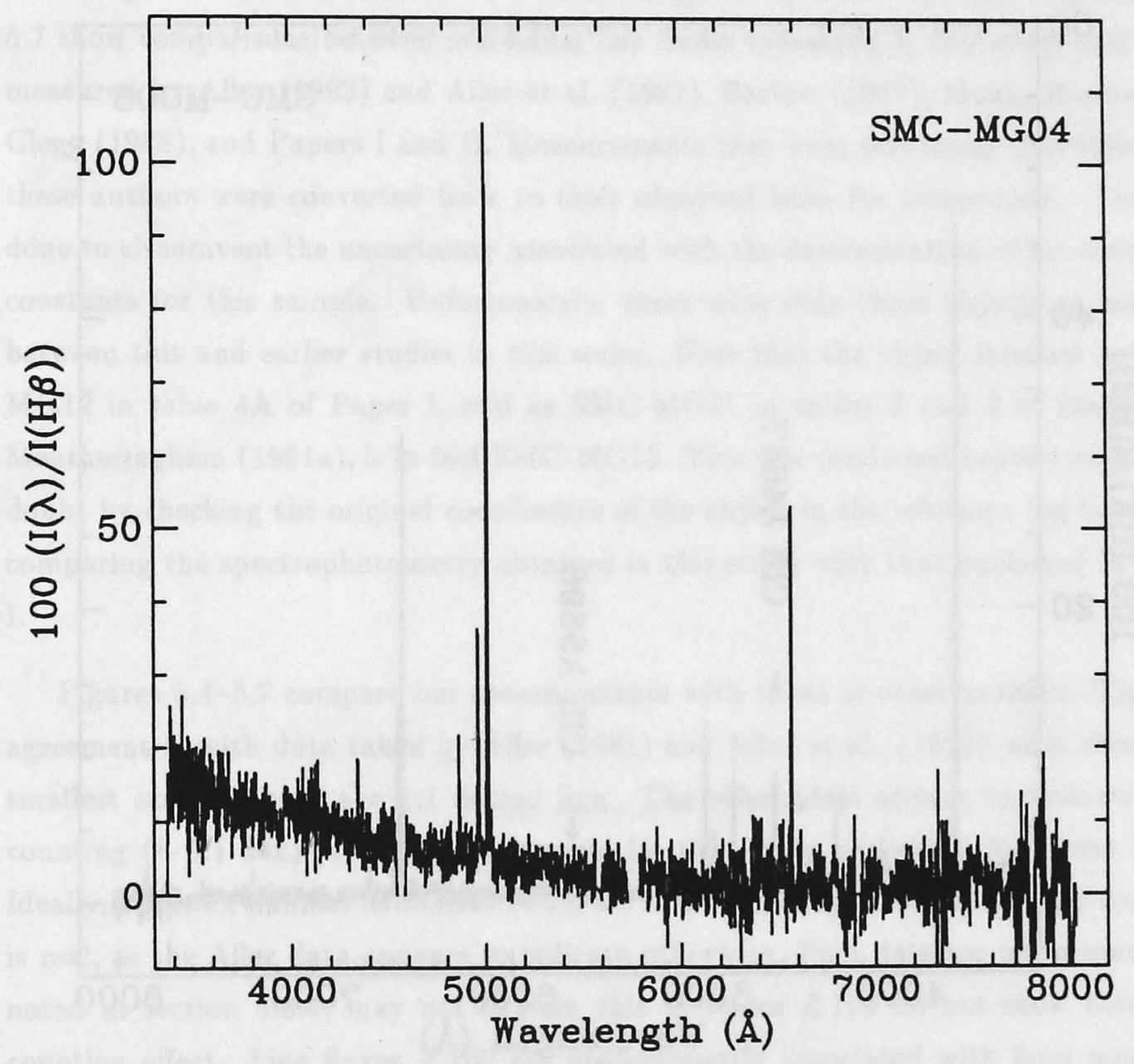

Figure 5.2: This object was found to have the least number of emission lines. Comparison between these spectra gives an indication of the variation in signal-to-noise between different objects. 


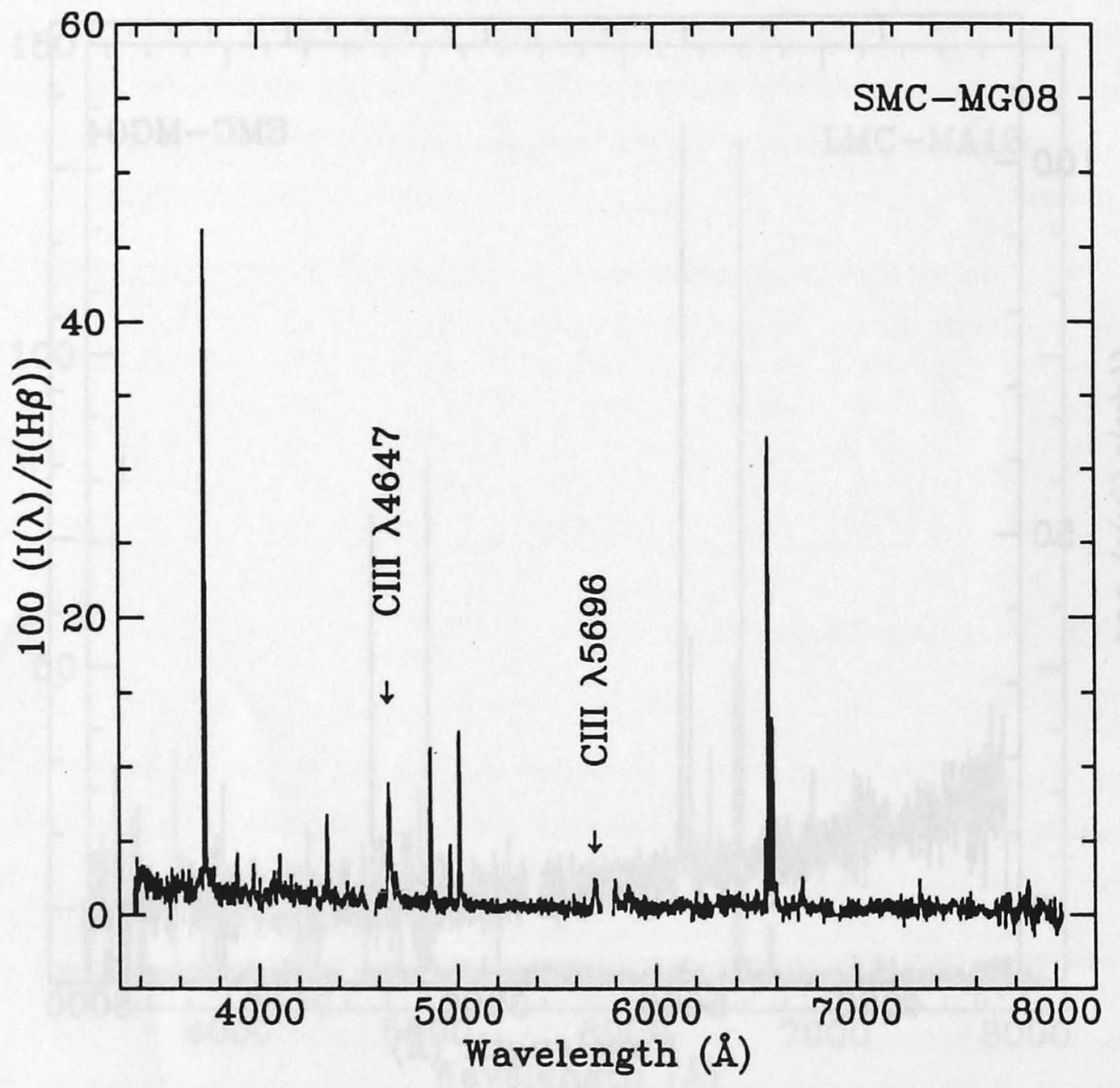

Figure 5.3: Flux calibrated, but not dereddened, composite DBS spectrum of SMCMG08. This object has WR-type emission present at $\lambda 4647 \AA$ and $\lambda 5696 \AA$, believed to be due to C III. This object has also been observed by Monk et al. (1988), and classified as having a WC8 central star. 


\subsubsection{Other Studies}

The majority of the objects presented in this study are newly discovered and therefore no published spectrophotometry exists for them. However, of the small sample of SMP objects observed, some have been investigated by other authors. Figures 5.45.7 show comparisons between individual line fluxes measured in this study and those measured by Aller (1983) and Aller et al. (1981), Barlow (1987), Monk, Barlow, and Clegg (1988), and Papers I and II. Measurements that were previously dereddened by these authors were converted back to their observed form for comparison. This was done to circumvent the uncertainty associated with the determination of the reddening constants for this sample. Unfortunately, there were only three objects to compare between this and earlier studies in this series. Note that the object labelled as SMCMG12 in table 4A of Paper I, and as SMC-MG01 in tables 2 and 3 of Dopita and Meatheringham (1991a), is in fact SMC-MG13. This was confirmed beyond reasonable doubt by checking the original coordinates of the object in the telescope log book, and comparing the spectrophotometry obtained in this study with that published in Paper I.

Figures 5.4-5.7 compare our measurements with those of other authors. The best agreement is with data taken by Aller (1981) and Aller et al. (1983), as it shows the smallest scatter about the 1:1 dotted line. The other plots appear to indicate overcounting $(\sim 0.1$ dex $)$ in the measurements for this study, especially for fluxes $\lesssim 100$. Ideally, a greater number of objects would aid in determining whether this over-counting is real, as the Aller data appears to indicate otherwise. Poor detector performance, as noted in section 5.3.4, may not explain this as fluxes $\gtrsim 100$ do not show this overcounting effect. Line fluxes $\gtrsim 100$ are predominantly associated with lines measured blueward of $\mathrm{H} \beta$. If these lines were subject to atmospheric dispersion or wavelengthdependent seeing effects, then we would be expect them to suffer under-counting with respect to the other studies noted.

If one makes a closer comparison between the individual objects, then the various data sets agree reasonably well. Looking at nebular diagnostics, such as the electron temperature derived from the [O III] line ratio, and line intensities of $\lambda 4686 \AA$ and $\lambda 5007 \AA$ used in determination of the excitation class, all agree with their counterparts in this study to within the quoted errors.

The only major discrepancy remaining concerns the reddening constant values. There appears to be no systematic effect. Some values agree within the errors, such as 


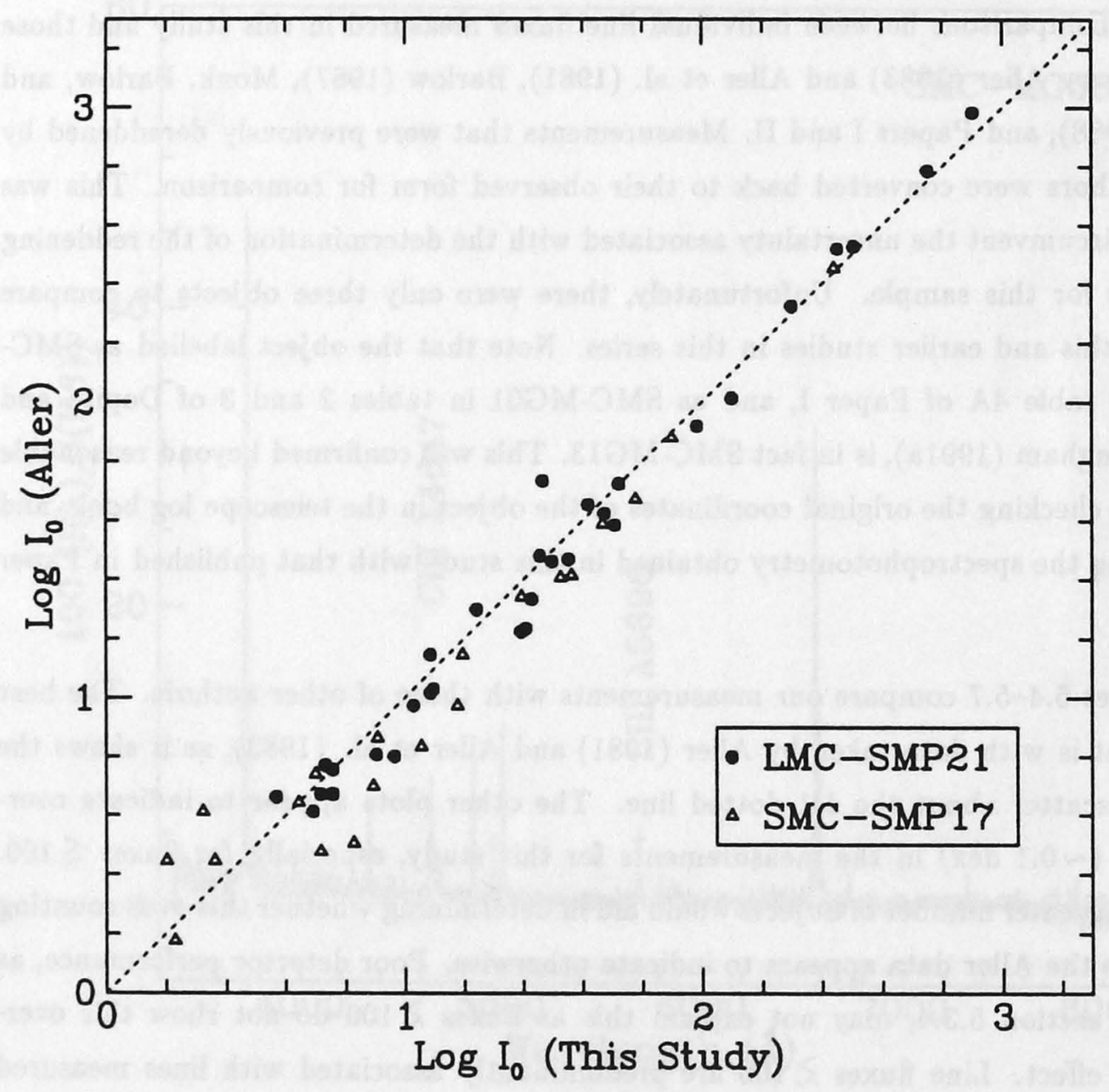

Figure 5.4: Comparison of measured line fluxes between this study and those of Aller et al. (1981) and Aller(1983). The dotted line represents the 1:1 locus and is not a fit. 


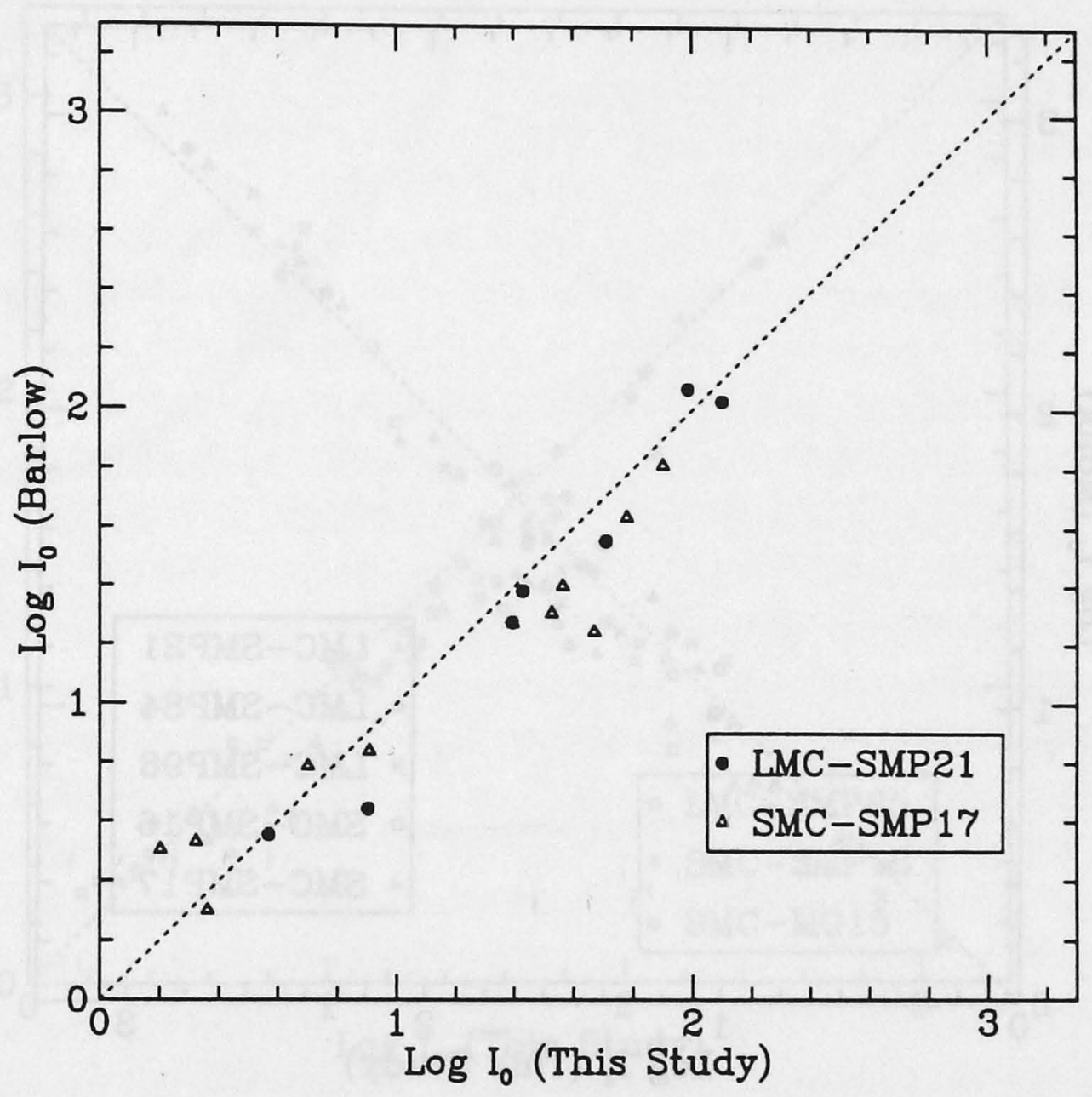

Figure 5.5: Comparison of measured line fluxes between this study and that of Barlow (1987). The dotted line represents the 1:1 locus and is not a fit. 


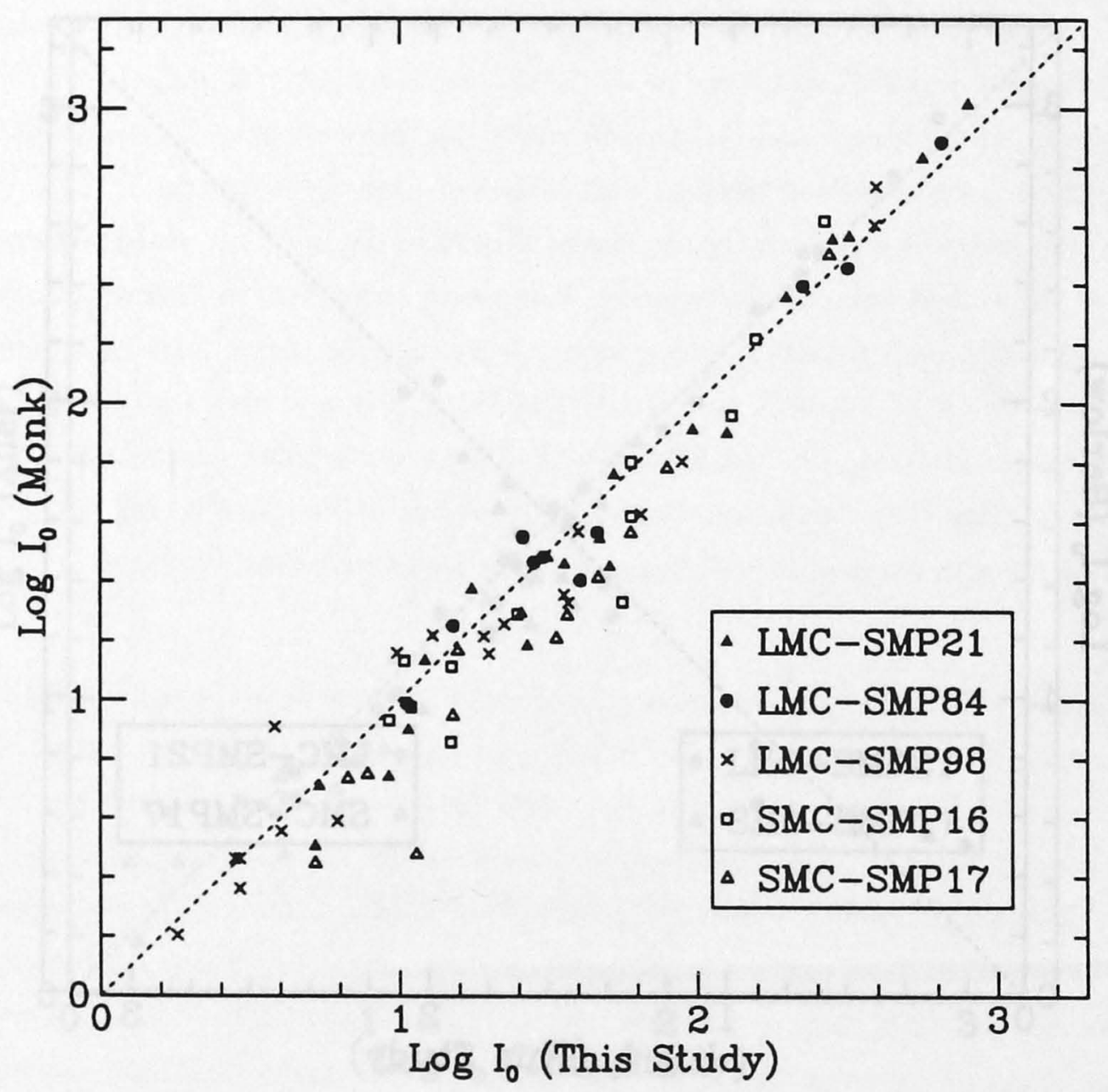

Figure 5.6: Comparison of measured line fluxes between this study and that of Monk, Barlow, and Clegg (1988). The dotted line represents the 1:1 locus and is not a fit. 


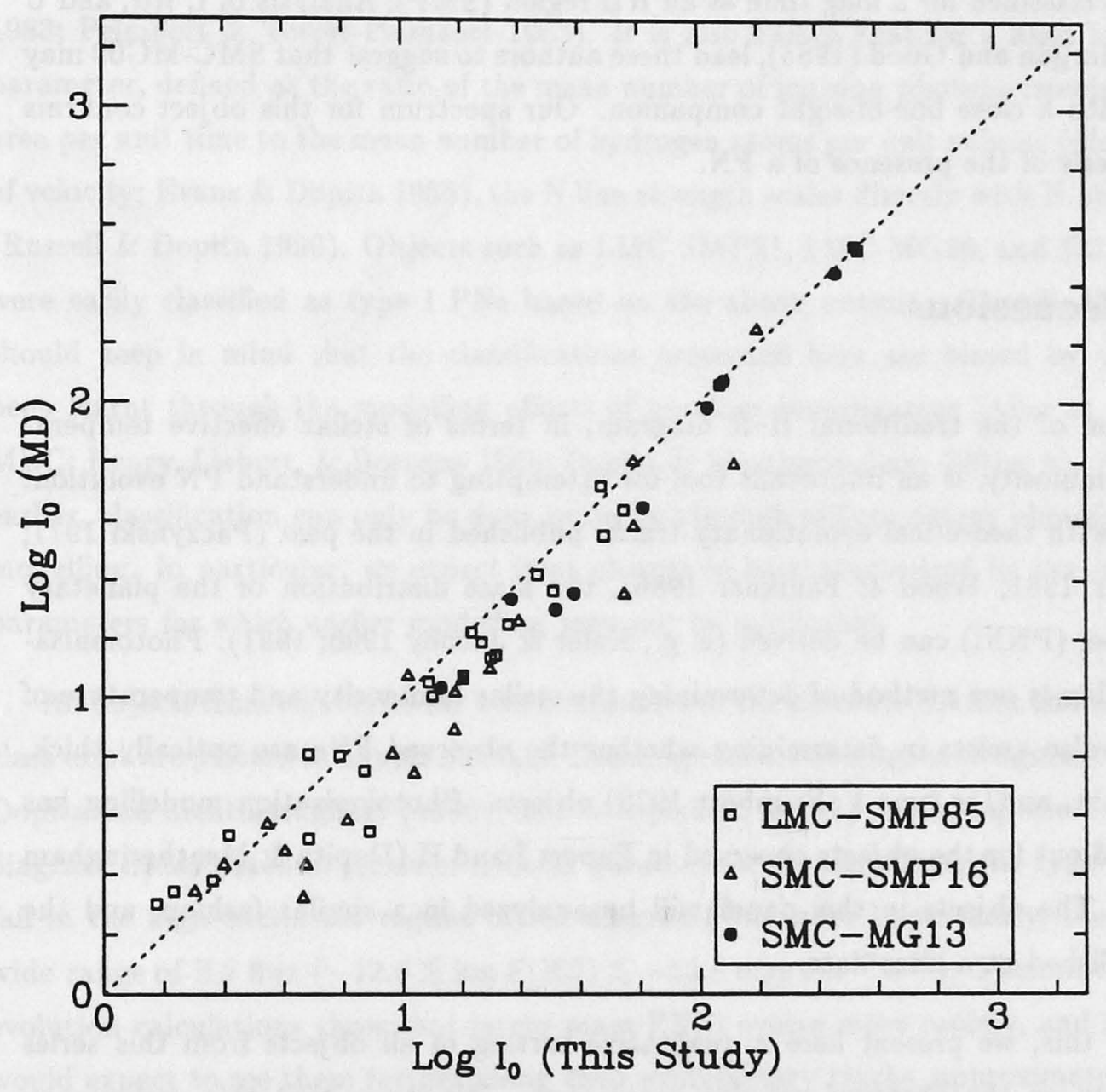

Figure 5.7: Comparison of measured line fluxes between this study and that of Meatheringham and Dopita (1991a, b). The dotted line represents the 1:1 locus and is not a fit. 
LMC-SMP21 in Aller (1983), but others are clearly subject to some effect which has not been corrected (e.g. $c=-0.84$ for SMC-SMP17 in this study, whereas Aller et al. (1981) and Barlow (1987) give a value $\sim 0$ ).

Finally, we would like to point out that the object SMC-MG09, otherwise known as $\mathrm{Ln} 339$, was classified for a long time as an $\mathrm{H}$ II region (SMP). Analysis of I, $\mathrm{H} \alpha$, and $\mathrm{U}$ plates by Morgan and Good (1985), lead these authors to suggest that SMC-MG09 may be a PN with a close line-of-sight companion. Our spectrum for this object confirms the hypothesis of the presence of a PN.

\subsection{Discussion}

Construction of the traditional $\mathrm{H}-\mathrm{R}$ diagram, in terms of stellar effective temperature and luminosity, is an important tool for attempting to understand PN evolution. Combined with theoretical evolutionary tracks published in the past (Paczyński 1971; Schönberner 1981; Wood \& Faulkner 1986), the mass distribution of the planetary nebula nuclei (PNNi) can be derived (e. g., Kaler \& Jacoby 1990; 1991). Photoionisation modelling is one method of determining the stellar luminosity and temperature of $\mathrm{PNNi}$, and also assists in determining whether the observed PNe are optically thick, optically thin, and/or type I (Peimbert 1978) objects. Photoionisation modelling has been carried out for the objects observed in Papers I and II (Dopita \& Meatheringham 1991a; b). The objects in this paper will be analyzed in a similar fashion, and the results published at a later date.

Despite this, we present here a qualitative sorting of all objects from this series based simply on the observed nebular parameters. We stress that this preliminary investigation of our results should in no way be regarded as definitive-a detailed physical analysis is warranted.

Objects in this study have been noted as optically thin following the description given by Dopita and Meatheringham (1991b). To summarize, [O I] and [N I] lines weaken and then disappear as an object becomes more optically thin. Singly ionised $\mathrm{He}, \mathrm{O}$, and $\mathrm{N}$ species also weaken. This is accompanied by an increase in the [O III] and [NeIII] intensities with respect to $\mathrm{H} \beta$. For very optically thin objects, [O III] decreases and $\mathrm{He}$ II $\lambda 4686 \AA$ increases with respect to $\mathrm{H} \beta$.

Similarly, following from the results found in Dopita and Meatheringham (1991a; b) once more, objects were classified as type I if they had [N II] $\lambda 6583 \AA$ strengths 
comparable to, or greater than, $\mathrm{H} \alpha$, and again, He II $\lambda 4686 \AA$ had to be present. In the high excitation class régime, strong [N $\mathrm{II}$ ] emission is indicative of nitrogen-rich $\mathrm{PNe}$, as optically thin objects will tend to have diminished [N II] emission (Osmer 1976; Dopita \& Meatheringham 1991b). A significant proportion of Type I PNe have been observed to be bipolar, and hence, more likely to be optically thin (Calvet \& Peimbert 1983; Peimbert \& Torres-Peimbert 1983). It is also known that for a fixed ionisation parameter, defined as the ratio of the mean number of ionising photons crossing a unit area per unit time to the mean number of hydrogen atoms per unit volume (dimensions of velocity; Evans \& Dopita 1985), the N line strength scales directly with N abundance (Russell \& Dopita 1990). Objects such as LMC-SMP21, LMC-MG20, and SMC-MG12 were easily classified as type I PNe based on the above criteria. Overall, the reader should keep in mind that the classifications presented here are biased by what has been learnt through the modelling efforts of previous investigators (Aller et al. 1987; MBC; Henry, Liebert, \& Boroson 1989; Dopita \& Meatheringham 1991a; b). As stated earlier, classification can only be done properly through self-consistent photoionisation modelling. In particular, we expect faint objects to be characterised by low ionisation parameters for which earlier modelling may not be applicable.

All objects from this series for which measures of the absolute $\mathrm{H} \beta$ flux and excitation class exist are plotted in figures 5.8-5.9. These figures are analogous to figures 8 and 9 in Dopita and Meatheringham (1990), and as explained in that paper, represent the H-R diagram transformed in terms of nebular parameters. By definition, the type I objects fall in the high excitation régime of the diagram, but more importantly, they span a wide range of $\mathrm{H} \beta$ flux $\left(-12.6 \lesssim \log F(\mathrm{H} \beta) \lesssim-13.8 \mathrm{ergs} \mathrm{cm}^{-2} \mathrm{~s}^{-1}\right)$. Theoretical stellar evolution calculations show that larger mass PNNi evolve more rapidly, and hence we would expect to see them further along their evolutionary tracks, approximately where we see the type I objects of this series (Renzini 1979). This supports the hypothesis that type I PNe have larger mass nuclei, and therefore, larger mass progenitors (Peimbert \& Serrano 1980; Dopita \& Meatheringham 1990).

There is a relatively large proportion of optically thick objects, compared to the previous two studies in this series, having low flux and low excitation. These objects appear to be too faint to be evolving along a path of constant luminosity, and too cool to be evolving along a fading path as appears defined by the other objects in that régime. It is possible that these objects may have excitation classes $\gtrsim 5$, but because of their overall faintness, He II $\lambda 4686 \AA$ was not detected. A similar group of objects is observed in the case of the SMC near (E.C., $\log \mathrm{F}(\mathrm{H} \beta))=(3,-14)$. 
The overall appearance of the SMC transformed H-R diagram is less striking due to the smaller number of $\mathrm{PNe}$ observed. There is also a larger proportion of optically thin objects in this sample. The greater difficulty in trying to model these objects translates to a larger error in trying to place these objects in an H-R diagram. Specifically, errors increase as the optical depth in the nebula decreases (Dopita \& Meatheringham 1991b). From past studies, optically thin objects have been observed over a broad region of the H-R diagram, and so no clear conclusions can be made on their evolutionary characteristics.

In figures $5.10-5.11$ we plot the correlation between $\mathrm{T}_{\text {eff }}$ and the excitation class for the LMC and SMC, respectively. Measurements from Papers I and II have been included. This correlation is the basis for the energy balance method used in determining the stellar temperature (Stoy 1933; Kaler 1976; Preite-Martinez \& Pottasch 1983). Looking at the LMC case, the correlation between the two quantities for optically thick objects is evident (Dopita \& Meatheringham 1991a). Optically thin objects appear to fit the observed correlation for optically thick objects quite well. Two effects are immediately obvious which appear to detract from the correlation. Firstly, in the high excitation class régime, the type I objects span a considerable range in $\mathrm{T}_{e}([\mathrm{O} \mathrm{III}])$. More specifically, type I objects from this study appear to span a large range in $\mathrm{T}_{e}([\mathrm{O} \mathrm{III}])$, whereas type I objects from Paper II are concentrated towards high excitation class and high $\mathrm{T}_{e}([\mathrm{O} \mathrm{III}])$. Secondly, low excitation optically thick objects from this study appear to significantly broaden the correlation between the two quantities, PNe being hotter for a given excitation class. Both of these results are most likely the result of changing ionisation parameter. Our sample contains a large fraction of evolved, low density $\mathrm{PNe}$ for which the central star luminosity has fallen by a factor of perhaps 50 . If the fall in stellar luminosity and/or the increase in radius is sufficient, then the ionisation parameter will fall, resulting in a nebula of lower excitation but similar temperature. The broadening in the correlation may also be caused by lower metal abundance in the nebula if the faint PNe are drawn from a lower mass (older) population.

Again, as with figure 5.9, figure 5.11 for PNe in the SMC suffers from a lack of data points. For the optically thick objects, a similar correlation as in the LMC case is apparent between the two quantities. Also similarly, 4 of the 5 type I objects observed in the $\mathrm{SMC}$ are positioned in the high $\mathrm{T}_{e}([\mathrm{O} \mathrm{III}])$ régime. This is probably a selection effect, as [O III] $\lambda 4363 \AA$ is harder to detect. The effect is not so prominent in figure 5.9. There is a noticeable lack of high excitation objects, in general, which seems to support the observations of Feast(1968) and Webster(1976) of the LMC and SMC having 
different excitation distributions. However, the present data seems insufficient to make such a strong statement.

The dereddened [N II] $\lambda 6583 \AA$ and He II $\lambda 4686 \AA$ line fluxes are plotted against each other in figures $5.12-5.13$, for the LMC and SMC cases, respectively. Optically thin objects have negligible [N II] emission. Type I objects, with strong [N II] emission, form a clear subsample. Note that the point types of this subsample represent PNe from this study and Paper II, which specifically looked at fainter objects than in Paper I. This implies that these type I objects have larger PNNi, and are consequently observed further along their evolutionary tracks due to their faster evolution. It is also interesting to note the broader range in $[\mathrm{N}$ II] intensities for $I$ (He II) $\lesssim 50$, introduced by objects in this sample. This suggests that these objects typically have a lower ionisation parameter which results in low ionisation lines becoming stronger (Russell \& Dopita 1990). This raises the possibility we have misclassified some PNe as type I objects.

Of the PNe observed in the LMC, $23 \%$ are classified as type I. In the SMC case, this figure is $16 \%$. It is known that the ratio $\lambda 6583 \AA / \mathrm{H} \alpha$ is generally smaller for the SMC than the LMC (Sanduleak et al. 1972). This result is important in tracing the chemical evolution in the Magellanic Clouds, and suggests two possibilities: either the SMC was always deficient in N, or that some process, more efficient in the SMC than the LMC, has destroyed some fraction of the $\mathrm{N}$ originally present. Currently, it is believed that the $\mathrm{N}$ and $\mathrm{He}$ enhancements in type I objects arise from the second and third dredge-up episodes which take place as the progenitors climb the asymptotic giant branch (hereafter, AGB; Renzini 1979; Renzini \& Voli 1981; Iben \& Renzini 1983). The second dredge-up phase occurs for AGB stars with $M \gtrsim 3 M_{\odot}$, and results in $\mathrm{N}$ and He enhancements. The third dredge-up phase occurs during the thermal pulsing phase when hydrogen and helium shell burning sources alternate in providing the primary energy source for the stars. The result is that $\mathrm{C} / \mathrm{O}$ and $\mathrm{He} / \mathrm{H}$ increase as $\mathrm{N} / \mathrm{O}$ decreases. However, for stars in this mass range, deep envelope convection is believed to bring the nitrogen produced from hot-bottom burning to the surface, and thus increase the surface N/O value (Kaler \& Jacoby 1989; Perinotto 1990; Walton et al. 1990). The lower incidence of type I objects in the SMC may be indicative of deep envelope convection not being as efficient as in the more metal-rich environment of the LMC.

This study has obtained fairly accurate spectrophotometry for the faint PNe in the Magellanic Clouds. Photoionisation modelling will allow us to trace the evolution in terms of temperature, luminosity, and composition. Many recent studies have shown 
that these faint $\mathrm{PNe}$ are predominantly associated with the more massive progenitors, and are important in placing constraints on stellar evolutionary theory. 


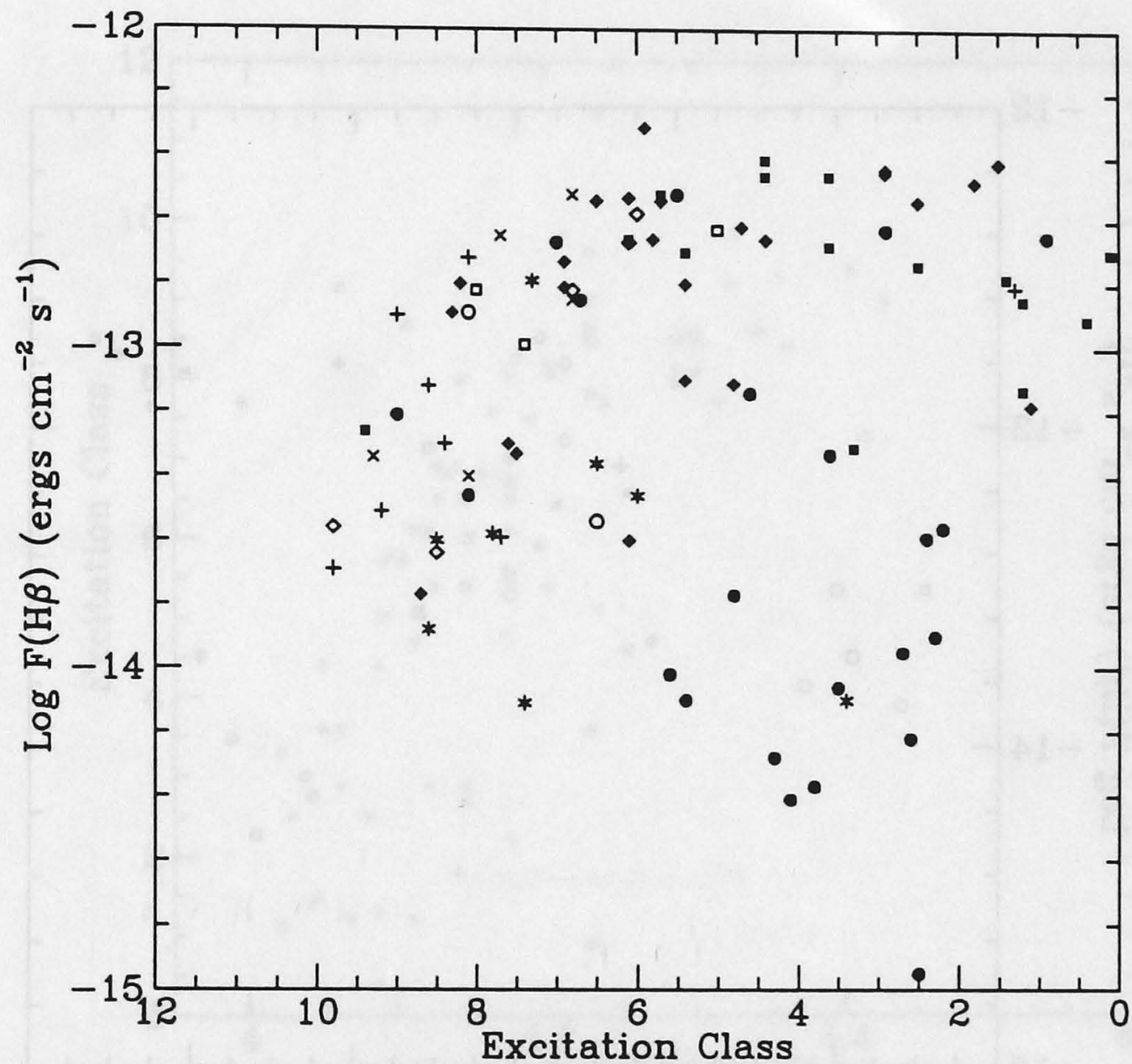

Figure 5.8: The transformed H-R diagram for the LMC PNe in terms of the nebular quantities of excitation class and nebular $\mathrm{H} \beta$ flux. One can see the horizontal part of the evolution up to excitation class $\sim 6$, and the beginning of the fading part in the excitation class range 6-10. There are nine point types defined as follows: from Paper I, ( $\square)$ optically thick, $(\square)$ optically thin, and $(\times)$ type I; from Paper II, $(\diamond)$ optically thick, $(\diamond)$ optically thin, and $(+)$ Type I; and from this study, $(\bullet)$ optically thick, $(\circ)$ optically thin, and (*) type I. See text for definitions of classifications. 


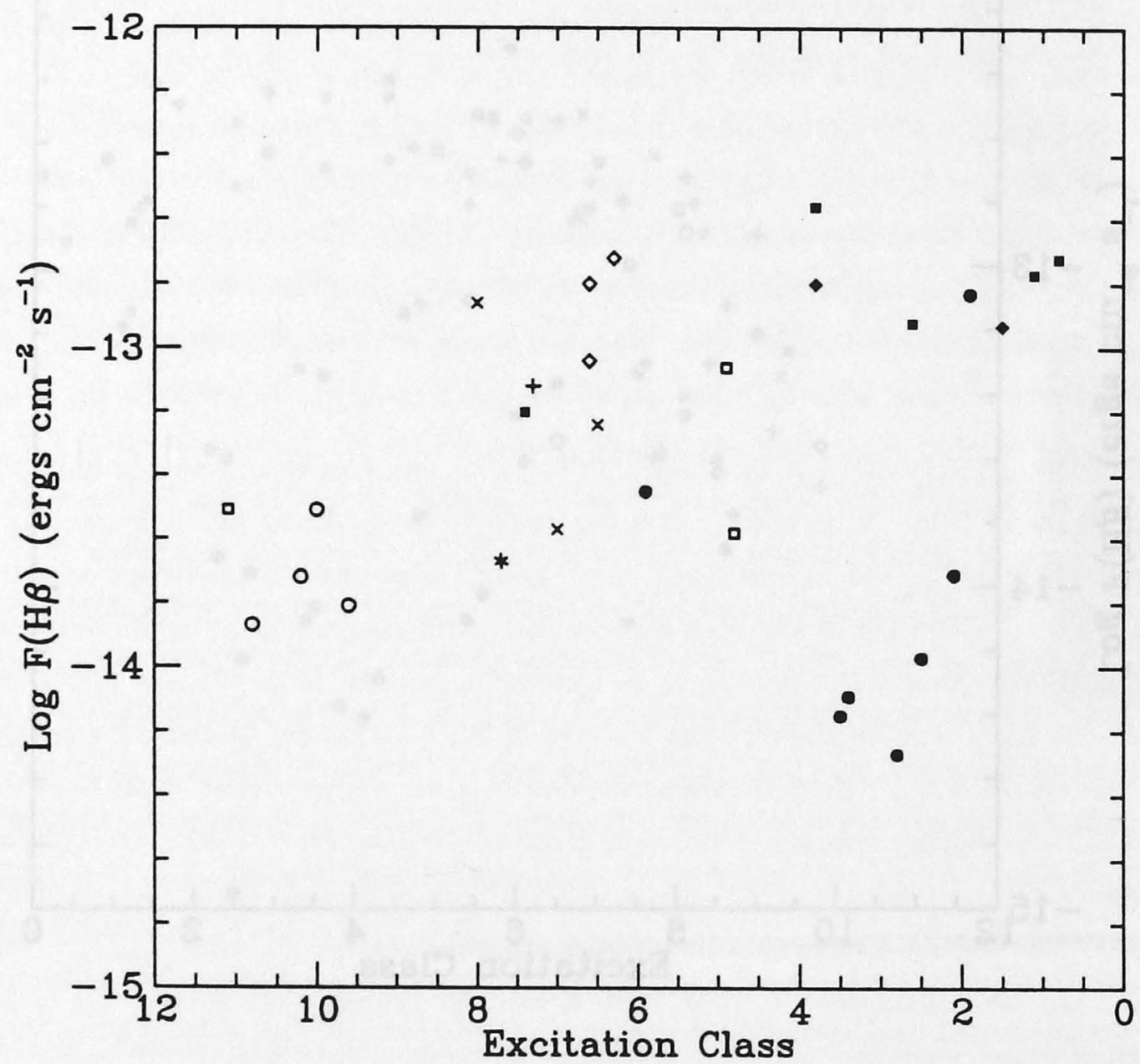

Figure 5.9: Same as figure 5.8 for objects in the SMC. The evolutionary sequence here is not so clear, primarily due to the smaller number of objects, and a larger fraction of optically thin objects. 


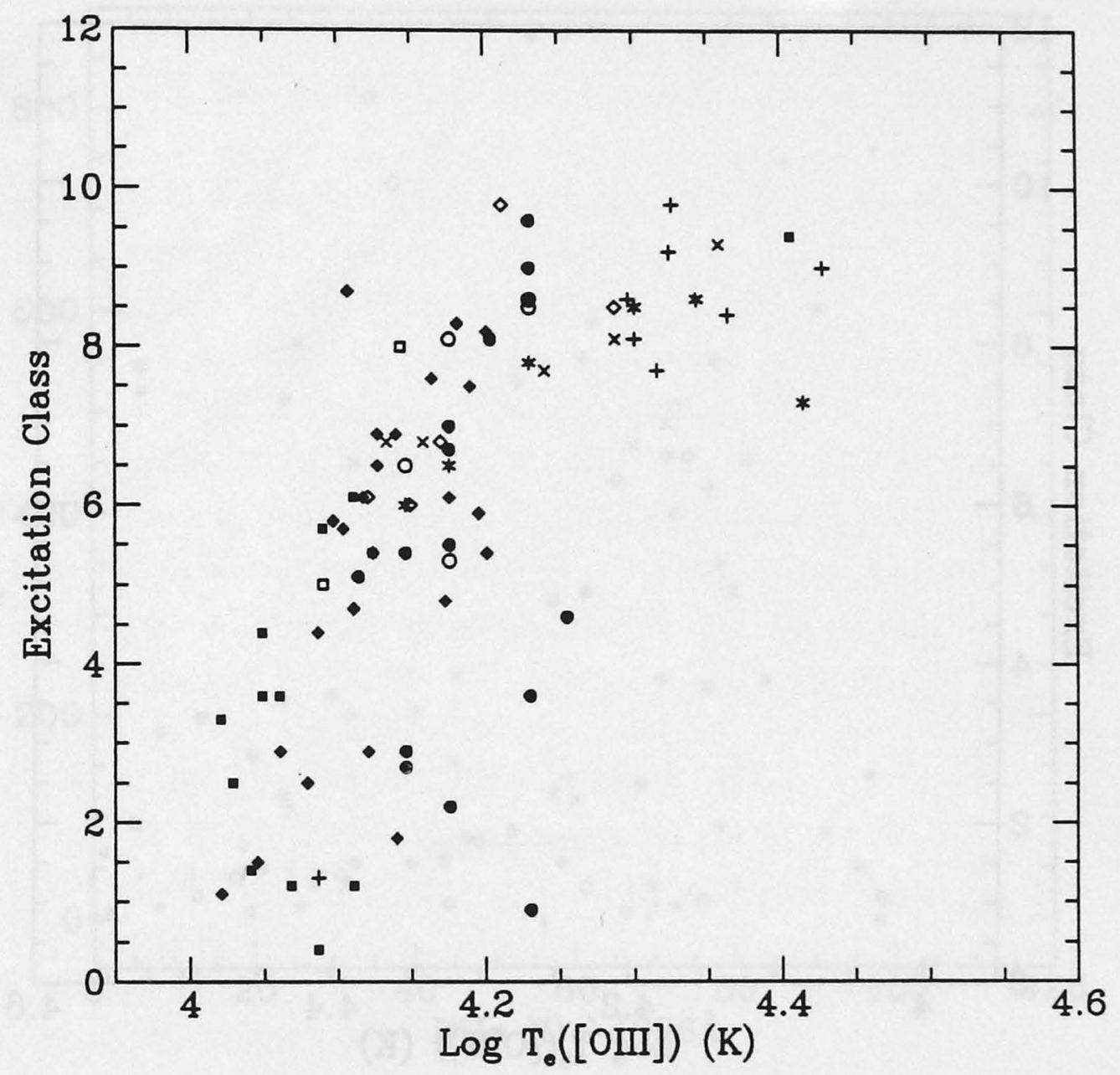

Figure 5.10: Electron temperature of the LMC PNe, calculated from the [O III] line ratio, plotted against the excitation class. Point types are as defined in fig. 5.8. The relationship appears linear, especially if the optically thin objects in the range $4.3 \lesssim$ $\log \mathrm{T}_{e} \lesssim 4.4$ are ignored. Also, the low excitation-high $\mathrm{T}_{e} \mathrm{PNe}$ are indicative of a lower ionisation parameter for these objects. 


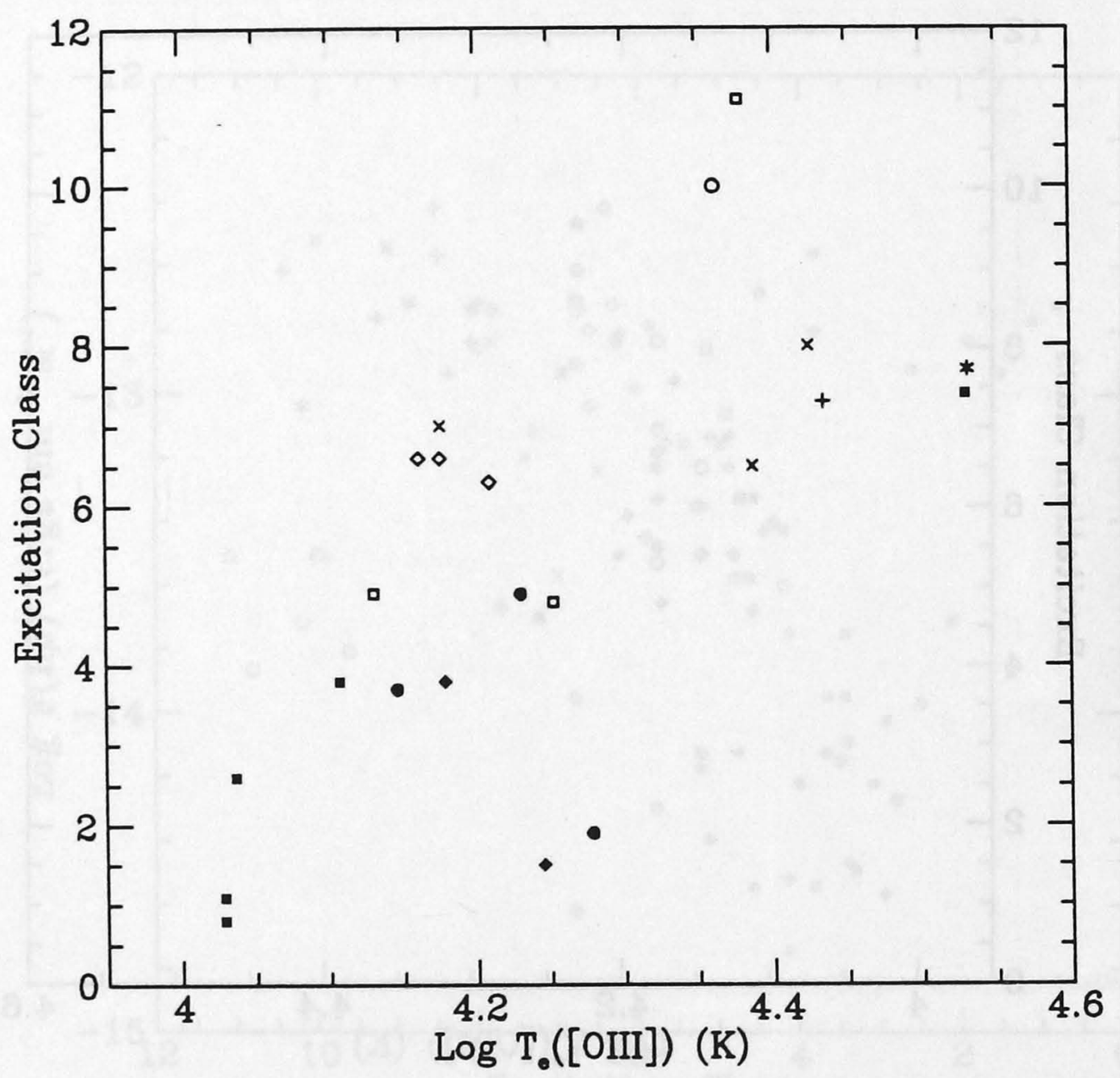

Figure 5.11: Same as figure 5.10 for objects in the SMC. Despite the lack of points, there appears to be more scatter as one moves to higher excitation class and higher $\mathrm{T}_{e}$. However, it should be noted that the majority of points in this region are optically thin. 


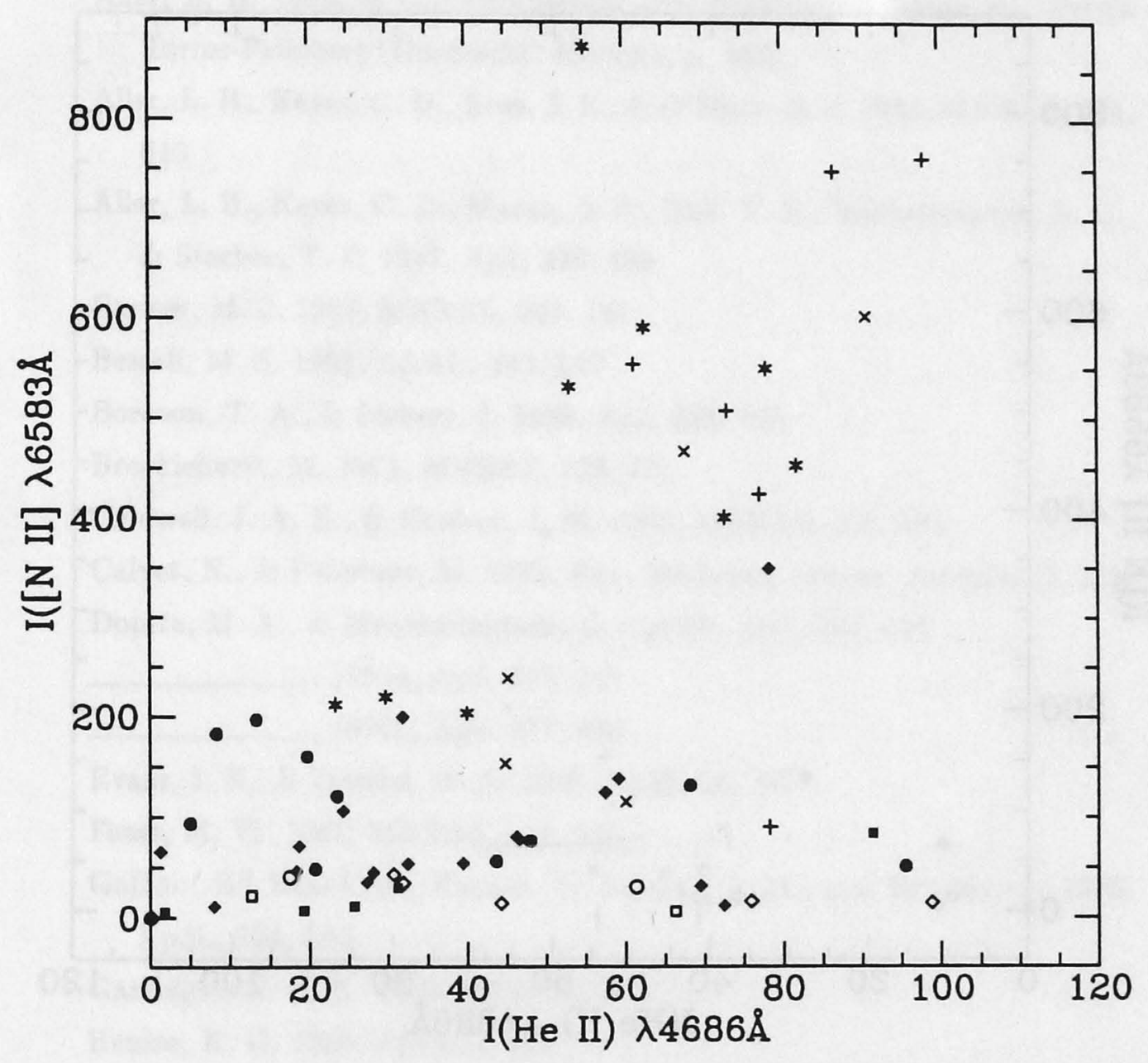

Figure 5.12: Plot of the He II $\lambda 4686 \AA$ line intensity against that of [N II] $\lambda 6583 \AA$ for $\mathrm{PNe}$ in the LMC. Point types are as defined in fig. 5.9. Objects with very strong [N II] emission form a clear subsample. 


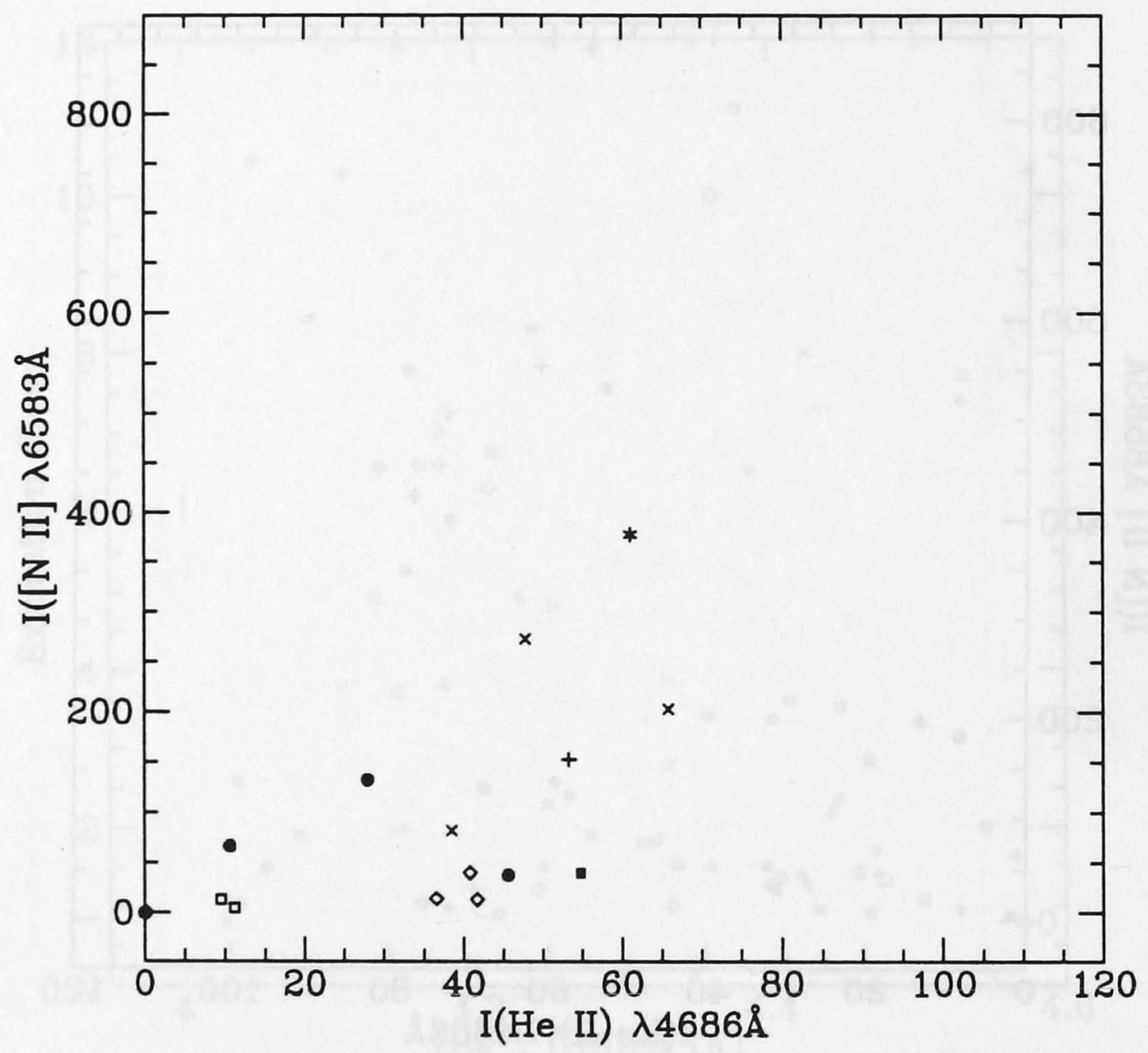

Figure 5.13: Same as figure 5.12 for objects in the SMC. Type I objects are seen towards higher He II line strengths but of the small number of objects denoted, none appear for He II line strengths $\gtrsim 70$. 


\subsection{References}

Acker, A., Köppen, J. Stenholm, B., Raytchev, B. 1991, ApJS, 89, 237

Aller, L. H. 1983, ApJ, 273, 590

Aller, L. H. 1989, in IAU Symposium 131, Planetary Nebulae, ed. Sylvia Torres-Peimbert (Dordrecht: Kluwer), p. 567.

Aller, L. H., Keyes, C. D., Ross, J. E., \& O'Mara, B. J. 1981, MNRAS, 194, 613

Aller, L. H., Keyes, C. D., Maran, S. P., Gull, T. R., Michalitsianos, A. G., \& Stecher, T. P. 1987, ApJ, 320, 159

Barlow, M. J. 1987, MNRAS, 227, 161

Bessell, M. S. 1991, A\&AL, 242, L17

Boroson, T. A., \& Liebert, J. 1989, ApJ, 339, 844

Brocklehurst, M. 1971, MNRAS, 153, 471

Caldwell, J. A. R., \& Coulson, I. M. 1986, MNRAS, 218, 223

Calvet, N., \& Peimbert, M. 1983, Rev. Mexicana Astron. Astrofis., 5, 319

Dopita, M. A., \& Meatheringham, S. J. 1990, ApJ, 357, 140 , 1991a, ApJ, 367, 115 1991b, ApJ, 377, 480

Evans, I. N., \& Dopita, M. A. 1985, ApJS, 58, 125

Feast, M. W. 1968, MNRAS, 140, 345

Gallino, R., Busso, M., Picchio, G. Raiteri, C. M., and Renzini, A. 1988, ApJL, 334, L45

Gascoigne, S. C. B. 1969, MNRAS, 146, 1

Henize, K. G. 1956, ApJS, 2, 315

Henry, R. B. C., Liebert, J., and Boroson, T. A. 1989, ApJ, 339, 872

Hollowell, D., \& Iben, I., Jr. 1989, ApJ, 340, 966

Iben, I., Jr., \& Renzini, A. 1983, ARA\&A, 21, 271

Jacoby, G. H. 1980, ApJS, 42, 1

Kaler, J. B. 1976, ApJ, 210, 843

Kaler, J. B., \& Jacoby, G. H. 1990, ApJ, 362, 491

$$
\text { ., 1991, ApJ, 382, } 134
$$

Lindsay, E. M. 1961, AJ, 66169

1963, Irish AJ, 6, 127

Lindsay, E. M., \& Mullan, D. J. 1963, Irish AJ, 6, 51

Meatheringham, S. J., \& Dopita, M. A. 1991a, ApJS, 75, 407 1991b, ApJS, 76, 1085 
Meatheringham, S. J., Dopita, M. A., \& Morgan, D. H. 1988, ApJ, 329, 166

Monk, D. J., Barlow, M. J., \& Clegg, R. E. S. 1988, MNRAS, 234, 583

Morgan, D. H., \& Good, A. R. 1985, MNRAS, 213, 491 , 1992, A\&AS, 92, 571

Morgan, D. H. 1992, in preparation

Oke, J. B. 1974, ApJS, 27, 21

Osmer, P. S. 1976, ApJ, 203, 352

Osterbrock, D. E. 1989, in Astrophysics of Gaseous Nebulae \& Active Galactic Nuclei (University Science Books; Mill Valley), p. 118

Paczyński, B. 1971, Acta Astronomica, 21, p. 417

Peimbert, M. 1978, in IAU Symposium No. 76, Planetary Nebulae: Observations and Theory, ed. Y. Terzian (Dordrecht: Reidel), p. 215

Peimbert, M., \& Serrano, A. 1980, Rev. Mexicana Astron. Astrof., 5, 9

Peimbert, M., \& Torres-Peimbert, S. 1983, in IAU Symposium No. 103, Planetary Nebulae, ed. D. R. Flower (Dordrecht: Reidel), p. 233

Perinotto, M. 1990, ApJS, 76, 687

Preite-Martinez, A., \& Pottasch, S. R. 1983, A\&A, 126, 31

Pottasch, S. R. 1984, in Astrophys. \& Space Sci. Lib., vol 107, Planetary Nebulae (Dordrecht: Reidal), p. 95

Renzini, A. 1979, in IAU 4th European Regional Meeting in Astronomy,

Stars \& Star Systems, ed. B. E. Westerlund (Dordrecht: Reidel), p. 155

Renzini, A., \& Voli, M. 1981, A\&A, 94, 175

Rodgers, A. W., Conroy, P., \& Bloxham, G. 1988, PASP, 100, 626

Rodgers, A. W., van Harmalen, J., King, D., Conroy, P., \& Harding, P. 1988, PASP, 100, 841

Russell, S. C., \& Dopita, M. A. 1990, ApJS, 74, 93

Sanduleak, N. 1984, in IAU Symp. 108, in Structure and Evolution of the Magellanic Clouds, eds. S. van der Bergh, \& K. S. de Boer (Dordrecht: Reidel), 231

Sanduleak, N., MacConnell, D. J., \& Philip, A. G. D. 1978, PASP, 90, 621

Sanduleak, N, \& Pesch, P. 1981, PASP, 93, 431

Schönberner, D. 1981, A\&A, 103, 119

Stone, R. P. S., \& Baldwin, J. A. 1983, MNRAS, 204,347

Stoy, R. H. 1933, MNRAS, 93, 588

Walton, N. A., Barlow, M. J., Monk, D. J., \& Clegg, R. E. S., in IAU Symposium No. 148, The Magellanic Clouds, eds. R. Haynes \& D. 
Milne (Dordrecht: Reidel), p. 334

Webster, B. L. 1976, MNRAS, 174, 513

Westerlund, B. E., \& Smith, L. F. 1964, MNRAS, 127, 449

Whitford, A. E. 1958, ApJ, 63, 201

Wood, P. R., Bessell, M. S., \& Fox, M. W. 1983, ApJ, 272, 99

Wood, P. R., Bessell, M. S., \& Dopita, M. A. 1986, ApJ, 311, 632

Wood, P. R., \& Faulkner, D. J. 1986, ApJ, 307, 659

Wood, P. R, Meatheringham, S. J., Dopita, M. A., \& Morgan, D. H. 1987, ApJ, 320, 178 


\section{Chapter 6}

\section{The Kinematics of Planetary Nebulae in the Outer Fields of the Large Magellanic Cloud}

\subsection{Introduction}

Planetary Nebulae (PNe) are believed to form from low to intermediate mass stars which undergo a mass-loss process as they evolve up, and off, the asymptotic giant branch (AGB). As such a star evolves to higher temperatures after leaving the AGB, it becomes hot enough to ionize the expanding shell of material surrounding it. The emission-like nature of the resultant $\mathrm{PNe}$ makes them excellent test objects for studying far-off systems, such as the Galactic Centre and other galaxies.

Although the study of their precise behaviour is an ongoing process, substantial progress has been made in the past decade in trying to understand these objects (see for example IAU Symposium 131, 1989). Observations of Galactic PNe have been hampered by reddening and distance scale problems. The Magellanic Clouds offer an ideal opportunity to observe a group of $\mathrm{PNe}$ which form a large luminosity-limited sample at a common and externally determined distance.

Naturally, a major concern is that the Magellanic Clouds are not an isolated, dynamically undisturbed system. There has been considerable research into the area of tidal interaction between the Magellanic Clouds and with the Galaxy. Simulations by Murai and Fujimoto (1980) suggest that a major event may have occurred as late as $\sim 10^{8}$ years ago. Various kinematical studies have lent support to this theme. H I surveys of the region clearly show some complex form of interaction (Mathewson \& Ford 
1984, \& references therein; Rohlfs et al. 1984, Mathewson, Ford \& Visvanathan 1986).

The Small Magellanic Cloud (SMC) has a rather confusing radial velocity field as seen in the $21 \mathrm{~cm}$ line of $\mathrm{HI}$, characterized by a double peak structure with a velocity splitting of $30-40 \mathrm{~km} \mathrm{~s}^{-1}$ (Hindman 1967; McGee \& Newton 1981; Bajaja \& Loiseau 1982; Mathewson \& Ford 1984). The PNe in the SMC, which offer an older subsystem to study than the gas, seem to indicate that the SMC was subject to some tidal interaction, and that the stellar and gaseous components differed in response to the event (Dopita et al. 1985).

The situation in the LMC is not so clear and has consequently earned a much more intense investigation (see Westerlund 1991 for an overview). Recent kinematical studies have been carried out using the HI gas (Rohlfs et al. 1984; Rohlfs \& Luks 1991), CO molecular clouds (Cohen et al. 1988), PNe (Meatheringham et al. 1988; hereafter MDFW; Meatheringham 1991), CH stars (Hartwick \& Cowley 1988, 1991; Cowley \& Hartwick 1991), young and old clusters (Freeman, Illingworth, \& Oemler 1983 (hereafter, FIO); Freeman 1984; Olszewski et al. 1988; Schommer 1991), and long period variables (Hughes, Wood, \& Reid 1990, 1991).

To summarize the above work: The oldest long period variables (periods $=100$ to 225 days) have flattened-spheroidal kinematics; and, the old FIO clusters and $\mathrm{CH}$ stars yield a different rotation solution to the other remaining studies-the difference in position angle of the line of nodes being $49^{\circ}$, and systemic velocity being lower by approximately $18 \mathrm{~km} \mathrm{~s}^{-1}$.

The original cluster data of FIO has been recently reanalysed, yielding new ages and velocities, rectifying the discrepancy in systemic velocity, but not position angle of the line of nodes (Olszewski et al. 1988; Schommer 1991). With regards to the $\mathrm{CH}$ stars, these objects may be younger than originally proposed (Hughes, Wood \& Reid 1991).

Early studies of PNe in the LMC have yielded radial velocities for a total of $35 \mathrm{PNe}$ (Feast 1968; Webster 1969; Smith \& Weedman 1972). Recently, MDFW carried out an extensive kinematical study of the LMC using new data for $94 \mathrm{PNe}$. They found the PNe population forms a flattened disk with an almost identical rotation solution to that of the H I. Following the reasoning used by Schommer (1991), it may be instructive to study the kinematics of a sample of PNe in the outer fields of the LMC. Our sample, which consists of 11 new objects, offers no better statistics than the old cluster group used by FIO and Schommer (1991). Consequently, we have combined the observations 
presented here with those of MDFW to see whether their rotation solution still holds in the outer disk.

\subsection{Observations and Data Reduction}

\subsubsection{Selection of Objects}

Our sample consists of 16 objects recorded by Morgan (1992) on an UK Schmidt telescope objective prism survey of the outer fields of the LMC. The extensive identification procedure used to find PNe candidates is described in Morgan and Good (1992). Of these 16 objects, 5 have been identified as belonging to the list of Sanduleak, McDConnell, and Phillips (1978; hereafter SMP). One other object, listed as A4 in MDFW, is object No. 47 in table 2 of Morgan and Good (1992). The remaining 10 objects are classified as new. Optical spectroscopy exists for these objects and is published elsewhere (Vassiliadis et al. 1992).

The spatial distribution of this sample is depicted in figure 6.1. The original MDFW sample, totalling $94 \mathrm{PNe}$, is also shown.

\subsubsection{The Observations}

The observations were obtained in 1990 November using the 2.3-meter Advanced Technology Telescope at Siding Spring Observatory, operated by the Australian National University. The spectrograph was a Perkin-Elmer échelle with a 79 line $\mathrm{mm}^{-1}$ échelle grating, and a 316 line $\mathrm{mm}^{-1}$ cross disperser blazed at $7500 \AA$. The detector used was an uncoated, $416 \times 578$ pixel, GEC CCD. In conjunction with a slit width of $150 \mu \mathrm{m}$, the whole system gave a resolution (FWHM) of $11.5 \mathrm{~km} \mathrm{~s}^{-1}$ at the [O III] $\lambda 5007 \AA$ line. In comparison to the Photon Counting Array (PCA) detector system used by MDFW, which gave a sampling rate of $0.083 \AA$ pixel $^{-1}$, the CCD offered a poorer sampling rate of $0.116 \AA$ pixel $^{-1}$. Exposure times varied from 400 to 2000 seconds. The raw detections are presented in figure 6.2.

\subsubsection{Reduction Procedure}

The data were reduced using version 2.9.1 of the Image Reduction Analysis Facility (IRAF). The standard CCD reduction techniques of dividing through by a mean flatfield and a median bias frame were carried out. Nebular observations were interspersed 


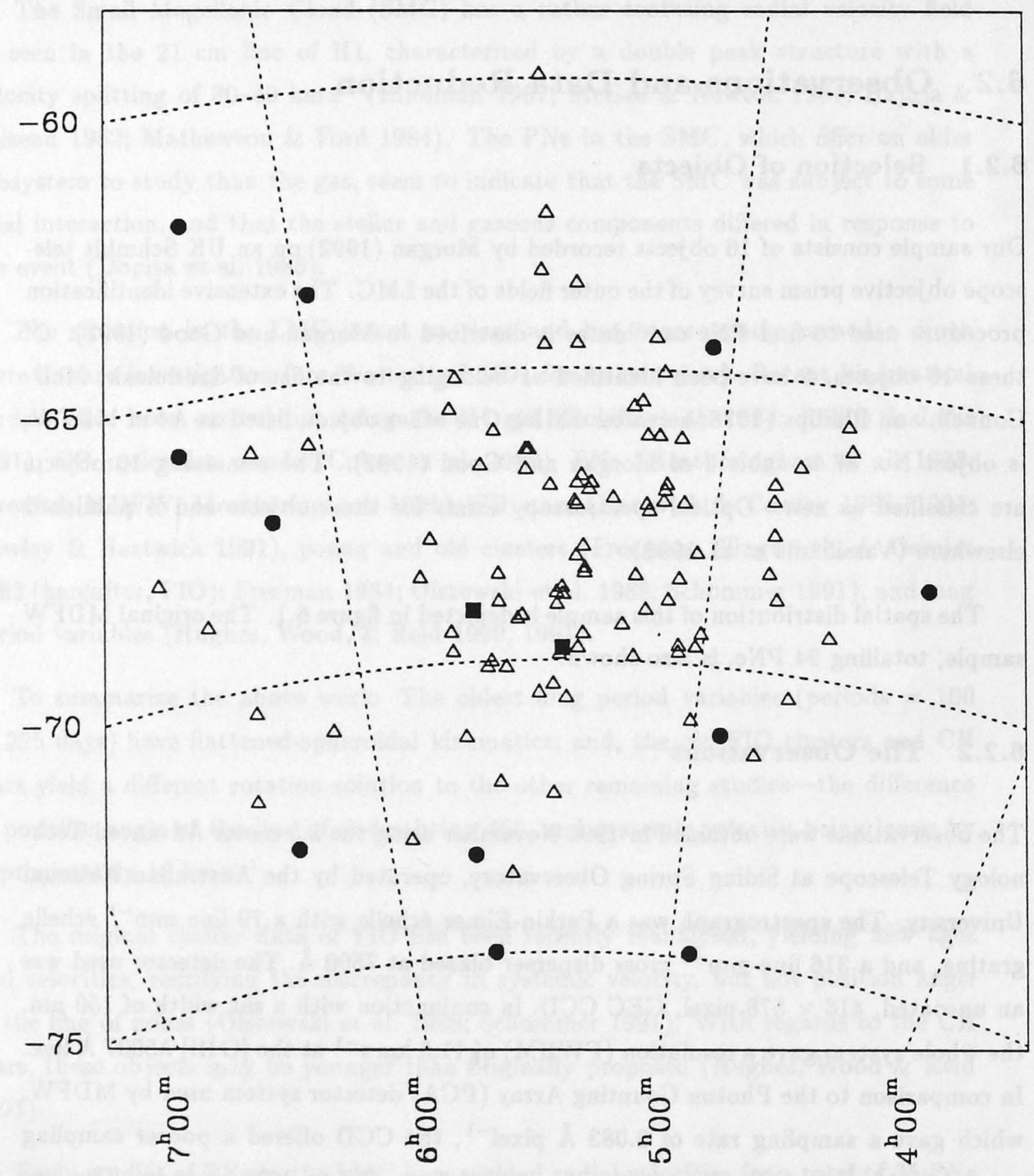

Figure 6.1: The spatial distribution of PNe across the face of the LMC. Filled circles represent the new $\mathrm{PNe}$ presented in this paper and the open triangles represent the MDFW sample. The filled box at centre represents the optical optical centre of the LMC, with the position of 30 Dor indicated by the same symbol to the left of it. 
with Thorium-Argon arc lamp exposures throughout the observing schedule to record any instrumental drifts. In general, every three nebular observations were bracketed with arc lamp exposures.

The CCD was orientated such that the dispersion axis coincided with the long axis of the detector. The resultant spectral lines from test arc exposures were thus parallel to the short axis of the CCD. The exact orientation of the detector was accomplished by trial-and-error using arc exposures, until the FWHM of the spectral lines were minimised in the region where [O III] $\lambda 5007 \AA$ was expected to be observed. No further straightening of the orders was attempted in the reduction procedure.

The echelle order to be used covered approximately $65 \AA$ : from $4975 \AA$ to $5042 \AA$. Because of the slanting pattern of the echelle orders across the face of the CCD, the central, uncontaminated portion of the order required was extracted, being 200 pixels in length. All spatial increments having appreciable signal were reduced to one-dimensional form.

Treating this reduced portion of the CCD as essentially being linear, the standard deviation of the wavelength fitting procedure averaged 0.09 pixels $(\sim 0.01 \AA)$, which transforms to a velocity error of $0.7 \mathrm{~km} \mathrm{~s}^{-1}$. The dispersion solutions were calculated and applied to each nebular observation. The pixel-shift between successive arc exposures was found to average 0.04 pixels $(\sim 0.005 \AA$ ), corresponding to a velocity error of $0.3 \mathrm{~km} \mathrm{~s}^{-1}$.

The [O III] profiles were fitted by a single gaussian. The resulting fits are overlayed on the corresponding spectra displayed in figure 6.2. Because of the relatively poor signal-to-noise ratio in some spectra, the application of multiple gaussian fits does not appear to be justified.

\subsubsection{Accuracy}

Combining the errors from our reduction procedure, the estimated uncertainty in the radial velocity measurements is $1.0 \mathrm{~km} \mathrm{~s}^{-1}$. Of course, there is also the added uncertainty due to photon shot noise. In MDFW, this error is represented as $\pm \sigma / N^{1 / 2}$, where $\sigma$ is the $e$-folding width of the Gaussian fit and $N$ is the number of photons in the profile (Bevington 1969). Individual errors are calculated and recorded accordingly in table 6.1. These error measurements range from 0.5 to $5.4 \mathrm{~km} \mathrm{~s}^{-1}$. The average shot noise error is $2.5 \mathrm{~km} \mathrm{~s}^{-1}$. Hence the total error in the radial velocity measurements is the tabulated shot noise error plus the inherent uncertainty in the reduction procedure 


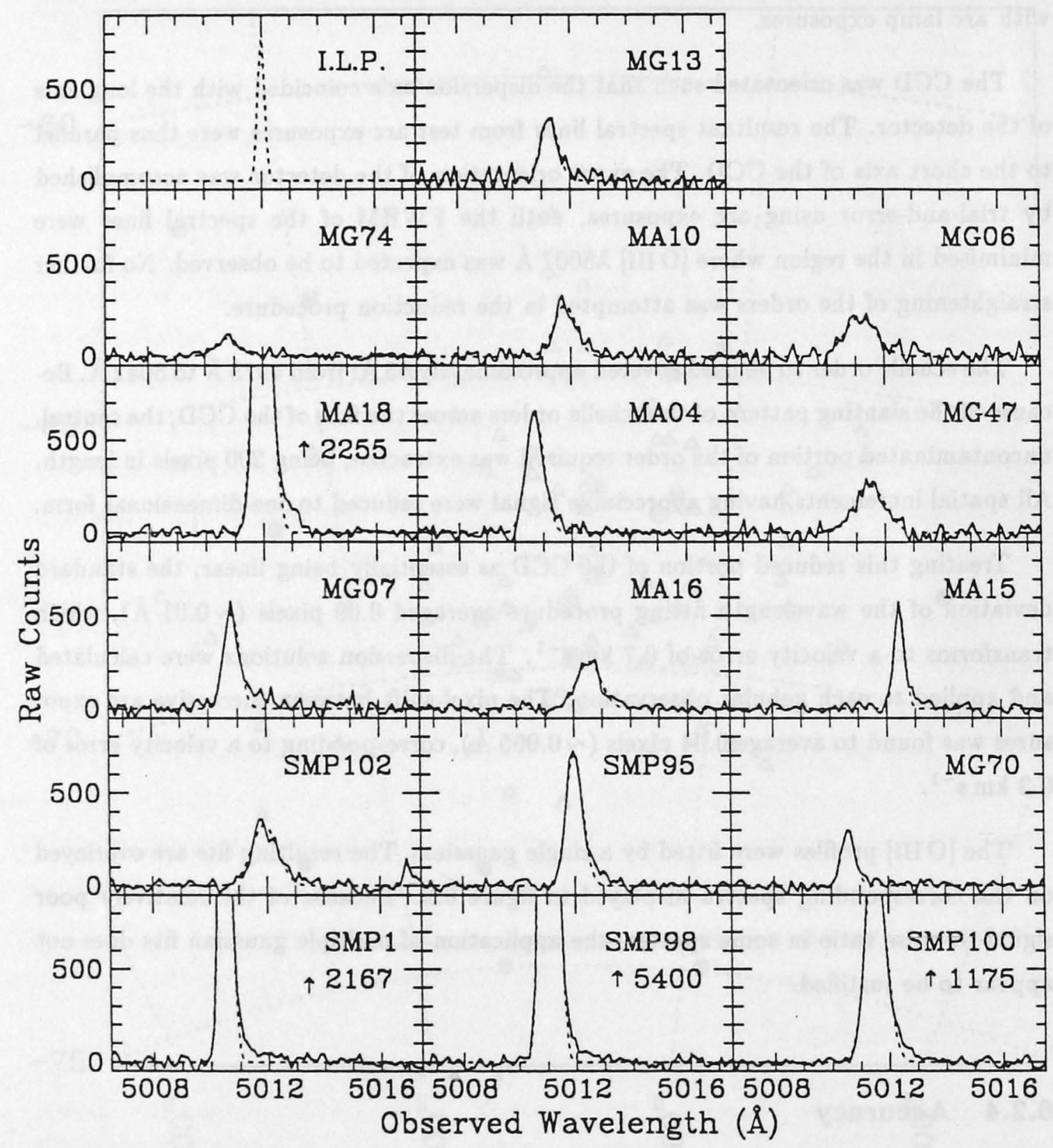

Figure 6.2: Observed [O III] emission line profiles for the sample of outer-field LMC $\mathrm{PNe}$ (solid lines). Gaussian fits to the observed profiles are superimposed as dotted lines. The identification of each object is indicated in the top right hand corner of each paner. For those profiles which do not fit on the scale used, the peak number of counts is indicated below the name of the object. The instrumental line profile (I.L.P.) is shown in the top left panel. 
of $1 \mathrm{~km} \mathrm{~s}^{-1}$.

\subsection{Results}

\subsubsection{Radial Velocities}

Using the time of observation, the observed radial velocity measurements were converted to velocites in the local standard of rest (LSR) frame. These velocities were then, in turn, converted to the Galactic standard of rest (GSR) frame, assuming a canonical LSR motion of $250 \mathrm{~km} \mathrm{~s}^{-1}$ about the Galactic center.

$$
V_{G S R}=V_{L S R}+250.0 \sin \left(l^{I I}\right) \cos \left(b^{I I}\right)
$$

Since the sample of objects presented in this paper are at a relatively large distance from the LMC center (i. e., $\gtrsim 5^{\circ}$ ), a correction must be made for the transverse motion of the LMC (Feitzinger et al. 1977; Prevot et al. 1989). To be as consistent as possible with the work of MDFW, we have adopted a value of $275 \pm 65 \mathrm{~km} \mathrm{~s}^{-1}$. The corrected velocities, $\mathrm{V}_{c}$, and all of the velocities mentioned above, are presented in table 6.1.

Comparing measurements of $\mathrm{V}_{L S R}$ between this study and that of MDFW, we find a mean absolute difference of $4.7 \mathrm{~km} \mathrm{~s}^{-1}$ for the 5 coincident objects listed in table 6.2.

\subsubsection{Expansion Velocities}

Expansion velocities for the $\mathrm{PNe}$ are derived from the widths of the gaussian fits to the spectral profiles. The velocity dispersion is corrected for the instrumental half-width, assuming it adds in quadrature.

It is fortunate that all of the fits to the spectral profiles appear to be satisified by single component gaussians as this simplifies the definition of what is meant by expansion velocity. We have defined the expansion velocity, $\mathrm{V}_{\text {exp }}$, as half the full width at one-tenth maximum intensity. Thus $V_{\text {exp }}$ is equivalent to 0.911 times the measured velocity dispersion corrected for the instrumental half-width (see Dopita et al. 1985). The resultant expansion velocities for this sample of PNe are presented in table 6.3.

As a comparison between the expansion velocities obtained in this study and that of MDFW, we find a mean absolute difference of $2.6 \mathrm{~km} \mathrm{~s}^{-1}$ (table 6.3).

The object SMP102 has been excluded from the calculation of the mean absolute 
TABLE 6.1

SAmple Data ${ }^{a}$

\begin{tabular}{lccccccrr}
\hline \hline Object & $\begin{array}{c}\text { R.A. } \\
(1950.0)\end{array}$ & $\begin{array}{c}\text { Decl. } \\
(1950.0)\end{array}$ & $\log F(H \beta)$ & E.C. & $\mathrm{V}_{\text {exp }}$ & $\mathrm{V}_{L S R}$ & $\mathrm{~V}_{G C R}$ & $\mathrm{~V}_{\mathrm{c}}$ \\
\hline SMP98 & 061842.2 & -731115.6 & -12.52 & 5.5 & 24.4 & $236.8 \pm 0.5$ & 23.3 & -2.8 \\
MA04 & 042449.7 & -694907.7 & -13.27 & 9.0 & 36.2 & $211.7 \pm 1.8$ & 18.6 & 40.2 \\
MA10 & 060934.5 & -684312.9 & -13.63 & 8.4 & 47.4 & $280.6 \pm 3.2$ & 64.5 & 36.4 \\
MA15 & 061323.7 & -635947.4 & -12.65 & 0.9 & 17.4 & $320.0 \pm 1.2$ & 100.3 & 84.5 \\
MA16 & 055532.3 & -643052.2 & -13.24 & 9.6 & 52.2 & $324.7 \pm 3.5$ & 108.6 & 99.1 \\
MA18 & 062101.2 & -660629.5 & -12.65 & 7.0 & 32.6 & $292.8 \pm 0.9$ & 72.7 & 52.2 \\
MG70 & 053947.8 & -750155.0 & $\ldots$ & $\ldots$ & 25.3 & $207.9 \pm 2.1$ & 1.9 & -12.7 \\
MG07 & 045534.7 & -750431.0 & $\ldots$ & 5.1 & 32.1 & $219.3 \pm 1.9$ & 20.4 & 18.8 \\
MG74 & 054224.7 & -744238.0 & $\ldots$ & 0.9 & 49.7 & $217.2 \pm 5.4$ & 10.6 & -4.4 \\
MG13 & 050251.3 & -652710.0 & $\ldots$ & $\ldots$ & 32.8 & $247.4 \pm 2.4$ & 45.4 & 60.5 \\
MG06 & 045506.5 & -725105.0 & $\ldots$ & $\ldots$ & 67.5 & $241.2 \pm 4.3$ & 41.6 & 45.0 \\
\hline
\end{tabular}

${ }^{\mathrm{a}} \mathrm{H} \beta$ flux and excitation class (E.C.) information from Vassiliadis, Dopita \& Morgan (1992). Objects with MA prefix from Morgan(1992).

Objects with MG prefix from Morgan \& Good(1992). 
TABLE 6.2

LSR VELOCITY COMPARISON ${ }^{a}$

\begin{tabular}{lcc}
\hline \hline Object & $\begin{array}{c}\mathrm{V}_{\text {LSR }} \\
\text { (MDFW) }\end{array}$ & $\begin{array}{c}\mathrm{V}_{\text {LSR }} \\
\text { (This Study) }\end{array}$ \\
\hline SMP 1 & 209.1 & 200.1 \\
SMP 95 & 290.9 & 292.2 \\
SMP 100 & 270.1 & 264.6 \\
SMP 102 & 286.5 & 288.8 \\
A 4 & 254.2 & 259.3 \\
Mean absolute difference $=4.7$ \\
\hline \multicolumn{2}{c}{ All measurements in $\mathrm{km} \mathrm{s}^{-1}$. }
\end{tabular}

TABLE 6.3

EXPANSION Velocity CoMPARISON ${ }^{\text {a }}$

\begin{tabular}{lcc}
\hline \hline Object & $\begin{array}{c}\mathrm{V}_{\text {exp }} \\
\text { (MDFW) }\end{array}$ & $\begin{array}{c}\mathrm{V}_{\text {exp }} \\
\text { (This Study) }\end{array}$ \\
\hline SMP 1 & 17.2 & 17.0 \\
SMP 95 & 31.2 & 34.1 \\
SMP 100 & 46.4 & 41.8 \\
SMP 102 & 41.2 & $56.9^{\mathrm{b}}$ \\
A 4 & 68.8 & 66.0 \\
Mean absolute difference $=2.6$ \\
\hline all measurements in km s ${ }^{-1}$. \\
'
\end{tabular}


difference. Upon closer inspection, the [O III] $\lambda 5007 \AA$ profile of this object may be treated as having two components. Assuming the FWHM for each component is equal, we derive $\mathrm{V}_{\text {exp }}=42.3 \mathrm{~km} \mathrm{~s}^{-1}$, a value more in agreement with the MDFW measurement. Meatheringham has confirmed the two component structure for the profile of this object: The components are separated by $30 \mathrm{~km} \mathrm{~s}^{-1}$ and have an amplitude ratio of 5:1.

We must stress that this two component identification was only accomplished in hindsight, as our resolution is too low to be definitive in such cases. The possibility remains that some of the broad, low signal-to-noise profiles may, in fact, be made up of two components.

\subsubsection{Rotation Solution}

With this information in hand, it remains to derive the rotation solution as given by equation 2 of MDFW. The procedure is to minimize the residuals in this equation using a nonlinear $\chi^{2}$-minimization routine from the software package MINUIT (James $\&$ Roos 1975). Two free parameters need to be minimized with respect to the velocity residuals: The position angle of the kinematic line of nodes, $\theta_{0}$, and the Galactocentric velocity of the LMC, $V_{0}$.

Three rotation solutions were calculated: The first corresponds to the 11 new PNe; The second uses the whole of the combined sample, and the third corresponds to all the PNe in the combined sample greater than $4^{\circ}$ from the LMC center. The results are presented in table 6.4, together with the original solution from MDFW. All results are identical within the errors.

\subsection{Discussion}

The question of whether the second, old, disk (FIO) exists still has no definitive answer. However, the results presented here, and the results of other kinematical studies in recent years appear to be making the existence of such a structure dubious. As stated by FIO, a two-disk structure is dynamically unstable and could be no older than $\sim 1$ Gyr.

Based on the work of Wielen (1977), relating the observed velocity dispersion of various disk populations in the Galaxy and their age, it is believed that the PNe, $\mathrm{CH}$ stars, intermediate age (225 to 450 days) long period variables, and old cluster populations in the LMC have ages of the order of $\sim 4$ Gyrs (Hughes, Wood \& Reid 
1991). If this is the case, then this intermediate age population precludes the possibility that any twisting has taken place due to tidal torques initiated by a recent ( $\sim 0.2 \mathrm{Gyrs}$ ago) close encounter of the LMC with the SMC. Any change in kinematics must have taken place at least 4 Gyrs ago.

Our data appear to support the rotation solution derived by MDFW. Our sample of 11 objects is an effective increase in the MDFW sample of approximately $11 \%$. More importantly, since our sample predominantly falls at distances greater than approximately $5^{\circ}$ from the optical center of the LMC, we are essentially adding weight to the outer fields of the LMC when deriving our rotation solution. Ignoring the velocity dispersions, we can state that no significant difference exists between the $\mathrm{HI} P \mathrm{PNe}$ kinematics, following from the analysis made by MDFW for their sample.

Referring to the results in table 6.4, it is obvious there are no significant differences between the three rotation solutions. Firstly, the solution based on only the 11 new PNe should be treated with caution because the sample is so small. The other two solutions show that the same rotation solution is stable from the center of the LMC to approximately $8^{\circ}$ radius (the maximum extent of the combined sample).

Our results seem to yield no support for the findings of FIO, Schommer (1991) and Cowley and Hartwick (1991), that there exists an old age component ( $\gtrsim 1 \mathrm{Gyr}$ ) with a rotation axis inclined $\sim 50^{\circ}$ to the young and intermediate populations. Since the work of FIO, several of the old clusters have had their ages redetermined to $\lesssim 4$ Gyrs (Jensen et al. 1988; Frantsman 1988; da Costa 1990, 1991). The reanalysis by Schommer (1991) has also shown that some published velocities suffer from systematic errors $(\sim$ $18 \mathrm{~km} \mathrm{~s}^{-1}$ ). With this correction, the systemic velocities agree for all clusters but the line of nodes is still rotated for the oldest clusters ( $\gtrsim 10 \mathrm{Gyrs})$. This constitutes such a small sample ( $\sim 10$ objects) that little can be said conclusively with this data. It should also be pointed out that the new clusters found by Olszewski et al. (1988), beyond the boundary defined by the Hodge and Wright atlas (1967), are heavily concentrated north and south of the bar (see figure 2, Olszewski et al. 1988). The spatial distribution of the $\mathrm{PNe}$ studied in this paper give a relatively more uniform spatial distribution across the face of the LMC.

The similarity in the CH star rotation solution (Hartwick \& Cowley 1988, 1991; Cowley \& Hartwick 1991) with the FIO cluster result suggests that the ages of the two populations are comparable. That is, the $\mathrm{CH}$ star population in the LMC is younger than its analagous Galactic counterpart. The reanalysis by Hughes, Wood and Reid 
(1991) proposed that the $\mathrm{CH}$ stars in the LMC could be similar to the young, $\mathrm{CH}$-like stars, observed by Yamashita (1975) to have disk kinematics. For the inner $2^{\circ}$ field, these authors find that the only disagreement between the populations is in the lower systemic velocities of the $\mathrm{CH}$ stars and also the FIO clusters. However, the uncertainty introduced by small sample statistics cannot be avoided. Also, as stated before, the reanalysis by Schommer (1991) found that several of the FIO cluster velocities suffered from systematic errors.

A main factor in studies of this nature, which is clearly evident from the literature, is that a definitive statement cannot be made about the outer-field kinematics when the samples considered only consist of about 10 objects. The work presented here has been combined with previous $\mathrm{PN}$ observations to yield an important result which has proved to be allusive in the past. 
TABLE 6.4

Rotation Solutions

\begin{tabular}{lcll}
\hline \hline Sample & $\mathrm{N}^{\mathrm{a}}$ & $\mathrm{V}_{0}{ }^{\mathrm{b}}$ & $\theta_{0}{ }^{\mathrm{c}}$ \\
\hline New PNe & 11 & 40 & 164 \\
New + MDFW PNe & 106 & 42 & 168 \\
New + MDFW PNe $\left(\mathrm{R} \geq 4^{\circ}\right)$ & 30 & 46 & 170 \\
MDFW PNe & 95 & 42 & 170 \\
\hline
\end{tabular}

${ }^{a}$ Number of objects.

${ }^{\mathrm{b}} \mathrm{In} \mathrm{km} \mathrm{s}^{-1}$.

${ }^{\mathrm{c}}$ In degrees. 


\subsection{References}

Bajaja, E., \& Loiseau, N. 1982, A\&AS, 48, 71

Bevington, P. R. 1969, in Data Reduction and Error Analysis in the Physical Sciences (New York: McGraw-Hill)

Cohen, R. S., Dame, T. M., Garay, G., Montani, J., Rubio, M., Thaddeus, P. 1988, ApJL, 331, L95

Cowley, A. P., \& Hartwick, F. D. A. 1991, ApJ, 373, 80

da Costa, G. S. 1991, in IAU Symp. 148, The Magellanic Clouds, ed. R. F. Haynes \& D. K. Milne (Dordrecht: Kluwer), p. 183

Dopita, M. A., Ford, H. C., Lawrence, C. J., \& Webster, B. L. 1985, ApJ, 296,390

Feitzinger, J. V., Issertedt, J., \& Schmidt-Kaler, Th. 1977, A\&A, 57, 265

Feast, M. W. 1968, MNRAS, 140, 345

Frantsman, J. L. 1988, Ap. Space Sci., 145, 251

Freeman, K. C. 1984, in IAU Symp. 108, Structure and Evolution of the Magellanic Clouds, ed. S. van den Bergh \& K. S. de Boer (Dordrecht: Reidel), p. 107

Freeman, K. C., Ilingworth, G., \& Oemler, A. 1983, ApJ, 272, 488

Hartwick, F. D. A., \& Cowley, A. P. 1988, ApJ, 334, 135 , 1991, in IAU Symp. 148, The Magellanic Clouds, ed. R. F.

Haynes \& D. K. Milne (Dordrecht: Kluwer), p. 77

Hindman, J. V. 1967, Australian J. Phys., 20, 147

Hodge, P. W., \& Wright, F. W. 1967, The Large Magellanic Cloud, (Smithsonian Pub. No. 4699; Washington, DC; Smithsonian Press)

Hughes, S. M. G., Wood, P. R., \& Reid, N. 1991a, in IAU Symp. 148, The Magellanic Clouds, ed. R. F. Haynes \& D. K. Milne (Dordrecht: Kluwer), p. 81 , 1991b, AJ, 101, 1304

James, F., \& Roos, M. 1975, Comput. Phys. Comm., 10, 343

Jensen, J., Mould, J., \& Reid, N. 1988, ApJS, 67, 77

Mathewson, D. S., \& Ford, V. L. 1984, in IAU Symp. 108, Structure and Evolution of the Magellanic Clouds, ed. S. van den Bergh \& K. S. de Boer (Dordrecht: Reidel), p. 125

Mathewson, D. S., Ford, V. L., \& Visvanathan, N. 1986, ApJ, 301, 664

McGee, R. X., \& Newton, L. M. 1981, Proc. Astr. Soc. Australia, 4, 189 Meatheringham, S. J. 1991, in IAU Symp. 148, The Magellanic Clouds, ed. 
R. F. Haynes \& D. K. Milne (Dordrecht: Kluwer), p. 89

Meatheringham, S. J., Dopita, M. A., Ford, H. C., \& Webster, B. L. 1988, ApJ, 327, 651

Morgan, D. H. 1992, (in preparation)

Morgan, D. H., \& Good, A. R. 1992, A\&AS, 92, 571

Mould, J R., \& da Costa, G. S. 1988, in Progress and Opportunities in Southern Hemisphere Optical Astronomy, ASP Conf. Series, vol. 1, ed. V. M. Blanco \& M. M. Phillips (San Francisco: ASP), p. 197

Murai, T., \& Fujimoto, M. 1980, Pub. Ast. Soc. Japan, 32, 581

Olszewski, E., Harris, H., Schommer, R., \& Canterna, R. 1988, AJ, 95, 84

Prévot, L., Rousseau, J., \& Martin, N. 1989, A\&A, 225, 303

Rohlfs, K., Kreitschmann, J., Siegman, B. C., \& Feitzinger, J. V. 1984, A\&A, 137, 343

Rohlfs, K., \& Luks, Th. 1991, in IAU Symp. 148, The Magellanic Clouds, ed. R. F. Haynes \& D. K. Milne (Dordrecht: Kluwer), p. 63

Sanduleak, N., MacConnell, D. J., Philip, A. G. D. 1978, PASP, 90, 621

Schommer, R. A. 1991, in IAU Symp. 148, The Magellanic Clouds, ed. R. F. Haynes \& D. K. Milne (Dordrecht: Kluwer), p. 171

Smith, H. C., \& Weedman, D. W. 1972, ApJ, 177, 545

Vassiliadis, E., Dopita, M. A., \& Morgan, D. H. 1992, ApJ, submitted

Webster, B. L. 1969, MNRAS, 143, 79

Wielen, R. 1977, A\&A, 60, 263

Westerlund, B. E. 1991, in IAU Symp. 148, The Magellanic Clouds, ed. R. F. Haynes \& D. K. Milne (Dordrecht: Kluwer), p. 15

Yamashita, Y. 1975, PASJ, 27, 325 


\section{Chapter 7}

\section{A Theoretical Calibration of the Planetary Nebular Cosmic Distance Scale}

\subsection{Introduction}

The luminosity function of planetary nebulae (PNe) has been shown to be a very promising extragalactic distance indicator (Jacoby, Ciardullo, \& Ford 1990). Up to the present time, no compelling evidence for a metallicity dependence on the luminosity function of PNe has yet been detected (Jacoby, Ciardullo, \& Ford 1988; Jacoby, Walker, \& Ciardullo 1990). However, before this indicator achieves total acceptance, it must be shown that any sensitivity to metallicity of the host galaxy stellar population is either negligible or can be calibrated and removed. A comparable situation exists for other common and more widely-known candles such as RR Lyrae stars and Cepheids.

Jacoby (1989) suggested that the peak [O III] luminosities of an ensemble of PNe experience a modest $\sim Z^{0.5}$ dependence. Provided that the method is applied differentially, between objects with metallicity differences of $30 \%$ or less, then errors from this source would be relatively unimportant. This is the case, for example, when distances for giant Virgo ellipticals in the Virgo Cluster are derived using the bulge of M31 as a calibrator. However, any attempt to apply the technique to a wider range of metallicities could lead to a significant error if the method is this sensitive to metallicity variations.

Jacoby's (1989) conclusion was based on a very limited numerical experiment in which it was assumed that the luminosities of the central stars are unaffected by metal- 
licity. Sandage \& Tammann (1990), however, correctly point out that the dependence of the central star core mass and luminosity with metallicity cannot be ignored if the models of Lattanzio (1986) are adopted. Those models, which refer to giant stars, rather than to planetary nebulae nuclei (PNNi) suggest that the core masses of central stars should decrease with metallicity such that $L \sim Z^{-0.25}$. More recent models by Brocato et al. (1989) yield similar expectations but with a stronger $L \sim Z^{-0.5}$ dependence. Thus, to the extent that core mass on the giant branch and PNNi core mass are related, the planetary nebula (PN) system (nebula plus exciting star) might be expected to display a net power law luminosity dependence with an index of between 0.0 (for the Brocato et al. model) and +0.25 (for the Lattanzio model).

In this paper, we examine the effects on the luminosity of PNe in more detail using improved nebular modeling techniques (see Section II). In particular, we consider the behaviour of the [O III] and $\mathrm{H} \beta$ emission lines, which have both been used as distance estimators in the past (Pottasch 1990). In addition, we consider the effects of metallicity variations on the mass of the central star. Thus, the modeled PN is treated as a selfconsistent system. The model results provide the added bonus that, provided that the nebular temperature is known, it is possible to derive the nebular metallicity, and the temperature and luminosity (and therefore mass) of the central star from minimal spectroscopic data.

\subsection{Photoionization Modelling}

Recently, Dopita \& Meatheringham (1991a,b), have derived the stellar and nebular parameters of a large sample of Magellanic Cloud PNe through self-consistent photoionization modelling. This work demonstrated that, for the optically thick PNe in the Magellanic Clouds, at least, the stellar parameters are simply related to the excitation class, $E$, defined as a continuous variable by Dopita \& Meatheringham (1990):

$$
\begin{aligned}
E & =0.45 \frac{F(\lambda 5007)}{F(H \beta)}, & & 0.0 \leq E<5.0 \\
& =5.54\left(0.78+\frac{F(\lambda 4686)}{F(H \beta)}\right), & & 5.0 \leq E<10.0
\end{aligned}
$$

with this definition, a least-squares fit to some 66 objects gives:

$$
\begin{aligned}
\log \left(T_{\text {eff }}\right)= & (4.489 \pm 0.017)+(0.1124 \pm 0.0025) E \\
& -(0.00172 \pm 0.00037) E^{2}
\end{aligned}
$$

with a correlation coefficient of 0.98 , and, with a correlation coefficient of 0.96 : 


$$
\begin{aligned}
\log \left(\frac{L}{L_{\odot}}\right)= & \log \left(\frac{L_{H \beta}}{L_{\odot}}\right)+(2.32 \pm 0.02)-(0.179 \pm 0.016) E \\
& +(0.035 \pm 0.004) E^{2}-(0.00166 \pm 0.00035) E^{3}
\end{aligned}
$$

For Magellanic Cloud abundances, these equations provide a useful transformation between the parameters of the observed Hertzsprung-Russell (H-R) plane; $\log \left(L_{\mathrm{H} \beta} / L_{\odot}\right)$ and $E$, and the theoretical $\mathrm{H}-\mathrm{R}$ parameters; $\log \left(L / L_{\odot}\right)$ and $\log \left(T_{\text {eff }}\right)$. However, at other metallicities these simple relationships will break down, in particular, in the excitation class range $0.0<E<5.0$, because the $[\mathrm{O} \mathrm{III]}] / \mathrm{H} \beta$ ratio is itself dependent on metallicity as well as upon the stellar temperature. It is therefore important to investigate the metallicity dependence before drawing general conclusions.

To this aim we have constructed a grid of model PNe covering a range of stellar temperatures and metallicities using the generalised modelling code MAPPINGS (Binette, Dopita \& Tuohy, 1985). In general, the spectrum of an optically-thick ionised region depends not only on these, but also upon the ionisation parameter, $Q$, defined as (Evans \& Dopita, 1985) $Q=N_{L y-c} / 4 \pi \bar{r}^{2} N_{H}$; where $N_{L y-c}$ is the number of Lyman continuum photons emitted by the central source, and $\bar{r}$ is the mean radius of the ionised nebula with hydrogen particle density $N_{H}$. Only the upper end of the luminosity function of PN is observed in external galaxies. These objects will have luminosities generally larger than $1000 L_{\odot}$. For this group of PN in the Magellanic Clouds, Dopita \& Meatheringham (1991a,b) found $Q$ to lie in the range $6 \times 10^{7}<Q<6 \times 10^{8} \mathrm{~cm} \mathrm{~s}^{-1}$, with an average of $\sim 2 \times 10^{8} \mathrm{~cm} \mathrm{~s}^{-1}$. At these high values of the ionisation parameter, and with the high temperatures of the central star which characterise PNe, the ionisation parameter has only a second-order effect on the output spectrum.

For our grid, we adopt values of the ionisation parameter, stellar luminosity, and nebular gas pressure typical of the brighter PNe in the Magellanic Clouds, or of the brighter PNe in the Galactic Bulge (Webster, 1988; Pottasch \& Acker 1989; Tylenda et al. 1991) viz.:

$$
\frac{L}{L_{\odot}}=5000 ; \quad Q=2 \times 10^{8} \mathrm{~cm} \mathrm{~s}^{-1} ; \quad T_{e} N_{H}=10^{8} \mathrm{Kcm}^{-3}
$$

With these parameters, we computed the emission line spectrum of isobaric model $\mathrm{PNe}$ in photoionisation equilibrium. The central star was assumed to have a black 
body distribution in frequency. Stars with effective temperature lower than $10^{5} \mathrm{~K}$ have the spectrum cut off above 4 Ryd to simulate $\mathrm{He}^{+}$blanketing. To be sure, this may appear to be a rather crude approximation to the ionising spectrum, but this has been found to work very satisfactorily in the case of the Magellanic Cloud PNe, and there remain important uncertainties and disagreements within and between the various model atmosphere computations. In particular, the importance of non-LTE effects, and our knowledge of the effect of the extension of the atmosphere by stellar winds on the distribution of ionising radiation has, in the past, been quite uncertain. Recent work on unified spherical non-LTE model atmospheres, which include a self-consistent treatment of both the photospheric and the wind regions show that the effect of this redistribution is to restore the emergent spectrum towards the Black Body distribution (Gabler, Kudritzki, \& Mendez 1991).

The solar abundance set adopted has the following relative numbers of atoms, $H$ : $\mathrm{He}: \mathrm{C}: \mathrm{N}: \mathrm{O}: \mathrm{Ne}: \mathrm{Mg}: \mathrm{Si}: \mathrm{S}: \mathrm{Cl}: \mathrm{Ar}=1: 0.1: 1.04 \times 10^{-3}: 8.7 \times 10^{-5}$ : $6.9 \times 10^{-4}: 9.8 \times 10^{-5}: 4.0 \times 10^{-5}: 3.8 \times 10^{-5}: 1.9 \times 10^{-5}: 1.8 \times 10^{-7}: 4.0 \times 10^{-6}$. This is solar except for the $\mathrm{C} / \mathrm{O}$ ratio, which is taken as 1.5 to approximately account for the dredge-up of carbon on the AGB. At high metallicity (defined by $[\mathrm{Fe} / \mathrm{H}] \mathrm{ratio}$ ), it is known that there are few, if any, $\mathrm{C}$ stars. This suggests that the $\mathrm{C} / \mathrm{O}$ ratio is probably less than unity in these systems, which will have some effect on the [O III] flux computed from our models. Dopita \& Meatheringham $(1990 ; 1991 \mathrm{a}, \mathrm{b})$ have investigated this effect in the context of attempts to model type I PNe in the Magellanic Clouds, and find that the temperature and optical spectrum is remarkably insensitive to the assumed $\mathrm{C} / \mathrm{O}$ ratio. We return to this point in section 7.6 .3 , below, where we find the same result. In our grid of models the helium abundance is kept fixed with respect to hydrogen, and the abundances of the other elements are scaled with respect to this set. Families of models have been computed at $0.1,0.15,0.2,0.3,0.5,0.7,1.0,1.5$ and 2.0 times solar abundances. For each of these abundance sets, we have computed models with the following set of temperatures; $35,000,40,000,45,000,50,000,55,000,60,000$, $70,000,80,000,90,000,100,000,110,000,120,000$ and 140,000 K. For fixed central star luminosity, and over the whole abundance range, this temperature range encompasses the luminosity maximum in both the $\mathrm{H} \beta$ and in the [O III] $\lambda 5007 \AA$ lines. 


\subsection{Results}

\subsubsection{The $\mathbf{H} \beta$ Flux}

Equation 7.3 implies that, for optically thick PNe, the absolute $\mathrm{H} \beta$ flux should be a reliable indicator of stellar luminosity, provided that the excitation class is known. Even in the cases where the excitation class is not known, it is still possible to compute an approximate luminosity, since the conversion efficiency from stellar luminosity to luminosity in the $\mathrm{H} \beta$ line only varies by a factor of at most 2. From our grid, we find that the variation of this conversion efficiency is even less. In figure 7.1, we show the conversion efficiency factor as a function of stellar effective temperature and of metallicity. The peak efficiency occurs at a stellar effective temperature of near $70000 \mathrm{~K}$, and a maximum of just under $1 \%$ of the luminosity of the star is converted to $\mathrm{H} \beta$ photons. Note that the variation in the conversion efficiency factor is quite small. At a given temperature, metallicity variations of a factor of 10 make less than $10 \%$ change, and the variation with temperature is only $50 \%$.

\subsubsection{The [O III] Flux}

The variation of the [O III] $\lambda 5007 \AA$ line intensity with metallicity and temperature is stronger and more complex than is the case for the Balmer lines. This is because the [O III] $\lambda 5007 \AA$ line is one of the most important coolants in the nebula. Figure 7.2 shows the luminosity conversion efficiency factor as a function of stellar effective temperature and of metallicity. At high metallicities, up to $15 \%$ of the luminosity of the central star is re-radiated in the [O III] $\lambda 5007 \AA$ line alone. The effective temperature of the central star at which the luminosity conversion efficiency factor is maximised is somewhat dependent on the metallicity, ranging from $100,000 \mathrm{~K}$ at high metallicity, down to near $80,000 \mathrm{~K}$ for $\log [Z]=-1.0$. We therefore expect that the most [O III] luminous $\mathrm{PNe}$ in any galaxy will be characterised by a fairly narrow range of effective stellar temperatures, corresponding to excitation classes 4-6, approximately, since objects with lower effective temperatures will have much lower luminosity conversion efficiency factors, and will therefore appear fainter. In the case of PNe with much hotter central stars, the intrinsic luminosity of the central star will be lower as a result of hydrogen exhaustion.

The electron temperature as measured by the [O III] $\lambda \lambda 4363 / 5007 \AA$ ratio is sensitive to both metallicity and to stellar effective temperature. Hotter stars deliver a 


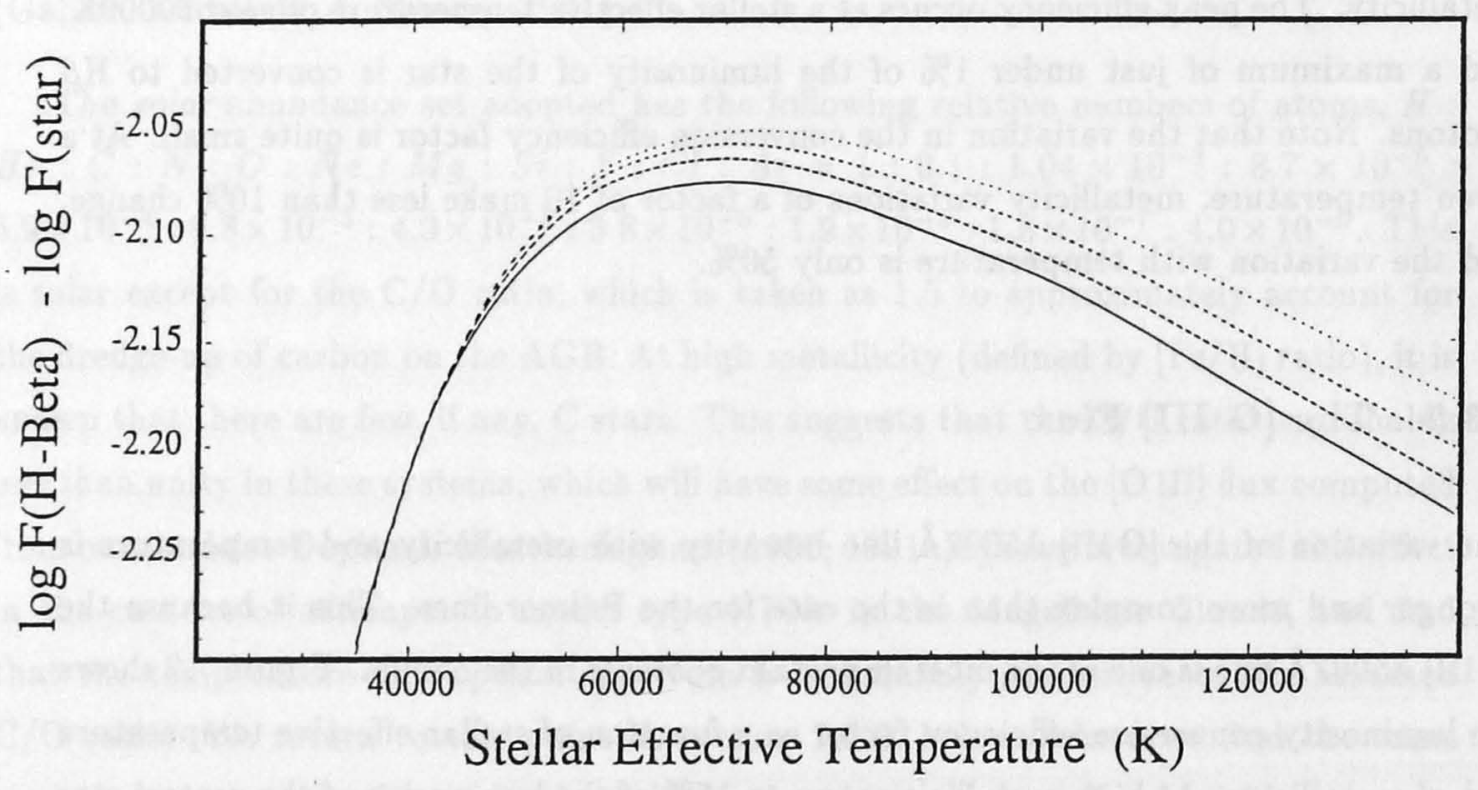

Figure 7.1: The conversion efficiency of stellar flux to $\mathrm{H} \beta$ luminosity in optically-thick $\mathrm{PNe}$ models as a function of stellar effective temperature. The lines are for different oxygen abundances in the nebula, as follows: $2 \times$ solar, solid line; solar, long-dashed line; $0.3 \times$ solar, short-dashed line; and $0.1 \times$ solar, dotted line. 


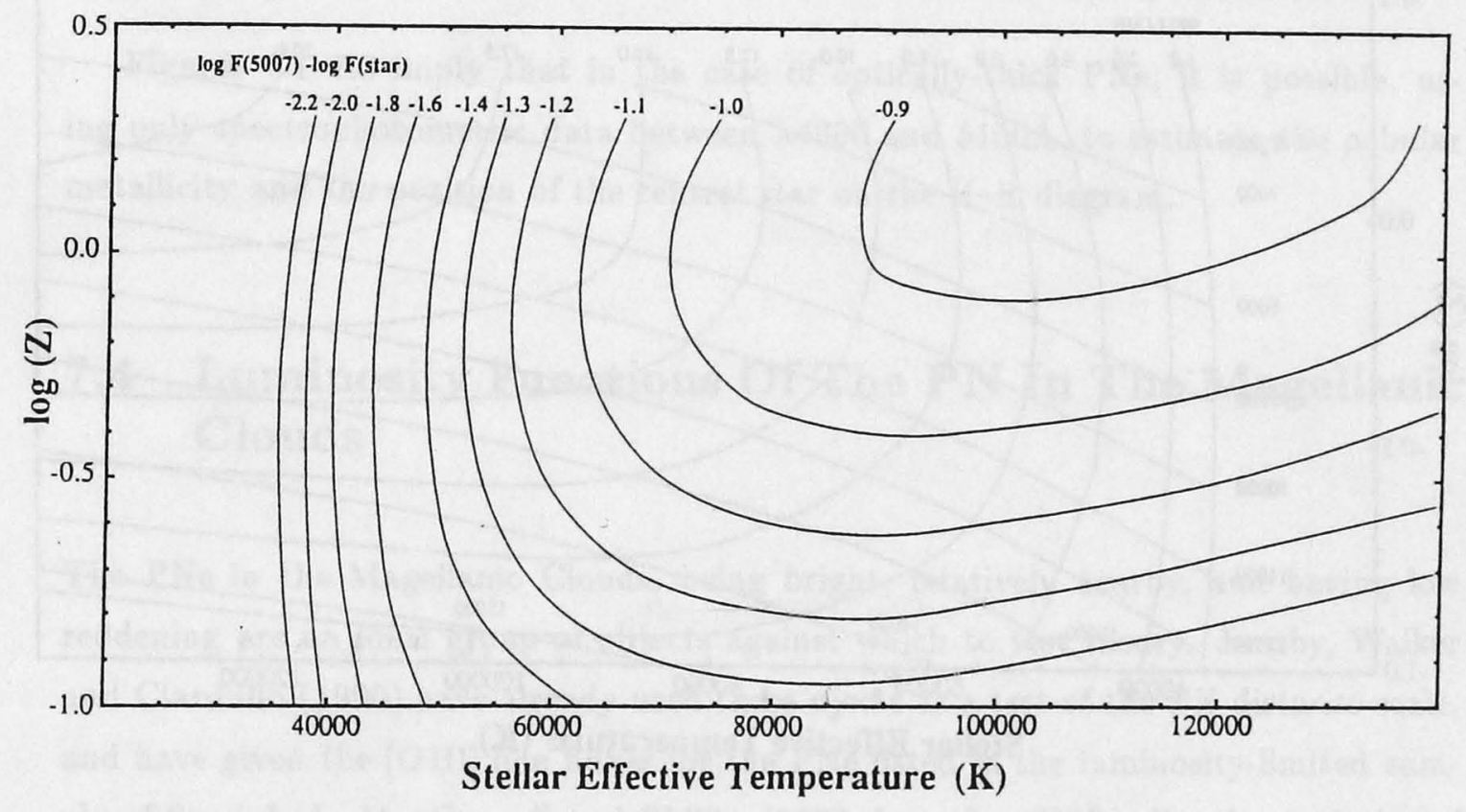

Figure 7.2: Conversion efficiency of stellar flux to [O III] $\lambda 5007 \AA$ luminosity in opticallythick PNe models as a function of both stellar effective temperature and metallicity. 


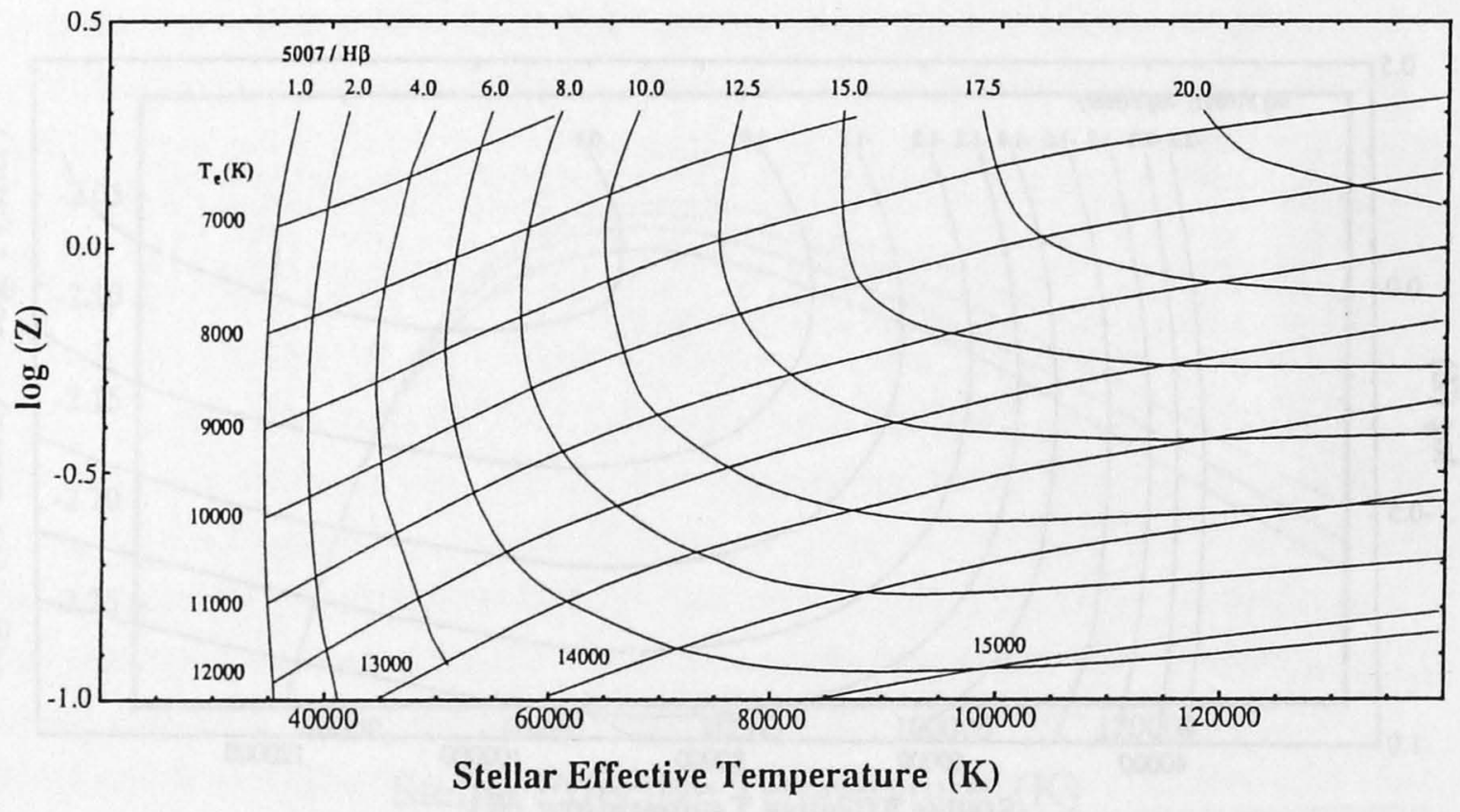

Figure 7.3: The grid defined by the electron temperature as measured by the [O III] lines and by the $[\mathrm{O} \mathrm{III}] / \mathrm{H} \beta$ line ratio as a function of both stellar effective temperature and metallicity. Nebular and stellar conditions determined in this figure can be used to estimate the stellar luminosity using Figure 7.2. 
greater energy input to the nebula per photoionisation, resulting in hotter nebulae, and higher metallicity nebulae are cooled more efficiently by emission lines, producing cooler nebulae. On the other hand, figures 7.1 and 7.2 show that the $[\mathrm{OIII}] / \mathrm{H} \beta$ ratio is a strong function of temperature, and is also somewhat dependent on metallicity. This opens the possibility of using $\mathrm{T}_{e}$ and the $[\mathrm{O} \mathrm{III}] / \mathrm{H} \beta$ ratio as a diagnostic to localise $\mathrm{PNe}$ on the $\left(T_{\text {eff }}, \log [Z]\right)$ plane. The results of our calculations are shown on Figure 7.3. Generally, the measured $\mathrm{T}_{e}$ and the $[\mathrm{O} \mathrm{III}] / \mathrm{H} \beta$ ratio can be used as diagnostic for both $\mathrm{T}_{\text {eff }}$ and $\log [Z]$. However, in the region $T_{\text {eff }}>90,000 \mathrm{~K}$ and $\log [Z]<-0.5$, the grid is not very useful for determination of the stellar temperature. However, this can be measured in this region using the $\mathrm{He} I \mathrm{I} \lambda \lambda 4686 \AA / \mathrm{H} \beta$ ratio, according to equations 7.1 and 7.2.

Figures 7.1-7.3 imply that in the case of optically-thick PNe, it is possible, using only spectrophotometric data between $\lambda 4300$ and $5100 \AA$, to estimate the nebular metallicity and the position of the central star on the H-R diagram.

\subsection{Luminosity Functions Of The PN In The Magellanic Clouds}

The PNe in the Magellanic Clouds, being bright, relatively nearby, and having low reddening are an ideal group of objects against which to test theory. Jacoby, Walker and Ciardullo (1990) have already used these ojects as a test of the PN distance scale, and have given the [O III] line fluxes for the PNe listed in the luminosity-limited sample of Sanduleak, MacConnell and Phillip (1978; hereafter SMP). For the majority of these objects, Meatheringham, Dopita and Morgan (1988) and Wood et al. (1987) had already measured the $\mathrm{H} \beta$ flux, and, for a large fraction of this sample, high quality spectrophotometry exists (Monk, Barlow \& Clegg 1988; Meatheringham \& Dopita 1991a, b; Dopita, Vassiliadis \& Morgan 1992).

Using these data, we have constructed (figures $7.4 \mathrm{a}, \mathrm{b}$ ) the distribution of points on the (emission-line-flux, excitation-class) planes for both the [O III] and $\mathrm{H} \beta$ lines. As Dopita and Meatheringham (1990) have pointed out, these can be thought of as H-R diagrams transformed to observable coordinates. As expected, the [O III] flux peak occurs near excitation class 6 , and the effect of the transformation of the H-R diagram implied in figure 7.2 is quite evident.

The raw cumulative luminosity functions derived from the [O III] and $\mathrm{H} \beta$ line fluxes 

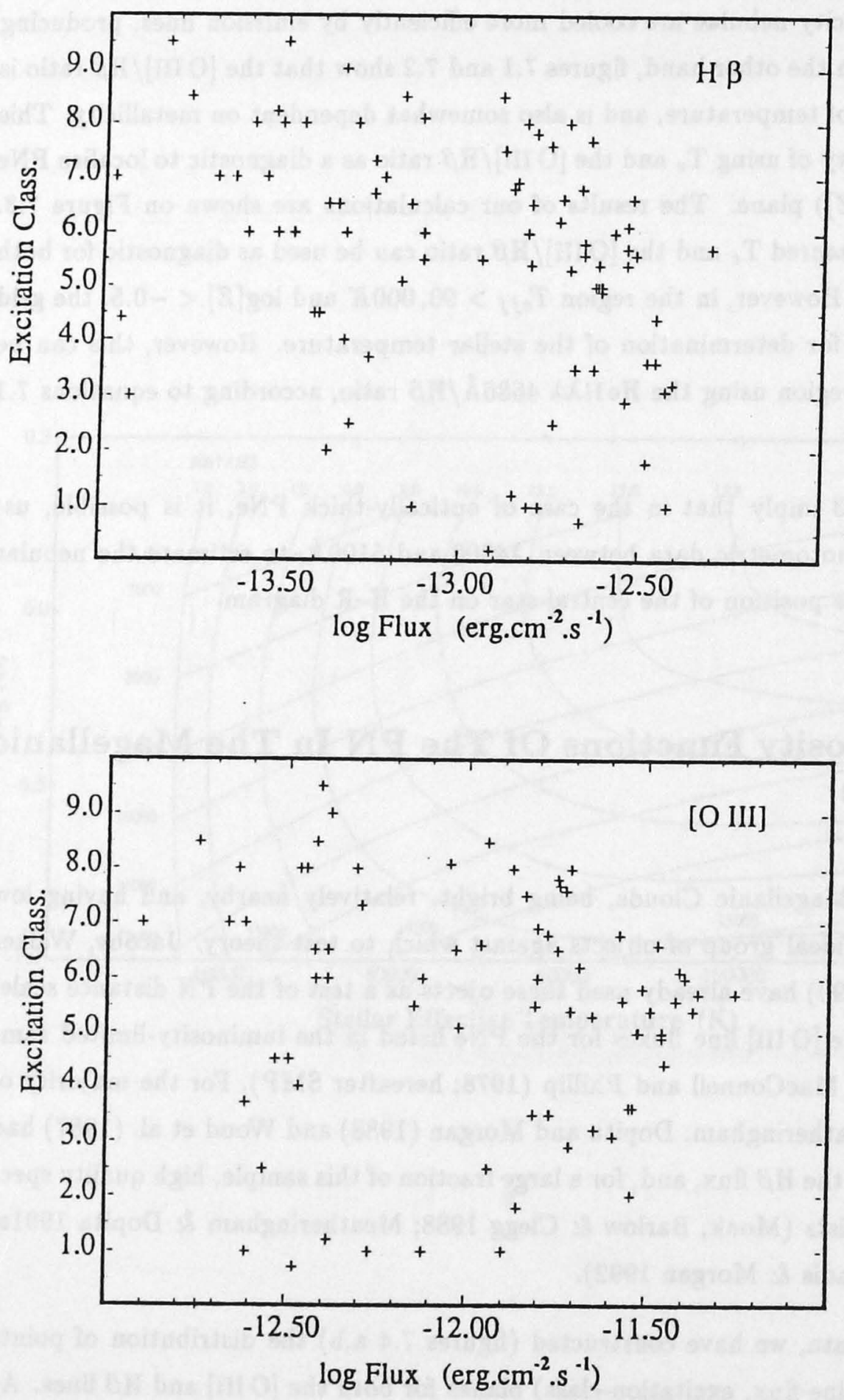

Figure 7.4: Raw $\mathrm{H} \beta$ and [O III] PN luminosities as a function of excitation class. Note that the [O III] luminosities peak at excitation class 5-6. 

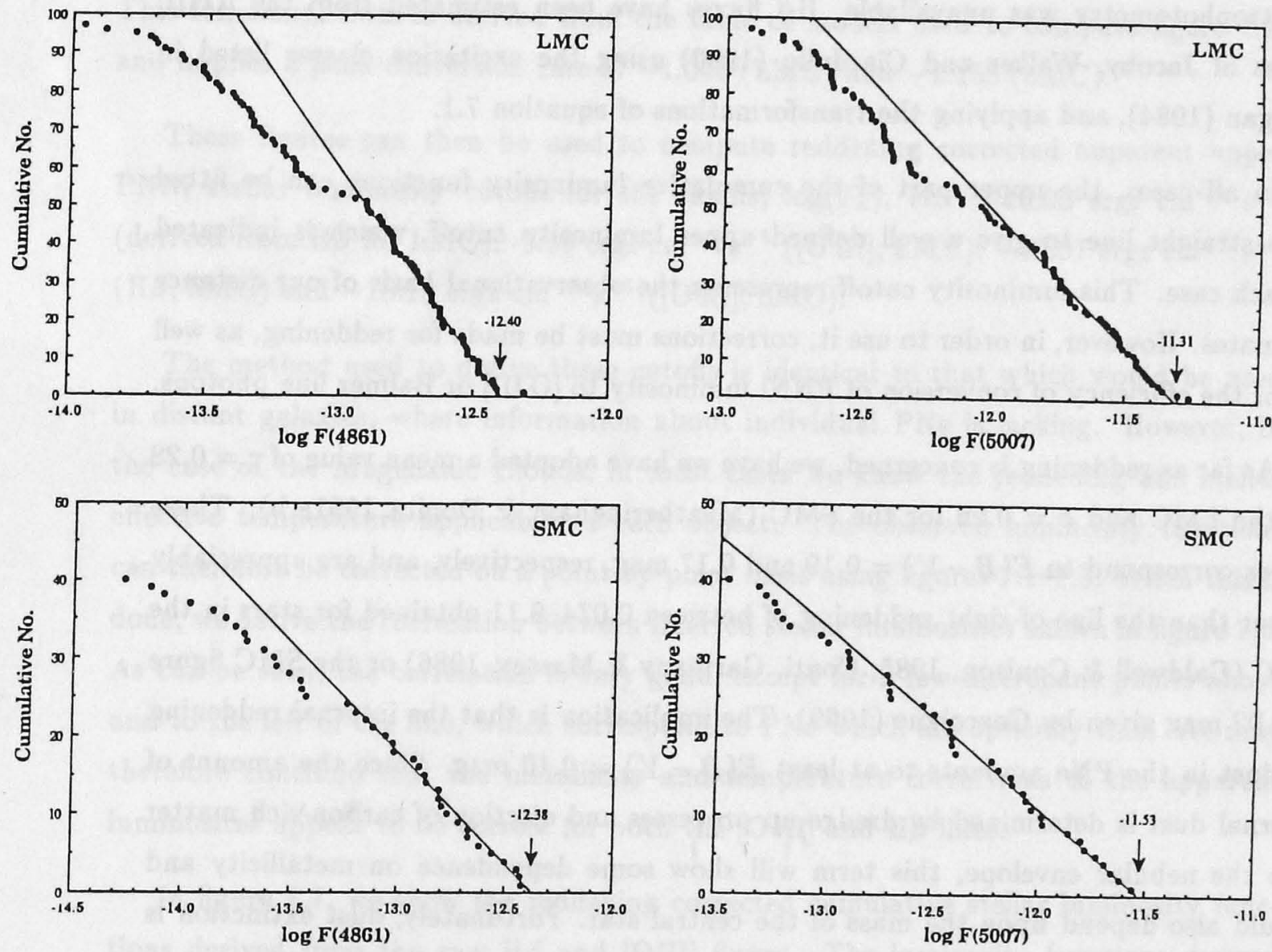

Figure 7.5: The apparent $\mathrm{H} \beta$ and [O III] cumulative absolute luminosity functions for the LMC and the SMC, uncorrected for reddenning. In each case the fit ot the upper end of the function is shown, along with the inferred upper luminosity cutoff. 
are shown in figures $7.5 \mathrm{a}-\mathrm{d}$. These have been derived from the observational data base of Meatheringham, Dopita and Morgan (1988), Wood et al. (1987), Jacoby, Walker and Ciardullo (1990) and Dopita et al. (1992). Extra [O III] or $\mathrm{H} \beta$ fluxes have been derived from the spectrophotometry of Monk, Barlow and Clegg (1988) and Meatheringham and Dopita (1991a, b). In addition, for a small number of objects for which detailed spectrophotometry was unavailable, $\mathrm{H} \beta$ fluxes have been estimated from the [O III] fluxes of Jacoby, Walker and Ciardullo (1990) using the excitation classes listed in Morgan (1984), and applying the transformations of equation 7.1.

In all cases, the upper part of the cumulative luminosity functions can be fitted by a straight line to give a well defined upper luminosity cutoff, which is indicated in each case. This luminosity cutoff represents the observational basis of our distance estimates. However, in order to use it, corrections must be made for reddening, as well as for the efficiency of conversion of PNNi luminosity to [O III] or Balmer line photons.

As far as reddening is concerned, we have we have adopted a mean value of $c=0.28$ for the LMC and $c=0.26$ for the SMC (Meatheringham \& Dopita 1991a,b). These values correspond to $E(B-V)=0.19$ and $0.17 \mathrm{mag}$, respectively, and are appreciably higher than the line-of-sight reddening of between $0.074-0.11$ obtained for stars in the LMC (Caldwell \& Coulson, 1985; Conti, Garmany \& Massey, 1986) or the SMC figure of $0.02 \mathrm{mag}$ given by Gascoigne (1969). The implication is that the internal reddening by dust in the PNe amounts to at least $E(B-V)=0.10 \mathrm{mag}$. Since the amount of internal dust is determined by dredge-up processes and ejection of carbon-rich matter into the nebular envelope, this term will show some dependence on metallicity and should also depend upon the mass of the central star. Fortunately, dust extinction is already sufficiently small that the differential in the internal reddening would not have a serious effect on distance estimates. However, a correction must be made for internal dust extinction in the derivation distances to other galactic systems.

In order to make a correction for the efficiency of conversion of stellar flux to observable photons, we adopt the mean $\mathrm{O} / \mathrm{H}$ abundances of the $\mathrm{PNe}$ in the Magellanic Clouds derived Meatheringham \& Dopita (1991a; b), namely, $2.0 \times 10^{-4}$ by number for the LMC, and $1.2 \times 10^{-4}$ by number for the SMC. These correspond to $\log [O / H]=-0.54$ for the LMC, and -0.75 for the SMC. We should point out that these figures should be distinguished from the mean stellar metallicity, which is dominated by $[\mathrm{Fe} / \mathrm{H}]$. For these, we adopt values of $\log [Z]=-0.32$ (LMC) and -0.64 (SMC) (Russell \& Dopita, 1991). 
From figure 7.1 , at these metallicities, the peak logarithmic conversion efficiency of stellar flux to flux in the $\mathrm{H} \beta$ line is -2.065 (LMC), and -2.05 (SMC). The peak conversion efficiency of stellar flux to $\lambda 5007 \AA$ photons is conveniently given by:

$$
(\log F(5007)-\log F(\text { star }))_{\text {peak }}=-0.8791+0.1459 \log \left[\frac{O}{H}\right]-0.3013\left[\frac{O}{H}\right]^{2} .
$$

This functional form is derived from the table of models used to compute figure 7.2, and implies a peak conversion rate of -1.046 (LMC) and -1.158 (SMC).

These figures can then be used to compute reddening corrected apparent upper PNNi stellar luminosity cutoffs for the clouds, $\log \left(F_{*}\right)$, viz. -10.05 ergs $\mathrm{cm}^{-2} \mathrm{~s}^{-1}$ (derived from $\mathrm{H} \beta$ for LMC), $-9.99 \mathrm{ergs} \mathrm{cm}^{-2} \mathrm{~s}^{-1}$ ([O III], LMC), $-10.07 \mathrm{ergs} \mathrm{cm}^{-2} \mathrm{~s}^{-1}$ $(\mathrm{H} \beta, \mathrm{SMC})$ and $-10.12 \mathrm{ergs} \mathrm{cm}^{-2} \mathrm{~s}^{-1}$ ([O III], SMC).

The method used to derive these cutoffs is identical to that which would be used in distant galaxies, where information about individual PNe is lacking. However, in the case of the Magellanic Clouds, in most cases we know the reddening and stellar effective temperature applicable to each object. The observed luminosity functions can therefore be corrected on a point-by-point basis using figures 7.1-7.3. When this is done, we derive the correlation between inferred stellar luminosities shown in figure 7.6. As can be seen, the correlation is very good, except for a few discrepant points above and to the left of the line, which correspond to PNe which are optically thin. We may therefore conclude that the metallicity and temperature corrections to the apparent luminosites appear to be correct for both the [O III] and $\mathrm{H} \beta$ lines.

In figure 7.7 , we show the reddening corrected cumulative stellar luminosity functions derived from the raw $\mathrm{H} \beta$ and [OIII] fluxes. The luminosity functions derived from the two lines are, to all intents and purposes, identical. The rollover in the slope of the luminosity function at lower luminosities is a consequence of incompleteness in the sample. Note that, near the apparent upper stellar luminosity cutoff, $\log \left(F_{*}\right)$, the cumulative luminosity function is not so well fitted by a straight line. This is a consequence of the inclusion of low temperature PNNi with high internal reddening which, although not the most luminous objects in the raw sample, turn out to be the most luminous when corrected for extinction and the conversion efficiency to nebular emission. As a consequence, $\log \left(F_{*}\right)$ is somewhat higher than that inferred from the raw sample, $-9.80 \pm 0.03 \mathrm{erg} \mathrm{cm} \mathrm{cm}^{-2} \mathrm{~s}^{-1}$ (LMC) and $-9.90 \pm 0.06 \mathrm{erg} \mathrm{cm}^{-2} \mathrm{~s}^{-1}$ (SMC). The difference between the apparent and true $\log \left(F_{*}\right)$ is similar in both clouds, +0.23 for the LMC, and +0.2 for the SMC. If not accounted for, this could be an important source of zero-point error in more distant systems. 


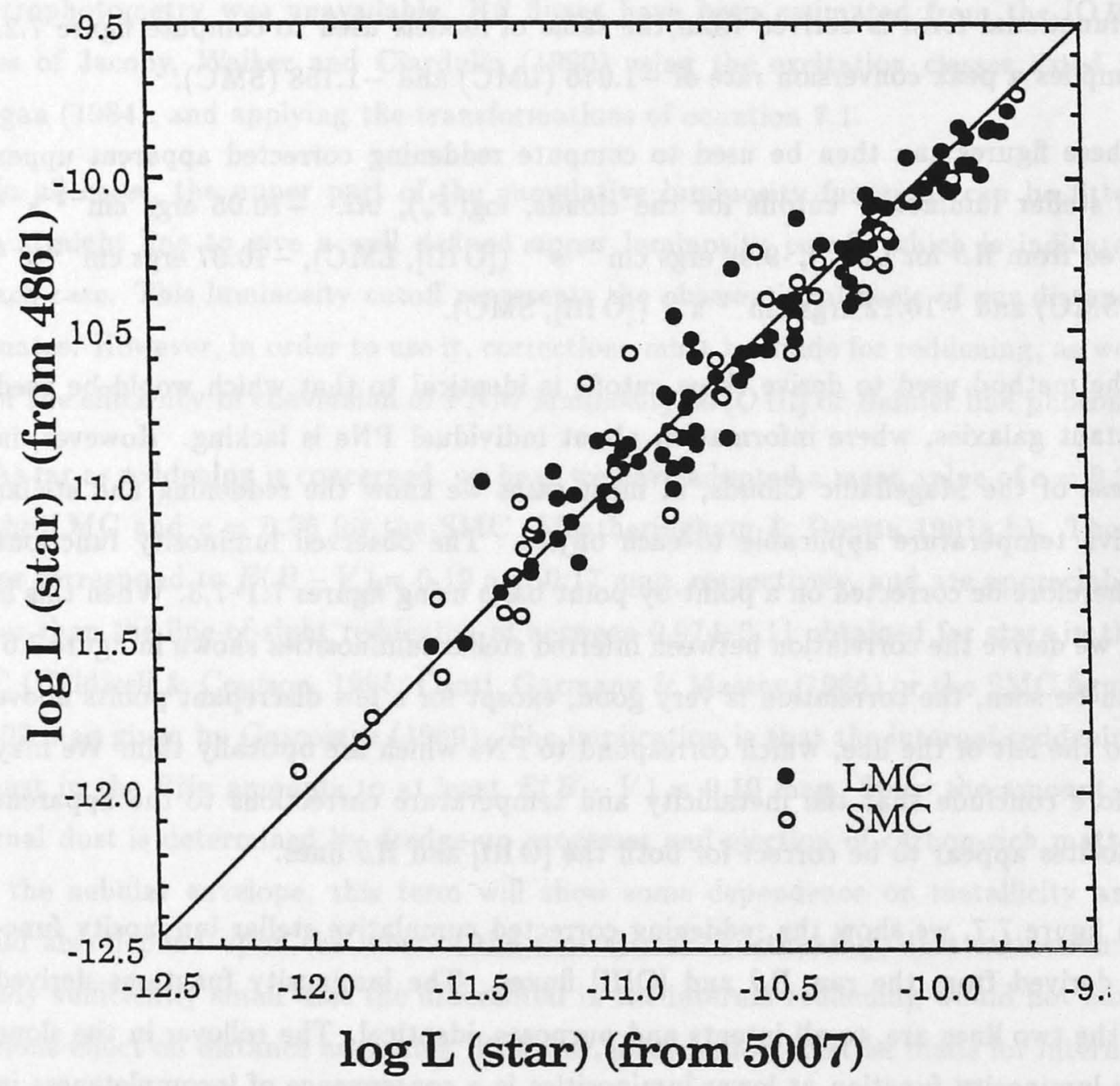

Figure 7.6: The relationship between apparent reddenning-corrected stellar fluxes of individual $\mathrm{PNe}$ in the LMC and the SMC derived from the [O III] line fluxes, or from the $\mathrm{H} \beta$ line fluxes, using relationships of figures 7.1-7.2. The units of flux are erg $\mathrm{cm}^{-2}$ $\mathrm{s}^{-1}$. The points which lie some distance above and to the left of the relationship are in all cases optically thin PNe. It is clear that either line can be used equally well to derive the stellar flux, indicating that the corrections for metallicity and excitation are good. 

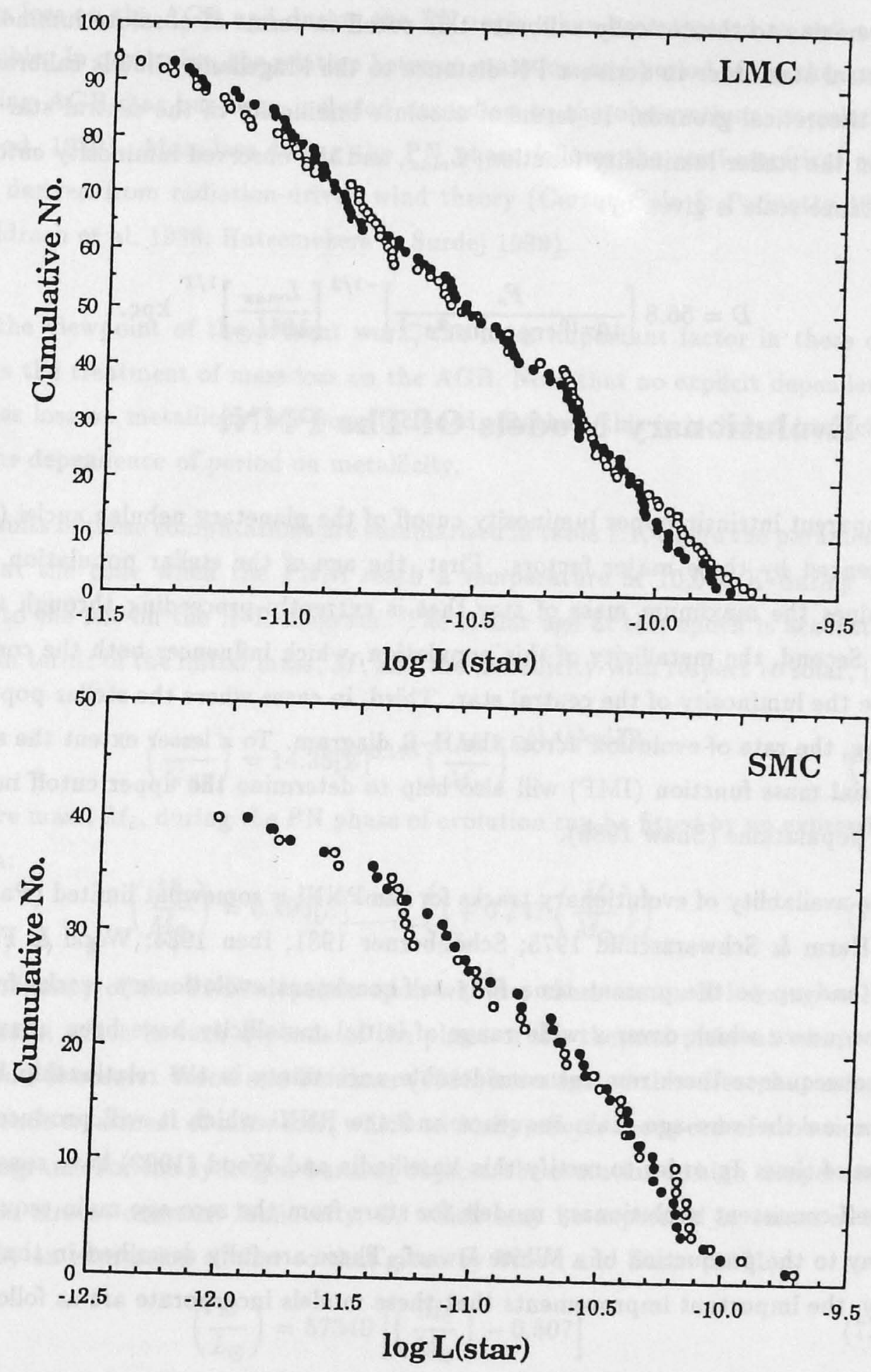

Figure 7.7: The true cumulative apparent stellar luminosity functions for the LMC and SMC corrected for reddenning, metallicity and excitation. The units of flux are erg $\mathrm{cm}^{-2} \mathrm{~s}^{-1}$. 
It remains to theoretically calibrate this cutoff in terms of absolute luminosity of the central star order to derive a PN distance to the Magellanic Clouds calibrated on purely theoretical grounds. In terms of absolute luminosity of the central star at the cutoff in the stellar luminosity function, $L_{\max }$, and the observed luminosity cutoff, $F_{*}$, the distance scale is given by:

$$
D=56.8\left[\frac{F_{*}}{10^{-10} \text { ergs cm }^{-2} s^{-1}}\right]^{-1 / 2}\left[\frac{L_{\max }}{10^{4} L_{\odot}}\right]^{1 / 2} \mathrm{kpc} .
$$

\subsection{Evolutionary Models Of The PNNi}

The apparent intrinsic upper luminosity cutoff of the planetary nebulae nuclei (PNNi) is influenced by three major factors. First, the age of the stellar population, which determines the maximum mass of star that is currently proceeding through the PN phase. Second, the metallicity of this population, which influences both the core mass and the the luminosity of the central star. Third, in cases where the stellar population is young, the rate of evolution across the H-R diagram. To a lesser extent the slope of the initial mass function (IMF) will also help to determine the upper cutoff in young stellar populations (Shaw 1989).

The availablity of evolutionary tracks for the PNNi is somewhat limited (Paczynski 1971; Harm \& Schwarzschild 1975; Schönberner 1981; Iben 1984; Wood \& Faulkner 1986), and up to the present time fully self-consistent evolutionary tracks from the main sequence which cover a wide range of initial metallicity have been unavailable. As a consequence there remains considerable uncertainty in the relationship between the star on the zero-age main sequence and the PNNi which it will produce in the fullness of time. In order to rectify this Vassiliadis and Wood (1992) have constructed fully self-consistent evolutionary models for stars from the zero-age main sequence all the way to the production of a White Dwarf. These are fully described in that paper. Briefly, the important improvements that these models incorporate are as follows:

1. Abundances and opacities appropriate to SMC, LMC and the Galaxy have been used (Huebner et al. 1977; Russell \& Bessell 1989; Russell \& Dopita 1990).

2. The updated Caughlan and Fowler (1988) reaction rates have been used.

3. The Castellani et al. (1985) treatment of semi-convection during the horizontal branch evolution has been incorporated. 
4. Mass loss on the AGB and during the PN regime has been treated as well as is possible. In particular, the relation between mass-loss and period of the thermally pulsing AGB star has been included according to the observational correlation (Wood, 1989). Mass-loss during the PN phase follows the semi-empirical relation derived from radiation-driven wind theory (Cerruti-Sola \& Perinotto 1985; Pauldrach et al. 1988; Hutsemekers \& Surdej 1989).

From the viewpoint of the present work, the most important factor in these calculations is the treatment of mass-loss on the AGB. Note that no explicit dependence of this mass loss on metallicity has been included. Rather, this is included implicitly through the dependence of period on metallicity.

The results of these computations are summarised in table 7.1, where the parameters are given at the time when the $\mathrm{PNNi}$ reach a temperature of $10,000 \mathrm{~K}$ during the transition to the left on the H-R diagram. The stellar age at this epoch is accurately expressed in terms of the initial mass, $M$, and the metallicity with respect to solar, $[Z]$, by:

$$
\left(\frac{\tau}{G y r}\right)=14.35[Z]^{0.195}\left(\frac{M}{M_{\odot}}\right)^{-(3.4+\log [Z])}
$$

and the core mass, $M_{c}$, during the PN phase of evolution can be fitted by an expression of the form:

$$
\left(\frac{M_{c}}{M_{\odot}}\right)=0.493[Z]^{-0.035}\left(1+0.147\left(\frac{M}{M_{\odot}}\right)\right)
$$

The luminosity of the PNNi depends upon whether these are on H-burning or Heburning tracks, which in turn depends of the phase of the thermal pulse at which the transition to PN occurs. Wood and Faulkner (1986) investigated this effect, but using a relatively crude treatment of mass-loss, which critically affects the speed of evolution in the H-R diagram. For the hydrogen-burning objects, the evolution to high temperature occurs at an almost constant luminosity, $L$, which may be expressed in terms of the core mass by an expression similar to that given by Wood and Zarro (1981);

$$
\left(\frac{L}{L_{\odot}}\right)=57340\left[\left(\frac{M_{c}}{M_{\odot}}\right)-0.507\right]
$$

Note that the metallicity dependence of PNNi luminosity is relatively weak. Equations 7.2 and 7.3 show that this dependence cannot be treated as a simple power law. However, if such a crude approximation must be made, then we have $L \sim Z^{-(0.3 \rightarrow 0.6)}$.

It is well known that the evolutionary track followed by the PNNi on the H-R diagram is strongly dependent upon the phase of the thermal pulse cycle at which 
TABLE 7.1

Calculated Parameters of PNNi

\begin{tabular}{lrrcc}
\hline \hline $\begin{array}{l}\text { Abundance Mix } \\
\text { and } \log [Z]\end{array}$ & $M_{Z A M S} / M_{\odot}$ & $\tau(\mathrm{Gyr})$ & $\begin{array}{c}\log \left(L / L_{\odot}\right) \\
\text { (H-burning) }\end{array}$ & $M_{\text {core }} / M_{\odot}$ \\
\hline Solar & 1.0 & 14.96 & 3.54 & 0.569 \\
0.000 & 1.5 & 3.65 & 3.73 & 0.600 \\
& 2.0 & 1.56 & 3.89 & 0.634 \\
& 2.5 & 0.956 & 3.98 & 0.677 \\
LMC & 1.0 & 11.05 & 3.60 & 0.578 \\
-0.32 & 1.5 & 3.11 & 3.88 & 0.620 \\
& 2.0 & 1.31 & 3.98 & 0.668 \\
& 2.5 & 0.784 & 4.01 & 0.679 \\
SMC & & & & \\
-0.64 & 1.0 & 8.90 & 3.64 & 0.593 \\
& 1.5 & 2.64 & 3.86 & 0.640 \\
& 2.0 & 1.16 & 3.92 & 0.672 \\
Low & 2.5 & 0.661 & 4.15 & 0.692 \\
-1.21 & & & & \\
& 1.0 & 7.21 & 3.78 & 0.623 \\
& 1.5 & 2.09 & 3.99 & 0.663 \\
\hline
\end{tabular}




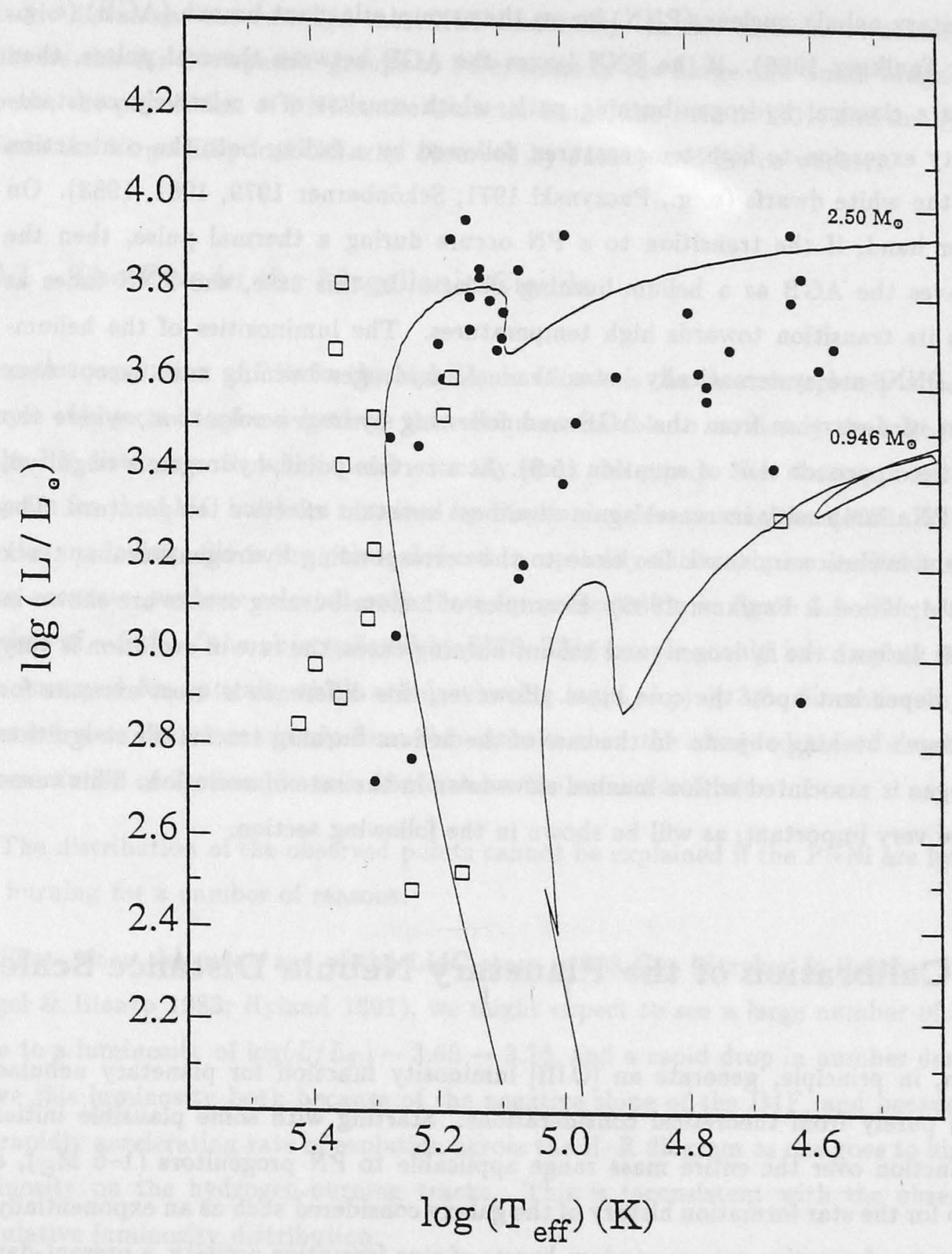

Figure 7.8: Theoretical and observed H-R diagram for PNNi in the LMC. The tracks represent helium-burning models from Vassiliadis and Wood (1992) and refer to stars having initial masses of 0.945 and $2.5 \mathrm{M}_{\odot}$, respectively. The observational points are for bright PNNi in the LMC having optically-thick nebulae and are taken from Dopita and Meatheringham (1991a,b). The Type I objects are shown as open squares. The observed points have been scaled in luminosity in order to force a fit between the clump of objects at $4.0 \geq \log \left(L / L_{\odot}\right) \geq 3.7 ; 5.2 \geq \log \left(T_{\text {eff }}\right) \geq 5.05$, and the position of the clump predicted in the region of hydrogen re-ignition on the helium-burning tracks. 
the planetary nebula nucleus (PNN) leaves the asymptotic giant branch (AGB) (e. g., Wood \& Faulkner 1986). If the PNN leaves the AGB between thermal pulses, then it follows a classical hydrogen-burning path, which consists of a relatively constantluminosity excursion to high temperatures, followed by a fading onto the contraction path to the white dwarfs (e. g., Paczynski 1971; Schönberner 1979, 1981, 1983). On the other hand, if the transition to a PN occurs during a thermal pulse, then the PNN leaves the AGB as a helium-burning object. In this case, the PNN fades as it makes its transition towards high temperatures. The luminosities of the heliumburning PNNi are systematically lower than the hydrogen-burning case except near the point of departure from the AGB and following hydrogen reignition, where the luminosities approach that of equation (5.3). At a certain point, hydrogen is reignited, and the PNn luminosity increases again at almost constant effective temperature. The subsequent evolutionary track lies close to the corresponding hydrogen-burning track (Iben 1984; Wood \& Faulkner 1986). Examples of helium-burning tracks are shown in figure 7.8. In both the hydrogen- and helium-burning cases, the rate of evolution is very strongly dependent upon the core mass. However, this difference is most extreme for the hydrogen-burning objects. In the case of the helium-burning tracks, the re-ignition of hydrogen is associated with a marked slow-down in the rate of evolution. This turns out to be very important, as will be shown in the following section.

\subsection{Calibration of the Planetary Nebula Distance Scale}

One can, in principle, generate an [O III] luminosity function for planetary nebulae (PNLF) purely from theoretical considerations. Starting with some plausible initial mass function over the entire mass range applicable to PN progenitors (1-5 $\mathrm{M}_{\odot}$ ), a scenario for the star formation history of the galaxy considered such as an exponentially decaying star formation rate or random bursts of star formation activity, a present-day stellar mass and age function can be derived. In turn, this can be combined with the evolutionary results given in equations 7.1-7.3 to yield a core-mass and age distribution for the central stars of $\mathrm{PNe}$ for any metallicity. We then require to know whether the $\mathrm{PNNi}$ are hydrogen or helium burning to predict the distribution of objects on the $\mathrm{H}$ $\mathrm{R}$ diagram. Finally, the results of figure 7.2 can be used to predict the corresponding luminosity distribution of objects in the [O III] line. A full analysis of this sort represents an effort beyond the scope of this work but will be attempted in a future paper.

It is more simple, however, to calculate the critical parameter in deriving extra- 
galactic distances: the cutoff in the PNLF luminosity. In this section, we attempt to calibrate this for five specific groups of $\mathrm{PNe}$, those in the Large and Small Magellanic Clouds, the population of PNe in the Galactic Bulge, the PNe in M31, and finally the $\mathrm{PNe}$ in the Virgo Elliptical Galaxies observed by Jacoby and his co-workers.

\subsubsection{The PNe in the Magellanic Clouds}

Since the population of PNe in the Magellanic Clouds is the most completely observed sample of objects at a fixed distance that we have available to us, clearly the calibration of the PN distance scale will depend critically upon this group. The observational H-R diagram for the LMC has been obtained by Dopita and Meatheringham (1991a,b) from nebular modelling. Since the points defined by optically-thick nebulae are liable to be more accurate, we have selected only these for presentation in figure 7.8 . The sample consists of $\sim 50 \%$ of the objects listed by SMP. This sample should be complete in the area surveyed for optically thick nebulae having $\log \left(L / L_{\odot}\right) \geq 3.2$. Since the objects observed were drawn randomly from this sample, then the observed points should be representative of the sample as a whole above the same flux limit.

The distribution of the observed points cannot be explained if the PNNi are hydrogen burning for a number of reasons.

First, since the mean age of the LMC stars is 3-8 Gyr (Stryker \& Butcher 1981; Frogel \& Blanco 1983; Hyland 1991), we might expect to see a large number of $\mathrm{PNe}$ close to a luminosity of $\log \left(L / L_{\odot}\right) \sim 3.65 \rightarrow 3.75$, and a rapid drop in number density above this luminosity both because of the negative slope of the IMF, and because of the rapidly accelerating rate of evolution across the H-R diagram as one goes to higher luminosity on the hydrogen-burning tracks. This is inconsistent with the observed cumulative luminosity distribution.

Second, the clump of points in the vicinity of $4.0 \geq \log \left(L / L_{\odot}\right) \geq 3.7 ; 5.2 \geq$ $\log \left(T_{\text {eff }}\right) \geq 5.05$ has no explanation on the basis of hydrogen-burning models which evolve through this portion of the $\mathrm{H}-\mathrm{R}$ diagram in less than 1000 years.

Third, on the basis of hydrogen-burning models, the type I PNe could not occur in the part of the H-R diagram that they are observed. These stars would have to have initial masses greater than $\sim 2.5 \mathrm{M}_{\odot}$ to be able to evolve PNNi with such high temperatures. These stars are not only rare compared with the lower mass PNNi, but also they evolve exceedingly rapidly in the region $\log \left(T_{\text {eff }}\right)>5.2$, and would fade 
to $\log \left(L / L_{\odot}\right) \leq 2$ within 1000 years. Even if we postulate a violent recent burst of star formation, we could not get the fraction of type I PNe up to the $\sim 25 \%$ which is observed.

All three of these problems has a natural solution if we postulate that the central stars are helium- rather than hydrogen-burners. First, the rate of evolution across the $\mathrm{H}-\mathrm{R}$ diagram up to the point of hydrogen reignition is much less dependent on mass, decreasing only by a factor $\sim 3$ from a $1.0 \mathrm{M}_{\odot}$ track to a $2.5 \mathrm{M}_{\odot}$ track. Second, the rate of evolution slows down dramatically in the vicinity of the hydrogen reignition point. As an example, the $2.5 \mathrm{M}_{\odot} \mathrm{LMC}$ track shown on figure 7.6 evolves from $\log \left(T_{\text {eff }}\right)=4.5$ to the hydrogen reignition dogleg in 2500 years. It then takes a further 5000 years negotiating the dogleg before fading to $\log \left(L / L_{\odot}\right)=2.6$ in another 5000 years. We therefore expect that the density of points observed near the reignition dogleg will be of order 5 times higher than elsewhere. The same argument applies for the tracks of more massive stars giving rise to the type I PNe. However, in these cases, the dogleg occurs on the fading part of the evolutionary track and at lower luminosity.

The slower rate of evolution of the less massive stars is presumably the reason why the hydrogen reignition clump does not extend to lower luminosity for optically-thick nebulae. The nebulae associated with these objects are less massive, and would therefore be expected to have a larger velocity of expansion than higher luminosity $\mathrm{PNe}$ having central stars with the same effective temperature. Given the slower rate of evolution across the H-R diagram, such nebulae will become optically-thin, leading to a dramatic decline in their emission-line luminosities. Such nebulae would be relatively uncommon in the SMP sample, but should be plentiful in the Jacoby (1980) sample. Even within the SMP sample, evidence for such an effect is present when the kinematical data from Dopita et al. (1985) and Meatheringham et al. (1988) is compared with the effective temperatures of Dopita and Meatheringham (1991a, b). Both optically thin and optically thick objects show a linear correlation on the $\left(\mathrm{v}_{\text {exp }}, \log \left(T_{\text {eff }}\right)\right)$ plane, but each has a different slope. At an effective temperature of $\log \left(T_{\text {eff }}\right)=4.4$, the mean expansion velocities of both classes of object are identical, $\sim 6 \mathrm{~km} \mathrm{~s}^{-1}$. However, at a temperature of $\log \left(T_{\text {eff }}\right)=5.0$, the optically thin objects have a mean expansion velocity of $37 \mathrm{~km} \mathrm{~s}^{-1}$, while the optically-thick objects have only reached a mean expansion velocity of $30 \mathrm{~km} \mathrm{~s}^{-1}$.

The result that the PNNi appear to be helium- rather than hydrogen-burning is fundamental to our understanding of PN evolution in general. The thermal pulse which gives rise to helium burning PN last $\sim 10$ times shorter than the interpulse 
duration. Clearly therefore, the enhanced luminosity during the thermal pulse is the most important factor leading to the formation of the PN shell. The enhanced mass-loss produced during the thermal pulse must occur at low velocity in order to be consistent with the observed expansion velocities of $6 \mathrm{~km} \mathrm{~s}^{-1}$ for the youngest PN.

The fact that the hydrogen reignition clump follows a well-defined trajectory in the H-R diagram allows us to calibrate the distance to the LMC by fitting the observed clump onto the trajectory estimated from the stellar models. This has been done in figure 7.8 by increasing the luminosities estimated by Dopita and Meatheringham $(1991 \mathrm{a}, \mathrm{b})$ by +0.06 dex. With this adjustment, the cumulative luminosity function for the optically-thick $\mathrm{PNe}$ is shown in figure 7.9. Fitting a straight line to the upper section gives $\log \left(L_{\max } / \mathrm{L}_{\odot}\right)=4.02 \pm 0.02$. Allowing for a \pm 0.02 dex absolute calibration error in the luminosities used to derive this, and a further \pm 0.02 dex error in conversion of the line flux to stellar flux, we estimate that the distance to the LMC is $47.2 \pm 3.3 \mathrm{kpc}$. Expressed as a distance modulus, this corresponds to $(m-M)_{0}=18.37 \pm 0.15$. This is in good agreement with the revised Cepheid distance modulus of the LMC derived by Feast (1988), $(m-M)_{0}=18.52$.

The age of the stars which determine $L_{\max }$ can be estimated from equations 7.1-7.3 to be $\sim 0.8$ Gyr.

The distance of the SMC can be established in a differential manner with respect to the LMC. The observational value of $\Delta \log \left(F_{*}\right)$ between the LMC and the SMC is $-0.07 \pm 0.03$ from the (raw-cumulative-number, luminosity-plot), or $-0.10 \pm 0.05$ from the corrected version of the same plot. On the other hand, assuming the population which determines $L_{\max }$ has the same age in the SMC as in the LMC, then from equations 7.1$7.3 \Delta \log \left(L_{\max } / L_{\odot}\right)=0.055 \pm 0.010$. From these numbers, the difference in the distance modulii of the two systems will be $0.35 \pm 0.12 \mathrm{mag}$. Thus the distance modulus of the SMC is estimated to be $18.72 \pm 0.17$ corresponding to a distance of $55.5 \pm 4.3 \mathrm{kpc}$. Again, the PN distance scale is somewhat shorter than the Cepheid scale, Feast (1988) finding $(m-M)_{0}=18.83 \pm 0.15$.

We summarise the adopted values of the key parameters which determine the PN distance scale and the distance estimates themselves in table 7.2. 


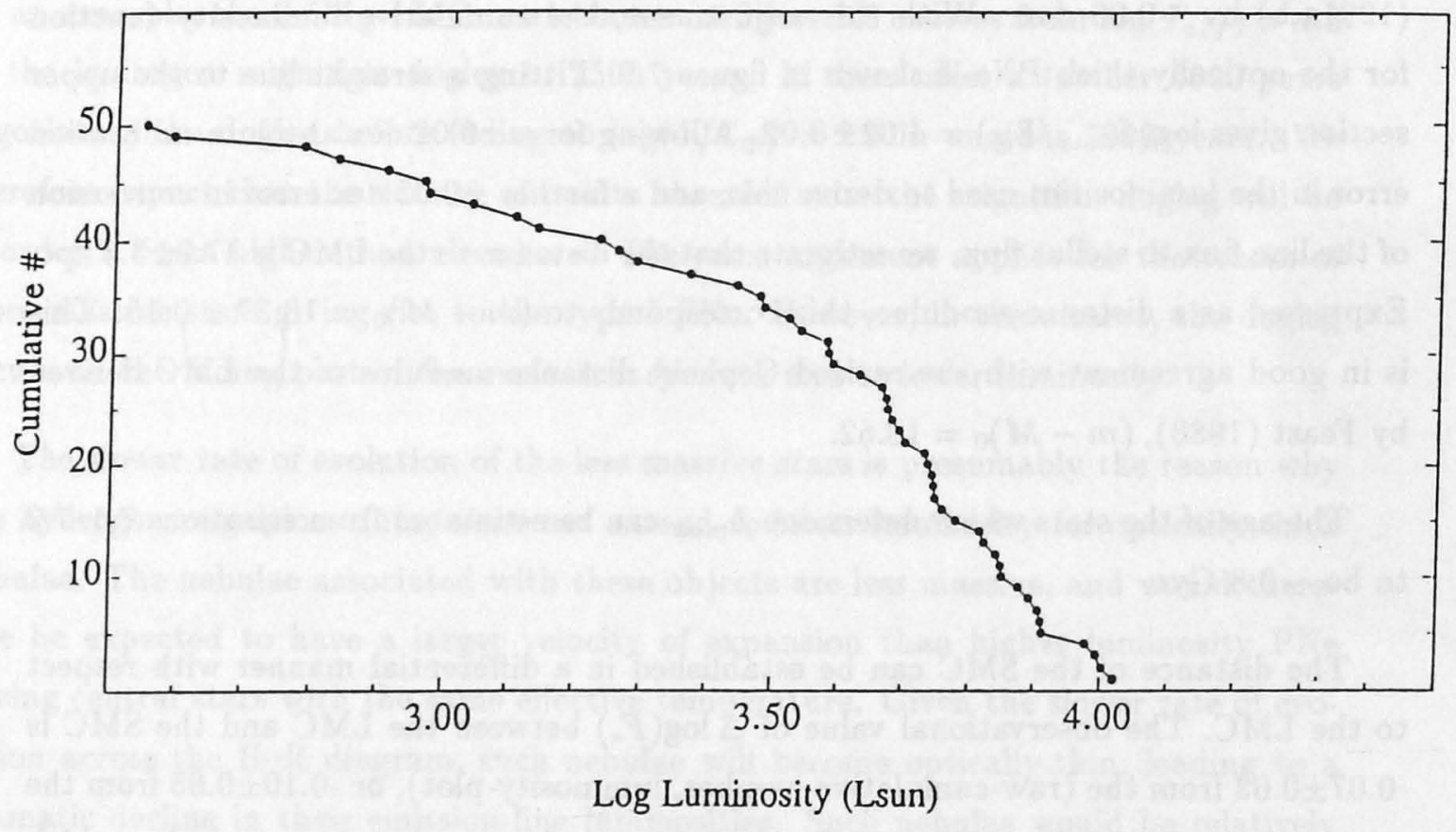

Figure 7.9: The intrinsic cumulative stellar luminosity function derived from the sample of optically-thick nebulae in the LMC shown on figure 7.6. The well defined upper luminosity cutoff is calibrated absolutely, and is used as the basis of the distance estimates given in the remainder of this paper. 


\subsubsection{The PNe in the Galactic Bulge}

The luminosity function of the PNe in the Galactic Bulge can be investigated from their radio continuum emission, in particular, from their emission at $5 \mathrm{GHz}$ (Gathier et al. 1983; Isaacman 1984; Zijlstra 1989; Pottasch 1990). The luminosity function derived by this route is essentially the same as the luminosity function derived from the $\mathrm{H} \beta$ luminosity, since the radio continuum and the $\mathrm{H} \beta$ emission are, to first order, proportional to each other (Pottasch 1984, 1990);

$$
\frac{S(5 G H z)}{F(\mathrm{H} \beta)}=2.82 \times 10^{12}\left[\frac{T_{e}}{10^{4} K}\right]^{0.53} Y
$$

where $S(5 G H z)$ is the radio flux in $\mathrm{mJy}, F(\mathrm{H} \beta)$ is the $\mathrm{H} \beta$ luminosity in ergs $\mathrm{cm}^{-2} \mathrm{~s}^{-1}$, $\mathrm{T}_{e}$ is the electron temperature, and $\mathrm{Y}$ is the number of electrons per hydrogen atom,

$$
Y=1+\frac{N\left(H e^{+}\right)}{N(H)}+2 \frac{N\left(H e^{++}\right)}{N(H)} \text {. }
$$

We have taken the $5 \mathrm{GHz}$ luminosity distribution given in Pottasch (1990), adopted $\overline{T_{e}} \sim 9000 \mathrm{~K}$ from figure 7.3 , used an average $\bar{Y}$ of 1.15 and have assumed a $\mathrm{H} \beta$ luminosity conversion factor of 2.11 from figure 7.1 to derive a cumulative luminosity function for the Galactic Bulge. This is shown in figure 7.10. Once again, the upper portion of the cumulative luminosity function for Galactic Center PNe is a straight line with a well defined upper luminosity cutoff at $\log \left(F_{*}\right)=-8.52 \pm 0.05$. The errors in this estimate do not account for selection effects in the sample, nor does it allow for the variation in the nebular temperature and the degree of helium ionisation from object to object, each of which directly affects the radio flux. These effects are likely to somewhat increase our estimated errors.

The maximum luminosity, $L_{\max }$, is influenced by both the metallicity of the youngest stars in the Galactic Bulge, and also by their age. From the work of Webster (1988), we know that metal-rich PNe are reasonably common in the Bulge, typically of order 1.5 times solar in terms of oxygen abundance. If recent star formation has occurred, and that $L_{\max }$ is not determined by the mean age of the stellar population in the Bulge, and in this case we would only have to take into account the effect of metallicity on core mass in the determinination of $L_{\max }$. However, this is not trivial. To do this, we have used equations 7.2 and 7.3 to derive the ratio of the $L_{\max }$ for the Galactic Center and for the LMC. This procedure carries with it the caveat that equation 7.3 refers to hydrogen-burning stars, rather than helium-burning objects. However, the dogleg in the helium-burning tracks is caused by the re-ignition of hydrogen, which, if 


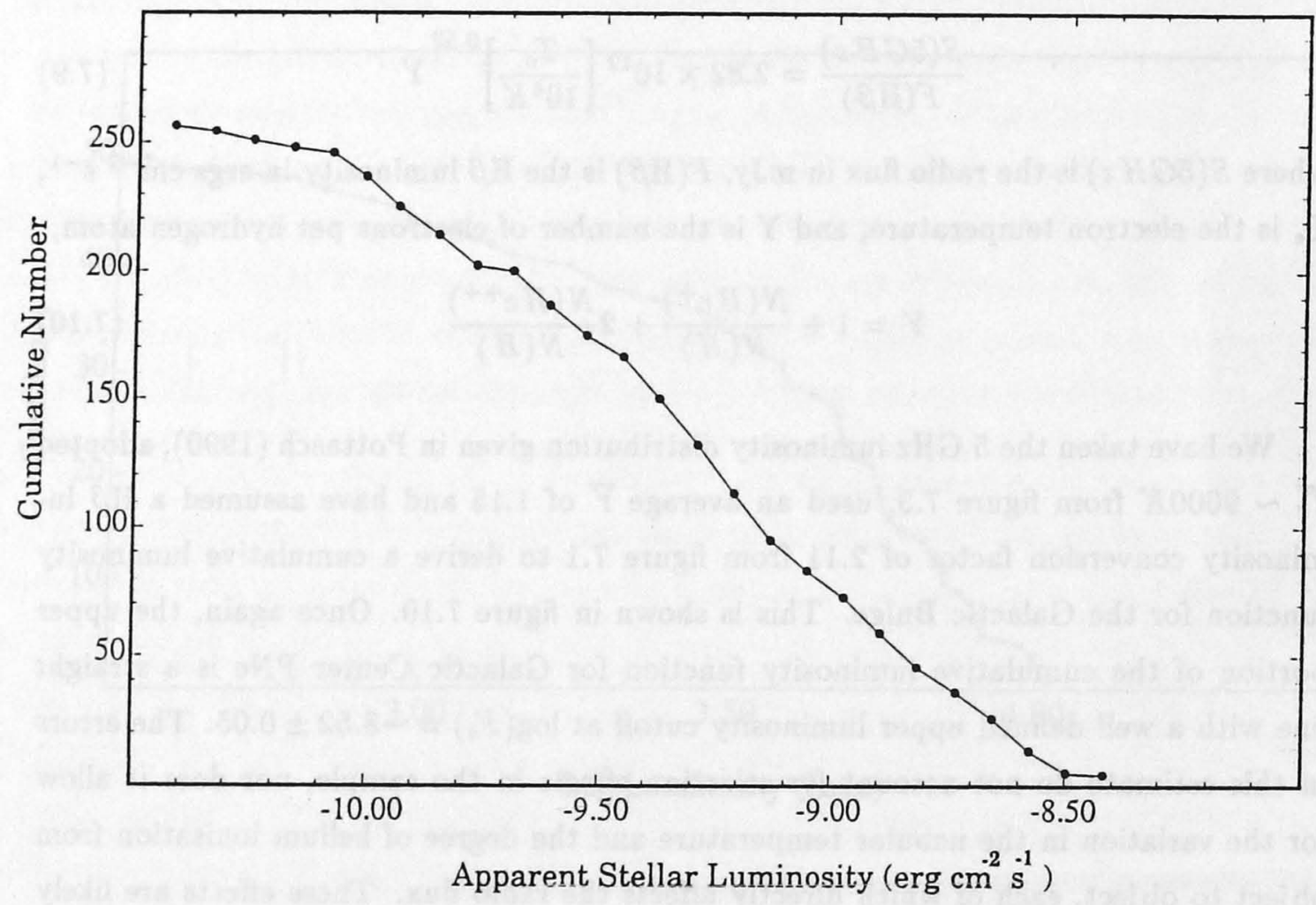

Figure 7.10: The cumulative apparent luminosity function for the PNe in the Galactic Bulge. 
allowed to proceed to equilibrium, would return the PNNi to the corresponding hydrogen burning track. Using a mean $\log [Z]=+0.20$ for the luminous Galactic Bulge PNe, we find that the ratio of the $L_{\max }$ for the Galactic Center to that of the LMC should be $0.78 \pm 0.04$, giving a value of $L_{\max }($ GalacticBulge $)=8100 \pm 1100 L_{\odot}$. This value is somewhat unreliable, because the trajectory followed by the hydrogen reignition dogleg is itself a function of metallicity. If this effect is taken into account, the maximum luminosity may well be as low as $5500 \mathrm{~L}_{\odot}$.

In fact, both of these estimates will be too high if the population giving rise to the most luminous $\mathrm{PNe}$ is old. Just how old can be estimated from the dynamical age of the $\mathrm{OH}-\mathrm{IR}$ stars which, in a metal-rich environment like the Galactic Bulge, are presumably the immediate precursors of PNe. This has been done by te Lintel Hekkert (1990), who found that the dynamically young group of $\mathrm{OH}-\mathrm{IR}$ stars have a mean dynamical age of 5 Gyr. In this case, from equations 7.1-7.3 $L_{\max }($ GalacticBulge $)=5450 \pm 1000 L_{\odot}$. We adopt this value as our best estimate guess for $L_{\max }$. Clearly, any improvement in the theoretical estimate of $L_{\max }$ would allow a corresponding improvement in the distance estimate, since the observational cuttoff is far better determined than its theoretical value.

In table 7.2 , we give the distance estimate with these two extreme assumptions about the stellar age to show the sensitivity of our results on this parameter. Our adopted distance to the Galactic Bulge is $7.6 \pm 0.7 \mathrm{kpc} ;(m-M)_{0}=14.4 \pm 0.2$. This is in good agreement with the value of $7.5 \pm 0.9 \mathrm{kpc}$ derived from globular clusters by Racine and Harris (1989), or the mean value of $7.7 \pm 0.7$ (Reid 1989), but clearly the older value of $\sim 8.5 \mathrm{kpc}$ (Harris 1976; Gunn et al. 1979; Quiroga 1981) is not excluded by our estimate.

\subsubsection{The PNe in M31}

The rich PNe system in M31 and its companion dwarf elliptical galaxies has been very extensively studied by Ciardullo et al. (1989). The cutoff which they derived was converted to an apparent stellar luminosity cutoff, $F_{*}$, by correcting for mean extinction and for metallicity. For the reddening correction, we have taken $A(5007)=$ $3.56 E(B-V)$ and have used an mean $E(B-V)$ of 0.11 .

The correction for metallicity is somewhat more difficult, since although the $[\mathrm{Fe} / \mathrm{H}]$ is quite well known, the $[\mathrm{O} / \mathrm{H}]$ is not so well determined. We have taken $[\mathrm{O} / \mathrm{H}]=0.3$. However, errors in this quantity will make little difference to the conversion efficiency of 
stellar flux to [O III] flux, since equation 7.4 and figure 7.2 both indicate that the conversion efficiency is near its maximum. In this case, the conversion efficiency to $\lambda 5007 \AA$ luminosity is $13.7 \%$ and remains constant to within $15 \%$ for metallicities between twice solar and one fourth solar. These estimates are slightly increased if the PNNi did not evolve from $\mathrm{C}$ stars, which is likely in this higher metallicity regime. It is well known that in the Galaxy, for example, the ratio of the oxygen-rich and carbon-rich AGB stars is a strongly changing function of Galactocentric radius (Habing 1988; Jura \& Kleinmann 1989, 1990). Within the solar circle, the large majority of AGB stars are of the oxygen-rich type. If the $\mathrm{C} / \mathrm{O}$ ratio is taken to be solar $(0.6)$ rather than 1.5 , the maximum luminosity conversion factor is increased by a factor of 1.09 to $15 \%$. We have adopted this value to derive the figure given in table 7.2 .

As for the Galactic Bulge, the age of the stars giving rise to the most luminous $\mathrm{PNe}$ remains the most uncertain factor. In table 7.2, we show the effect of assuming that star formation terminated $10 \mathrm{Gyr}$ ago compared with the assumption that the youngest population has the same age as the OH-IR sources in our own Galaxy, 5 Gyr. This makes a $0.28 \mathrm{mag}$ difference in distance modulus. Since we have no reason to prefer either of these estimates, we adopt the mean, with correspondingly greater error.

Once again, we find the PN distance scale to be somewhat smaller than the Cepheid distance (Welch et al. 1986; Freedman \& Madore 1990); by $0.18 \mathrm{mag}$ in distance modulus. From the comparison of M31, LMC, and the SMC, it is clear that there is a systematic and metallicity independent zero-point shift of $0.14 \mathrm{mag}$, on average, between the two distance scales.

\subsubsection{The PNe in the Virgo Ellipticals}

The PNe in Virgo Ellipticals have been studied by Jacoby, Walker and Ciardullo (1990). In table 7.2 , we have derived the mean apparent stellar luminosity cutoff, $F_{*}$, in exactly the same fashion as for M31.

Once again, effectively all the uncertainty in the distance derivation is in the calculation of the intrinsic luminosity, $L_{\max }$, of the stellar population which currently defines $F_{*}$. In table 7.2 , we investigate two extreme assumptions, first, that this population is like that of the Galactic Bulge, and second, that it is a $10 \mathrm{Gyr}$ population with $[\mathrm{Fe} / \mathrm{H}]=$ +0.3 . This makes a difference of some $0.6 \mathrm{mag}$. Again, at the current time we have no reason to prefer one above the other of these assumptions, so we take the mean, giving the PN distance to the Virgo Cluster of $15.0 \pm 2.0 \mathrm{Mpc}$, or $(m-M)_{0}=30.86 \pm 0.30$. 
This result is in startlingly good agreement with that found by Jacoby, Walker and Ciardullo (1990), $(m-M)_{0}=30.84$. However, this should not be so surprising, as this distance was found by comparing M31 and Virgo in a diffential manner. Clearly, the stellar populations of these two systems are sufficiently similar for such a procedure to be valid. Our distance is also in good agreement with that given by the Tully-Fisher method, $(m-M)_{0}=30.85 \pm 0.40$, but is somewhat short compared with those derived by a number of other methods (see review by van den Bergh, 1989). We can shift our PN distance scale to a Cepheid distance scale by adding the +0.14 mag. offset found above, to give $D_{0}=15.8 \pm 2.1 M p c$, or $(m-M)_{0}=31.00 \pm 0.30$. This still gives a short distance scale, with a Hubble Constant (from Visvanathan 1990) of $84 \pm 11$ $\mathrm{km} \mathrm{s}^{-1} \mathrm{Mpc}^{-1}$.

Based on this discussion, it is clear that, by making the appropriate corrections for metallicity and age, we have established relative distances of the LMC, the SMC, the Galactic Bulge, M31 and the Virgo cluster by the use of the cutoff in the PNLF. This calibration can be derived equally well from the [O III] or Balmer line fluxes. Distances so derived are, within the errors, similar to those of the Cepheid distance scale, where available. We conclude that the techniques for deriving extragalactic distances are well based upon stellar evolution and nebular plasma theory.

\subsection{Conclusions}

In this paper, we have shown that nebular modelling allows us to correct for metallicity and excitation effects to derive intrinsic apparent stellar luminosity functions of PNn using either observations in the [O III] lines, or in the Balmer lines (or, equivalently, in the radio continuum). From these, it is clear that the upper luminosity cutoff is a good measure of the distance to the galaxy, provided that corrections for the age of the stellar population, and its metallicity can be accounted for.

This we have done, using theoretical evolutionary tracks which self-consistently deal with the evolution from the main sequence all the way through to the PN phase. Applying these to the observational H-R diagram of the LMC, and the luminosity functions of the LMC, SMC, Galactic Bulge, M31, and Virgo cluster populations, we have been sucessful in deriving distance modulii to each of these systems.

We find that the PN distance scale shows a systematic zero-point offset with respect to the Cepheid distance scale which appears to be independent of the metallicity or age 
of the parent stellar population.

The PN results favour a short distance scale on which the Virgo cluster lies within the range 13-18 kpc, and, correcting for the Cepheid zero-point offset, we find the Hubble Constant is to be $84 \pm 11 \mathrm{~km} \mathrm{~s}^{-1} \mathrm{Mpc}^{-1}$.

Further refinement of the PN distance scale is dependent not so much upon the accuracy with which the upper luminosity cutoff can be established, but rather on a knowledge of the theoretical luminosity of the stars which are responsible for this cutoff. This in turn depends upon an accurate understanding of the age of this stellar population, and upon the core mass / initial mass relationship. These improvements are principally dependent upon the theory of stellar mass-loss and its dependence upon metallicity. 
TABLE 7.2

The Planetary Nebula Distance Scale.

\begin{tabular}{|c|c|c|c|c|c|c|c|c|}
\hline Galaxy & $\log [\mathrm{Fe} / \mathrm{H}]$ & $\mathrm{E}(\mathrm{B}-\mathrm{V})$ & $\begin{array}{r}\log \\
\text { (app.) }\end{array}$ & $\begin{array}{l}F_{*} \\
\text { (true) }\end{array}$ & $\begin{array}{r}\text { Age } \\
\text { (Gyr) }\end{array}$ & $\log \left(L_{\max } / L_{\odot}\right)$ & $\overline{(m-M)}$ & $\begin{array}{l}\mathrm{D} \\
(\mathrm{kpc}) \\
\end{array}$ \\
\hline Gal. Bulge & +0.2 & $\ldots$ & -8.50 & -8.50 & $\begin{array}{l}0.8 \\
5.0\end{array}$ & $\begin{array}{l}3.910 \\
3.737 \\
\text {. Adopted: }\end{array}$ & $\begin{array}{l}14.80 \\
14.36 \\
14.4 \pm 0.2\end{array}$ & $\begin{array}{l}9.11 \\
7.46 \\
7.6 \pm 0.7\end{array}$ \\
\hline LMC & -0.32 & 0.18 & -10.03 & -9.82 & 0.8 & $\begin{array}{c}4.020 \\
\text { Cepheid }^{1,2,3} \text { : }\end{array}$ & $\begin{array}{l}18.37 \pm 0.15 \\
18.51 \pm 0.15\end{array}$ & $\begin{array}{l}47.2 \pm 3.3 \\
50.3 \pm 3.4\end{array}$ \\
\hline SMC & -0.64 & 0.17 & -10.10 & -9.90 & 0.8 & $\begin{array}{l}4.075 \\
\text { Cepheid }\end{array}$ & $\begin{array}{l}18.72 \pm 0.17 \\
18.83 \pm 0.15\end{array}$ & $\begin{array}{l}55.5 \pm 4.3 \\
58.3 \pm 3.9\end{array}$ \\
\hline M31 & +0.3 & 0.11 & -12.59 & -12.59 & $\begin{array}{r}5.0 \\
10.0\end{array}$ & $\begin{array}{l}3.614 \\
3.500 \\
\text {..Adopted: } \\
\text {. Cepheid }{ }^{4,5} \text { : }\end{array}$ & $\begin{array}{l}24.28 \\
24.00 \\
24.14 \pm 0.20 \\
24.32 \pm 0.20\end{array}$ & $\begin{array}{l}718 \\
630 \\
673 \pm 60 \\
730 \pm 50\end{array}$ \\
\hline Virgo & $\begin{array}{l}+0.2 \\
+0.3\end{array}$ & 0.02 & -15.21 & -15.21 & $\begin{array}{r}5.0 \\
10.0 \\
\ldots \ldots \\
\ldots .0 n \\
\end{array}$ & $\begin{array}{l}\quad 3.737 \\
\quad 3.500 \\
\text {... Adopted: } \\
\text { epheid scale: }\end{array}$ & $\begin{array}{l}31.14 \\
30.55 \\
30.86 \pm 0.30 \\
31.00 \pm 0.30 \\
\end{array}$ & $\begin{array}{l}16900 \\
12900 \\
15000 \pm 2000 \\
15800 \pm 2100\end{array}$ \\
\hline
\end{tabular}

References:

1. Feast, M.W. 1988, in The Extragalactic Distance Scale, ASP Conference Series No. 4, eds. S. van den Bergh and C.J. Pritchet, Brigham Young U. Press:Provo, p9.

2. Laney, C.D. and Stobie, R.S. 1988, quoted in reference 1.

3. Feast, M.W., and Walker, A.R. 1987, Ann. Rev. Ast. Ap., 25, 345.

4. Welch, D.L., McAlary, C.W., McLaren, R.A., and Madore, B.F., 1986, Ap.J., 305, 583.

5. Freedman, W. L., and Madore, B.M. 1990, Ap.J., 365, 186. 


\subsection{References}

Binette, L., Dopita, M. A., \& Tuohy, I. R. 1985, ApJ, 297, 476

Brocato, E., Matteucci, F., Mazzitelli, I., \& Tornambè 1990, ApJ, 349, 458

Caldwell, J.A.R., \& Coulson, I.M. 1986, MNRAS, 218, 223

Castellani, V., Chieffi, A., Pulone, L., \& Tornambe, A. 1985, ApJ, 296, 204

Caughlan, G.R., \& Fowler, W.A. 1988, Atomic \& Nuclear Data Tables, 40, 283

Cerruti-Sola, M., \& Perinotto, N. 1985, ApJ, 291, 237

Ciardullo, R., Jacoby, G.H., Ford, H.C., \& Neill, J.D. 1989, ApJ, 339, 53

Conti, P.S., Garmany, C.D., \& Massey, P. 1986, AJ, 92, 48

Dopita, M.A., Ford, H.C., Lawrence, C.J., \& Webster, B.L. 1985, ApJ, 296, 390

Dopita, M.A. \& Meatheringham, S. J. 1990, ApJ, 357, 140

Dopita, M.A. \& Meatheringham, S. J. 1991a, ApJ, 367, 115 ., 1991b, ApJ, 377, 480

Dopita, M. A., Vassiliadis, E., \& Morgan, D. H., 1992, (in preparation)

Evans, I. N., \& Dopita, M. A. 1985, ApJS, 58, 125

Feast, M.W. 1988, in The Extragalactic Distance Scale, ASP Conference Series No. 4, ed. S. van den Bergh \& C.J. Pritchet (Provo: Brigham Young Univ.), p. 9

Feast, M.W., \& Walker, A.R. 1987, ARA\&A, 25, 345

Freedman, W. L., \& Madore, B.M. 1990, ApJ, 365, 186

Frogel, J.A., and Blanco, V.C. 1983, Ap. J., 274, L57.

Gabler, R., Kudritski, R.P., \& Mendez, R. 1991, in Stellar Atmospheres:

Beyond Classical Models, ed. L. Crivellari, I. Hubeni \& D.G. Hummer, NATO ASI Series, p. 143

Gascoigne, S. C. B. 1969, MNRAS, 146, 1

Gathier, R., Pottasch, S.R., Goss, W.M., \& van Gorkam, J.H. 1983, A\&A, 128,325

Gunn, J.E., Knapp, G.R., \& Tremaine, S.D. 1979, AJ, 84, 1181

Habing, H.J. 1988, A\&A, 200, 40

Harm, R., \& Schwarzschild, M. 1975, ApJ, 200, 324

Harris, W.E. 1976, AJ, 81, 1095

Heubner, W.F., Merts, A.L., Magee, N.H., \& Argo, M.F. 1977, Astrophysical Opacity Library, Los Alamos Scientific Observatory Report, LA6760-M. 
Hutsemekers, D., \& Surdej, J., 1989, A\&A, 219, 237

Hyland, A.R. 1991, IAU Symp. 148, in The Magellanic Clouds, eds R.

Haynes \& D. Milne, (Kluwer: Dordrecht), p. 125

Iben, I., Jr. 1984, ApJ, 277, 333

Isaacman, R. 1984, MNRAS, 208, 399

Jacoby, G. H. 1980, ApJS, 42, 1

Jacoby G.H., 1989, ApJ, 339, 39

Jacoby, G. H., Ciardullo, R., \& Ford, H. C. 1988, in The Extragalactic

Distance Scale, ASP Conf. Ser., vol 4, ed. S. van den Bergh \& C. J.

Pritchet (Bringham Young U.: Provo), p. 42

Jacoby, G.H., Ciardullo, R., \& Ford, H.C. 1990, ApJ, 356, 332

Jacoby, G.H., Walker, A. R., \& Ciardullo, R. 1990, ApJ, 365, 471

Jura, M., \& Kleinmann, S.G., 1989, Ap. J., 341, 359. 1990, ApJ, 364, 663

Laney, C. D., \& Stobie, R. S. 1986, MNRAS, 222, 449

Lattanzio, J.C. 1986, ApJ, 311, 708

Meatheringham, S.J., Dopita, M.A., \& Morgan, D.H. 1988, ApJ, 329, 166

Meatheringham, S.J., \& Dopita, M.A.1991a, ApJS, 75, 407 , 1991b, ApJS, 76, 1085

Monk, D.J., Barlow, M.J., \& Clegg, R.E.S. 1988, MNRAS, 234, 583

Morgan, D.H. 1984, MNRAS, 208, 633

Paczyński, B. 1971, Acta Astron., 21, no. 4, p. 417

Pauldrach, A., Puls, J., Kudritski, R.P., Mendez, R.H., \& Heap, S.R., 1988, A\&A, 207, 123

Pottasch, S. R. 1984, Planetary Nebulae: a study of late stages of stellar evolution, Astrophys. \& Space Sci. Lib., vol. 107 (Kluwer: Dordrecht)

Pottasch, S. R. 1990, A\&A, 236, 231

Pottasch, S. R., \& Acker, A., 1989, A\&A, 221, 123

Quiroga, R.J. 1980, A\&A, 92, 186

Racine, R., \& Harris, W. E. 1989, AJ, 98, 1609

Reid, N. 1989, Astrophys. Space Sci., vol. 156, p. 73

Russell, S.C., \& Bessell, M.S. 1989, ApJS, 70, 865

Russell, S.C., \& Dopita, M.A. 1990, ApJS, 74, 93

Russell, S.C., \& Dopita, M.A. 1991, ApJS, (in press)

Sanduleak, N., MacConnell, D.J., \& Philip, A.G.D. 1978, PASP, 90, 621 (SMP) 
Sandage, A., \& Tammann, G.A. 1990, ApJ, 365, 1

Schönberner, D. 1981, A\&A, 103, 119

Schönberner, D. 1983, A\&A, 272, 708

Shaw, R.A. 1989 in IAU Symp. 131, Planetary Nebulae, ed. S. Torres-

Piembert (Kluwer:Dordrecht), p. 473

Schonberner, D. 1981, A\&A, 103, 119

Stryker, L.L., \& Butcher, H.R. 1981, in IAU Colloq. 68, ed. A.G. Davis Philip \& D.S. Hayes, (L. Davis: Schenectady), p255.

te Lintel Hekkert, P. 1990, The Evolution of OH/IR stars \& their Dynamical Properties, Ph. D. Thesis, Sterrewacht Leiden, University of Leiden.

Tylenda, R., Stasinska, G., Acker, A., \& Stenholm, B. 1991, A\&A, 246, 221 van den Bergh, S. 1989, in The Extragalactic Distance Scale, ASP Conf.

Ser., vol. 4, ed. S. van den Bergh \& C. J. Pritchet (Bringham Young Univ.: Provo), p. 375

Visvanathan, N. 1990, Australian J. Phys., 43, 189

Vassiliadis, E., \& Wood, P.R. 1992, in preparation (Chapter 2)

Webster, B.L. 1988, MNRAS, 230, 377

Welch, D.L., McAlary, C.W., McLaren, R.A., \& Madore, B.F., 1986, ApJ, 305,583

Wood, P.R., 1991, in Frontiers of Stellar Evolution, ASP Conf. Ser., (in press)

Wood, P.R., \& Faulkner, D.J. 1986, ApJ, 307, 659

Wood, P. R., Meatheringham, S. J., Dopita, M. A., \& Morgan, D. H. 1987, ApJ, 320, 178

Wood, P. R., \& Zarro, D. M. 1981, ApJ, 247, 247

Zijlstra, A.A. 1989, A Radio Study of Planetary Nebulae, Ph.D. Thesis, University of Groningen. 


\section{Chapter 8}

\section{Summary and Suggestions for Further Work}

In this thesis dissertation, a wide range of aspects of the evolution from the the AGB phase into the PN regime has been studied. It is evident that a clear evolutionary path is not a trivial exercise to determine: There are many observational facts ranging from stellar nucleosynthesis to dynamical evolution of circumstellar material that must be reconciled into a generalized scenario of evolution.

We have presented a grid in mass and metallicity of AGB evolutionary calculations, believed to be representative of the observed Mira variables and OH/IR stars seen in the Galaxy and the Magellanic Clouds. The calculations clearly showed that a superwind phase develops over the later stages of AGB evolution, and its extent critically depends on the initial main sequence mass, and subsequently core mass. The calculations accurately predict the luminosities at the tips of AGBs in Magellanic Cloud clusters.

Our calculations failed to reproduce the existence of carbon stars at the low luminosities observed in the Magellanic Clouds. This suggests that convection was not sufficiently efficient in dredging carbon-rich material from the stellar interior to the surface. Some authors have noted this problem previously, and have accordingly increased the mixing-length to pressure-scale-height ratio in some artificial manner. An alternative is to consider a formalism such as envelope undershoot (Alongi et al. 1991). Convective extension is important when deep envelope convection is present on the first and asymptotic giant branches. New opacities (Rogers \& Iglesias 1992) may also help in making convection more efficient at low luminosities.

Following from the AGB calculations described above, we have presented a wide 
range (again, in mass and metallicity) of PNN evolutionary tracks calculated from models evolved continuously from the main sequence. These evolutionary tracks span a range in metallicity so that valid analyses can be made of stellar populations which are metal poor with respect to the Sun.

The next step to be undertaken with the calculations we have presented is to convolve them with an initial mass function and star formation rate appropriate for the LMC. This will yield a theoretical luminosity function which can be compared with the available observations. This, in turn, may help in distinguishing whether PNNi leave the AGB primarily burning hydrogen or helium. Similarly, the PNN tracks presented can be used in a model of galactic evolution (Brocato et al. 1989) to discern the relative energy contributions of $\mathrm{H}$ and He burning PNNi and explain the UV excess seen in elliptical galaxies.

A long standing problem of studying the evolution of PNe is obtaining a complete luminosity-limited sample of objects. This is being currently addressed, primarily by Dopita and coworkers for the Magellanic Clouds, and Pottasch and coworkers for the Galactic Bulge. Low extinction along the line-of-sight towards the Magellanic Clouds means that fainter PNNi can be observed in the Clouds than in the Galactic Bulge, despite the former being further away. Accurate spectrophotometry has allowed objects to be identified as $\mathrm{PNe}$ and to be placed in the H-R diagram through the use of photoionisation modelling techniques. During the observing time available during this thesis, we only managed to observe a quarter of the latest list of possible PN candidates in the LMC published by Morgan and Good (1992). This means that there are at least 60 objects that remain to be observed which, if they are true PNe, have nuclei down to $\sim 100 \mathrm{~L}_{\odot}$. Naturally, the Hubble Space Telescope will prove an important tool for studying these faint PNe.

A further test of the hydrostatic evolutionary sequences calculated in this thesis is to examine the hydrodynamics of the expelled material during both the AGB and PN phases. We have conducted a preliminary investigation of the wind behaviour on the AGB over nearly two helium shell flash cycles. Ideally, this should be repeated with more mesh points to follow the development of the shock features, and for more helium shell flashes to examine the occurrence of multiple circumstellar shells. The hydrodynamic behaviour of the expelled material can also be examined during the PN phase when the photoionisation and fast wind effects need to be taken into account. The evolution of the central star would be described by the theoretical evolutionary tracks presented in this thesis. A major use of this work would be to constrain the 
AGB to PN transition time.

There is clearly still a large volume of work to complete, both observationally and theoretically, to provide an accurate description of the evolution from an AGB star to a PN.

8.1 References

Alongi, M., Bertelli, G., Bressan, A., \& Chiosi, C. 1991, in IAU Symp. 148, The Magellanic Clouds, eds. R. Haynes, \& D. Milne (Dordrecht: Kluwer), 327

Brocato, E., Matteucci, F., Mazzitelli, I., Tornambè, A. 1989, Ap\&SS, 156, 159

Morgan, D. H., \& Good, A. R. 1992, A\&AS̄, 92, 571

Rogers, F. J., \& Iglesias, C. A. 1992, ApJS, 79, 507

281 